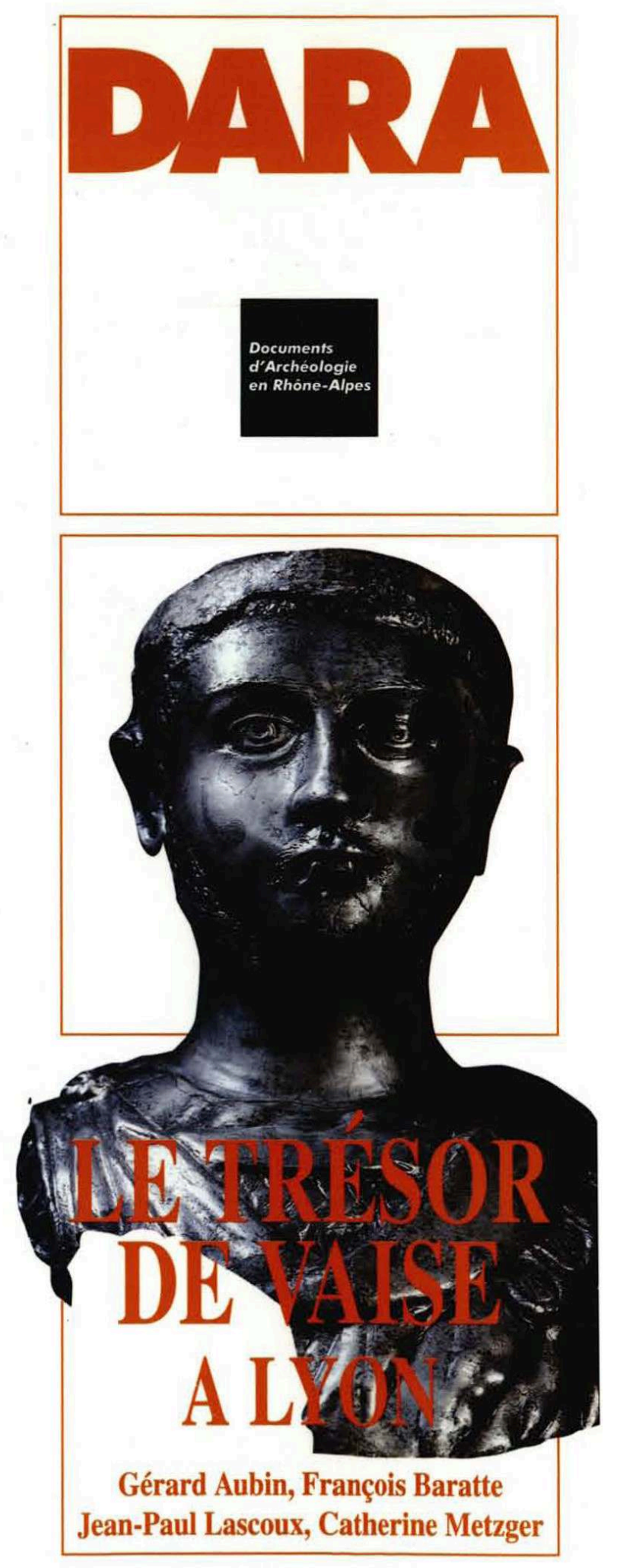




\section{Le trésor de Vaise à Lyon (Rhône)}

\section{Gérard Aubin, François Baratte, Jean-Paul Lascoux et Catherine Metzger}

DOI : 10.4000/books.alpara.1946

Éditeur : Alpara

Lieu d'édition : Lyon

Année d'édition : 1999

Date de mise en ligne : 2 juin 2016

Collection : DARA

ISBN électronique : 9782916125411

\section{Sbooks}

http://books.openedition.org

\section{Édition imprimée}

ISBN : 9782906190214

Nombre de pages : 192

\section{Référence électronique}

AUBIN, Gérard ; et al. Le trésor de Vaise à Lyon (Rhône). Nouvelle édition [en ligne]. Lyon : Alpara, 1999

(généré le 12 janvier 2021). Disponible sur Internet : <http://books.openedition.org/alpara/1946>. ISBN : 9782916125411. DOI : https://doi.org/10.4000/books.alpara.1946.

Ce document a été généré automatiquement le 12 janvier 2021. Il est issu d'une numérisation par reconnaissance optique de caractères.

(c) Alpara, 1999

Conditions d'utilisation :

http://www.openedition.org/6540 
Un trésor parle toujours à l'imaginaire : outre sa valeur intrinsèque, la noblesse des matériaux et l'esthétique des objets qui le composent ne manquent pas de frapper l'esprit qui ne peut s'empêcher d'évoquer le destin du propriétaire, disparu sans récupérer ses biens cachés en prévision de jours meilleurs...

Au-delà de la part du mystère, la découverte du trésor de Vaise dans le cadre d'une fouille archéologique préventive - celle qui a précédé la réalisation de la ZAC Charavay - et sa composition - statuettes, bijoux, vaisselles et monnaies - permettent de mettre en relation le contexte de son enfouissement avec les recherches menées par des spécialistes de ces diverses catégories d'objets, mais encore avec les étapes de sa patiente restauration.

Trésor d'un sanctuaire, magot d'un antique Harpagon ou bien familial patiemment épargné, réserve d'un orfèvre destinée à la refonte ou butin d'une bande de voleurs ? Sans pour autant apporter de réponse définitive, cette étude aborde ces différentes hypothèses. Elle est aussi l'occasion de comparer ce trésor à des découvertes analogues, en particulier celles survenues à Lyon dans le passé, et de le replacer dans une perspective historique. 
SOMMAIRE

\section{Remerciements}

Les auteurs

Préface

Robert Turcan

Introduction

L'environnement archéologique du trésor de Vaise Jean-Paul Lascoux, Pierre Jacquet et Woijtek Widlak

Le site de Charavay

Le site et son environnement

Les dépôts

\section{Catalogue raisonné du trésor de vaise}

La vaisselle du trésor de Vaise

François Baratte

1. Coupe

2. Petit plateau

3. Petit plateau

4-19. Les cuillers et les petits ustensiles

La statuaire du trésor de Vaise

François Baratte

Les bijoux du trésor de Vaise

Catherine Metzger

33. Bracelet

34. Bracelet

35. Collier

36. Paire de bracelets

37. Paire de pendants d'oreilles

38. Paire de pendants d'oreilles

39. Bague

40. Bague

41. Médaillon monétaire

Les monnaies du trésor de Vaise Gérard Aubin

Catalogue des monnaies

Nature et datation du trésor de Vaise à Lyon

Un ou deux dépôts?

Annexe

François Planet 
Bibliographie

Crédits des illustrations (type annexe)

Résumé

SUMMARY

RESUMEN

RIASSUNTO 


\section{Remerciements}

1 Comme toute recherche, celle-ci n'aurait pu aboutir sans l'aide généreuse de nombreux collègues auxquels nous tenons ici à exprimer notre gratitude: M. J. Lasfargues, tout d'abord, qui a bien voulu nous accueillir avec patience et confiance au Musée de la civilisation gallo-romaine, à Lyon, pour examiner à plusieurs reprises les objets du trésor ; le Service archéologique municipal de la ville de Lyon pour son étroite collaboration et son concours dans le domaine de l'iconographie.

2 Mais aussi tous ceux qui ont accepté de se pencher très amicalement sur les questions que nous leur avons soumises ou qui nous ont fourni très libéralement informations, documents ou photographies: M. Amandry (Directeur du cabinet des médailles, BNF, Paris), J.-Ch. Balty (Professeur à l'université de Paris IV), F. Bérard (ENS, Paris), M. Bergmann (Professeur à l'université de Gottingen), H. A. Cahn (Bâle), M. Christol (Professeur à l'université de Paris I), D. Cliquet (Conservateur du patrimoine au Service régional de l'archéologie de Basse-Normandie), K. Fittschen (Directeur de l'Institut archéologique allemand à Athènes), H. Guiraud (Professeur à l'université de Toulouse Le Mirail), R. Hanoune (Université Charles de Gaulle - Lille III), C. Johns (Conservateur au British Museum à Londres), A. Kaufmann (Archäologisches Seminar der Universität, Bâle), E. Kiinzl (Directeur de la section romaine du Romische Germanisches Zentralmuseum à Mayence), J. Meissonnier (Conservateur du patrimoine au Service régional de l'archéologie de Bourgogne), F. Planet (Chargé du médaillier de Lyon), G. Platz (Conservateur au Staatliche Antikensammlung de Berlin) et P. Zanker, (Directeur de l'Institut archéologique allemand de Rome).

3 Enfin, nos derniers remerciements vont à M. Ch. Thioc (MCGR), qui a effectué la plupart des clichés des objets du trésor de Vaise. 


\section{Les auteurs}

1 Gérard Aubin : Inspecteur général de l'archéologie.

2 François Baratte : Professeur à l'université de Paris IV-Sorbonne.

3 Jean-Paul Lascoux : Ingénieur au Service archéologique municipal de Lyon.

4 Catherine Metzger: Conservateur en chef au Département des antiquités grecques, étrusques et romaines du Musée du Louvre, Paris.

5 Xavier Loriot: Maître de conférences à l'université de Paris IV-Sorbonne.

6 Pierre Michel : Professeur émérite à l'université Claude Bernard, Lyon I.

7 François Planet : Chargé du médaillier du Musée des Beaux-Arts, Lyon.

8 Atelier de restauration G. Chapotat, C.R.E.A.M. à Vienne (Isère) : P. Chantriaux, M.-H Kappes, V. Langlet-Marzloff, J.-B. Latour, P. Pliska.

9 La coordination de cet ouvrage a été assurée par Gérard Aubin 


\section{Préface}

\section{Robert Turcan}

1 Un "trésor" a toujours le double prestige de son contenu et de son mystère. Dans un musée, le prix même du contenu - espèces monétaires, objets ouvrés dans un métal noble - s'impose à l'œil et à l'esprit du visiteur, qui songe à la valeur qu'avait pour son propriétaire cet échantillon d'une fortune plus ou moins notable, serré, mis au secret en attendant des jours meilleurs. Mais qui sait, après tout? Ne s'agirait-il point du produit d'un vol provisoirement dissimulé ? d'un recel de pièces et (ou) de bijoux destinés à la fonte? Les interrogations surgissent et pullulent sur les raisons, les circonstances de cette dissimulation intentionnelle, comme sur les causes qui ont pu empêcher le dissimulateur de venir rechercher son précieux dépôt.

Dans le cas de numéraire, les composantes de la "cachette" fournissent des indices sur la nature du lot : caisse militaire, économies d'un particulier, prélèvement ponctuel sur la circulation en cours, sélection d'un amateur (car l'Antiquité eut elle aussi ses "collectionneurs")... L'état des monnaies et la date des plus récentes nous informent parfois assez précisément sur le temps et la fin d'une thésaurisation.

Dans le cas de vaisselle ou de statuaire, la fonction culinaire, votive ou cultuelle des objets, leur homogénéité ou leur disparité soulèvent d'autres questions. De toute façon, le terrain de la trouvaille nous apparaît alors comme tout aussi précieux que le dépôt lui-même.

4 Rares, au total, demeurent les trésors dont la découverte est matériellement bien située, dans le chantier d'une fouille programmée et menée à son terme. Celui de la place Camille-Jouffray à Vienne (Isère) en était récemment un excellent exemple. Celui de Vaise présente non seulement l'intérêt majeur d'un trésor mixte (monnaies et objets, en deux parties apparemment solidaires), mais l'avantage d'être strictement localisé, dans un contexte dûment étudié et analysé par les archéologues du secteur. N'oublions pas cependant la définition du Code civil (art. 716) : "Le trésor est toute chose cachée ou enfouie... découverte par le pur effet du hasard". Même dans une intervention programmée, le hasard a sa part. Apparu sous le godet d'un engin mécanique actionné pour "l'enlèvement d'une berme située contre et sous un mur moderne", le premier lot de Vaise a évidemment surpris les fouilleurs et, malgré la 
vérification attentive des déblais, ils n'écartent pas "l'éventualité que quelques fragments aient pu échapper" à leur vigilance : ce qui expliquerait l'état incomplet de certains éléments. Mais le second dépôt a fait l'objet d'une surveillance minutieuse, et l'histoire de l'habitat où ils dormaient tous deux depuis dix-sept siècles ou plus a été passée au crible d'une stratigraphie critique aussi exacte que possible (J.-P. Lascoux, P. Jacquet, W. Widlak). C'est d'ailleurs cette fouille du site de Charavay qui a permis de reconsidérer l'état de nos connaissances sur ce secteur de Vaise censé (jusqu'en 1990) n'avoir abrité aucune installation humaine.

Les traitements appliqués avec patience et précaution aux objets pour leur restauration ne sont pas moins instructifs que l'environnement archéologique (P. Chantriaux, M.-H. Kappes, V. Langlet-Marzloff, J.-B. Latour, P. Pliska). Ils nous éclairent, en effet, sur les techniques mêmes de fabrication. "Rendre sa lisibilité à l'objet, lui restituer une cohérence suffisante, stabiliser les processus de dégradation": beaucoup de "restaurateurs" actuels devraient s'inspirer d'une aussi juste définition de leur métier.

7 L'inventaire de la vaisselle et de la statuaire (F. Baratte), des bijoux (C. Metzger), des monnaies (G. Aubin) ne laisse rien à désirer. Car non contents de mesurer, de décrire et d'identifier, les auteurs de la publication savent nous mettre en perspective le double dépôt de Vaise, moyennant d'intelligents parallèles qui en soulignent les singularités. Ensemble modeste, en somme, et (nous dit-on) relativement "homogène", dont une partie proviendrait "d'un sanctuaire plutôt que d'un laraire privé" : peut-être lié pour un temps aux dévotions d'un collège de ratiarii acheminant des marchandises entre Lausanne et Yverdon, comme nous le suggère la dédicace gravée sur le socle d'une statuette d'Hélios... Mais des malandrins ont pu rassembler ce butin en vue de la refonte, à en juger par la présence de fragments divers (deux têtes, deux bras, un coude, une aile, un débris de couronne tourelée).

Quant aux monnaies, on y discerne deux ensembles curieusement séparés par une interruption de vingt-trois ans : l'un va de 69 à 218, l'autre de 241 à 258 . Mais trentetrois antoniniens du premier "lot" datent de 215-217 : produit d'un donativum ? Enquête évidemment passionnante. Tel quel, en tout cas, ce dépôt "ne correspond à aucun autre trésor connu dans la région Rhône-Alpes". A cet égard, l'annexe de F. Planet (Trésors anciens du Rhône et de la région Rhône-Alpes) fournit opportunément au lecteur des termes de comparaison.

9 Les contributions de M.-Cl. Depassiot (atelier de restauration G. Chapotat), X. Loriot, P. Michel, des tableaux, des appendices, des notes techniques permettront aux chercheurs de travailler encore sur ce matériel qui n'a pas livré le fin mot de toutes ses énigmes, à commencer par l'identité du buste impérial: Gallien? J'aurais personnellement tendance à en douter. Mais il faut bien reconnaitre que l'artisanat provincial schématise l'iconographie officielle. Quoi qu'il en soit, il s'agit d'un témoignage remarquable sur le culte du souverain au IIIe siècle de notre ère.

Ce numéro 17 des DARA nous offre donc ainsi un beau document d'histoire. Il devrait susciter d'autres publications sur le patrimoine archéologique d'une région qui, durant les quatre ou cinq dernières décennies, a révélé les richesses d'un sous-sol trop souvent méconnu, sinon saccagé naguère encore! Mais le directeur des antiquités de la région Rhône-Alpes que j'ai été il y a maintenant déjà un quart de siècle se réjouit de constater que les choses ont changé. Ce magnifique ouvrage nous en donne la preuve éclatante et réconfortante. 
AUTEUR

ROBERT TURCAN

Membre de l'Institut 


\section{Introduction}

1 Pendant longtemps, on a gardé de la plaine de Vaise l'image qu'en donnait l'Intendant Général d'Herbigny à la fin du XVIIe s., une paroisse ingrate, régulièrement inondée et ensablée, peu propice à la culture :

"C'est un climat froid, malsain et fort marécageux... et l'on ne peut pas y vivre longtemps à cause du mauvais air. Ce sont des terres fort sujettes aux eaux de la Saône, qui les inondent, qui les sablent et qui gâtent les fonds. Il s'y recueille fort peu de blé parce qu'il y a peu de terres labourables, le principal fruit consiste en herbage, et encore y a-t-il peu de jardins" (Guillemain 1961, p. 14-15).

2 Naturellement, cette image était aussi projetée sur la période antique. La position topographique de la plaine de Vaise, située entre la Saône, la colline de Fourvière et le plateau de la Duchère, en faisait un quartier suburbain en marge des principaux centres de peuplement: la ville haute de Fourvière, les quartiers fluviaux de la presqu'île, le sanctuaire fédéral de Condate. La documentation rassemblée par les archéologues se résumait à des vestiges funéraires et au passage d'une voie du réseau d'Agrippa. Vaise, en périphérie de la colonie, avait essentiellement une fonction de transit et de nécropole.

Un précédent volume de la collection des DARA (Delaval et al. 1995) a fait justice de cette vision. La publication des résultats de quatre fouilles vaisoises conduites entre 1985 et 1991, accompagnée d'un bilan critique de la documentation ancienne et des acquis récents, a renouvelé l'approche historique d'un quartier que l'on découvre largement occupé durant la protohistoire et doté dans l'antiquité d'un carrefour de voies, d'un bourg (vicus) et peut-être d'un port. Vaise, "la porte nord de Lyon". Ce renversement de perspectives est dû aux résultats de nombreuses fouilles préventives rendues nécessaires par le remodelage du quartier depuis 1980 et le renouvellement du bâti.

La Zone d'Aménagement Concertée (Z.A.C.) Charavay constitue l'une de ces opérations de transformation. Un premier projet immobilier, rue du Chapeau rouge, dans la partie sud-est de la plaine, est précédé de deux campagnes de sondages, en 1987 et 1990, qui détectent la présence de vestiges gallo-romains et précisent leur nature et leur extension, amenant ainsi l'Etat (Direction régionale des affaires culturelles - service régional de l'archéologie) à prescrire la réalisation, sous son contrôle scientifique, d'une fouille préventive. En 1991, une convention passée entre les partenaires des 
fouilles préalables à la construction prévoit que le financement de ces dernières serait à la charge des aménageurs: la société d'équipement de la région lyonnaise (S.E.R.L.), propriétaire du terrain et la C.O.P.R.A., promoteur immobilier.

5 Les fouilles ont été effectuées d'août 1991 à mars 1992 par une équipe de l'Association pour les fouilles archéologiques nationales (A.F.A.N.) sous la direction de J.-R Lascoux (Service archéologique municipal). Les deux dépôts étudiés dans cet ouvrage ont été découverts en mars 1992, donc en fin d'intervention : le premier un peu brutalement, le 17 mars, lors d'une vérification stratigraphique faite à l'engin mécanique; le second, plus tard, a fait l'objet d'une fouille. La discrétion qui a entouré ces découvertes et la très rapide prise en charge des objets par J. Lasfargues, conservateur en chef du musée, ont permis d'assurer avec une grande efficacité leur prélèvement et leur stabilisation. La restauration, financée par le Département du Rhône, a été confiée à l'Atelier municipal de restauration Gabriel Chapotat, de Vienne, sous la responsabilité de M.-C1. Depassiot. Dès cette étape, les auteurs de l'étude ont pu suivre au laboratoire les étapes $\mathrm{du}$ nettoyage et du remontage et faire leur profit des observations techniques. Depuis lors, ces deux dépôts sont conservés au musée de la civilisation gallo-romaine de Lyon, après que la S.E.R.L., propriétaire du terrain lui ait fait don de sa part, et que l'Etat y ait par convention déposé la sienne.

6 Le trésor de Vaise, appellation désormais consacrée par l'usage, est un dépôt mixte, regroupant de manière classique vaisselle d'argent, bijoux et monnaies, et de manière plus exceptionnelle, des statuettes en argent. À cet égard, il acquiert une place tout à fait originale dans le corpus des trésors d'orfèvrerie gallo-romains du IIIe s. Il présente aussi l'intérêt majeur d'être doté d'un contexte archéologique, même si les précisions chronologiques tirées de la stratigraphie ne permettent pas de répondre à toutes nos interrogations.

7 Les données acquises sur le terrain, présentées synthétiquement, montrent l'évolution qui conduit d'une grande demeure rurale à la création, au Ier s., d'un îlot urbain composé d'habitats, d'ateliers et d'entrepôts, puis, dans des conditions mal définies, à sa transformation au début du IVe s. en officine de potier. Est-ce dans un contexte de déclin du quartier que les dépôts sont enfouis et abandonnés?

Nous avons choisi de consacrer la plus grande part de l'ouvrage à un catalogue raisonné des pièces des trésors : étude typologique, stylistique, iconographique, et dans certains cas technique. La détermination des minéraux des bijoux a été effectuée par spectroscopie (Université Claude Bernard, Lyon I). En revanche, divers problèmes matériels nous ont amené à renoncer ici à une étude métallographique, qui au demeurant pourra être effectuée lorsque sera menée une étude d'ensemble sur les alliages et la provenance de l'argent utilisé dans l'orfèvrerie gallo-romaine. La conclusion sur la nature du trésor résulte d'une réflexion commune entre les différents auteurs, mais aussi des suggestions qui nous ont été faites par plusieurs de nos collègues.

9 Encore un mot sur la présentation de l'ouvrage. À côté de l'édition des trésors, nous avons souhaité donner au lecteur non spécialiste, à l'aide d'encadrés ou d'annexes, sur la dynastie des Sévères, les événements de 260 ou les autres trésors régionaux, l'occasion de satisfaire ses curiosités. 


\title{
L'environnement archéologique du trésor de Vaise
}

\author{
Jean-Paul Lascoux, Pierre Jacquet et Woijtek Widlak
}

1 La plaine alluviale de Vaise décrit, sur la rive droite de la Saône, un long arc tendu aux pieds des massifs du Mont-d'Or et des Monts du Lyonnais, avant de se resserrer brusquement à l'est, à l'entrée du défilé de Pierre Scize (fig. 1). Elle est isolée, de fait, par des reliefs que l'on ne saurait considérer toutefois comme des obstacles infranchissables puisque des axes naturels de communication la relient, soit à la presqu'île par la rive droite de la Saône, soit à Fourvière par les talwegs qui échancrent les plateaux.

2 C'est au sud-est de cette plaine qu'est établi le site de Charavay, lieu d'une opération d'archéologie préventive ${ }^{1}$ et de la découverte des deux dépôts d'orfèvrerie présentés dans cet ouvrage. 


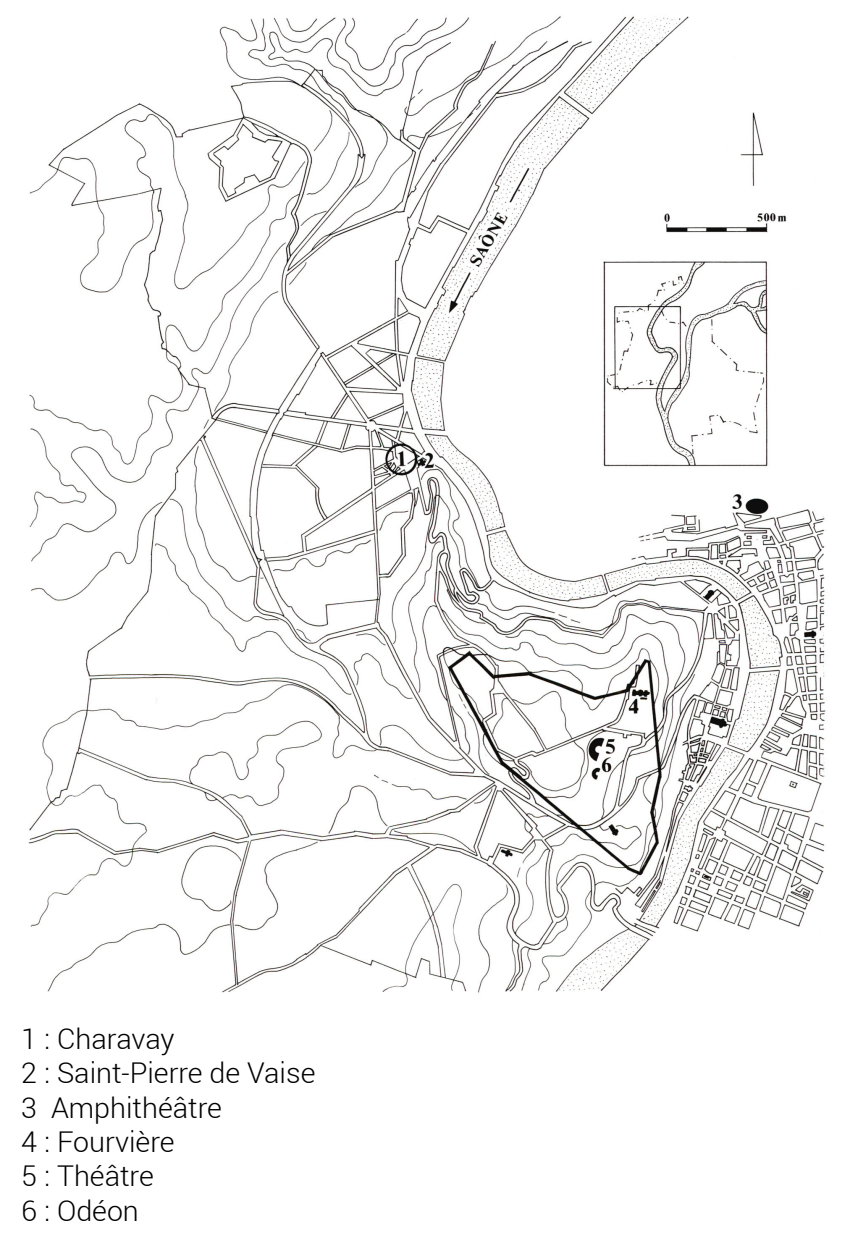

\section{Présentation de l'étude}

Cette étude se propose d'analyser les données archéologiques qui permettraient de préciser, avec celles des autres contributions de cet ouvrage, quels furent les auteurs et les circonstances de l'enfouissement des deux dépôts. Pour cela, trois approches ont été privilégiées. Elles sont complémentaires et remédient dans une certaine mesure au déficit de l'information. La première, consacrée à l'évolution des formes de l'occupation du site, aborde la question de la destination des différents bâtiments ou ensembles de construction qui se sont succédés. Elle examine aussi les arguments en faveur du maintien ou de l'abandon de l'habitat entre le IIe s. et le IIIe s. ap. J.-C. ; ce point étant particulièrement important pour appréhender le contexte des deux dépôts. La seconde porte sur les comparaisons entre le site de Charavay et les autres sites de la plaine de Vaise. Elle détermine les grandes composantes de l'occupation de la plaine et la manière dont celles-ci ont évolué. Cette approche, pour le IIIe s. et le IVe s. ap. J.-C., modifie notablement les conclusions que l'on aurait été amené à porter au vu du seul site de Charavay. La troisième concerne les dépôts proprement dits. Elle expose les éléments de chronologie, les traces de leur dissimulation et leur composition.

Cette étude emprunte à un mémoire de maîtrise (Lascoux 1994) la chronologie et les restitutions du site de Charavay. Elle se réfère également aux synthèses développées dans ce travail universitaire et dans le volume de la collection des Documents d'archéologie en Rhône-Alpes consacré à Vaise à l'époque antique (Delavai et al. 1995), 
complétées par les résultats des découvertes les plus récentes. Parmi cette documentation on mettra en garde le lecteur contre le caractère provisoire des chronologies et des interprétations des rapports de fouille et, d'autre part, contre les hypothèses de restitution des occupations du site de Charavay étayées parfois par trop peu de preuves matérielles. Toutefois, les limites imposées à cette étude sont ailleurs. Elles sont consécutives aux destructions du site, en particulier à l'arasement des sols du IIIe s. et IVe s. ap. J.-C., qui ne permettent pas de disposer de faits précis quant à la nature des contextes et à la chronologie relative des deux dépôts.

\section{Le site de Charavay}

6 L'évolution du site pendant l'Antiquité se décompose en trois périodes du Ier s. av. J.-C., au IVe s. ap. J.-C.

7 La plus ancienne se rapporte à une occupation effective du site, dont les traces sont insuffisantes pour en comprendre la destination.

8 Les vestiges de la seconde, datés vers 30 av. J.-C. au plus tôt, appartiennent à un établissement de type rural, peut-être à vocation agricole.

9 Les constructions de la dernière période s'inscrivent à l'intérieur de deux îlots qui, créés à la fin du règne d'Auguste ou au début de celui de Tibère, se maintiennent jusqu'au IVe s. ap. J.-C. Pendant le Ier s. ap. J.-C., la densité des constructions à l'intérieur des parcelles ne cesse de croître, puis se stabilise aux siècles suivants. Au IVe s. ap. J.-C., l'installation d'un atelier de potier marque une mutation importante de l'occupation avant l'abandon du site.

\section{Les tout premiers vestiges}

La première occupation gallo-romaine s'intercale entre une couche contenant du mobilier de La Tène finale et l'apparition de l'occupation suivante, vers 30 av. J.-C. Le mauvais état de conservation des vestiges ne facilite par leur interprétation (fig. 2). Un foyer et quelques trous de poteaux, sans organisation particulière, témoignent peutêtre de l'existence d'un habitat dans la partie est du site (fig. 2, A). Au sud-ouest, une vaste dépression peu profonde, dont l'origine est probablement naturelle, conserve des traces d'aménagements spécifiques. Concentrées au nord-ouest de la dépression, ces traces suggèrent que seule cette partie était utilisée. L'intérieur de la dépression est tapissé d'une couche organique près de laquelle sont implantés deux trous de poteaux. À l'extérieur, un alignement de cinq trous de poteaux double partiellement sa limite nord. Plus à l'ouest, trois négatifs oblongs conservaient les traces de planches de bois carbonisées (fig. 2, B) ; restes modestes, vraisemblablement, d'un platelage, ayant eu pour fonction de stabiliser l'accès à cette dépression. 


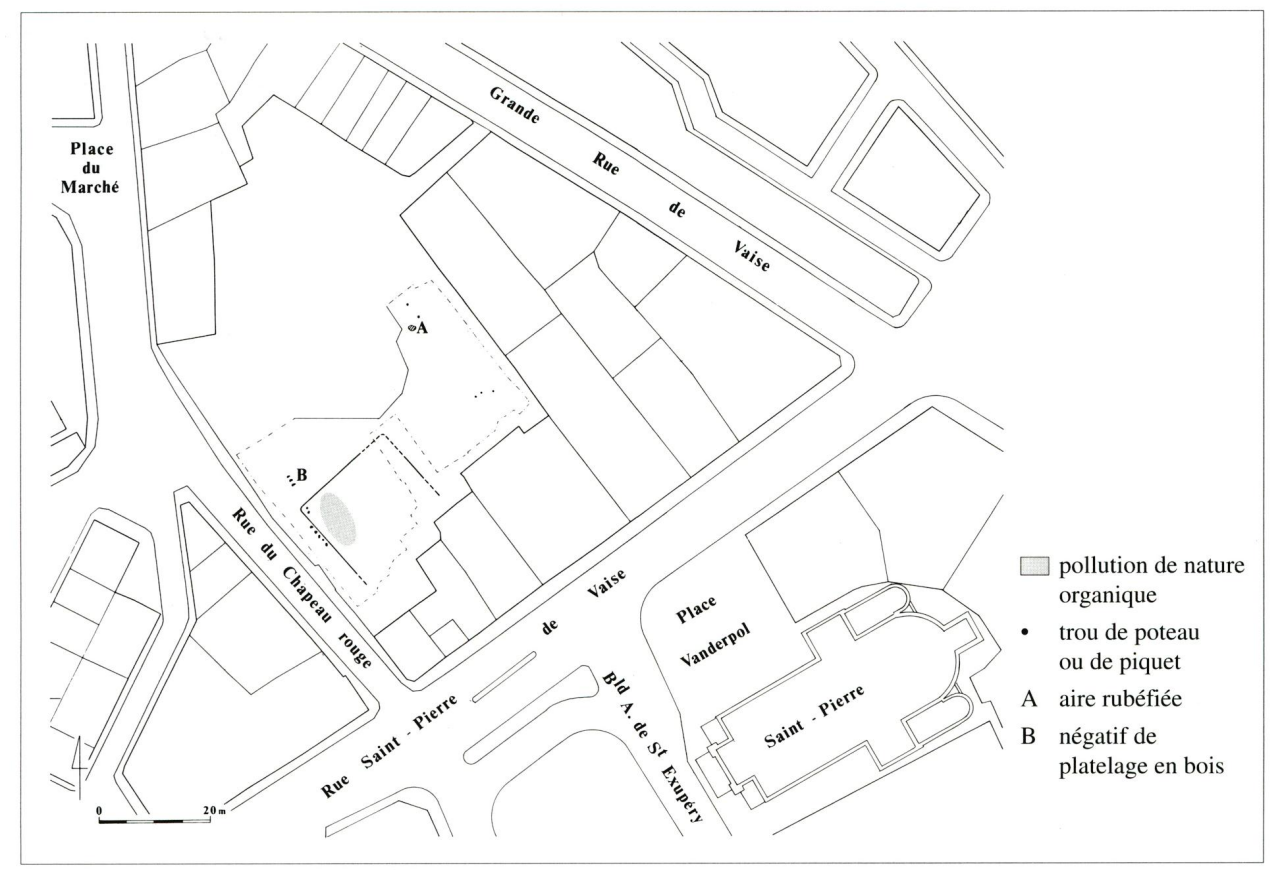

\section{Une vaste demeure à caractère rural}

11 Vers 30 av. J.-C., les installations précédentes sont abandonnées pour une nouvelle construction dont les vestiges, quoique fragmentaires, permettent de reconnaître le plan et la destination du bâtiment. Ils se répartissent en deux ensembles distincts tant par leur organisation spatiale, leur technique de construction, que par leur équipement.

Le premier, à l'est, se compose d'une série de pièces contiguës disposées en L, délimitant un espace ouvert dont le sol est constitué essentiellement de matière organique (fig. 3, A). Les murs, dont la largeur n'excède pas 0,10 m, sont en terre sans trace d'ossature de bois. Les sols intérieurs sont, au nord, en terre battue, à l'ouest, en petits fragments de schistes pollués par des matières organiques. Aucune de ces pièces n'est équipée de foyer.

13 À l'ouest, le second ensemble (fig. 3, B) s'organise sur un espace ouvert possédant un mur bahut, témoin de l'existence d'un portique ou d'un jardin. Autour de cet espace central s'élevaient au moins trois corps de bâtiment dont un seul a laissé des traces. Ses murs, construits également en terre, sont, contrairement à ceux de l'ensemble précédent, raidis par une ossature de bois dont les dimensions et l'organisation des empreintes en négatif suggèrent qu'il comportait un étage. D'autre part, outre les sols de terre battue, ce corps de bâtiment conservait un sol construit et des foyers qui équipaient les pièces. Cette occupation peut être interprétée comme un habitat, élevé sur un espace d'agrément, articulé à une partie utilitaire. Sa restitution par symétrie, selon l'axe médian du mur bahut, porte sa surface à $2000^{\mathrm{m} 2}$ minimum, ce qui incite à considérer que ces deux ensembles appartiennent à une vaste demeure rurale, dont ils constitueraient la pars rustica et la pars urbana. 


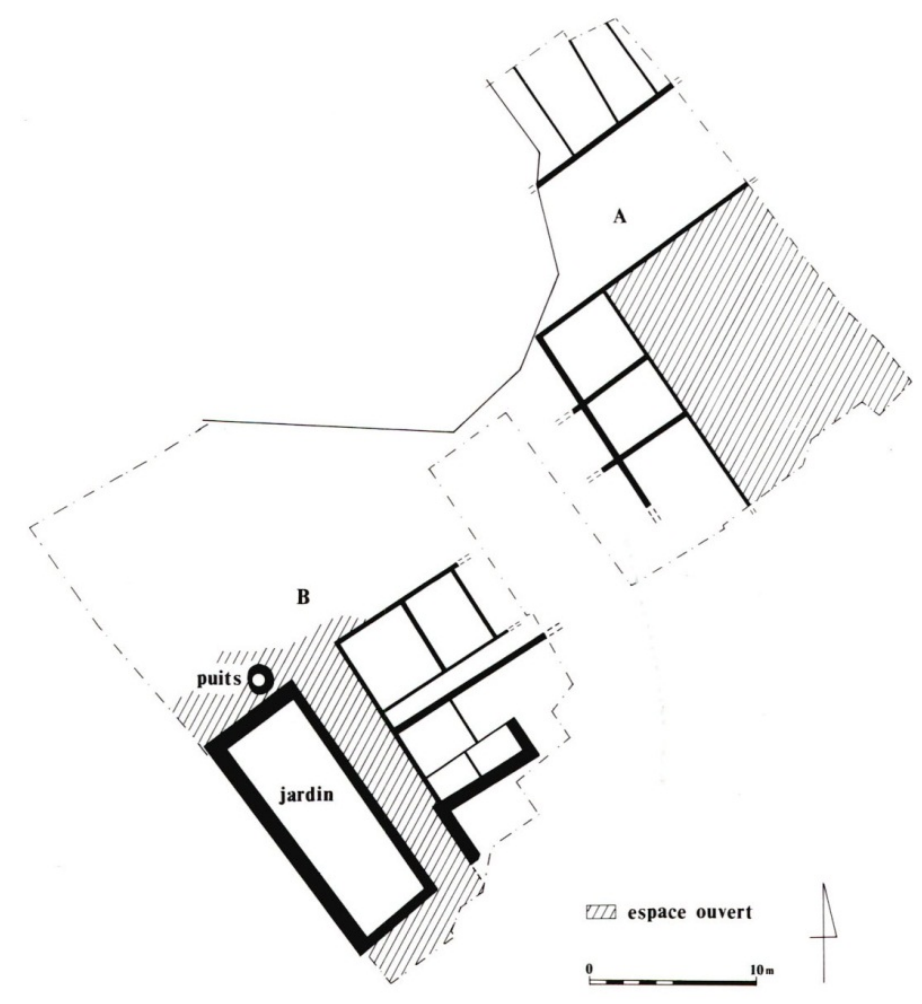

\section{L'apparition d'un urbanisme}

Après la destruction du bâtiment de la période précédente, les nouvelles constructions sont élevées à l'intérieur de deux îlots incomplets dont les façades occidentales ouvrent sur une voie nord-sud (fig. 4). La largeur de sa chaussée, selon l'axe de symétrie de l'égout du IIe s. ap. J.-C., est approximativement de 42 pieds $(12,40 \mathrm{~m})^{2}$, soit une valeur supérieure de 1,5 pied à celle des voies de grande communication, par exemple la voie d'Aquitaine mesurant 41 pieds. Seule une voie, dans le secteur de Vaise, peut répondre à cette définition, la voie de l'Océan. La largeur de la voie est-ouest (fig. 4), séparant les deux îlots, est moins importante 21 pieds (environ $6 \mathrm{~m}$ ), c'est-à-dire comparable à celle de certaines rues découvertes à l'intérieur de la colonie. 


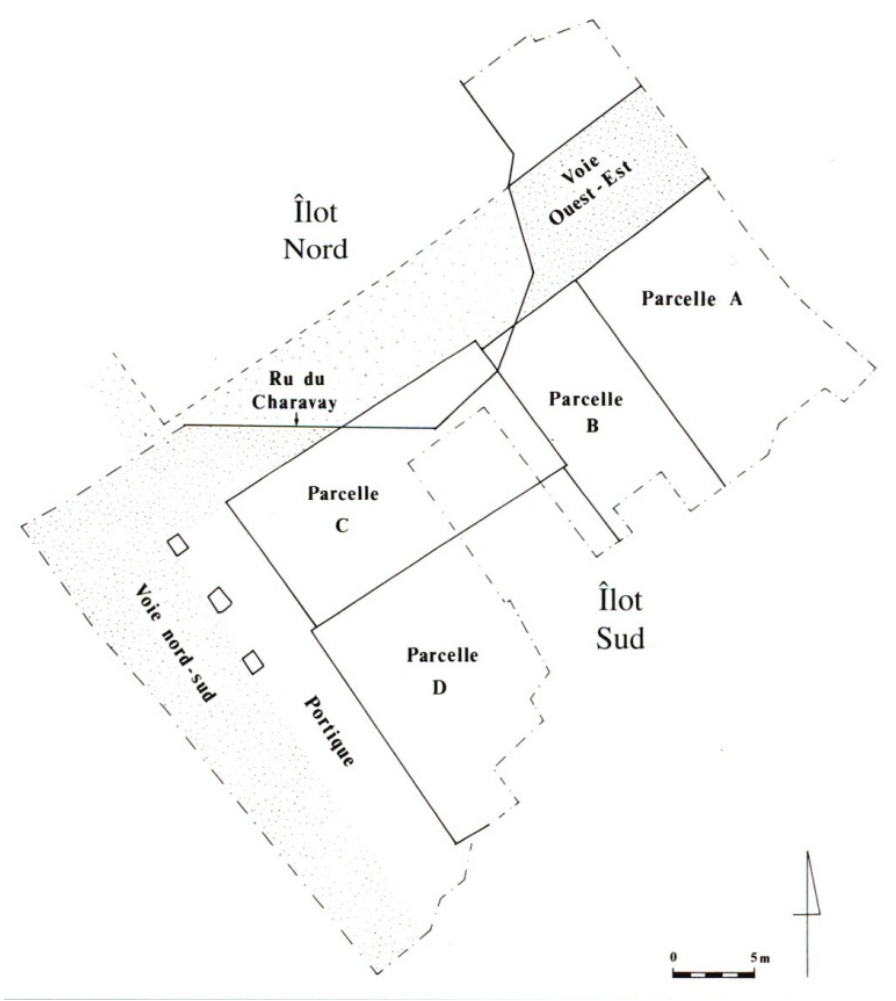

15 Les façades occidentale et septentrionale de l'îlot sud, le plus complet, s'étendent sur une longueur de $25 \mathrm{~m}$ et $40 \mathrm{~m}$ portique inclus. La façade occidentale, dont on est assuré du prolongement hors des limites de la fouille, peut être prolongée sans risque d'erreur des deux entraxes des piliers du portique. Sa longueur portée ainsi à 116 pieds, soit de peu inférieure à 120 pieds, suggère que l'îlot s'inscrit à l'intérieur d'un îlot théorique de la dimension d'un jugère (240 x 120 pieds soit $71 \mathrm{~m}$ x 35,50 m).

D'autre part, l'intérieur de l'îlot sud est divisé, sur la façade septentrionale, en plusieurs parcelles dont le fond pourrait s'appuyer sur l'axe médian est-ouest de l'îlot. Néanmoins, l'espace restreint alors réservé au développement des constructions à l'arrière de ces parcelles incite à retenir avec réserve une telle hypothèse. De l'évolution des parcelles et de leur construction se dégagent les différentes caractéristiques du bâti.

\section{Évolution de l'habitat et des entrepôts de l'îlot sud}

\section{L'évolution au ler s.}

17 L'habitat de la parcelle À est composé à l'origine d'un corps de bâtiment comprenant un minimum de six pièces aux sols de terre battue et équipées pour quelques-unes de foyers dont seules les soles de brique étaient conservées (figs. 5, 6a). Disposé en front de rue, le bâtiment ouvre au sud sur un espace ouvert dont les sols sont composés de fragments de schiste et/ou de granite. Ses fondations sont en granite, liées au mortier. Le fait qu'elles soient peu profondes et étroites privilégie l'hypothèse d'une construction de plain-pied plutôt que celle d'une construction à étage, néanmoins possible en raison de la trame relativement dense des fondations. 
18 Au milieu du Ier s. ap. J.-C., ce bâtiment est reconstruit à l'identique. La présence dans les fondations, d'encoches rectangulaires de faible section, atteste l'utilisation de montants en bois dans son élévation. Leur entraxe régulier, suffisant pour accueillir des ouvertures, renvoie à un procédé de construction à ossature de bois sans étage, ce que suggère aussi l'absence de traces de supports de fort gabarit, en particulier aux angles. On retrouve à l'intérieur de ce bâtiment les équipements déjà mentionnés à l'état précédent (fig. 6b).

5- Les îlots au ler s. ap. J.-C.

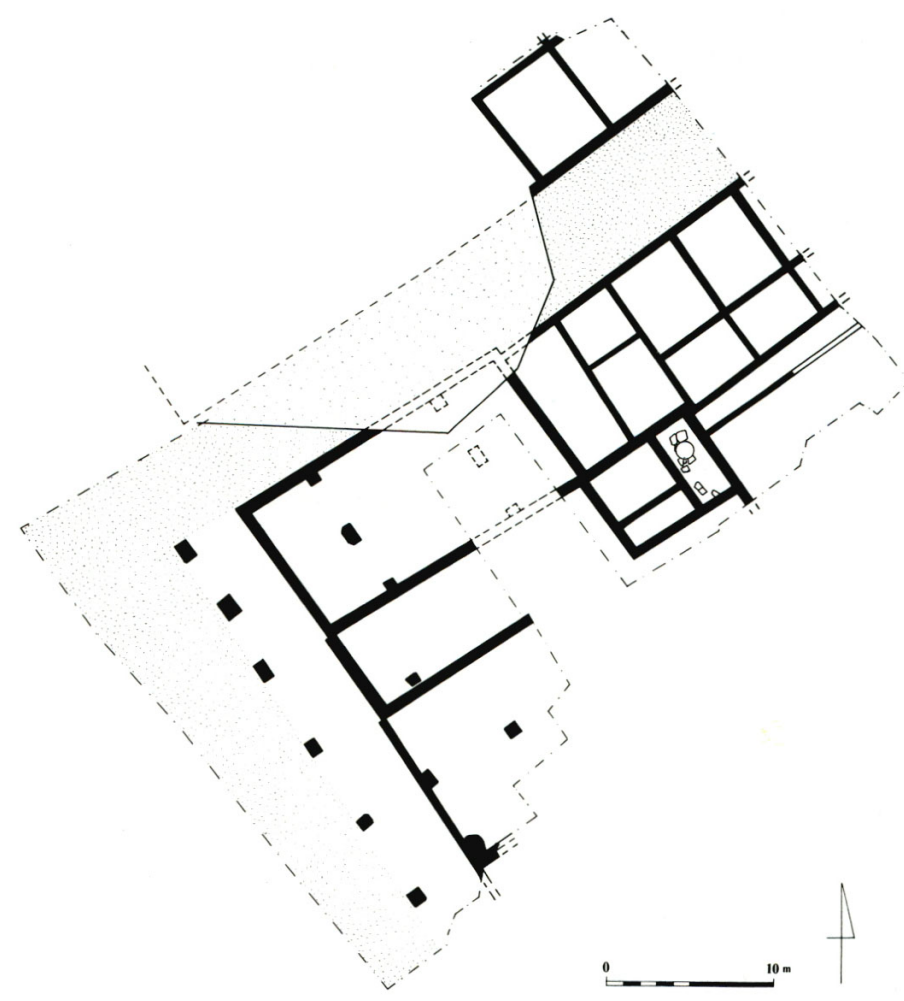

19 La dernière évolution est marquée par la construction d'un portique ou d'une galerie sur la cour. Cet ajout ne modifie apparemment en rien la destination du bâtiment sur rue (fig. 6c). 
6- L'évolution de l'habitat de la parcelle À au ler s. ap. J.-C.
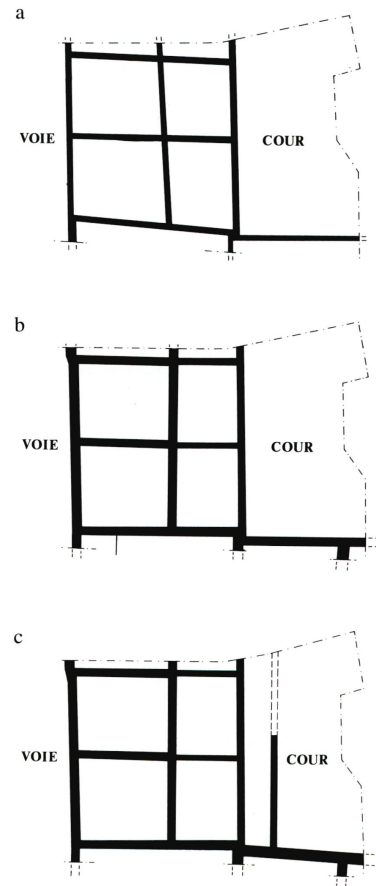

20 L'habitat de la parcelle B est implanté tout d'abord en front de rue puis s'étend ensuite, au cours du Ier s. ap. J.-C., en fond de parcelle. Du premier état du bâtiment sur rue, seule la façade septentrionale est conservée.

21 Le second état du bâtiment reconstruit à son emplacement est plus complet. Celui-ci s'insère entre les murs est et ouest des parcelles mitoyennes sur lesquels il s'appuie directement, comme ce sera également le cas pour les constructions qui lui succéderont. Il subsiste de ce second état la façade sur rue et une cloison intérieure (fig. 7a). Sa façade sur cour devait être approximativement dans l'alignement des autres façades, c'est-à-dire à l'endroit où un changement dans la composition des sols suggère la transition entre un espace couvert et un espace ouvert. Sa façade sur rue possédait une ossature bois dont les montants verticaux reposaient sur des dalles de granite. Cette technique de construction devait permettre de stabiliser l'élévation en terre de la façade qui était désolidarisée du reste du bâtiment. Outre cette exception, les autres fondations sont des radiers très étroits, formés d'une seule assise de pierre et de fragments de briques qui supposent une construction sans étage.

Au milieu du Ier s. ap. J.-C., le troisième état correspond à la reconstruction complète du bâtiment sur rue. Son espace intérieur est subdivisé en deux pièces. La première, dans l'angle sud-est, est dotée d'un sol de terrazzo et d'un décor peint. La seconde est équipée d'une banquette chauffante disposée contre la façade sur rue (fig. $7 \mathbf{b})$. Le reste de cette pièce, outre sa fonction propre, devait également permettre la desserte de la cour et du nouveau bâtiment construit en fond de parcelle. Ce bâtiment arrière, dont on ne possède qu'un plan partiel est subdivisé au moins en deux pièces. Ces nouvelles constructions possèdent des fondations larges construites en granite lié au mortier. Les matériaux et les dimensions suggèrent qu'elles ont pu recevoir un étage, d'autant que 
les portées sont suffisamment courtes pour justifier l'emploi, sans mur intermédiaire, de poutres d'un seul tenant.

Dans la seconde moitié du Ier s. ap. J.-C. l'habitat de la parcelle B est de nouveau reconstruit dans sa totalité. Le bâtiment sur rue (quatrième état) est alors divisé en trois pièces dont les cloisons de terre reposent sur des sablières de bois (fig. 7c). La cour, réduite au tiers de sa surface, est équipée d'un puits. Le reste est subdivisé en deux pièces dont l'une possède une sole de foyer construite en brique. Tous les sols des pièces sont en terre battue. Les fondations sont faites de schiste lié au mortier. Comme précédemment, les matériaux, les dimensions et les portées suggèrent que l'habitat de cette parcelle était encore doté d'un étage. L'évolution du bâtiment arrière est inconnue.

7- L'évolution de l'habitat de la parcelle B au ler s. ap. J.-C.

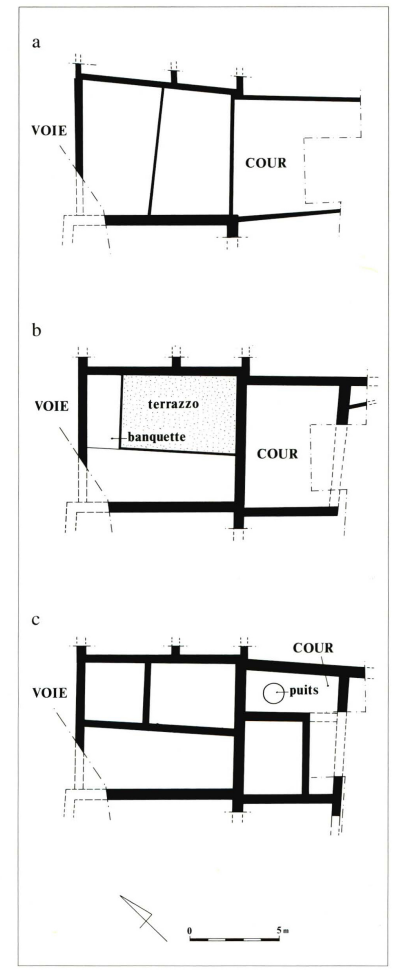

La transformation des entrepôts des parcelles $C$ et $D$ diffère nettement de celle de l'habitat qui vient d'être décrit. Elle aboutit cependant, elle aussi, à une densification des constructions dans les parcelles. La parcelle $C$ est occupée dès l'origine par un bâtiment qui ne sera jamais modifié. À l'opposé, la parcelle D est initialement un espace ouvert avant d'être construit, puis divisé en deux parcelles D1 et D2.

Le bâtiment de la parcelle C'est une construction de forme rectangulaire, sans aucune subdivision interne, dont la façade occidentale est doublée d'un portique (fig. 8). Elle est dotée d'un système de reprise de charges assuré par des piliers engagés qui, disposés symétriquement sur les faces internes des murs nord et sud, devaient être destinés à recevoir les fermes d'une charpente ou les poutres maitresses d'un plancher. Cette hypothèse, qui suppose l'existence d'un ou plusieurs étages, est justifiée par l'observation des fondations y compris celles des piliers du portique, puissantes et profondes. On retire l'impression d'un projet délibéré de créer un espace d'un seul 
tenant en rez-de-chaussée, peut-être un entrepôt, ou tout du moins un local à vocation commerciale, surmonté d'un ou plusieurs étages, éventuellement réservé à l'habitat. Selon ce point de vue, il pourrait donc s'agir d'un immeuble de rapport.

La parcelle D était soit initialement non lotie, soit un espace ouvert servant de cour à l'habitat attenant au sud (fig. 8a). Elle est occupée ensuite par un (fig. 8b), puis par deux entrepôts (fig. 8c) après avoir été probablement aliénée dans la seconde moitié du Ier ap. J.-C. Le plan des bâtiments, même partiel, n'offre aucune ambiguïté quant à leur destination. Il se rapporte à un type d'entrepôt connu et abondamment commenté. Les fondations de leurs piliers, maçonnées mais peu profondes, permettent d'écarter l'existence d'un étage. Il en est de même pour les piliers des portiques construits dans la seconde moitié du Ier s. ap. J.-C..

8- L'évolution de l'habitat de la parcelle $C$ et des entrepôts de la parcelle D au ler s. ap. J.-C.

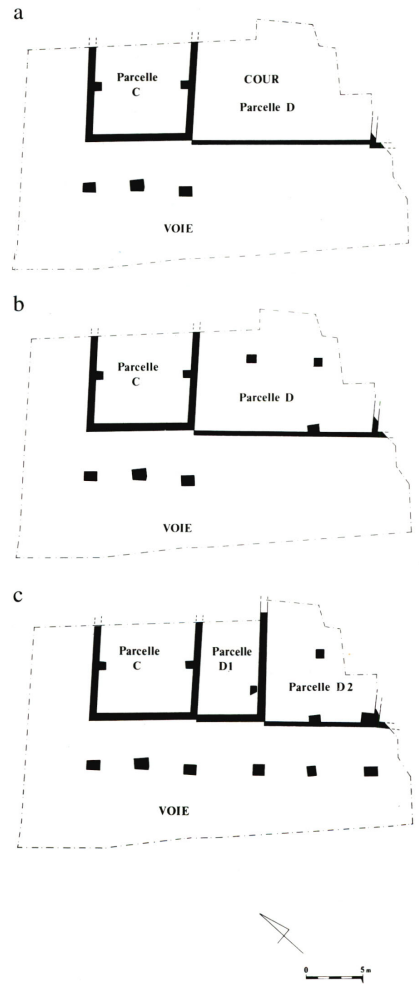

\section{L'évolution au lle s.}

27 Le début du IIe s. ap. J.-C. est marqué par une vague de reconstructions et par la création d'un grand collecteur sous la chaussée de la voie de l'Océan (fig. 9). L'emploi d'un même mortier dans les fondations de l'îlot nord, de la parcelle À et dans les maçonneries de l'égout, indique probablement que ces travaux se rapportent à un projet d'ampleur, dont néanmoins les entrepôts de l'îlot sud sont exclus. La densité des constructions à l'intérieur des parcelles À et $\mathrm{B}$ atteint son maximum. Les techniques de construction et les plans n'évoluent guère cependant par rapport au siècle précédent. 


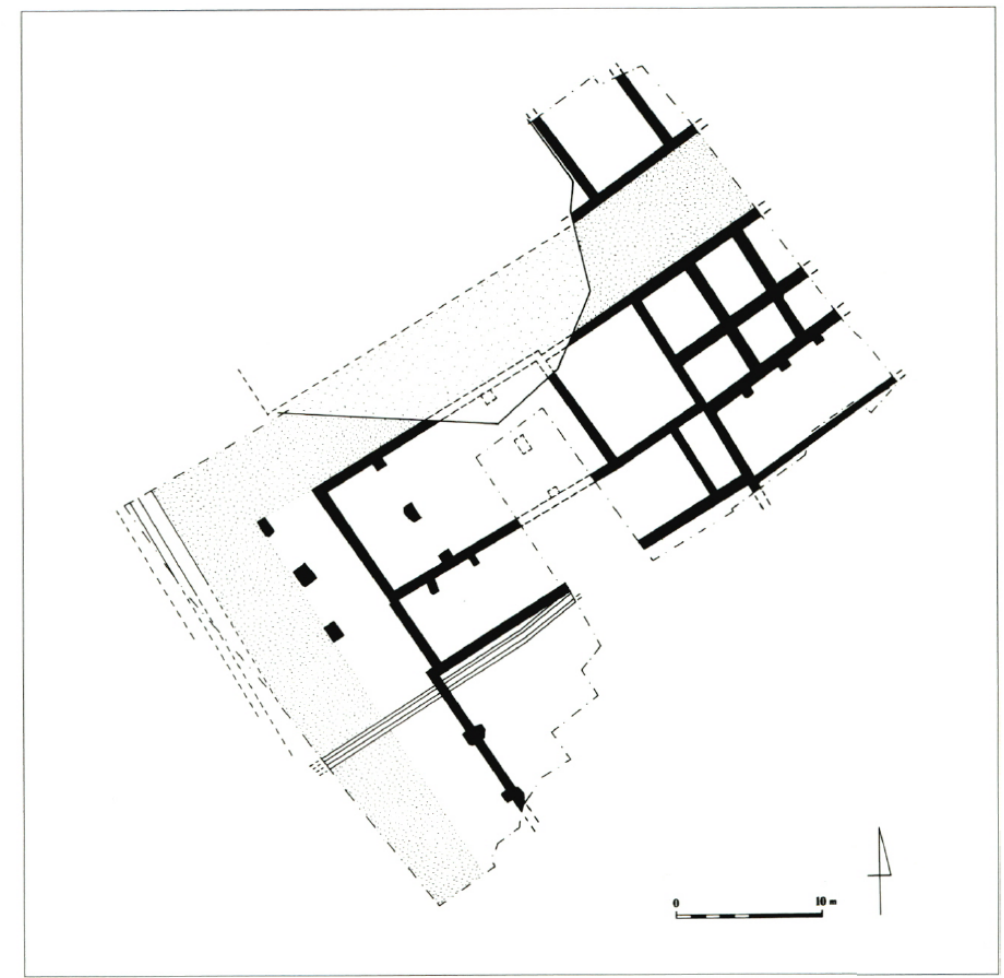
comprend alors toujours un minimum de six pièces dont les dimensions relativement faibles et la puissance des fondations indiquent qu'il était, peut-être, doté d'un étage. À l'arrière de ce bâtiment, une série de trois piliers appuyés contre sa façade méridionale et un mur situé plus au sud, se prêtent au moins à deux restitutions différentes de l'organisation de l'espace à l'emplacement de la cour de l'état précédent: dans la première, les piliers engagés seraient un décor de façade et le mur sud appartiendrait à un autre bâtiment, en fond de parcelle; dans la seconde, ces piliers supporteraient la charpente d'un bâtiment occupant l'espace de la cour d'origine. L'emploi de briques de section semi-circulaire pour les piliers, matériau souvent réservé aux éléments décoratifs, invite à privilégier la première hypothèse. L'absence de toute subdivision et de tout équipement particulier à l'intérieur du nouveau bâtiment, quelle que soit l'hypothèse que l'on privilégie, indique que celui-ci était destiné à un tout autre usage que domestique, peut-être à un entrepôt.

Malgré des destructions ultérieures qui ne permettent de comprendre ni son organisation interne ni ses équipements, l'habitat de la parcelle B est également reconstruit selon un plan de masse identique. Il subit néanmoins quelques évolutions. La cour, toujours équipée d'un puits, est désormais dallée. Plus remarquable, la partie habitat à l'arrière du bâtiment sur rue s'étend à l'ouest au détriment d'une parcelle mitoyenne comme en témoigne la disparition du mur nord-sud de l'état précédent, mais aussi la reconstruction et le prolongement du mur est-ouest.

Sur la façade occidentale, la disparition des niveaux du Ile s. ne permet pas de juger de l'évolution du bâtiment de la parcelle C. Les deux entrepôts des parcelles D1 et D2 perdurent et ne subissent que des transformations ponctuelles. Les plus importantes sont la disparition des portiques qui leur étaient associés et la reconstruction de la 
façade de la parcelle D2. Les reprises de charges de cette nouvelle façade par des dalles prises dans la maçonnerie illustrent le retour à des techniques de construction du début du Ier s. ap. J.-C. Bien que la structure de bois puisse laisser supposer la présence d'un étage, l'absence de supports intermédiaires internes suggère plutôt une construction en rez-de-chaussée.

\section{Les mutations de la fin de l'Antiquité}

31 L'arasement général du site ayant entraîné la disparition des niveaux les plus récents, peu d'indices permettent d'établir l'existence formelle d'une occupation au cours du IIIe s. ap. J.-C. Cependant, celle-ci apparaît comme très probable, même si aucune reconstruction, ni évolution parcellaire, n'eut lieu avant la période suivante. En effet, on peut supposer que l'habitat et les entrepôts de l'époque antérieure sont encore en élévation compte tenu de la localisation de l'un des trésors dans l'angle intérieur du bâtiment sur rue de la parcelle B et du fait que les fosses n'investissent les parties construites et les espaces ouverts, qu'à partir du IVe s. ap. J.-C. Par ailleurs, le fait que l'atelier de la période suivante réutilise des bâtiments existants et que la voirie perdure jusqu'au début du IVe s. ap. J.-C. montre aussi que la forme urbaine était suffisamment prégnante pour avoir servi de cadre à une occupation ultérieure.

32 Au début du IVe s. ap. J.-C., une modification significative du parcellaire, dont les limites étaient relativement stables depuis près de trois siècles, intervient consécutivement à l'installation d'un atelier de potier sur les parcelles À et B (fig. 10). Celui-ci remploie le bâtiment sur rue de la parcelle À et se dote à l'arrière d'un nouvel édifice. Il installe également ses fosses de décantation de l'argile sur la parcelle B dont les constructions sont alors détruites. Le bâtiment sur me, destiné à la fabrication, est équipé de deux fours dont l'un de facture soignée, à sole circulaire, servait incontestablement à la cuisson des céramiques. Le bâtiment arrière, doté de supports intermédiaires divisant l'espace en deux rangées de quatre travées minimum, servait probablement d'entrepôt ou de lieu de séchage.

L'officine étant la seule trace repérée d'une occupation, on suppose que le site est alors pratiquement déserté tout en offrant encore au regard quelques éléments de son organisation passée, comme la voirie. Enfin, après l'abandon de l'atelier, soit vers le milieu du IVe s. ap. J.-C., du mobilier épars atteste encore une fréquentation du site jusqu'à la fin du IVe ou au début du Ve s. ap. J.-C. La disparition des niveaux, lors de l'arasement du site, ne permet d'en préciser ni la nature ni la chronologie exacte. 


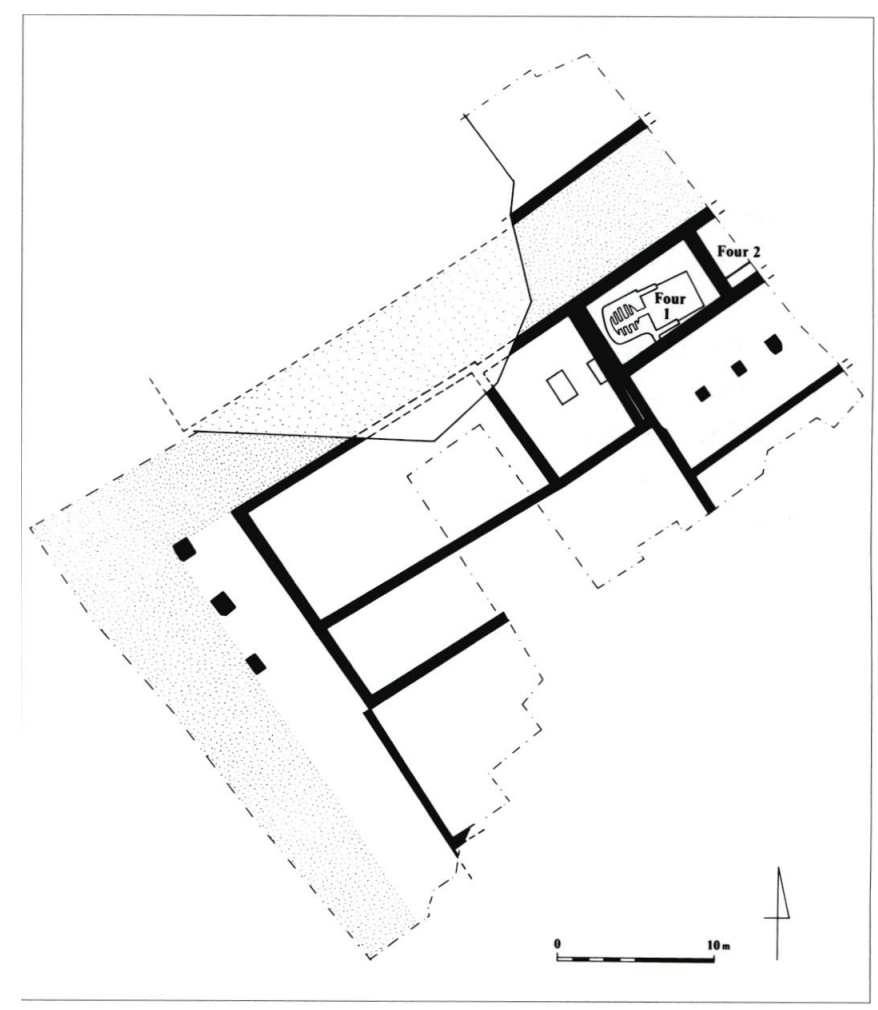

\section{Les caractéristiques de l'habitat de Charavay} une activité commerciale ou artisanale, et une partie habitat en fond de parcelle. Les boutiques sont inexistantes à Charavay.

bitat du Verbe Incarné s'organise autour d'une cour, rappelant en cela une disposition d'origine italique, qui perdurera pendant toute l'Antiquité. À Charavay, au contraire, la cour se réduit progressivement et apparait comme un espace tampon entre deux constructions; ceci pourrait indiquer que la parcelle est partagée entre plusieurs occupants et que l'habitat de Charavay a été peu influencé par les traditions romaines. En ce sens également, on fera observer que les cours des parcelles À et B n'ont jamais reçu d'aménagements les désignant comme un espace à part entière à l'exemple des citernes, des dispositifs de récupération des eaux ou encore des laraires placés dans les cours des habitats du Verbe Incarné. 
Enfin, les techniques de construction, les éléments de confort et de décor de l'habitat de Charavay, fréquents dans l'architecture domestique lyonnaise, traduisent, plus encore que les plans, un habitat commun, sinon modeste. Les sols du Ier s. ap. J.-C., les seuls conservés, sont essentiellement en terre battue, alors que les revêtements de sol construits sont relativement nombreux dans certains habitats du Verbe Incarné et sont généralisés dans les pièces de l'habitat de la terrasse médiane de la rue des Farges. Les traces d'enduits peints sont peu nombreuses. Seuls, deux exemples ont été conservés qui appartiennent au bâtiment sur rue de la parcelle B. L'un est contemporain de la pièce au sol de béton, l'autre de la dernière évolution du bâtiment à la fin du Ier s. ap. J.-C. Ces deux exemples, une plinthe monochrome de couleur verte et un enduit moucheté, ne traduisent pas, à l'évidence, l'existence d'un décor exceptionnel. Les foyers dont on ne possède aucune trace d'élévation ne se distinguent pas de ceux découverts à Lyon. Seule, une banquette chauffante dans le bâtiment sur rue de la parcelle B, qui souligne encore la particularité de cet habitat, se retrouve dans quelques très rares habitats du Verbe Incarné et de Saint-Vincent (Rapport, Lascoux 1987).

\section{Le site et son environnement}

\section{Le site et la plaine de Vaise}

Les hypothèses des archéologues du XIXe s. et de la première moitié du XXe s. ont fortement marqué jusqu'à une époque très récente l'approche scientifique de cette partie du site antique de Lyon. Ainsi, considérant qu'une vaste nécropole s'étendait dans sa partie sud, la plupart des auteurs ont rejeté la plaine de Vaise en dehors du territoire stricto sensu de la cité (Allmer 1888, Steyert 1895). Par ailleurs, jugée insalubre et par conséquent impropre à l'installation humaine, elle était supposée n'avoir abrité aucune occupation (Audin 1956). Avec le développement des recherches sur les rapports de la colonie et de son territoire, l'extension de la zone de protection archéologique en 1991 et la multiplication des projets d'urbanisme, plusieurs opérations archéologiques récentes ont en partie modifié les hypothèses consacrées par l'usage (Delaval et al. 1995). Néanmoins, les recherches réalisées dans la partie sud de la plaine (par exemple, place Valmy), à l'emplacement de la nécropole, se sont encore inscrites dans la continuité des auteurs anciens, tout en précisant les chronologies et les associations entre le funéraire et l'habitat. L'étude du site de Charavay permet, dans une certaine mesure, de reconsidérer l'histoire de la plaine et sa place dans celle du site lyonnais, mais aussi de poser en termes différents les rapports entre habitat et zones funéraires.

\section{Les occupations initiales de la plaine de Vaise}

En l'état actuel des connaissances, les deux premières périodes de Charavay sont à considérer comme deux occupations successives et isolées dans la plaine de Vaise. Pour la première période, l'absence de toute trace d'occupation et de fréquentation au-delà du site semble indiquer qu'un espace restreint et une partie infime des ressources de la plaine étaient exploités à ce moment-là (fig. 11A). 
11- Evolution des occupations de la plaine de Vaise selon les dernières fouilles.

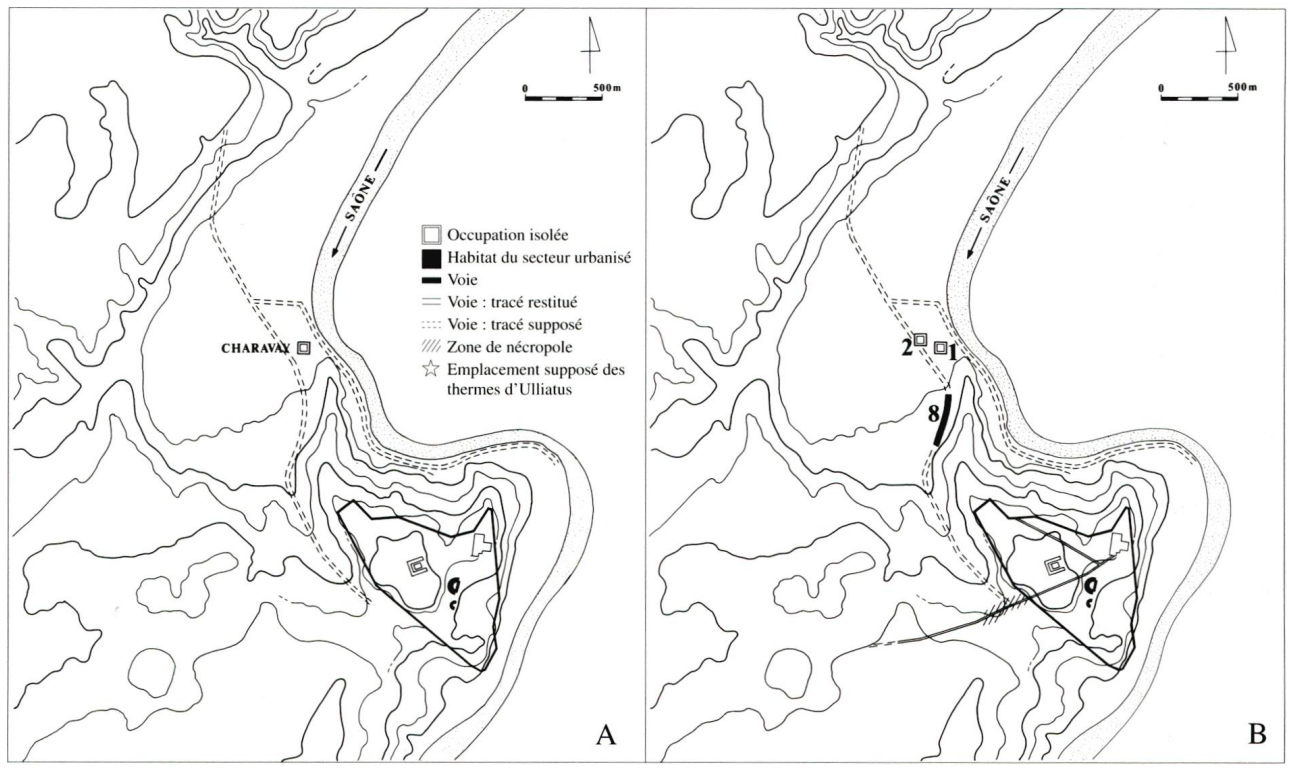

A : vers 30 av. J.-C.

$B$ : époque augustéenne

C : début ler s. ap. J.-C.

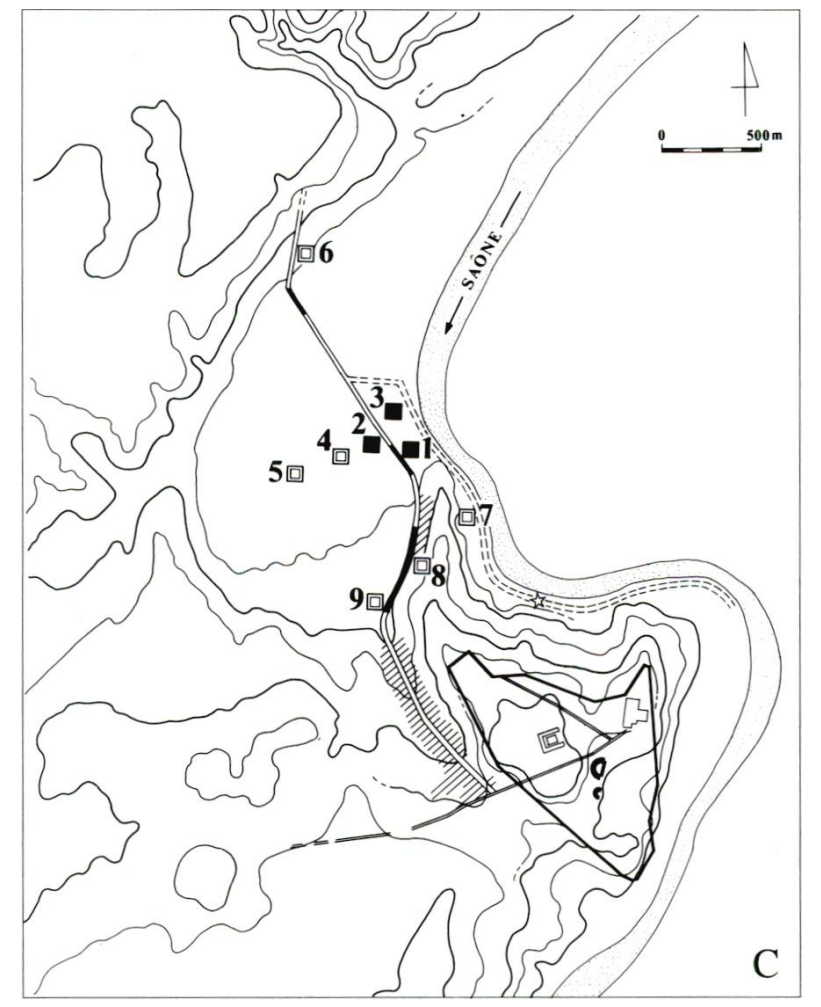
1: Charavay
2: Valmy
3: Marietton
4 : Blanchisserie
5 : Horand
6 : Échangeur de Vaise
7 : Arloing
8: Rhodiacéta
9: Gorge-de-Loup 
41 Au contraire, la villa qui succède suggère une activité plus large sur le territoire (fig. 11B). Des traces, parfois ténues, témoignent en faveur de cette interprétation. Ainsi, un dépotoir et des structures fossoyées, à Gorge de Loup (Rapport, Chastel 1987 ; Bellon 1995, p. 160-161), attestent des travaux d'assainissement et des activités humaines au pied du versant septentrional de l'éperon de Loyasse dans la seconde moitié du règne d'Auguste. De même, les sites de la rue du docteur Horand (Rapport, Bellon 1996, p. 50) et des Blanchisseries (Rapport, Rahatsoz 1995, p. 42-44), plus tardivement à la fin de l'époque augustéenne, ont livré quelques fragments de céramique, à défaut de tout autre vestige, qui signalent peut-être une fréquentation de la partie centrale de la plaine. Enfin l'apparition, sur Valmy et Marietton (Rapport, Célestin-Le Nezet 1993, p. 23-42, Rapport, Monin 1995, p. 48-51), à l'ouest de Charavay, d'un premier habitat, suivie rapidement de la construction de trois autres, entre la fin du Ier s. av. et le début du Ier s. ap. J.-C., témoigne, soit d'un développement lié à la présence de la villa de Charavay soit d'une anticipation de l'évolution à venir (fig. 11C).

Le secteur urbanisé et les occupations dispersées au ler s. ap. J.-C.

C'est au début du Ier s. ap. J.-C. que s'opère une première mutation de l'occupation de la plaine de Vaise qui se manifeste par l'émergence d'un secteur urbanisé, suivie, une décennie plus tard, par l'apparition de plusieurs occupations isolées. Cette évolution traduit un essor de l'occupation de la plaine alors traversée par une voie de grande communication, en l'occurrence la voie de l'Océan sur laquelle se développe un noyau urbain (fig. 12).

\section{Le secteur urbanisé}

43 L'importance de cette urbanisation peut être appréciée par l'analyse des différents fragments de trame qui la composent et en fonction des limites qui lui sont imposées par la Saône et le versant de l'éperon de Loyasse. Le site de Valmy, dont les bâtiments s'élèvent sur le côté ouest du prolongement de la voie de l'Océan, en fait partie. L'absence de constructions, au-delà des limites des fonds de parcelles, indique que celui-ci ne s'étendait pas vers l'ouest (Rapport, Celestin-Le Nezet 1993, p. 43-59); ce que confirment aussi les résultats négatifs des suivis archéologiques de la partie est du tènement de la Rhodiacéta (Rapport, Chastel 1987) et de la tranchée ouverte de la rue M. Berthet (Rapport, Tarras 1992). Si le site de Valmy se compose de parcelles mitoyennes que l'on peut assimiler à un urbanisme de rue, les îlots de Charavay, à l'est de la voie, indiquent en revanche un urbanisme qui s'est développé selon un quadrillage régulier. Eu égard aux dimensions supposées de l'îlot sud ( 240 x 120 pieds), quatre ou huit îlots peuvent être restitués de Charavay jusqu'au droit de Valmy, selon que l'on considère que la Saône empruntait ou non un tracé plus occidental pendant l'Antiquité. Le site de Marietton, organisé sur des orientations différentes, représente sa marge la plus septentrionale. Le relief constitue rapidement un obstacle naturel à son extension au sud. Enfin, au sud-est, l'absence de vestiges sur le site du quai Arloing, au début de notre ère (Pelatan 1971), suppose que le plan d'urbanisme ne s'est pas étendu à l'intérieur du défilé de Pierre Scize.

Si les parcelles de Charavay sont essentiellement destinées à l'habitat et à des entrepôts, celles de Valmy et de Marietton sont plutôt le lieu d'une occupation mixte associant habitat et artisanat, en particulier des métaux (Rapport, Célestin-Le Nezet 
1993, p. 9 ; Rapport, Monin 1995, p. 5455). Cette distribution apparente des activités entre la périphérie, où sont rejetées les activités nuisibles et dangereuses, et un centre, occupé par l'habitat, conforterait l'idée d'un secteur urbain relativement étendu et organisé dont les limites restent à préciser. Il pourrait s'agir d'un nouveau quartier de la colonie, voire d'un nouveau pôle de peuplement. En tout état de cause, les reconstructions incessantes et la densification des bâtiments traduisent le dynamisme de cette urbanisation.

12- Reconstitution du plan d'urbanisme autour de Charavay

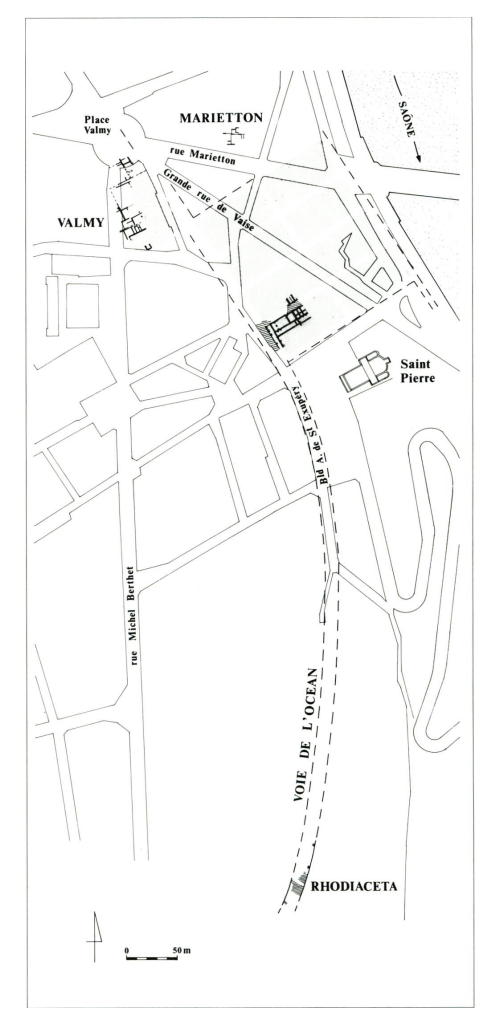

\section{Les occupations dispersées}

Par ailleurs, si les occupations les plus anciennes ne se rapportent pas directement à une valorisation des potentialités de la plaine, les occupations isolées montrent au contraire que ses ressources naturelles et agricoles ont été largement exploitées (fig. 11C). Toute une série de découvertes récentes signalent la variété des activités pratiquées sur son territoire au cours du Ier s. ap. J.-C. L'utilisation des ressources aquifères est attestée ainsi sur de nombreux sites, d'abord à l'ouest, à Gorge de Loup, avec un premier bassin, daté entre le deuxième quart et le milieu du Ier s. ap. J.-C., puis trois autres qui, disposés en série, sont attribués à une fullonica fonctionnant pendant le dernier quart du Ier s. ap. J.-C. (Bellon 1995, p. 138-156). Ces structures témoignent de l'utilisation de la nappe aquifère des plateaux, comme l'indiqueraient les différents captages observés dans le talweg de la rue Pierre Audry (Rapports, Gisclon 1995, p. 14 ; Lasfargues 1973). Mais, d'autres exemples suggèrent l'utilisation de l'eau à d'autres fins. Il en est ainsi de l'aqueduc du Chalin, sur le site de l'échangeur de l'autoroute A7 au nord-ouest de la plaine, dont ni la date de construction, ni la vocation ne sont connues. C'est également ce que l'on peut supposer des vestiges installés en bordure d'un talweg 
sur le site des Blanchisseries, bien qu'aucune preuve matérielle n'atteste formellement une occupation artisanale, (Rapport, Rahatsoz 1995, p. 42-45 et 63). Par contre, au centre de la plaine, sur le site de la rue du Docteur Horand, à la fin du Ier s. ap. J.-C., un complexe de biefs et de bassins, creusés directement dans le substrat, se rapporte aux installations d'une meunerie hydraulique (Battiga 1997). L'exploitation agricole de la plaine transparait à travers les analyses palynologiques indiquant la culture de la vigne et de céréales (Bellon 1995, p. 164). Une activité d'extraction du granite des balmes, dans le défilé de Pierre Scize est également supposée avant l'apparition d'installations artisanales associées à des habitats et des entrepôts sur le site du quai Arloing (Tranoy 1995, p. 184-193). Enfin, une grande domus (maison aux Xenia), bâtie vers 25 ap. J.-C. sur le site de la Rhodiacéta, abrite un artisanat de la métallurgie entre les règnes de Claude et de Vespasien (Plassot 1995, p. 94-96).

\section{Le secteur urbanisé et les occupations dispersées au lle s. ap. J.-C.}

L'occupation de la plaine est affectée par de profondes mutations au IIe s. ap. J.-C. qui apparaissent comme un recentrement sur les activités artisanales et commerciales du secteur urbain (fig. 13A). Certes des raisons d'ordre économique ou social peuvent expliquer cette mutation, mais on ne peut pas exclure que des raisons plus pratiques, comme le tarissement des sources ou une modification du régime des rus, aient entraîné l'abandon des points d'occupations isolés, en majorité liés à des artisanats utilisant l'eau.

13-Évolution des occupations de la plaine de Vaise.

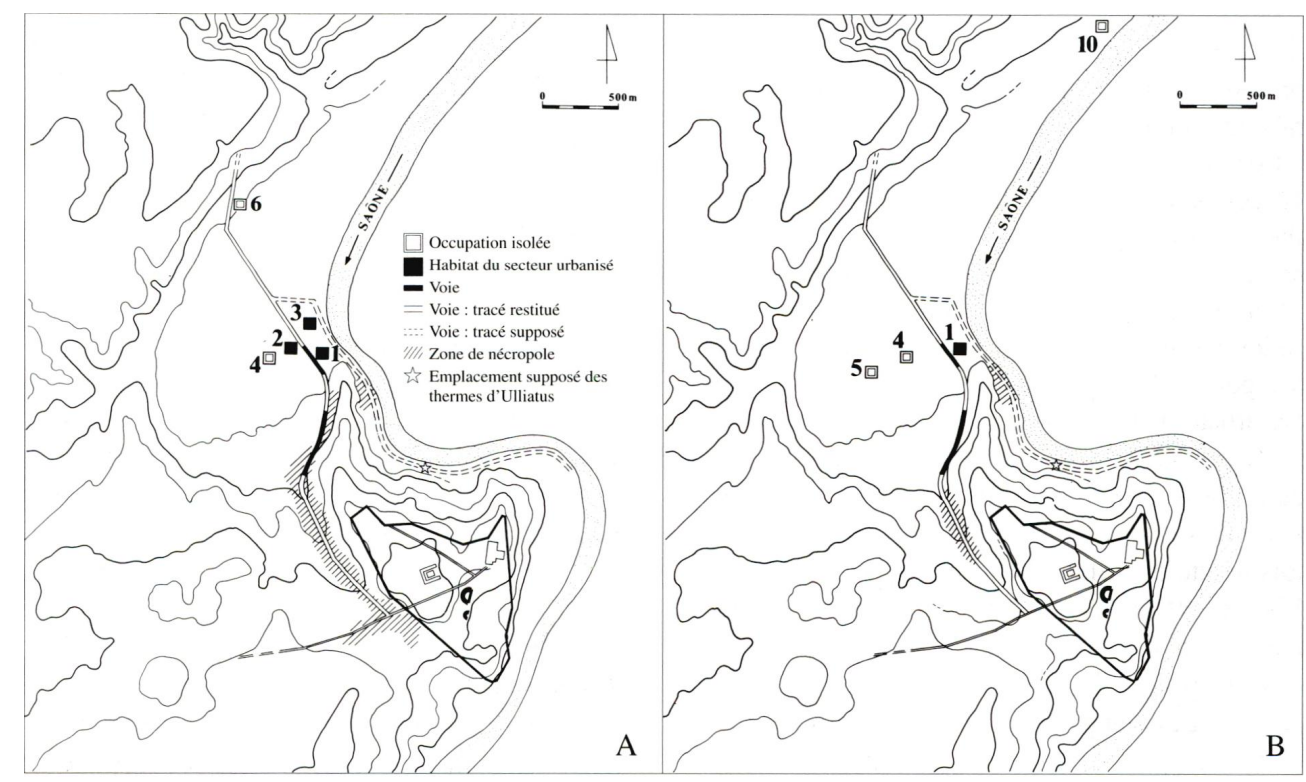
A : au lle s. ap. J.-C.
B : à la fin du llle s. et au IVe s. ap. J.-C.
1 : Charavay
2: Valmy
3 : Marietton
4 : Blanchisserie
5 : Horand
6 : Échangeur de Vaise
10 : Masson 

du Ier s. ap. J.-C., et le début du IIe s. ap, J.-C., excepté toutefois le site des Blanchisseries (Rapport, Rahatsoz 1995, p. 49-53). Au débouché du vallon de la rue Pierre Audry, la domus de la Rhodiacéta est abandonnée après le règne de Vespasien (Plassot 1995, p. 122). Les équipements artisanaux de la station Gorge de Loup le sont également vers 120 ap. J.-C. (Bellon 1995, p. 155-157). Au nord de Charavay, le site de la rue du Docteur Horand est délaissé au plus tard au milieu du IIe s. ap. J.-C. (Rapport, Bellon 1994, p. 38-40 et p. 73-79). D'autre part, au sud, dans le défilé de Pierre Scize, quai Arloing, bien que l'occupation se poursuive, les premières sépultures d'une zone funéraire, qui ne cessera de s'étendre, apparaissent au début du IIe s. ap. J.-C. (Tranoy 1995, p. 231-240).

À l'intérieur du noyau urbain plusieurs reconstructions traduisent la poursuite de l'essor de cette partie de la plaine. Comme le suggère le site de Charavay, un nouveau projet d'urbanisme pourrait être à l'origine des différents travaux. En effet, la reconstruction quasi générale dont fait l'objet le site, à la fin du Ier ou au début du IIe s. ap. J.-C. et l'installation d'un grand collecteur sous la chaussée de la voie nord-sud au même moment, ne sont pas les seuls arguments en faveur d'une telle hypothèse. Ainsi, à Valmy, des travaux de stabilisation et d'assainissement, l'augmentation du nombre des parcelles, leur construction ou reconstruction et leur agrandissement à l'ouest à la même époque vont également dans ce sens (Rapport, Célestin-Le Nezet 1993, p. 59-78). Il est à signaler, hors le cas de Marietton dont l'occupation est trop mal conservée (Rapport, Monin 1995, p. 48 et 60), que les mêmes activités à l'intérieur des parcelles se maintiennent pendant toute cette période.

\section{L'évolution de la plaine de Vaise à la fin de l'Antiquité}

La topographie de l'occupation du IIIe s. ap. J.-C. reprend dans sa forme celle du siècle précédent. Hormis le site des Blanchisseries (Rapport, Rahatsoz 1995, p. 53-63) et le quai Arloing (Tranoy 1995, p. 202-206), la plaine ne présente toujours pas d'autres occupations isolées sur son territoire. Le secteur urbanisé, encore occupé, est en net déclin, voire partiellement abandonné (fig. 13B).

50 Cependant, l'appréciation de l'état réel n'est pas aisée, car tous les sites ont fait l'objet d'arasements. Seul celui de Charavay livre quelques indices en faveur du maintien d'une occupation. Au contraire, le site de Valmy est abandonné et investi par des sépultures à partir du IIIe s. ap. J.-C. (Rapport, Celestin-Le Nezet 1993, p. 79-87). Marietton l'est apparemment également à la fin du IIe s. ap. J.-C., quoique ce site ait livré un antoninien qui pourrait indiquer qu'il est encore fréquenté dans la seconde moitié du IIIe s. ap. J.-C. (Rapport, Monin 1995, p. 60).

$51 \mathrm{Au}$ IVe s. ap. J.-C., la topographie héritée des siècles précédents cède la place à une nouvelle organisation où dominent les zones funéraires des sites du quai Arloing (Tranoy 1995, p. 231-240) et de Valmy (Rapport, Celestin-Le Nezet 1993, p. 79-87). Des sites connus au Ier s. ap. J.-C. sont à nouveau occupés (rue du docteur Horand et impasse Masson). L'occupation du secteur urbain reste difficile à saisir à partir du seul cas de Charavay. L'atelier de potier qui y prend place se situe peut-être en marge d'un noyau désormais réduit ou à l'intérieur d'une vaste zone entièrement dédiée au funéraire. 


\section{Les dépôts}

Les deux dépôts étaient enfouis à l'intérieur et dans l'angle sud-ouest du bâtiment sur rue de la parcelle B (fig. 14), en léger retrait le long du mur ouest. Distincts non seulement par leur position relative, ils le sont également par les objets qui les composent. Sans entrer dans le détail de leur inventaire, le premier dépôt, ou dépôt 1 , est constitué, pour l'essentiel, de statuettes et fragments de statuettes qui sont peutêtre des objets votifs. Le second, ou dépôt 2, peut être qualifié de dépôt d'effets personnels. Il est constitué de bijoux, d'ustensiles de table, de petits objets et d'une bourse qui contenait plusieurs dizaines de monnaies.

Les deux dépôts ont été découverts lors de l'enlèvement d'une berme située contre et sous un mur moderne. Ces travaux, réalisés à l'aide d'un engin mécanique et destinés à compléter l'information déjà recueillie pour les niveaux les plus anciens, n'ont fait l'objet d'aucune disposition particulière, tout au moins jusqu'à la découverte du premier trésor. Bien que celui-ci soit apparu sous le godet de la pelle, on peut considérer qu'aucune de ses pièces ne manque en raison des vérifications d'usage faites dans les déblais. Toutefois, on ne saurait écarter l'éventualité que quelques fragments aient pu échapper à notre vigilance, expliquant ainsi l'état incomplet de quelques-uns des objets. Le second trésor, découvert sous la surveillance d'un archéologue, est au contraire complet et n'a subi aucune atteinte.

14- Situation des deux dépôts dans l'habitat de la parcelle B (état fin ler s. ap. J.-C.)

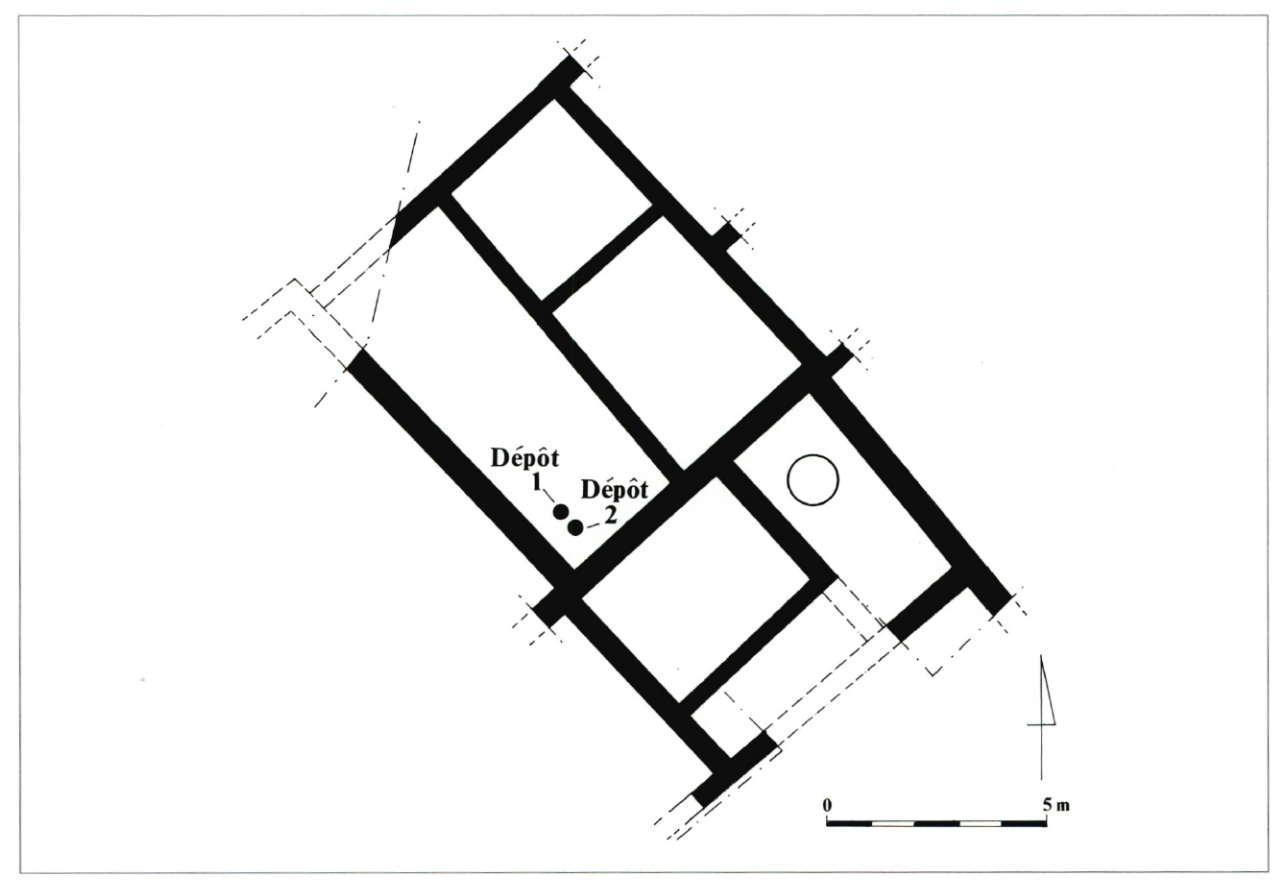

mode d'enfouissement

Les différences dans l'organisation et dans la composition des objets des deux dépôts permettent de supposer que leur enfouissement n'est pas simultané et qu'il n'obéit pas aux mêmes motivations. 


\section{le dépôt 1} aucune trace n'a subsisté, mais que l'organisation des objets atteste. Les statuettes étaient en effet disposées tête-bêche afin de réduire leur encombrement ; dans le même esprit, les autres objets avaient été étroitement imbriqués entre eux et formaient avec les statuettes un tout compact. La position inclinée du dépôt indique une excavation au profil tronconique, comme celle que l'on peut obtenir en creusant un trou avec les mains ou un petit outil de fortune. Le creusement de la cache et l'organisation du dépôt suggéraient un acte exécuté dans la précipitation. Il est également curieux que des objets votifs, auxquels était attaché un respect particulier, aient été traités d'une telle manière.

\section{le dépôt 2}

Lors de la découverte on a observé que les objets du dépôt 2 avaient été réunis à l'intérieur d'un coffre dont le volume était conservé en partie en négatif ${ }^{3}$. Il ne subsistait rien des matériaux qui avaient servi à sa confection si ce n'est quelques pièces métalliques, très oxydées, qui pourraient appartenir au dispositif qui maintenait les parois entre elles. Outre les objets, ce volume était en partie comblé par des matériaux provenant des couches dans lesquelles le coffre avait été installé et qui s'étaient introduites à l'intérieur après décomposition ou rupture du couvercle et des parois. La bourse, qui contenait les monnaies, reposait directement sur le fond, à proximité de quelques menus objets. Les pendants d'oreilles, le collier, les bracelets et un pendentif monétaire étaient posés sur celle-ci en position d'équilibre. À côté de ce premier ensemble, étaient disposés les plats en argent posés les uns sur les autres, et sur lesquels avaient été placées les cuillers regroupées en un seul ensemble (fig. 15). L'organisation, l'ordre et la position d'équilibre des objets indiquent que ceux-ci ont été placés à l'intérieur du coffre après l'installation de celui-ci en terre dans une position parfaitement horizontale. L'enfouissement a été probablement prémédité par l'occupant du bâtiment de la parcelle $B$, avant que ne surviennent des événements dramatiques, ou en prévision de ceux-ci. Le fait qu'il s'agisse de mobiliers et d'objets personnels renforce cette hypothèse. 


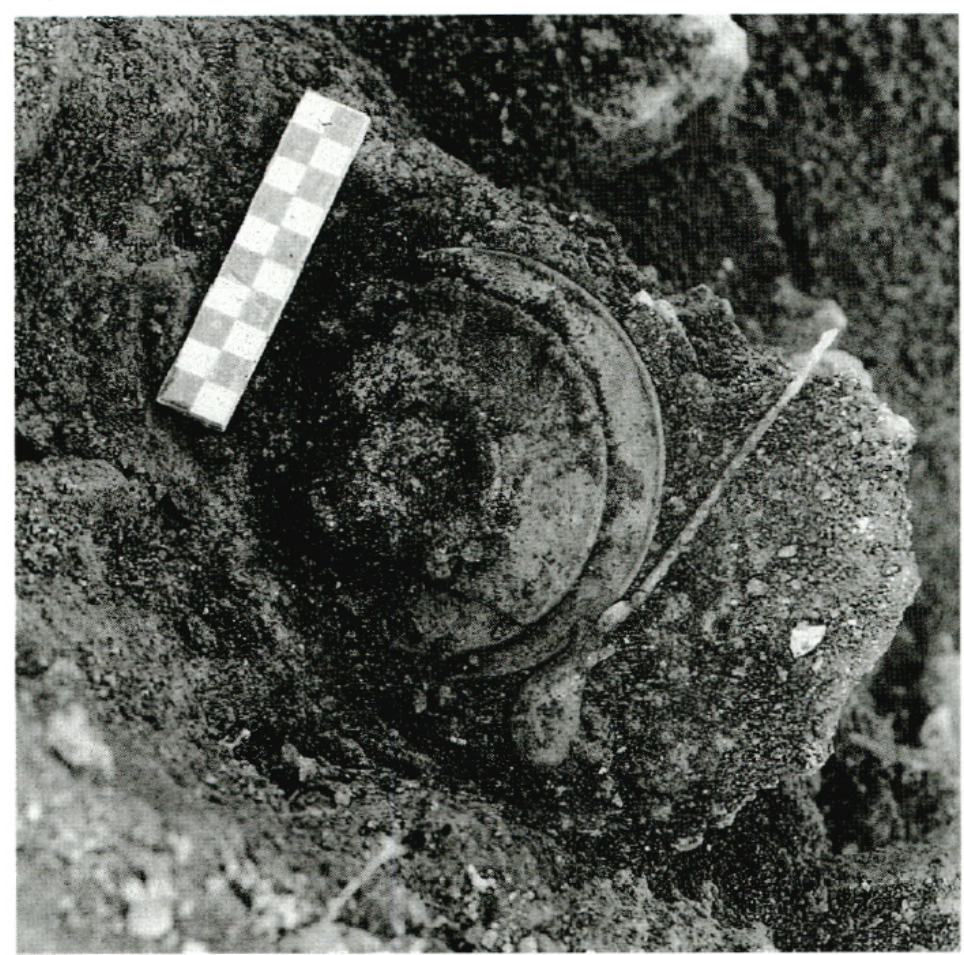

\section{Datation des dépôts}

57 Les deux dépôts ont été découverts sous la première assise de fondation d'un mur moderne qui recoupe le bâti de la fin du Ier-début IIe s. ap. J.-C. de la parcelle B. Ainsi, hormis le fait que le dépôt 1 recoupe la fosse 1161 située directement sous le niveau de décapage, toute autre tentative de raccordement stratigraphique s'avère impossible. Cet obstacle peut être toutefois aisément contourné par les monnaies du dépôt 2 et le mobilier de la fosse 1161 qui permettent d'insérer ces deux dépôts dans les évolutions du site comprises entre la fin du IIIe et le début du IVe s. ap. J.-C. De par sa chronologie, la présentation du mobilier de l'atelier de potier s'impose donc. Toutefois, en l'absence de tout niveau, les deux dépôts ne peuvent être attribués stratigraphiquement ni à l'atelier, ni à l'habitat. Tout au plus peut-on considérer à titre d'hypothèse, en particulier pour le dépôt 2, que les circonstances de son enfouissement et la nature de ses objets plaident pour sa contemporanéité avec la dernière occupation de l'habitat.

\section{Le dépôt 1}

La fosse, recoupée par le dépôt de statuettes, a livré un mobilier en grande partie résiduel, en particulier des sigillées du Ier s. ap. J.-C., de la céramique à paroi fine, ou bien encore les types 7 et 8 d'amphores gauloises (Laubenheimer 1985, p. 385) (fig. 16). Les céramiques les plus récentes appartiennent aux productions d'origine africaine ; parmi celles-ci, une forme Hayes 50 produite à partir de 230 ap. J.-C. situe le comblement de la fosse au plus tôt à cette date. On remarquera également, à l'appui de la chronologie proposée, la forte proportion d'amphores gauloises et la présence du type 4 que l'on rencontre à partir de l'époque flavienne et dont la production et la 
commercialisation se développent très largement jusqu'au IIIe s. ap. J.-C. (Laubenheimer 1985, p. 391).

16- Inventaire du mobilier de la fosse 1161

\begin{tabular}{|c|c|c|c|c|c|c|c|c|c|c|c|c|c|c|}
\hline \multicolumn{2}{|c|}{ Parois. fines } & \multicolumn{3}{|c|}{ Sigillées } & \multicolumn{2}{c|}{ CRA } & Afr & \multicolumn{3}{c|}{ Communes } & \multicolumn{3}{c|}{ Amphores } & Dol \\
\hline Es & Ens & SgIta & SgGs & SgLe & CIB & NI & & Cs & Cc & Cr & Gles & D20 & NI & \\
\hline 1 & 1 & 1 & 18 & 2 & 3 & 1 & 3 & 64 & 41 & 5 & 51 & 4 & 77 & 1 \\
\hline
\end{tabular}

Es : engobe sablé ; Ens : engobe non sablé ; Sglta : sigillées italiques; SgGs : sigillées de Gaule du Sud ; SgLe : sigillées de Gaule du Centre (Lezoux); CRA : céramiques à revêtement argileux; C1B : Claire B; Afr : sigillées et commune africaines; $\mathrm{Cs}$ : commune sombre ; $\mathrm{Cc}$ : commune claire ; $\mathrm{Cr}$ : commune rouge; Gles : gauloise ; D20 : Dressel 20 ; NI : non identifié ; Dol : dolium.

Avec un Service I en italique ; un Drag17a, 24/25, 29, 35/36, deux Drag 18/31 et un Rit 8 en Gaule du Sud ; une forme Desbat 15 (?) en Claire B; une forme Hayes 50 production africaine; deux marmites à lèvre horizontale, quatre ovoïdes à col lisse, un ovoïde à épaule carénée, un ovoïde à col côtelé et quatre écuelles en commune sombre ; un mortier à lèvre pendante en commune claire ; une marmite à lèvre horizontale en commune rouge ; une forme Gauloise 4, 8 et 7 ainsi qu'une forme Dressel 7/11.

\section{Le dépôt 2}

Le dépôt 2 a été enfoui au plus tôt dès le début de la seconde moitié du IIIe s. ap. J.-C. Les monnaies qu'il contenait, au nombre de quatre-vingt une, couvrent en effet une période comprise entre le règne de Vitellius ( $69 \mathrm{ap}$. J.-C.) et le règne conjoint de Valérien et Gallien (253-260 ap. J.-C.). À cette série s'ajoute un aureus de Gordien III, monté en médaillon (238-244 ap. J.-C.) (cf. infra).

\section{Le mobilier de l'atelier de potier}

L'atelier possède un mobilier qui permet de fixer avec une relative précision son fonctionnement dans le temps. Parmi les faits en relation directe avec son installation, la tranchée du four principal a livré un follis de Constantin qui situe l'installation de cet équipement au plus tôt entre 310 et 311 . Par ailleurs, la céramique tardive, mais plus encore la série monétaire contenue dans le comblement de deux fosses de décantation de l'argile suggèrent que son abandon a eu lieu à la fin du IVe ou au début du Ve s. ap. J.-C. (fig. 17). L'occupation du site pendant le fonctionnement de l'atelier est attestée également par des fosses contenant en particulier les formes Lamb. 37 et 45 produites au IVe et Ve s. ap. J.-C.

\section{7- Inventaire du mobilier des fosses de décantation}

\begin{tabular}{|c|c|c|c|c|c|c|c|c|c|c|c|c|c|c|}
\hline \multicolumn{2}{|c|}{ P. fines } & \multicolumn{3}{|c|}{ Sigillées } & \multicolumn{2}{c|}{ CRA } & Afr & \multicolumn{3}{c|}{ Communes } & \multicolumn{3}{c|}{ Amphores } & Dol \\
\hline Es & Ens & SgIta & SgGs & SgLe & CIB & Tard & & Cs & Cc & Cr & Gles & D20 & NI & \\
\hline & & & 5 & & 1 & 2 & & 101 & 7 & 1 & & & 9 & \\
\hline
\end{tabular}

Ibidem que le tableau 16 sauf pour la céramique tardive (Tard : céramique à revêtement argileux de la fin de l'Antiquité).

Avec deux Drag 29 et un Drag 35/36 en Gaule du Sud ; une forme non identifiée de cruche à bec tréflé en Claire $B$; une forme non identifiée en CRÀ tardive ; une marmite à lèvre horizontale, un ovoïde à épaule carénée, un ovoïde à col lissé, deux formes de vases globulaires à décor lissé en trois registre sur panse et une assiette en commune sombre.

61 Il faut également signaler une série monétaire importante comprenant une imitation frappée entre 270 et 300, un antoninien de Claude II le Gothique (268-270), un moyen bronze Ae 2 de Magnus Maximus (383-387), un petit bronze Ae 3 de Gratien (367-375), 
un second petit bronze du même empereur, et enfin un petit bronze Ae 3 frappé entre 350 et 400 .

\section{Le traitement de conservation-restauration}

Les quatre ensembles distincts constituant le trésor de Vaise présentaient des états de conservation bien différents. Chaque lot a fait l'objet d'examens préalables afin de déterminer le traitement le plus adapté à appliquer.

\section{La statuaire}

L'ensemble de statuettes était le plus gravement dégradé au moment de la découverte. Les pièces creuses, réalisées à partir de minces tôles d'argent battues travaillées au repoussé et assemblées par soudure, n'avaient pas résisté aux contraintes de l'enfouissement; elles nous sont parvenues disloquées et brisées.

\section{Examen préalable}

Après un travail préliminaire de tri des nombreux fragments et la recherche des assemblages, les différentes pièces ont pu être individualisées. Un examen précis des socles et des traces de soudure qu'ils portaient a permis de les associer sans doute possible à la statuette correspondante. Huit menus fragments isolés et illisibles recollaient entre eux pour reconstituer la tête barbue d'un Jupiter.

Le matériau constitutif des statues est un argent vraisemblablement très bien titré - les dépôts de corrosion verts du cuivre étaient rares -, mais aussi profondément corrodé. La surface de tous les fragments était à peu près uniformément recouverte d'une couche granuleuse gris foncé de produits de corrosion de l'argent (oxydes, sulfures, mais aussi chlorures responsables d'une dégradation plus grave dite "argent corné"), d'une épaisseur de 1 à $2 \mathrm{~mm}$ alors que la tôle d'origine ne dépassait pas ellemême le millimètre (fig. 18). 
18- Buste impérial, avant traitement

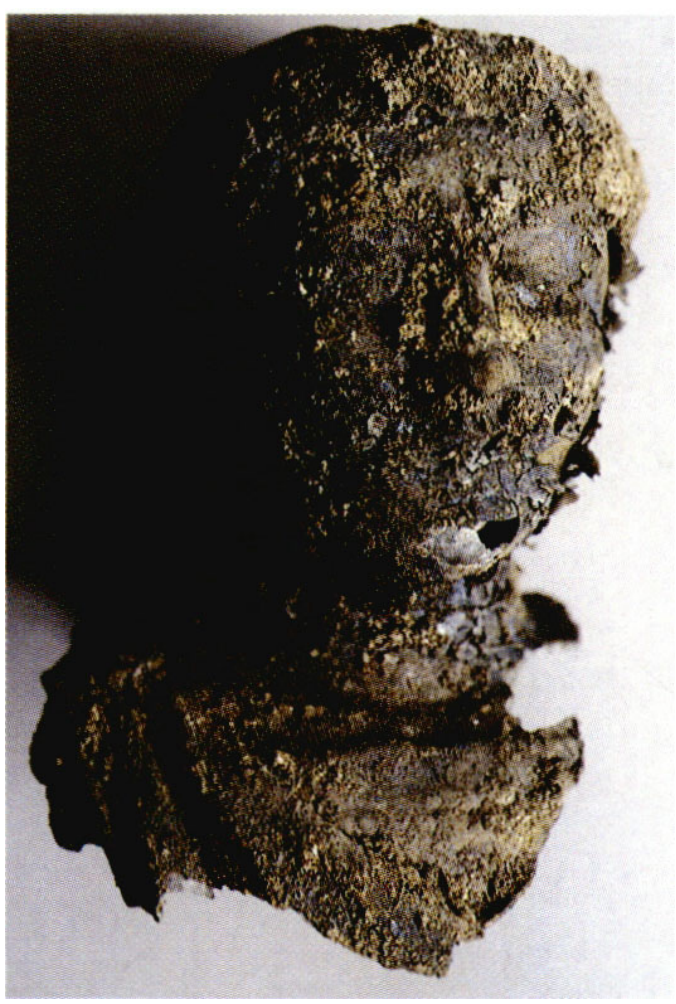

\section{Traitement}

Plusieurs procédés visant à éliminer la couche de corrosion pour retrouver la surface d'origine ont été testés sur de petits fragments : le dégagement au scalpel risquait de rayer la surface sous-jacente plus tendre que les dépôts ; quant aux nettoyages chimiques qui agissent sans distinguer entre les produits de corrosion externes et ceux présents au sein du matériau, ils faisaient perdre à la tôle d'argent toute cohésion interne.

C'est un procédé mixte, associant méthodes électrochimique et manuelle, qui a donné les meilleurs résultats. Les fragments ont d'abord subi un traitement de réduction électrolytique ${ }^{4}$, ce qui a rendu la couche de corrosion externe moins compacte et moins adhérente. Le dégagement au scalpel a ensuite pu être pratiqué aisément sous loupe binoculaire, sans que la surface d'origine ne soit lésée.

Les dorures des vêtements, drapés ou socles, masquées par la corrosion de l'argent ont été nettoyées à l'aide de compresses chimiques.

Le plus souvent, seul l'extérieur a été dégagé. La couche de corrosion qui subsiste au revers reste un renfort utile alors que la mince tôle d'origine est tellement fragilisée. Les parties comme les drapés, visibles et donc nettoyés des deux côtés, sont d'une minceur et d'une faiblesse extrêmes.

Les remontages et consolidations par entoilage du revers ont été effectués avec des résines acryliques ou époxy sur tissu de verre. Quelques comblements limités ont 
été nécessaires pour renforcer des parties trop lacunaires. Enfin, les statues ont été recollées sur leur socle à l'aide d'un adhésif réversible.

\section{Remarques technologiques}

Les interventions de restauration sont souvent l'occasion d'observer les détails des techniques de fabrication. Ainsi, les petits éléments - pieds de statues ou avantbras avec leurs cornes d'abondance et autres attributs - sont pour la plupart en argent massif. Ils ont été coulés à part avant d'être assemblés au membre creux. Un liseré noirâtre correspondant à des restes de soudure oxydés marque nettement l'endroit de la jonction à la cheville ou au bras. La même ligne noirâtre a pu être observée le long de la plupart des bras et des jambes. Elle indique là aussi l'endroit où le tube constituant le membre a été fermé sur lui-même par soudure.

La statue de Fortuna présentait, quant à elle, sur toute sa hauteur côté gauche, une ligne verticale de sels verts de cuivre. C'est donc une brasure au cuivre qui a dû être appliquée ici, les autres soudures étant plus vraisemblablement constituées d'un alliage à base d'argent, plomb, étain ${ }^{5}$.

Des gros tenons en alliage cuivreux, des remplissages en plomb dont la corrosion a d'ailleurs provoqué des poussées et des dommages sur la tôle d'argent, garnissent fréquemment les bras et les épaules pour aider à l'assemblage de ces parties plus lourdes sur les corps creux.

Les radiographies $\mathrm{X}$ effectuées montrent l'existence à l'intérieur des têtes de tiges métalliques dont la fonction ne peut être précisée : servaient-elles au maintien de la tête sur le corps, ou à la fixation d'éléments rapportés comme les rayons d'une couronne solaire pour Apollon, le diadème et le collier serpentiforme de la Fortuna?

\section{La vaisselle}

Deux types de produits de corrosion pouvaient être observés sur les différentes pièces d'argenterie : des dépôts verts de sels de cuivre d'une part, de l'autre des produits granuleux gris-violacé caractéristiques de l'argent corrodé (chlorures et/ ou sulfures d'argent).

La présence de cuivre allié avec l'argent répond à une nécessité technique : l'argent pur est un métal trop malléable pour la fabrication d'objets, même d'apparat ; l'adjonction d'une petite quantité de cuivre en augmente considérablement la dureté.

L'argent corrodé perd son élasticité et devient cassant. La grande coupe au Mercure, quelques cuillers également présentaient fissures, fractures et lacunes.

Ce sont des procédés de nettoyage chimique qui ont été appliqués à ce lot ; dans un premier temps un bain dans une solution d'acide formique pour toutes les pièces portant des sels de cuivre, puis un traitement au thiosulfate d'ammonium pour éliminer cette fois les dépôts de corrosion d'argent. Ces nettoyages chimiques sont conduits conjointement avec des dégagements mécaniques au scalpel, des 
brossages et des polissages doux. De fréquents examens sous loupe binoculaire permettent de contrôler la progression du travail.

Pour ne pas fragiliser davantage la coupe au Mercure, profondément corrodée et cassante, le nettoyage du revers a été laissé inachevé, au profit d'un dégagement complet de l'intérieur et de son médaillon gravé. L'extérieur a conservé ses dépôts d'oxydes d'argent noirs (fig. 19).

19- La coupe au Mercure en cours de nettoyage

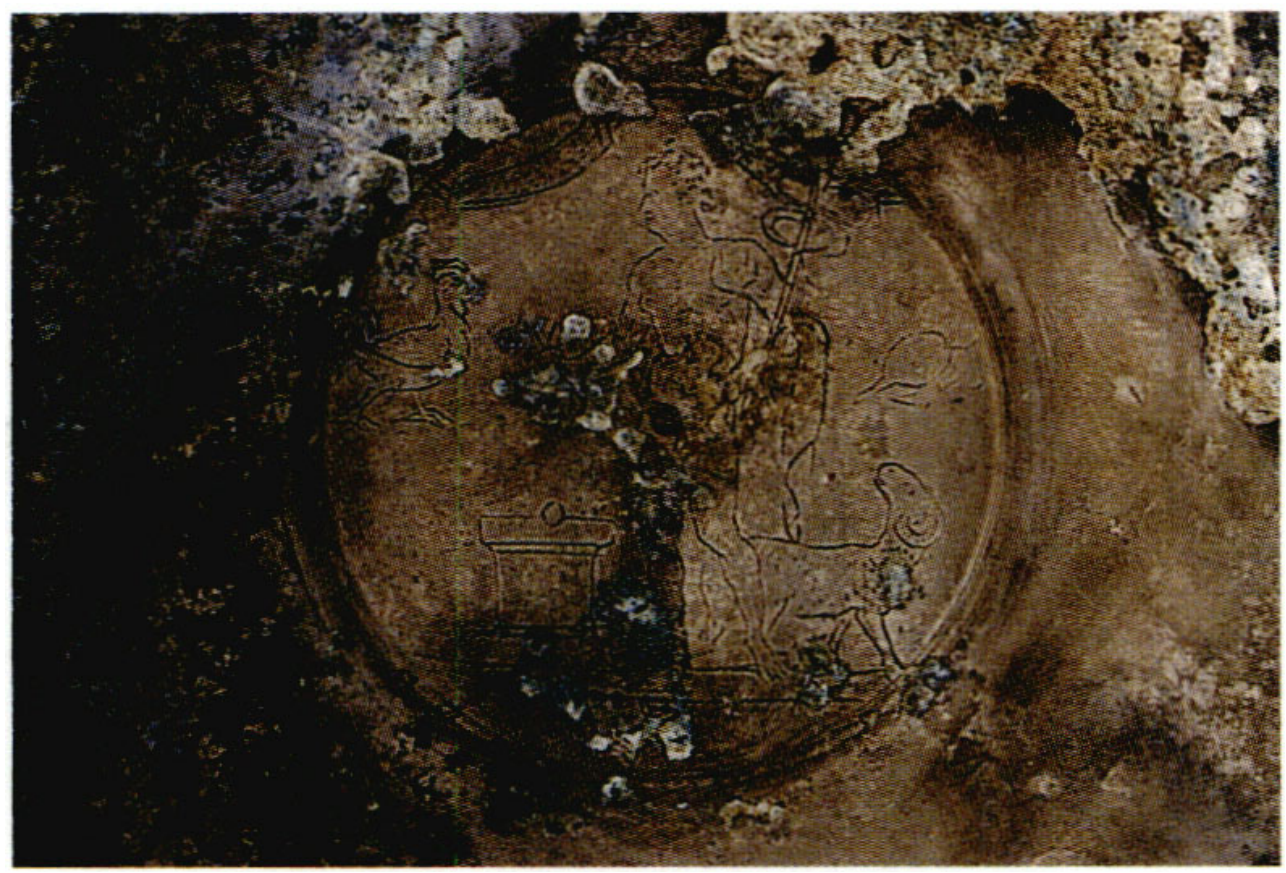

Le plus petit plateau, constitué d'une âme en alliage de cuivre plaquée d'une feuille d'argent décorée au repoussé, a posé un problème spécifique. Le cuivre s'est corrodé ; la feuille d'argent couverte de sels est soulevée, fissurée et repose en fait sur un espace vide. Dégager la surface sans l'écraser ou la transpercer a été particulièrement délicat et l'aspect en est moins flatteur que celui des objets massifs. Le traitement des pièces les plus altérées est toujours une recherche de compromis entre les nécessités du nettoyage et le souci de ne pas fragiliser davantage l'objet.

\section{Les bijoux}

L'or, qui est inaltérable dans tous les milieux naturels, apparaissait intact sous quelques concrétions brunâtres provenant du sol. Les seuls dommages subis étaient d'ordre mécanique. Certains anneaux ou éléments d'articulation des pendants d'oreille étaient tordus, ouverts ou cassés. L'une des extrémités de pendeloque manquait. Un bracelet torsadé était déformé, avec une déchirure qui a d'ailleurs permis d'observer la manière dont le bijou était fabriqué : huit fils d'or torsadés sont enroulés sur un tube central. 
Plus généralement, à l'exception des deux bagues qui sont massives, les éléments en or sont creux. Ils ont été réalisés à partir de minces feuilles d'or battues et roulées sur elles-mêmes, dans le souci d'économiser le matériau précieux.

Un autre détail technique intéressant a pu être remarqué : la réparation antique de certains maillons du collier, par rajout d'une plaquette d'or.

Les dépôts ont été nettoyés délicatement à l'aide d'alcool et de pointes en matériaux tendres afin de ne pas rayer la surface de l'or. Les anneaux de suspension endommagés des boucles d'oreille ont été reconstitués en résine transparente ; les perles de nacre, très pulvérulentes, imprégnées d'un consolidant. Les déformations subies ont été respectées : l'or est trop fragilisé pour être détordu sans se déchirer davantage.

\section{Les monnaies}

Elles ont été retrouvées agglomérées entre elles par des produits de corrosion de couleur verte, caractéristiques des sels de cuivre.

On sait que le titre des monnaies d'argent varie beaucoup avec les fluctuations économiques et que la proportion de cuivre dans l'alliage est souvent importante. Moins noble que l'argent, le cuivre se corrode préférentiellement, ce qui explique la présence d'une volumineuse corrosion de cuivre à la surface des monnaies.

C'est un procédé de nettoyage chimique qui a été appliqué ici. Un bain de courte durée dans une solution d'acide formique dilué, qui dissout tous les sels de cuivre sans attaquer l'argent et ses composés, a permis de désolidariser les monnaies et de dégager l'essentiel des dépôts de corrosion pour retrouver la surface d'argent restée métallique.

Les nettoyages chimiques toujours suivis de rinçages prolongés, doivent être conduits avec précautions car si l'argent est chimiquement intact, il a perdu beaucoup de sa cohésion physique avec l'élimination des composés de cuivre qui lui étaient intimement liés dans l'alliage initial. Dans le cas des monnaies les moins bien titrées, le traitement a été interrompu plus tôt pour préserver la surface affaiblie et pulvérulente ; le dégagement a alors été achevé manuellement, au scalpel sous loupe binoculaire.

Les patients traitements appliqués au trésor de Vaise ont permis de le rétablir dans son intégrité, en répondant au triple objectif que doit poursuivre toute intervention de conservation-restauration : rendre sa lisibilité à l'objet, lui restituer une cohésion suffisante, stabiliser les processus de dégradation du matériau. 
20- La statuette de la déesse aux oiseaux en cours de dégagement

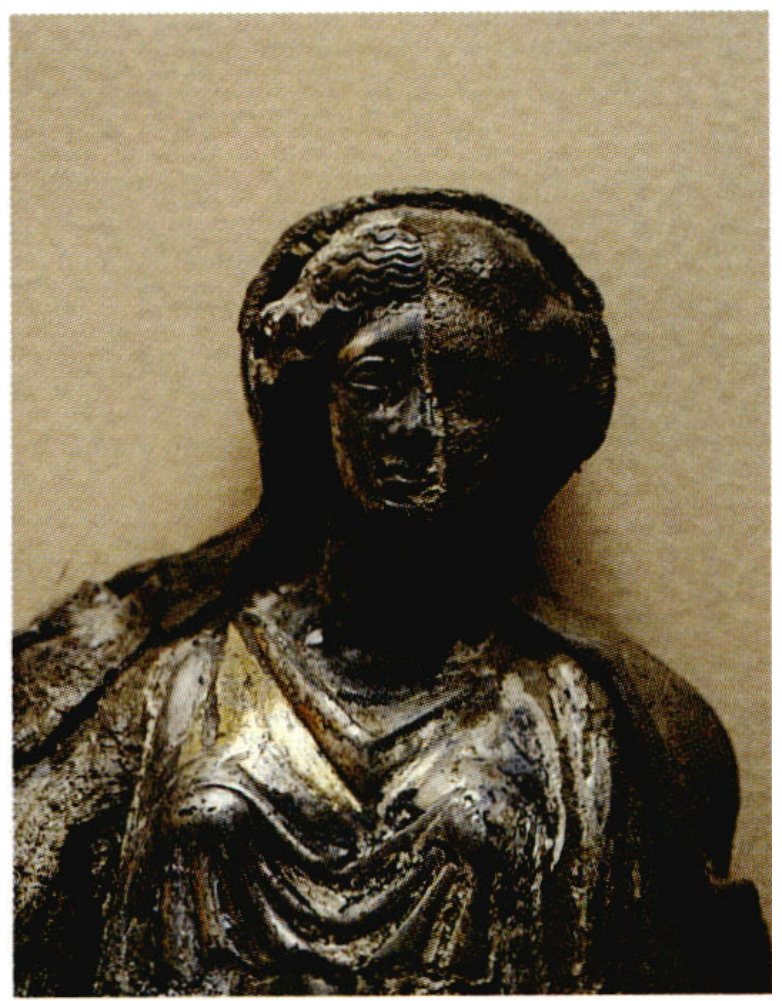

Comparé aux traitements souvent ingrats que le restaurateur mène sur les métaux moins précieux, ce travail prend un caractère particulièrement spectaculaire (fig. 20). L'éclat restitué à l'argent ferait presque oublier la réalité matérielle des objets qui, sous une apparence de splendeur retrouvée, restent très fragiles et sont profondément altérés dans leur constitution physique et chimique.

Atelier de restauration C.R.E.A.M. G. Chapotat, 11 rue du 24 avril 1915

Estressin, Vienne 38200

\begin{tabular}{|c|c|}
\hline DÉPÔT1 & DÉPÔT 2 \\
\hline 20 Buste masculin, argent & 1 Coupe, argent \\
\hline Inv. $n^{\circ} 93110425$ & Inv. $\mathrm{n}^{\circ} 9311041$ \\
\hline 21 Statuette d'Apollon-Hélios, argent doré & 2 Petit plateau à marli, bronze plaqué argent \\
\hline Inv. n 93110420 & Inv. nº 9311043 \\
\hline 22 Statuette de Fortuna, argent doré au feu & 3 Petit plateau à marli, argent \\
\hline Inv. n93110421 & Inv. nº 9311042 \\
\hline $\begin{array}{l}23 \text { Statuette d'une déesse aux oiseaux argent doré } \\
\text { au feu }\end{array}$ & 4 à 17 Lot de 14 cuillers, argent \\
\hline Inv. $n^{\circ} 93110422$ & Inv. nº 9311044 à 17 \\
\hline
\end{tabular}




\begin{tabular}{|c|c|}
\hline $\begin{array}{l}24 \text { Tête de divinité, argent } \\
\text { Inv. n } 93110424\end{array}$ & $\begin{array}{l}18 \text { Manche et anneau, argent } \\
\text { Inv. n'931104 } 18\end{array}$ \\
\hline $\begin{array}{l}25 \text { Tête masculine, argent } \\
\text { Inv. } n^{\circ} 93110423\end{array}$ & $\begin{array}{l}\text { 19 Tige avec « marteau », argent } \\
\text { Inv. nº } 93110419\end{array}$ \\
\hline $\begin{array}{l}26 \text { Aile, argent } \\
\text { Inv. } n^{\circ} 93110426\end{array}$ & $\begin{array}{l}35 \text { Collier, or et émeraudes } \\
\text { Inv. } n^{\circ} 9312013\end{array}$ \\
\hline $\begin{array}{l}27 \text { Fragment de couronne tourelée, argent } \\
\text { Inv. } n^{\circ} 93110427\end{array}$ & $\begin{array}{l}36 \text { Paire de bracelets, or } \\
\text { Inv. } n^{\circ} 9312018 \text { et } 9\end{array}$ \\
\hline $\begin{array}{l}28 \text { Lunule, argent doré } \\
\text { Inv. n } 93110428\end{array}$ & $\begin{array}{l}37 \text { Paire de pendants d'oreilles, or et pierres } \\
\text { fines } \\
\text { Inv. } n^{\circ} 9312016 \text { et } 7\end{array}$ \\
\hline $\begin{array}{l}29 \text { Bras gauche l'index tendu, argent } \\
\text { Inv. } n^{\circ} 93110430\end{array}$ & $\begin{array}{l}38 \text { Paire de pendants d'oreilles, or et pierres } \\
\text { fines } \\
\text { Inv. } n^{\circ} 9312014 \text { et } 5\end{array}$ \\
\hline $\begin{array}{l}30 \text { Bras droit tenant une patère, argent } \\
\text { Inv. } n^{\circ} 93110429\end{array}$ & $\begin{array}{l}39 \text { Bague, or } \\
\text { Inv. } n^{\circ} 93120110\end{array}$ \\
\hline 31 Fragment de bras : coude, argent & $\begin{array}{l}40 \text { Bague à intaille, or et nicolo } \\
\text { Inv. n } 93120111\end{array}$ \\
\hline 32 Fragments de feuilles, argent & $\begin{array}{l}41 \text { Médaillon monétaire, or } \\
\text { Inv. } n^{\circ} 93120112\end{array}$ \\
\hline $\begin{array}{l}33 \text { Bracelet torsadé, argent } \\
\text { Inv. } n^{\circ} 9312012\end{array}$ & $\begin{array}{l}42 \text { Lot de } 81 \text { monnaies, argent, } \\
\text { Inv. } n^{\circ} 9311051 \text { à } 81\end{array}$ \\
\hline $\begin{array}{l}34 \text { Bracelet serpentiforme, argent } \\
\text { Inv. } n^{\circ} 9312011\end{array}$ & \\
\hline
\end{tabular}

\section{Ces objets sont conservés au Musée de la civilisation gallo-romaine, 17 rue} Cleberg 69005 Lyon

\section{NOTES}

1. Les terrains étaient situés des $\mathrm{n}^{\circ} 1$ à 17 de la rue du Chapeau Rouge (Lyon 69009). Ils couvraient une superficie de $3000 \mathrm{~m}^{2}$, dont seulement $2000 \mathrm{~m}^{2}$ ont été fouillées. Ce site est aujourd'hui occupé par les immeubles 1 à 9 de la rue du Chapeau Rouge. 
P. Jacquet (AFAN), était responsable de secteur; Woijtek Widlak (AFAN), responsable de l'étude de la céramique antique. Nous remercions A. Audra et P. Mathey (bénévoles) qui ont effectué l'étude des monnaies isolées du site.

2. La valeur habituelle du pied romain est de $0,2957 \mathrm{~m}$ (0,296 $\mathrm{m}$ en chiffre rond).

3. Long. $25 \mathrm{~cm}$, larg. $21 \mathrm{~cm}$, profondeur conservée $20 \mathrm{~cm}$.

4. Électrolyse dans une solution potassique; les sulfures d'argent se réduisent à un potentiel d'environ - $1300 \mathrm{mV}$ ESS, les chlorures d'argent d'environ - $400 \mathrm{mV}$ ESS. Les traitements électrolytiques sont menés au C.R.E.A. de Vienne avec l'aide matérielle et scientifique du Laboratoire EDF-VALECTRA, mécénat technologique et scientifique.

5. Michael Hughes et al.: Technologie de l'argenterie romaine, In: Trésors d'orfevvrerie gallo-romains 1989 , p. $26(21-28)$. 
Catalogue raisonné du trésor de vaise 


\section{La vaisselle du trésor de Vaise}

\section{François Baratte}

21- Le trésor de Vaise.

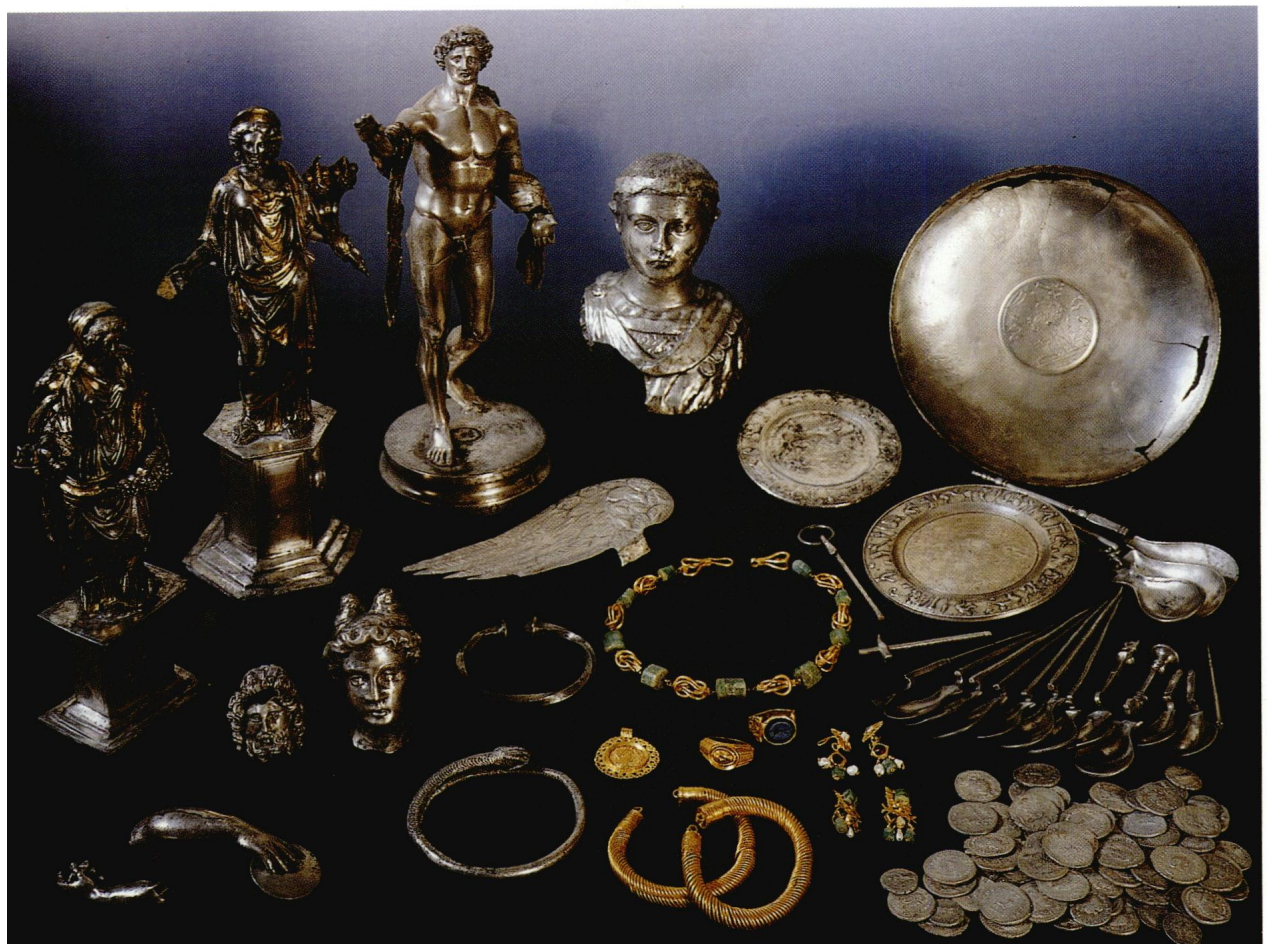

À gauche, les objets du dépôt 1, à droite ceux du dépôt 2

\section{Coupe}

Dimensions et état de conservation

Inv. 9311041

Argent. 
Diam. $20,3 \mathrm{~cm} ; \mathrm{h} .2,5 \mathrm{~cm}$; diam. du pied $6,5 \mathrm{~cm} ; \mathrm{h}$. du pied 0,3 cm environ; diam. du médaillon central $6 \mathrm{~cm}$.

Poids 203,30 g.

2 Nous n'avons pas relevé de graffiti. L'état de conservation n'est pas excellent. Le métal est fragile et la coupe présente deux lacunes importantes le long du rebord, d'où partent de nombreuses fissures, consolidées à l'araldite (voir rapport de restauration).

\section{Description}

3 Coupe basse très ouverte (fig. 22 à 23). À l'intérieur du pied et du médaillon, de même diamètre, le fond est plat. Puis les parois se développent pour former une calotte au galbe régulier. Le pied annulaire est bas et assez large ; le revers est lisse.

22- Coupe $\mathrm{n}^{\circ} 1$ au Mercure, diam. 20,3 cm

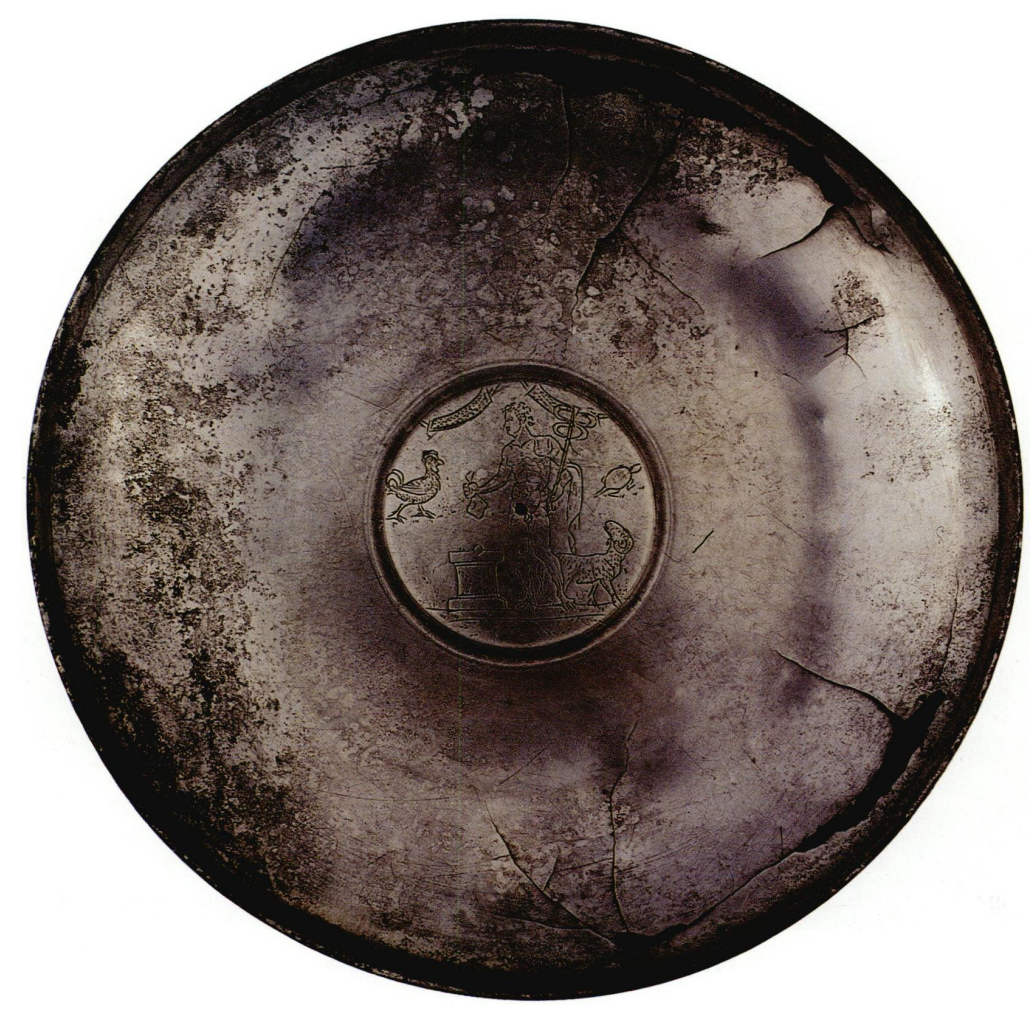

23- Coupe $n^{\circ} 1$ au Mercure, profil

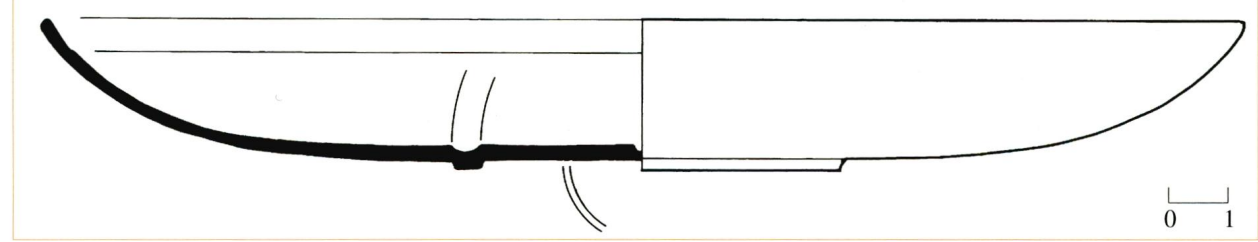

4 Traces de polissage au tour exécuté après la réalisation du décor figuré : le pointeau a laissé une trace profonde sur la face, au centre, qui empiète sur l'anatomie de Mercure, tandis que les contours du médaillon rognent un des sabots du bélier et la tête de la 
tortue ; au revers deux cercles concentriques (diam. 2,6 cm) correspondent à la fixation du tour.

5 La partie intérieure est lisse, à l'exception d'une ligne gravée qui souligne le rebord et de la partie centrale. La première délimite la lèvre $(\mathrm{h} .0,8 \mathrm{~cm})$ qui ne distingue pas autrement. Une moulure en creux entre deux rainures, correspondant à l'emplacement du pied, cerne le médaillon qui présente un décor figuré entièrement gravé (fig. 24). À l'intérieur d'un cadre abstrait constitué par le cercle du médaillon à la partie supérieure duquel sont accrochées deux guirlandes, Mercure est représenté debout sur une double ligne de sol. Le dieu est nu, la partie inférieure du corps de face, le torse de trois quarts, la tête de profil vers la droite ; sa chlamyde est passée sur l'épaule gauche et enroulée autour du bras. La tête nue est coiffée en courtes mèches. La main droite étendue tient à poignée la bourse. $L a$ main gauche tient un grand caducée dans la saignée du coude.

6 À la droite du dieu, au sol, se dresse un autel sur les flancs duquel se croisent deux guirlandes et sur lequel est posée une pomme de pin (?). À sa gauche, derrière lui, un bélier est debout vers la droite, la tête levée et retournée vers Mercure. À mi-hauteur, à droite, une tortue se dirige vers l'extérieur du médaillon, à gauche un coq est en marche vers le dieu. Les deux animaux reposent sur un sol constitué par des séries de courtes hachures juxtaposées par groupe de deux ou trois, que l'on retrouve à l'intérieur de la bande de sol sur laquelle se dresse le dieu (où elles dessinent des herbes) et sur l'autel de part et d'autre de la pomme de pin.

24- Coupe $n^{\circ} 1$ au Mercure, le médaillon

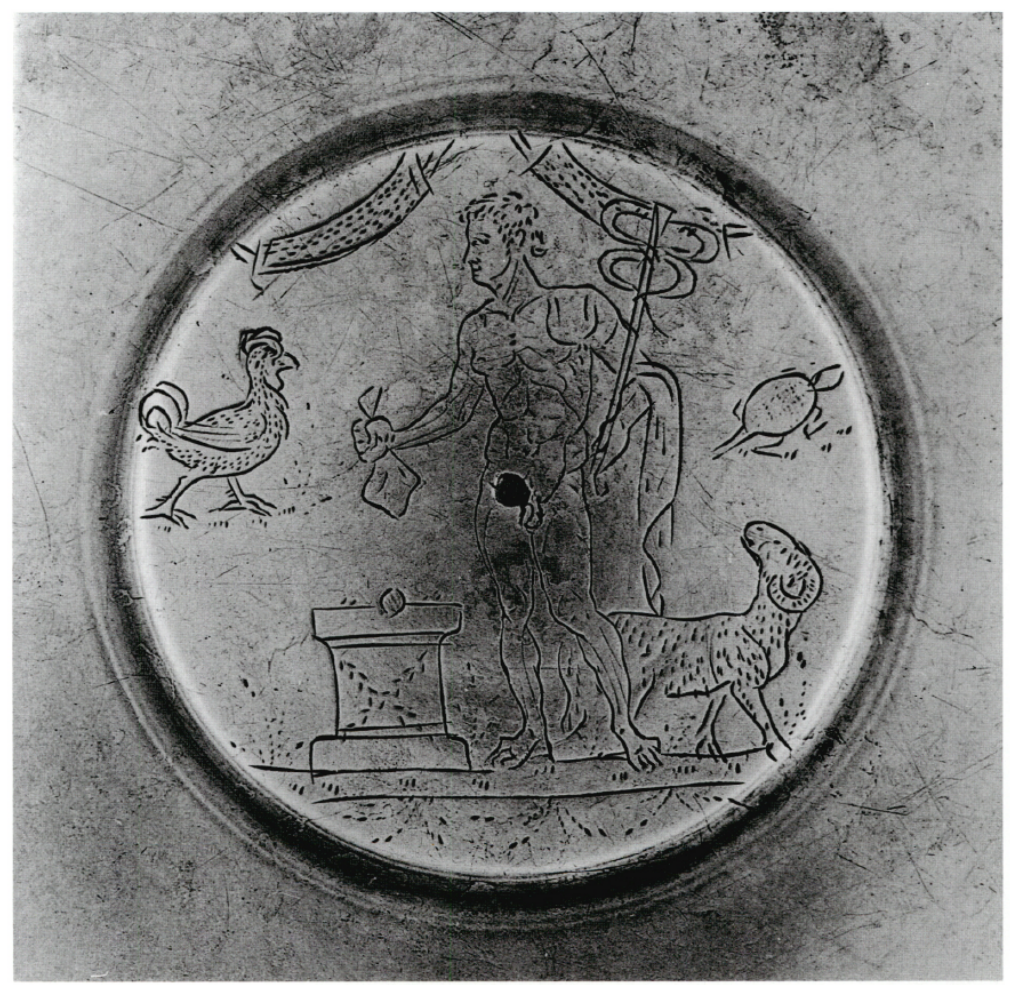




\section{Analyse stylistique et commentaire iconographique}

7 D'une manière générale le décor est caractérisé par la multiplication des lignes hachurées qui remplissent les guirlandes supérieures, dessinent le plumage du coq et la toison du bélier, les guirlandes sur l'autel, des touffes d'herbe très schématiques au sol ou des festons à l'exergue. La carapace de la tortue est suggérée par de fins pointillés. Mercure est dessiné très fermement par des contours nettement incisés; sa musculature est indiquée avec soin par des lignes plus ou moins profondes.

Prise individuellement chaque figure est bien campée, sauf la tortue, assez caricaturale. Mais la composition est médiocre: elle ne tient aucun compte des proportions des différents éléments les uns par rapport aux autres; on la comparera, pour mieux en saisir par contraste les caractéristiques, au médaillon d'une des coupes de Berthouville dont la disposition d'ensemble n'est pas sans analogie (Babelon $1916 \mathrm{n}^{\circ} 18$; Trésors d'orfêvrerie, $\mathrm{n}^{\circ}$ 21) : le dieu est dans la même attitude et le même costume (fig. 25); il est flanqué d'une tortue et d'un coq qui sont tous deux posés sur des cippes; le bélier est remplacé par un bouc, l'autel n'existe pas (l'un des cippes occupe sa place) et un arbre complète à gauche la scène. La composition, malgré quelques variantes, est bien évidemment la même, et remonte à un modèle identique. S. Boucher (Boucher 1976, p. 114) a bien mis en évidence la parenté de la figure du dieu avec celle qui apparaît sur un tambour de colonne sculpté de l'Artémision d'Éphèse, exécuté à la fin du IVe s. av. J.-C. (conservé au British Museum. Une bonne reproduction dans J. Charbonneaux, R. Martin, F. Villard, Grèce classique, Paris, 1969, fig. 256) et sur laquelle Hermès ramène Alceste des enfers (c'est tout au moins une des interprétations qui a été proposée) : la position du corps de Mercure, la tête levée, le geste des deux bras en dérivent manifestement. Le traitement de la musculature, exprimée sur les deux médaillons d'argent avec des moyens très différents, un relief très sculptural à Berthouville, un dessin gravé à Vaise, pourrait même être un souvenir du relief de marbre. Mais des modifications non négligeables avaient été apportées au schéma d'origine. Le bras gauche était d'abord passé derrière le dos, appuyé sur les reins, drapé dans la chlamyde; dans la version romaine, la main droite, qui tenait le kerykion, le bâton du héraut, est plus étendue et présente la bourse ; le caducée est passé de l'autre côté. Le réalisateur de cette nouvelle figure a ajouté en outre les animaux. Le jeune éphèbe de Berthouville conserve encore le souvenir précis du mouvement qui animait le dieu sur la colonne d'Éphèse ; il est à Lyon parfaitement statique. 


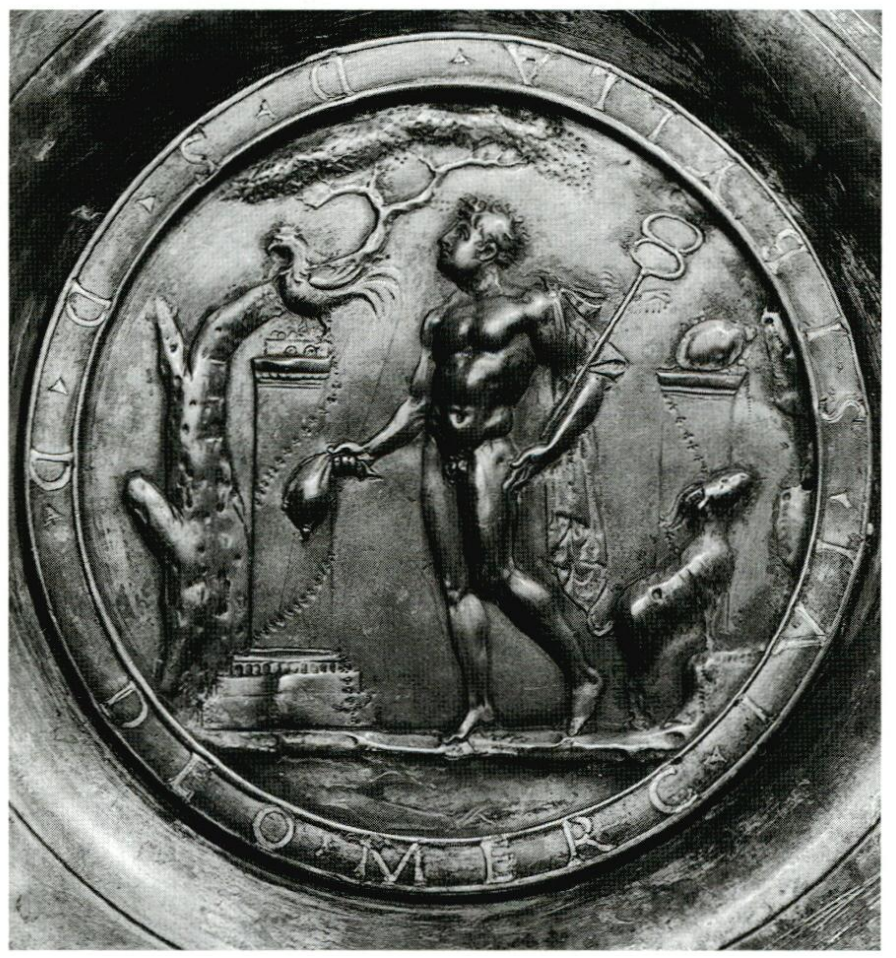

Paris, B.N.F. Cl. du musée

9 Sur une autre coupe de Berthouville (Babelon 1916, $\mathrm{n}^{\circ}$ 16), le dieu est assis, et porte dans la chevelure deux petites ailes, absentes sur les deux pièces d'orfèvrerie déjà citées, un détail qui souligne leurs liens avec le modèle grec. Les trois animaux, coq, tortue et bouc, sont encore présents, comme l'autel et sa pomme de pin, placés cette fois à droite.

On n'insistera pas ici sur le succès de Mercure en Gaule, où plus de 200 représentations sont connues; il a déjà été souligné à maintes reprises (Boucher 1976; B. Combet Farnoux, dans Les dieux de la Gaule romaine, 14 avril - 15 juin 1989, Luxembourg, p. 55-57). Nous rappellerons seulement qu'il apparaît sous deux formes différentes : en pied, comme ici, ou bien trônant sur un rocher, les jambes croisées, la main droite appuyée sur le caducée, la gauche tenant la bourse et reposant sur le genou, une transcription de la statue colossale en bronze, création fameuse au milieu du Ier s. ap. J.-C. du sculpteur et orfèvre Zénodore pour la sanctuaire des Arvernes au Puy-de-Dôme (Pline l'Ancien, Histoire naturelle, 34, 45-47).

11 L'argenterie fournit un certain nombre de représentations de Mercure, dont nous donnons ici l'inventaire :

1. Lyon-Vaise. Médaillon de coupe. Mercure debout de trois quarts. Décor gravé (fig. 24).

2. Berthouville (Eure). Emblema de coupe. Mercure debout de trois quarts. Décor en relief (Babelon 1916, $\mathrm{n}^{\circ}$ 16) (fig. 25).

3. Berthouville. Emblema de coupe. Mercure assis. Décor en relief (Babelon 1916, $\mathrm{n}^{\circ}$ 18).

4. Berthouville. Emblema de coupe. Mercure et Maia (?) en buste sur un fleuron d'acanthe au-dessus d'un caducée. Décor en relief (Babelon 1916, nº 17 ; Trésors d'orfevrerie, $\mathrm{n}^{\circ} 23$ ). 
5. Berthouville. Emblema de coupe. Mercure debout de face, appuyé sur le caducée. Décor en relief (Babelon 1916. $\mathrm{n}^{\circ}$ 19).

6. Berthouville. Sur le manche d'un simpulum. Mercure debout de trois quarts. Décor en relief (Babelon 1916, $n^{\circ} 12$ ).

7. Sans provenance connue. Turin, Museo di Antichità, $n^{\circ}$ 54231. Patère. Au sommet du manche, buste de Mercure; sur le fond, corne d'abondance, autel et pomme de pin, tortue. bourse et caducée. Décor en relief (Ori e argenti dell'Italia antica, Turin, Palazzo Chiablese, juin-août 1961, n 689).

8. Sans provenance connue. Turin, Museo di Antichità, $n^{\circ} 5424$. Patère. Au sommet du manche, buste de Mercure avec pétase et caducée. Décor en relief (Ori e argenti, $\mathrm{n}^{\circ}$ 693, pl. LXXX).

9. Sans provenance connue. Turin, Museo di Antichità, $n^{\circ}$ 5423. Patère. Sur le manche Mercure de face, très juvénile, le caducée au creux du bras gauche, la main droite levée et portée à la tête. Décor en relief (Ori e argenti, nº 694, pl. LXXX).

10. Provenance précise inconnue; "au pied de la montagne du Salève" (HauteSavoie). Il pourrait s'agit d'une pièce du trésor de Reignier, auquel appartiennent peut-être aussi certaines des patères du musée de Turin. Patère. Au sommet du manche, Mercure en buste portant le pétase. Décor en relief (Revue du Louvre, 5/6, décembre 1994, p. 89).

11. Sans provenance précise. Turin, Museo di Antichità, $n^{\circ}$ 5416. Patère. Sur le manche, Mercure en pied. Décor gravé, plutôt sommaire (Ori e argenti, $\mathrm{n}^{\circ} 690$ ).

12. Les Granges (Aube), disparu. Coupe. Mercure debout entre deux colonnes avec le pétase, le caducée et la bourse ; à ses pieds, un coq et une tortue. Décor gravé.

13. Chaourse (Aisne). Médaillon de coupe. Mercure de face, des ailes dans la chevelure, tenant le caducée et la bourse, entre un coq et un bélier. Décor en relief (Trésors d'orfêvrerie, $\mathrm{n}^{\circ}$ 77) (fig. 26).

14. Saint-Jouin-sur-Mer (Seine-Maritime), disparu. Médaillon au fond d'un plat "creux et oblong comme une cuvette". Mercure "posé sur un pied, un caducée à la main avec des ailes aux pieds et à la tête, entouré d'un cercle doré de la grandeur d'une pièce de 5 francs."

15. Autun (Saône et Loire), disparu. Dans un trésor trouvé en 1614, une cuiller. Dans le cuilleron Mercure assis sur un rocher, entouré d'un coq, d'un bélier et d'une tortue. Décor en relief. Une cuiller identique, sans doute moderne, au Cabinet des Médailles de la Bibliothèque Nationale de France (F. Baratte, Le trésor d'orfèvrerie découvert à Autun en 1614, BSNAF, 1992, p. 201-211).

16. L'identification avec Mercure d'un des personnages de la coupe découverte en 1929 à Lyon, assis devant une table sur laquelle est posée une bourse, une autre bourse à la main, est très discutable. L'homme est presque nu, un manteau passé dans le dos et sur la cuisse gauche, assis probablement sur un siège dont l'un des pieds, le seul visible, est en forme de silhouette humaine, les bras levés (un Atlante? Il s'agit pour Ch. Picard d'un des montants de la table, ce qui parait peu probable). Une autre bourse - une tortue a-t-on dit parfois - tombe sur la table, lâchée par un oiseau (un corbeau ?).Le personnage a le plus souvent été identifié à Mercure, parfois même à Mercure-Toutatès. S. Boucher a fait remarquer à juste titre l'absence des attributs les plus caractéristiques du dieu, le caducée et les ailettes (Trésors d'orfêvrerie, $\mathrm{n}^{\circ} 11$; Boucher 1976, p. 194-195). 


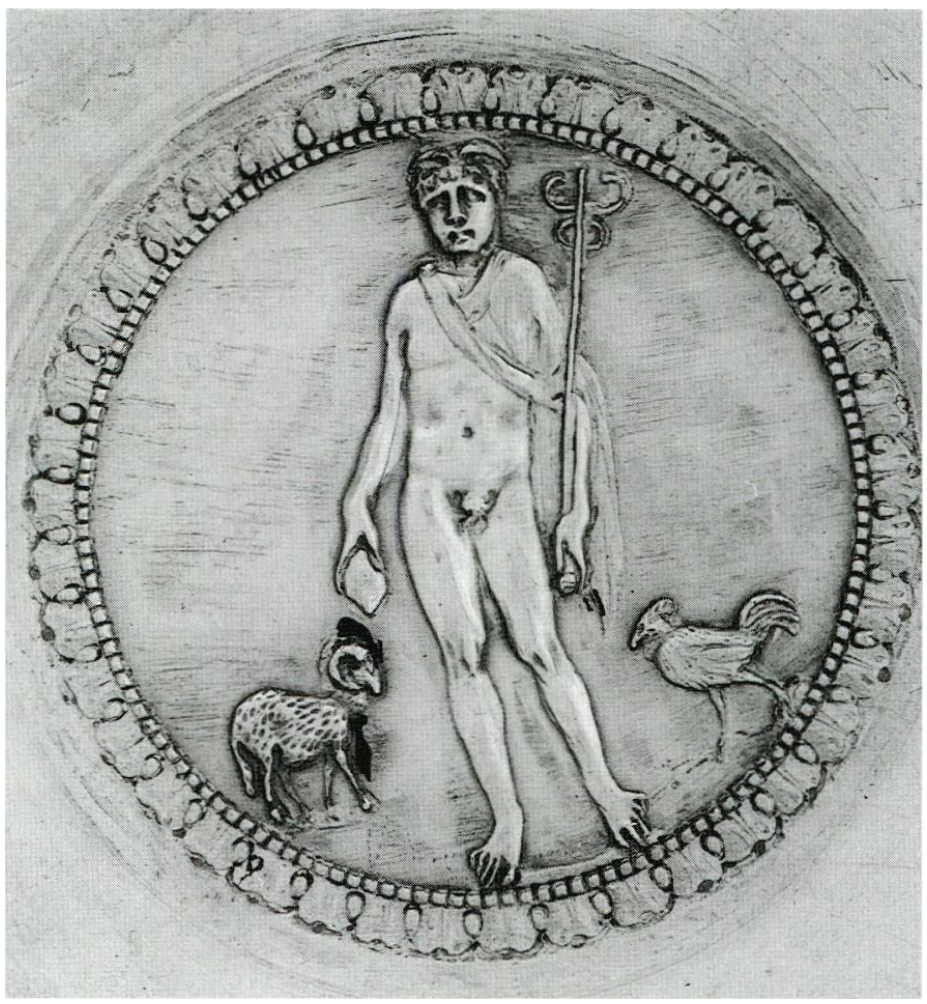

LONDRES. BRITISH MUSEUM. COURTESY TRUSTEES OF THE BRITISH MUSEUM

12 L'attitude du dieu sur la coupe de Vaise, debout entre un autel et un bélier, une bourse dans la main droite, le caducée au creux du bras gauche, une chlamyde enroulée autour du même bras, accompagné du coq et de la tortue, le rapproche avec quelques variantes de quelques-uns des objets de notre liste : les deux coupes de Berthouville ( $\mathrm{n}^{\circ} 2$ et 3 de notre liste), celle de Chaourse ( $\left.n^{\circ} 13\right)$, celle des Granges ( $\left.n^{\circ} 12\right)$, connue seulement par une description comme celle de St-Jouin ( $\left.n^{\circ} 14\right)$, et deux des quatre manches de patère conservés à Turin ( $n^{\circ} 9$ et 11$)$.

Seuls le décor des Granges et celui d'une des patères de Turin sont gravés : il s'agit là d'une technique moins prestigieuse que le relief, qu'il soit au repoussé ou fondu; les exemples dans l'argenterie romaine en sont rares : on la rencontre sur une coupe dans le trésor de Karnak, caché au début du IIIe s. ap. J.-C., pour une figure de divinité égyptisante (H. Mielsch, Romisches Silber aus Àgypten, dans Das Haus lacht vor Silber, H.H. von Prittwitz, H. Mielsch (éd.), Bonn, 1997, p. 49, fig. 9), sur deux autres coupes à Berthouville sans figures humaines (Babelon 1916, $\mathrm{n}^{\circ} 20$ et 21), sur deux autres encore à NotreDame d'Allençon (F. Baratte, Le trésor d'argenterie gallo-romaine de Notre-Dame d'Allençon (Maine-et-Loire), 40e suppl. à Gallia, Paris, 1981, $\mathrm{n}^{\circ} 7$ et 8), ainsi que sur un miroir du même ensemble (ibid., $\mathrm{n}^{\circ}$ 39). La grande coquille du trésor de Rethel est décorée de cette manière (Trésors d'orfèvrerie, $\mathrm{n}^{\circ} 116$ ) : au fond, en médaillon, Epona est représentée entre deux chevaux, tandis qu'à l'extérieur on voit un homme portant une amphore. Le seul plat conservé du trésor de Mâcon en offre un exemple original : une scène de sacrifice dans un réseau de motifs géométriques (Trésors d'orfêvrerie, $\mathrm{n}^{\circ} 133$ ).

Difficiles à dater, la plupart de ces objets sont à placer sans doute aux IIe-IIIe s., de même que le grand plat trouvé dans une tombe à Piestany, en Slovaquie, qui combine des reliefs sur le marli et la gravure sur le médaillon central, une scène de serment sur 
un porcelet (C. Wölfel, Die Lanx von Stráže, dans Das Haus lacht vor Silber, p. 162, fig. 8) : les personnages sont en grande partie incrustés sur le fond d'argent, mais les détails sont bien gravés, annonçant ainsi une façon de faire volontiers utilisée plus tard. Au IVe s., la technique continue d'être employée, de manière parfois spectaculaire, en raison du goût qui s'affirme alors pour les décors plats et colorés : le grand plat de Cesena (P. E. Arias, Il piatto argenteo di Cesena, Annali della Scuola Archeologica di Atene, 24-26, 1946-1948, p. 309-344) est orné de cette manière (on y voit notamment un banquet en plein air), de même que la partie centrale de deux coupes fragmentaires de Traprain Law, en Écosse, représentant une néréide sur un monstre marin et une Vénus anadyomène, et d'un plat en forme de cœur sur lequel apparaît un poisson (A. O. Curie, The Treasure of Traprain, 1923, $n^{\circ} 30$, p. 36-39, et $n^{\circ} 44$, p. 49, et $n^{\circ} 208, p .72$ ). La dorure intervient alors souvent abondamment, et l'effet est meilleur que sur des objets plus anciens, la coupe de Vaise notamment, sur lesquels la gravure apparait plutôt comme un pis-aller par rapport au relief, et le décor comme un pseudo-emblema remplaçant incomplètement les médaillons, rapportés ou non, dont les coupes de Berthouville et ou celle de Chaourse donnent de bons exemples.

La coupe de Vaise fournit ainsi une représentation supplémentaire de Mercure en Gaule, qui se rattache, comme on l'a vu plus haut, à un type dérivé d'un modèle grec du IVe s. av. J.-C. Elle met en évidence, une fois encore, la faveur dont le dieu jouit en Gaule, qu'il doit à son assimilation à une divinité gauloise importante. Sa présence dans la trouvaille de Vaise n'implique pas plus qu'à Chaourse le caractère religieux du dépôt. À Berthouville en revanche, à Saint-Jouin et aux Granges, des dédicaces sur les autres objets indiquent clairement la nature votive de l'image de Mercure.

\section{Petit plateau}

\section{Dimensions et état de conservation}

$16 \quad$ Inv. 9311043

Bronze plaqué d'argent.

Diam. 10,5 cm; diam. du pied 4,5 cm; long, de la frise $1,2 \mathrm{~cm}$.

Poids $144,3 \mathrm{~g}$.

Nous n'avons pas relevé de graffiti.

18 L'état de conservation est relativement bon, mais la feuille d'argent, fendue par endroits, sur la tranche du plateau et à l'intérieur, notamment au ressaut entre le fond et le marli, comporte quelques manques sur les reliefs; elle adhère mal au bronze et apparaît comme froissée sur presque toute sa surface.

\section{Description}

19 Petit plateau à pied annulaire bas (fig. 27). Le fond plat est relié par un ressaut légèrement concave au marli, qui est lui-même à peine convexe. La tranche du rebord est verticale. Les différentes parties de l'objet sont soigneusement marquées par des arêtes vives. Le rebord est décoré d'une frise de reliefs entre deux moulures. La première, à l'extérieur, est faite d'un listel sur lequel ont été sommairement dégagées des perles carrées; la seconde, à l'intérieur, comportait sans doute le même décor, mais, soit usure, soit maladresse de l'exécution, les perles ne sont presque pas visibles. 


\section{7- Petit plateau $n^{\circ} 2$}

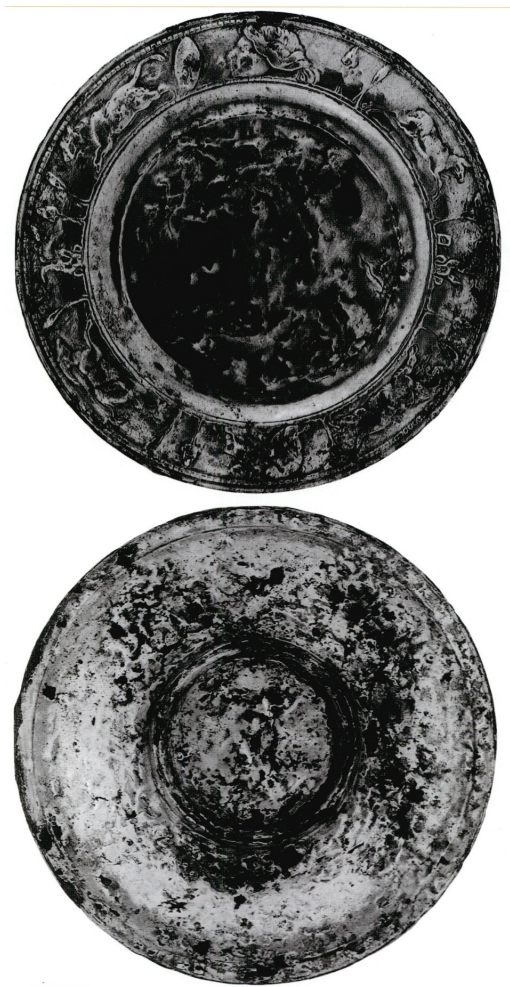

Diam. 10.5 cm, bronze plaqué d'argent, face et revers

Le décor est fait d'une succession d'éléments habituels sur ce type de frise, divisée ici en deux sections égales par deux têtes bachiques sans cou (fig. 28). La première, tournée vers la gauche, est précédée d'un tympanon; on y reconnaîtra donc une ménade. Les cheveux, tirés sur la nuque où ils sont rassemblés en une sorte de catogan, sont organisés en outre en deux rouleaux de part et d'autre du front qui entourent la tête vers l'arrière, passant par-dessus le catogan. La seconde tête, moins nette, est sans doute celle d'un jeune satyre tourné vers la droite; les cheveux mi-longs sont ramenés vers la nuque et dressés en un toupet très dru au-dessus du front; aucun accessoire ne l'accompagne. 


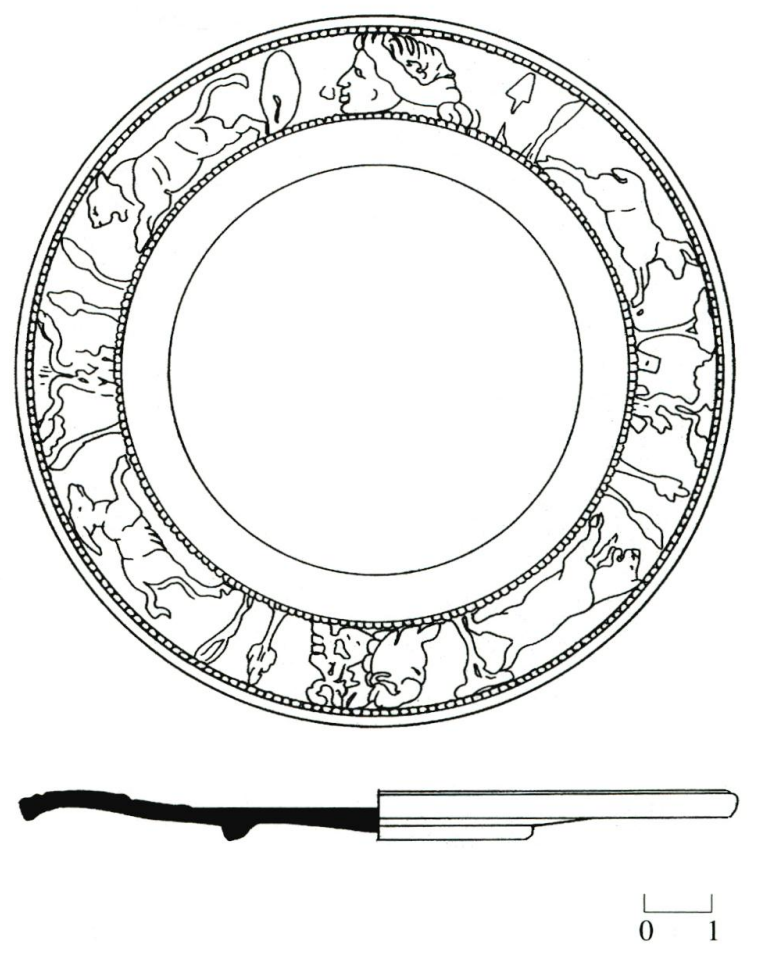

Profil et relevé du décor

21 Chacun des deux segments ainsi déterminés est à son tour divisé en deux en son milieu par un arbre noueux dont la ramure se répartit en deux branches divergentes aux extrémités feuillues. Ces arbres sont à leur tour environnés d'autres arbustes en forme de cyprès ou surmontés au contraire d'une large corolle plate : trois cyprès d'un côté de la frise, deux cyprès et un "pin" de l'autre côté.

De part et d'autre de ces deux bosquets deux animaux s'élancent l'un vers l'autre : un lion face à une antilope aux longues cornes d'un côté, un lion opposé à une sorte de biche de l'autre. On note encore la présence de deux arbres devant le satyre et derrière la ménade et d'un arbre au feuillage fait de deux touffes divergentes derrière le satyre. Un petit carré (rocher, accessoire ?) est placé au sol à côté d'un des arbres.

\section{Commentaire}

Le plateau appartient bien évidemment au même groupe que le suivant. Le commentaire concernant la forme et le décor sera donc présenté plus loin. On doit cependant attirer l'attention ici sur la technique de cet objet, en bronze plaqué d'argent. Aucune étude n'a encore été consacrée à ce procédé qui permet d'économiser le métal précieux. Si de petites pièces, comme des cuillers ou des récipients à usage semble-t-il culinaire, paraissent avoir été enduites d'une fine pellicule de métal blanc (ainsi plusieurs objets de la trouvaille des Ilettes, près d'Annecy : Trésors d'orfêvrerie, $\mathrm{p}$. 211-212), les objets plus importants sont véritablement plaqués : l'âme en bronze est recouverte d'une feuille d'argent plus ou moins épaisse, qui l'épouse d'autant plus étroitement que c'est elle qui porte le décor; la technique est alors apparentée à celle de la dorure à la feuille, telle qu'on la rencontre sur certaines pièces d'argenterie (par 
exemple une coupe du trésor de Rethel: Trésors d'orfêvrerie, $\mathrm{n}^{\circ}$ 108), et est utilisée également pour la statuaire (statuette de Fortuna de Sainpuits, Trésors d'orfèvrerie, $\mathrm{n}^{\circ}$ 129). La qualité du travail se mesure à la manière dont le placage adhère au bronze : dans le cas du plateau de Vaise, la feuille de métal précieux s'est en partie détachée du fond, ce qui lui donne un aspect froissé caractéristique. La manière différente dont travaillent les deux métaux en contact l'un avec l'autre peut aussi expliquer des défauts dans l'état de conservation. On peut penser enfin que certains objets aujourd'hui exclusivement en bronze ont en fait perdu totalement leur placage ou leur couverte de métal blanc: ainsi peut-être pour un grand plat ovale de Billig, au Rheinisches Landesmuseum de Bonn (M. Menninger, Die romischen Silbergefâsse, dans Das Haus lacht vor Silber, op. cit., $\mathrm{n}^{\circ} 4$, p. 105-107, fig. 11), ou pour le plat circulaire en bronze décoré de reliefs au centre et sur le marli au musée Bargoin de Clermont Ferrand (inédit).

Même si elles n'ont pas été systématiquement recensées, on connaît un certain nombre d'exemples de pièces de vaisselle en bronze plaquées d'argent, découvertes souvent mêlées à de l'argenterie : le trésor de Rethel contenait un grand plat circulaire sans décor recouvert de deux feuilles d'argent (Trésors d'orfêvrerie, $\mathrm{n}^{\circ}$ 120), celui de Saulzoir un plat circulaire fragmentaire au rebord orné de reliefs (Trésors d'orfêvrerie, $\left.\mathrm{n}^{\circ} 106\right)$; plusieurs objets plaqués appartenaient au trésor de Chaourse : un miroir et deux petits plateaux (Trésors d'orfèvrerie, $\mathrm{n}^{\circ}$ 65-66 et 86). À Niederbieber, deux plats sont plaqués : l'un sans autre décor qu'une moulure de perles et de pirouettes sur le rebord (E 18123), l'autre (E 18122) dont le marli porte une frise d'un type traditionnel (quatre masques séparant des animaux se poursuivant); les deux objets pourraient bien avoir constitué une paire comme on en trouve dans d'autres trésors, qui oppose un plat creux à rebord et un plat horizontal sans marli (M. Menninger, dans Das Haus lacht vor Silber, op. cit., p. 113-123, no 12 et 13, fig. 36-42). Par sa technique, le plateau de Vaise est proche de ce petit groupe.

Ces objets plaqués posent un intéressant problème, celui de leur valeur. Mêlés à l'argenterie, ils se confondaient aisément avec elle. Leur rôle parait être de tromper l'observateur, en faisant illusion sur leur qualité réelle. Intacts, ils devaient y parvenir. Mais on peut aussi s'étonner de les trouver dans des trésors comme celui de Chaourse, où ils ne représentent pratiquement rien. Substituts de l'argenterie, ils n'étaient peutêtre pas aussi mal considérés que nous pouvons le penser.

\section{Petit plateau}

\section{Dimensions et état de conservation}

Inv. 9311042

Argent.

Diam. 12,3 cm; diam. du pied $5 \mathrm{~cm}$; largeur de la frise $1,5 \mathrm{~cm} ; \mathrm{h}$. du pied $0,3 \mathrm{~cm}$.

Poids 167, $20 \mathrm{~g}$.

L'objet, sans doute fondu, a été terminé au tour : les trous de pointeau, au centre, sont si accentués qu'ils ont traversé le métal de part en part. On observe aussi au revers une zone particulièrement polie sous le pied (diam. $1,9 \mathrm{~cm}$ ) cernée par une rainure très nette (fig. 29). 
28 L'état de conservation est très bon: les reliefs présentent une certaine usure, mais le décor est intact ; le métal est piqueté sur la face et au revers, à l'extérieur du pied.

29 a- Petit plateau $n^{\circ} 3$

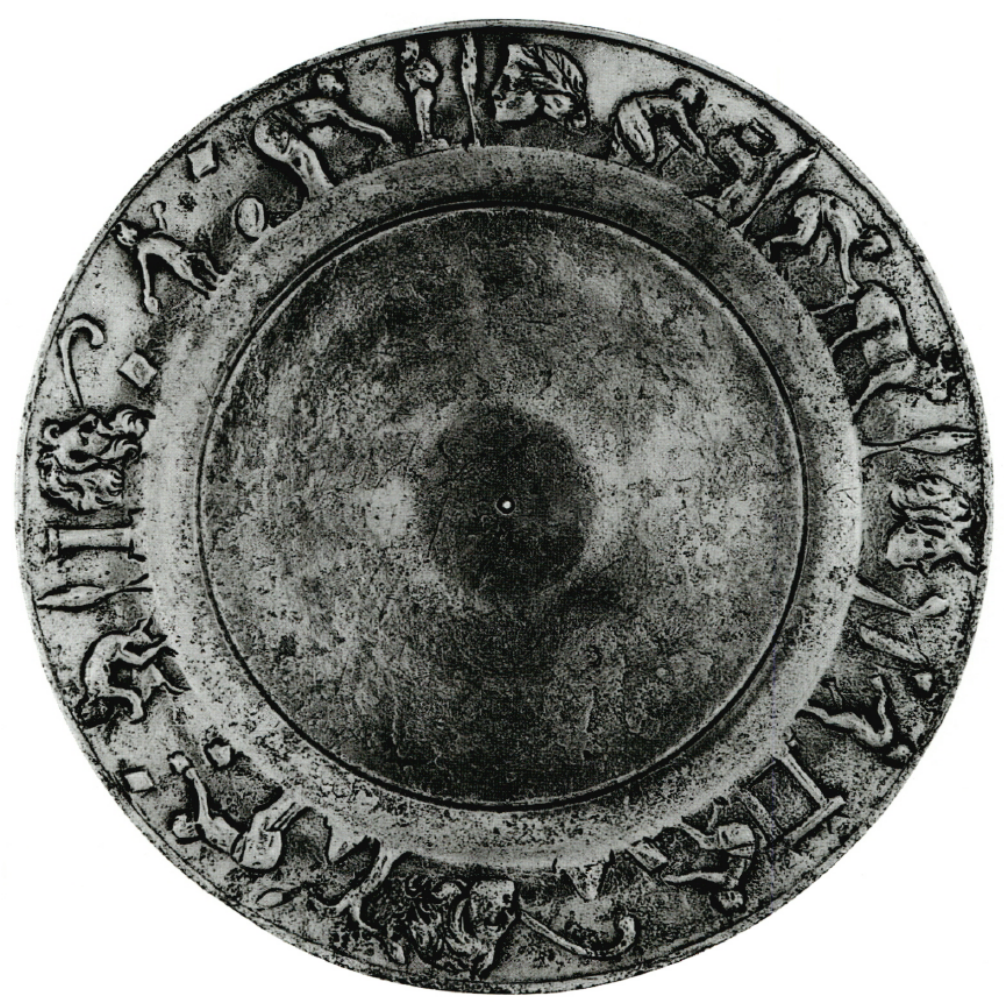

Face, diam. $12,3 \mathrm{~cm}$

$29 \mathrm{~b}$ - Petit plateau $\mathrm{n}^{\circ} 3$

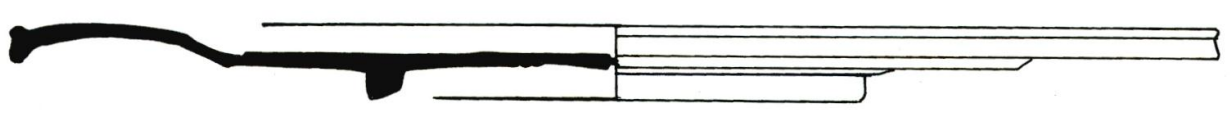

Profil 


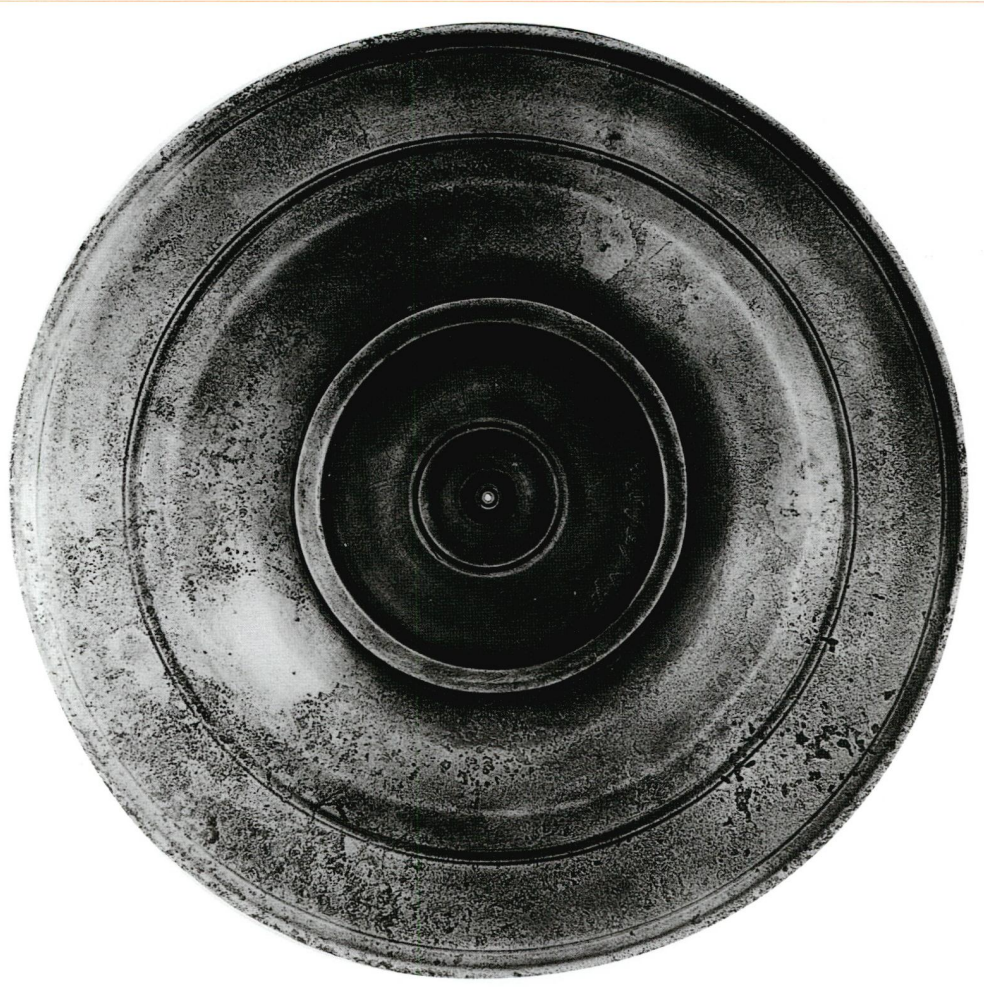

Revers, diam. $12,3 \mathrm{~cm}$

\section{Les inscriptions}

Quatre graffiti sont visibles au revers (fig. 30) : une indication de poids (a) et deux noms $(b-c)$ à l'intérieur du pied, un nom sous le rebord $(d)$ :

a - indication pondérale en lettres pointillées entre deux palmes. $P($ ondo) semis uncia scripula (quinque), soit 183,04 g.

b- un graffiti très fin à la pointe. On lit : TAVRAV[

c- un graffiti gravé à la pointe, fait de lettres enchevêtrées, irrégulières et sommairement tracées. Je lis : SECI[

d- un graffiti finement gravé à la pointe, assez soigneusement tracé : COCNCO[

\section{Description}

Petit plateau à pied annulaire bas. Le fond est plat, en léger creux par rapport au marli convexe. Ce dernier, bordé une moulure en faible relief, est doté d'une lèvre pendante.

Les différentes parties de l'objet sont soigneusement distinguées. À l'intérieur, le fond, délimité par une rainure nette, est relié au marli par un ressaut concave. Au revers, plusieurs rainures soulignent le passage du fond au rebord et la limite extérieure de ce dernier.

Le décor est constitué d'une frise de reliefs sur le marli ; quatre têtes bachiques (a-d) la divisent en autant de secteurs égaux entre lesquels sont représentés des scènes rustiques, en partie cultuelles (fig. 29a et 31). 

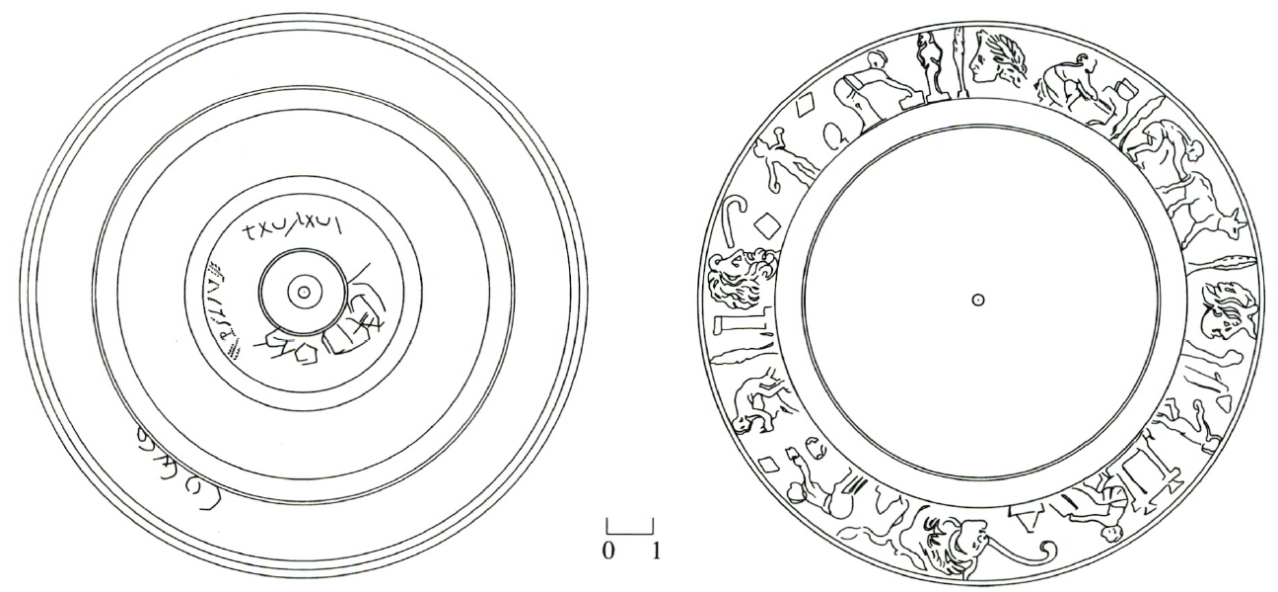

\section{Les têtes bachiques}

1. Une femme vêtue d'un vêtement long serré à la taille est agenouillée devant un four sur lequel est posé une marmite et à l'intérieur duquel elle enfourne du bois ou un récipient.

2. Un personnage masculin en tunique courte s'apprête à traire un animal. L'absence de queue et la position du paysan qui trait les mamelles entre les pattes arrière désignent de préférence une chèvre. Devant le quadrupède, un cyprès.

b-c: Un premier personnage s'avance vers la droite d'un pas dansant. $\mathrm{Nu}$ (?), il tient le bras droit rejeté en arrière, un objet rectangulaire (un instrument de musique ?) à la main ; le bras gauche n'est pas visible. Devant lui un petit édicule - une sorte d'autel ? formé d'un socle quadrangulaire, de deux colonnettes et d'un plateau sur lequel sont posés deux vases tronconiques. Il s'agit peut-être d'une table si l'on admet une différence volontaire d'échelle avec le personnage.

Devant cette installation, un personnage masculin tourné vers la droite, une tunique courte autour des reins, s'accroupit pour prendre, poser ou verser quelque chose sur un objet rectangulaire placé en oblique devant lui ; plus loin, sous le pedum du satyre (c), un objet en forme d'ombilic. 
c-d : Derrière le satyre, un arbre à deux branches divergentes; à son pied, un autre objet en forme d'ombilic. Puis, tourné vers la droite, une femme vêtue du liknon - une sorte de tablier - par-dessus sa tunique et voilée se penche en avant pour tendre un petit vase qu'elle tient par une anse ; au sol, un autre vase (?); en l'air, un objet en forme de losange. Faisant face à la femme, un homme maîtrise en le chevauchant un quadrupède (mouton, porc?) dont il tient la tête. Derrière lui, un cyprès puis une colonne sur laquelle est posée un globe ou un cadran solaire.

$\mathrm{d}-\mathrm{a}$ : Sous le pedum du satyre, un objet rectangulaire. Puis viennent deux personnages tournés vers la droite. Un homme tout d'abord, debout en tunique courte, statique, le bras droit en arrière tenant un objet arrondi comme le fait le bras gauche levé. Devant lui, au sol, une masse ronde, en l'air un objet rectangulaire. Plus loin un sacrifice rustique: une femme en tunique longue se penche au-dessus d'un van (?) pour le dévoiler, la tête tournée vers un hermès, sans doute un Priape ithyphallique; derrière, un cyprès.

\section{Commentaire}

41 La forme des deux petits plateaux est l'une des plus répandues dans l'orfèvrerie romaine, tant en argent qu'en bronze argenté, en Gaule en particulier. La liste dressée naguère à l'occasion de la publication du trésor de la place Camille Jouffray à Vienne en recensait quatorze (F. Baratte, A. Le Bot-Helly et al, Le trésor de la place Camille Jouffray à Vienne (Isère), 50e suppl. à Gallia, Paris, 1990, n 5-6, p. 53-64). Elle en compte désormais dix-sept après les découvertes de Vaise, dont nous redonnons la liste classée en fonction du décor de chacun de ces objets :

1. Pompéi. Conservé au Museo Archeologico Nazionale de Naples, inv. 110850. diam. 9,4 cm. poursuites d'animaux. Inédit, signalé dans Baratte et al. 1990, p. 57, a. 2. Italie. Conservé au Metropolitan Museum of Art de New York (Rogers Fund, inv. 18.145.37). Diam. 12,6 cm, poids $164,4 \mathrm{~g}$. Quatre têtes : deux satyres et deux ménades entre lesquelles courent quatre animaux, deux chiens, un lion et une antilope. Un listel simple en encadrement extérieur du marli. A. Oliver, Silver for the Gods, Toledo, $1977, \mathrm{n}^{\circ} 99$; Baratte et al. 1990, p. 57, i.

3. Italie (fig. 32). Conservé au musée du Louvre, AGER (ancienne collection Campana, Bj 1955). Diam. 10,8 cm. Quatre têtes (deux satyres âgés et deux satyres jeunes), entre lesquelles courent quatre animaux, deux lions, un cheval et un chevreuil. Un filet simple en encadrement du marli. A. De Ridder, Musée du Louvre. Catalogue des bijoux antiques, Paris, 1924, n 1955 ; Baratte et al. 1990, p. 57, h. 


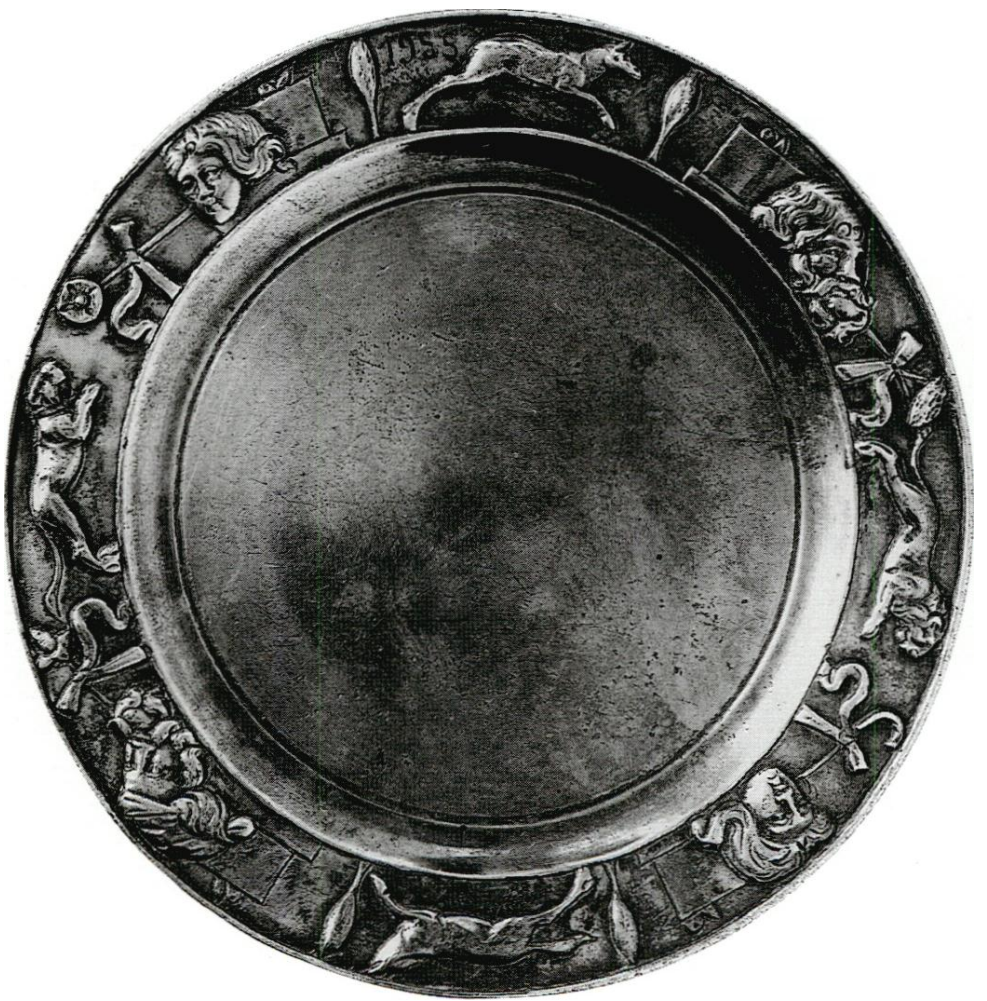

Paris, Musée du Louvre, AGER Bj 1955. Cl. Chuzeville

4. Italie (ou Hongrie ?). Conservé au Kunsthistorisches Museum de Vienne. Diam. $10,4 \mathrm{~cm}$. Quatre têtes (deux ménades, deux satyres, l'un âgé, l'autre jeune) entre lesquelles courent quatre animaux, deux lions, un cervidé, un cheval (?). Un listel simple en encadrement extérieur du marli. Fr. Drexel, Alexandrinische Silbergefâsse der Kaiserzeit, Bonner Jahrb. 1909, p. 184, n 9, pl. VIII, 2; W. Kubitschek, Grabfunde in Untersiebenbrunn, Jahrbuch fur Altertumskunde, 5, 1911, p. 72, n 14, fig. 43 ; Fr. Mutz, Das Kunstdrehen des Metalls bei den Römern, Munich, 1972, p. 86-87, fig. 202 ; Baratte et al. 1990, p. 57,j.

5. France ? Conservé au musée du Petit-Palais, Paris (ancienne collection Dutuit). Diam. $12,8 \mathrm{~cm}$. Quatre têtes (deux satyres et deux ménades) entre lesquelles courent quatre animaux, un lion, une lionne, une antilope et un cerf, au milieu d'arbres et de différents accessoires. Deux baguettes perlées encadrent le marli. W. Fröhner, Collection Auguste Dutuit. Bronzes antiques, Paris, 1897, $\mathrm{n}^{\circ} 122$, pl. 111 ; Baratte et al. 1990, p. 57, g.

6. Simféropol (Ukraine). Conservé au musée de Simféropol (inv. 97.504). Diam. 12 $\mathrm{cm}$. Le décor du marli est réparti en deux secteurs égaux de part et d'autre de deux paires de têtes bachiques placées nuque à nuque; dans chaque secteur, deux animaux affrontés (lion et antilope) ou se poursuivant (lion et cervidé) au milieu d'arbres et de constructions. Moulure d'oves schématiques sur le rebord. V. V. Kropotkine, Objets importés romains d'Europe occidentale, dans Archeologia SSSR, Moscou, 1970, p. 21, n² 21, et n 742, pl. 81,1-3 ; Baratte et al. 1990, p. 57,1.

7. Provenance inconnue. Conservé au Musée national de Belgrade. Diam. 12,2 cm. Poids 153,63 g. Listel simple (?) en encadrement extérieur du marli (aucune des photographies publiées n'est satisfaisante). Quatre têtes bachiques entre lesquelles sont disposés deux petits " tempietti », des arbustes et quatre animaux (dont deux griffons ?). Antique silver from Serbia, éd. I. Popovic, Belgrade, 1994, $\mathrm{n}^{\circ} 201$; I. Popovic, Miscellanea Argentea, Starinar, 48, 1997, p. 80-86.

8. Lyon-Vaise. Bronze plaqué d'argent (cf. supra). 
9. Thil (Haute-Garonne) (fig. 33). Conservé au British Museum (G\&R 1824. 4-89-72). Diam. 13,8 cm, poids 302,3 g. Le décor, doré, comporte quatre têtes bachiques (deux satyres jeunes, deux satyres âgés) entre lesquelles sont disposés six animaux, deux isolés et quatre groupés deux par deux : un lion et un chien poursuivant chacun un cerf. Moulure de perles et de pirouettes en encadrement extérieur du marli, moulure de petites perles à l'intérieur. M. Feugère-M. Martin, le trésor d'argenterie gallo-romain de Thil, dans F. Baratte (éd.), Argenterie romaine et byzantine, Paris, 1988, p. 66, n 3 ; Baratte et al. 1990, p. 57, f ; Trésors d'orfêvrerie, n² 201.

\section{3- Thil, petit plateau}

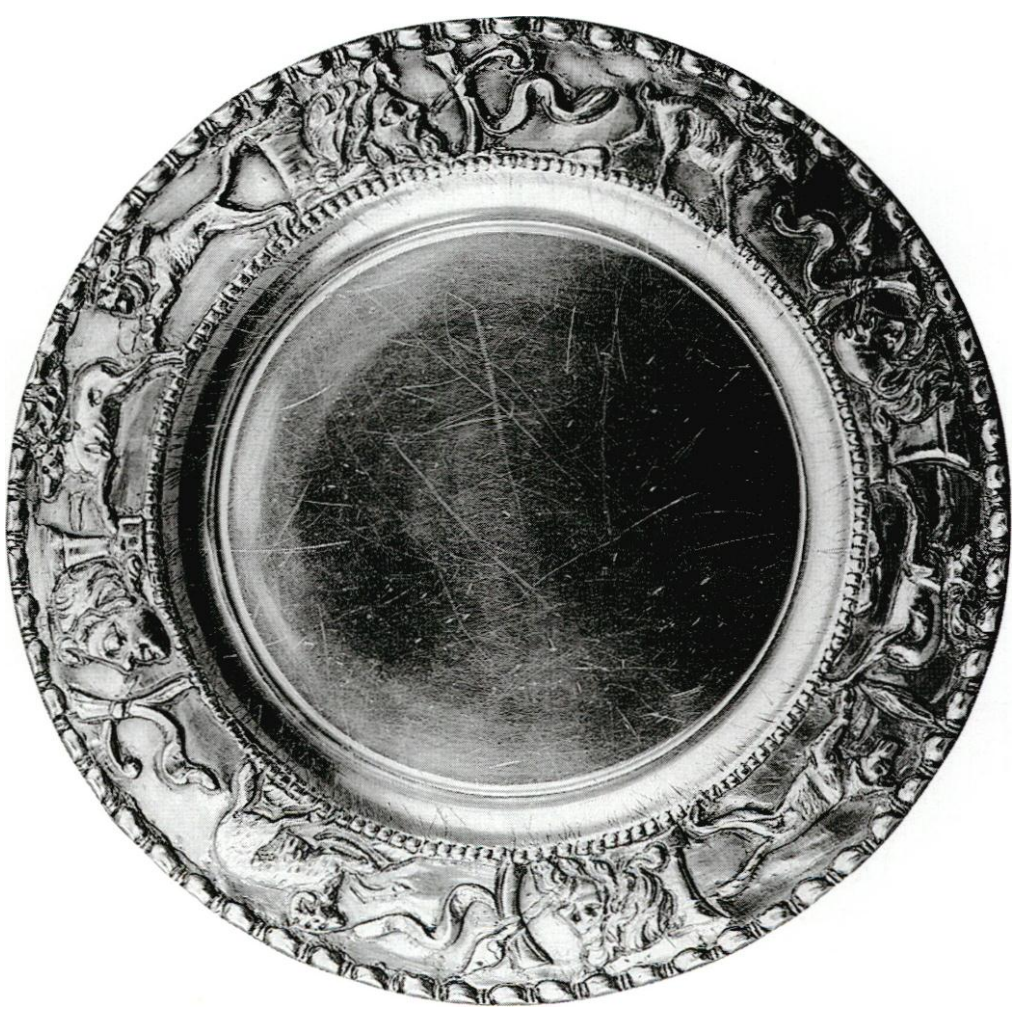

Argent doré

Londres, British Museum. Courtesy Trustees of The British Museum

10. Houdain-lès-Bavay (Nord) (fig. 34). Conservé au musée du Petit-Palais, Paris (ancienne collection Dutuit). Diam. 11,2 cm. Quatre têtes (deux ménades et deux satyres âgés) entre lesquelles sont disposés quatre animaux au repos : un sanglier, un cerf, un bélier et un bouc. Des deux animaux domestiques, le premier est accompagné d'un berger assis, au repos, le second d'un berger assis jouant de la flûte. Moulure d'oves et de rais-de-cœur très simplifiés et listel simple en bordure extérieure du marli. Fröhner 1897, n 121, pl. 111 ; Baratte et al. 1990, p. 57, b; Trésors d'orfèvrerie $\mathrm{n}^{\circ} 103$; CAG. Nord. p. 281, n 17. 


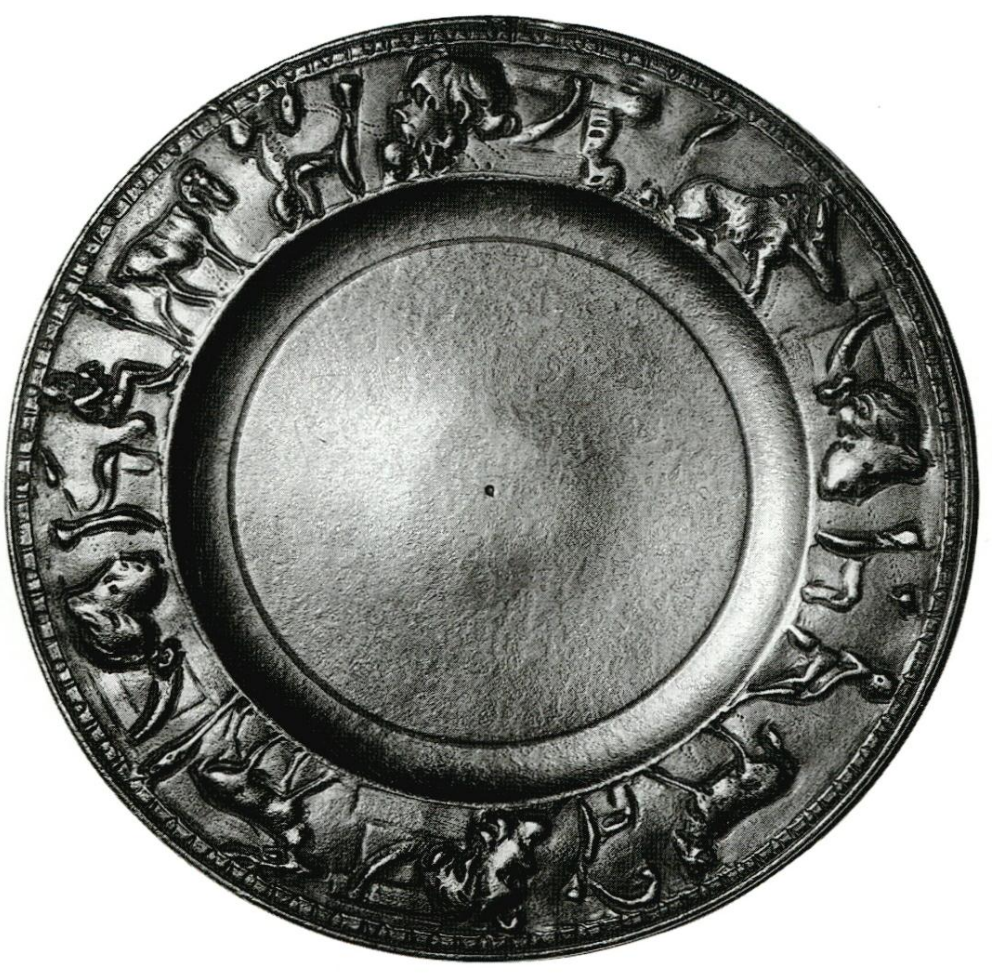

Paris, Musée du Petit-Palais. Cl. Bulloz

11. Chalon-sur-Saône (Saône-et-Loire). Conservé au Museo Lazaro Galdiano à Madrid (ancienne collection Combette, inv. 2154). Diam. 12,1 cm. Quatre têtes, groupées deux par deux: à deux reprises, un satyre âgé (ou Pan luimême) accompagné d'une ménade; sur les deux secteurs du marli ainsi déterminé, un personnage couché (un homme ou une femme) nourrit un animal (un lion, une antilope); derrière lui, un autre animal (un lion, un cervidé). Pas de moulure en bordure du marli. Vienne 1990, p. 57, c ; F. Baratte, Un plato galorromano de plata en el museo Lazaro Galdiano, Goya, 222, 1991, p. 322-330, fig. 1-3.

12. Vienne (Isère). Conservé au musée municipal, Vienne. Diam. 12,6 cm ; poids 132,23 g. Quatre arbres entre lesquels sont couchés six personnages: quatre ménades groupées deux à deux, une cinquième isolée (Ariane ?) ainsi que Dionysos et sa panthère. Un listel sommairement perlé en encadrement extérieur du marli, une moulure de petites perles à l'intérieur. Baratte et al. 1990, n 5, p. 53-57.

13. Vienne (Isère) (fig. 35). Conservé au musée municipal, Vienne. Diam. 13,1 cm, poids 224,63 g. Le décor est réparti sur le marli d'une part (A), sur son raccord avec le fond d'autre part (B). A : frise de monstres marins ; B : frise bachique complexe dont les différents éléments (des groupes de personnages) sont répartis en quatre secteurs inégaux séparés les uns des autres par une colonne. Une moulure perlée en encadrement extérieur du marli. Baratte et al. 1990, $\mathrm{n}^{\circ}$ 6, p. 58-64; Trésors d'orfêvrerie, $\mathrm{n}^{\circ} 172$. 


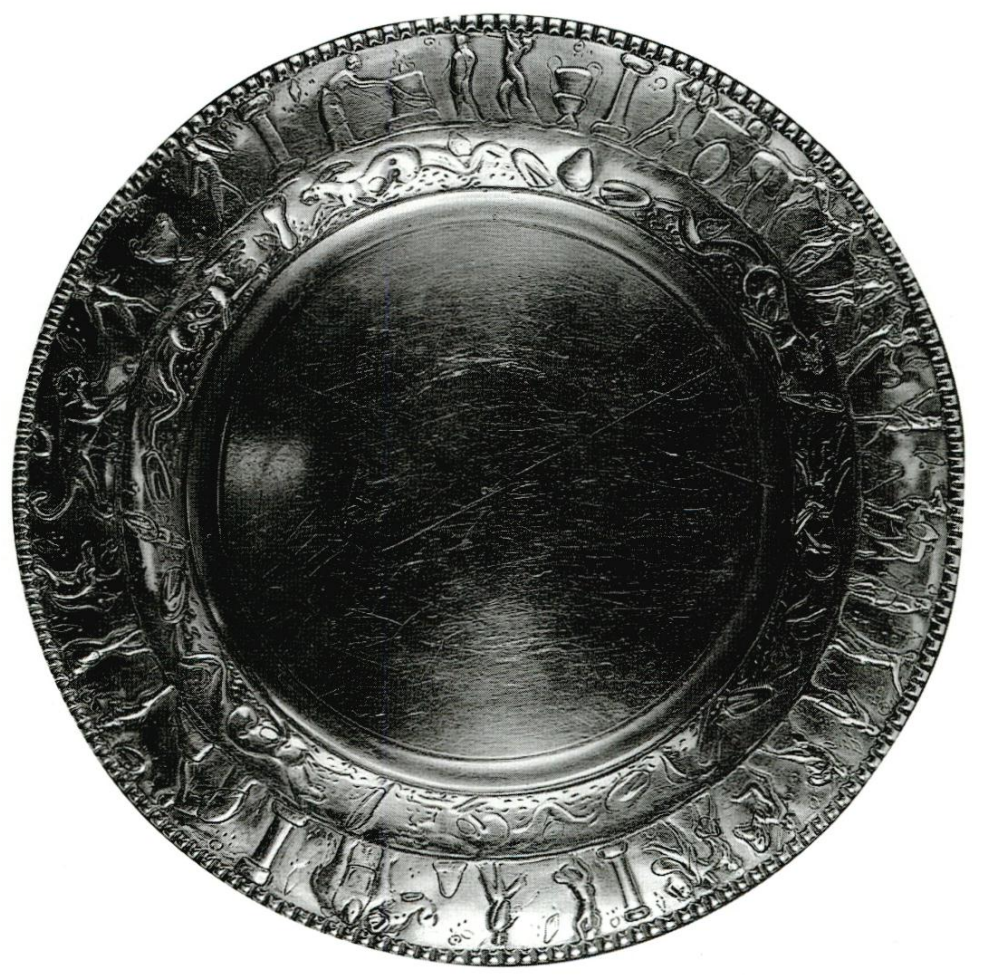

Vienne, musée municipal. Cl. P. Plattier.

14. Lyon-Vaise. Argent (cf. supra)

15. Baguinéthi (Géorgie). Diam. 10,8 cm. Sur le marli, frise de poissons, d'animaux marins, d'oiseaux et d'accessoires de pêche. Un listel simple en encadrement extérieur du marli. K. Matchabély, La toreutique de la Géorgie dans l'Antiquité tardive, Tbilissi, 1976, p. 67, pl. 20 ; F. Baratte, La coupe aux poissons du musée du Louvre, Revue du Louvre, 1979, p. 9, fig. 8 ; Baratte et al. 1990, p. 57, k; F. Baratte, Une nouvelle nature morte aux poissons: le plat d'argent de Mathay, Antiquités nationales, 26, 1994, p. 140.

16. Graincourt-lès-Havrincourt (Pas-de-Calais). Conservé au musée du Louvre, AGER (Bj 2211). Diam. 13,8 cm, poids 267 g. Deux rinceaux d'acanthe dorés. Listel et kymation stylisé à l'extérieur du marli, moulure perlée à l'intérieur. Baratte et al. 1990, p. 57, d ; Trésors d'orfevvrerie, $\mathrm{n}^{\circ} 90$.

17. Saint-Genis-Pouilly (Ain). Conservé au Musée d'Art et d'Histoire, Genève (inv. C 1372). Diam. $12 \mathrm{~cm}$; poids $96 \mathrm{~g}$. Six motifs végétaux stylisés. Moulure perlée et kymation stylisé à l'extérieur du marli. Baratte et al., p. 57, e; Trésors d'orfèvrerie, $\mathrm{n}^{\circ}$ 155.

La comparaison des profils de ces différents objets, de ceux qui ont été publiés tout au moins (fig. 36), fait apparaître une très grande similitude entre eux, quel que soit le décor. Le jeu, infime, porte notamment sur le diamètre du pied et donc sa position par rapport au rebord. Mais il est difficile de mettre en évidence des séries distinctes. Les dimensions sont très proches les unes des autres : l'exemplaire le plus petit est celui de Pompéi $(9,4 \mathrm{~cm})$, le seul nettement à part en raison de ce diamètre assez réduit, mais aussi de son décor, puisqu'il ne présente que des animaux. À l'inverse, ceux de Thil et de Graincourt (13,8 cm l'un et l'autre) se détachent du reste du groupe, dont les quatorze autres pièces s'échelonnent entre 10,8 cm (exemplaire du Louvre) et 13,1 cm (exemplaire à double frise de Vienne). Les poids, qui ne sont connus que pour quelques- 
uns des plateaux, sont en revanche très divers. Ils vont de $96 \mathrm{~g}$ pour celui de Saint-Genis à $302,30 \mathrm{~g}$ - presque une livre - pour celui de Thil. On observera seulement que l'exemplaire conservé à NewYork, provenant d'Italie, pèse à peu de choses près le même poids (168,4 g, soit une demi-livre) que celui de Vaise $(167,20 \mathrm{~g})$, à peine plus petit. En revanche le plateau à frise unique de Vienne pèse $36 \mathrm{~g}$ de moins, bien que de mêmes dimensions.

36- Profils comparés de six plateaux en argent.

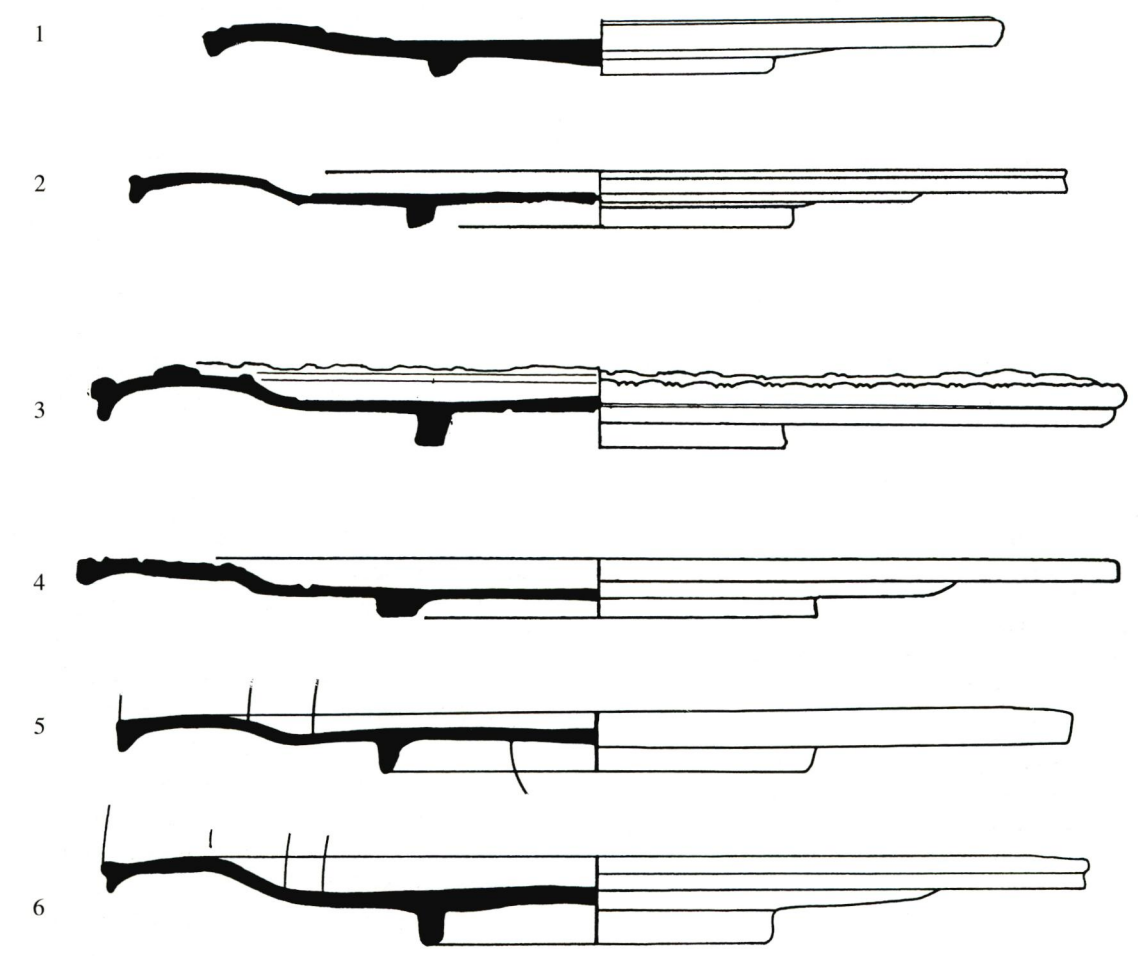
1. Vaise
2. Vaise
3. Thil
4. Graincourt
5. Vienne
6. Vienne (éch. 1/1)

Ce sont les moulures d'encadrement du marli qui présentent le plus de différences : huit plateaux ne possèdent qu'un listel simple en léger relief, mais sans aucun décor sur la bordure extérieure, les quatre provenant d'Italie, ceux de Chalon-sur-Saône, de Vaise (le plateau en argent), de Baguinéthi et de Belgrade. Un seul offre des oves, celui de Simféropol, peut-être le plus ancien (milieu du 1er s. ap. J.-C. ?), un autre une moulure perlée (le plateau de Vienne à double frise). Deux exemplaires ont une double moulure à l'extérieur, celui de Saint-Genis (une baguette perlée et un kymation) et celui du musée du Petit-Palais découvert en France (deux baguettes perlées). Cinq enfin possèdent deux moulures d'encadrement, une à l'intérieur, l'autre à l'extérieur : un listel et des rais-de-cœur à Houdain-lès-Bavay, une baguette perlée et un kymation complété par un listel à Graincourt, une moulure de petites perles et une baguette perlée à Vienne (plateau avec Dionysos et Ariane), une moulure de petites perles et un astragale à Thil, 
une moulure de petites perles et une baguette perlée sur le plateau en bronze argenté de Vaise. Mais il paraît difficile d'opérer des regroupements sur cette seule base.

$\mathrm{Du}$ point de vue du décor, plusieurs catégories apparaissent immédiatement à l'examen. On mettra à part les deux plateaux à décor végétal, ceux de Graincourt et de Saint-Genis, d'ailleurs très différents l'un de l'autre: le premier porte un double rinceau d'acanthe de type illusionniste, très soigné mais qui reprend des motifs de feuilles et de plantes bien reconnaissables que l'on rapprochera du décor de la coupe à collerette du même trésor (Trésors d'orfèvrerie, $\mathrm{n}^{\circ}$ 94) pour la structure générale - mais les fleurons sont uniformément constitués de pétales rayonnants - et plus encore de l'une de celles du trésor de Chaourse (Trésors d'orfêvrerie, $\mathrm{n}^{\circ}{ }^{74}$ ) qui présente une alternance analogue de fleurs à pistil allongé disposées têtes en haut puis tête en bas, ou de la frise extérieure du seau du même ensemble de Chaourse (Trésors d'orfêvrerie, $\mathrm{n}^{\circ}$ 48) sur lequel toutefois ces éléments alternent avec des fleurs rayonnantes. Quant au plateau de Saint-Genis, les courts segments végétaux juxtaposés très stylisés qui le décorent se retrouvent sur la collerette d'une autre coupe de Chaourse (Trésors d'orfevrerie $\mathrm{n}^{\circ}$ 75), sur laquelle ils se combinent avec des têtes bachiques d'une allure très particulière et avec des monstres marins; mais la structure des rinceaux est très proche, leur facture étant sans doute plus schématique à Chaourse qu'à Saint-Genis.

On distinguera également des autres plateaux celui de Baguinéthi, pour une double raison : la présence sur le marli d'un décor de nature morte marine tout d'abord, qui se retrouve sur toute une série d'autres objets, grands plats - comme celui de Graincourt (Trésors d'orfèvrerie, $\mathrm{n}^{\circ}$ 87) - petites coupes, et même sur une oenochoé en bronze et un fragment de rebord de plat rectangulaire en céramique sigillée claire africaine et sur le rebord d'un autre grand plat circulaire en argent de Baguinéthi (sur le thème de la nature morte marine sur les pièces d'orfèvrerie et la céramique, nous renvoyons pour la bibliographie en dernier lieu à $\mathrm{F}$. Baratte, Une nouvelle nature morte aux poissons : le plat de Mathay, Antiquités nationales, 26, 1994, p. 135-144) le met à part des autres plateaux. Mais la facture très particulière de ces reliefs contribue par ailleurs aussi à en faire un objet assez original : si les différents motifs apparaissent sur d'autres pièces d'argenterie découverts ailleurs dans l'empire romain, plutôt dans sa partie occidentale (Gaule, Italie, Roumanie, Afrique également pour le fragment de plat en sigillée claire : Atlante delle forme ceramiche. I. Ceramica fine nel bacino mediterraneo (mediotardo impero), Rome, 1981, p. 143, pl. 71, 1), ils sont traités sur le plateau de Baguinéthi de manière plus schématique, certains des oiseaux notamment. Il en va de même, on le remarquera, pour le décor végétal qui figure sur le marli d'une coupe découverte au même endroit (Matchabély 1976, pl. 21) que l'on peut rapprocher des rinceaux segmentés du plateau de Saint-Genis. Les nombreuses pièces d'argenterie provenant de Géorgie, et plus précisément de ce site particulier, représentent une production originale, très proche par bien des côtés de celle des orfèvres de l'Occident romain (même si l'influence sassanide est aussi manifeste à bien des égards) par son répertoire, mais de facture un peu différente : imitations locales d'objets produits ailleurs - mais où les artisans auraient-ils vu les modèles ?-ou importation à partir d'un ou de centres qui restent à localiser.

Parmi les treize autres plateaux, un premier critère de classement mérite d'être retenu: la présence ou l'absence de têtes bachiques sur le marli comme éléments organisateurs du décor. Ces têtes devraient être replacées dans une étude d'ensemble reprenant la totalité des pièces de vaisselle, en argent et en bronze, sur lesquelles elles 
apparaissent : leur type, généralement sans cou, mais plus proche de têtes réelles que de masques à proprement parler, et leur nature (il y a parfois d'autres têtes que des têtes bachiques) sont en effet à prendre en considération, mais dans un cadre plus large que celui de cette publication.

Pour nous limiter aux petits plateaux, on observera que l'exemplaire de Pompéi n'en possède pas ; mais la simplicité de son décor le met à part. En dehors de lui, seules les deux pièces de Vienne ont cette particularité ; les dix autres utilisent ce motif, mais sous des formes diverses; sans même considérer la variété purement formelle des têtes, ménades, satyres jeunes et plus âgés, on remarquera le plateau de Chalon sur lequel les quatre têtes sont regroupées deux à deux et adossées : une formule que l'on retrouve notamment sur le grand plat du trésor de Vienne (Trésors d'orfêvrerie, $\mathrm{n}^{\circ} 174$ ) ou sur celui de Léda à Graincourt (Trésors d'orfêvrerie, $\mathrm{n}^{\circ}$ 88). Mais l'usage des têtes dionysiaques ne constitue pas une particularité des plateaux de petites dimensions; il est bien plus largement répandu dans la vaisselle de métal, mais ailleurs aussi, jusque sur des frises architecturales comme à Lillebonne (L. Harmand, Dionysos à Lillebonne, BSNAF, 1968, p. 121-132), et ne saurait être analysé que dans un plus large contexte. Sur le plateau de Vaise en bronze plaqué d'argent, l'allure et la présentation des têtes bachiques rentrent dans la série la plus courante, mais elles ne sont qu'au nombre de deux, ce qui est exceptionnel.

Une autre distinction doit intervenir entre les plateaux dont le décor ne comporte que des animaux, sauvages ou domestiques, et ceux sur lesquels interviennent également, de manière diverse, des figures humaines, ou bien mêlées aux animaux précédemment évoqués, ou bien occupant à elles seules toute la frise.

Le premier groupe compte neuf exemplaires: ceux de Pompéi et de Thil, les trois provenant sans doute d'Italie et conservés au Louvre, à New-York et au Kunsthistorisches Museum de Vienne, celui provenant peut-être de France (au musée du Petit-Palais), ceux de Simféropol et de Belgrade et le plateau en argent de Vaise. Le second groupe en compte cinq; deux mêlent personnages et animaux: deux figures couchées, dionysiaques peut-être, à Chalon, deux bergers à Houdain-lès-Bavay.

50 Sur les trois derniers, les personnages occupent la place essentielle: sur l'un des plateaux de Vienne on rencontre des figures bachiques étendues, parmi lesquelles Dionysos et Ariane; sur le second exemplaire du même ensemble, que distingue par ailleurs l'existence d'une seconde frise de monstres marins, et sur celui de Vaise ce sont des scènes rustiques et cultuelles plus complexes.

51 Parmi tous les plateaux à décor figuré (personnages et animaux) interviennent enfin des différences de facture, parfois suffisamment nettes pour en mettre certains à part, ou pour au contraire en rapprocher d'autres. L'exemplaire de Thil, notamment, est caractérisé par une exécution de qualité, mais très vigoureuse, encore soulignée par un emploi abondant de la dorure. Les reliefs, plutôt accentués, ont des contours particulièrement nets et les détails eux-mêmes, les mèches de la chevelure des satyres par exemple, se détachent avec force. On remarquera qu'il s'agit aussi, avec l'exemplaire de Simféropol, d'un des deux objets qui se distinguent par une inscription en grec, en lettres pointillées très soignées : une indication pondérale sur les deux, complétée sur celui de Simféropol par deux inscriptions se référant aux possesseurs. On restera prudent toutefois sur les conclusions à tirer de cette observation qui n'implique évidemment pas une supériorité technique des orfèvres grecs. 

composition est moins dense et plus aérée - mais on tiendra compte de l'usure assez prononcée de cet objet. L'exemplaire du Kunsthistorisches Museum de Vienne en est très proche, comme celui de la collection Campana au Louvre, l'un et l'autre bien conservés. Mais le dernier est de facture plus souple, plus raffinée, presque maniérée dans le traitement de certaines figures. Cette recherche se manifeste aussi dans le choix qui a été fait par l'orfèvre de présenter les quatre têtes bachiques de trois quarts et non mécaniquement de profil strict comme dans la plupart des cas, ce qui leur donne davantage de volume. Cette disposition, rare, est aussi celle qui revient, partiellement, sur l'un des grands plats du trésor de Vienne, avec les mêmes effets. Sur le plateau de Chalon, qui combine animaux et personnages, la facture est en revanche assez mécanique, maladroite même, dans le traitement des têtes comme des figures étendues, beaucoup mieux maîtrisées sur celui du trésor de Vienne à frise simple. Le plateau en bronze argenté de Vaise pour sa part est caractérisé par des formes arrondies, presque massives, très différentes par exemple de celles de Houdainlès-Bavay ou de Thil, par une composition relativement aérée (on rappellera qu'elle ne comporte que deux têtes bachiques), et par des accessoires parsemés en assez grand nombre sur le fond. La facture est plutôt soignée, en dépit des déformations qu'a introduites le décollement partiel de la feuille d'argent sur l'âme en bronze.

On rapprochera enfin les uns des autres trois objets sur lesquels les personnages se présentent sous la forme de petites silhouettes rapidement esquissées : les plateaux de Houdain-lès-Bavay, de Vienne (exemplaire à double frise) et de Vaise (exemplaire en argent). Le premier se range un peu à part ; les différents éléments figurés, de qualité, sont fortement modelés, mais sont aussi cernés assez profondément ; les détails en sont souvent marqués à grands coups d'outils qui creusent de manière rapide les volumes et contribuent comme les cernes à accrocher la lumière. Mais, comme sur les deux autres objets, les personnages qui apparaissent ici, deux bergers, sont seulement esquissés, sans aucun détail. À Vaise, entre les quatre têtes de petites dimensions vivement dessinées, quatre groupes d'images ont été représentés, des scènes empruntées à la vie campagnarde, la traite d'une vache ou la cuisson dans un four notamment, mais qui ont pour la plupart une tonalité religieuse ; évidente pour l'offrande à Priape, elle est également sensible, par exemple, dans la scène d'égorgement d'un animal. Comme à Houdain-lès-Bavay, tous les personnages sont réalisés sous forme de silhouettes et l'usure des reliefs, qui n'est pas excessive, ne suffirait pas à expliquer cette absence de détails, encore plus flagrante sur le dernier plateau de Vienne, celui à double frise. La tonalité religieuse y est encore davantage marquée : une partie des figures appartient directement au thiase bachique et plusieurs scènes d'offrande ou de sacrifice y sont présentées ; le répertoire est bien évidemment celui des « paysages sacrés et idylliques ", mais il évoque aussi, dans le cas de l'assiette à double frise de Vienne et de celle en argent de Lyon-Vaise, les cortèges qui apparaissent sur les flancs des coupes à relief en terre cuite sorties des ateliers de Corinthe à l'époque impériale (M. Bats, Dionysiastai. À propos de vases corinthiens à représentations dionysiaques d'époque romaine, Revue Archéologique, 1981, p. 3-26). On ne saurait affirmer toutefois, comme on l'a fait parfois (Ph. Filtzinger, D. Planck, B. Cämmerer, Die Romer in Baden-Württemberg, Stuttgart, 1986, 3e éd., p. 589, à propos de la coupe aux têtes bachiques et aux Amours de Stettfeld: « aus dem Mysterienkult des Gottes Bacchus ») que ces objets aient une véritable valeur 
liturgique: ils traduisent davantage une simple religiosité, si présente dans l'iconographie romaine.

Ces figures rapidement esquissées suscitent un rapprochement avec les décors muraux. Elles évoquent en effet directement celles qui peuplent une partie des paysages idylliques de la peinture campanienne ou certaines frises, mises en place par de grandes taches de couleurs sans modelé ni contours fermes. Bien représenté à Pompéi ou à Rome - la «frise jaune » de la Maison de Livie en donne un belle illustration (R. Bianchi Bandinelli, Rome. Le centre du pouvoir, Paris, 1969, fig. 136)-, ce goût particulier se rencontre plus tard encore : la villa de Dar Bue Ammera, en Tripolitaine, en offre plusieurs exemples de qualité (R. Bianchi Bandinelli, Rome. La fin de l'art antique, Paris, 1970, fig. 241), marqués évidemment par un sens de l'espace qui est absent sur les pièces d'argenterie. On peut également rappeler pour le Ier s., à en croire tout au moins Pline l'Ancien (Histoire Naturelle, XXXIII, 157), le goût des Romains pour une vaisselle aux reliefs usés qui peut ainsi prétendre à une ancienneté, réelle ou supposée, que plus de trois siècles plus tard Sidoine Apollinaire s'est plu lui aussi à souligner (c'est l'aspect terni des objets en argent qui, dans le texte de Sidoine Apollinaire - Ep., I, 2, 6-7; Carmina, XVII, v. 7-12-, suggère leur antiquité) et qui devait rehausser la distinction des propriétaires : peut-être le goût de ces figures comme en ombres chinoises sur les trois plateaux d'argent, s'il traduit fidèlement une certaine sensibilité esthétique, permettait-il en même temps au maître de maison des effets mondains qui rentraient pour beaucoup dans l'engouement dont la vaisselle d'argent jouissait dans le monde romain.

L'analyse qui peut être menée sur le décor de ces plateaux fait apparaître quelques similitudes qui n'autorisent pas cependant à définir les contours d'ateliers particuliers. Les provenances elles-mêmes ne sont pas révélatrices. Sur les exemplaires, neuf (peutêtre dix) proviennent certainement de Gaule, quatre d'Italie (mais un seul de façon assurée), un d'Ukraine, un dernier de Géorgie. La plupart comportent des graffiti en latin, indications pondérales ou noms du propriétaire, deux, ceux de Thil et de Simféropol, des inscriptions en grec; ces dernières ne suffisent pas à assurer l'existence d'une production dans la partie orientale du Bassin Méditerranéen.

Quant à la chronologie, elle n'est pas facile à établir ; quelques points cependant sont à souligner :

- l'objet le plus ancien devrait être celui de Simféropol. Si son allure ne présente rien de particulier, l'une des inscriptions qu'il porte fait en effet référence à la reine Gépaepyris, une souveraine du royaume du Bosphore au milieu du Ier s. ap. J.-C. ;

- le plateau de Pompéi doit être antérieur à l'éruption de 79 ;

- plusieurs autres appartiennent à des trésors dont l'enfouissement est daté avec vraisemblance de la seconde moitié du IIIle s. :

- la trouvaille de St-Genis était accompagnée de monnaies de Galba à Gallien ;

- celle de Vaise de monnaies allant jusqu'au règne conjoint de Valérien et Gallien ;

- les trésors de Graincourt, de Vienne et de Thil sont placés dans la même période (la seconde moitié du IIIe s.) sur des critères typologiques et stylistiques. La cachette d'un trésor n'implique pas, il est vrai, une date identique, ni même voisine pour les objets qu'il contient.

Mais la grande ressemblance du plateau de Simféropol avec la plupart des autres exemplaires montre bien la difficulté d'établir une chronologie précise pour ce groupe au décor semble-t-il très conservateur. C'est sur des critères techniques - le développement de la vaisselle de bronze plaquée d'argent au IIIe s. - et sur la 
conception du décor - la complexité d'une frise à personnages multiples - que nous proposerons de placer dans le courant du IIIe s. les deux pièces de Vaise. Mais pour parvenir peut-être à une datation plus précise de ces plateaux, il conviendrait en fait de reprendre l'analyse de l'ensemble des décors bachiques du même type dans la vaisselle de bronze et d'argent, comme dans la céramique qui en donne d'intéressants exemples.

Reste la question de la fonction : elle n'est pas aisée à définir. Les dimensions réduites, une dizaine de centimètres au plus pour la partie centrale, la seule utile, pourraient en faire peut-être des supports pour d'autres objets, vases à boire, récipients pour des sauces, plutôt que des présentoirs pour des aliments, ce qui ne saurait toutefois être totalement exclu. L'inscription grecque qui figure au revers de l'exemplaire de Thil indique, si l'on suit l'interprétation de M. Martin, que ce dernier appartenait initialement à un ensemble de huit pièces: "Ces huit (patères) (pèsent) 7 livres 4 onces ", soit 2401,30 g (M. Martin, dans M. Feugère-M. Martin, Le trésor d'argenterie de Thil (Haute-Garonne), dit « trésor de Caubiac, dans Argenterie romaine et byzantine, 1988, p. 75). On observera cependant qu'ils n'ont jamais été trouvés par groupe de plus de deux dans un même trésor, à Vaise comme à Vienne, et qu'ils ne constituent jamais, dans ces deux cas, de paire véritable : à Vaise, l'un est en argent et l'autre en bronze argenté, et dans l'un et l'autre de ces deux trésors le décor des quatre plateaux est très différent.

On posera en outre la question des rapports de ce groupe de plateaux à marli horizontal avec les "assiettes" de même module, mais sans rebord et sans décor autre parfois qu'un astragale sur la lèvre et un motif niellé au centre : les trésors de Reims (Trésors d'orfêvrerie, $\mathrm{n}^{\circ}$ 97-99) et de Chaourse (Trésors d'orfêvrerie, $\mathrm{n}^{\circ}$ 62-66) en fournissent plusieurs exemples qui se distinguent en outre des plateaux qui viennent d'être étudiés par une forme différente, légèrement creuse, uniformément concave. Les deux catégories semblent en fait sans lien l'une avec l'autre; si l'on peut envisager une différenciation des fonctions entre un plateau doté d'un marli horizontal comme le fond, et un autre sans rebord et légèrement concave, dans la pratique l'usage était peut-être analogue, le creux des "assiettes" n'étant pas suffisant pour les empêcher de jouer le rôle de présentoir. Mais une semblable distinction de forme intervenait aussi pour les plats à proprement parler, parmi lesquels on distinguait des séries sans marli, creuses, ou plates, et des séries dotées d'un rebord, creuses le plus souvent, mais plates aussi parfois : le trésor de Rethel en offre les meilleurs exemples, puisque les objets en question portent alors un décor identique (Trésors d'orfêvrerie, $\mathrm{n}^{\circ}$ 113-114).

\section{4-19. Les cuillers et les petits ustensiles}

\section{Catalogue}

61 La trouvaille de Vaise comporte quatorze cuillers et deux petits ustensiles fragmentaires, disposés en vrac, semble-t-il, au-dessus des deux ensembles de vaisselle d'argent et de la bourse de monnaies qui, enfermés dans une caisse en bois, constituaient à côté des statuettes le second dépôt du trésor.

On observera d'emblée, avant de présenter chacune des cuillers, que si plusieurs sont proches les unes des autres, elles ne forment aucun véritable service contrairement à ce qui se produit parfois; le groupe est, comme bien souvent, passablement disparate. 
Par ailleurs, toutes, par la forme en bourse plus ou moins accentuée de leur cuilleron, appartiennent aux séries attribuées avec vraisemblance au IIIe s.

\section{Cochlear}

$63 \quad$ Inv. 9311044

Argent (les dimensions de toutes les cuillers ont été rassemblées dans un tableau) (fig. 56).

Excellent état de conservation. L'extrémité du manche est tordue.

64 Cuilleron en bourse, de proportions assez allongées (la longueur est environ le double de la largeur). Il est bordé par un étroit méplat. L'étranglement arrière est net. Le manche, effilé, de section rectangulaire, est rattaché au cuilleron par un élément en arc de cercle d'où se dégage vers l'avant une sorte d'ergot (fig. 37).

65 Deux graffiti dans le cuilleron : au-dessus de l'axe, MES[(l'abréviation d'un nom ?) ; vers l'avant deux hastes verticales soulignées d'un trait horizontal, qui correspondent peutêtre aux lettres LI. Les deux hastes, si elles ont isolées, pourraient représenter un numéro d'ordre dans une série : 2 ?

\section{7- Cochlear $n^{\circ} 4$}

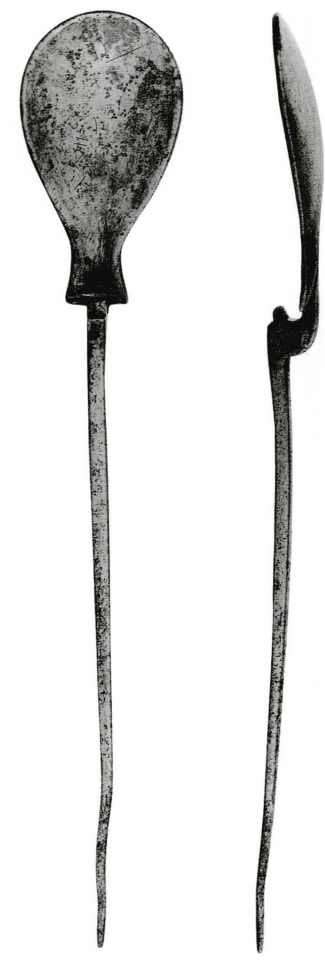

\section{5. cochlear}

Inv. 9311045

Argent.

Excellent état de conservation. Très légère usure à l'avant.

Cuilleron en forme de bourse, de proportions allongées (la longueur est le double de la largeur). Il est bordé par un étroit méplat. L'étranglement arrière est marqué et allongé. Le revers est à facettes. Deux larges incisions en $\mathrm{V}$ à l'arrière du cuilleron. Le 
manche effilé, de section circulaire, est rattaché au cuilleron par un élément en quart de cercle (fig. 38).

38- Cochlear $n^{\circ} 5$

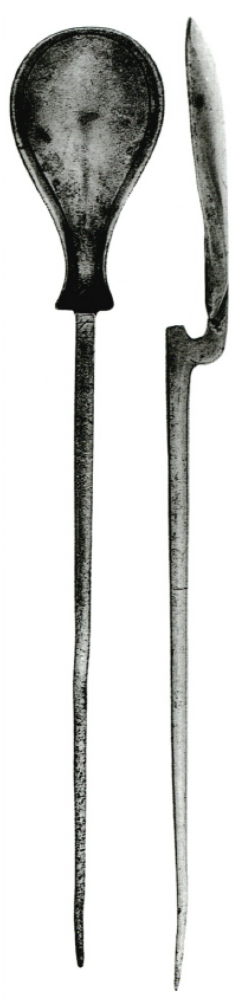

Trois graffiti dans le cuilleron : à l'intérieur MES(...) et une croix ; au revers (fig. 39), en grandes lettres GRECI(...). Le premier, sans doute l'abréviation d'un nom, apparaît déjà dans la cuiller $\mathrm{n}^{\circ} 4$. La croix pourrait être un numéro d'ordre : 10 ?

39- Cochlear $n^{\circ} 5$, revers du cuilleron avec le graffiti

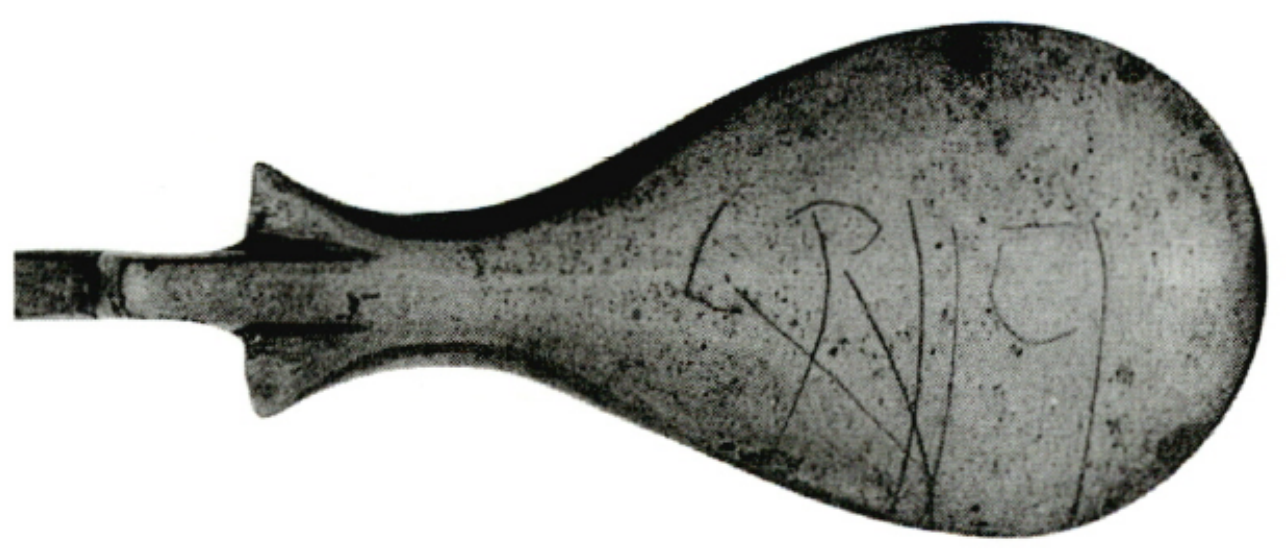




\section{Cochlear}

$69 \quad$ Inv. 9311046

Argent.

Excellent état de conservation.

70 Cuilleron en bourse très soigné : le rebord est cerné par une ligne gravée et par une incision en $\mathrm{V}$ à l'extrémité. La partie arrière est allongée et l'étranglement peu marqué. Le manche effilé, de section circulaire, est rattaché au cuilleron par un élément en arc de cercle (fig. 40).

40- Cochlear $\mathrm{n}^{\circ} 6$

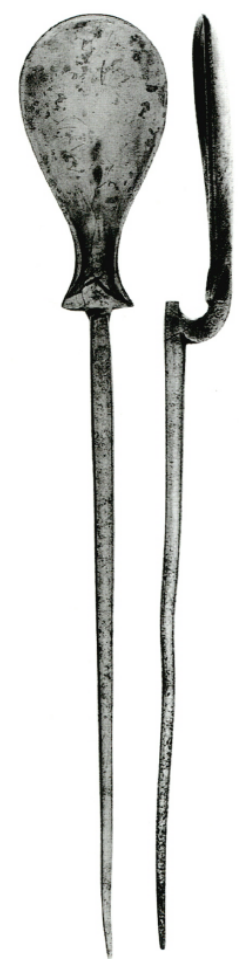

71 Plusieurs graffiti : au revers du cuilleron, on voit de grandes incisions qui ne forment cependant pas de lettres. Sur chaque côté, une incision en croix. Sur le côté gauche de l'attache, en fines lettres pointillées (h. $2 \mathrm{~mm}$ ), SEX(...), probablement l'abréviation (Sextus par exemple) du nom d'un des possesseurs.

\section{Cochlear}

$72 \quad$ Inv. 9311047

Argent.

Excellent état de conservation.

73 Cuilleron en bourse de proportions assez trapues. Il est bordé par un étroit méplat. L'évasement de la partie arrière est assez accentué. L'extérieur est légèrement facetté. Le manche effilé, de section circulaire, est relié au cuilleron par un élément en arc de cercle, auquel il est lui-même rattaché en biseau (fig. 41). 
74 Dans la partie avant du cuilleron, un graffiti presque imperceptible, XI, peut-être un numéro d'ordre : 11 ? (des numéros analogues apparaissent, très soigneusement tracés, sur l'attache du cuilleron de quelques-unes des cuillers du «trésor de Porto »: S. Hauser, Spàtantike und frühbyzantinische Silberloffel. Bemerkungen zur Produktion von Luxusgütern im 5. bis 7. Jahrhundert, JbAC, Ergänzungsband 19, Münster, 1992, n 50-52, p. 104, pl. 9a).

41- Cochlear $n^{\circ} 7$

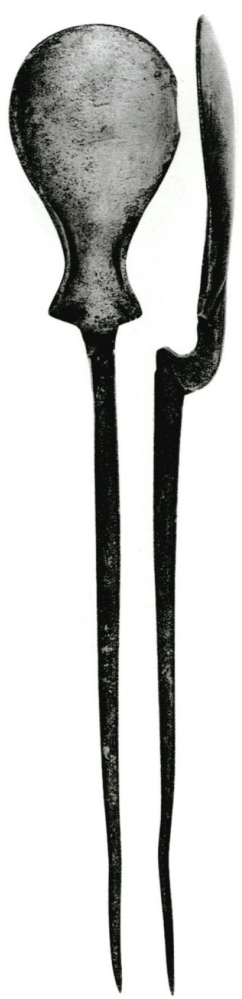

\section{Cochlear}

Inv. 9311048

Argent.

Excellent état de conservation.

Cuilleron en bourse bordé d'un méplat. L'étranglement arrière est nettement marqué. Le manche effilé, de section circulaire, se termine en saillie au-dessus du cuilleron auquel il est rattaché par un élément en arc de cercle (fig. 42).

Pas de graffiti. 
42- Cochlear $n^{\circ} 8$

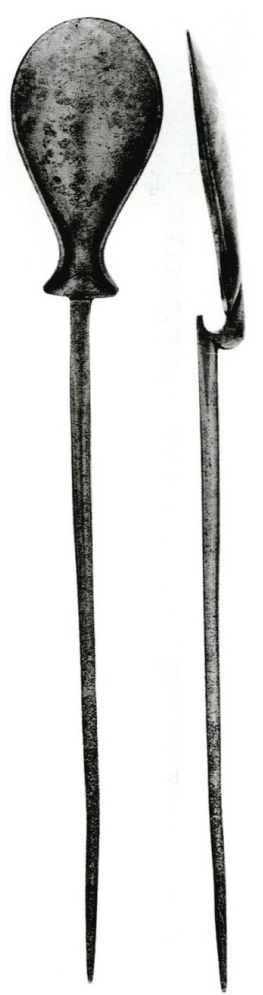

\section{Cochlear}

\section{Argent.}

Excellent état de conservation.

79 courte, est marquée par une incision en V. Au centre du cuilleron, un motif estampé que nous interprétons comme une fleur très stylisée, en forme de trident: une tige courte et renflée, un cœur globulaire et trois petits pétales étroits (fig. 44). Le revers du cuilleron est facetté. Le manche effilé, de section cylindrique, est raccordé au cuilleron par un élément en arc de cercle écrasé.

80 Au revers du cuilleron, un graffiti incisé en grandes lettres maladroites : GRECI (...), le même nom qui apparaît déjà sur la cuiller $n^{\circ} 5$ 
43- Cochlear $n^{\circ} 9$

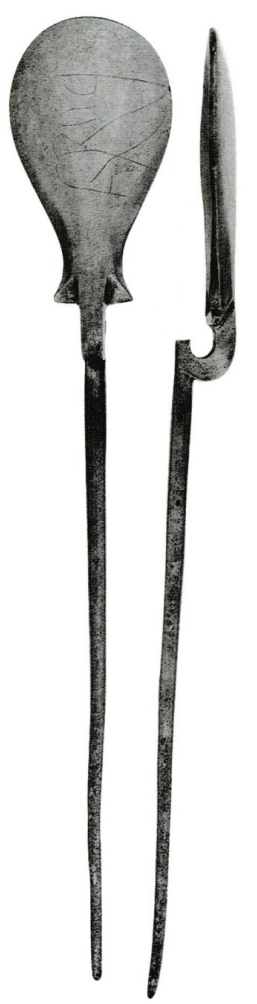

44- $\operatorname{Cochlear} \mathrm{n}^{\circ} 9$, détail du cuilleron avec un motif estampé

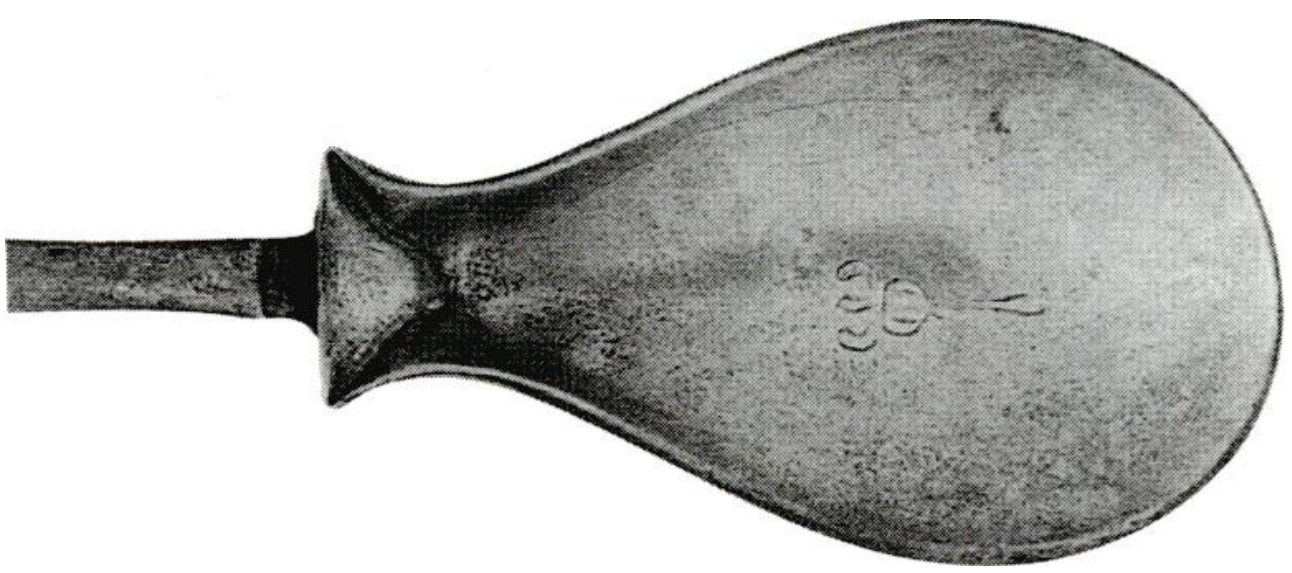

10. Cochlear

$81 \quad$ Inv. 93110410

Argent.

82 Le cuilleron, cassé un peu au-delà de son raccord avec le manche, a disparu. Il était sans doute en bourse, mais très peu resserré à l'étranglement, qui n'est pratiquement pas marqué et au-delà duquel la partie arrière, courte, ne s'élargit pas (fig. 45).

83 Manche effilé en deux parties : la première, de section circulaire, se termine par un élément en balustre très schématisé, au-delà duquel, vers le cuilleron, se développe une partie plus courte, plate, qui se rétrécit vers l'avant. Le raccord au cuilleron se faisait 
par un élément en arc de cercle, bien distinct du manche qui est mouluré à son extrémité supérieure.

45- Cochlear $\mathrm{n}^{\circ} 10$

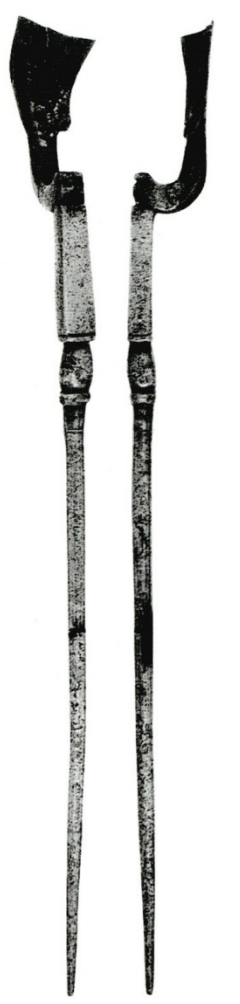

11. Cochlear

Inv.93110411

Argent.

Bon état de conservation. Néanmoins la pointe du manche est cassée.

Cuilleron en bourse sans étranglement ni élargissement à la partie arrière (fig. 46). Son rebord est souligné par une ligne incisée qui se développe à l'avant en une fleur de lys. Le manche effilé est en deux parties: la première, de section octogonale, dont l'extrémité libre manque, se termine de l'autre côté par un élément en balustre qui se poursuit par une partie plate de section rectangulaire; du côté du cuilleron auquel il est rattaché par un élément en arc de cercle, le manche s'achève par une petite moulure à sa partie supérieure.

Deux graffiti, l'un dans le cuilleron, l'autre à l'extérieur, qui donnent le début d'un même nom : $\operatorname{CO}(. .$.$) (fig. 47).$ 
46- Cochlear $n^{\circ} 11$

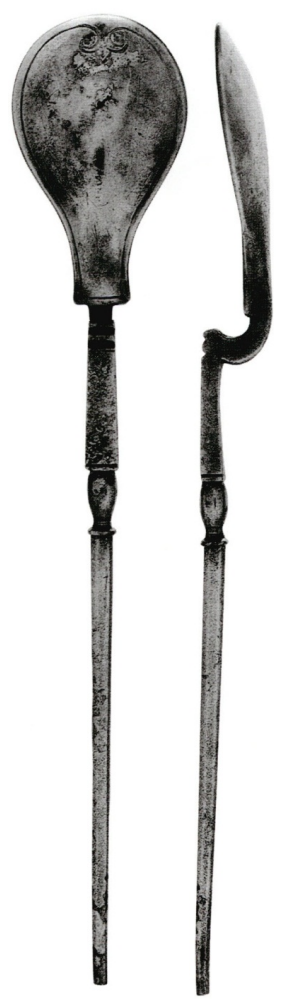

47- Cochlear $n^{\circ} 11$, détail du cuilleron (revers)

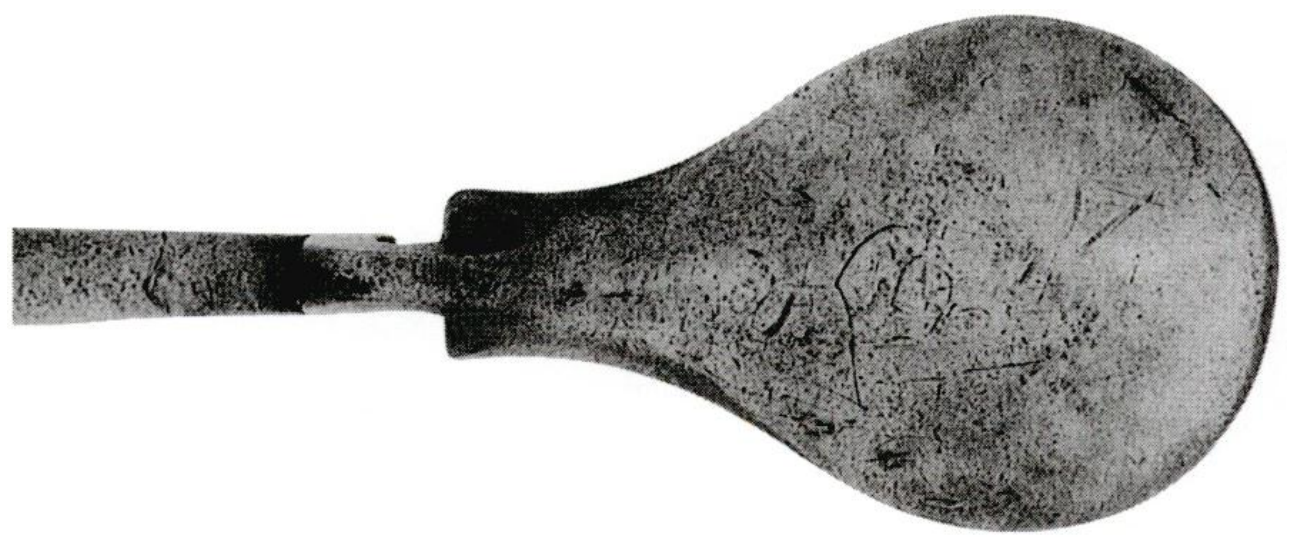

12. Cochlear

Inv. 93110412

Argent.

Toute la première partie du manche manque. Le cuilleron, à la surface corrodée, est cassé en deux aux deux tiers de sa longueur ; il est recollé. Il est analogue à celui de la cuiller $n^{\circ} 11$, légèrement plus petit toutefois. Le décor en est identique. Le manche lui aussi devait être semblable à celui de la cuiller précédente; il est cassé au début du balustre. Il se termine également par une petite moulure à la partie inférieure (fig. 48).

Dans le cuilleron, un graffiti: $\operatorname{COR}(. .$.$) qui donne le début du même nom que sur la$ cuiller $n^{\circ} 11$. Sous la partie plate, un autre graffiti : PO. 
48- Cochlear $n^{\circ} 12$

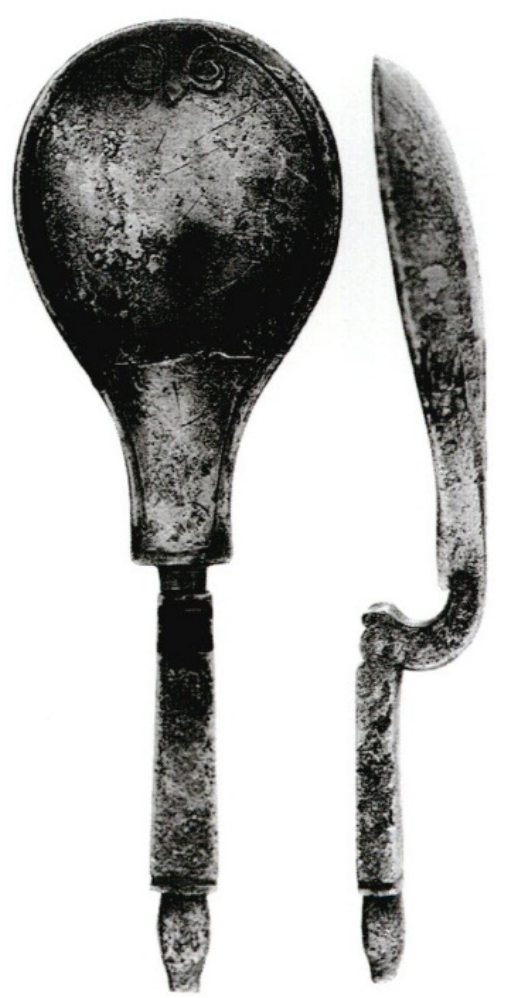

13. Ligula

$90 \quad$ Inv. 93110413

Argent.

Très bon état de conservation.

91 Cuilleron en bourse analogue à ceux des cuillers $n^{\circ} 11$ et 12 , sans étranglement ni élargissement à la partie arrière ; il présente le même décor en fleur de lys. Le manche se termine par un empilement de deux balustres surmontés d'une petite boule. Il offre ensuite une partie de section octogonale qui s'achève par un nouveau balustre. Vient ensuite une partie plate. Raccordé au cuilleron par un élément en arc de cercle, il se termine par une petite moulure à sa partie supérieure, en saillie au-dessus du cuilleron (fig.49). 


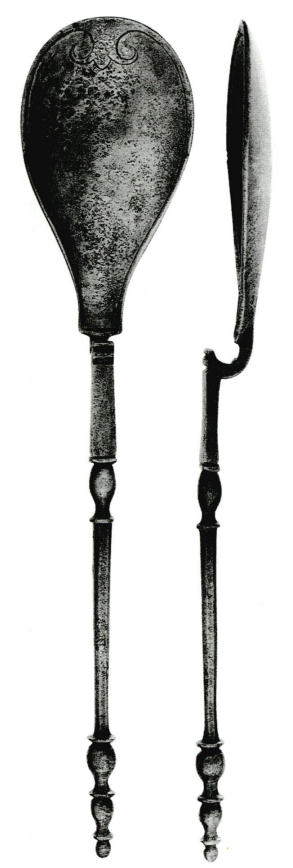

92 Deux graffiti identiques, très ténus, à l'intérieur et à l'extérieur du cuilleron, le début du même nom déjà présent sur les cuillers nº 11 et $12:$ CO (...).

\section{Ligula}

$93 \quad$ Inv. 93110414

Argent.

Très bon état de conservation. Le cuilleron est légèrement usé à l'avant.

94 Cuilleron en bourse bordé par un étroit méplat. La partie arrière, dont l'étranglement est à peine sensible, est très large et marquée par une profonde incision en $\mathrm{V}$. Le revers est orné de trois séries de deux lignes parallèles, la première sur l'axe du cuilleron, les deux autres divergeant de part et d'autre vers l'avant (fig. 50).

Le manche, de section circulaire, s'élargit progressivement vers l'arrière; de son extrémité arrondie sort un petit bouton terminal. Il est rattaché au cuilleron par un élément en arc de cercle écrasé formant un ergot saillant.

Dans le cuilleron, deux graffiti : un nom déjà rencontré à plusieurs reprises CO (...) et une lettre isolée, T. Au revers, deux autres graffiti: de nouveau CO (...) et une succession de quatre lettres PXPI, que l'on interprétera peut-être comme une indication pondérale donnant le poids de plusieurs pièces analogues. Le poids assez élevé de cette cuiller $(34,70 \mathrm{~g})$ ne correspond cependant pas au 1/10e de la livre. 


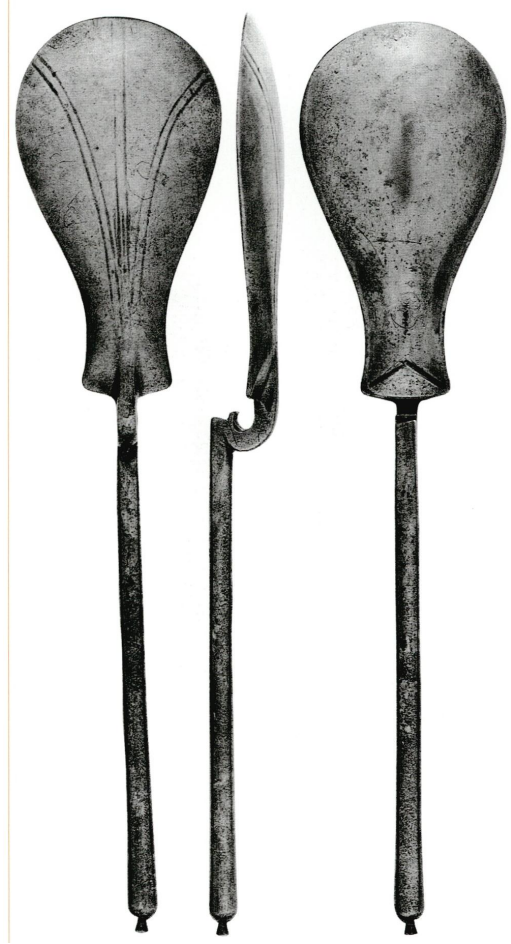

\section{Cuiller à double fonction}

Inv. 93110415

Argent.

La cuiller est en très bon état de conservation. Mais l'ustensile en fer qui était fixé à l'extrémité du manche a disparu; il n'en reste que la soie cylindrique.

Cuilleron en bourse de la forme la plus courante, assez trapu (la largeur représente plus de la moitié de la longueur). Un élément en volute, en nette saillie au-dessus du cuilleron et d'où se détache un petit appendice, le rattache au manche. Celui-ci est court et massif: au-delà d'un élément d'astragale, une tige (L. 2,4 cm) de section hexagonale s'achève par une pièce en forme de cratère stylisé à l'intérieur duquel est plantée la soie qui appartenait probablement à une lame de couteau (fig. 51).

Il n'y a pas de graffiti. 
51- Cuiller $n^{\circ} 15$

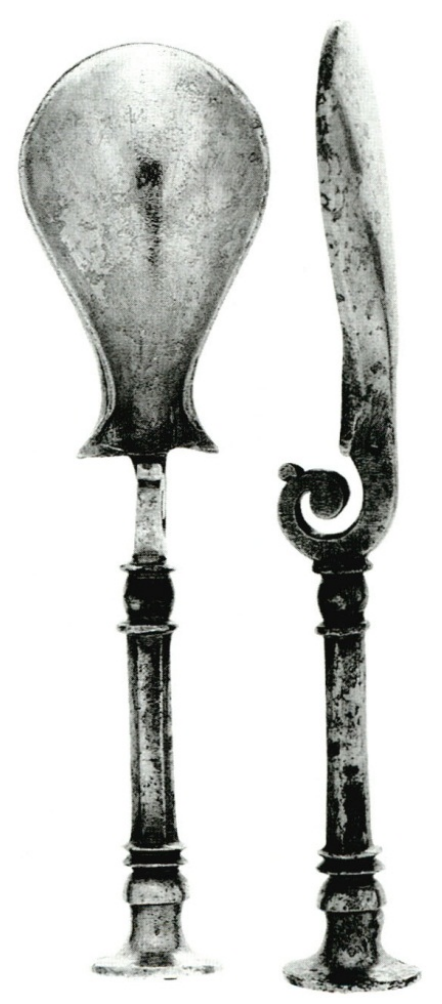

\section{Cuiller à double fonction}

$\operatorname{lnv.} 93110416$

\section{Argent.}

La cuiller est en très bon état de conservation. La pièce en fer qui la prolongeait, sans doute une lame de couteau, a toutefois disparu.

101 Cuilleron en bourse très trapu et très arrondi. L'étranglement arrière est bien marqué, de même que l'élargissement terminal. Le cuilleron s'achève en pointe vers le manche. Le revers comporte trois facettes. Une volute massive le relie au manche. Celui-ci se présente sous l'aspect d'un double balustre de section octogonale qui s'achève à son extrémité en s'évasant. Dans la platine ainsi ménagée venait se fixer, dans un plan parallèle à celui du cuilleron, une lame en fer maintenue sans doute par une soie fixée dans le manche, mais aussi, de chaque côté, par deux éléments soudés au manche, ajourés et traités en pelte à leur extrémité (fig. 52).

102 Il n'y a pas de graffiti. 
52- Cuiller $n^{\circ} 16$ et cuilleron
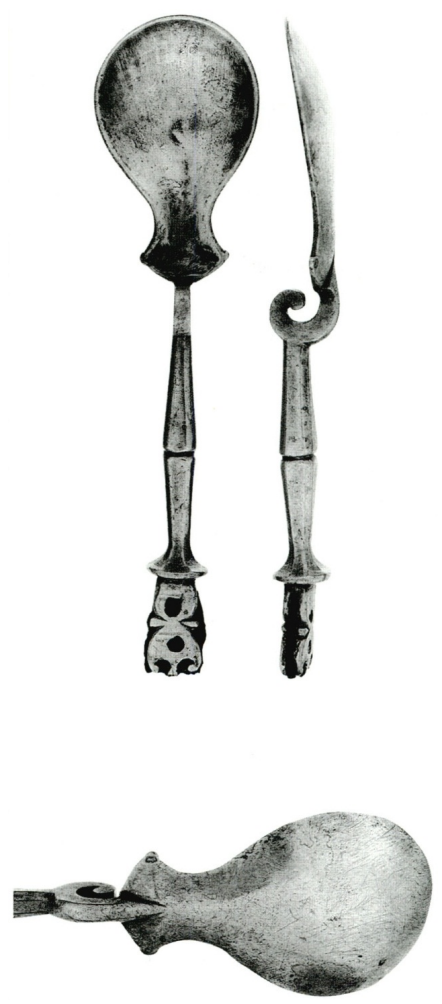

\section{Cuiller}

Inv. 93110417

Argent.

Excellent état de conservation.

Le cuilleron est en forme de bourse, du type le plus répandu. Deux incisions en $\mathrm{V}$ marquent le talon. Toute la partie avant est bordée d'une bande de triangles niellés. Au centre, un svastika niellé de chaque extrémité duquel pendent deux petits appendices parallèles terminés par un petit triangle. D'un des angles sortent également deux appendices simples (fig. 53).

C'est un motif en tête de griffon qui rattache le cuilleron au manche. Celui-ci est complexe : au-delà d'un élément en forme de lyre, il comporte une partie en balustre de section octogonale (à pans concaves) terminée par un astragale et une pièce facettée portant un disque; un second disque identique au premier marque le début d'une deuxième partie: deux éléments d'un astragale, puis l'extrémité d'une patte munie d'un sabot dont les pinces sont nettement marquées.

Un graffiti en grandes lettres incisées est visible au revers du cuilleron : GRECI, un nom qui revient aussi sur les cuillers $n^{\circ} 5$ et 9 . 


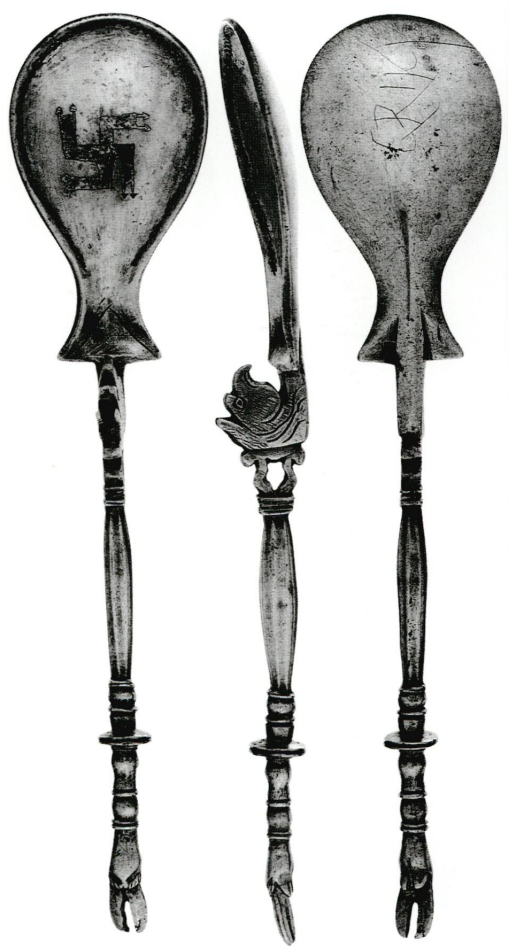

107 En plus des quatorze cuillers deux petits ustensiles incomplets figuraient dans le second dépôt de Vaise :

\section{Manche et anneau}

Inv. 93110418

Argent.

L. 8,5 cm, diam. anneau $2 \mathrm{~cm}$, diam. tige $0,25 \mathrm{~cm}$.

Poids 9,6 $\mathrm{g}$.

Il s'agit d'une tige de section octogonale, cassée à l'une de ses extrémités, au-delà d'une bague moulurée. De l'autre côté, au-delà d'une seconde bague moulurée, la tige se poursuit en col de cygne qui s'achève par une tête de canard stylisée, aux yeux globuleux et au large bec plat. Dans le repli du col de cygne est passé un anneau de suspension (fig. 54 et 61).

110 La tige se prolongeait donc vers le bas par la partie utilitaire de l'instrument, une passoire probablement. 
54- Manche et anneau $n^{\circ} 18$

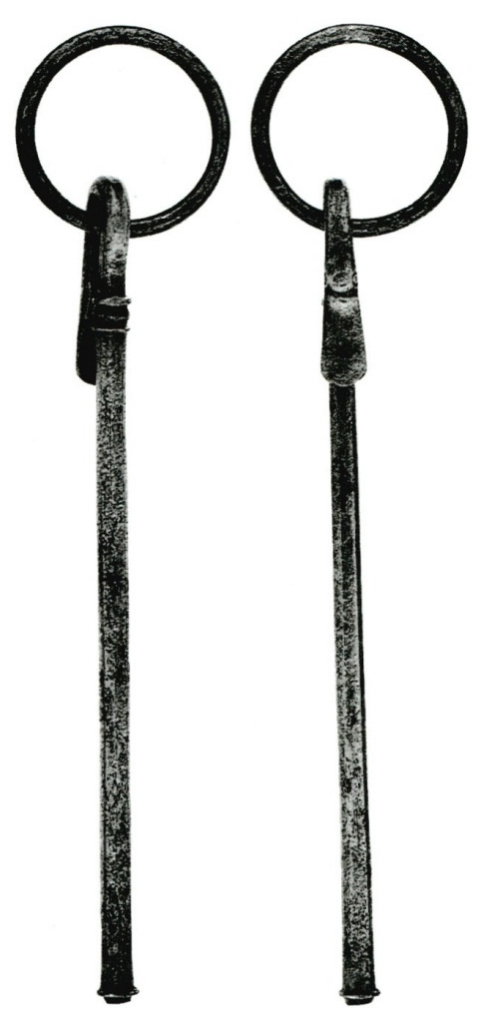

19. ustensile fragmentaire
cassée dans sa partie inférieure, au-delà d'une bague moulurée; elle l'est aussi,
vraisemblablement, à son extrémité supérieure qui se rétrécit. À $5,8 \mathrm{~cm}$ de la bague inférieure, elle porte, comme si elle était enfilée sur elle, une excroissance perpendiculaire en forme de petit marteau, parallélépipédique d'une côté, en pointe légèrement recourbée de l'autre (fig. 55 et 61). 


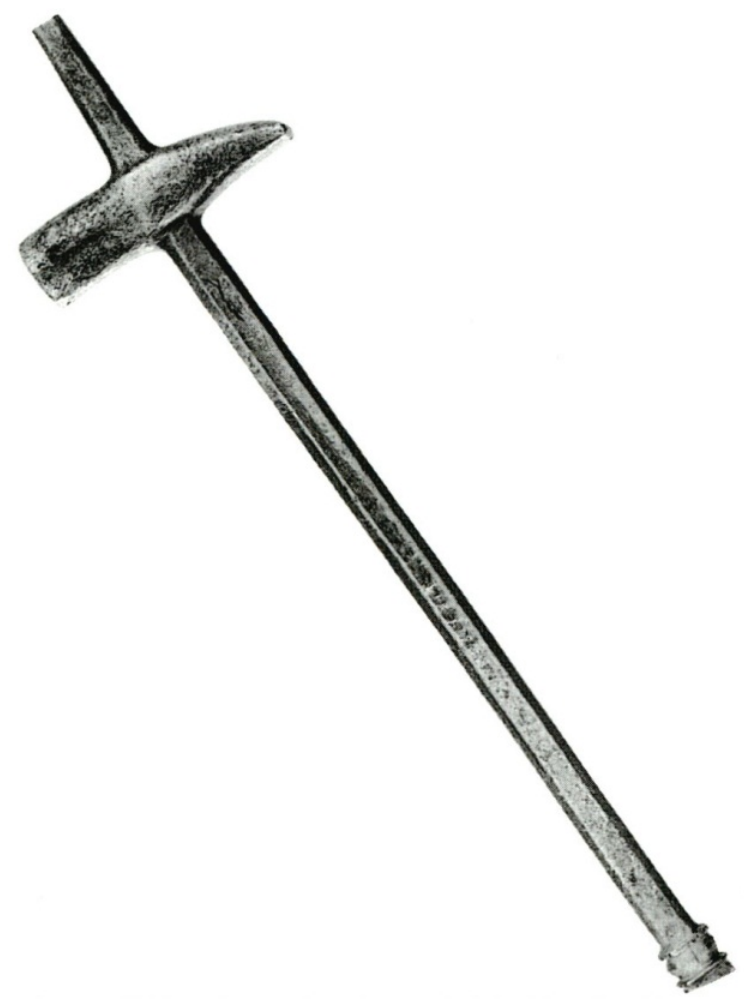

56- Dimensions des cuillers du trésor de Vaise

\begin{tabular}{|c|c|c|c|c|c|}
\hline $\mathrm{N}^{\circ}$ d'inventaire & Poids (g) & Longueur (cm) & $\begin{array}{l}\text { Longueur du } \\
\text { cuilleron }(\mathrm{cm})\end{array}$ & $\begin{array}{c}\text { Largeur du } \\
\text { cuilleron }(\mathrm{cm})\end{array}$ & $\begin{array}{l}\text { Longueur du } \\
\text { manche }(\mathrm{cm})\end{array}$ \\
\hline 9311044 & 17.80 & 17 & 5.30 & 2.90 & 11.75 \\
\hline 9311045 & 29.50 & 18.70 & 5.80 & 2.90 & \\
\hline $93 \quad 110417$ & 38.70 & 16 & 6 & 3.50 & 8.60 \\
\hline 93110416 & 21.80 & 11 & 4.50 & 2.70 & 9.50 \\
\hline 93110415 & 32.10 & 11 & 4.90 & 3 & 4.75 \\
\hline 9311048 & 17.90 & 17 & 4.90 & 2.60 & \\
\hline 9311046 & 17.50 & 16.80 & 5 & 2.60 & \\
\hline 93110410 (cassée) & 11 & 13.90 & & & 11.60 \\
\hline 93110412 & 10.05 & 7.50 & 4.20 & 2.50 & \\
\hline 93110413 & 32 & 18.50 & 7 & 4 & 11.50 \\
\hline 93110414 & 34.70 & 16.80 & 7 & 3.80 & \\
\hline 9311049 & 21.80 & 17.40 & 5.20 & 3 & \\
\hline 93110411 & 17 & 14.70 & 4.40 & 2.60 & \\
\hline 9311047 & 18.10 & 15.30 & 4.70 & 2.70 & \\
\hline
\end{tabular}

\section{Commentaire}

\section{La tige au marteau}

Le dernier ustensile présenté paraît de prime abord surprenant : on ne comprend ni la nature de l'outil qui le terminait, ni son usage exact. Un parallèle existe cependant, découvert en 1911 dans les fouilles de Lauriacum (Enns), en Autriche (M. von Groller, Grabung in Lager Lauriacum im Jahre 1911, Der romische Limes in Österreich, XIII, 1919, p. 20, fig. 10, $n^{\circ} 6$ ). L'objet, en argent lui aussi, est très étonnant (fig. 57) ; il se présente en effet comme une fourchette à trois dents montée sur une tige complexe faite d'éléments successifs; elle comporte précisément une bague moulurée dans sa partie inférieure ; vers le haut est insérée une pièce elle aussi en forme de marteau (ou de pic), 
comportant deux parties, toutes deux de section octogonale, l'une plus allongée et à l'extrémité arrondie, l'autre s'évasant vers l'arrière. Le raccord de la tige aux dents de la fourchette se fait de manière compliquée : il prend l'aspect d'une lyre. Quant à la partie finale, son allure évoque, mais à une toute autre échelle, les enseignes de beneficiarii : elle comporte en effet un élément en forme de cœur percé de deux trous circulaires que l'on rapprochera par exemple d'une extrémité de hampe en bronze ( $h$. $24 \mathrm{~cm}$ ) conservée au musée du Caire (J. Strzygowski, Catalogue général des antiquités égyptiennes du Musée du Caire. Koptische Kunst, Vienne, 1904, nº 9165, p. 301, fig. 340) (fig. 58).

57- Enns/Lauriacum. Fourchette.

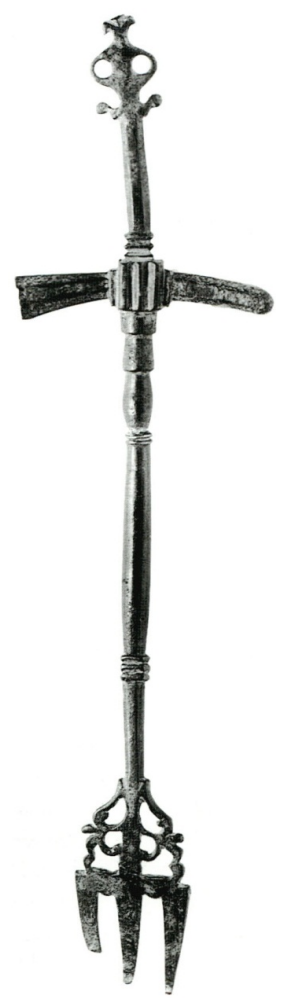

Vienne, Kunsthistorisches Museum. Cl. du musée 


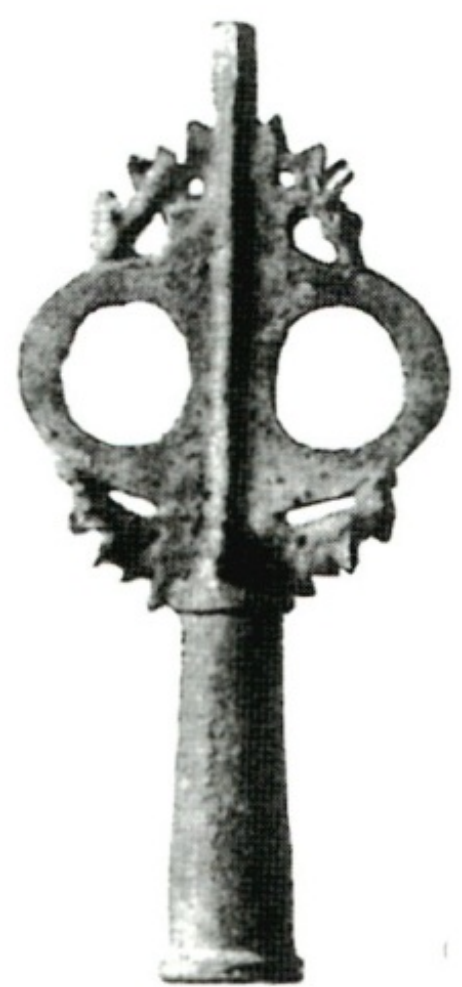

Le Caire, musée national, d'après Strzygowski

117 On connaît maintenant un nombre relativement élevé de fourchettes en argent : à celles que nous avions rassemblée à l'occasion de la publication du trésor de la place Camille Jouffray à Vienne (Baratte et al. 1990, p. 80-81), notamment les deux appartenant chacun à des ustensiles à usage multiple, l'un trouvé dans une tombe à Vintimille, l'autre longtemps dans le commerce des antiquités, s'ajoutent encore deux exemplaires inédits; le premier provenant des fouilles des grands thermes nord de Cimiez (au musée de Cimiez), le deuxième conservé à Cologne, au RömischGermanisches Museum. Les fourchettes de Vienne et de Cimiez se terminent à leur partie supérieure par une pointe: elles auraient pu servir à manger des coquillages. C'est de cette manière que l'objet d'Enns a été décrit dans la courte notice qui en constitue la publication: "Gabel mit Hammer", "fourchette avec marteau"; celle-ci aurait servi pour les huîtres, celui-là pour les coquillages. On imaginera un outil analogue à Vaise, en reconstituant à titre d'hypothèse les trois dents d'une fourchette à l'extrémité inférieure, surmontées par un décor. Mais le poids très faible (moins de 10 $\mathrm{g}$; la fourchette de Lauriacum ne doit pas être beaucoup plus lourde) semble empêcher que le "marteau" ait servi à écraser les coquillages, sauf ceux qui étaient fragiles comme des escargots.

\section{La tige au col de cygne}

118 Le second ustensile pourrait bien être une passoire; l'anneau en effet indique que l'objet pouvait être suspendu, au doigt éventuellement comme le fait l'échanson représenté sur l'un des grands panneaux peints qui ornaient la salle à manger d'une maison découverte à la fin du XVIIIe s. à Rome sur le Coelius, dans le secteur de 
l'Ospedale Maggiore, près du Latran (G. Cassini, Pitture antiche ritrovate... in una vigna accanto il s. ospedale di S. Giovanni in Laterano, l'anno 1780, Rome, 1783 ; F. Baratte, La vaisselle de bronze et d'argent sur les monuments figurés romains, BSNAF, 1990, p. 89-95. Le panneau signalé ici a été commenté par M. Martin, dans H. Cahn, A. Kaufmann-Heinimann, Der spätrömische Silberschatz von Kaiseraugst, Derendingen, 1984, p. 112 ; bonne photographie dans R. Bianchi Bandinelli, Rome. La fin du monde antique, Paris, 1970, fig. 86). La peinture date sans doute de la fin du IVe s. Mais, M. Martin l'a bien montré à propos des passoires du trésor de Kaiseraugst (M. Martin, dans Cahn, Kaufmann-Heinimann, Kaiseraugst, p. 101-121), ce type d'ustensile commence à apparaitre, sous sa forme individuelle, dès la fin du IIIe s., comme le montrent les exemplaires de La Alcudia (prov. Alicante), en Espagne (Martin, op. cit., p. 101, fig. 54, 1), de Strâze en Slovaquie (Martin, op. cit., p. 101, fig. 54, 2) et, peut-être un peu plus tard, de Glinisce près de Kertch (Martin, op. cit., p. 101, fig. 54, 3). Le trésor de la place Camille Jouffray à Vienne en contient également une, assez originale puisque la tige est remplacée par un dauphin (Baratte 1990 et al., nº 19, p. 78-79).

119 L'aspect de l'exemplaire de Vaise ne peut guère être reconstitué avec certitude : la tige n'est pas trop courte pour que l'on pense que la passoire n'ait pas été fixée immédiatement sous la bague moulurée. L. sous la tête de canard $7 \mathrm{~cm}$; L. de la tige de la passoire de Strâze : 7,5 cm (9 cm à La Alcudia). Mais si l'on prend pour modèle les exemplaires de Kaiseraugst (L. $14 \mathrm{~cm}$ ) on sera tenté de restituer sous la bague encore une certaine longueur de tige, éventuellement torsadée (fig. 59).

59- Kaiseraugst, passoire.

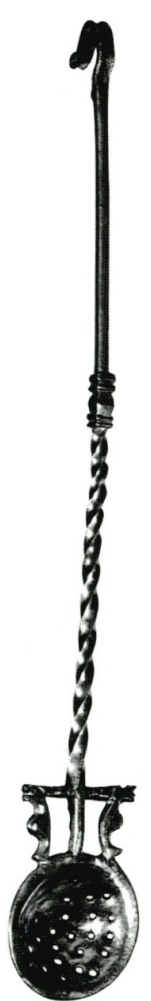

Römermuseum Augst. Cl. E. Schulz

120 L'attache en col de cygne ne surprend guère : on la trouve couramment, plus grande, sur les puisoirs dans l'orfèvrerie grecque des IVe-IIIe s. av. J.-C. ; mais surtout elle est à 
la mode, après une période d'abandon, pour les grandes ligulae des IVe-Ve s. ap. J.-C. (Martin, op. cit., p. 87-91).

On observera que le trésor de Vienne a livré un ustensile identique à bien des égards à celui de Vaise : la tige, complétée par un anneau, est fragmentaire; elle s'achève par une petite moulure en panneton de clé, mais se prolongeait au-delà, peut-être par une passoire (Baratte 1990 et al., p. 85, n² 23, fig. 59).

\section{Les cuillers}

Les cuillers de Vaise posent d'intéressants problèmes. Si elles ne constituent pas un ensemble homogène, tant s'en faut, on peut néanmoins les regrouper en plusieurs ensembles distincts soit en raison de leur forme, soit en raison des graffiti qu'elles portent, sans que les deux types de classement se recoupent exactement (fig. 60).

60- Cuillers du trésor de Vaise

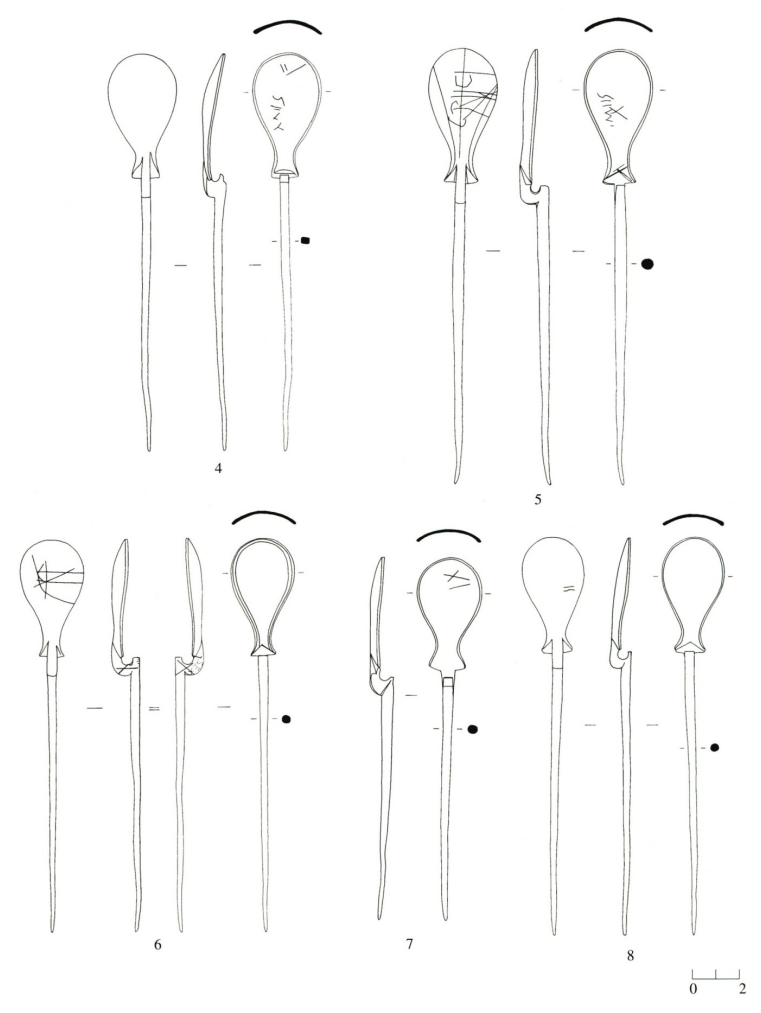

\section{Les graffiti}

Quatre noms reviennent sur neuf cuillers, $\operatorname{COR}(. .),. \operatorname{GRECI}(.),. \operatorname{MESC}(.),. \operatorname{SEX}(. .$.$) , tracés à$ la pointe sur le cuilleron sauf dans un cas $\left(n^{\circ} 6\right)$; l'inscription figure alors sur l'attache du cuilleron au manche, en lettres pointillées

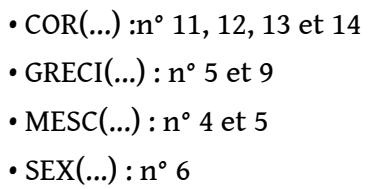

Une des cuillers, $\mathrm{n}^{\circ} 5$, (un cochlear de type ordinaire) porte deux noms à la fois, GRECI et MESC. Ce dernier nom revient sur deux cochlearia de dimensions analogues, mais qui 
diffèrent par des détails de l'attache du manche au cuilleron. GRECI figure sur deux cochlearia presque identiques, y compris par l'aspect facetté du revers du cuilleron. L'un des cuillerons est un peu plus grand que l'autre, qui porte lui-même en son fond un motif floral estampé. COR revient enfin sur quatre cuillers, trois fois sous la forme CO, une fois sous une forme un peu plus complète. Deux des cochlearia, bien que différant légèrement par les dimensions, constituent manifestement une paire en raison de la forme du cuilleron et de son décor (fleur de lys gravée à l'avant). S'y ajoute une grande ligula au manche terminé par deux petits balustres empilés et au cuilleron sans étranglement, comme celui des deux cochlearia, décoré lui aussi d'une fleur de lys. Ces trois objets appartiennent à une même série. Le quatrième enfin est une ligula au manche terminé par un petit bouton. 
61- Les ustensiles 


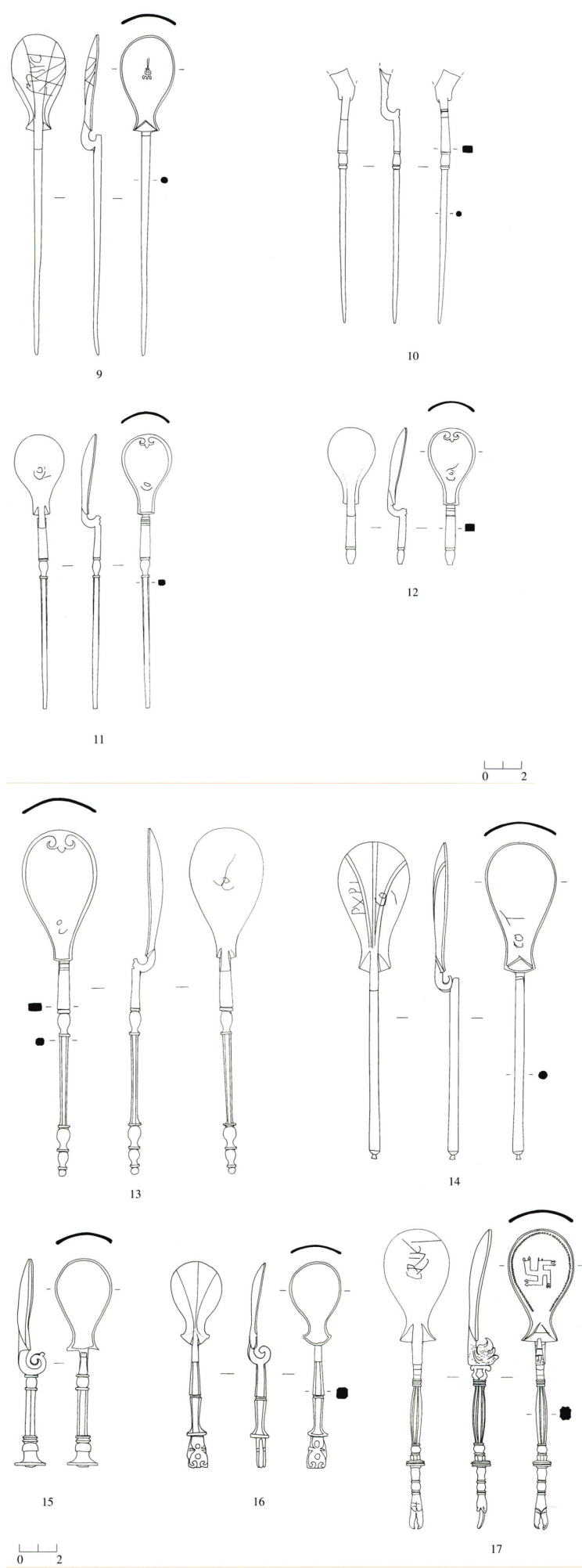




\section{0}
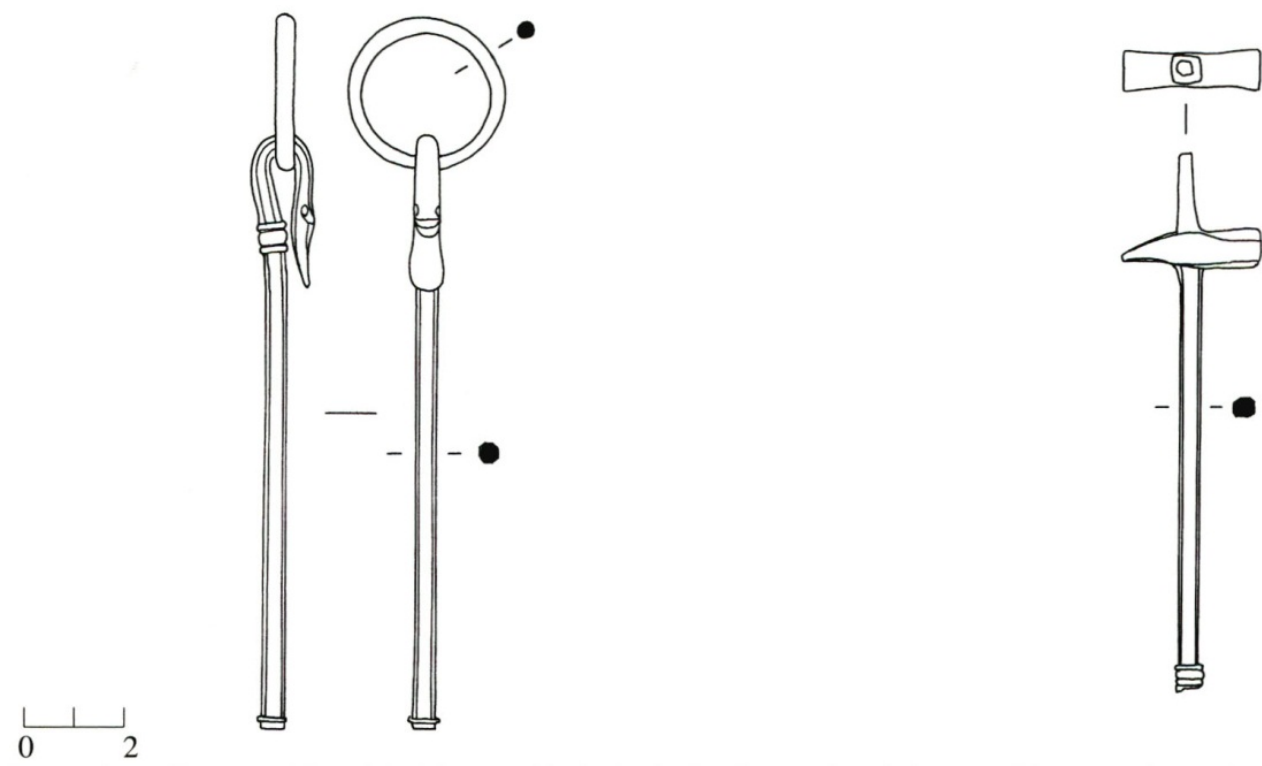

\section{Forme et décor}

Les quatorze cuillers ont leur cuilleron en bourse, avec des variantes : elles prennent place ainsi au sein des séries du IIIe s. (Sur l'évolution d'ensemble des cuillers, cf. M. Martin, Essloffel, dans Cahn, Kaufmann 1984, p. 76-82). On compte deux ligulae ( $\mathrm{n}^{\circ} 13$ et 14), huit cochlearia ( $\mathrm{n}^{\circ} 4,5,6,7,8,9,10$ et 11$)$ et sans doute une neuvième ( $\left.\mathrm{n}^{\circ} 12\right)$ dont le manche est cassé, et trois cuillers d'un type particulier. La distinction, qui repose sur les textes antiques eux-mêmes (cf. Thesaurus Linguae Latinae, s.v. cochlear et ligula) n'est pas très aisée à établir. L'usage moderne range parmi les cochlearia les cuillers à manche effilé, qui servirait ainsi à extraire les mollusques de leur coquille; la ligula serait à l'inverse une cuiller au manche à l'extrémité arrondie ou moulurée. Mais elle apparaît aussi comme un cochlear de grandes dimensions. (Sur ces distinctions, E. Riha, W. B. Stern, Die römischen Loffel aus Augst und Kaiseraugst, Forsch. in Augst 5, Augst, 1982, p. 10).

La première de ces cuillers particulières $\left(n^{\circ} 17\right)$ combine plusieurs particularités que l'on retrouve ailleurs ; à un grand cuilleron en bourse aux proportions très équilibrées, décoré d'un motif niellé, elle joint un manche complexe : une attache en tête de griffon, une tige épaisse en balustre à facettes, une terminaison en sabot d'ongulé.

127 Nous ne connaissons pas d'exemplaire aussi complet, qui possède notamment l'extrémité en sabot effilé qui permettait de piquer ou de gratter, et qui joue donc le même rôle que la pointe des cochlearia (on observe que le sabot, de profil, ressemble davantage à une griffe). Mais cette terminaison a été exécutée à part : elle comportait une petite platine circulaire, qui s'adaptait sur celle de l'autre partie du manche, et deux éléments d'astragale; elle a donc pu se détacher sur les cuillers analogues conservées, qu'il faudrait examiner toutes pour en reconnaitre la structure. Le sabot lui-même n'est pas un motif rare : on le retrouve à l'extrémité d'autres cuillers, souvent plus anciennes, provenant des trouvailles de Campanie en particulier, mais il est aussi plus largement diffusé ; on en connaît ainsi un exemplaire d'un type du IIe s. à Ouréki en Géorgie (Matchabély 1976, pl. 25), un autre, très proche à Nova Bozurna en Serbie (Antique silver front Serbia, $\mathrm{n}^{\circ} 196$, p. 286) ; (le cuilleron est moins arrondi, en pointe vers l'avant, mais les deux cuillers possèdent un élément plat au raccord du manche et du 
cuilleron); deux exemplaires plus anciens (1ère moitié du Ier s.) ont été retrouvés à Jabucje en Serbie (Antique silver from Serbia, $\mathrm{n}^{\circ}$ 160-161, p. 265), de forme analogue aux cuillers de Nova Bozurna et d'Ouréki, mais sans l'élément plat et sans méplat autour du cuilleron. D'autres ont été retrouvés en Russie du Sud et en Syrie (D. E. Strong, Greek and Roman Gold and Silver Plate, Londres, 1966, p. 156), mais aussi à Xanten, dans un petit ensemble de vaisselle d'argent dont l'enfouissement est daté par des monnaies des années 260 (F. Gelsdorf, Der Schatzfund aus Xanten, dans Das Haus lacht vor Silber, fig. 6 et p. 143) (fig. 62). Un exemplaire trouvé au Mont Tourveon, près de Beaujeu (Rhône) possède un grand cuilleron ovale et un manche en balustre particulièrement riche, relié au cuilleron par un élément en forme de pince dont les branches viennent embrasser une volute ( $c f$. liste infra).

62- Xanten, deux cuillers en argent.

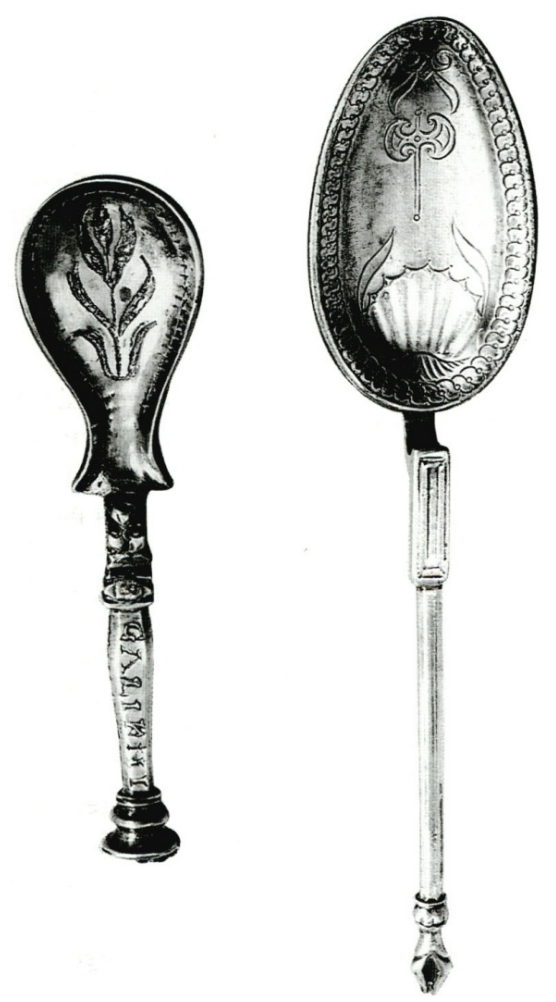

Bonn, Rheinisches Landesmuseum. Cl. du musée

Si l'on fait abstraction de cette partie terminale, la cuiller $n^{\circ} 17$ entre dans la même série que les deux autres de Vaise déjà évoquées ( $n^{\circ} 15$ et 16$)$, à fonction multiple. La platine du manche recevait la soie d'une lame en fer. Une série assez fournie de cuillers de ce type est connue, dans laquelle les cuillerons sont souvent niellés, et le raccord du manche au cuilleron est fréquemment assuré par une tête de lion. Nous en donnons ici une première liste, certainement incomplète :

- Cologne-Bickendorf, dans une tombe à incinération datée par des monnaies des années $280(\mathrm{H}$. Hellenkemper, Trésors romains, trésors barbares, Bruxelles, 1979, nº 8, p. 78-79 ; Gelsdorf, op. cit., p. 144). L $7 \mathrm{~cm}$. Tête de lion, cuilleron niellé : une bande de triangles sur le pourtour, un candélabre végétal au centre et une inscription niellée, ADELFI. Manche en balustre. (Römisch-Germanisches Museum Köln, $24326)$.

- Cologne (ancienne coll. Forst). L 10,7 cm. Tête de griffon, manche en balustre décoré de bandes de triangles niellés. Cuilleron niellé : bande de triangles sur le pourtour, touffe végétale au centre. Ouverture à l'extrémité pour une soie (RGM Köln. M 1091) (fig. 63b). 
- Cologne. L 10,2 cm. Tête de lion; cuilleron niellé : une ligne de postes sur le pourtour, rinceau au centre. Manche en balustre, ouverture dans la platine pour une soie. (RGM Köln, M 25 482) (fig. 63a) ;

- Cologne. Avant-train de lion à l'attache du manche et du cuilleron. Tige du manche en patte de lion terminée par une tête de fauve. Soie à l'extrémité du manche. Cuilleron niellé : bande de triangles sur le pourtour, canthare d'où sortent des feuillages au centre (fig. 63c).

- Cologne (ancienne coll. du Schnütgen-Museum). L 11,8 cm. Cuilleron en amande, niellé : ligne de postes en bordure, rinceau au centre, tête de lion à l'attache. Manche en balustre assez sommaire, platine à l'extrémité et traces d'une soie en fer. (RGM Köln, M 30151 ).

- Cologne (anc. coll. Niessen). L 10,9 cm. Tête de griffon. Pas de décor dans le cuilleron. Manche en balustre cannelé, restes d'une lame en fer. (RGM Kôln, inv. 3861).

- Cologne. L 6,7 cm. Tête de lion. Cuilleron en amande; au centre, inscription niellée : VTERE FELIX. Manche : tige de section hexagonale, extrémité en forme de vase, traces d'une soie. (RGM Kôln. M 26 118).

\section{3- Cologne, trois cuillers en argent.}

a
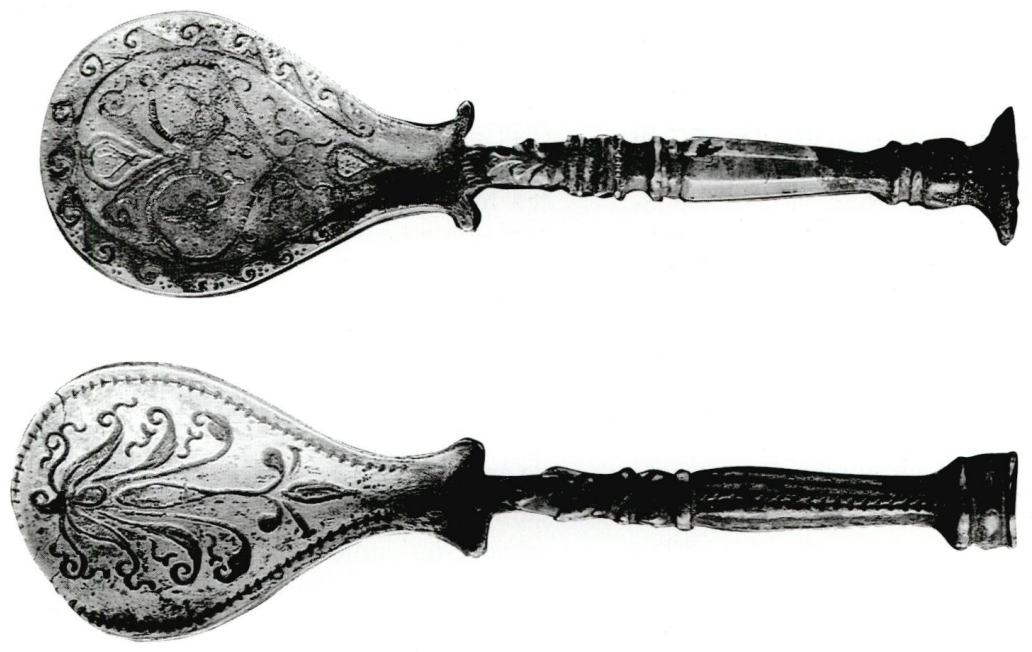

$\mathrm{c}$

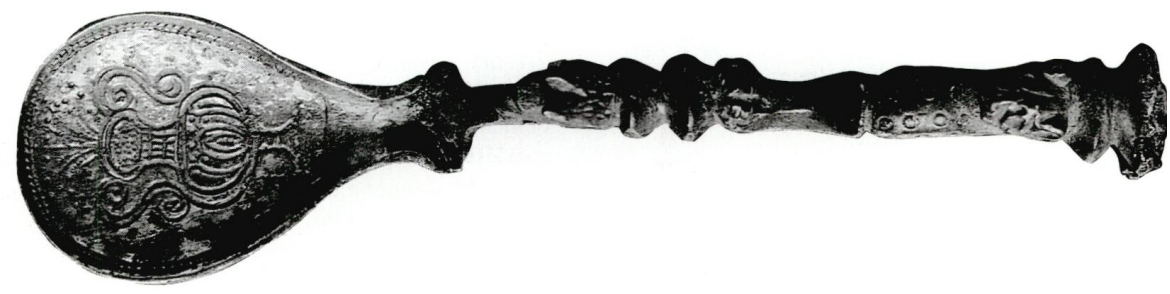

Cologne. Römisch-Germanisches Museum. Cl. du musée

- Cologne ? (anc. coll. Lückger, inv. 81). L 9,5 cm. Tête de lion, cuilleron niellé : bande de triangles en bordure, touffe végétale au centre. Manche en balustre, traces d'une soie. (RGM Köln).

- Cologne ? (anc. coll. Lückger, inv. 88). Exemplaire très sommaire (antique ?). Tête de lion ou de griffon, manche en balustre cannelé (RGM Köln).

- Mayence, sur le Kästrich. L 14,8 cm. Cuilleron ovale, à l'intérieur duquel est gravé un motif figuré : une panthère devant un arbre, en partie doré. Tête de lion, première partie du manche en balustre terminée par une platine circulaire sur laquelle est fixée une longue tige en os avec un petit anneau d'or à l'extrémité (fig. 64). Mittelrheinisches Landesmuseum, inv. R 2125.

-Worms. Tête de lion très stylisée. Cuilleron en bourse, niellé : une bande de triangles en bordure ; volutes végétales au centre avec traces de dorure. Sur le manche en balustre à quatre pans, décor niellé : alternativement un rinceau et une ligne de triangles.

-Worms. Cuilleron en bourse simplifié, sans étranglement. Pas de tête d'animal, mais une volute fixée entre les deux branches d'une pince qui se dégage du manche. Ce dernier, de section carrée, comporte un décor niellé, alternativement des postes et des triangles. On le rapprochera d'un autre manche en bronze incrusté d'argent découvert à Genainville, identifié comme appartenant à un miroir. Sur les quatre faces de la partie de section carrée alternent les deux mots de l'inscription VTERE FELIX et un décor géométrique, des postes et des feuillages très stylisés (Gallia, 19, 1961, p. 294-295). 
- Lyon, musée des Beaux-Arts. Cuilleron en bourse, niellé ; ligne de triangles en bordure, rinceau à l'intérieur. Tête de lion. Manche en balustre de section carrée. Sur les quatre faces, ligne de triangles niellés. L'extrémité en forme de petit vase est prolongée par une lame de couteau en fer dans le même plan que le cuilleron. L totale $18 \mathrm{~cm}$, L de la cuiller $11 \mathrm{~cm}$ (A. Comarmond, Description des antiquités et objets d'art contenus dans les salles du Palais des arts de la ville de Lyon, Lyon, 1855-1857, nº 79, p. 494, $\mathrm{n}^{\circ} 79$ ).

- au Mont Tourveon près de Beaujeu (Rhône), dans une petite trouvaille avec la cuiller signalée plus haut. Cuilleron en bourse ; bordure de triangles niellés, au centre, rinceau végétal. Manche en balustre terminé par une tête de griffon. La seconde partie est lisse et effilée (anc. coll. Claudius Cote, vente Drouot, 4 décembre 1936, $\mathrm{n}^{\circ}$ 45, pl. III. Coll. privée en Grande-Bretagne).

- Helpston (Peterborough, Grande-Bretagne). Christie's, vente du 20 mai 1981 (British Museum, PRB 1984. 5 - 1. 1). Cuilleron en bourse, niellé : postes en bordure, au centre un sanglier en partie doré. Manche complexe: personnage masculin assis devant un pilier, un lagobolon dans la main droite, la gauche posée sur un lièvre. Le manche se termine par un petit chapiteau surmonté d'un vase. Restes d'une soie en fer. L 11, 5 cm. (C. Johns, A Roman Silver Spoon from Helpston, Britannia 1982, p. 309, pl. 32A).

- Trèves, St-Irminen. Cuilleron d'un type particulier. La forme de base est la bourse, mais l'évasement du talon est remplacé par deux petits appendices en volutes. Décor niellé : candélabre végétal simplifié, à l'intérieur d'une ligne de petits triangles emboîtés. L'attache du cuilleron est en volute. Le manche s'y raccorde par un élément en forme de pince. Il se développe ensuite en balustre pour se terminer par un élément en forme de vase. Restes d'une soie en fer. (H. Ciippers, Kurtrierisches Jahrbuch, 16, 1976, p. 97-98, fig. 1 ; S. Martin-Kilcher, Ein silbernes Schwertortband mit Niellodekor, Jahresberichte aus Augst und Kaiseraugst, 5, 1986, fig. 15-1).

- Xanten, dans un petit ensemble caché peu après 260. Cuilleron en bourse niellé, décoré d'un candélabre végétal à l'intérieur d'une ligne de triangles. Tête de lion à l'attache du cuilleron, manche en balustre portant un décor niellé : sur une des faces, une inscription (GALIKNI), sur les autres, une ligne de triangles. À l'extrémité du manche, restes d'une soie en fer (Gelsdorf, op. cit., fig. 6, p. 143) (fig. 62).

- Preuseville (Seine-Maritime), trouvaille fortuite isolée. Cuilleron en bourse niellé, décoré d'un candélabre végétal à l'intérieur d'une ligne de triangles. Tête de lion à l'attache du cuilleron, manche court en balustre, qui se prolongeait par une lame métallique (restes d'une soie en fer), (F. Baratte, La vaisselle d'argent d'époque romaine en Normandie, dans L'art en Normandie. Actes du XXVIe Congrès des sociétés historiques et archéologiques de Normandie, Caen, 1992, II, p. 51).

À cette série de cuillers en argent s'ajoutent encore plusieurs exemplaires en bronze souvent argenté ou recouvert d'une pellicule de métal blanc qui montrent la faveur dont ce type particulier a pu jouir : ainsi dans la tombe 87 de la Viktorstrasse à Xanten, (RLMB inv. 70 0267, 10 ; Gelsdorf, op. cit., p. 144) et pour quelques pièces conservées à Cologne.

La manière dont se présentaient ces objets à manche double lorsqu'ils étaient complets reste discutée. Plusieurs exemplaires intacts montrent que dans un certain nombre de cas au moins c'est une terminaison tout à fait ordinaire qui prolongeait le manche, en matière organique - ce qui justifiait pleinement la présence d'une soie en fer à la jonction des deux parties - ou en métal. La cuiller de Mayence à manche en os déjà mentionnée illustre le premier cas (fig. 64); du second on connait un certain nombre d'exemples, parmi lesquels nous ne citerons qu'une cuiller de Niederbieber (E 1020) antérieure donc aux années 260 - dont l'attache du manche avec le cuilleron se faisait par l'intermédiaire d'une volute, ce qui la rapprochait des deux cuillers de Vaise $\mathrm{n}^{\circ} 15$ et 16 (Oliver $\left.1977, \mathrm{n}^{\circ} 108\right)$.

64- Mayence, cuiller en argent et en ivoire.

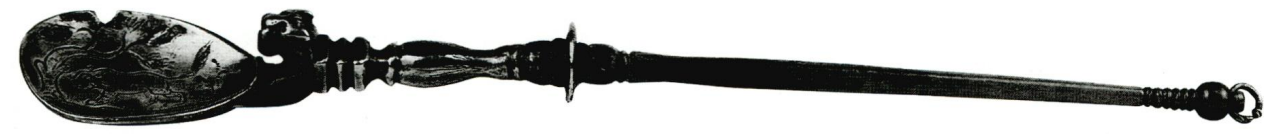


La seconde partie du manche toutefois peut prendre une autre allure que celle d'une tige à bouton ou d'une simple pointe, comme le montre la présence du sabot sur la cuiller de Vaise $\mathrm{n}^{\circ}{ }^{17}$. Ce bel exemple est original par son traitement, qu'il s'agisse $d u$ sabot qui peut aussi servir de griffe, du balustre et de la tête de griffon, ou de la présence d'un petit élément en lyre servant de transition entre le manche et cette dernière. Si nous ne connaissons pas d'autre attestation de ce détail, on pourra le rapprocher de la terminaison en pince que l'on retrouve plus fréquemment, sur la cuiller de Trèves-St Irminen déjà citée, mais aussi sur des exemplaires de Mayence (Rheinisches Landesmuseum), de Worms, de la Saalburg (en bronze), de Lyon-Quai Pierre Scize (musée des Beaux-Arts, X 237), ou sur la cuiller du Mont Tourveon déjà citée. Il s'accompagne souvent d'un manche long, effilé et richement tourné.

Dans le cas des cuillers à manche double la partie manquante correspondait aussi parfois à un autre instrument, comme on l'a déjà fait observer, sans doute souvent un couteau ; contrairement à ce qui a été récemment affirmé (Gelsdorf, op. cit., p. 144) il en existe en effet un exemple au moins : la cuiller à tête de lion du musée des Beaux-Arts de Lyon, sur laquelle la lame en fer est toujours conservée. La cuiller de Vaise $n^{\circ} 16$ en offre par ailleurs une attestation très vraisemblable, puisque l'attache ajourée encore en place ne peut convenir à la fixation d'un élément cylindrique de petit diamètre : elle maintient une lame plate, cassée il est vrai, mais qui se prolongeait de toute évidence, en lame de couteau probablement.

132 En ce qui concerne le raccord du manche au cuilleron, le lion est mieux représenté que le griffon; il revient en effet encore sur quelques objets particuliers, d'une forme différente, comme une cuiller en or provenant d'Entrains dans la Nièvre (Trésors d'orfèvrerie, $\mathrm{n}^{\circ} 128$ ). D'une manière générale, on notera que l'utilisation d'une tête d'animal à l'extrémité du manche est également fréquente à la fin de l'Antiquité et au début de l'époque byzantine (sur ce point, et pour les références aux exemples cités $c f$. Hauser 1992) : des sangliers par exemple sur un lot de sept cuillers des VIe-VIIe s. sans provenance précise (environs d'Antioche ?) à Dumbarton Oaks (Hauser, $n^{\circ} 136142$ ), un lion sur trois cuillers de Canoscio (Hauser, $\mathrm{n}^{\circ}$ 144-146), datées, elles, sans doute du VIe s., ou bien encore sur une cuiller sans provenance du Musée d'art et d'histoire de Genève (Hauser, $n^{\circ}$ 148, pl. 3ld-e).

133 Le griffon est plus rare sur les cuillers du IIIe s. : un exemplaire déjà cité de la petite trouvaille du Mont Tourveon en offre une autre attestation. Mais il faut attendre surtout la fin de l'Antiquité pour rencontrer ce motif : ainsi à Krefeld-Gellep dans une tombe masculine, à Crema, à Desana et à Isola Rizza (3 exemplaires) en Italie du Nord (Hauser, $\mathrm{n}^{\circ}$ 1-6, pl. 4). Mais au IVe s. déjà, ce principe décoratif est fréquent : le trésor de Thetford, pour ne citer qu'un exemple parmi beaucoup d'autres, en offre plusieurs attestations (Johns, Potter 1983, $\mathrm{n}^{\circ}$ 68-72, p. 121-125) et le goût se prolonge effectivement au Ve s. (trouvaille de Canterbury, C. Johns, T. Potter, Antiquaries Journal 65, 1985, p. 318, n C 8-9, fig.7).

S'il est donc certain, comme l'avait déjà observé D. Strong (1966, p. 178), que cette manière de placer une tête d'animal au raccord entre le manche et le cuilleron apparaît dans le courant du IIIe s. avec les cuillers dont le trésor de Vaise fournit un exemple, on notera que les formes plus tardives, dès le IVe s., sont tout à fait différentes, plus sobres: les manches complexes de cette série particulière si représentée à Cologne qu'on est tenté d'en situer l'origine dans un atelier sur place ne semblent guère avoir rencontré d'écho par la suite. 
Les deux cuillers $\mathrm{n}^{\circ} 15$ et 16 sont, du point de vue de la forme, une variante de la cuiller $n^{\circ} 17$ : la tête d'animal est remplacée par une volute comme sur d'autres exemplaires du même type, ceux de Trèves St-Irminen ou du Mont Tourveon par exemple. Mais ce choix est particulièrement intéressant, dans la mesure où il est appelé à un grand succès au IVe s., où la volute est l'un des éléments les plus courants pour assurer la jonction entre les deux parties essentielles de la cuiller, le manche et le cuilleron.

La présence d'un décor dans le cuilleron, sans être fréquent, est bien attesté, parfois sous la forme de motifs en creux ou en léger relief. Ils sont souvent purement ornementaux (une coquille par exemple), mais parfois davantage figurés : une pelte et des feuillages sur une cuiller de Wiesbaden (H. Schoppa, Die romische Kaiserzeit, Wiesbaden, 1963, p. 28), un canthare sur un exemplaire du Rheinisches Landesmuseum de Bonn, de provenance inconnue, (inv. 1519), une double hache sur une des cuillers du petit trésor de Xanten (fig. 62), mise en rapport avec Sabazios (Gelsdorf, op. cit., fig. 6, p. 141-142), ou bien encore une panthère pour la cuiller de Mayence à manche en os mentionnée plus haut (fig. 64), deux gladiateurs dans le cuilleron en bourse d'une cuiller de la trouvaille d'Hagenbach (H. Bernhard et al., Der romische Schatzfund von Hagenbach, Mayence, 1990, p. 32, fig. 19). Faute d'une étude directe ou d'analyses, il est difficile de dire si certains éléments de ce décor en creux, qui occupent le plus souvent le fond d'un cuilleron de forme ovale, n'ont pas pu être niellés.

137 Les décors niellés sont les plus abondants, liés pour la plupart au groupe qui vient d'être étudié (celui des objets à manche en balustre complétés par un élément dans un autre matériau) ; on rencontre toutefois des exemples de cuillers de forme différente :

- une cuiller du Rheinisches Landesmuseum de Bonn, inv. 1520. Cuilleron en bourse, attache en arc de cercle, manche effilé d'une seule pièce. Décor niellé dans le cuilleron: à l'intérieur d'une bande de postes, candélabre végétal.

- Zülpich-Enzen, dans une tombe de la seconde moitié du IVe s. Cuilleron en bourse, attache en arc de cercle, manche effilé d'une seule pièce. Dans le cuilleron, à l'intérieur d'une ligne de triangles emboîtés, candélabre végétal très stylisé (Gallien in der Spätantike, Mayence, 1980, $n^{\circ}$ 178,1, p. 130).

- Zakrzow (Pologne). Cuilleron en bourse, attache en arcde-cercle. Manche d'une seule pièce, terminé en biseau. Dans le cuilleron, à l'intérieur d'une bande de postes, candélabre végétal simplifié (W. Grempler, Der Fund von Sackrau, Brandenburg an der Heide, 1887, pl. V, 2 ; K. Majewski, Importy rzymskie w Polsce, Cracovie, 1960, pl. 30).

La faveur dont jouissent ces décors niellés est à mettre bien évidemment en rapport avec celle qu'ils rencontrent au même moment en Gaule sur les plats et les coupes. La forme du cuilleron a imposé en règle générale un répertoire assez original. Si l'on excepte le canthare d'une cuiller de Cologne, il s'agit de variations sur des motifs végétaux : des rinceaux se développant sur toute la surface, ou bien une sorte de touffe ou de candélabre végétal encadré de pousses, un élément extrait des grands rinceaux couvrants que l'on rencontre aussi bien sur un grand plat niellé de Graincourt que sur deux grands plats de Rethel. Exceptionnelle est l'utilisation à Vaise du svastika, un motif souvent représenté sur les plats et les coupes, et que l'on retrouve sur une autre cuiller (au Rheinisches Landesmuseum de Bonn), mais de manière très simplifiée : dans un cuilleron circulaire, une série de perforations (il s'agit donc plus d'une petite passoire que d'une véritable cuiller) dessinent ce signe. Pour l'avoir étudié ailleurs en détail (F. Baratte, La vaisselle d'argent en Gaule dans l'Antiquité tardive, Paris, 1992, p. 96-111), nous n'y reviendrons pas ici, en rappelant toutefois les occurrences et en soulignant qu'il possède sur la cuiller de Vaise un caractère particulier : l'extrémité des bras est garnie de petits appendices en forme de flèches, que nous ne connaissons pas sur d'autres pièces d'argenterie, mais qui évoquent des motifs de broderie. 
Svastika à extrémités pleines :

- Chatuzange, plat circulaire (Trésors d'orfêvrerie, $\mathrm{n}^{\circ}$ 194) (fig. 65)

- Wettingen, plat circulaire (Chr. Simonett, Der römische Silberschatz aus Wettingen, Zeitschrift fur Schweizerische Archdologie und Kunstgeschichte, 1946, p. 13-14, n 2, pl. 2,4). Svastika à extrémités échancrées :

- Chaourse, plat circulaire (Trésors d'orfêvrerie, $\mathrm{n}^{\circ} 78$ )

- Chatuzange, coupe (ibid., $\mathrm{n}^{\circ} 194$ )

- Graincourt, coupe (ibid., $\mathrm{n}^{\circ}$ 92)

- Neupotz, plat circulaire (E. Kiinzl, Der Alamannenbeute aus dem Rhein bei Neupotz, Mayence, 1993, 2, Diam. 17, p. 23-24, pl. 32).

- Reims, 4 petits plateaux circulaires (Trésors d'orfêvrerie $n^{\circ}$ 98) (fig. 66).

Svastika à extrémités échancrées et pendentifs en flèche :

- Lyon-Vaise, cuiller $\mathrm{n}^{\circ} 17$.

65- Chatuzange, plat niellé.

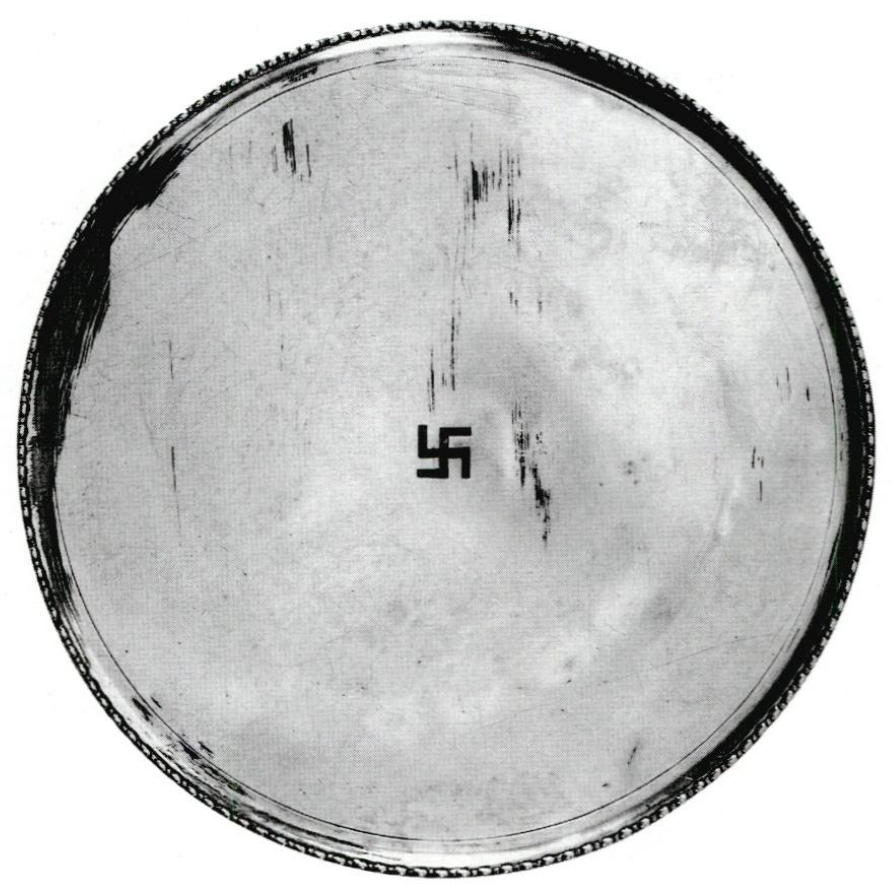

LONDRES, BRITISH MUSEUM. COURTESY TRUSTEES OF THE BRITISH MUSEUM 


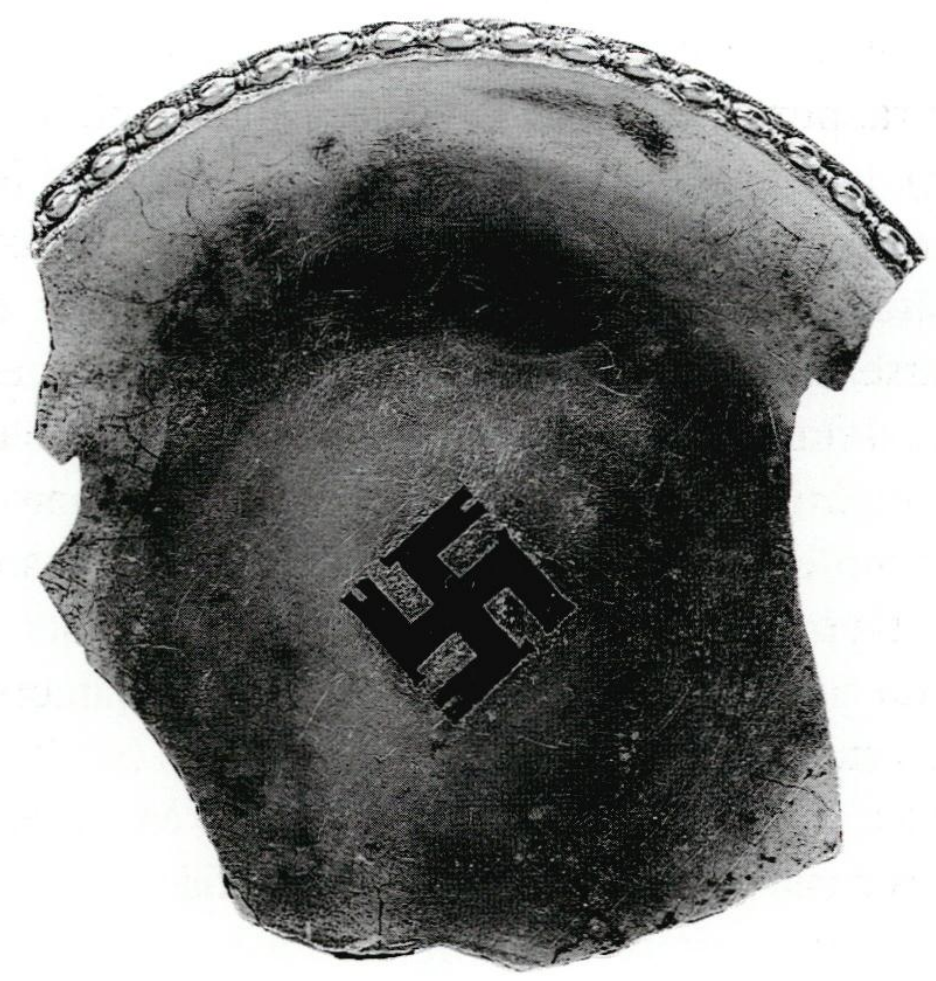

Coll. privée

139 En revanche la bordure de triangles emboîtés les uns dans les autres, bien attestée comme les postes aussi bien pour encadrer des médaillons que pour garnir des manches de petits ustensiles (dont des cuillers), est fréquent, on l'a vu, autour des cuillerons.

Les exemplaires précisément datés de ces cuillers sont rares : ce sont les alentours de 260 que l'on peut attribuer comme date d'enfouissement aux cuillers de Xanten et de Niederbieber, sur la foi des monnaies qui accompagnaient les deux dépôts; celle de Cologne-Bickendorf provient d'une tombe des années 280. La tombe de Zülpich est près d'un siècle plus tardive ; mais la cuiller niellée devait faire figure d'antiquité dans le mobilier funéraire. En effet ce n'est pas le seul exemple de cuillers du IIIe s. dans des ensembles du IVe s., la récente trouvaille de Saint-Ouen-du-Breuil (Seine-Maritime), encore inédite, datée précisément du milieu du IVe s. par des monnaies, contenait un petit lot de cuillers tout à fait analogues aux cochlearia de Vaise $n^{\circ} 4,5$ et 8 .

141 Il ne fait guère de doute que les cuillerons en bourse sont essentiellement une production du IIIe s. et que, sauf exceptions comme celles qui viennent d'être signalées, elles passent ensuite de mode au début du IVe s. C'est la date (le IIIe s) que l'on assignera à la plupart des cuillers à manche en balustre, qui s'accorde bien avec la période pendant laquelle sont en faveur les décors niellés du type de ceux utilisés.

Quant à leur origine, le seul indice précis est fourni par l'analyse de la carte de répartition: Cologne a livré à elle seule plus de la moitié des exemplaires (même compte tenu des incertitudes sur un certain nombre de provenances); la plupart des autres se regroupent dans la vallée du Rhin : c'est là qu'on cherchera les ateliers qui ont produit ces objets assez particuliers. 
Plusieurs éléments entrent en ligne de compte lorsqu'il s'agit de caractériser des cuillers : la forme du cuilleron, celle du manche (un élément parfois peu discriminant), la position du cuilleron par rapport au manche (dans le même plan ou non), la forme de l'élément qui éventuellement les relie l'un à l'autre. Les dimensions et le poids ont également leur importance. Si les cuillerons en bourse appartiennent avant tout au IIIe s., il est plus difficile d'en dater précisément les variantes (talon étroit, sans étranglement ni élargissement à l'arrière) dans la mesure même où ce n'est pas le seul type existant au même moment.

144 La présence d'un élément plat plus ou moins épais sur certains des cochlearia de Vaise ( $n^{\circ} 10$ et 11$)$ les rattache plutôt à des séries relativement précoces dans le IIIe s., en les rapprochant de certaines cuillers du Ier s. (les développements du IIe s. sont mal connus, faute de trésors suffisamment datés). Une des ligulae de Xanten dotée d'un tel élément a été récemment datée d'avant 235 , mais sur des raisons iconographiques (présence d'une double hache rapportée à Jupiter Dolichenus dont le culte décline brutalement en Germanie après cette date) dont on peut discuter (Gelsdorf, op. cit., p. 141-142).

145 La plupart des autres cochlearia de Vaise sont caractérisées par un manche effilé et une attache en arc de cercle relativement peu développée (notamment $n^{\circ} 4$ ), parfois assez écrasé ( $n^{\circ} 8$ et 9), mais toujours ouvert, qui se situe plutôt au début du processus d'évolution qui conduit aux cuillers de la fin de l'Antiquité où le raccord entre cuilleron et manche a une grande importance.

146 Quant aux volutes déjà signalées sur deux des cuillers à manche en balustre, elles connaîtront un grand succès au IVe $s$. notamment : ainsi un lot de cuillers du trésor de Kaiseraugst les utilise-t-il. Mais leur évolution a déjà été bien étudiée.

147 La trouvaille de Vaise compte deux ligulae. La première $\left(\mathrm{n}^{\circ} 14\right)$ ne se distingue des cochlearia analogues que par un manche un peu plus épais s'élargissant vers l'arrière et se terminant par un petit bouton, comme on en rencontre sur la cuiller d'Hagenbach déjà citée ou sur une autre de Brestovic en Serbie (au cuilleron en bourse, mais très arrondi, comme sur le cochlear $n^{\circ} 7$ de Vaise : Antique silver from Serbia, op. cit., $\mathrm{n}^{\circ} 213, \mathrm{p}$. 295).

148 Des boutons de ce type se rencontrent plus tard encore, au VIe s. notamment, sur une cuiller de provenance inconnue à Londres par exemple, ou bien à Lambousa (Hauser 1992, pl. 40-41). La seconde ligula a un manche plus complexe. S'il est bien rattaché au cuilleron par un élément en arc de cercle, il offre à l'avant une partie de section rectangulaire puis, au-delà d'un petit balustre, une tige carrée à pans coupés terminée par un double balustre. Le trésor de Rudnik en Serbie a livré deux ligulae qui s'achèvent de manière analogue, l'une avec un seul balustre, l'autre - au manche guilloché - avec deux (Antique silver from Serbia, $\mathrm{n}^{\circ} 209$ et 210, p. 293-294) : une particularité dont l'écho se conserve encore au VIe et au VIIe s. sur des cuillers bien différentes pourtant par leur allure, à Crema (Hauser 1992, pl. 4b), Krefeld-Gellep (Id., pl. 4a) ou Antioche (Id., pl. 31a-b), ou bien encore, mais un peu adaptées, avec un manche beaucoup plus travaillé, à Lambousa (Chypre) (Id., pl. 44-47), Lampsaque (Id., pl. 23-29) et Sutton Hoo (Id., pl. $17 b)$, parmi de très nombreux exemples. La mode est manifestement de nouveau à ces extrémités terminées par un bouton plus ou moins complexe.

149 Il n'en demeure pas moins que les quatorze cuillers de Vaise appartiennent toutes, sans ambiguïté, aux séries du IIIe s. 


\section{Conclusion}

150 Il est difficile de dire à quoi correspond exactement le lot d'argenterie de Vaise : deux petits plateaux, une coupe basse, quatorze cuillers et deux autres ustensiles dont sans doute une passoire et peut-être une fourchette : de la vaisselle de table ? Sans doute à l'origine, mais l'ensemble paraît un peu hétéroclite. La présence de Mercure sur la coupe et l'existence de statuettes de divinités en argent pourrait faire songer à des objets votifs. Rien ne s'y oppose, mais aucun élément non plus ne confirme l'hypothèse. Réunion fortuite, dans une cachette, du contenu d'un laraire et de quelques pièces de vaisselle de table? C'est aussi une possibilité. Rien ne permet cependant de trancher entre les différentes solutions envisageables. 


\title{
La statuaire du trésor de Vaise
}

\author{
François Baratte
}

\section{Buste masculin}

$1 \quad$ Inv. 93110425

\section{Argent.}

$H .17 \mathrm{~cm}$; h. de la tête $8,6 \mathrm{~cm}$; large, de la tête $7,6 \mathrm{~cm}$; épaisseur de la tête $7,2 \mathrm{~cm}$.

$2 \mathrm{Au}$ moment de la découverte la tête était recouverte d'une épaisse couche de concrétions. On observait un manque à la pointe du menton et dans l'oreille droite, et un petit enfoncement sous la pommette gauche et au sommet du crâne. Une large pièce comprenant une partie de la cuirasse et du paludamentum et couvrant l'épaule gauche était détachée, ainsi que plusieurs petits fragments de manteau.

Aujourd'hui, après restauration ( $c f$. encart supra), le buste est en assez bon état (fig. 67). Les manques ont été bouchés et les parties détachées remises en place. La surface a été nettoyée. Le rebord du vêtement est cassé et déchiqueté. Un grand morceau du paludamentum manque du côté droit du buste. Plusieurs fissures affectent le métal, très fragile. 


$$
12
$$


67d- Buste en argent

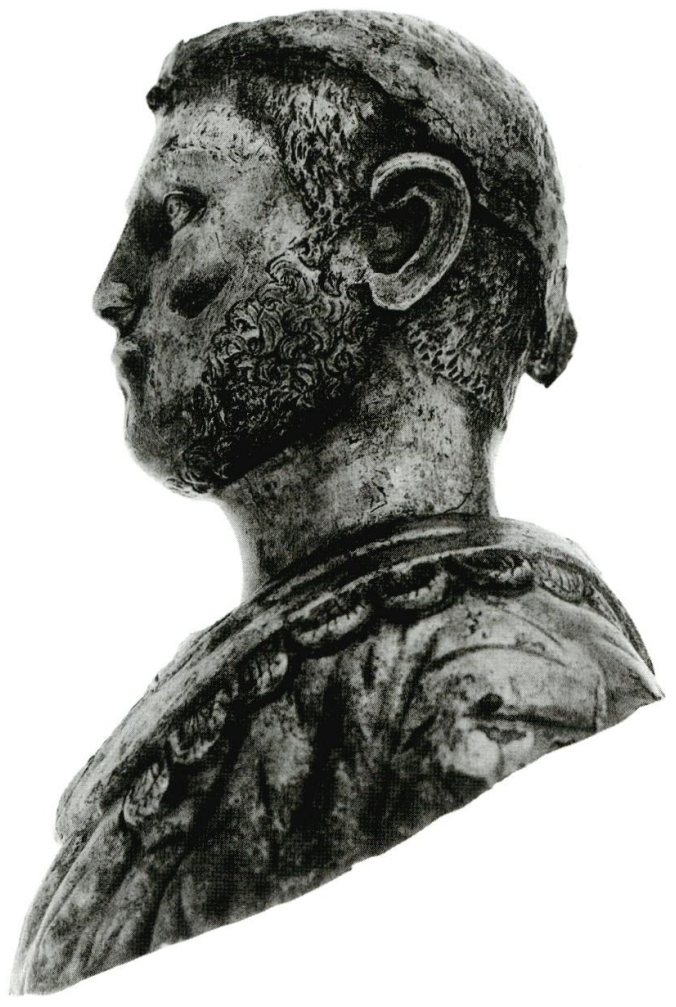

4 Le buste est celui d'un personnage semble-t-il plutôt jeune, portant cuirasse et paludamentum. La tête, posée sur un cou massif et cylindrique, est de face; les volumes et les traits sont fortement géométrisés. À peine plus haute que large, elle s'inscrit dans un cube. Le visage, très régulier, est triangulaire, mais ses contours sont faits de courbes molles et continues. Le modelé est presque inexistant; on tiendra compte cependant du fait que les photographies existantes écrasent plutôt les reliefs. Le front lisse est à peine marqué par deux petites rides horizontales. Les arcades sourcilières sont particulièrement discrètes, très faiblement arquées ; quelques coups de burin, très espacés, indiquent les sourcils. L'arête du nez droit et mince est dans le prolongement du front ; le nez lui-même s'élargit à la base que marque un profond sillon labio-nasal, qui se poursuit sous la lèvre inférieure. Les yeux sont réguliers, cernés par de minces paupières ; la pupille, ovale, est marquée par un creux bien net.

5 La bouche est plutôt petite, aux coins tombant; son dessin est très caractéristique. La lèvre inférieure, petite, est encadrée par un profond sillon qui s'élargit de chaque côté : une façon de faire qui n'est pas sans rappeler un autre objet du trésor de Vaise, la tête aux deux "cornes" (infra $\mathrm{n}^{\circ} 24$ ). Le menton est assez fort. Les oreilles, pour leur part, sont tout à fait schématiques, aux lobes en creux, en forme de haricot. Le personnage porte une barbe abondante, mais courte, bouclée, dont les mèches sont soigneusement dessinées et individualisées par des sillons au ciseau dans une masse en léger relief qui prolonge la chevelure. Continue, elle dégage cependant le menton, sous lequel elle passe. Une courte moustache rase, dessinée par des incisions, souligne la lèvre supérieure.

6 La chevelure est peu épaisse, formant une calotte tout autour de la tête, ainsi qu'une frange régulière au-dessus $\mathrm{du}$ front et sur la nuque. Elle est traitée par des 
scarifications sommaires au burin, contrastant avec le soin apporté à la réalisation de la barbe.

7 Une couronne, faite d'une mince feuille d'argent, est posée dans les cheveux, assez bas sur le front; les deux extrémités en sont repliées l'une sur l'autre. L'état est trop mauvais pour pouvoir préciser sa nature exacte ; sans doute s'agissait-il de feuillage, de laurier probablement, dont les feuilles, trop minces, ont été en grande partie cassées.

8 Le vêtement comprend un manteau militaire, le paludamentum, drapé sur le torse. Agrafé sur l'épaule droite par une grosse fibule circulaire faite d'un bouton central entouré d'un bourrelet torsadé, il couvre le buste de manière assez régulière, presque symétrique, un large pli formant comme une sorte de col tout autour. Une série d'éléments en languettes arrondies, huit à gauche, cinq à droite où il y a une cassure, sortent de sous le pli sur les épaules, constitués chacun d'un bourrelet torsadé entourant une partie centrale ornée d'incisions en palmettes. On penserait donc au décor des épaulières de la cuirasse portée sous le manteau ; mais elles auraient été alors mal comprises : on peut imaginer que l'artisan a eu sous les yeux un modèle sur lequel le manteau était jeté en travers de l'épaule, découvrant encore la cuirasse et ses ptéryges en dessous. Le disque en argent trouvé à Niederbieber, qui appartenait sans doute à une enseigne de la Cohors VII Raetorum, en offre un bel exemple (fig. 68), puisque c'est la tenue que porte l'empereur représenté vainqueur en fort relief au centre, Caligula a-t-on dit, ou Salonin (H. G. Horn, Cohors VII Raetorum equitata. Signumscheibe aus Nierderbieber, Das Rheinische Landesmuseum Bonn, 4/82, p. 82 sqq ; E. Künzl, Zwei silberne Tetrarchenporträts im RGZM und die römischen Kaiserbildnisse aus Gold und Silber, Jahrbuch des römisch-germanischen Zentralmuseums Mainz, 30, 1983, p. 385-386, pl. 73, 1); elle est très proche de celle du personnage de Vaise, et comprend une cuirasse rigide moulante par-dessus laquelle est passé le paludamentum. Le rapprochement des deux fait ressortir la confusion introduite par l'orfèvre du buste lyonnais. Ce dernier porte en outre une cuirasse souple à écailles, bordée par une large bande plus rigide, portée sur un tissu plus léger visible autour du cou. 


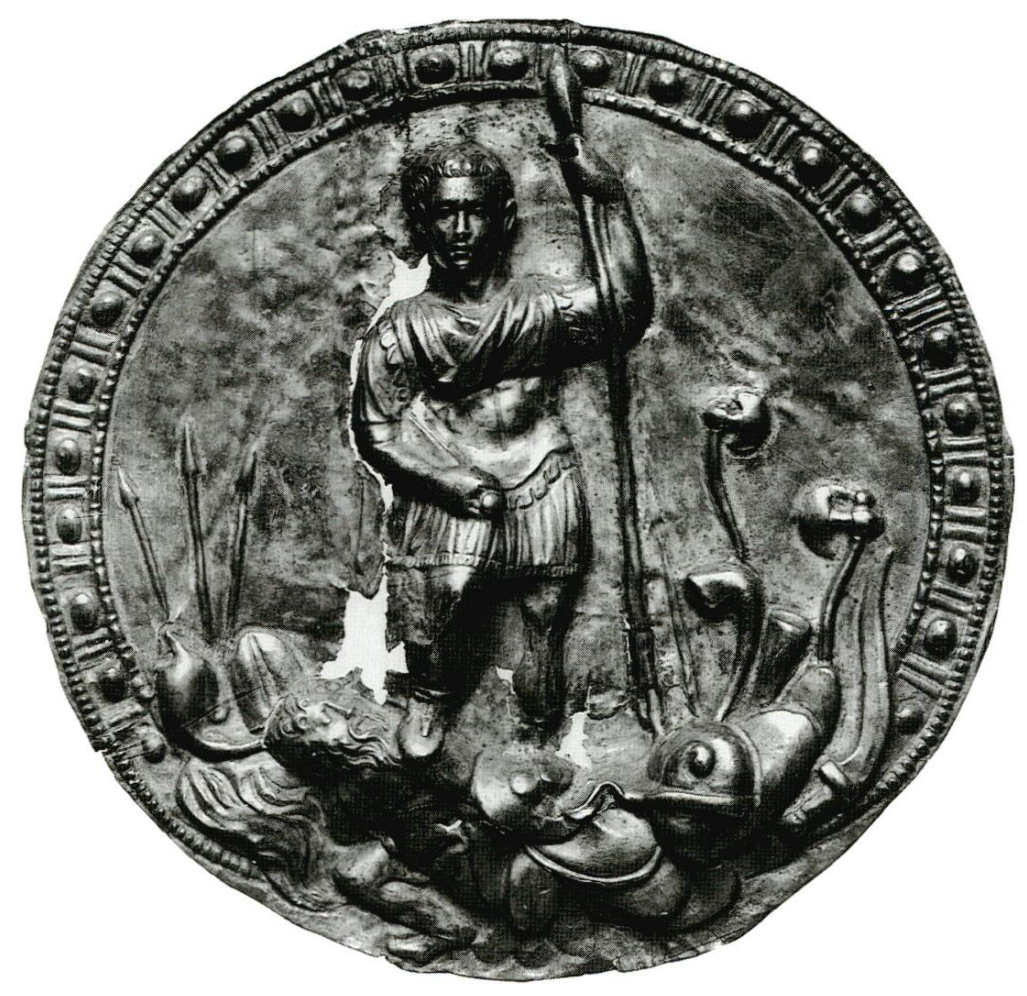

Bonn, Rheinisches Landesmuseum. Cl. du musée

9 La statuaire en argent était beaucoup plus nombreuse dans le monde romain qu'on ne l'imagine souvent. Seul le buste de Lucius Vérus du trésor de Marengo (G. Bendinelli, Il tesoro di argenteria di Marengo, Turin, 1937, p. 11-16; G. Sena Chiesa, dans L. Mercando [dir.], Archeologia in Piemonte, II, L'età romana, Turin, 1998, p. 359-368) conserve le souvenir matériel des statues et des bustes de grandeur naturelle qui ornaient les bâtiments et les places publiques. Mais les inscriptions et les textes sont relativement nombreux à les mentionner : on rappellera seulement le passage des Res Gestae (IV, 24) dans lequel Auguste se fait gloire d'avoir fait fondre les statues de métal précieux qui lui avaient été élevées à Rome - 120 environ selon ses dires, en pied, équestres, dans un quadrige - pour en faire des ex-voto en or en l'honneur d'Apollon (D'autres attestations ont été réunies par G. Lahusen, Schriftquellen zum romischen Bildnis I. Textstellen. Von den Anfängen bis zum 3. Jahrhundert n. Chr., Brême, 1984). En Gaule même, 4 statues sont ainsi mentionnées à Arles (CIL XII 197), Nîmes (CIL XII 3058), Vienne (CIL XII5864) et Narbonne (CIL XIII4316).

10 Cet honneur, lorsqu'il s'agissait de statues complètes, était évidemment coûteux en raison du poids d'argent qu'il réclamait, bien supérieur à celui d'un service de vaisselle de table de belle ampleur (on rappellera que le trésor de Boscoreale, enseveli en 79 ap. J.-C. par l'éruption du Vésuve, représente environ $25 \mathrm{~kg}$ d'argent, ce qui paraît insuffisant pour une statue de taille réelle, si mince soit le métal, sauf à utiliser la technique du placage) : on comprend donc qu'il soit attesté plus rarement pour des personnes privées.

11 L'existence de bustes, tout au moins, est avérée, sans qu'on puisse toutefois être assuré de leur taille : mentionnons, pour les empereurs, le groupe de bustes en argent signalé à Ostie dans l'inventaire d'un collège, quatre d'Antonin le Pieux, deux de Marc Aurèle et 
de Lucius Vérus, accompagnés, on le notera, de statuettes de la Victoire et de Fortuna (A. Degrassi, La dedica alla Fortuna Melior del Tesoro di Marengo, Athenaeum, XVII, 1939, p. 227-232) ou bien les cinq imagines impériales en argent qui apparaissent sur une dédicace offerte à l'époque tibérienne par un praefectus castrorum d'Egypte (C. Letta, La dinastia dei Cozi e la romanizzazione delle Alpi occidentali, Athenaeum, LXIV, 1976, p. 37-76). Parmi tous les textes qui mentionnent des œuvres de cette nature, un est particulièrement significatif parce qu'il fait connaître le testament de Dasumius, consul en 108, un personnage bien connu par la qualité de certains de ses légataires, Tacite, Pline le Jeune. Or, parmi tous les legs mentionnés, il est fait état de statues du défunt signa - d'or et d'argent, et de portraits - imagines - en argent. On peut discuter du sens exact des deux termes utilisés (encore que signum ne puisse guère être compris que comme "statue"; imago est moins précis), mais le sens général ne prête pas à confusion; ce testament (CIL VI 10229, 1. 74) est d'ailleurs confirmé par un passage du Digeste (XXXIV, 2, 6, 2) qui discute de dispositions analogues en plaçant les imagines argenteas, les portraits d'argent, sur le même plan que les portraits de marbre. L'exemple d'une grande statuette de Fortuna $(\mathrm{h} .22 \mathrm{~cm}$ ) découverte il y a quelque années à ClermontFerrand (Gallia Informations. Préhistoire et Histoire, 1989 - 1, p. 36-39, fig. 22) montre il est vrai qu'on pouvait pratiquer de substantielles économies en reprenant à échelle réduite la technique des sculptures acrolithes, c'est-à-dire en ne faisant pas exécuter en argent la totalité de l'œuvre: seule la tête, les bras et des éléments de draperie en effet sont en métal précieux. Mais nous ne connaissons pas d'exemple d'un tel procédé pour une statue de grandes dimensions.

Bien plus nombreux étaient les portraits de dimensions moyennes, comme celui de Vaise : les exemplaires conservés le montrent, qu'il s'agisse de portraits impériaux ou privés. Ces bustes en argent obtenus par martelage ont été étudiés à plusieurs reprises : en 1968 F. Braemer, examinant principalement sous l'aspect technique un groupe d'objets exécutés de cette manière, s'était intéressé en particulier aux quelques portraits découverts de part et d'autre des Alpes (Braemer 1968, p. 329344). Plus récemment, E. Kiinzl a repris entièrement cette question (Künzl 1983, p. 381-402), puis G. Sena Chiesa en republiant le buste de Marengo (op. cit., dans Archeologia in Piemonte). Le buste de Vaise, comme la plupart d'entre eux, pose deux problèmes principaux : celui de sa présentation, qui dépend en partie de sa fonction; celui de son identité. La technique d'exécution et sa qualité influent pour leur part sur les réponses que l'on peut apporter à ces interrogations.

Le caractère relativement médiocre de la facture, sensible tout particulièrement lorsqu'on examine la face, molle et schématique (le profil est un peu plus énergique et plus marqué) complique en effet singulièrement l'identification du personnage. L'âge notamment n'est pas évident: l'homme semble relativement jeune, mais ce pourrait n'être qu'une impression due à l'incapacité de l'orfèvre à rendre plus précisément son apparence : on rappellera à ce propos le passage bien connu d'une lettre de Fronton à Marc Aurèle, dans lequel il explique à son ancien disciple combien certes ses portraits, partout présentés, sont nombreux, mais combien aussi ils peuvent donner de lui une image déformée, peu ressemblante et pour tout dire épouvantable (ad M. Caes. 4, 12, 4, éd. C. H. Haines, Loeb Classical Library, I, 1919, p. 206 : « cum interim numquam tua imago tam dissimilis ad oculos meos in itinere accidit, ut non ex ore meo excusserit rictum osculei et somnum »). 
Plusieurs éléments le caractérisent: la présence d'une couronne tout d'abord, rapportée, très usée (les feuilles sont à l'heure actuelle à peu près indistinctes), mais qui paraît bien avoir été assez sommaire (elle est fixée par un simple pincement du bandeau à l'arrière) ; celle de la cuirasse et du paludamentum, si maladroits soient-ils, ensuite. La présence simultanée de la couronne et du costume militaire conforte l'idée du caractère impérial du personnage. Physiquement, celui-ci est caractérisé par la forme de sa tête, plutôt massive, dont on tiendra compte cependant avec une grande prudence, mais surtout par la présence d'une moustache très discrète et par le contraste très net entre la chevelure et la barbe. La première est traitée par grandes incisions, presque a penna sur une surface pratiquement sans relief : on la comparera à la coiffure du buste en argent de Velleia au musée de Parme (fig. 69), elle aussi a penna et dotée de sourcils qui évoquent, en plus réguliers, ceux de Vaise (le personnage est glabre, mais le traitement du portrait n'est pas sans analogie avec la tête de Vaise : F. d'Andria, Un bustino d'argento da Veleia, Rendiconti. Atti delia Pontificia Accademia Romana di Archeologia, 40, 1967/1968, p. 103 sqq, fig. 1-4 ; Braemer 1968, p. 335-337, fig. 10-12). Les mèches bouclées de la barbe sont pour leur part soigneusement individualisées: si elles sont traitées seulement au burin, c'est-à-dire plates, elles ressortent néanmoins sur une masse en relief. On observera que cette barbe couvre assez largement les joues et qu'elle passe sous le menton, mais qu'elle dégage complètement le cou: se trouvent ainsi exclus, en apparence au moins, plusieurs empereurs du IIIe s. sur les effigies monétaires desquels on remarque la même opposition entre une chevelure rase et une barbe à longues mèches. C'est la cas par exemple de Carin (283-285), sur ses monnaies (belle illustration sur un aureus de 284, J. P. C. Kent, B. Overbeck, A. U. Stylow, Die romischen Miinzen, Munich, 1973, 564v) ou sur le portrait du musée du Capitole qui lui a souvent été attribué (Kl. Fittschen, P. Zanker, Katalog der romischen Porträts in den Capitolinischen Museen, I, Mayence, 1985, n¹17, p. 141-142, pl. 145). Ce détail il est vrai peut ne pas apparaître sur toutes les représentations du même souverain, ce qui complique la réflexion : sur un médaillon d'or de Carus, son fils apparait avec une barbe arrêtée au ras du cou (M. Wegner, Das römische Herrscherbild. Gordianus III. bis Carinus, Berlin, 1979, pl. 58e). 

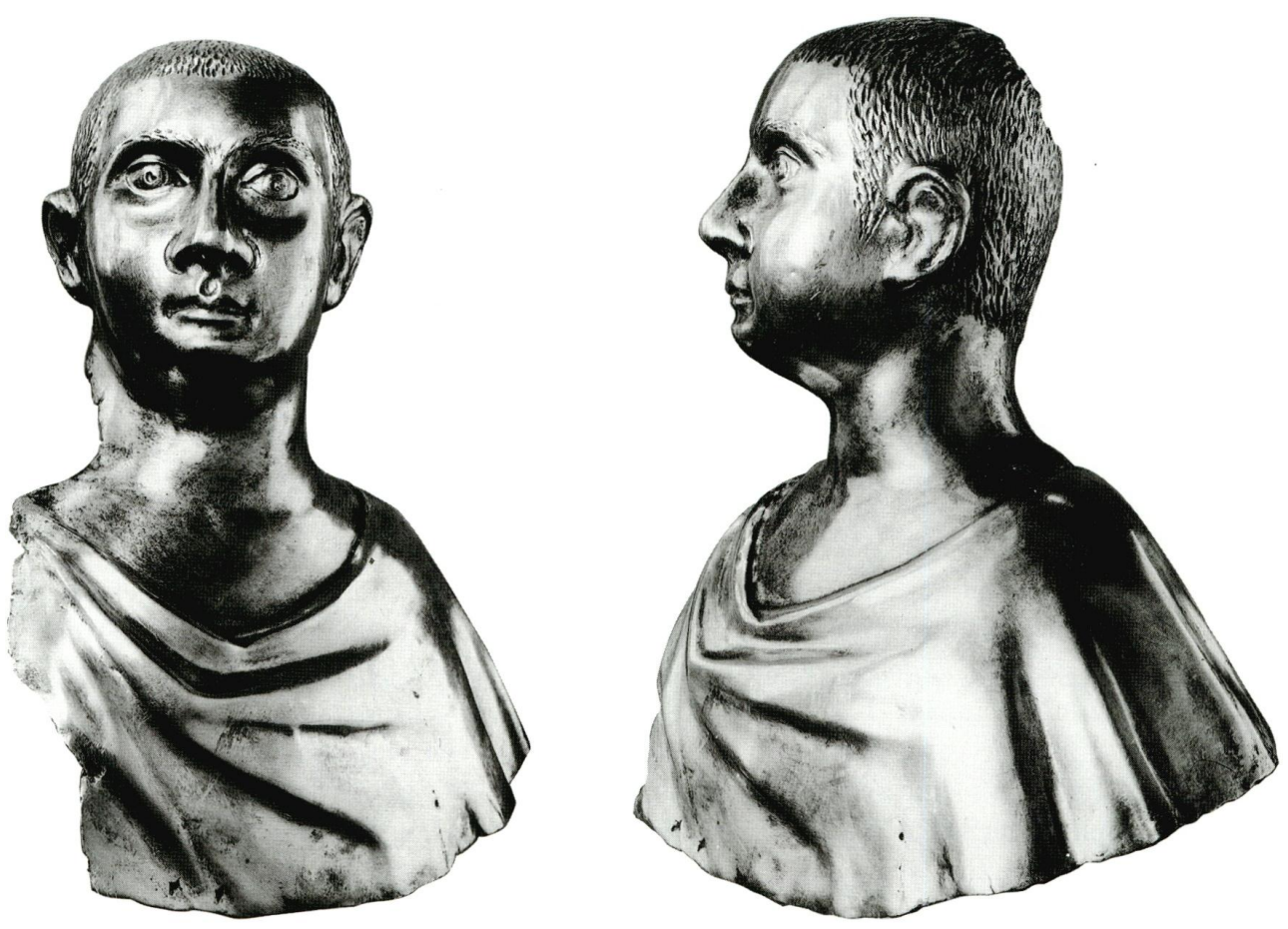

Une fois écartés les empereurs dont l'apparence ne peut s'accorder avec celle du buste en argent (absence de barbe ou de moustache, barbe ou chevelure non conformes à celles du portrait), il n'en reste guère qui puissent entrer en ligne de compte ; mais il est difficile de trancher entre eux, d'autant plus que la datation du buste ne s'impose pas, que la physionomie n'est manifestement pas d'une ressemblance très grande et que les portraits en ronde bosse clairement identifiés manquent souvent pour la période concernée. À première vue assez différents de l'image que donne le buste de Vaise, les deux fils de Septime Sévère, Caracalla et Géta, méritent d'être pris sérieusement en considération, le premier en particulier : le visage large correspond assez bien aux portraits du type "Tivoli» (d'après un buste trouvé dans la Villa d'Hadrien: H. B. Wiggers, Caracalla. Geta. Plautilla, Berlin, p. 87, pl. 11d); mais le contraste entre barbe et chevelure n'est alors jamais aussi marqué. Il va sans dire qu'à Lyon, où Caracalla est né, et dans le contexte du trésor dont les monnaies accordent une place particulière aux fils de Septime Sévère et notamment à celui-ci, la présence d'un de ses bustes serait particulièrement significative. Mais la part de hasard dans la localisation du trésor est trop importante pour que le lieu de sa découverte puisse constituer un argument solide. À considérer le format de la tête, enfin, Géta pourrait aussi convenir - en tout cas en prenant en considération ses portraits les plus tardifs ; mais il serait à tous égards moins satisfaisant que Caracalla.

Si la facture très lisse du visage, presque sans modelé, peut rappeler Sévère Alexandre, la barbe bouclée l'exclut, encore que certaines de ses monnaies ne soient pas loin d'en suggérer l'apparence. Il en va de même pour Volusien (Wegner 1979, pl. 36c ; la barbe paraît un peu raide). D'une manière générale, il semble que l'on doive préférer pour le buste de Vaise une datation après le milieu du siècle.

Peu d'empereurs portent alors une barbe bouclée et floconneuse. L'un offre des similitudes intéressantes avec le buste de Vaise : Gallien. La tête n'a, il est vrai, rien de très caractéristique. On observera tout de même que le profil, inscrit dans un rectangle 
très ramassé, au menton et au sommet du crâne arrondis, peut évoquer les portraits de jeunesse de l'empereur; quant à la face, très géométrique, elle n'est pas tellement dissemblable de celle du portrait bien connu du Musée des Thermes (bonne photographie dans R. Bianchi Bandinelli, Rome. La fin de l'art antique, Paris, 1970, fig. 24) : la structure ne s'oppose donc pas à une identification avec Gallien. La barbe floconneuse (c'est ce que l'orfèvre a voulu suggérer), les cheveux ras, a penna, et la moustache très discrète orientent vers le type I de Gallien : les deux bustes du Musée du Capitole (inv. 360) et du Palais Braschi (inv. 487) en offrent deux exemples intéressants sur lesquels la chevelure aux mèches à peine dégagées d'une masse peu épaisse pourrait avoir été rendue par l'orfèvre au moyen de quelques incisions, compte tenu du caractère schématique et, peut-être, provincial du buste en argent (ce dernier point doit être sérieusement pris en considération, d'autant plus que les erreurs dans le détail des vêtements, cuirasse et surtout paludamentum, soulignent l'inexpérience de l'artisan). On peut donc envisager, à titre d'hypothèse et avec prudence (car la barbe tend à déborder sur le cou), cette identification, d'autant plus que d'autres portraits contemporains, privés ceux-là, comme une belle tête de Detroit (M. Bergmann, Studien zum romischen Porträt des 3. Jahrhunderts n. Chr., Bonn, 1977, pl. 18, 3) montrent nettement l'opposition entre la coiffure traitée a penna et la barbe bouclée. Mais on ne perdra pas de vue que d'autres empereurs, plus tardifs, pourraient aussi avoir été représentés : le cas de Carin, déjà évoqué, mérite attention, la structure de la tête étant plus massive et donc plus proche du buste de Vaise. Mais peut-être faut-il, plus modestement, laisser ouverte l'identification, qui pose en tout cas la question de savoir de quel modèle l'orfèvre a pu disposer.

Le buste de Vaise, avec ses $17 \mathrm{~cm}$ de hauteur, appartient à la catégorie des portraits impériaux en ronde bosse de petites dimensions, dont on connaît des exemples aussi bien en métal précieux qu'en pierre dure (portrait de Trajan en calcédoine de l'Antikensammlung de Munich) ou en verre (Auguste et Constance II du RömischGermanisches Museum de Cologne : D. Harden, H. Hellenkemper, K. Painter, D. Whitehouse, Glass of the Caesars, Corning, Londres, Cologne, 1987, n 2, p. 21-22 et n ${ }^{\circ}$ 4, p. 24) qui tout en ayant le caractère d'objets précieux devaient être plus répandus qu'il n'y paraît aujourd'hui, avec des fonctions diverses. Aux bustes en métal, or ou argent, réunis il y a quinze ans par E. Künzl, on ne peut ajouter qu'un seul exemple, en argent, assez grossier comme travail (il était, il est vrai, très déformé au moment de la découverte). Il s'agit sans doute de Licinius: le petit buste appartenait au trésor de vaisselle découvert sur les bords de la Mer Noire, conservé à la Pràhistorische Staatssammlung de Munich (J. Garbsch, B. Overbeck, Spatantike zwischen Heidentum und Christentum, Munich, 1989, $\mathrm{n}^{\circ}$ S10, p. 58-64).

On rappellera ici, après d'autres, la brève liste :

en or :

- buste de Marc Aurèle (h. $33,5 \mathrm{~cm}$, poids $1590 \mathrm{~g}$ ), caché devant le temple du Cigognier à Avenches (Suisse) (Künzl 1983, p. 394, pl. 81, 1).

- buste de Septime Sévère (h. $25 \mathrm{~cm}$ ) en cuirasse et paludamentum, de Didymoteichon (Grèce) (ibid., p. 395, pl. 81, 2-3).

- à ces deux portraits on hésitera à ajouter le petit buste masculin $(\mathrm{h} .4,2 \mathrm{~cm}) \mathrm{du}$ trésor d'orfèvrerie de Monaco, aujourd'hui disparu, dont la facture est si sommaire qu'il est difficile d'y reconnaître avec certitude un empereur, et plus précisément encore un tétrarque (A. Héron de Villefosse, Trésor de Monaco. Notice sur les bijoux, dans Mémoires de la Société Nationale des Antiquaires de France, 1879, p. 203; Kiinzl 1983, pl. 81,4-5). 
en argent :

- buste de Galba, en cuirasse et paludamentum (h. $41 \mathrm{~cm}$ ), découvert à Herculanum, très déformé au moment de la trouvaille, plusieurs fois restauré, récemment encore (W. Geominy, Cl. Franchi, Die Silberbiiste des Kaisers Galba. Il busto argenteo del imperatore Galba, Bonn, 1995 ; plus succinct : À l'ombre du Vésuve. Paris, Musée du Petit Palais, 1995-1996, n 59, p. 172).

- buste de Lucius Vérus de Marengo (h. 55,3 cm), en cuirasse et paludamentum, déjà cité.

- buste de Gallien ( ?) (h. $17 \mathrm{~cm}$ ) de Lyon-Vaise.

- buste de Trébonien Galle (?) de Brigetio (h. 26,3 cm) (Е. B. Thomas [éd.], Archäologische Funde in Ungarn, Budapest, 1956, p. 246-247 ; Kiinzl 1983, p. 399, pl. 85, 2).

- buste de Magnence, en bronze argenté (h. 17,5 cm) en cuirasse et paludamentum, découvert dans la Saône à Chalonsur-Saône (Braemer 1968, p. 341-344, fig. 18-20).

- 2 bustes de tétrarques (11 et $12 \mathrm{~cm}$ ) au Rômisch Germanisches Zentralmuseum de Mayence, en cuirasse et paludamentum, provenant peut-être d'Asie Mineure (Kiinzl 1983, p. 381-393, Spatantike, op. cit., n 7-8, p. 69-70).

- buste de Licinius ( ?) à Munich, provenant des bords de la Mer Noire, déjà cité.

On voit, à la lecture de cette liste, que l'exemplaire de Vaise occupe avec le Magnence de Chalon une position intermédiaire entre les bustes de taille naturelle, ou presque, ceux de Galba et de Lucius Vérus, voire celui de Trébonien Galle déjà plus petit, et les effigies de petite taille, les trois tétrarques de Mayence et de Munich.

On considérera également que, du point de vue de la fonction, ils doivent être rapprochés à la fois des portraits impériaux en bronze de petit module, eux-mêmes parfois dorés, comme le bel exemplaire découvert en Pannonie (h. $12 \mathrm{~cm})$, attribué par R. Delbrueck à Valentinien II (autour de 388) et plus récemment par J. Meischner à Théodose II (vers 410-420), et des portraits privés en argent, de dimensions analogues et souvent de qualité remarquable, comme les portraits de Velleia, déjà cité, et de Vaison-la-Romaine (Braemer 1968, p. 337-339, fig. 13-16) (fig. 70). 
70- Vaison-la-Romaine, buste en argent.

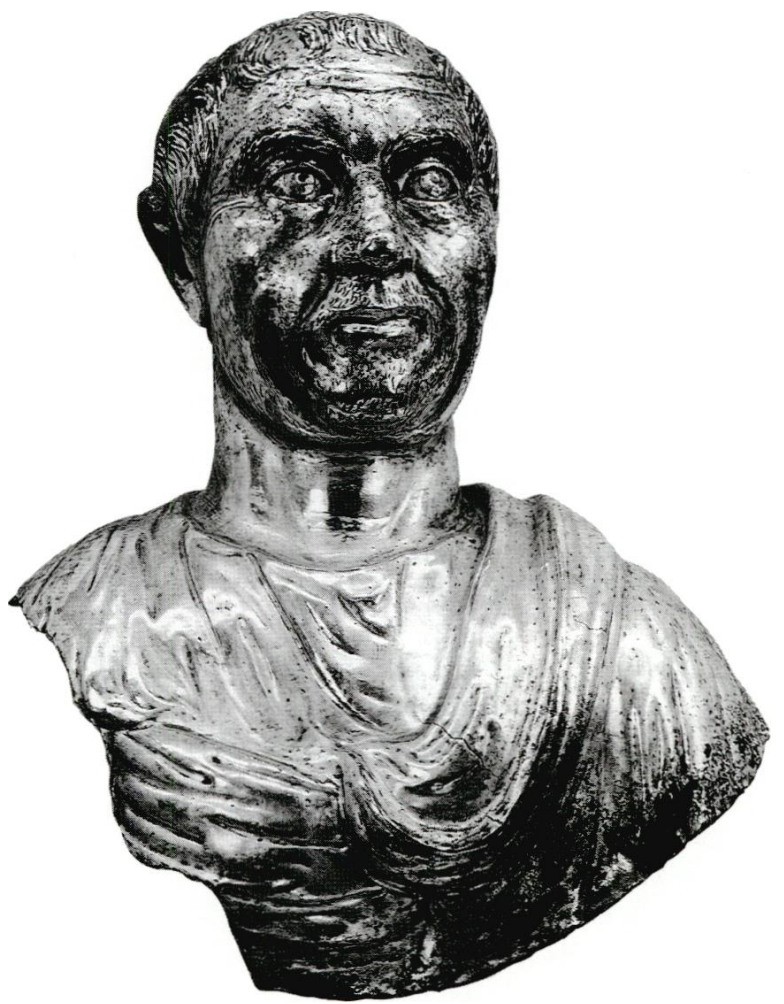

Vaison, musée archéologique. Cl. Chuzeville

21 En ce qui concerne les plus grands de ces bustes, impériaux mais aussi privés, il ne fait guère de doute qu'ils étaient l'équivalent pur et simple des portraits sculptés: ils appartenaient certainement à ces imagines argenteas item marmoreas, ces portraits d'argent et de marbre destinés à être placés, selon le Digeste (XXXIV, 2, 6, 2), (in) porticum publicam. Pour d'autres, les plus petits, ils pouvaient orner différentes pièces décoratives (sur ces emplois, voir tout particulièrement les remarques de E. Künzl 1983, p. 385-393), des phalères éventuellement, comme on le voit à Xanten (Kiinzl 1983 p. 386, $n^{\circ} 19$ ), ou des pièces de vaisselle, des coupes comme les nombreux exemples connus en argent, à Boscoreale (portraits masculin et féminin: Baratte 1986, p. 15-17) ou à Mzcheta en Géorgie (K. Matchabély, Les coupes d'argent d'Armaziskhévi, Géorgie, Tbilissi, 1970, pl. 10-14); c'était aussi la fonction, sans doute, du buste du Musée Thorvaldsen à Copenhague, un portrait privé plutôt qu'une effigie d'Antonin le Pieux auquel on a parfois songé (N. Hannestad, Thorvaldsen's Small Silver Head - A Ruined Tondo Portrait, Meddel. Thorvaldsens Mus., 1982, p. 27 sqq). Le buste en verre de Constance II, à Cologne, devait lui aussi occuper le centre d'une coupe destinée aux distributions impériales. Pour les bustes impériaux de dimensions analogues $(10 \mathrm{~cm}$ environ) on a pensé également à un couronnement d'enseigne militaire ou de sceptre.

Le cas le plus délicat est celui des bustes de dimensions intermédiaires comme celui de Vaise. Le profil de la partie arrière et l'ampleur de celle-ci (on la comparera au buste de Velleia) suggèrent davantage la fixation sur une surface plane que sur un support vertical (qui en ferait un portrait indépendant). Mais son inclinaison par rapport au buste lui-même paraît indiquer que la plaque qui le recevait n'était pas très inclinée : elle ne devait guère faire un angle de plus de $30^{\circ}$ avec l'horizontale pour préserver la verticalité de la tête. Il s'agissait donc d'une sorte de présentoir, un clipeus ou même 
une coupe de grand diamètre, qui ne pouvait lui-même être directement accroché au mur ou posé sur une table, mais qui était incliné. Une telle supposition n'a cependant rien de surprenant lorsqu'on observe que la coupe en argent du trésor de Boscoreale au fond de laquelle était fixée un portrait d'homme (un possesseur du trésor ou l'un de ses ancêtres) devait, pour offrir la meilleure vision du buste, être présentée de la sorte.

Le buste de Vaise constituait ainsi soit un don fait par l'empereur lui-même dans le cadre de ses largesses, soit un objet offert à la vénération dans un contexte impossible à préciser davantage, public ou bien privé (on songe aussi à des associations), puisque la possession et la présentation de bustes impériaux par des personnes privées sont clairement attestées par les textes antiques y compris dans les laraires ou dans des contextes funéraires (G. Lahusen, Untersuchungen zur Ehrenstatue in Rom. Literarische und epigraphische Zeugnisse, Rome, 1983, p. 37-38).

\section{Statuette D'apollon - Hélios}

\section{Dimensions et état de conservation}

Inv. 93110420

Argent doré.

H. de la statuette $27,5 \mathrm{~cm} ; h$. du visage $2,2 \mathrm{~cm}$. $h$. du socle $2,5 \mathrm{~cm}$; diam. du socle à sa partie supérieure: 10,2 cm ; diam. à sa partie inférieure $11,2 \mathrm{~cm}$. Poids $737 \mathrm{~g}$.

La statuette, la plus grande du trésor, était relativement mal conservée ; complète, elle était néanmoins détachée de son socle et en plusieurs morceaux, cassée par endroits ou tout au moins très fragilisée : le bras droit était détaché et en trois fragments, comme la jambe du même côté ; il manque un morceau à l'extérieur de la cuisse.

Le socle était cassé en plusieurs fragments qui ont été recollés. L'ensemble était assez corrodé (pour le processus et les étapes du nettoyage, voir supra et L'usure du temps, $1997, \mathrm{n}^{\circ} 15$, p. 34).

\section{Présentation matérielle}

\section{Description}

27 Statuette masculine debout (fig. 71). L'homme, jeune, est en appui sur sa jambe droite, solidement planté : un axe vertical descend de la tête au pied. La jambe gauche est nettement en retrait: le pied, soulevé, repose sur l'extrémité des orteils. Un léger hanchement est perceptible, complété par une torsion du buste vers la droite qui accompagne le mouvement de la tête, encore plus net. Le bras droit est levé ; la paume de la main est ouverte. La main gauche qui pend le long du corps tient une sphère. Le personnage est nu; il porte un manteau jeté sur l'épaule gauche, qui s'enroule autour du bras correspondant. Un pan de vêtement étroit, isolé, replié en zigzag, est enroulé autour du bras droit.

La tête, sans être levée, est fermement dirigée vers l'avant. Plutôt petite, elle est plantée sur un cou massif, haut et large ; le menton est presque inexistant, légèrement en retrait; le nez, long et large à la base, est dans le prolongement d'un front large, mais relativement peu dégagé. Le modelé du visage est mou et assez inexpressif. La bouche est petite et entrouverte. Les yeux globuleux sous des arcades sourcilières 
obliques ont leur pupille marquée d'un léger creux. Les oreilles sont invisibles sous les cheveux abondants qui encadrent le visage d'une ligne de boucles ondulées en arrière desquelles est posé un bandeau continu. Au-dessus de la tête, ils sont tirés et presque lisses, à peine incisés. Sous le diadème, le chevauchant, cinq trous relativement larges ; dans l'axe du corps, au-dessus du bandeau, un trou est encore garni d'un appendice métallique (fer ?) : le personnage portait une couronne radiée.

71a- Statuette d'Apollon-Hélios, hauteur 27,5 cm

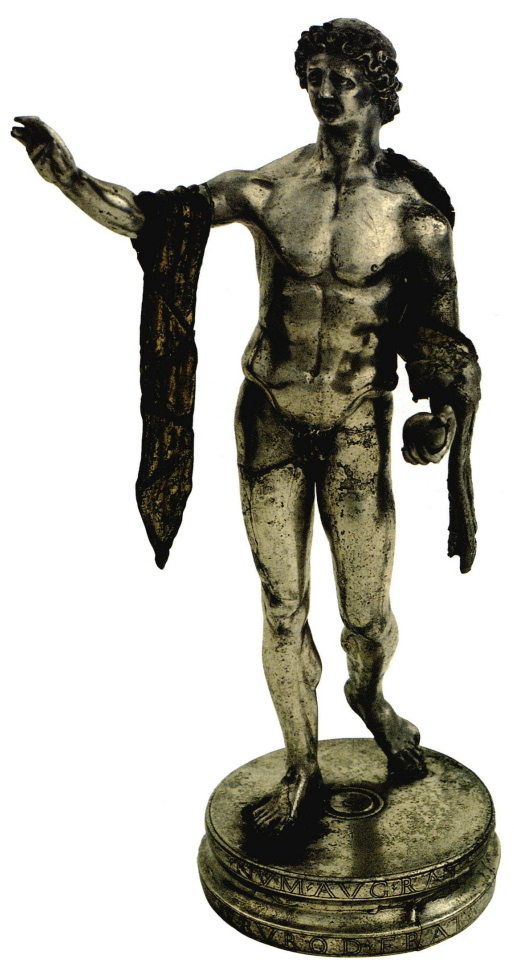


71b- Statuette d'Hélios

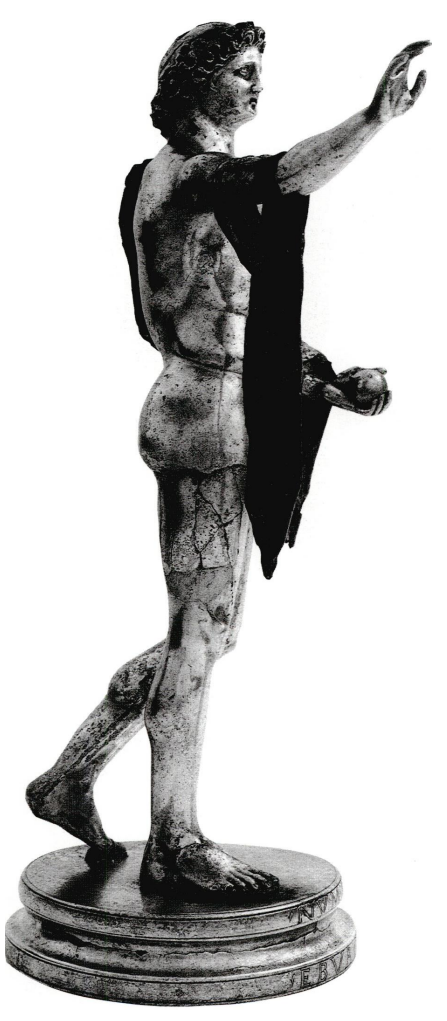

$71 c$ et d- Statuette d'Hélios

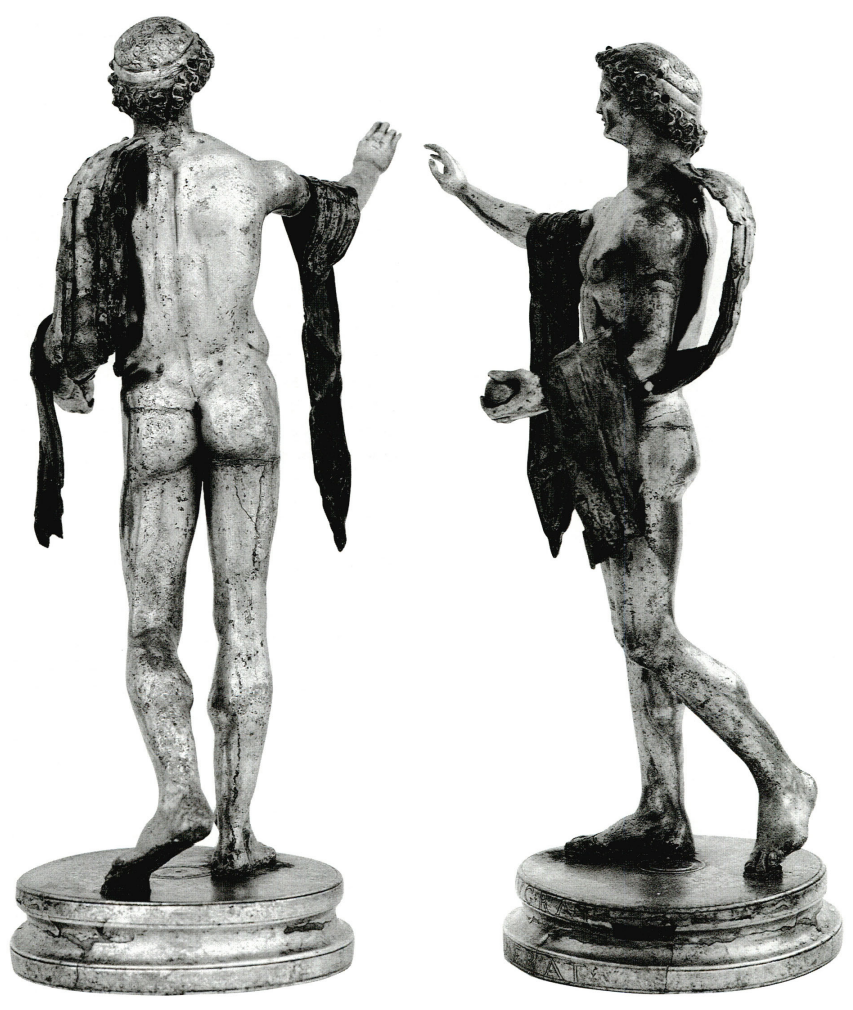




\section{Techniques de fabrication} était visible sur la surface, intert-être destiné à un tenon). Le circulaire (fig. 72) offre des traces de tournage : au centre, une marque de pointeau entourée d'un petit cercle (diam. $2,5 \mathrm{~cm}$ ) dessiné par une moulure en creux. Il présente un profil attique: deux moulures saillantes, ici verticales, séparées par une moulure concave. La moulure supérieure est en léger retrait par rapport à la moulure inférieure. Elles portent l'une et l'autre une inscription en lettres très soigneusement incisées, et dont les contours ont été redoublés.

\section{2- Statuette d'Hélios, profil du socle et relevé de l'inscription}

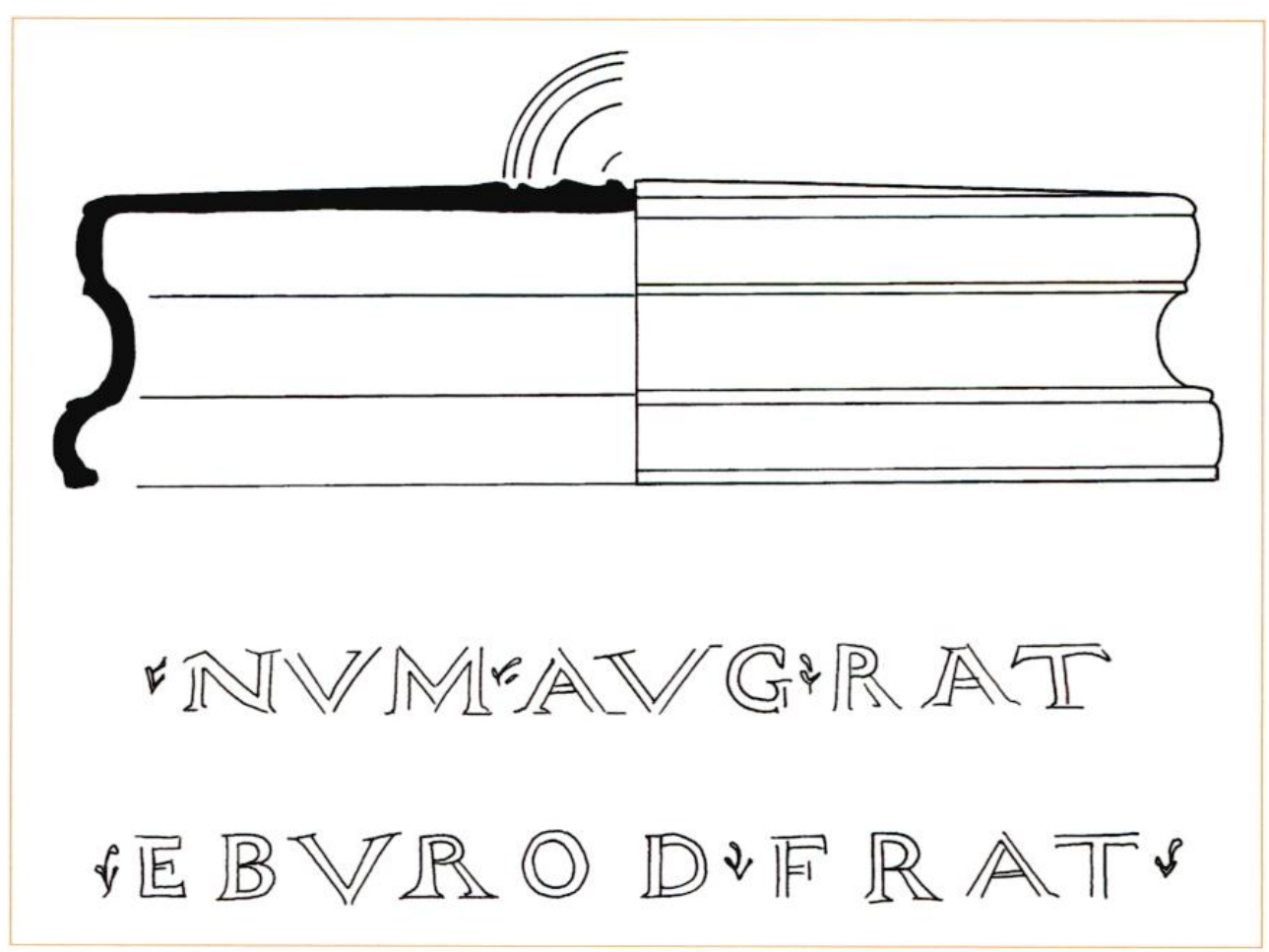

\section{L'inscription}

La hauteur des lettres est de 0,6 cm. Les différents mots sont séparés par un motif en flèche, une sorte d'hedera très stylisée.

NVM(ini) AVG(usti) RAT(iarii)

EBVROD(unenses) FRAT(res?) 
La restitution du texte (fig. 72) présente quelques difficultés, en particulier pour le dernier mot de chaque ligne ${ }^{6}$.

Il s'agit clairement d'une dédicace au numen d'un empereur qui n'est pas autrement désigné, faite par des personnes originaires d'Eburodunum, dont l'origine prête à discussion. Trois localités peuvent en effet prétendre à ce nom, Embrun dans les Hautes-Alpes, sur la Durance, Yverdon (Suisse, canton de Vaud) à l'extrémité sud-est du lac de Neuchâtel, ou bien encore Yvoire, en Haute-Savoie, sur le lac Léman. En ce qui concerne les deux derniers sites, la discussion, souvent vive, n'a pas été encore été tranchée entre les spécialistes; il s'agit en particulier d'identifier l'Ebrudunum Sapaudiae mentionné dans la Notitia Dignitatum. D. van Berchem, dans un article publié il y a un peu plus de 60 ans, a rappelé les arguments en faveur d'Yverdon, un nœud de communications particulièrement important où est stationnée, à la fin de l'Antiquité, une unité militaire, la flottille des barcarii (Ebrudunum - Yverdon, station d'une flottille militaire au Bas-Empire, Revue d'histoire suisse, 17, 1937, p. 83-95 [= Les routes et l'Histoire, Univ. de Lausanne, publications de la Faculté des Lettres XXV, Genève, 1982, p. 265 274], avec la bibliographie concernant Yverdon ; id., L'établissement des Burgondes en Sapaudia, dans Centre européen d'études burgondo-médianes, 14, 1972, p. 59-64 [= Les routes, en part. p. 280-281]).

Sans revenir sur cette discussion, qui ne peut pas être tranchée ici, on observera que la fin de la première ligne de l'inscription de Vaise peut aisément être développée en Rat(iarü) Eburod(unenses) : les dédicants de la statuette seraient ainsi les membres d'une corporation de nautes qui acheminaient les marchandises, notamment sur le trajet entre Lausanne et Yverdon. D. van Berchem a souligné le caractère essentiel de ce parcours qui correspond au passage entre les bassins du Rhin et du Rhône : les ratiarii assuraient donc au besoin le portage des marchandises, à la manière des ratiarii superiores mentionnés sur une inscription de Genève (CIL XII 2597). Ni Yvoire, qui n'a pas eu la même importance comme nœud de communications, ni Embrun, où la Durance n'a jamais été naviguable, n'ont pu jouer ce rôle.

7 Reste le dernier mot du texte : $\operatorname{FRAT}($.$) , le plus énigmatique. M. Christol a bien voulu$ nous suggérer une séduisante hypothèse, un développement en frat(res) qui associerait ainsi à Hélios une seconde statuette, celle de sa sœur Diane-Luna: le texte se serait donc prolongé sur une autre base. Un tel groupement, s'il n'est pas attesté par des œuvres conservées, n'aurait cependant rien d'étonnant.

L'inscription se traduirait donc ainsi :

“Au numen de l'empereur, les pilotes de barque d'Eburodunum (ont dédié ces statuettes) du frère et de la sœur [...]".

On connaît de nombreux cas de dédicaces de statuettes en métal à des divinités inscrites sur leur base: en argent, on signalera l'exemple encore inédit de la base fragmentaire découverte dans un fanum à Dompierre-sur-Authie (Somme), de forme analogue à celle de Vaise (mais les tores y sont bien arrondis), consacrée à Rosmerta ; les lettres sont alors niellées. Si les dédicaces au numen impérial sont banales dans l'épigraphie monumentale, elles sont beaucoup plus rares pour des documents de cette nature : manifestation intéressante de la diffusion du culte impérial, célébré ici par une association professionnelle, si le développement de l'inscription proposé est correct. 


\section{Analyse iconographique et datation}

En l'absence d'indications plus précises fournies par l'inscription qui ne nomme pas l'empereur auquel la statuette est dédiée, on ne peut déterminer le destinataire de cet hommage, ni apprécier s'il existait un rapport précis entre lui et la divinité représentée, Hélios (et peut-être Séléné). On rappellera seulement qu'à la fin du IIe s. et au IIIe s. plusieurs empereurs avaient voué un culte particulier au soleil : Aurélien évidemment, mais déjà auparavant Caracalla et Elagabal. Dans l'iconographie, ce n'est guère avant la fin du IIe s. que se répandent les images d'Hélios seul, hors de son quadrige (sur l'iconographie de Sol/Hélios, cf. LIMC, s.v. Sol [C. Letta] et Hélios); rares en sont les exemples antérieurs. Ils montrent en général le dieu, la main droite levée (sur une peinture pompéienne, elle tient le fouet), la gauche portant un sceptre ou un globe. À partir des Sévères, un type presque unique s'impose, celui qui est utilisé ici : la main droite est levée, la gauche tient divers attributs, globe ou fouet. C'est ainsi que se présente notamment un Sol en bronze aujourd'hui au Kestner Museum de Hanovre. La statuette, dédiée par M. Aurelius Sila(nus), peut être datée de la fin du IIe s. (fig. 73) : sa présentation est presque identique à celle de l'exemplaire de Vaise, le traitement apparaissant toutefois moins mou que celui de la version en argent. Elle constitue sans doute le plus proche parallèle à l'exemplaire de Vaise, à côté de quelques autres statuettes plus lourdes et maladroites dans leur attitude, souvent présentées la jambe droite en retrait, et portant la chlamyde en travers du torse (LIMC, s.v. Sol, $\mathrm{n}^{\circ} 114-117$ : statuettes de Boscoreale au Metropolitan Museum de New York, de Montdidier au Louvre (fig. 74), du Cabinet des Médailles de la Bibliothèque Nationale de France, de Sainte-Colombe à Genève et de Rhodes au British Museum). Un exemplaire inédit à New York, vente Sotheby's, 8 décembre 1995, nº 100. 
73- Statuette d'Hélios, bronze.

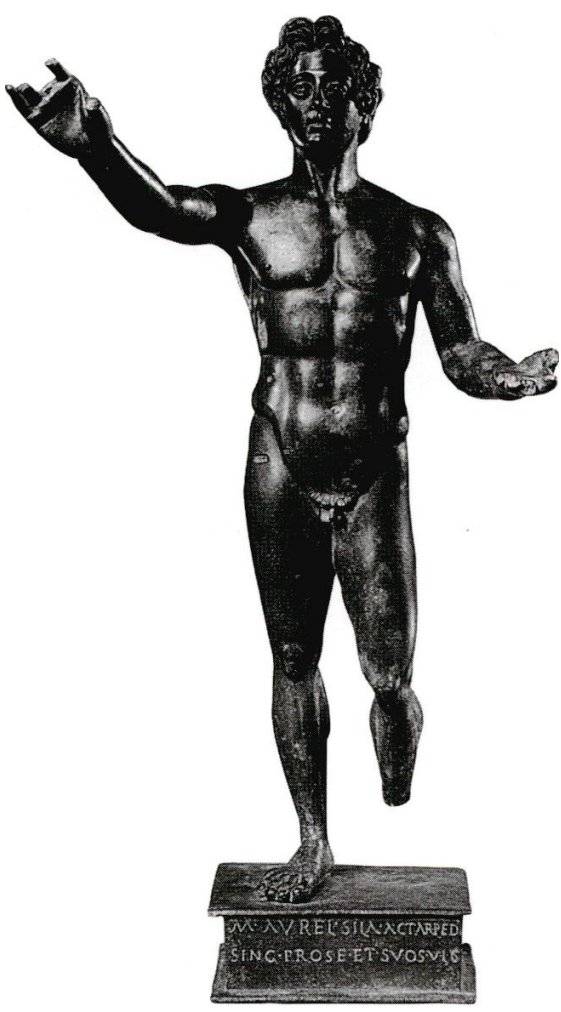

HANOVRE, KESTNER MUSEUM. CL. KUNSTWERKE DER ANTIKE.

74- Montdidier, statuette d'Hélios, bronze.

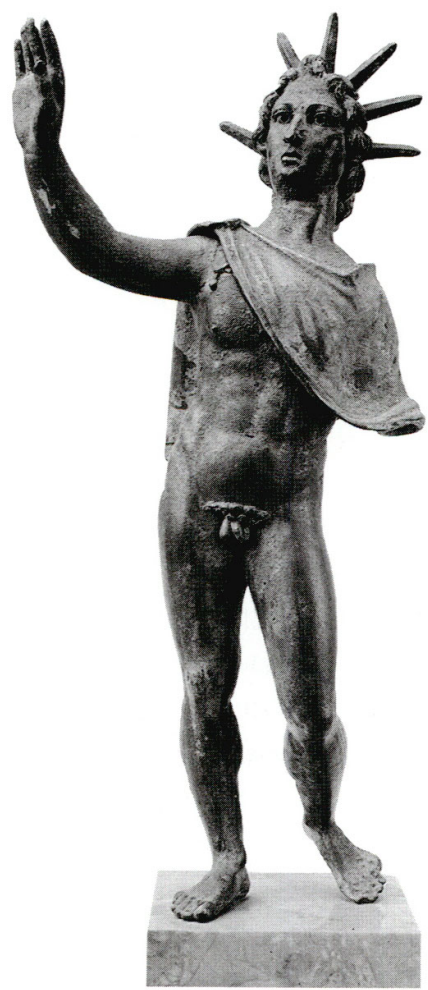

Paris, Musée du Louvre. Cl. Chuzeville 
En dehors des bizarreries du vêtement, quelques points caractérisent la statuette de Vaise. C'est d'abord l'ampleur du retrait du pied gauche, qui accentue le mouvement de marche en avant en lui donnant un caractère déterminé. Mais c'est aussi le déhanchement qui accompagne cette attitude, que souligne encore la torsion du corps vers la droite. On remarque également combien le bras gauche est abaissé : quel que soit l'objet que porte le dieu, une lance ou un globe, la main est en général plutôt tendue vers l'avant. Hélios est de proportions élancées, ce qui contraste avec l'allure parfois un peu tassée de plusieurs autres statuettes: celle offerte par M. Aurelius Silanus, mais plus encore, par exemple, celle passée en vente à New York en 1995. L'effigie de Vaise est isolée : s'il existe plusieurs autres statuettes en argent, elles sont pour la plupart féminines, et vêtues. On ne peut guère citer comme parallèle qu'un des deux Mercure du trésor de Berthouville, de plus haute taille et sans rapport véritable (Trésors d'orfêvrerie, $\mathrm{n}^{\circ}$ 27) (fig. 75). L'analyse stylistique en est d'autant plus délicate qu'il n'est pas aisé de comparer des œuvres réalisées dans des matériaux différents (l'argent et le bronze), donc suivant des techniques différentes. La mollesse du travail est néanmoins frappante à Vaise, en particulier pour le buste, et elle ressort davantage encore à la comparaison avec la statuette de Hanovre, comme la facture de la chevelure: assez proche dans son principe de celle de l'Hélios en bronze, elle ne possède pas le même aspect exubérant qui rapproche la seconde des portraits d'Alexandre.

75- Berthouville, statuette de Mercure.

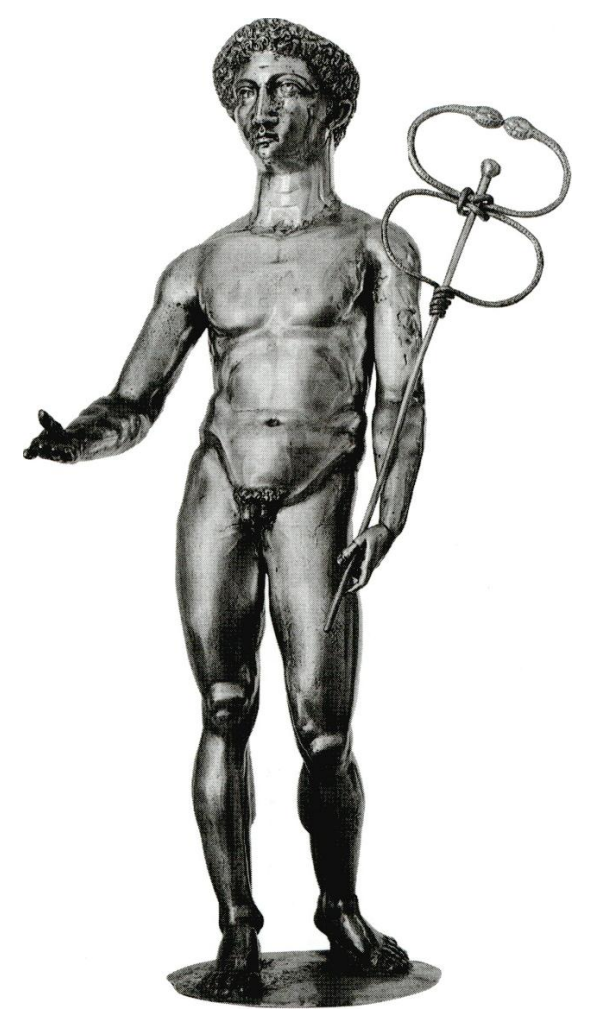




\section{Le socle}

Le socle de cette statuette, caractérisé par sa forme circulaire, ses proportions basses, une mouluration très sobre de type attique - une scotie entre deux tores - se rattache à une série relativement fournie, en bronze comme en argent : une statuette de Génie en bronze de Weissenburg (Kellner-Zahlhaas 1993, $n^{\circ}$ 13, p. 57) fournit ainsi un exemple que ses proportions mettent en marge du groupe ; il est en effet relativement haut pour un diamètre réduit, et chacune des trois moulures prend une épaisseur particulière. Plusieurs socles d'argent, en revanche, sont bien plus proches de celui de Vaise: la statuette de déesse de Saint-Honoré-les-Bains (Nièvre), proche par sa taille $(23 \mathrm{~cm})$ de l'Hélios de Vaise (fig. 81), n'en est guère éloignée (Braemer 1968, p. 346-348, fig. 2325), ni l'un des Mercure du trésor de Mâcon, pourtant plus petit (h. totale $14 \mathrm{~cm}$ ) (Trésors d'orfêvrerie, $\mathrm{n}^{\circ}$ 137) (fig. 76) ; une divinité féminine conservée au Louvre, provenant de Tortose en Syrie (A. de Ridder, Catalogue sommaire des bijoux antiques, Paris, 1924, $\mathrm{n}^{\circ}$ 2089), présente pour son socle la même succession de moulures, mais le retrait de la première est bien plus considérable que sur les deux précédentes (fig. 82). Le socle de la Vénus de Kaiseraugst est, sur ce point, intermédiaire (Cahn-Kaufmann 1984, p. 318). On ajoutera enfin à ces quelques exemples celui, fragmentaire, découvert à Dompierresur-Authie (cité supra). Mais on observera aussi que le socle de Vaise se distingue de tous ceux qui viennent d'être cités par le profil des deux tores, presque plats.

\section{6- Mâcon, statuette de Mercure.}

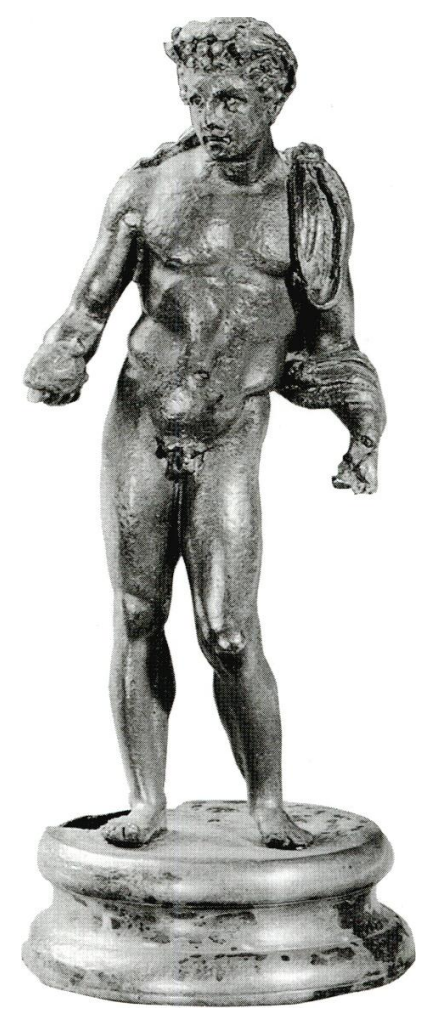

LONDRES, BRITISH MUSEUM. COURTESY TRUSTEEST OF THE BRITISH MUSEUM 


\section{Fortuna}

Inv. 93110421

Statuette féminine sur un socle hexagonal.

Argent doré.

H. totale $28,7 \mathrm{~cm}$.

\section{Dimensions, état de conservation et techniques de fabrication}

\section{Le socle}

Dimensions du socle : h. $8,8 \mathrm{~cm}$; dimensions de chacun des six panneaux : $3,3 \mathrm{~cm} \mathrm{X}$ $6,6 \mathrm{~cm}$; dimensions de la plaque de fond en alliage cuivreux : long. 10,8 cm, larg. 9,5 $\mathrm{cm}$; h. de la base $1,6 \mathrm{~cm}$, saillie $1,8 \mathrm{~cm}$. Hauteur de la partie supérieure $0,8 \mathrm{~cm}$, saillie $0,6 \mathrm{~cm}$.

Le socle a été retrouvé en plusieurs éléments dissociés qui ont été réassemblés (fig. 77).

\section{7- Statuette de Fortuna}

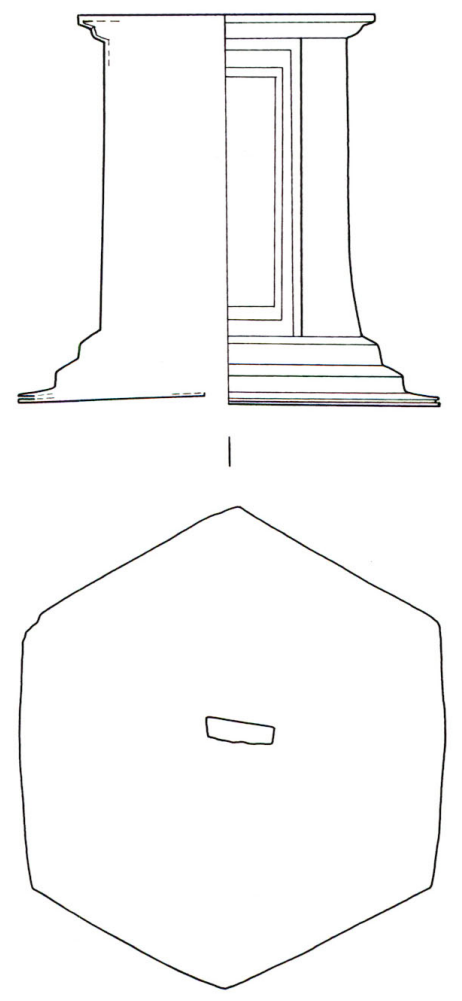

PROfIL DU SOCLE

En tôle d'argent, de section hexagonale, il repose sur une large base à trois degrés en forte saillie. La plaque formant le fond, percée approximativement en son centre d'une fente rectangulaire, était soudée. Chaque panneau est encadré d'une moulure en léger creux, dorée.

La partie supérieure est constituée d'une tôle d'argent en saillie plus faible, posée sur une moulure, repliée et sertie sur le socle. 


\section{La statuette}

Dimensions de la statuette : h. $19,7 \mathrm{~cm}$; long. du bras droit $4,6 \mathrm{~cm}$; h. de la tête (de

la pointe du menton au-dessus des cheveux) $2,3 \mathrm{~cm}$; larg. max. de la tête $1,9 \mathrm{~cm}$.

47 La statuette est exécutée en tôle d'argent travaillée au repoussé. Les deux bras sont pleins; ils étaient fixés par soudure et renforcés par deux tenons, suivant une technique fréquemment utilisée sur les statuettes en argent d'une certaine dimension (Fortuna de Chaourse, déesse de Toledo, déesse de Saint-Honoré-les-Bains).

48 Comme sur la statuette de la déesse aux oiseaux, une place importante est accordée ici à la dorure : outre les moulures des panneaux du socle, le diadème, la phiale, les fruits de la corne d'abondance et les feuillages de sa partie inférieure ainsi que les vêtements sont abondamment dorés au feu; on remarque ainsi une large bande en bordure du manteau, le gros pli en travers de la taille, l'encolure de la tunique et une bande dans son axe, du haut en bas. La statuette, détachée de son socle, était en état relativement bon au moment de la découverte, à l'exception d'une lacune dans le cou. Les bras, dessoudés, étaient restés en place. Très corrodé, l'objet a été nettoyé (sur la restauration, cf. supra).

\section{Description}

50 La jeune femme est debout de face en appui sur la jambe gauche (fig. 78) ; la droite est légèrement fléchie. Les pieds, chaussés de sandales, sortent à peine de sous la tunique. La déesse porte une tunique très longue, ample sur le buste (les manches couvrent les bras jusqu'au coude), plus serrée autour des jambes. L'encolure forme un pli autour du cou. Un manteau, passé sur l'épaule gauche, forme à sa partie supérieure un pan en écharpe dans le dos avant de retomber en larges plis ; à l'avant il constitue un large pli en travers de la taille, avant de se déployer comme un tablier en deux pans superposés et en sens opposé. Ce drapé compliqué n'a pas été rendu de manière parfaitement cohérente par l'orfèvre. 
78a- Statuette de Fortuna, hauteur $28,7 \mathrm{~cm}$

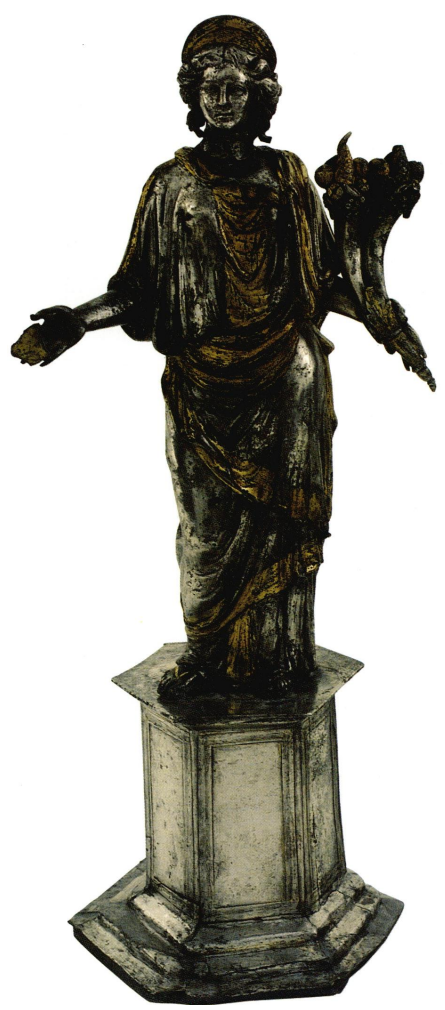

$78 \mathrm{~b}$ et c- Statuette de Fortuna
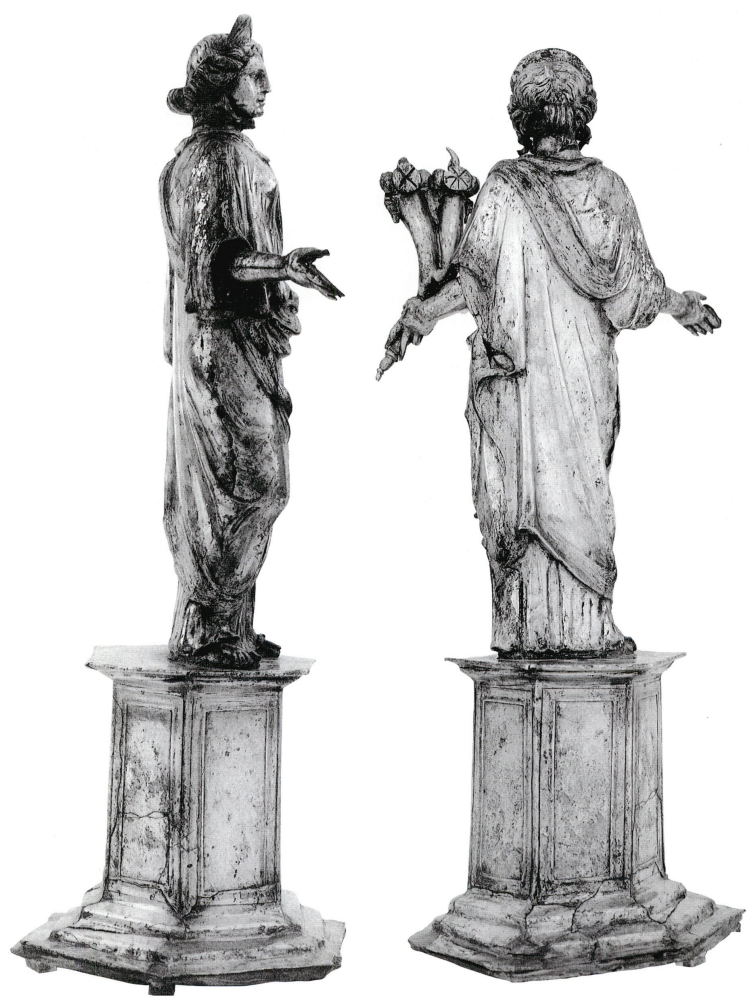

51 Le bras droit est tendu vers l'avant. La main tenait une phiale dont il ne reste plus que le fond. La main gauche, dans la même position, tenait une double corne d'abondance 
issue d'un culot d'acanthe et d'où sortent à profusion toutes sortes de fruits, pavots, raisins, pommes, pomme de pin, grenades, et de fleurs.

La tête est dirigée vers l'avant, légèrement tournée vers la droite. Elle porte un diadème. La chevelure abondante est coiffée en longues mèches ondulées, épaisses, tirées vers l'arrière; elles se rassemblent pour former des bandeaux assez lâches audessus des tempes et des oreilles qu'elles cachent, et un chignon sur la nuque. Des bandeaux s'échappe une sorte de ruban qui passe très souplement sous le menton. Le visage arrondi, presque carré, est plutôt massif ; le menton est large, le nez court ; les yeux sont profondément enfoncés dans les orbites. Un coup d'outil marque les pupilles. La bouche, petite, est entrouverte.

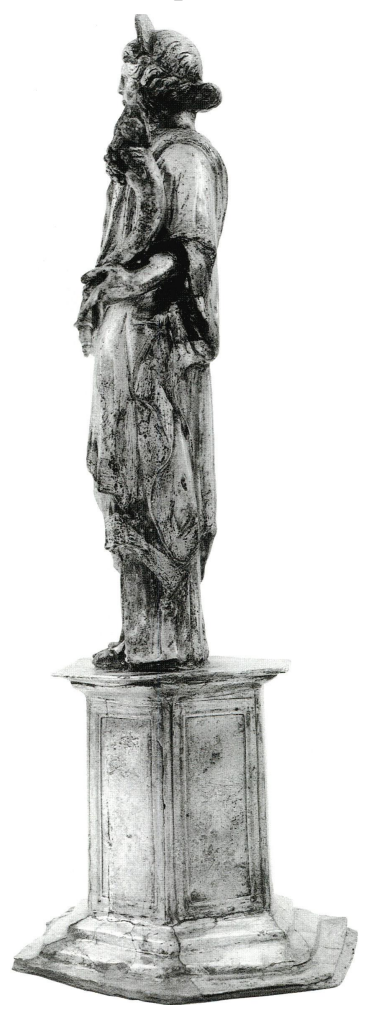

78d- Statuette de Fortuna

\section{Commentaire iconographique et stylistique}

\section{L'iconographie}

La phiale et la corne d'abondance désignent la déesse comme une Fortuna (sur l'iconographie de Tyché/Fortuna, cf. LIMC, VIII, 1997, s.v. Tyché [L. Villard], p. 115-125 ; ibid., s.v. Tyché/Fortuna [F. Rausa], p. 141). Si la phiale n'est pas l'attribut le plus fréquent (le personnage tient souvent un gouvernail), on en rencontre néanmoins d'autres exemples. La corne d'abondance est plus traditionnelle, mais elle prend ici une forme particulière puisqu'elle est double, une forme bien attestée ailleurs: on en trouve un exemple sur une statuette en argent de Regensburg en Bavière, qui représente Fortuna diadémée, et la main droite abaissée; la déesse tenait alors sans doute la ramegouvernail (LIMC VIII, s.v. Fortuna, $n^{\circ} 69$ ). Une feuille d'acanthe sert à dissimuler à Vaise 
la séparation de l'une et de l'autre cornes, peu esthétique sans doute puisque les deux ont une extrémité commune, soigneusement moulurée.

Ces cornes doubles existent en particulier dans l'iconographie hellénistique, dans laquelle elles sont parfois entrecroisées au lieu d'être jumelles, superposées en léger décalage ou séparées par un motif médian (sur leur signification et la faveur dont elles ont pu jouir, cf. en particulier H. Möbius, Alexandrin und Rom, Munich, 1964, p. 26-29). L'art ptolémaïque les a particulièrement appréciées : la double corne apparaît en tant que telle dans la grande procession organisée par Ptolémée Philadelphe à Alexandrie (Athénée, V, 202); mais surtout elle figure fréquemment sur les monnaies des Ptolémée, où elle prend la valeur d'un symbole religieux et dynastique; elle représente sans doute le couple royal divinisé (M. Nilsson, Bulletin de l'Académie de Danemark, 1909, p. 168-173). Les monnaies lagides et quelques autres monuments mettent en outre en évidence le lien qui peut exister entre cet emblème, Isis et Sarapis (J. Charbonneaux, Sarapis et Isis et la double corne d'abondance, dans Hommages à Waldemar Deonna, coll. Latomus, XXVIII, Bruxelles, 1957, p. 131-141). Sur certaines monnaies du souverain séleucide Démétrios I Sôter, c'est Tyché qui tient le rôle qu'ont en Egypte les deux autres divinités (E. Babelon, Catalogue des monnaies grecques de la Bibliothèque Nationale. Les rois de Syrie, Paris, 1890, p. 97-98, $\mathrm{n}^{\circ} 765$, pl. XVII, 1). On rencontre également la double corne, par exemple, entre les mains d'Arsinoé II sur une série d'œnochoés en faïence (D. Burr Thompson, Ptolemaic Oinochoai and Portraits in Faïence, Londres, 1973).

L'art romain a repris à son compte le motif de la double corne sous toutes ses formes, croisées, parallèles ou bien issues d'un unique culot, sans qu'il y ait, semble-t-il, de différence de signification. Marc Antoine, de façon logique, l'utilise sur des deniers frappés en Orient : c'est alors un caducée qui sépare les deux cornucopiae, comme sur certains autels (E. Sydenham, The coinage of the Roman Republic, Londres, 1952, $\mathrm{n}^{\circ} 1189$, p. 192). Dans le domaine politique, Auguste lui-même s'est saisi du symbole, dès avant 27 av. J.-C. : une pâte de verre du Kunsthistorisches Museum de Vienne le représente en buste au-dessus d'un globe entre deux cornes d'abondance entrecroisées, faisant ainsi pièce à son rival (C. Maderna-Lauter, "Glyptik ", dans Kaiser Augustus und die verlorene Republik, Berlin, 1988, p. 453 et $n^{\circ}$ 245). Ses successeurs, julio-claudiens notamment, reprendront volontiers le motif. Ainsi Tibère salue la naissance des deux jumeaux Tiberius Gemellus et Germanicus par une émission de sesterces sur laquelle un caducée est encadré par deux cornes d'où sortent la tête des deux enfants, un type qui sera repris quelques 125 ans plus tard par Antonin pour commémorer la naissance de deux de ses enfants (belles illustrations dans J. P. C. Kent, B. Overbeck, A. U. Stylow, Die römischen Miinzen, Munich, 1973, $\mathrm{n}^{\circ}$ 155, pl. 41 et $\mathrm{n}^{\circ}$ 318, pl. 81). De même Caligula, trônant sur un camée de Vienne aux côtés de Roma (H. Kyrieleis, dans Archaologischer Anzeiger, 1970, p. 493-498; W.-R. Megow, Kameen von Augustus bis Severus Alexander, Berlin, 1987, p. 185, n A 60, pl. 15, 3). On évoquera encore un autre célèbre camée du Kunsthistorisches Museum sur lequel les bustes de Claude, d'Agrippine la Jeune, de Germanicus et d'Agrippine l'Ancienne couronnent une paire de doubles cornes (Megow, op. cit., p. 200-201, $\mathrm{n}^{\circ}$ A 81, pl. 31).

56 J. Charbonneaux a rappelé la postérité du motif (op. cit., p. 141, n. 3) qui manifeste tantôt "la fécondité de l'union impériale" lorsque, comme sur les monnaies de Tibère et d'Antonin le Pieux, deux bustes d'enfant couronnent une double corne encadrant le buste de Messaline sur un camée du Cabinet des Médailles (Megow, op. cit., p. 303304, n D 39, pl. 18, 1; l'identification des personnages a été récemment discutée sans raison 
véritable : Livilla pour D. Kasper, Drusilla pour W.-R. Megow), tantôt l'harmonie du couple impérial. Sur des monnaies d'Alexandrie, depuis Trajan, on voit en effet Homonoia portant une double cornucopia (R. Stuart Poole, British Museum Coins. The Ptolemies, Londres, 1883, n 428, pl. VIII ; n 965, pl. X; n 1238, pl. X; n 1958, pl. X); dans le monnayage d'Hadrien et d'Antonin le Pieux la même figure prend le nom latin de Concordia (H. Mattingly, Coins of the Roman Empire in the British Museum, Londres, III, p. CL, pl. 64, 18 ; IV, p. LI et LVI, pl. 4,2 et 24, 6).

La faveur dont jouit la double corne ne se limite pas au monde politique; on la retrouve sur les autels funéraires : ainsi à Rome, Vatican, Galerie des Candélabres, $\mathrm{n}^{\circ} 2671$, au revers duquel deux cornes encadrent un culot d'acanthe qui supporte un clipeus orné d'un buste, symbole d'immortalité à travers l'idée de fécondité (D. Boschung, Antike Grabaltäre aus den Nekropolen Roms, Berne, 1987, n 958. Vers 125). Mais elle apparait plus encore dans la petite plastique en bronze : plusieurs exemples, d'Isis ou d'Isis Tyché, souvent difficilement datables, montrent les deux déesses la tenant et portant dans l'autre main parfois le sistre, plus souvent le gouvernail. La Syrie, entre autres, en a fourni quelques-uns, comme une statuette de la collection de Clercq trouvée à Tortose (A. De Ridder 1924, p. 209, n 313). Mais on en retrouve ailleurs. Le redoublement de la corne paraît alors simplement un moyen d'insister sur l'abondance et la fécondité : la double cornucopia apparaît donc entre les mains d'autres figures divines, comme les Genius (un exemple à Straubing: H. Kunckel, Der romische Genius, Heidelberg, 1974, F VIII, 3, pl. 67) ou les Lares (un bel exemplaire en bronze dans le trésor de Weissenburg: Kellner, Zahlhaas 1993, n 14, p. 58-60, pl. couleurs), probablement pour les mêmes raisons, et la Tutela Panthée du trésor de Mâcon (fïg. 79) (Trésors d'orfêvrerie, $\mathrm{n}^{\circ} 140$ ) ; dans ce dernier cas, la double corne est aussi un moyen commode de placer deux bustes divins, Diane et Apollon. 


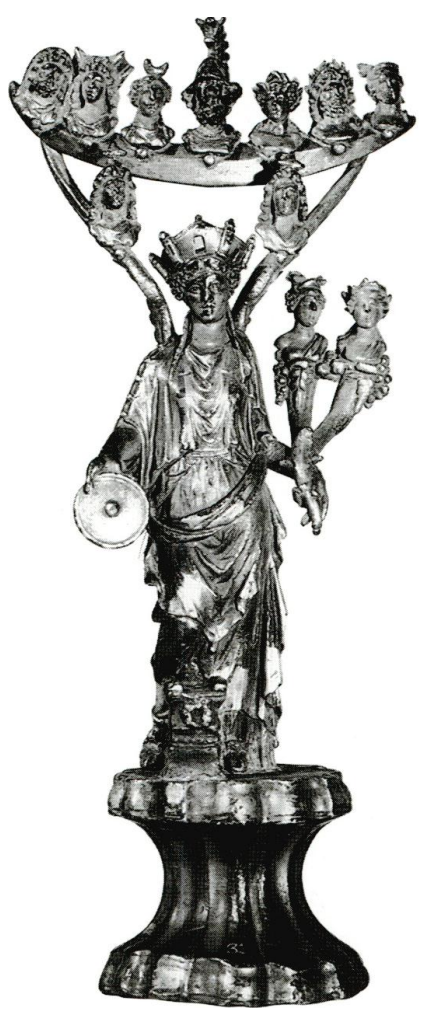

LONDRES BRITSIH MUSEUM. COURTESY TRUSTEES OF THE BRITISH MUSEUM

Il est donc difficile de préciser la nature de la déesse de Vaise, une Fortuna certes, mais avec quelques particularités. On notera ainsi la curieuse "mentonnière" dont nous n'avons pas trouvé d'équivalent, ainsi que le costume plus compliqué que d'ordinaire. Le drapé du manteau en particulier s'explique mal, ainsi que l'encolure légèrement repliée sur l'épaule droite. On observe aussi les deux groupes de plis lâches sur la tunique, tombant des épaules et des seins, de part et d'autre de la partie centrale plus plate; on s'interrogera aussi sur la raison d'être de la bande dorée médiane : simple élément décoratif laissé à l'interprétation de l'artisan, ou bien partie constitutive du vêtement? Le caractère anépigraphe du socle ne permet pas de préciser davantage.

Statuette votive, élément d'un laraire privé, la Fortuna de Vaise, comme la déesse aux oiseaux, pouvait faire partie purement et simplement d'un décor de table, comme la statuette de Chaourse, qui était sans doute accompagnée à l'origine dans le trésor d'une autre pièce, dont il ne subsiste aujourd'hui que le socle (Trésors d'orfêvrerie, $\mathrm{n}^{\circ} 84$ ), et d'un objet fonctionnel, mais figuré, le petit poivrier en forme de Noir (Id., $\mathrm{n}^{\circ} 81$ ).

\section{Analyse stylistique}

La facture est sensiblement différente de celle de la déesse aux oiseaux $\left(n^{\circ} 23\right)$ : les plis y sont moins prononcés, marqués certes, mais plus larges et plus plats; il suffira de comparer leur traitement sur le buste; drus et serrés d'un côté, ils sont moins nombreux, plus géométrisés sur l'autre (on observera par exemple les deux plis arrondis à leur base sur le côté gauche de la Tyché à la double corne). En revanche le traitement de la tête est plus fouillé : les mèches de la chevelure sont davantage 
creusées et les pupilles sont plus marquées. L'une et l'autre pourtant nous paraissent pouvoir être contemporaines : fin du IIe s. - première moitié du IIIe $s$.

61 On connaît une Fortuna en or $(\mathrm{h} .16,5 \mathrm{~cm})$, provenant d'Afrique du Nord, diadémée, tenant la corne d'abondance et la phiale ou le gouvernail, d'allure assez particulière (Kunstwerke der Antike. Auktion 34, Bâle, 6 mai 1967, p. 220). Une autre est en argent, un peu plus petite $(12,8 \mathrm{~cm})$, celle de Chaourse (A. Kaufmann-Heiniman, dans Trésors d'orfevrerie, $\mathrm{n}^{\circ}$ 83) (fig. 80), qui tenait aussi une corne d'abondance, et peut-être une phiale (le bras droit a disparu après la découverte) : l'attitude en est plus dynamique, le retrait du pied d'appui (le gauche) nettement plus marqué, la torsion du corps plus sensible. La physionomie est également moins figée. La statuette de Chaourse est manifestement plus proche des modèles classiques, même si elle présente des particularités dans son vêtement notamment (un chiton sans manteau). C'est d'ailleurs une certaine raideur qui caractérise la Fortune de Vaise. L'artisan a su avec une certaine compétence technique camper le personnage et rendre le détail du vêtement, dont les plis sont nombreux et plutôt adroits: s'ils apparaissent un peu moins plastiques que sur la déesse aux oiseaux, ils en sont en fait assez proches. Mais il est sans doute moins familier des modèles hellénisants, ce qui l'a conduit à des imprécisions ou même à des erreurs dans le costume. Il se distingue en cela non seulement de son confrère de Chaourse, mais aussi de l'auteur de la statuette de déesse du musée de Toledo, provenant du Liban (Oliver 1977, $\mathrm{n}^{\circ}$ 103). L'origine gauloise, proposée dans Trésors d'orfêvrerie, p. 136, nous paraît bien hypothétique en raison de la provenance de la pièce.

80- Chaourse, statuette de Fortuna.

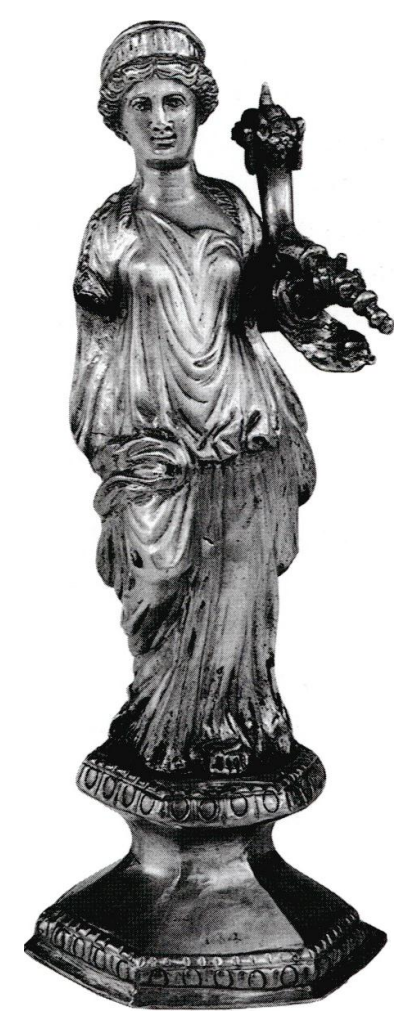

LONDRES, BRITISH MUSEUM. COURTESY TRUSTEES OF THE BRITISH MUSEUM 
Comparées aux pièces analogues, les deux sta-tuettes féminines de Vaise, la Fortuna et la déesse aux oiseaux, ont beaucoup de points communs en dépit de différences notables dans le traitement du visage notamment. Qu'il s'agisse de la Fortuna de Chaourse et de la déesse de Saint-Honoré (fig. 81) (Braemer 1968, p. 346-348), que rapproche le traitement des étoffes en plis drus et abondants qui suggère la matière et le volume sans reliefexcessif, ou de l'Aphrodite d'Antioche (M. C. Ross, A silver treasure from Daphné-Harbié, Archaeology, 6-1, 1953, p. 39), à laquelle s'appa-rente davantage la déesse de Toledo, aux plis de vêtements moins nombreux, et de celle de Tortose (au Louvre, Bj 2089) (fig. 82), une version simplifiée des deux précédentes, toutes traduisent, chacune à leur niveau technique, une autre sensibilité et un goût moins miniaturiste.

81- Saint-Honoré-les-Bains, statuette d'une déesse.

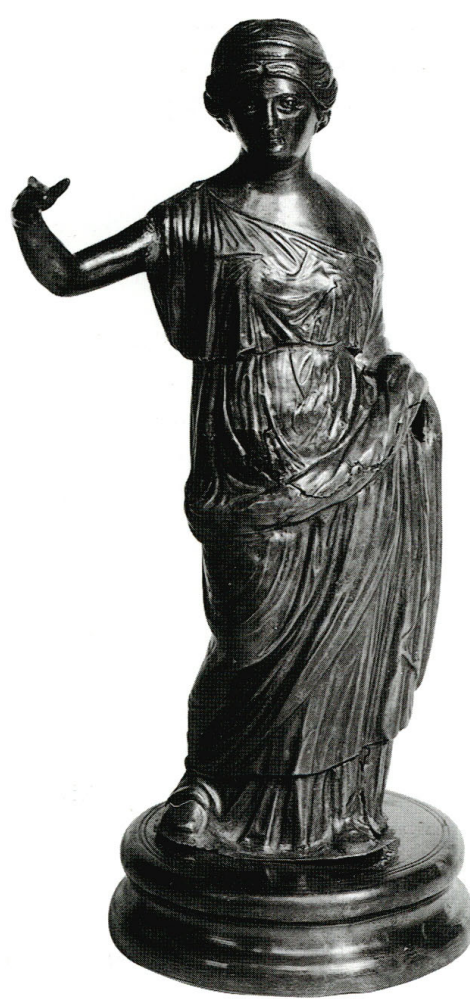

MUSÉE ROYAL DE MARIEMONT. CL. DU MUSÉE

Le socle, relativement haut par rapport à la sta tuette qu'il porte, a une forme assez complexe, que l'on retrouve, en argent, sous une statuette d'Isis dans le laraire de la villa rustica de Scafati, près de Pompéi (S. De Caro, Il Museo Archeologico Nazionale di Napoli, Naples, 1994, p. 252), évi demment plus ancien que les deux statuettes de Vaise. Le décor et les moulurations sont tout à fait analogues. Une des statuettes en bronze de la trouvaille de Champoulet, au musée des Antiquités nationales, donne un autre exemple d'un socle de même forme (Joffroy 1978, p. 795-814). 
82- Tortose, statuette de divinité.

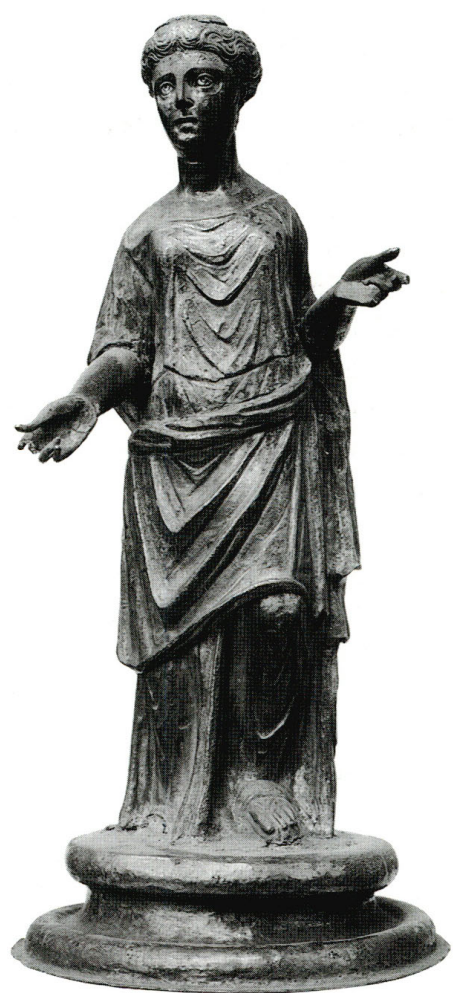

Paris musée du Louvre, AGER Bj 2089. Cl. Chuzeville

\section{Déesse aux oiseaux}

Inv. 93110422

Statuette féminine sur un socle rectangulaire.

Argent doré.

H. totale $23 \mathrm{~cm}$.

Dimensions, état de conservation et analyse technique

Le socle

Dimensions du socle: h. totale 5,3 cm; base : 7,2 $\mathrm{cm} \mathrm{X} \mathrm{5,3} \mathrm{cm} \mathrm{X} \mathrm{0,9} \mathrm{cm;} \mathrm{partie}$ supérieure $6,5 \mathrm{~cm} \mathrm{X} 5 \mathrm{~cm} \mathrm{X} \mathrm{0,6} \mathrm{cm} \mathrm{;} \mathrm{corps} \mathrm{du} \mathrm{socle} \mathrm{:} \mathrm{h.} 4,8 \mathrm{~cm} ; 4 \mathrm{~cm} \mathrm{X} \mathrm{2,7} \mathrm{cm.}$

Les moulures sont en forte saillie, $1 \mathrm{~cm}$ pour celles du haut, $1,3 \mathrm{~cm}$ pour celles de la base (fig. 83,84). 


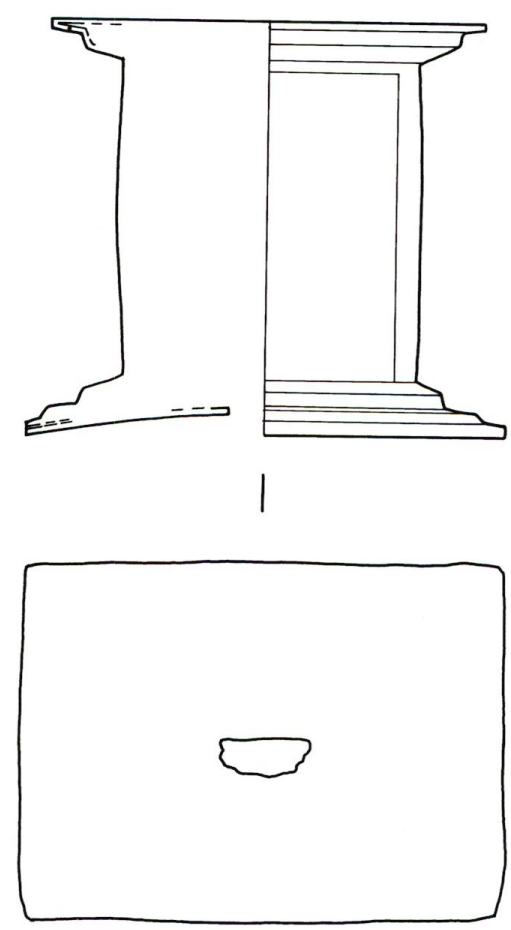
en argent de Boscoreale.

\section{La statuette}

Dimensions de la statuette : h. $16,3 \mathrm{~cm}$; h. de la tête, de la pointe du menton au voile, $2,3 \mathrm{~cm}$; larg. du visage $1,35 \mathrm{~cm}$.

La statuette est faite d'une feuille d'argent travaillée au repoussé. Le voile, exécuté à part, est rapporté, de même que le bras droit et la phiale aux oiseaux, la main gauche et les fruits dans le pli du vêtement. Le bras droit est plein et se termine par un tenon.

71 Au moment de la découverte, la statuette était dans un état médiocre, détachée de son socle et cassée en deux au niveau de la taille. Les bras étaient détachés et plusieurs fragments de draperie cassés, notamment celui renfermant les fruits. Le manteau était cassé dans le dos à hauteur du bassin.

72 La dorure, sans doute au feu, est très abondante ; elle couvre la bordure du vêtement, y compris dans le dos, en assez larges bandes, le repli du manteau, la coupe aux oiseaux et forme encore deux bandes verticales descendant de la poitrine jusqu'aux pieds. 


\section{Description et analyse stylistique}

73 La jeune femme est debout, de face, la jambe gauche très légèrement en avant (fig. 84) ; les pieds ne sont pas visibles, couverts par une longue tunique à manches courtes repliée sur la poitrine; elle dessine une étroite encolure en V. Les seins hauts sont moulés étroitement par le tissu qu'ils retiennent et qui dessine une série de plis profonds et arrondis. Un manteau plus court est drapé autour de la taille en formant un gros rouleau de plis qui remonte autour du bras gauche. La femme porte en outre une grande cape qui tombe droit dans le dos et lui couvre étroitement la tête en formant une sorte de capuchon.

84a- Déesse aux oiseaux, hauteur $23 \mathrm{~cm}$

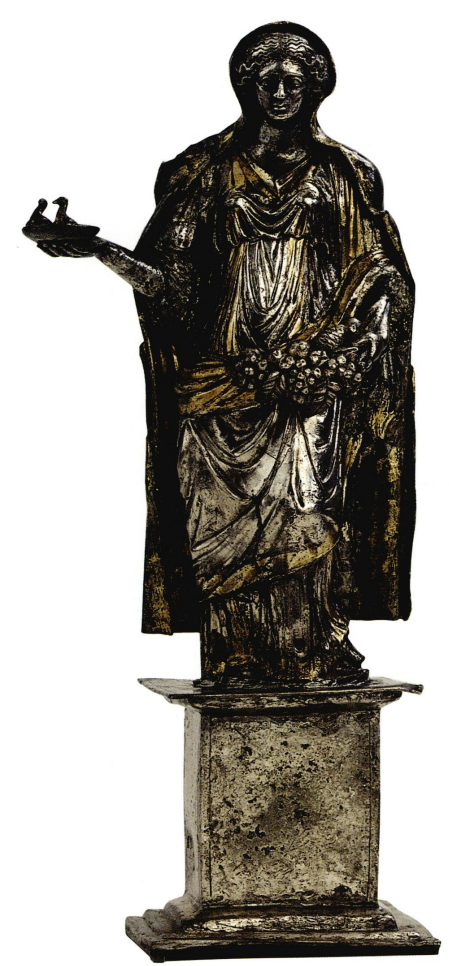



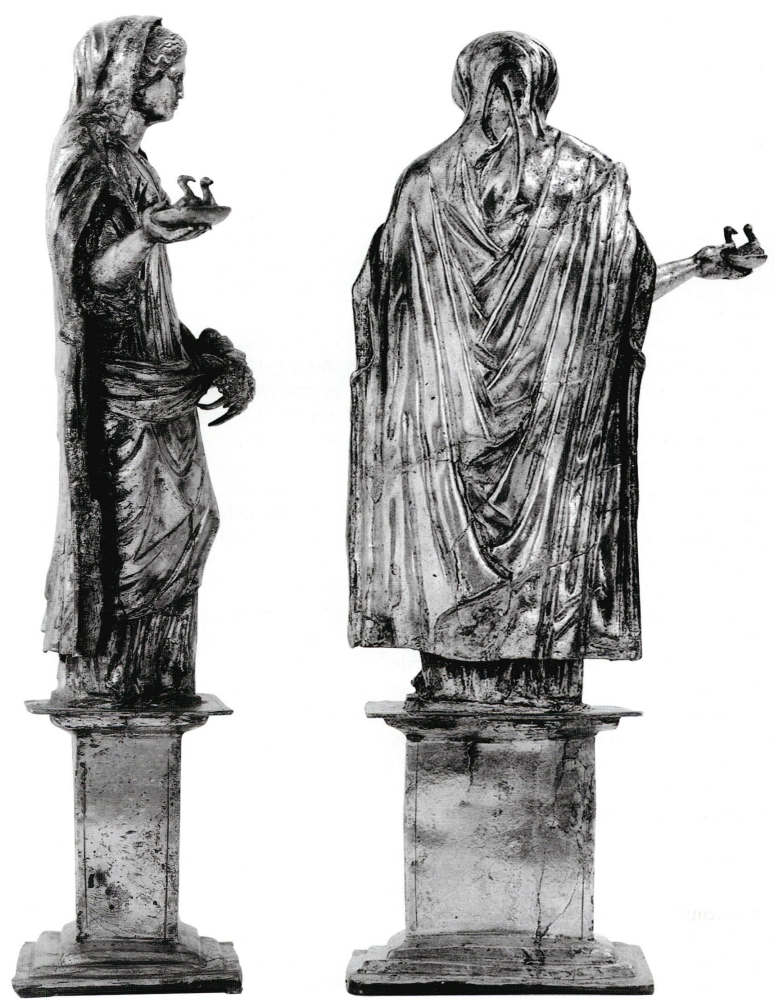

La main gauche, qui seule sort du manteau, retient un pan de vêtement rempli de fruits ; mais le dispositif n'est pas clair : on ne distingue pas vraiment dans quoi sont posés tous les fruits, ni ce que tient précisément la déesse, non pas un tissu ou un objet, semble-t-il, mais les plantes elles-mêmes, à la façon d'un bouquet. On reconnaît une pomme de pin, des raisins, des grenades, peut-être des épis et du feuillage, soigneusement individualisés; ces fruits surabondants donnent l'impression de déborder du tissu qui les contient. La main droite étendue vers l'avant tient une phiale dans laquelle sont posés deux oiseaux dont l'espèce est impossible à préciser (des colombes?).

La tête très ronde est tournée vers l'avant. Le visage est peu expressif, sans modelé véritable : la bouche est charnue, les lèvres sont bien dessinées sous un nez plutôt épais. Les yeux sont grands, dessinés par des paupières très nettes, mais sans pupilles. Le front est dégagé sous une chevelure qui encadre parfaitement l'arrondi du visage. Les cheveux sont répartis de part et d'autre d'une raie médiane en deux masses ondulées finement au-dessus du front, plus largement sur les tempes, et tirées vers l'arrière. Bien que rapportée, la cape était restée solidaire du reste de la statuette; on n'a donc pu vérifier si le revers, en dessous du vêtement, est effectivement travaillé.

L'exécution est soignée : la cape est faite d'une juxtaposition de grands plis travaillés directement dans la feuille de métal avec habileté et un réel effet décoratif. Sur le devant, le chiton et le manteau présentent des plis moins épais, mais néanmoins clairement modelés, disposés avec un souci que l'on peut qualifier de réaliste, sans monotonie en tout cas, plus drus et plus fins sur le chiton que sur le manteau: la statuette est l'œuvre d'un orfèvre de qualité qui maîtrise bien son métier et va au-delà d'une reproduction purement mécanique des schémas à sa disposition. Si la tête est régulière, le visage lisse et arrondi, le travail est dans l'ensemble plutôt nerveux. 
L'artisan est attiré par les effets plastiques et paraît rechercher à la fois le rendu des volumes et les jeux d'ombre et de lumière : le traitement des vêtements est significatif ; l'orfèvre lui a accordé une grande attention non seulement sur la face antérieure de la statuette, celle qui était immédiatement visible, mais aussi, ce qui est plus rare, à l'arrière; l'exécution de la cape est particulièrement intéressante: les grands plis qu'elle dessine ne sont pas une simple manière de suggérer l'apparence du tissu; ils témoignent aussi d'un véritable souci de volume, presque réaliste, comme l'indiquent les plis cassés qui parcourent l'axe du vêtement. La cape permet aussi d'encadrer la statuette d'une ligne d'ombre, à la manière de certaines sculptures de la fin du IIe s. Si la recherche n'est peut-être pas aussi délibérée que de la part des sculpteurs (que l'on pense - en choisissant volontairement un exemple extrême - au buste de Commode coiffé de la peau de lion du Palais des Conservateurs à Rome), elle traduit peut-être quand même un « Zeitstil », l'esprit d'une époque. C'est effectivement dans la seconde moitié du IIe s. que nous situerons la statuette.

Elle est, à bien des égards, sensiblement différente de la statuette de Fortuna (fig. 78) : le traitement des têtes est particulièrement significatif. Le visage est ovale, encadré par la masse de la chevelure qui accompagne la forme sur l'une, davantage charpenté sur l'autre, et surmonté par des mèches qui sont fortement individualisées, à la différence des ondulations sans grand relief de la première. On notera en outre que les globes oculaires de la Fortuna portent, eux, des pupilles en creux. Mais les deux statuettes témoignent cependant d'une sensibilité commune. Nous analysons plus loin leurs rapports avec les figures analogues: la comparaison fait apparaitre qu'elles ont en définitive entre elles davantage de points communs qu'avec les autres statuettes.

\section{Analyse iconographique}

La statuette est, à bien des égards, unique. Certains éléments en font clairement une déesse de l'abondance: l'attitude générale, la présence de la phiale, qui n'est pas toutefois l'attribut le plus fréquent, celle des fruits débordant du vêtement qui constituent un équivalent de la corne d'abondance, mais qui caractérisent aussi, plus souvent, les déesses assises nombreuses dans le domaine galloromain (Matronen und verwandte Gottheiten, Beihefte der Bonner Jahrbucher, 44, Cologne, 1987) ; placés dans leur giron, ils en font des Matres.

En revanche la cape est un vêtement inhabituel : on ne peut confondre le voile «à la romaine " qui couvre la tête de plusieurs déesses, Junon par exemple, et le manteau de la divinité de Vaise qui l'enveloppe complètement, formant en outre une sorte de capuchon. On observera également que la jeune femme ne porte dans sa chevelure ni bandeau ni diadème. Mais pour le reste le costume, tunique et manteau, est plutôt romain, même si l'artisan n'a pas su rendre clairement la superposition de ses différentes pièces

Toutefois l'élément le plus révélateur sur la personnalité de la figure divine représentée est l'existence de deux oiseaux posés dans la phiale. Celle-ci, instrument du sacrifice, est en même temps, comme la corne d'abondance que les déesses tiennent parfois dans l'autre main, un symbole de fécondité : elle peut être remplie de fruits, lorsqu'il s'agit des déesses-mères notamment. C'est le cas par exemple d'une statuette en bronze trouvée dans la Haute-Vienne (au musée des Antiquités nationales: S. Reinach, Catalogue du musée des Antiquités nationales, II, p. 164). Mais nulle part ailleurs le 
personnage n'est complété ainsi par des volatiles, qui en font immédiatement une des divinités aux oiseaux connues en Gaule. Celles-ci, masculines ou féminines, ont fait l'objet à plusieurs reprises d'enquêtes pour tenter d'en préciser la personnalité et la fonction (A. N. Newell, The Dove-Deity of Alesia and Serapis Moritasgus, Revue Archéologique, 1939, II, p. 133-158; W. Deonna, L'Art national de la Suisse romande, Genava, 1941, p. 119-131 ; A. Colombet, Les divinités aux oiseaux en Gaule et le dieu aux colombes d'Alésia, Mélanges Charles Picard, Paris, 1949, p. 224-239); sans doute est-il impossible de les réunir toutes dans un unique ensemble, en dépit des tentatives qui ont été faites en ce sens. Non seulement le sexe diffère, mais aussi les attributs : on rencontre aussi bien un dieu accompagné de Cerbère à Alise-Sainte-Reine (E. Espérandieu, Recueil général des bas-reliefs, statues et bustes de la Gaule romaine, $\mathrm{n}^{\circ} 7684$; figure analogue à Corgoloin, dans la même région : S. Deyts, dans Lavagne 1989, $\mathrm{n}^{\circ}{ }^{69}$ ) qu'une déesse assise, comme la statuette de la Haute-Vienne déjà citée ; quant aux oiseaux, ils peuvent entourer les divinités, perchés dans des arbres (Alise-Sainte-Reine, Espérandieu $n^{\circ}$ 7684) ou simplement disposés sur le fond de la stèle (autel d'Autun : Espérandieu $n^{\circ}$ 1895), ou bien être placés sur leurs épaules (Alise-Sainte-Reine, Espérandieu $n^{\circ} 2354,2355,2377,7280,7680$; stèle de Corgoloin) ou posés sur leur main (stèle de Sainte-Sabine [Côte-d'Or]: Espérandieu $\mathrm{n}^{\circ} 2224$; statuette de bronze à Besançon: P. Lebel, Catalogue des collections archéologiques de Besançon. V. Les bronzes figurés, Annales littéraires de l'Univ. de Besançon, 26, Paris, 1961, n 8, p. 20, pl. XI). On ne saurait exclure toutefois que sur un certain nombre de ces sculptures, souvent maladroites, la position apparente de l'oiseau, qui semble étroitement en rapport avec le personnage représenté, ne résulte pas d'une volonté délibérée de l'artisan. Les espèces représentées, enfin, sont diverses : échassiers, corbeaux, colombes; mais le plus souvent (c'est le cas ici) elles ne peuvent être formellement identifiées.

81 La statuette n'apporte guère d'élément nouveau sur les fonctions de la déesse, ni sur son identité : le socle est anépigraphe. On observera qu'un lien formel est établi entre la figure divine et les oiseaux, puisque ceux-ci sont indissociables d'un de ses attributs, la phiale. Comme sur la statuette de bronze de la Haute-Vienne - sur les genoux de laquelle sont posés deux volatiles - les éléments en rapport avec l'abondance sont mis en avant avec insistance, en particulier les fruits très abondants. Sans doute faut-il voir là l'indication la plus sûre sur la fonction du personnage, sans rentrer dans les débats plutôt stériles qui ont souvent accompagné les réflexions sur les divinités aux oiseaux.

\section{Tête}

Inv. 93110424

Argent.

H. 7,5 cm; larg. max. $5 \mathrm{~cm}$; h. de la tête de la base du cou au-dessus des mèches du front $5,6 \mathrm{~cm}$; larg. de la tête au niveau des oreilles $4,8 \mathrm{~cm} ; h$. du visage $4,7 \mathrm{~cm}$.

Très encroûtée et corrodée au moment de la découverte, la tête a été consolidée intérieurement avec de la gaze.

Il est difficile de se prononcer sur le sexe du personnage représenté. La tête est cassée au bas du cou. Le visage est ovale, très plein, peu modelé, posé sur un cou large (fig. 85). Le menton est arrondi, la bouche, petite, est légèrement entrouverte avec des lèvres charnues; le nez, plutôt court, est large, notamment à sa base où les narines épaisses sont soigneusement indiquées. Les arcades sourcilières sont peu accentuées et 
les sourcils ne sont pas détaillés. Les yeux sont indiqués par le contour des orbites, dessinés par deux paupières charnues. Les pupilles, en léger creux, sont en forme de peltes tournées vers le haut. Le front, largement dégagé, est surmonté par la chevelure : deux gros bandeaux de mèches ondulées tirées vers l'arrière de part et d'autre du front et réunies sur la nuque en un chignon sommaire. Au-dessus, la calotte crânienne est rapportée ; les mèches y sont dessinées très sommairement.

85- Tète de divinité, hauteur $7,5 \mathrm{~cm}$
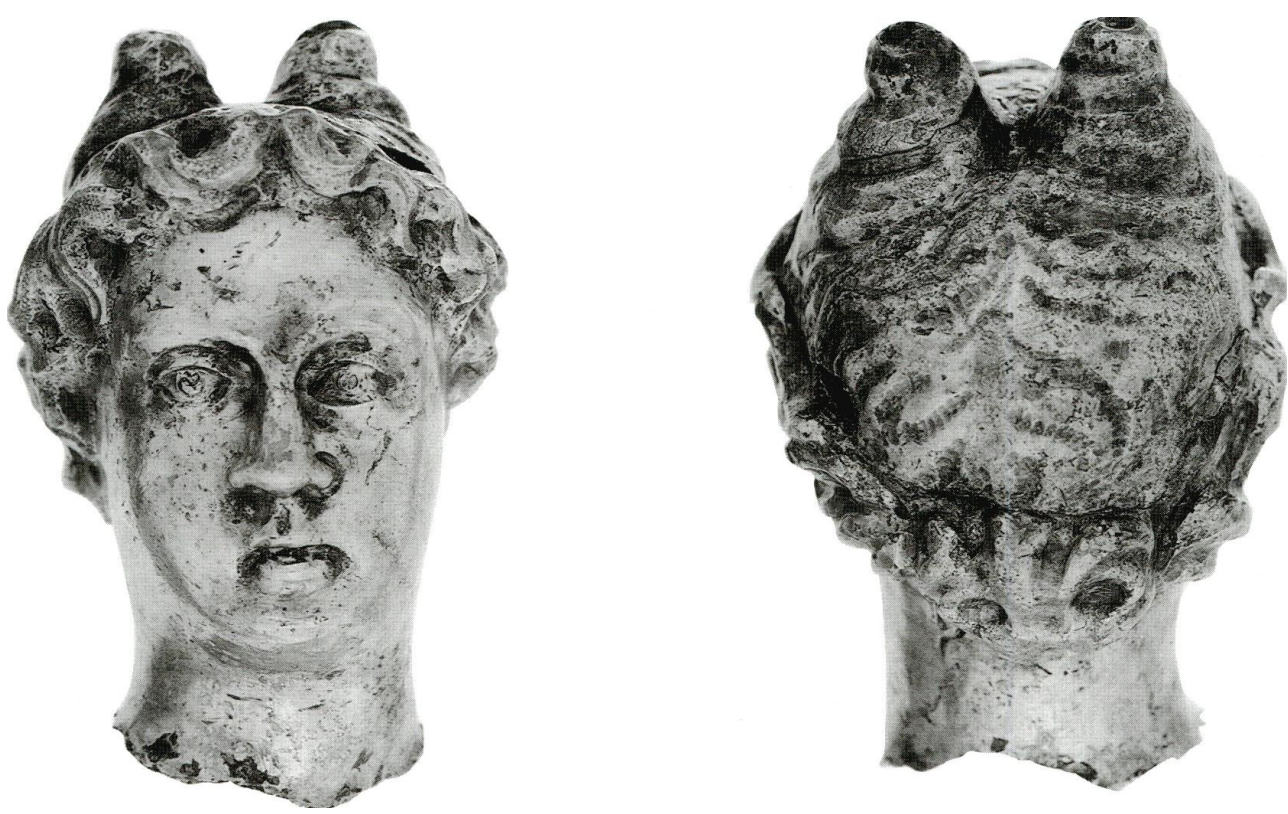

La tête de Vaise est exécutée au repoussé, en deux parties: le sommet de la calotte crânienne au-dessus des bandeaux de cheveux qui l'entourent est rapporté comme il l'était sur l'une des deux statuettes de Mercure en argent de Berthouville (Babelon 1916, nº 1, p. 73 ; Trésors d'orfêvrerie, $n^{\circ} 27$ ) et sur plusieurs autres statues en bronze de grandes dimensions : dans le cas du Mars de Coligny, de l'Apollon de Vaupoisson ou du Mercure de Thienne, la calotte a disparu (Boucher 1976, p. 56); à Womrath ou Weissenthurn, c'est elle qui est en revanche conservée (H. Menzel, Die römischen Bronzen aus Deutschland, III. Bonn, Mayence, 1986, n 170-171).

Dans le cas des statues en argent, le procédé s'explique aisément par des considérations techniques (sur ce point, Braemer 1968, p. 351-354) : il facilite grandement le travail au repoussé, notamment, dans le cas de Vaise, l'exécution des deux excroissances au sommet de la tête; mais il n'était pas indispensable puisque la tête féminine de Marengo est d'une seule pièce (Bendinelli 1937, p. 16, pl. VI ; Braemer 1968, p. 345 ; Baratte 1998, p. 369-379).

La coiffure se caractérisait par deux excroissances coniques posées au sommet de la tête et comme formées de mèches enroulées en spirales. Nous n'avons pu trouver de parallèle exact à cette curieuse présentation qui évoque davantage des sortes de cornes qu'un arrangement de la chevelure. Elle peut toutefois évoquer la forme que prend parfois la coiffure de certaines têtes féminines idéalisées d'inspiration hellénisante dont le trésor de Marengo fournit un exemple particulièrement significatif (fig. 86), puisque réalisé dans le même matériau et avec des dimensions équivalentes (h. totale $10 \mathrm{~cm}$; h. du visage $4,2 \mathrm{~cm}$; la tête est conservée de la même manière, arrachée au bas 
du cou. cf. supra). Tirés vers l'arrière depuis le sommet du visage tout en étant moins strictement organisés en bandeau, les cheveux sont rassemblés d'une part en un chignon assez souple sur la nuque, et en un double nœud au sommet de la tête d'autre part, plat dans sa partie médiane, mais gonflé à chaque extrémité. Les Victoires ont très fréquemment adopté ce type de coiffure, plus ou moins déformé (il n'est pas impossible que la tête de Marengo ait appartenu à une divinité de cette nature) : ainsi le bel exemple clipéophore d'Augst (A. Kaufmann-Heinimann, Die romischen Bronzen der Schweiz I: Augst und das Gebiet der Colonia Augusta Raurica, Mayence, 1977, n 75, pl. 77). Les statuettes de bronze en offrent de nombreuses attestations, sur lesquelles les deux extrémités du nœud de cheveux au sommet de la tête deviennent parfois plus importantes, constituant deux excroissances presque autonomes ou au contraire se transforment en une sorte de toupet; ainsi sur une statuette de médiocre qualité de Niederbronn en Alsace (B. Schnitzler, Strasbourg, musée archéologique. Bronzes antiques d'Alsace, Paris, 1995, n $\mathrm{n}^{\circ} 149$, p. 122). Mais jamais, même sur les objets les plus maladroits, le souvenir ne se perd complètement du lien qui existe entre les deux éléments proéminents.

86- Marengo, tête de divinité.
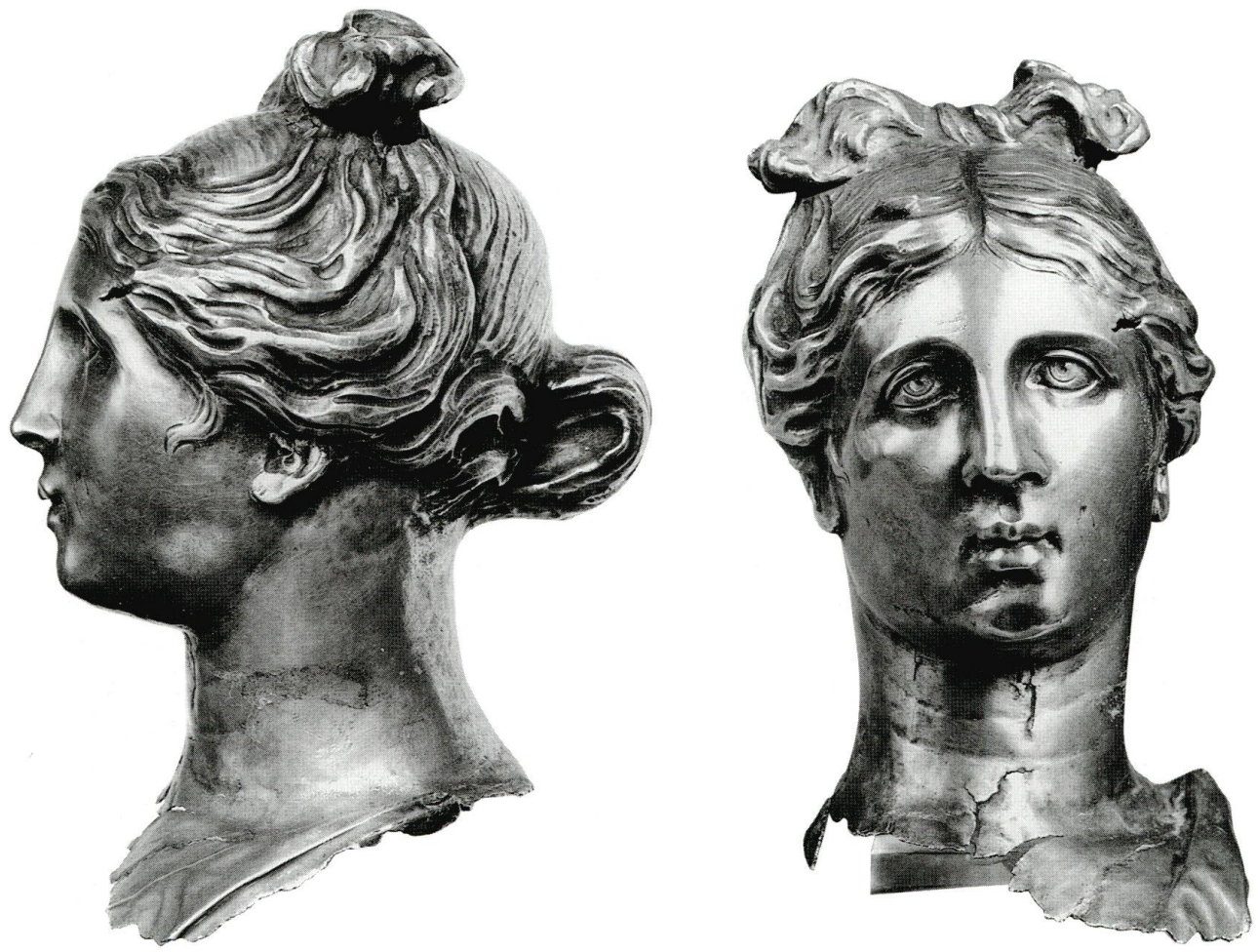

Turin, Museo di Antichità. Cl. Chuzeville

On doit observer encore qu'un type analogue de chevelure nouée se rencontre également dans le cas d'Apollon: celui découvert à Augst (insula 30), de qualité modeste, fournit l'exemple d'un nœud compliqué, mais très largement saillant audessus de la tête (Kaufmann-Heinimann, Augst, op. cit., n 8, pl. 7). Plus significatif encore est l'Apollon du trésor de Weissenburg (KellnerZahlhaas 1993, n 5, p. 36-38, pl. 16-18). Au sommet de la tête est posé un nœud en trois parties constituées chacune d'un rouleau : un élément médian horizontal de l'intérieur duquel se dégage en oblique vers le haut quatre autres éléments de grandes dimensions groupés deux par deux, tout 
à fait rigides et guère plus réalistes que les deux "cornes" de la tête de Vaise. A Weissenburg en tout cas, il s'agit bien d'un nœud, si extravagant qu'il paraisse. Peutêtre, faute de parallèle véritable, doit-on considérer qu'il s'agit à Vaise de la déformation ultime d'un nœud de cette nature mal compris par l'orfèvre, car trop loin des coiffures réellement en usage et effectivement interprété par lui comme des cornes; dans le détail cependant, chacune donne bien l'impression d'être constituée de mèches enroulées en spirales. Mais on ne perdra pas de vue qu'on peut effectivement rencontrer de telles originalités dans le traitement des chevelures: ainsi les deux longues mèches ondulées qui sortent au-dessus du front d'une têtemasque en bronze du trésor de Weissenburg en donnent-elles un exemple significatif (KellnerZahlhaas 1993, n 40, p. 81-82, pl. 70-71).

Une comparaison entre la tête de Vaise et celle de Marengo n'est pas sans intérêt étant donné les analogies formelles entre elles, qu'il s'agisse de leur nature ou de leurs dimensions. Mais l'une est sortie des mains d'un praticien de qualité, l'autre d'un artisan compétent certes, mais moins habile. Dans les deux cas, la tête s'inscrit dans un ovale. Mais à Marengo le modelé est beaucoup plus volontaire, alors qu'il est fort mou à Vaise. Les procédés techniques sont identiques, comme le montrent le traitement de la gouttière labio-nasale ou la manière de cerner assez profondément la base du nez et de traiter la zone des yeux. Sur les deux têtes les pupilles sont en creux, en forme de pelte. Mais à Marengo le nez est fin, l'arcade sourcilière aiguë, les paupières bien individualisées. Les mêmes outils sont mis en œuvre dans le traitement des mèches de la chevelure; mais leur action est davantage visible sur la tête de Vaise dont les mèches, qui tendent à être individualisées comme à Marengo, forment seulement une masse ondulée et passablement uniforme. La comparaison de la bouche, et plus particulièrement des lèvres, est tout à fait significative : nettes et charnues à Marengo, elles sont molles et presque indistinctes à Vaise.

Cette différence de qualité cependant n'implique pas une différence de date: les deux têtes sont vraisemblablement contemporaines, se situant l'une et l'autre pour des raisons stylistiques - le traitement de l'œil notamment - à la fin du IIe ou dans la première moitié du IIIe s.

\section{Tête masculine}

$91 \quad$ Inv. 93110423

Argent.

H. max. 4,3 cm; larg. $\max .3 \mathrm{~cm}$; profondeur $\max .3,7 \mathrm{~cm}$. La hauteur de la tête suggère une statuette d'une trentaine de centimètres.

92 Tête en argent de petites dimensions, travaillée au repoussé. Le cou a été arraché (fig. 87). L'état de conservation est très médiocre : il manque un morceau du front, ainsi qu'une grande partie de l'arrière de la tête. La face, brisée en plusieurs morceaux, a été restaurée. Toute la partie inférieure présente des traces d'arrachement. 


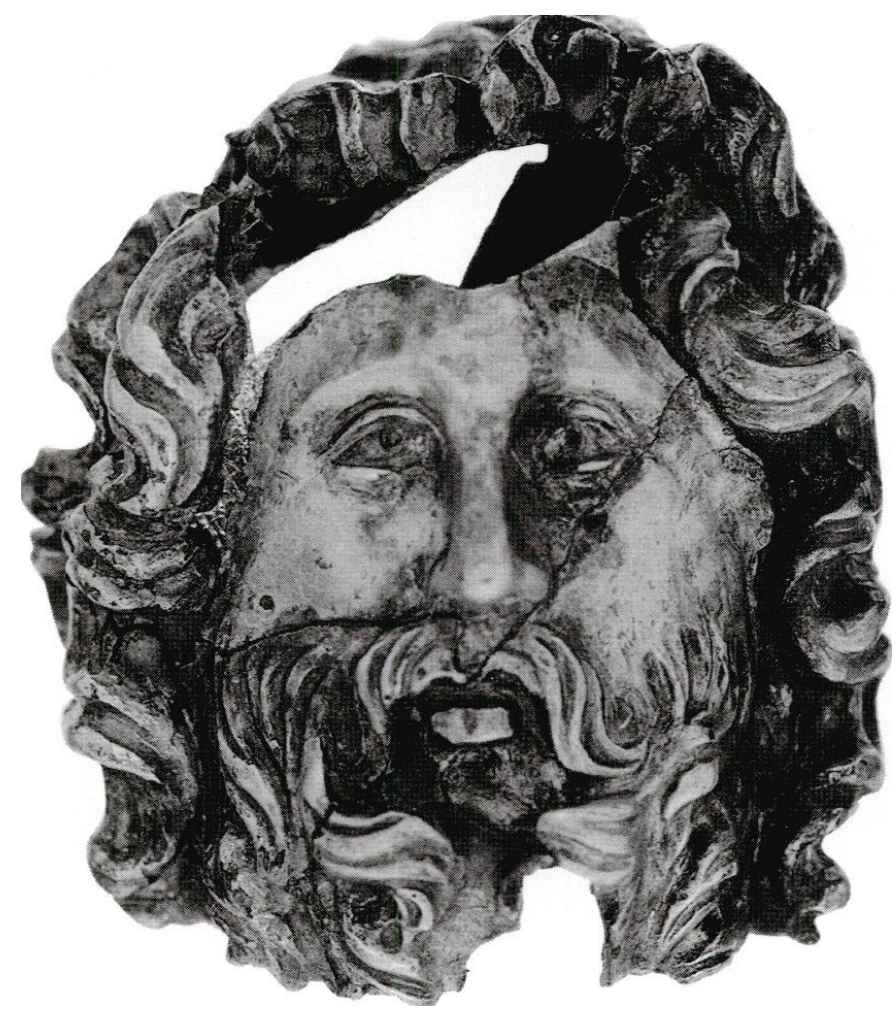

93 Tête d'un homme dans la force de l'âge, à la chevelure abondante (elle dissimule les oreilles), à la longue moustache et à la barbe touffue dont les longues mèches couvrent tout le bas des joues. Dans un crâne aux proportions presque cubiques, aussi profond que large (mais on tiendra compte des déformations dues à l'écrasement de l'objet), le visage n'occupe qu'une surface réduite ; toute l'attention est ici attirée par les mèches, d'autant plus que le travail est relativement médiocre: le modelé paraît presque inexistant.

La bouche, petite et très endommagée, est noyée sous la moustache. Le nez, allongé, est plutôt fin et se prolonge par deux arcades sourcilières aiguës sur lesquelles les sourcils ne sont pas indiqués. Les yeux sont rapprochés, assez petits, en amande, bordés par deux paupières charnues, en bourrelet ; la pupille circulaire est en creux.

Les mèches de la chevelure tombent en ondulant depuis le sommet de la tête : deux séries, très saillantes, traitées comme des festons géométriques, encadrent le visage, creusées par un canal intérieur large et profond qui leur donne du volume et les individualise. Le même traitement est appliqué à la barbe et à la moustache, dont les mèches cependant sont moins épaisses, plus minces et moins ondulées. Sur le sommet de la tête la chevelure est plus sobre ; des mèches couvrent la nuque.

Le type, une tête barbue plutôt solennelle d'allure, est celui des grandes divinités masculines, Jupiter, Poséidon, Neptune, Esculape notamment, ou même Sarapis dans sa présentation la plus hellénisante, comme sur un relief de la villa de Chiragan à MartresTolosane, même si, sur la tête de Vaise, il ne semble pas y avoir la possibilité d'un modius qui caractérise le dieu (Les dieux de la Gaule romaine, Luxembourg, 14 avril-15 juin 1989, n 96, p. 121-122, pl. couleurs p. 23 ; pour Esculape, $c f$. par exemple la statuette en bronze de Reims, ibid., $\mathrm{n}^{\circ} 15, \mathrm{p}$. 38). L'absence d'attributs interdit évidemment de préciser davantage, mais la présentation de détail de la chevelure fait pencher en 
faveur de Jupiter. La tête bouclée renvoie aux créations de la sculpture grecque du IVe s. av. J.-C., dont l'exemplaire de Vaise offre un bien médiocre écho.

97 On la rapprochera notamment des exemplaires en bronze de qualité bien supérieure, ceux de Brée en Belgique, de Dalheim au Luxembourg ou du Vieil-Evreux (S. et J.-P. Boucher, Musée d'Evreux. Bronzes antiques, I, statuaire et inscription, Evreux, 1988, n 7, p. 32-35) : la tête de Vaise en donne un équivalent beaucoup plus plat. Le Jupiter du VieilEvreux en particulier, assez proche par le foisonnement de la chevelure et d'une barbe envahissante qui couvre le bas du visage, montre tout ce que peut apporter un modelé puissant.

Le Jupiter du trésor de Mâcon (Trésors d'orfèvrerie, $\mathrm{n}^{\circ}$ 134) en donne une version en argent de dimensions réduites $(\mathrm{h}$. totale $6,5 \mathrm{~cm}$ ), à la chevelure et à la barbe particulièrement abondantes; elles le sont moins sur le buste du sanctuaire de Jupiter Dolichenus au Petit-Saint-Bernard (fig. 88), plus grand, mais plus figé aussi en dépit de sa belle facture au repoussé (Trésors d'orfèvrerie, $\mathrm{n}^{\circ} 188$ ); le modelé du visage est délibérément accentué, de manière à donner au dieu une physionomie impressionnante, mais les mèches, pourtant soigneusement dessinées et individualisées, sont aussi plus stylisées.

88- Petit-Saint-Bernard, buste de Jupiter Dolichenus.

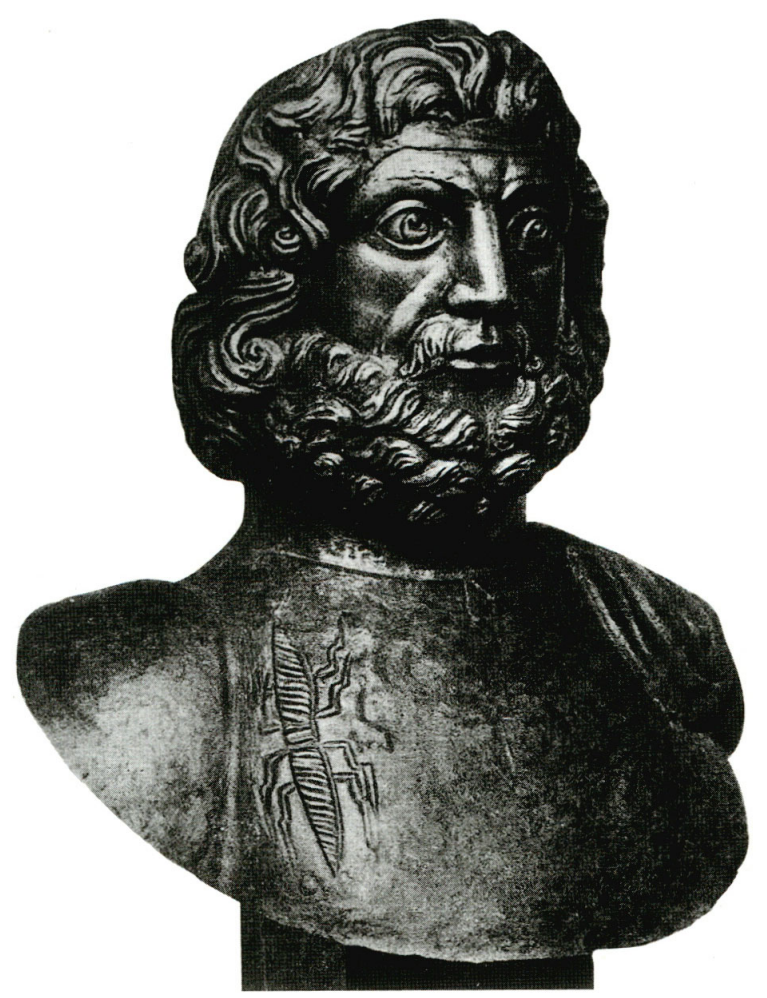

Aoste, Museo archeologico. Cl. du musée

La tête de Vaise, bien loin de la puissance du buste du Petit-Saint-Bernard, doit néanmoins en être contemporaine : fin du IIe - début du IIIe s. 


\section{Aile}

Argent.

H. 16,4 cm ; larg. max. $6,8 \mathrm{~cm}$; larg. sans l'attache $6 \mathrm{~cm}$.

101 Aile en tôle d'argent, de grandes dimensions : on peut estimer qu'elle appartenait à une statuette de 25 à $30 \mathrm{~cm}$ de hauteur (fig. 89). Arrondie à sa partie supérieure, elle est soigneusement dessinée : la découpe de l'extrémité des plumes apparaît à la partie basse ; leur détail est gravé sur l'une des faces, celle extérieure ; il apparaît en négatif sur l'autre, qui n'était pas destinée à être vue. Il s'agit d'une aile droite. Elle est munie vers le haut, à $3 \mathrm{~cm}$ du sommet, d'un petit tenon rectangulaire légèrement replié.

89 - Aile, hauteur $16,4 \mathrm{~cm}$

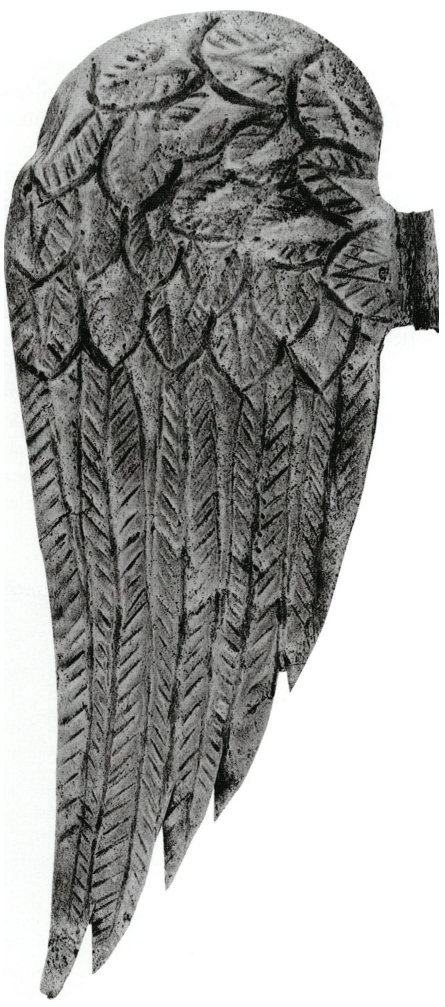

L'aile était au repos, verticale, la partie arrondie vers le haut.

Il est impossible de préciser la statuette dans le dos de laquelle elle était fixée : une Victoire? ou bien la Tutela dont nous possédons la couronne? Nous rappellerons en effet l'exemple de la Tutela en bronze de Vienne (S. Boucher, Vienne. Bronzes antiques, Paris, 1971, $\mathrm{n}^{\circ} 13$, p. 51-54) ou de celle, en argent, de Mâcon (Trésors d'orfêvrerie, $\mathrm{n}^{\circ} 140$ ) (fig. 79), deux divinités tourelées, tenant une patère dans la main droite étendue, et dont les ailes déployées supportent les bustes des dieux de la semaine. La présentation n'était évidemment pas aussi complexe dans le cas de la statuette de Vaise. 


\section{Fragment de couronne tourelée}

Inv. 93110427

Argent.

H. 2,5 $\mathrm{cm}$; h. du mur 2,3 cm; larg. d'un élément de mur 2,3 $\mathrm{cm}$; h. de la porte $1,7 \mathrm{~cm}$.

Fragment d'une couronne tourelée formée d'une alternance de tours et de sections de muraille percées d'une porte (fig. 90). Chaque tour se présente comme une sorte de colonne torsadée surmontée d'un couronnement aujourd'hui incomplet. La muraille comporte quatre assises de grand appareil terminées par une sorte d'arcade, et dans lesquelles s'ouvrent des portes cintrées.

90- Fragment de couronne tourelée

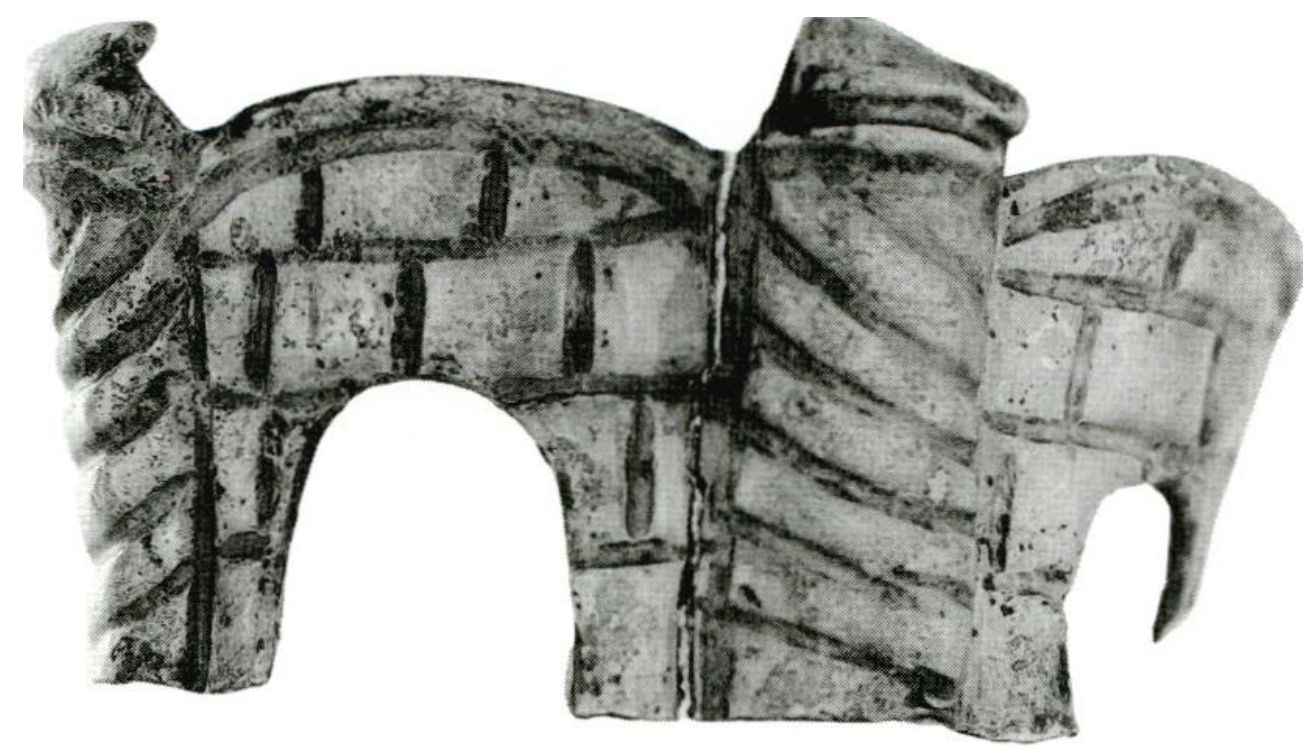

Deux tours sont conservées, dont les torsades s'enroulent en sens contraire; une section de muraille est complète, une autre fragmentaire.

Il est difficile de reconstituer exactement le diamètre de la couronne ( $3 \mathrm{~cm}$ env.) et le nombre de ses pans. Elle couronnait la tête d'une statuette féminine ou masculine de dimensions relativement importantes - $30 \mathrm{~cm}$ de hauteur environ - une Tyché (ou une Tutela) ou un Genius comme on en connaît de nombreux exemples, du Genius en bronze de Weissenburg (Kellner, Zahlhaas 1993, $n^{\circ} 13$, p. 56-57) et de celui en argent de Mâcon (Trésors d'orfèvrerie, $\mathrm{n}^{\circ} 141$; sur les Genius en général, $c f$. H. Kunckel, Der römische Genius, op. cit.) à la Tutela du Musée du Petit Palais à Paris (J. Petit, Bronzes antiques de la collection Dutuit, Paris, 1980, $\mathrm{n}^{\circ}$ 26) et à celles de Vienne (Boucher, Vienne. Bronzes antiques, op. cit., $\mathrm{n}^{\circ} 13$ et 13bis ; la dernière est en buste) et de Mâcon (fig. 79). La plupart de ces couronnes possèdent quatre tours, mais n'ont qu'une seule porte, dans l'axe audessus du front, cette face principale étant souvent caractérisée par la présence d'un fronton et par l'indication des assises, alors que les autres côtés sont plus sommaires. 


\section{Lunule}

Argent.

Envergure (de pointe à pointe) $2,5 \mathrm{~cm}$;épaisseur $0,15 \mathrm{~cm} ; \mathrm{h} .2,1 \mathrm{~cm}$.

Le revers, lisse, ne porte pas de traces de polissage, mais au contraire de nombreuses griffures. La face, cernée par un léger bourrelet, présente trois gros points dont le contour est dessiné par une ligne en creux. Elle est dorée. Aucune trace de soudure (fig. 91).

110 L'usage de cet objet est malaisé à établir : élément décoratif dont le revers n'était pas visible, il complétait peut-être une statuette en constituant un ornement. La valeur apotropaïque de la lunule et son rôle en tant qu'amulette ont été soulignés en particulier par H. Wrede (Lunulae im Halschmuck. Wandlungen, dans Studien zur antiken und neueren Kunst, 1975, p. 243-254).

\section{1- Lunule}
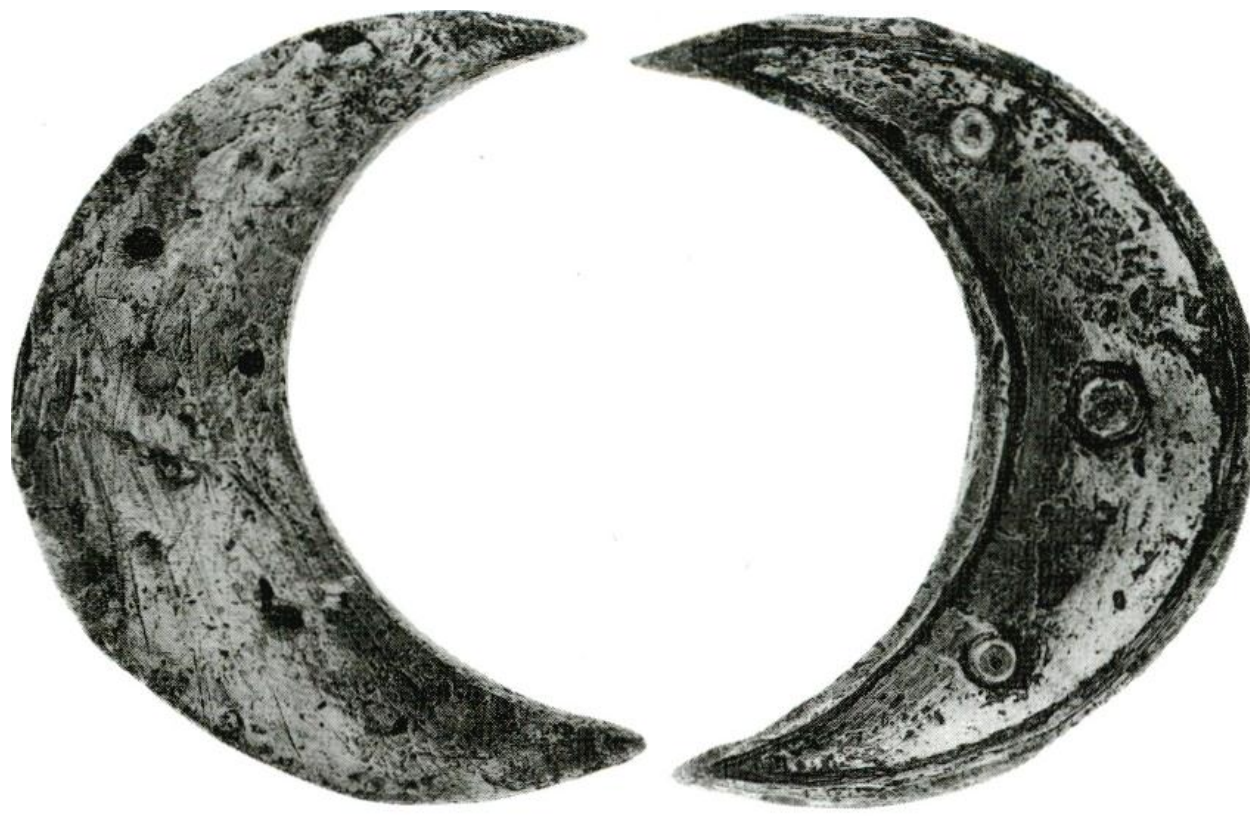

\section{Bras gauche}

$111 \ln v .93110430$

Argent. Bras gauche, cassé par pliure au-dessous du coude.

Sans doute fondu, l'objet est creux.

Long. max. 6,4 cm; diam. au poignet 0,9 cm. L'état de conservation est assez satisfaisant.

112 Bras gauche d'une statuette, étendu vers l'avant (fig. 92). Le pouce est serré, l'index étendu, les trois autres doigts repliés, comme si le personnage tenait l'extrémité d'une corne d'abondance ou un caducée : le bras pourrait ainsi appartenir à un Mercure 
analogue aux deux exemplaires du trésor de Berthouville (Babelon $1916 \mathrm{n}^{\circ} 1$ et 2), à un Lare ou à un Genius comme ceux de Weissenburg et de Mâcon, déjà cités.

92- Bras gauche de statuette, long. max. $6,4 \mathrm{~cm}$
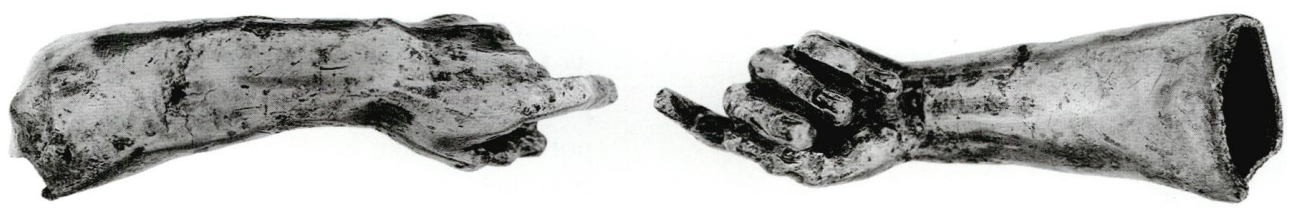

La facture, sans être très soignée (les doigts sont assez brutalement exécutés) est différente de celle de l'autre bras fragmentaire; le modelé est plus ferme, sur le bras lui-même, moins charnu, plus nerveux (on observe nettement les tendons sur la face inférieure du poignet) et sur la main dont l'ossature est parfaitement visible. Le caractère masculin paraît affirmé. On note que les ongles sont dessinés et profondément cernés.

\section{Bras droit}

$114 \operatorname{lnv} 93110429$

Argent. Bras droit, cassé juste au-dessus du coude.

Long, totale $9 \mathrm{~cm}$; long, de l'extrémité des doigts à la pliure du coude $8 \mathrm{~cm}$; diam. au poignet 1,3 $\mathrm{cm}$; diam. max. $2 \mathrm{~cm}$; diam. de la phiale $3,2 \mathrm{~cm}$.

115 Le bras est probablement fondu, sans doute en partie plein. Un gros tenon en fer de section carrée $(1,1 \mathrm{~cm})$ est enfoncé à l'intérieur (fig. 93). La phiale est percée au centre par le pointeau du tour. L'état de conservation est médiocre : deux grandes fissures parcourent longitudinalement le bras du poignet au coude à sa partie supérieure ; la phiale est abondamment rayée au revers.

Bras droit étendu tenant une phiale à ombilic entre le pouce et les quatre autres doigts écartés. Le bras, assez sommairement exécuté - le bras lui-même, plutôt charnu, est à peine modelé, les doigts sont grossièrement travaillés - était sans doute abaissé dans le geste de la libation. La phiale, un disque fait au tour, est lisse à l'extérieur, bordée par une ligne gravée à l'intérieur.

93a- Bras droit tenant une phiale, long. $9 \mathrm{~cm}$

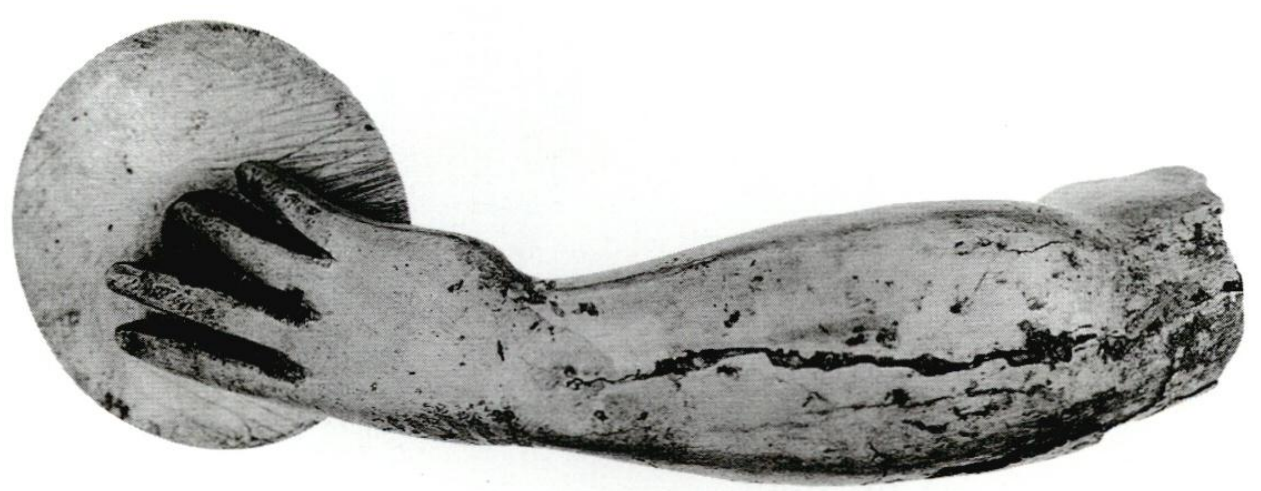




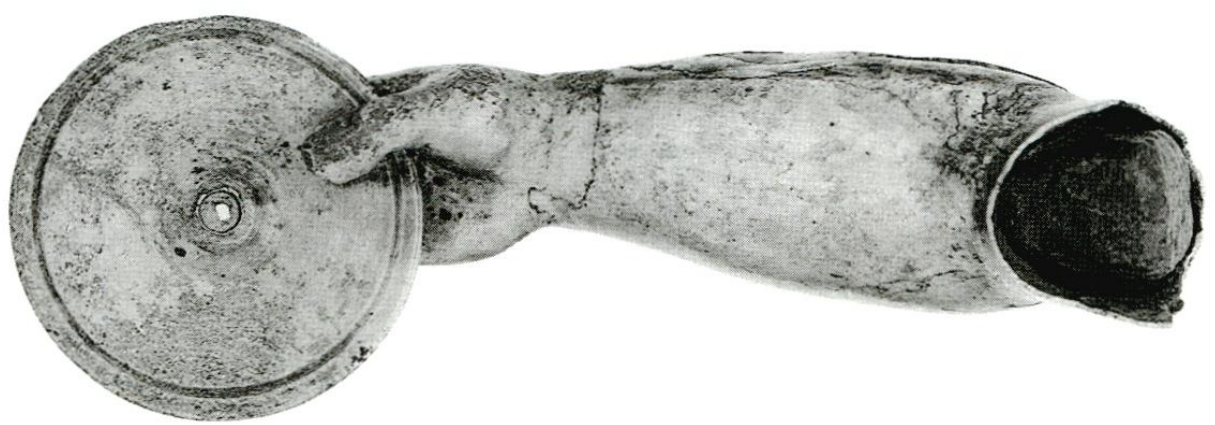

117 Il n'est pas possible d'attribuer avec certitude le bras à une des statuettes fragmentaires du trésor; peut-être appartenait-il à la Tyché dont on possède déjà la couronne tourelée. On imaginera une figure féminine du type de la Fortuna de Sainpuits (Nièvre), en bronze plaqué d'argent, coiffée d'un diadème (Trésors d'orfêvrerie, $\mathrm{n}^{\circ} 129$ ). La Tutela panthée du trésor de Mâcon en offre un autre exemple (fig. 79) (Trésors d'orfêvrerie, $\mathrm{n}^{\circ}$ 140), qui porte précisément une couronne tourelée. Mais le bras, même s'il n'est guère musculeux, pourrait aussi bien appartenir à un Génie, comme celui du même trésor de Mâcon (Trésors d'orfevrerie, $\mathrm{n}^{\circ}$ 141). Mais l'angle de la pliure au coude, trop faible, permet sans doute d'exclure tant un Lare tenant une phiale, comme à Weissenburg, qu'un togatus sacrifiant, dont le même trésor offre un exemple (Kellner-Zahlhaas, op. cit., $\left.\mathrm{n}^{\circ} 15, \mathrm{p} .60-61\right)$.

\section{Fragment de bras : coude}

Argent avec un remplissage en plomb.

Long, conservée $6,5 \mathrm{~cm}$; diam. $1,9 \mathrm{~cm}$.

119 Fragment de bras, cassé de part et d'autre du coude. D'un des côtés subsiste un noyau en plomb. Cet élément ne nous a pas paru se raccorder avec un des deux autres bras fragmentaires conservés (fig. 94).

94- Fragment de bras, coude

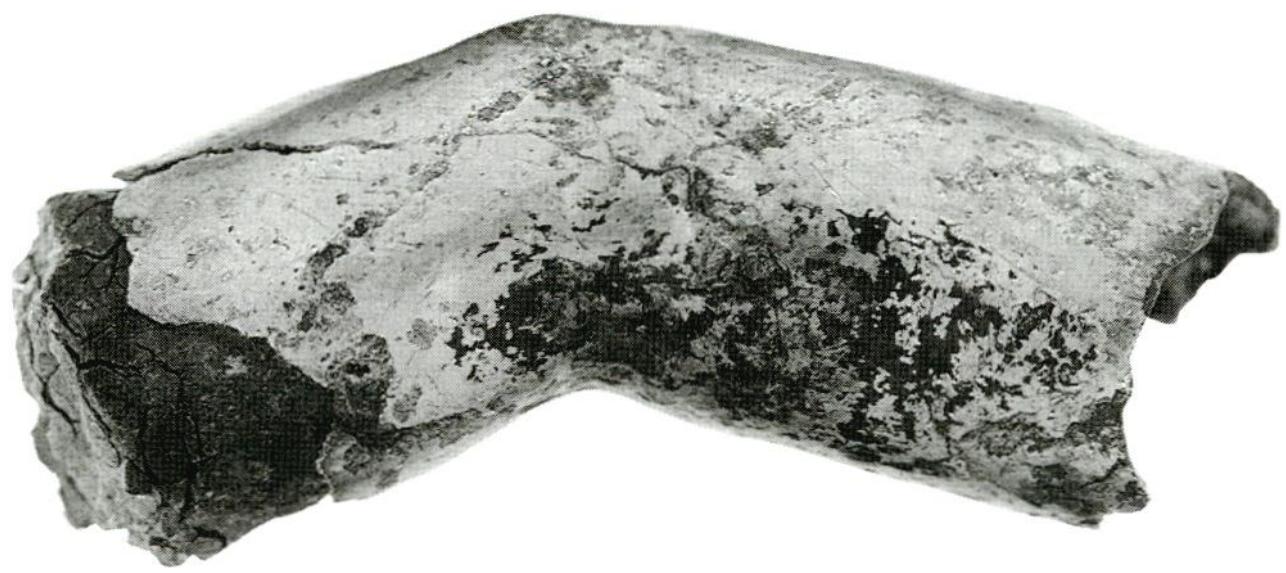




\section{Fragments de feuilles}

Argent.

Parmi tous les fragments (de draperie notamment) qui n'ont pu être remontés sur des objets plus importants, on en mentionnera encore trois : deux fragments probablement d'un même objet, en tôle dorée, plate et mince (dimensions respectives : $5,5 \mathrm{~cm} \times 2 \mathrm{~cm}$ et $4,5 \mathrm{~cm} \times 2 \mathrm{~cm}$ ), qui portent peut-être un décor de feuillage (palmes très stylisées). Un troisième, allongé (h. $5 \mathrm{~cm}$, largeur max. $1,8 \mathrm{~cm})$, cassé seulement à la partie inférieure, est plat et porte un décor de nervures. Il évoque l'extrémité d'une feuille votive du type le plus simple, comme il y en a dans la trouvaille de Vichy (au Musée des Antiquités nationales de SaintGermain-en-Laye) (Picard 1962, p. 10-30), mais peut constituer aussi, plus simplement, la pointe d'une draperie.

D’autres petits fragments, plats, pourraient appartenir à un socle.

\section{NOTES}

6. Je dois les remarques qui suivent aux observations que m'a généreusement communiquées $M$. Christol, auquel j'exprime toute ma gratitude. 


\section{Les bijoux du trésor de Vaise}

\section{Catherine Metzger}

1 Les bijoux du "trésor" de Vaise proviennent de deux lots distincts : d'une part, les deux bracelets en argent ( $\left.n^{\circ} 33-34\right)$ qui accompagnaient les statuettes du premier dépôt, d'autre part, tous les bijoux, en or et pierres de couleur faisant partie du dépôt $n^{\circ} 2$. Ces bijoux, en bon état de conservation, semblent assez représentatifs de la production de bijouterie à l'époque romaine.

\section{Bracelet}

$2 \quad$ Inv. 9312012

\section{Argent.}

Diam. ext. 7,5 cm; diam. int. $6,2 \mathrm{~cm}$; long, bouton $0,5 \mathrm{~cm}$.

Poids : 46,6 $\mathrm{g}$.

Bracelet ouvert formé d'une tige carrée légèrement torsadée, terminée par deux boutons pyramidaux (fig. 95). 


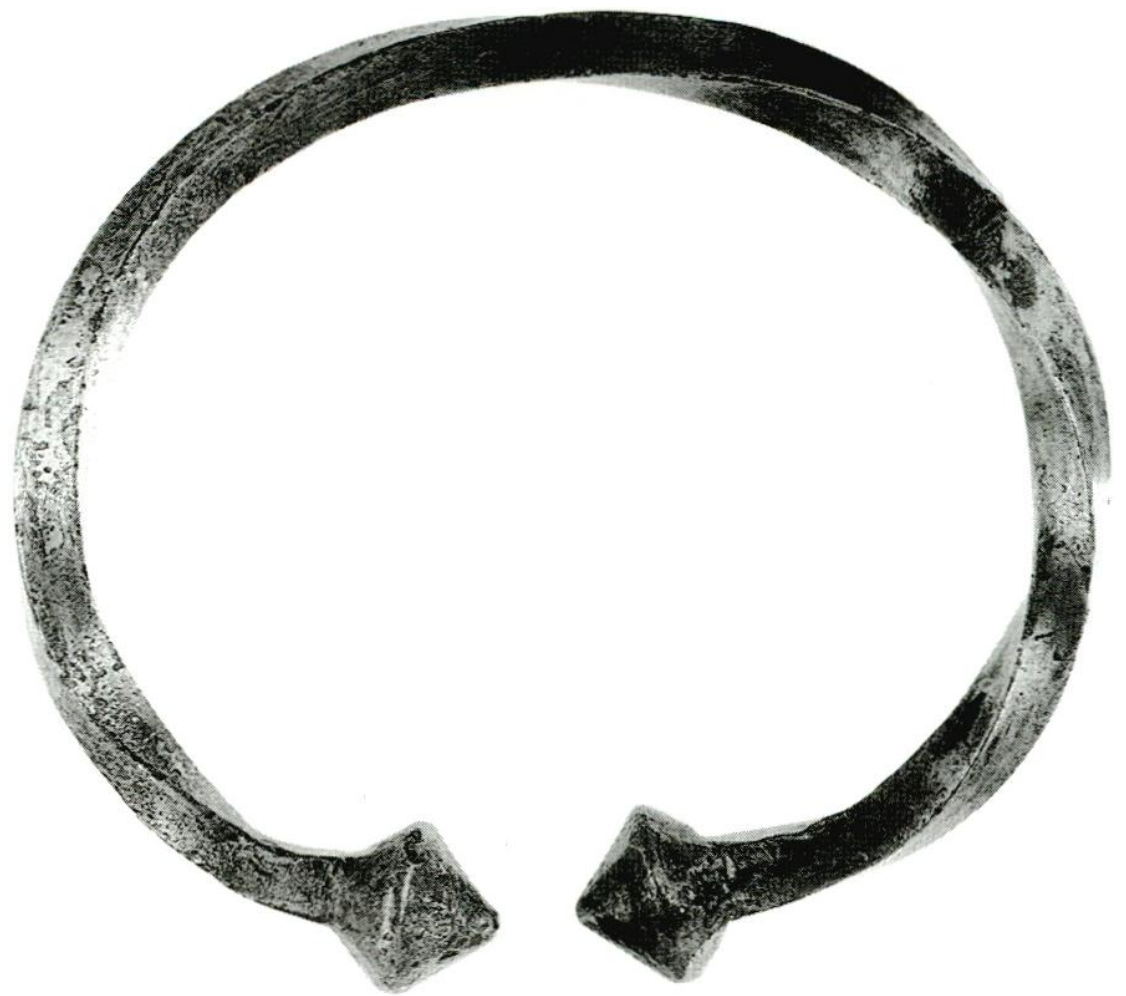

\section{Bracelet}

$4 \quad$ Inv. 9312011

\section{Argent.}

Diam. 8,5 cm environ;ép. de la tête :0,6 cm.

Poids : 73,7 $\mathrm{g}$.

5 Bracelet rigide serpentiforme à une seule spire, composé d'une tige de section ronde. La tête du serpent est bien rendue avec un système d'écailles diversifiées et la gueule ouverte. Le dessin des écailles s'interrompt sur le milieu du corps pour reprendre vers la queue effilée (fig. 96). 


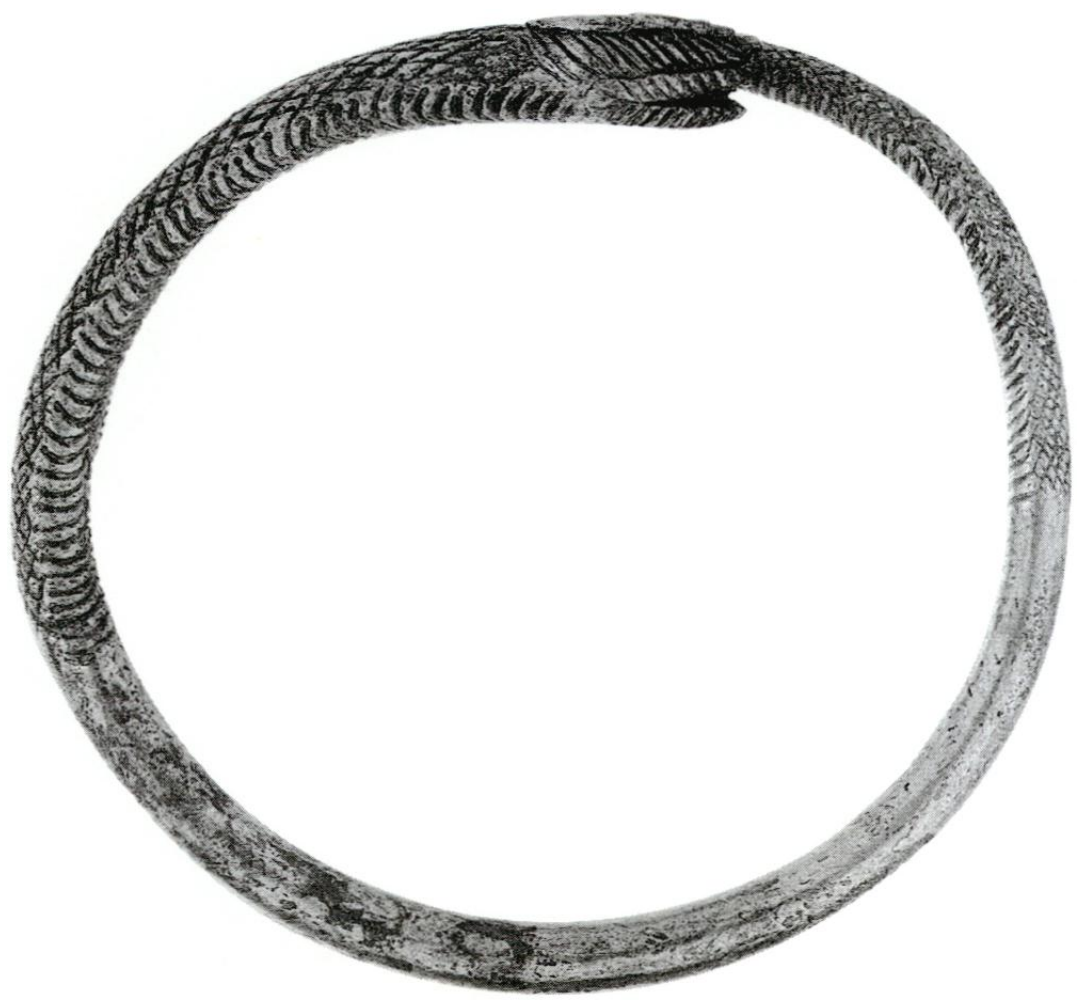

6 Nombreux en or, les anneaux serpentiformes sont soit des parures de bras, soit des anneaux de cheville. Ils sont surtout connus pour le début de l'Empire si l'on en juge par les exemplaires découverts à Pompéi et Herculanum, mais cette forme simple a dû se perpétuer aux IIe et IIIe s.

\section{Collier}

$7 \quad$ Inv. 9312013

Or et émeraudes.

Long $40 \mathrm{~cm}$; poids : 36,6 g.

Bon état de conservation.

8 Le bijou, complet, est constitué d'une alternance de 9 perles d'émeraude (béryl) et de 8 maillons d'or en forme de double nœud (fig. 97a et b).

9 Les perles d'émeraude en forme de prismes hexagonaux, de longueur irrégulière, sont percées de part en part et enfilées sur de petits fils d'or repliés en boucle aux extrémités.

10 Les maillons en double nœud de type communément appelé nœud d'Héraclès sont constitués de petits tubes d'or repliés pour obtenir la forme désirée et rassemblés à leurs extrémités dans des sortes de manchons à bout plat où viennent s'accrocher les fils supportant les émeraudes. Le fermoir est formé également de deux tubes d'or repliés en boucle avec manchon terminal auquel sont soudés pour l'un un crochet et pour l'autre un anneau (fig. 97b). 
97a- Collier en or et perles d'émeraude, long, $40 \mathrm{~cm}$

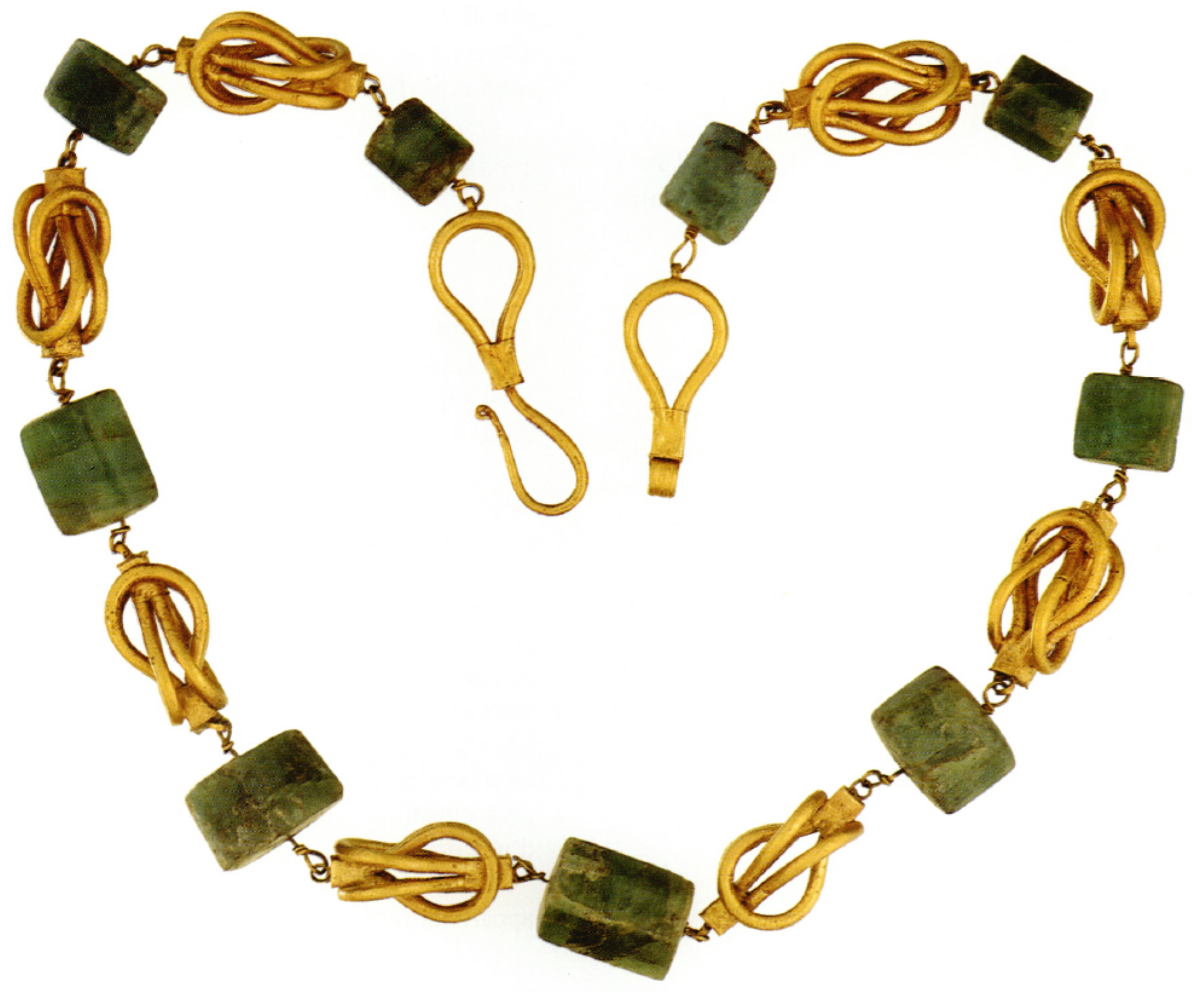

97b- Collier en or détail d'un nœud d'Héraclès
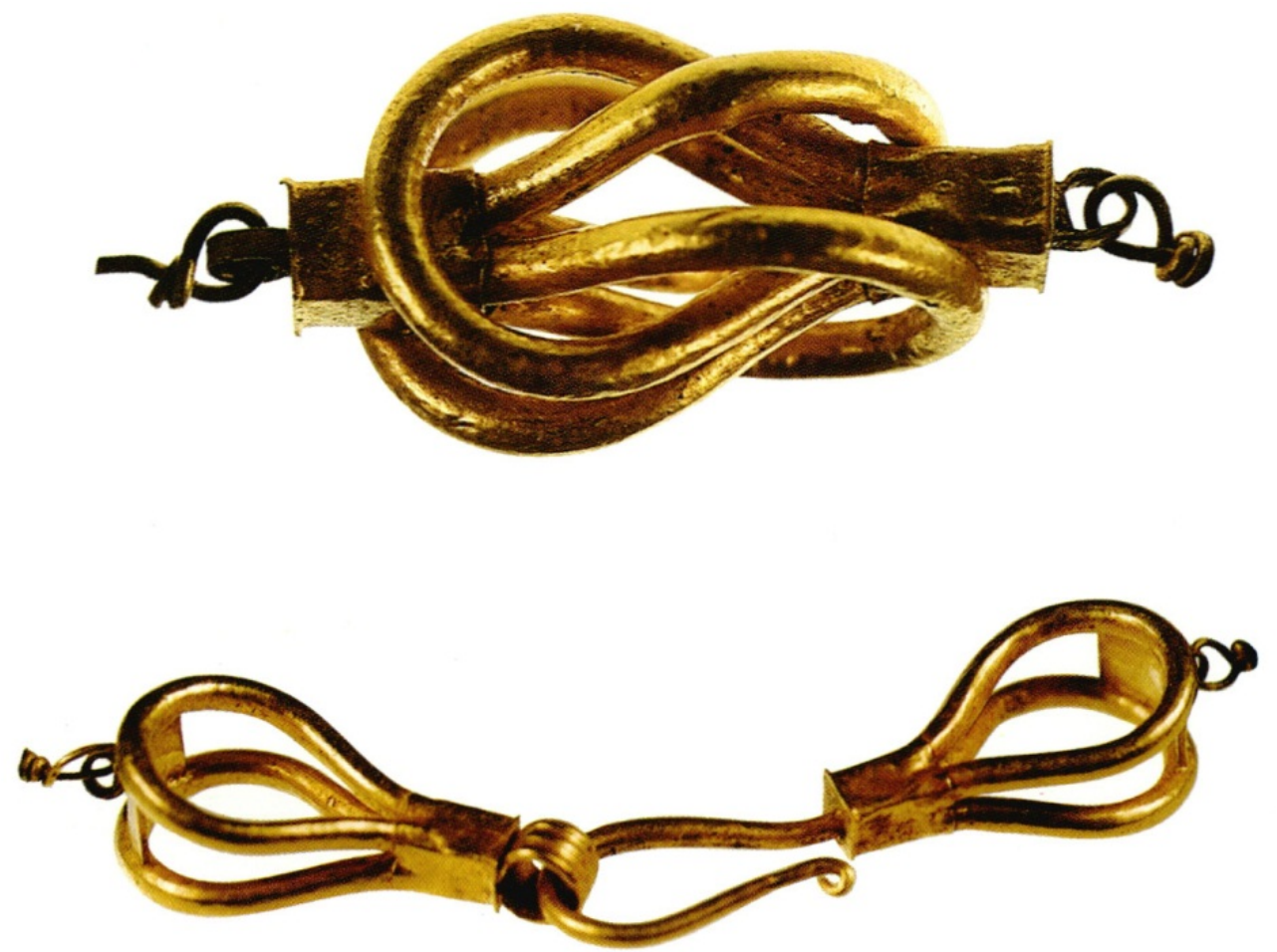

Détail du fermoir

11 Ce type de collier, relativement court avec ces nœuds d'Héraclès, connus depuis l'époque hellénistique où les fermoirs de bijoux (colliers ou diadèmes) adoptent 
fréquemment cette forme, alternant avec des éléments de pierre précieuse comme l'émeraude, est bien attesté en Gaule romaine. II semble participer à une recherche de polychromie à la mode un peu partout dans le monde romain et en particulier en Gaule au IIIe s. comme en témoignent des exemplaires retrouvés répartis sur différents points $\mathrm{du}$ territoire. Le trésor de Naix-auxForges (Meuse) (Lienard 1881, p. 16, pl. XXXIX, 8) comprend un collier de ce type (Cabinet des Médailles, Monuments d'or et d'argent $\mathrm{n}^{\circ} 130$ ) (fig. 98) avec d'autres bijoux dont un collier monétaire et des monnaies d'argent. Le trésor d'Eauze (Gers) (Schaad 1992, p. 17, fig. 1 et 2) comporte également un collier analogue. Un autre bijou de même type, mais beaucoup plus court (5 émeraudes et 4 nœuds d'Héraclès plus le fermoir), il s'agirait peut-être alors d'un bracelet, a été retrouvé avec d'autres bijoux dans les couches de démolition d'un fanum à Cracouville (Eure) (CAG. 27, p. $126 \mathrm{n}^{\circ}$ 285) (fig. 99).

98- Collier de Naix-aux Forges.

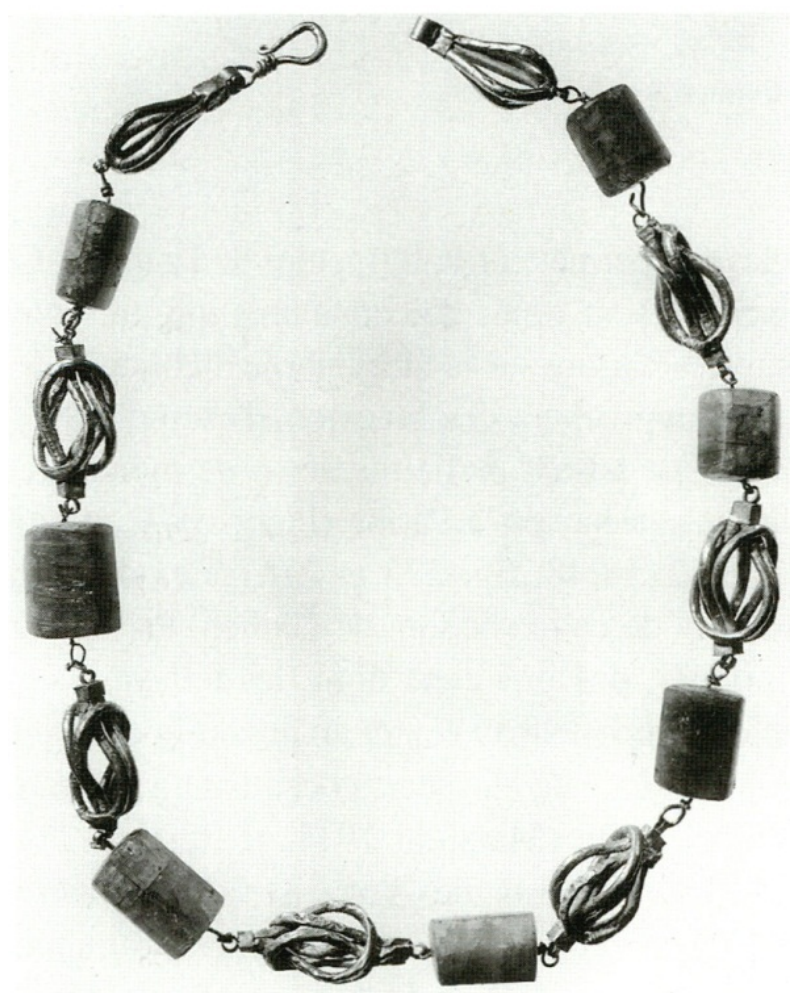

Cabinet des Médailles-Bibliothèque nationale de France 
99- Collier de Cracouville.

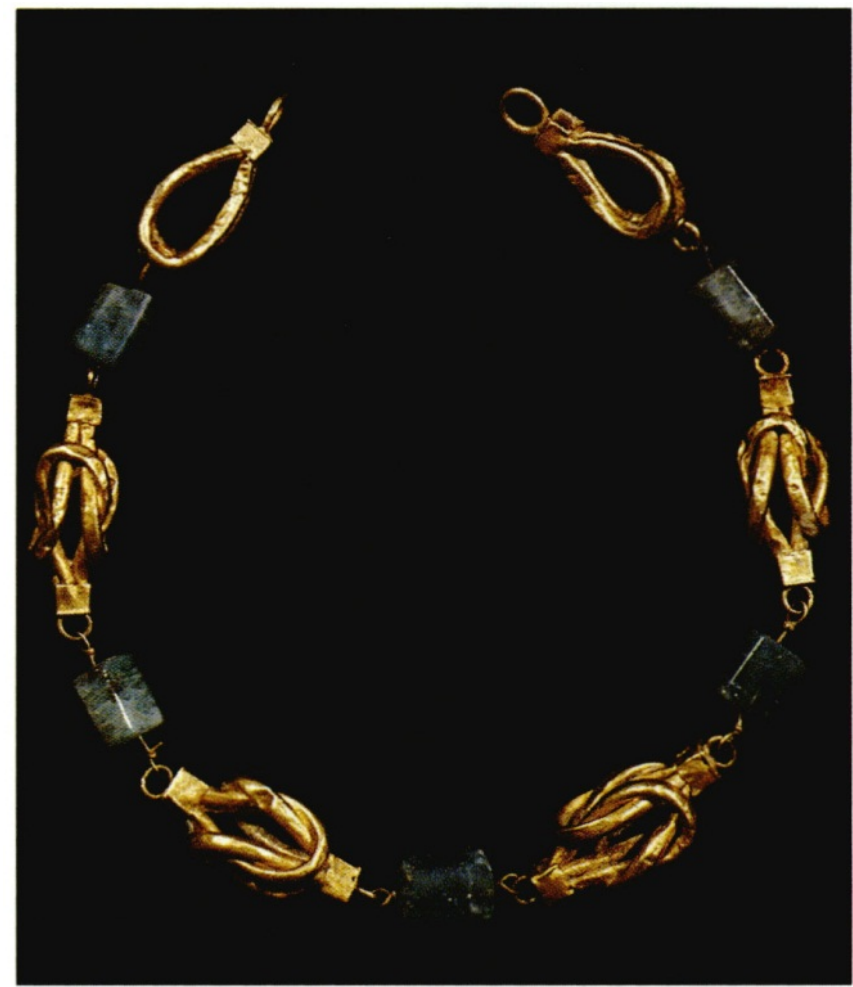

Musée d'Evreux. Cl. J.-P. Godais

\section{Paire de bracelets}

12 Inv. 9312018 et 9

Or.

Diam. 6,9 cm et 7,5 cm environ ; larg. : 0,9 cm.

Long, de manchons 1,3 à $1,4 \mathrm{~cm}$.

Poids 73 et $75 \mathrm{~g}$.

13 Ces bracelets à charnière sont constitués d'un fil d'or torsadé enroulé de façon serrée sur un tube d'or creux. Ce tube est brisé à l'intérieur d'un des bracelets, cela est visible au niveau du décalage des spires extérieures. Ce système de double anneau assure à la fois solidité et souplesse au bijou (fig. 100). 


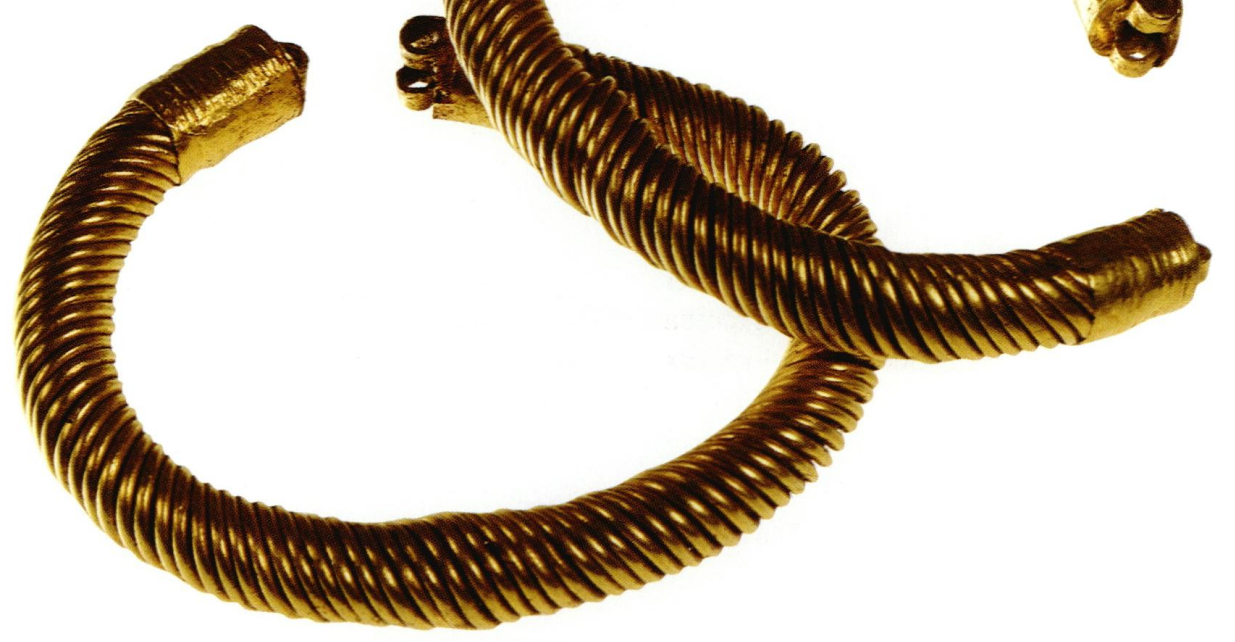

Des manchons cylindriques terminent les bracelets. Ils portent les anneaux dans lesquels se glissait la clavette permettant la fermeture de l'anneau. Ces manchons sont ornés de cannelures qui présentent des traces d'usure sur la face interne du bijou.

On rapprochera ces bracelets d'exemplaires du même type faisant partie du trésor de monnaies et de bijoux découvert à Parme (Pirzio 1992, p. 269271, n 245-248, fig. 273 et 276). Les dernières monnaies de ce trésor sont de Gallien. Par ailleurs, on connaît des bracelets dont des anneaux semblables comportent au centre un gros cabochon relié par un système de clavettes, constituant ainsi le fermoir de l'objet (Marshall 1911, p. 329, n 2813, pl. LXV). Le trésor des Lazaristes, découvert en 1841 à Lyon et daté du début du IIIe s., comportait des anneaux torsadés analogues avec fermoir et une monnaie de Commode montée dans une bâte (Comarmond 1844, pl. 1, $\mathrm{n}^{\circ} 1$ et 2).

\section{Paire de pendants d'oreilles}

Inv. 9312016 et 7

Or, émeraude, perle et pâte de verre.

Haut. 2,9 cm environ : larg. plaque carrée $1,7 \mathrm{~cm}$.

Poids $3,8 \mathrm{~g}$ et $3,4 \mathrm{~g}$.

L'extrémité d'une pendeloque manque sur un des pendants.

Ces pendants d'oreilles à crochet de suspension sont des bijoux composites. Un petit cadre carré en feuille d'or légèrement mouluré est découpé en son centre pour disposer une perle d'émeraude saillante maintenue par un petit fil d'or soudé au cadre. Un système de petites attaches en étroites bandes d'or maintient en dessous de cette plaque carrée un autre élément allongé en forme de losange aux extrémités bifides, 
débordant légèrement la largeur de la plaquette carrée. Viennent ensuite trois pendeloques parallèles constituées de petites bâtes carrées en or garnies de pâtes de verre auxquelles sont soudés trois granules d'or avec fil de suspension maintenant deux prismes d'émeraude encadrant une perle (fig. 101).

$\mathrm{Au}$ revers, des petites bandes d'or sont disposées en croix, renforçant ainsi la solidité de l'ensemble et permettant la fixation de la tige repliée qui sert de crochet de suspension (fig. 101).

Les différents éléments constitutifs des objets sont parfaitement solidaires les uns des autres et, contrairement à l'impression visuelle, les bijoux sont rigides.

21 Les parallèles ne manquent pas pour ces types de pendants d'oreilles. On peut en citer une paire dans le trésor des Lazaristes (Comarmond 1844, nº 17, 18 pl. I) (fig. 110). On notera en particulier la forme de la barre transversale entre le carré supérieur et les pendeloques et la disposition des perles et des pierres de couleur. Ce type ne semble cependant pas réservé à la Lyonnaise, des exemplaires très proches quoique incomplets ont été retrouvés dans les Balkans, montrant ainsi l'aire de diffusion de ces modèles de bijoux (Popovic 1996, $\mathrm{n}^{\circ}$ 85, 86).

101- Paire de pendants d'oreilles
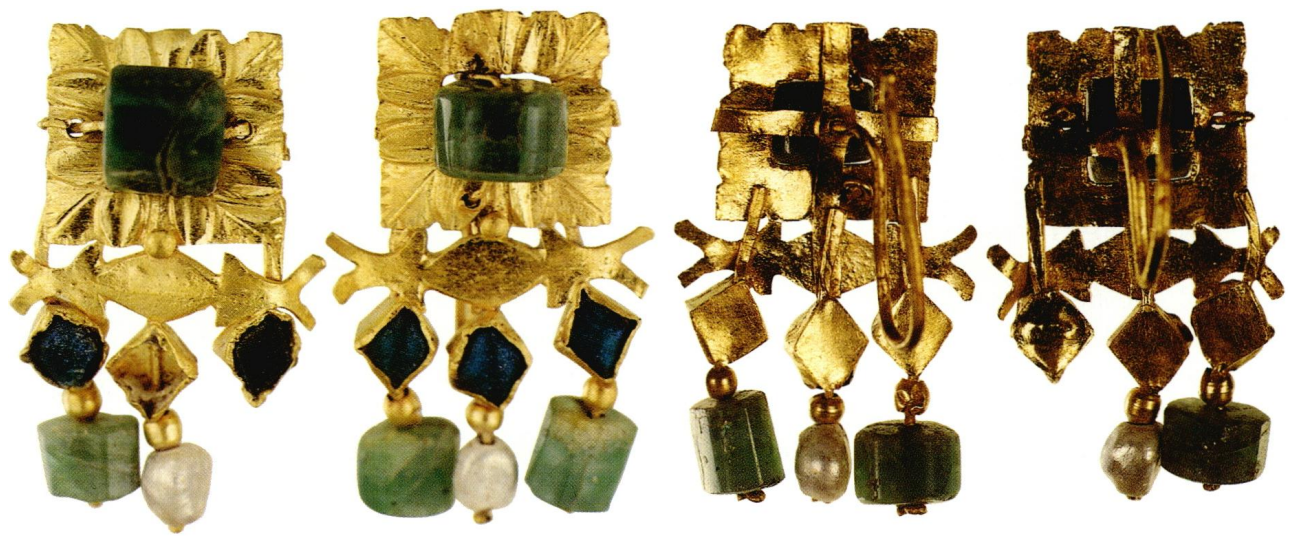

\section{Paire de pendants d'oreilles}

Inv. 9312014 et 5

Or, grenat, émeraude et perle.

Haut 3,6 cm env. ; diam. élément supérieur 1,4 cm.

Poids : $3,2 \mathrm{~g}$ l'une.

Ces pendants d'oreilles à crochet de suspension sont constitués de plusieurs éléments. La partie supérieure circulaire est une couronne de palmettes ajourées. Le centre est occupé par une perle passée sur un petit fil d'or soudé au diamètre interne de la couronne. Sur l'un des bijoux, la perle, cassée, laisse apparaître le fil d'or. Au revers, des étroites bandes d'or disposées en croix servent d'armature au disque ajouré permettant ainsi la fixation du crochet de suspension (renversé comme sur la paire $n^{\circ} 37$ ). Un anneau et un crochet relient cette couronne supérieure à un système de pendeloques mobiles. À une bâte carrée disposée sur la pointe et garnie d'un grenat taillé en pyramide, sont fixées trois petites pendeloques, une émeraude centrale encadrée de 
deux perles, toutes maintenues par de petits fils d'or repliés sur eux-mêmes à la base des pendeloques. L'ensemble constitue des bijoux légers et chatoyants (fig. 102).

102- Paire de pendants d'oreilles
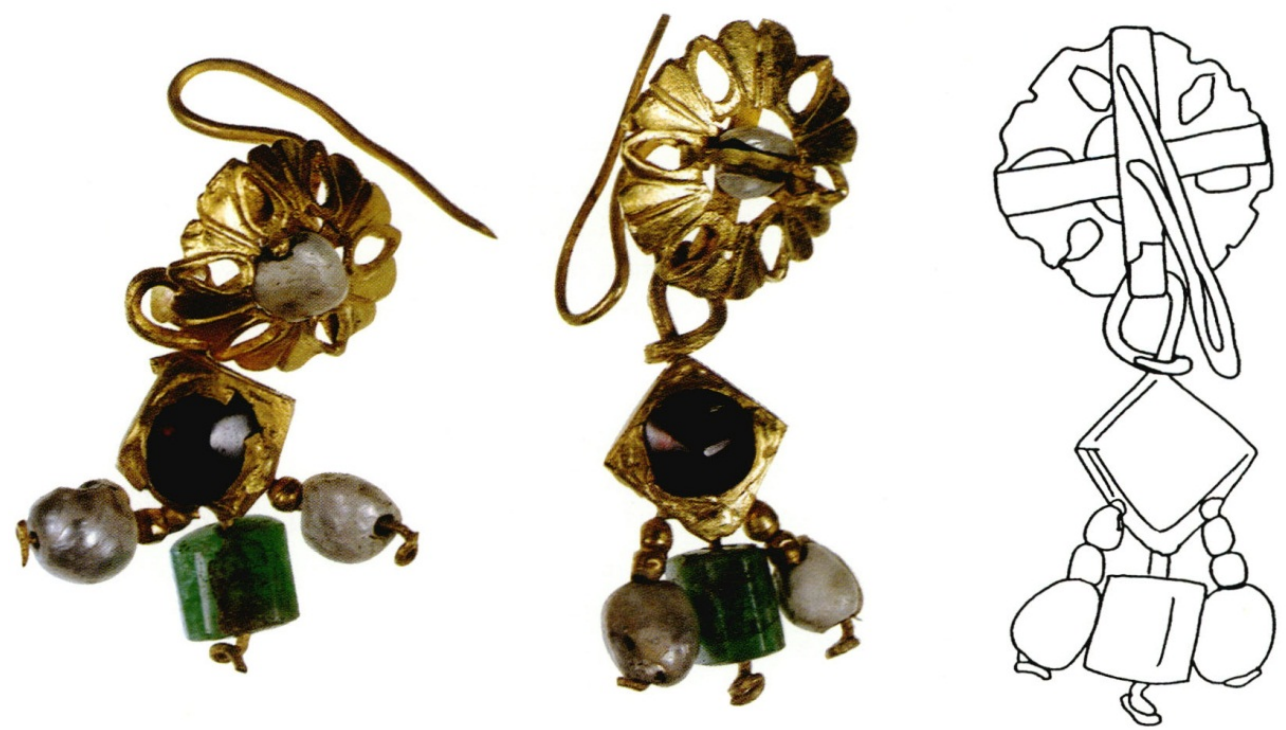

Ce type de pendant d'oreilles est loin d'être isolé ; on peut citer différents exemplaires analogues comme la paire de pendants d'oreilles qui proviendrait d'un «Trésor de Villardu ", selon le catalogue de la vente Guilhou (16-18 mars 1905, nº 198 a et b pl. VI). Il s'agit en fait du trésor de Coinces (Loiret) découvert en mars 1889 et encore incomplètement renseigné. Il aurait contenu au moins sept paires de pendants d'oreilles en or, deux bracelets en or, cinq bagues à intaille, trois médaillons monétaires d'Antonin le Pieux, MarcAurèle, Gordien III, etc. Cet exemplaire acquis par le Musée de Berlin a disparu au cours de la deuxième guerre mondiale. Un autre bijou du même type est également au Musée de Berlin (Greifenhagen 1970, I, pl. 50, n 3, inv. 11863, 185) provenant de Glinitsche (Russie méridionale) (fig. 103). D’autres bijoux très proches, à la fois par la couronne supérieure et par les pendeloques, se retrouvent dans les régions danubiennes (Popovic 1996, $\mathrm{n}^{\circ}$ 80, p. 177) et sont datés du IIIe s. 
103- Paire de pendants d'oreilles.
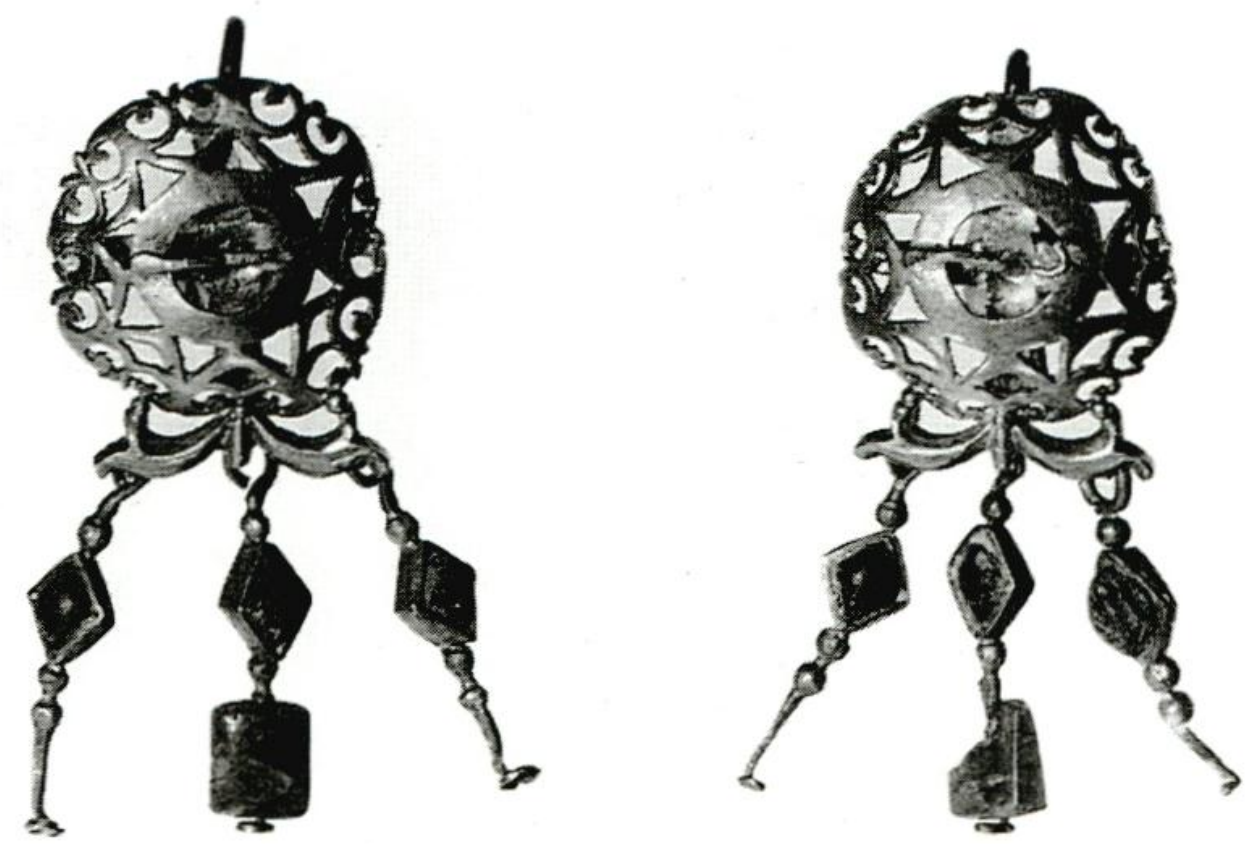

STAATLICHE MUSEUM, ANTIKENSAMMLUNg BERLIN, $\mathbf{N}^{\circ}$ INV. 11863,185. CL. JUTTA TIETZ

\section{Bague}

Inv. 93 I 20110

Or

Larg. max. 2,7 cm; long, chaton $1,6 \mathrm{~cm}$; diam. int. $1,85 \mathrm{~cm}$.

Poids : $27 \mathrm{~g}$.

L'anneau est massif, polygonal à l'extérieur, circulaire à l'intérieur. Il s'élargit aux épaules pour recevoir le chaton. Un décor de moulures ménage une large bande lisse au centre de l'anneau. Le chaton ovale, soudé sur l'anneau, présente dans un cadre saillant composé de deux bandes d'or en biseau, un décor en relief obtenu au repoussé dans une bordure perlée. Le motif central des deux mains croisées est une image de la dextrarum iunctio. Ce symbole de l'engagement, connu sur les monuments sculptés, apparaît aussi à partir du IIIe s. sur de petits objets comme les bagues que l'on considère alors comme des anneaux de mariage ou de fiançailles (fig. 104). 
104- Bague, vue d'ensemble, chaton
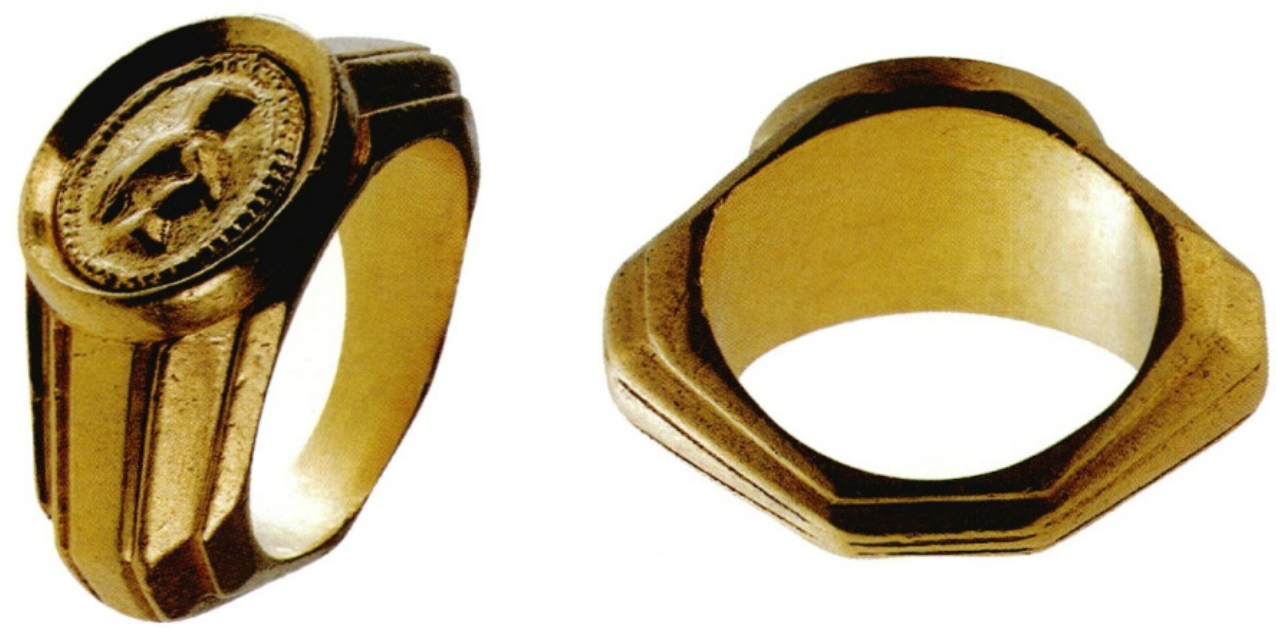

Le motif peut être soit en relief comme ici, soit gravé sur une intaille, sertie dans le chaton. Parmi les nombreux exemplaires d'époque romaine répertoriés souvent sans provenance assurée, on citera la bague du trésor de Thetford (Johns, Potter 1983, $\mathrm{n}^{\circ} 10$, p. 87). Une bague de Grovely Wood (Wiltshire) présente la particularité d'un chaton en or, très proche de celui de la bague de Vaise, disposé sur un anneau en argent (Johns 1996, p. 63, fig. 3. 24) (fig. 105).

105- Bague de Grovely Wood.

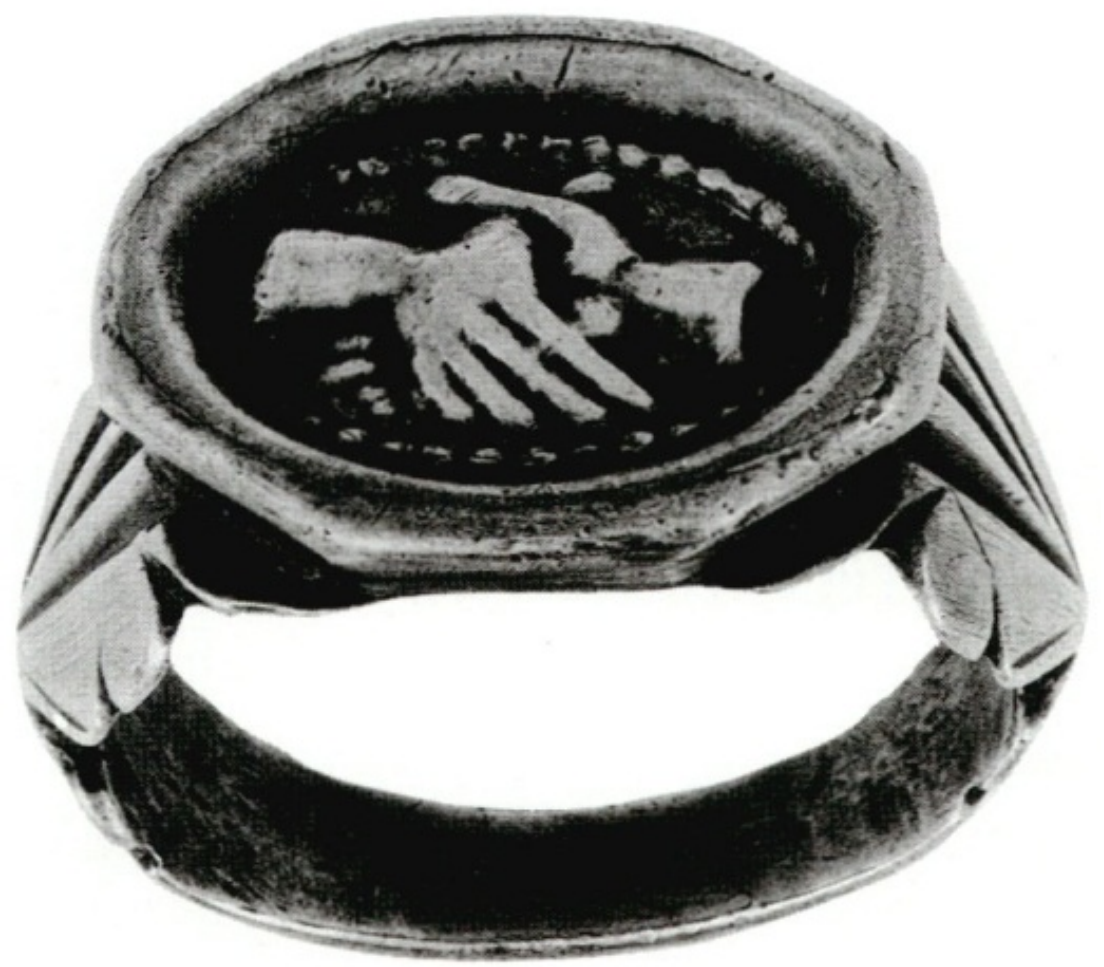

LONDRES, BRITISH MUSEUM, DÉPARTEMENT OF ROMANO-BRITISH ANTIQUITIES, N INV. 1911-10-261 


\section{Bague}

\section{Or et nicolo.}

Larg. max 2,4 cm ; larg. chaton $1,8 \mathrm{~cm}$; diam. int. $2 \mathrm{~cm}$.

Poids : $13,4 \mathrm{~g}$.

Bague à anneau circulaire, s'élargissant en triangle aux «épaules ", de chaque côté du chaton. Ces épaules présentent un décor ajouré de rinceaux et de peltes. Le chaton est constitué d'une intaille, un nicolo gravé ovale, dans une épaisse monture polygonale. Le motif figuré est un personnage masculin debout se déplaçant vers la droite, sur la pointe des pieds dans une attitude dansante. Le buste de face est enveloppé dans un manteau court à nombreux plis, maintenu par une main sur la poitrine. L'autre main tient une grande palme disposée en oblique. La tête semble ceinte d'un gros bandeau. La ligne de sol sur lequel se déplace le personnage est bien indiquée. La gravure est d'une très grande finesse, détaillant aussi bien la musculature du personnage que les plis du drapé. Par comparaison la tête paraît plus caricaturale (fig. 106).

106- Bague avec chaton, détail de l'épaule
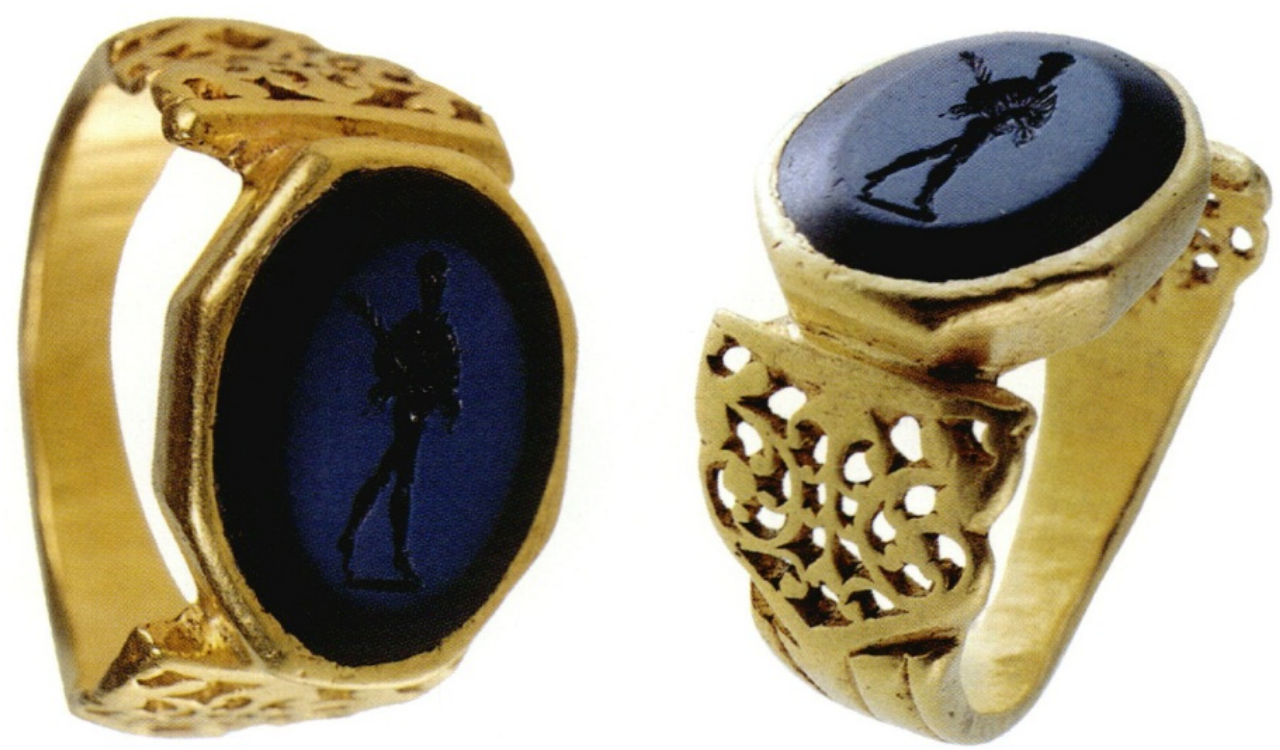

Le personnage ne paraît pas aisément identifiable. Le type de l'athlète portant une palme n'est en général pas vêtu. Le vêtement lui-même est mal reconnaissable. Nous ne sommes pas en présence d'une iconographie courante pour la glyptique romaine en Gaule. En revanche, la bague rentre dans des séries bien connues en Gaule (Guiraud 1988, p. 80, type 3) aussi bien pour la forme que pour le décor ajouré de l'anneau que l'on retrouve sur un exemplaire en argent de Mons-Boubert (Amandry et al. 1987, p. 35) ou encore sur une bague à chaton monétaire du Cabinet des Médailles $\left(n^{\circ} 508\right)$ où l'attache de l'anneau est du même type et le décor découpé en volutes végétales et peltes analogue à celui de la bague de Vaise. 


\section{Médaillon monétaire}

31

\section{Or.}

Diam. 3,2 cm ; haut, avec bélière $3,6 \mathrm{~cm}$.

Poids $10 \mathrm{~g}$.

La monnaie, un aureus de Gordien III, est prise entre deux étroits bandeaux d'or disposés en biseau. Une collerette circulaire est soudée à l'ensemble, le décor ajouré, découpé dans la feuille d'or, est constitué d'une suite de 18 motifs de peltes orientées vers l'extérieur de la collerette (fig. 107).

107- Médaillon monétaire
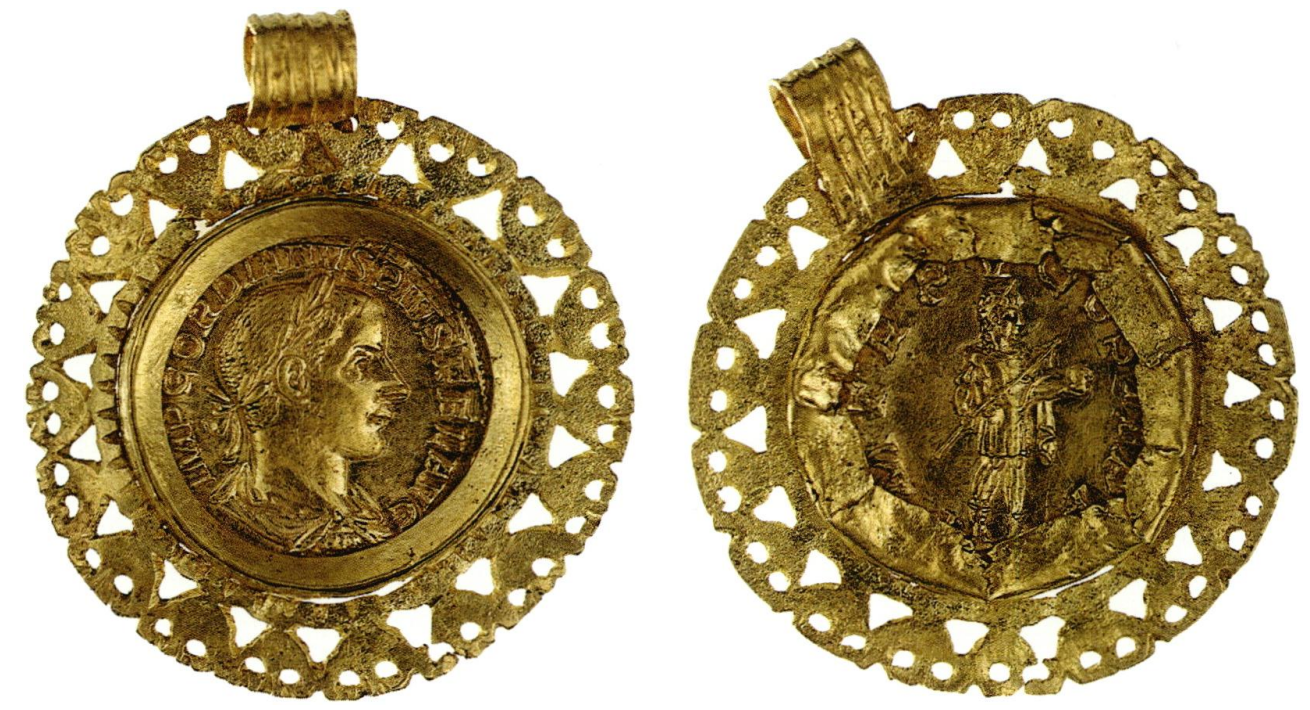

Agrandissement x 1,4

Une bélière de suspension constituée d'une mince bande d'or à cannelures est soudée au revers du médaillon et simplement rabattue sur l'avers. Comme toujours sur ce type de monture, le revers, non visible, est sommairement traité. Le biseau est grossièrement rabattu sur la monnaie, empiétant sur la légende et le décor ajouré n'est pas poli.

Les couronnes de peltes sont un des nombreux motifs de bordure utilisés pour des pendentifs variés en bijouterie romaine et en particulier pour les médaillons monétaires. On peut citer deux exemplaires du Cabinet des Médailles, des ciurei de Gordien III ( $n^{\circ} 156$ et 157 ) et deux médaillons appartenant à un collier monétaire apparu sur le marché antiquaire ces dernières années (Catalogue Greek and Roman Coins 1991, n² 264 e et f, p. 199-200) (fig. 108).

\section{Les bijoux monétaires}

Numismates et spécialistes de la bijouterie ont depuis longtemps noté l'utilisation, dans le monde romain, de monnaies d'or comme éléments décoratifs de bijoux. Les monnaies, des aurei le plus souvent, peuvent ainsi orner des chatons de bague, des fermoirs de bracelet ou encore des pendentifs de collier. Ce phénomène, peut être 
originaire d'Egypte et ce dès l'époque hellénistique (Vermeule 1975), est très répandu en Gaule romaine et particulièrement au IIIe s. De nombreuses trouvailles de trésors rassemblant monnaies et bijoux, dont des bijoux monétaires, ont en effet pu être répertoriées ces dernières années (Brenot, Metzger 1992). Ces bijoux monétaires utilisant la monnaie de la même manière qu'une intaille ou un camée, s'intègrent alors dans des séries de bijoux bien connues par leur typologie, leurs décors et leurs techniques de fabrication. Les bagues ornées d'une monnaie sont assez peu nombreuses. Elles s'inscrivent dans un groupe dont les anneaux s'élargissent pour rejoindre le chaton et dont les "épaules" triangulaires sont ornées soit de volutes moulées ou de motifs végétaux découpés. Le chaton est rond ou polygonal. Les monnaies sont placées du côté de l'effigie impériale. Un exemple particulièrement remarquable est une bague d'Autun dont le chaton est orné d'un aureus de Postume (Cabinet des Médailles, $\mathrm{n}^{\circ} 509$ bis ; Brenot, Metzger 1992, n² 23, pl. 4).

Rares également sont les bracelets garnis de monnaies. C'est pourquoi on insistera sur la paire de bracelets ayant fait partie du trésor des Lazaristes dont les fermoirs s'ornaient d'aurei de Commode (TAF V/l, p. 55, $\mathrm{n}^{\circ}$ 12) (fig. 125).

Les pendentifs de collier dont l'élément central est une monnaie constituent de loin la catégorie la plus abondante des bijoux monétaires. Ces médaillons sont formés d'une monnaie sertie dans une monture plus ou moins ouvragée, munie d'une bélière de suspension. Ce principe de fixation de la monnaie est simple : elle est maintenue entre deux bandes étroites d'or dont l'une est rabattue sur le revers. À cet ensemble est soudée une collerette circulaire ou polygonale décorée. Un petit ruban d'or décoré de cannelures moulées est fixé sur cette monture de manière à en permettre la suspension à une chaîne métallique ou à un cordon de cuir ou de tissu. On peut distinguer plusieurs types de décor des collerettes. Une première consiste en une alternance de petits arceaux plus ou moins ajourés et de triangles cannelés. Ce type est largement répandu. Une autre formule, plus rare semble-t-il est constitué d'une succession de peltes. Ce motif en forme d'écusson à la pointe plus ou moins arrondie et terminée par des volutes, est très fréquent dans le répertoire décoratif romain. Les pointes sont en général soudées à l'entourage de la monnaie et les volutes tournées vers l'extérieur de la collerette. C'est à ce type que se rattache le pendentif de Vaise.

Aux quelques exemplaires de comparaison déjà cités, on peut ajouter un médaillon, avec aureus de Caracalla, provenant de Rhénanie (Hamminkeln-Musée de Bonn, Brenot, Metzger 1992, n 78) et un médaillon avec aureus de Julia Domna (Lambèse-Musée d'Alger, Brenot, Metzger 1992, $\mathrm{n}^{\circ}$ 63). Ce type de bordure se retrouve aussi sur des pendentifs de colliers autres que les médaillons monétaires. Le collier à nombreux pendentifs du trésor de Naix (Lienard 1881, pl. XXXVIII, 1) offre pour les deux camées, l'un à l'effigie de Julia Domna, l'autre avec une Minerve casquée, des collerettes à peltes, d'un type plus complexe, il est vrai. Le pendentif monétaire de Vaise est seul de son espèce dans le trésor. La chaîne (ou le lien) à laquelle il était suspendu n'a pas été conservée. On connaît ainsi plusieurs exemples de bijoux monétaires isolés parmi d'autres éléments de parure découverts en Gaule. Mais l'usage était cependant fréquent de regrouper plusieurs médaillons sur une même chaîne (fig. 108). Le collier d'Eauze en compte trois (Schaad 1992, p. 22, 23), le collier de Naix en compte six, quatre médaillons monétaires et deux camées (Brenot, Metzger 1992, n 35, pl. 2). Enfin le trésor de Beaurains comprenait sept pendentifs avec des montures très ouvragées (Bastien, Metzger 1977, p. 177-186, pl. IX et X). Dans ces différents cas les médaillons se 
répartissent sur la chaîne grâce à de petits tubes d'or, sortes de coulants de séparation, évitant un chevauchement désordonné au centre du collier.

108- Collier monétaire.

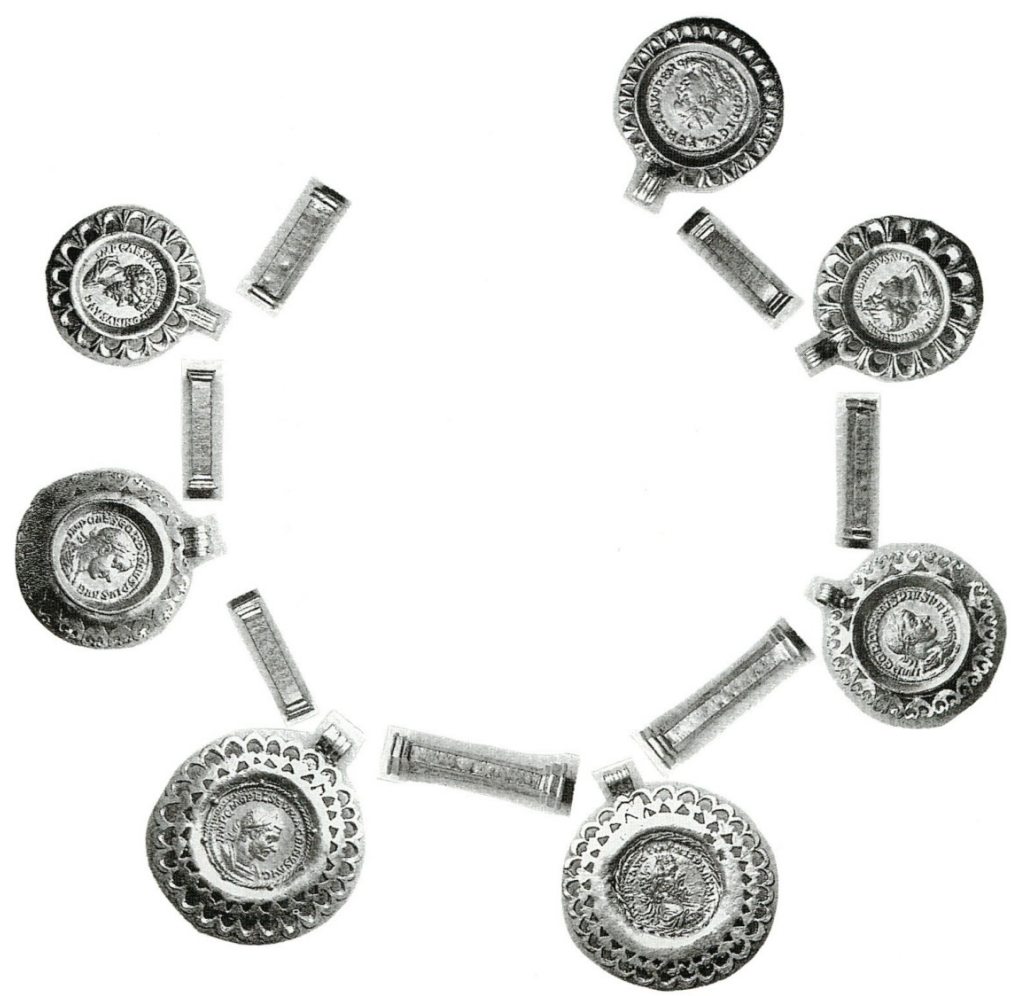

Essai de reconstitution, de gauche à droite, les monnaies d'or sont aux effigies de Marc Aurèle, Gordien III, Macrin, Septime Sévère, Gordien III, Hadrien, Valérien I. Marché antiquaire 1991

L'utilisation de la monnaie comme élément d'un bijou pose différents problèmes que l'on a, ces dernières années, essayé de cerner à défaut de les résoudre pleinement. Si l'on prend en compte d'abord les trésors avec provenance assurée regroupant bijoux et monnaies, on peut commencer à déterminer les périodes d'utilisation des bijoux monétaires. Ils semblent apparaître à partir de l'époque de Septime Sévère, comme le montre le trésor des Lazaristes, et disparaître au début du IVe s., en tout cas à l'intérieur des frontières de l'Empire. En territoire barbare, des bijoux monétaires sont encore enfouis au cours du Ve s. Des tableaux récapitulatifs classés par ordre chronologique d'enfouissement sont très clairs à ce sujet (Brenot, Metzger 1992, p. 349-359). On note le nombre important de monnaies au nom des dynasties antonine et sévérienne ainsi que des empereurs gaulois. Par ailleurs, on a pu dresser des cartes de la répartition géographique des trouvailles des bijoux monétaires en Gaule. Et l'on constate une densité particulièrement forte de bijoux monétaires du IIIe s., dans la province de Lyonnaise avec une concentration en deux grandes zones, la première entre Lyon et Autun, la seconde entre Orléans et Rouen (fig, 109). On remarque aussi une diffusion au Nord-Est vers la Belgique et la Germanie. À Lyon même et dans la région, en dehors du trésor des Lazaristes déjà cité, on connaît des trouvailles de bijoux monétaires : à Neuville-sur-Ain, un aureus de Victorin (TAF V/l, p. 29, n 31), à SaultBrenaz, un multiple d'or de Gallien (Brenot, Metzger 1992, n 9 ; TAF V/l, p. 33, n 41), 
enfin à Autun, deux bijoux monétaires d'Elagabale et d'Otacilie (Brenot, Metzger 1992, $\left.n^{\circ} 13\right)$.

109- Diffusion des bijoux monétaires jusqu'au Rhin dans la partie occidentale de l'empire aux trois premiers siècles

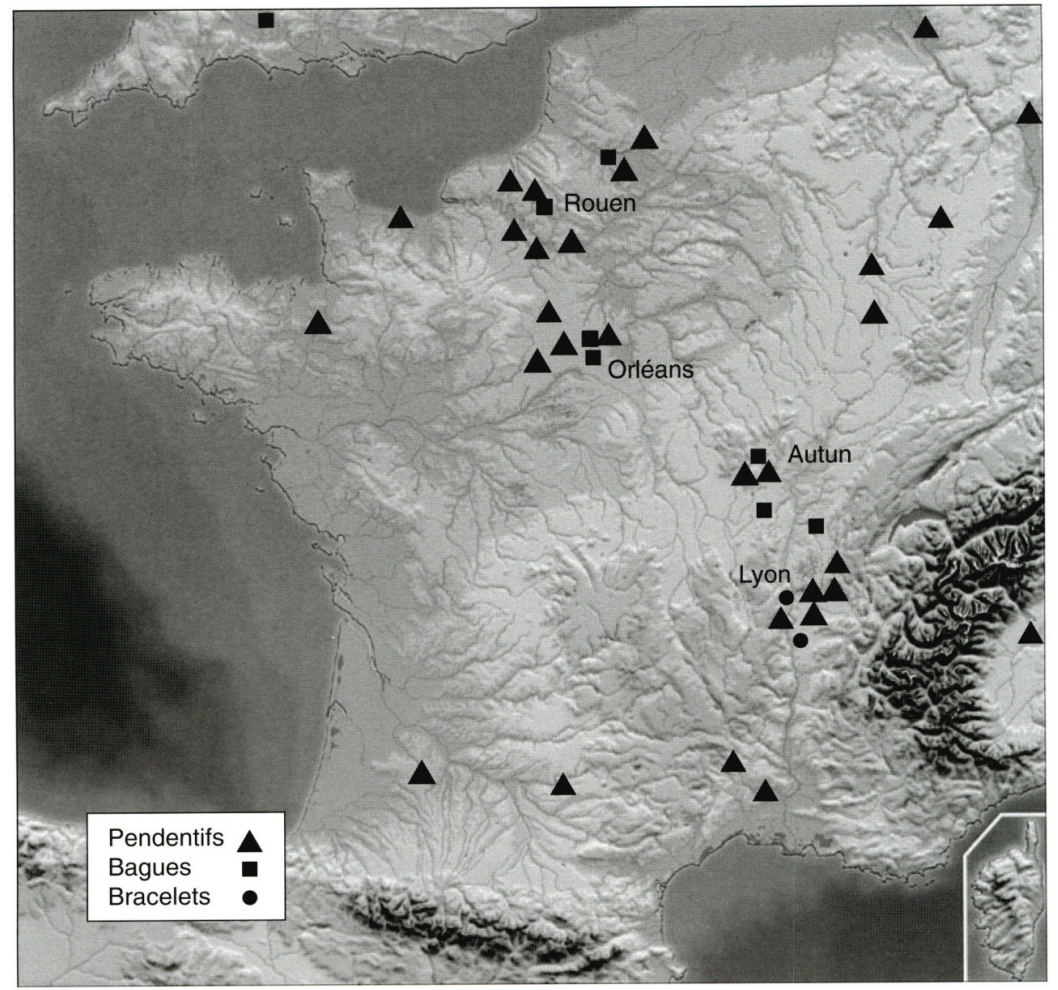

41 Répartition géographique et repères chronologiques ainsi délimités, d'autres problèmes demeurent comme celui, essentiel, de savoir pourquoi l'on a utilisé une monnaie comme élément de bijou. Diverses hypothèses ont été avancées pour expliquer cet usage détourné de la monnaie, ce retrait de la circulation. On a pensé à une forme de thésaurisation, bien limitée, il faut le reconnaître. On a aussi cherché à déterminer le caractère idéologique de ces bijoux portant l'effigie impériale (le revers n'est jamais visible) qui pourrait témoigner de l'attachement du possesseur du bijou à un empereur ou à une dynastie particulière. On remarquera toutefois que rien ne permet d'affirmer que les colliers à pendentifs monétaires soient destinés aux hommes. Ils ont très souvent été retrouvés, c'est le cas pour le trésor de Vaise, avec des bijoux typiquement féminins, colliers et pendants d'oreilles. Seules les bagues à chaton monétaire à anneaux de grandes dimensions pourraient être considérées comme des bijoux masculins.

Quelles que soient les raisons politiques, idéologiques ou plus simplement les phénomènes de mode qui aient incité à transformer des monnaies en objet de parure, il resterait à déterminer les ateliers fabriquant de tels objets. La concentration des trouvailles autour de certains pôles géographiques a conduit à imaginer des ateliers dans des villes comme Lyon ou Rouen, ce qui serait tout à fait plausible, mais n'impliquerait en aucun cas l'exclusivité de la fabrication. On a aussi envisagé à l'inverse, l'existence d'ateliers se déplaçant d'une région à l'autre. Cependant, sauf à découvrir véritablement un atelier d'orfèvre avec sa production, on ne peut vérifier aucune de ces hypothèses. 


\section{La bijouterie romaine} grands musées européens ont permis de décrire l'évolution des différents types de bijoux et les techniques de fabrication ou de décor en usage chez les orfèvres pendant toute la durée de l'empire romain. C'est ainsi que l'on distingue une bijouterie du Ier s. bien attestée à Pompei, à Herculanum et dans toute la zone dévastée par l'éruption du Vésuve en 79 de notre ère. On connaît à cette époque de très longues chaînes d'or portées en baudrier, des pendants d'oreilles et des bracelets composés de cupules d'or. L'emploi des perles et surtout des pierres de couleur est limité et les bijoux semblent relativement sobres par rapport à la luxueuse vaisselle d'argent contemporaine. On voit également apparaître au début de l'empire un phénomène particulier à la société romaine : la folie des bagues, rapportée par Pline (Histoire Naturelle XXXIII, 24). À l'origine l'anneau d'or distingue les membres de l'ordre équestre puis un phénomène de mode entraîne la multiplication de ce type de parure. On sait qu'il existait à Rome des collections de bagues ou dactyliothèques. Pendant toute la durée de l'empire, les types d'anneaux d'or ou d'argent à chatons gravés ou sertis sont extrêmement nombreux et variés.

Le goût pour la polychromie se développe au IIe s. et ne se démentira pas jusqu'à la fin de l'empire. Les pierres dures (cornaline, onyx, agate) ou précieuses (émeraude, améthyste, grenat) venues d'Orient sont largement utilisées en chatons de bague, éléments de colliers ou pendentifs variés. Un nouveau procédé décoratif qui apparaît au IIIe s. va connaitre un extraordinaire succès dans la bijouterie romaine : c'est ce que l'on appelle l'opus interrasile d'après un terme emprunté à Pline (Histoire Naturelle XII, 94) s'appliquant au travail du bois. Il s'agit d'un découpage de la feuille d'or suivant un dessin tracé au préalable. Ce travail qui comme une dentelle fait jouer les pleins et les vides, l'ombre et la lumière, décore aussi bien les bagues, les pendentifs et les bracelets du IIIe au Ve s.

À côté de cette évolution générale de la bijouterie et en dépit d'une certaine homogénéité des types de parure féminine à la mode dans le monde romain, on s'est attaché également à mettre en évidence les particularismes locaux suivant les différentes régions de l'empire. On a depuis longtemps noté l'abondance des bijoux représentés sur les stèles funéraires masculines et féminines de la région de Palmyre dans la province romaine de Syrie (Sadurska, Bounni 1994). Certains types de boucles d'oreilles ont été retrouvés en Syrie, au Liban et non dans la partie occidentale de l'empire. En Egypte, les portraits de bois peints posés sur les momies nous indiquent les bijoux en faveur dans cette province (Walker, Bierbrier 1997).

Récemment les particularismes de la Bretagne romaine ont bien été mis en évidence par C. Johns (Johns 1996). En Gaule, les comparaisons entre les différents trésors, soit connus depuis longtemps, soit publiés dernièrement permettent de dégager certains traits spécifiques. On a déjà cité le cas des bijoux monétaires particulièrement abondants au IIIe s. en Gaule.

Dans le cas du trésor de Vaise, on insistera sur le type de collier à maillons en forme de nœud d'Héraclès alternant avec des prismes d'émeraude que l'on ne retrouve pas ailleurs dans le monde romain, du moins sous cette forme, et qui doit constituer un bijou typiquement gallo-romain. Les bagues sont également plutôt caractéristiques 
d'une production de Gaule comme le montrent les études d'H. Guiraud (Guiraud 1988, p. 80 , type 3). En revanche bracelets et pendants d'oreilles ont des équivalents dans d'autres provinces de l'empire. On notera enfin, comme dans la plupart des trésors trouvés en Gaule, l'absence de fibule.

À côté de ces bijoux d'or et de pierres semi-précieuses, les deux bracelets d'argent, en dépit de leur simplicité, constituent des témoignages intéressants sur une bijouterie d'argent assez mal connue en Gaule comme dans l'ensemble du monde romain en raison sans doute de la fragilité même du matériau, beaucoup moins solide que l'or.

Ce petit lot d'objets de parure constitue vraisemblablement un ensemble de bijouterie familiale, ensemble cohérent, de bonne qualité, mais sans pièce exceptionnelle (ni par le type du bijou, ni par l'exécution) qui reflète la bijouterie à la mode dans les classes aisées en Gaule au milieu du IIIe s.

\section{Le trésor des Lazaristes}

Parmi les collections du Musée de la civilisation gallo-romaine se trouve un très important ensemble de appelé Trésor de Lyon ou Trésor des Lazaristes. Les objets ont été découverts en 1841 au cours de travaux de fondations entrepris sur un terrain appartenant aux Frères de la Doctrine chrétienne sur la colline de Fourvière entre leur chapelle et la montée St. Barthélémy, terrain possédé auparavant par les Lazaristes. Les objets rassemblés dans une cavité creusée dans un mur romain avaient manifestement été cachés intentionnellement. L'ensemble regroupait plus de 2000 monnaies d'argent de Vespasien à Septime-Sévère, quelques monnaies d'or et un lot important de bijoux d'or et pierres. Examinés dès la découverte par le Dr A. Comarmond, conservateur des musées archéologiques de Lyon, les bijoux entrèrent au Musée de Lyon en 1843, et furent publiés en 1844 par A. Comarmond.

Ces bijoux sont trois paires de pendants d'oreilles, (fig. 110) sept colliers dont une très longue chaîne double, trois paires de bracelets et un bracelet isolé de types variés, une petite fibule, six anneaux dont cinq très étroits (bagues d'enfant ?) et une curieuse chaîne plate terminée par des pendeloques peut-être, un ornement de coiffure. Quelques éléments épars, perles et fermoir de collier, une aiguille et une tige en os complétaient l'ensemble.

Ces bijoux d'une très grande qualité d'exécution peuvent se caractériser par l'emploi particulièrement abondant des pierres de couleur, grenat, émeraude, améthyste pour les colliers et pendants d'oreilles. Par ailleurs le corail utilisé pour la chaîne double, les perles et enfin la pâte de verre soulignent l'importance de la polychromie dans ces objets de parure.

Les bracelets témoignent en outre de la faveur dont jouissent les portraits impériaux avec le buste en relief de Crispine décorant une des paires, et les aurei de Commode enchâssés dans les fermoirs de deux autres bracelets (fig. 125). La présence des monnaies permet d'envisager un enfouissement à l'extrême fin du IIe s. 

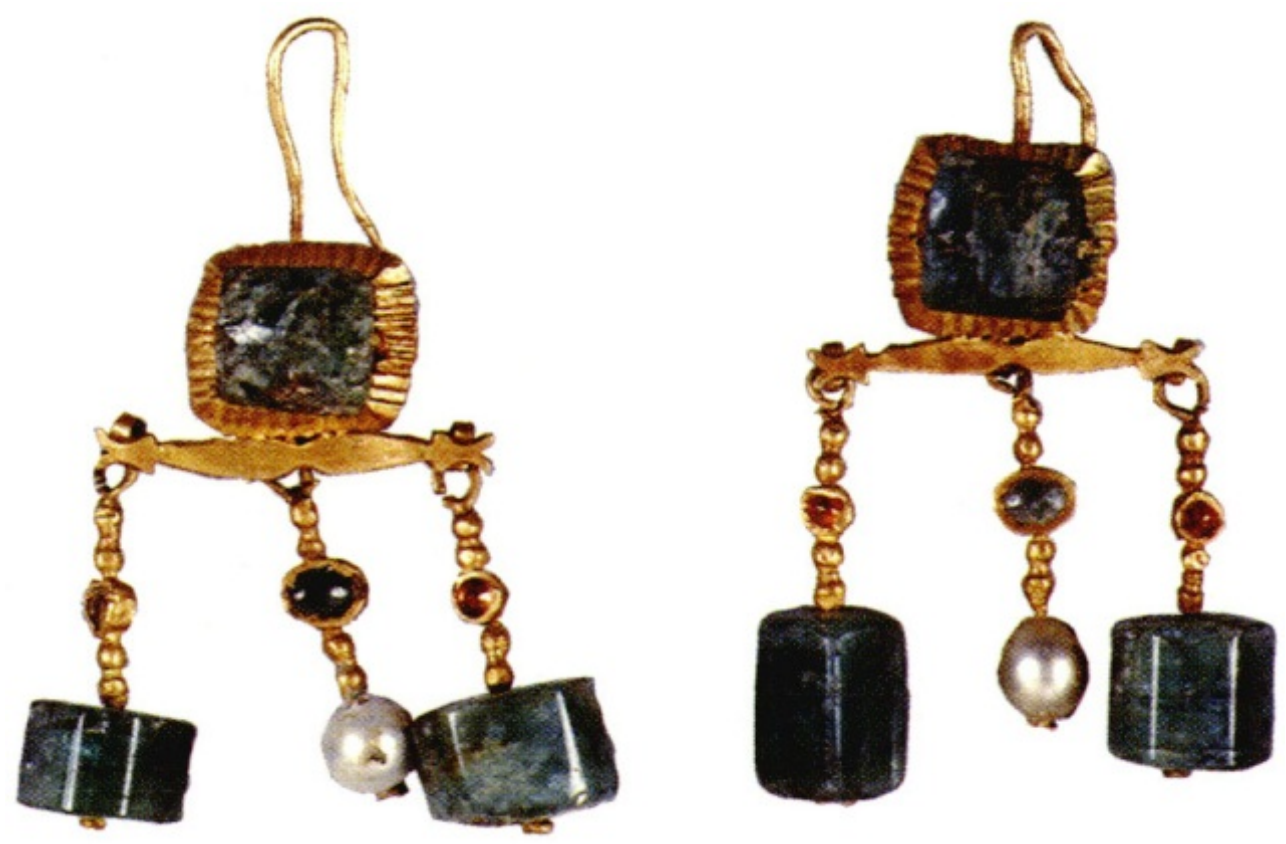

Trésor des Lazaristes.

Lyon, MCGR

\section{Cippe de Primilla}

Un cippe funéraire en calcaire au Musée de la civilisation gallo-romaine (Espérandieu $n^{\circ}$ 1787) montre dans une niche garnie d'une coquille un buste de jeune femme parée de bijoux. La défunte, Primilla, porte des pendants d'oreilles à plusieurs pendeloques, un collier de perles rondes et allongées ainsi qu'un bracelet (fig. 111). Elle tient dans la main gauche un coffret ouvert d'où elle retire un collier de perles rondes. Ce monument funéraire lyonnais, datable de la période 150/250, peut servir d'illustration des tendances de la bijouterie en Gaule romaine. 


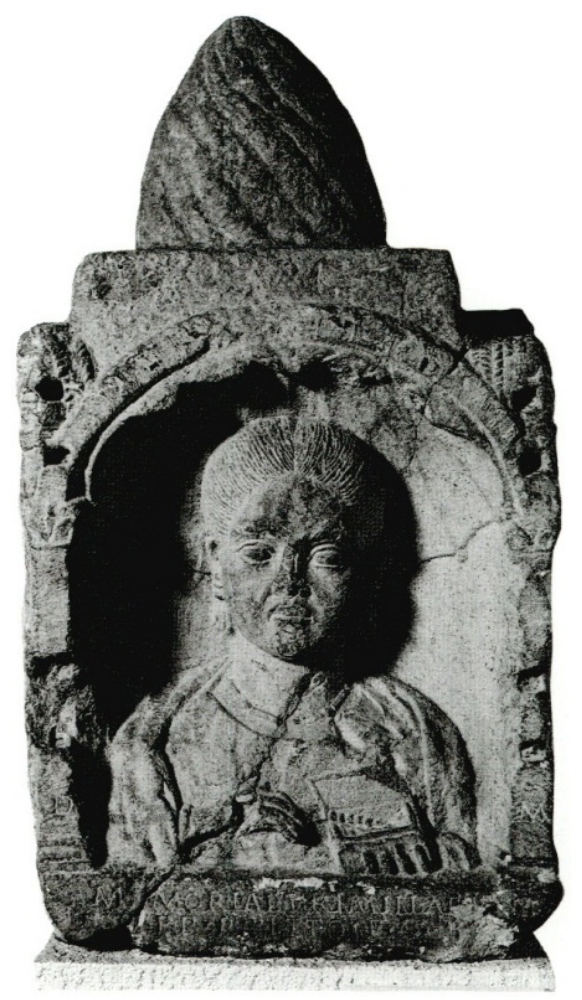

Inscription : D(iis) M(anibus), m[e]moriae Primillae, f[i]li[ae], Ter(entius) Pritto f(aciendum) c(uravit). Aux dieux Mânes, à la mémoire de Primilla, sa fille, Terentius Pritto a pris soin d'élever (ce monument).

Lyon, Musée de la civilisation gallo-romaine

\section{Gemmologie}

La détermination des minéraux des bijoux a été effectuée au laboratoire de physico-chimie des matériaux luminescents Claude (université Bernard, Lyon I) par M. Boudeulle, professeur, et G. Panczer, maître de conférences, au moyen de la spectroscopie "Raman".

L'effet "Raman", découvert en 1928 par le physicien indien C. V. Raman, est une réémission de lumière appelée par les physiciens diffusion inélastique car elle s'effectue avec changement de fréquence. Cette diffusion inélastique est indépendante de la longueur d'onde du rayonnement excitateur et ne dépend que du corps étudié dont elle permet ainsi l'identification. La technique, non destructive, consiste à envoyer sur l'échantillon une lumière excitatrice, fournie par un laser puissant, et focalisée par un microscope. La lumière diffusée par l'échantillon, après avoir traversé des dispositifs dispersifs, termine son trajet dans un détecteur permettant d'enregistrer instantanément son spectre. L'acquisition du spectre "Raman" peut ainsi caractériser sans ambiguïté la nature d'un minéral ou d'une gemme, par comparaison avec des spectres de référence. Les résultats obtenus sont les suivants :

- les minéraux du collier (catal. n 35 ) qui se présentent sous forme de prismes hexagonaux sont des béryls ;

- une paire de pendants d'oreilles (catal. $n^{\circ}$ 38) comporte aussi un béryl, encadré par deux perles fines. Le cabochon taillé de couleur rouge est un grenat de type 
rhodolite ;

- une autre paire de pendants (catal. $\mathrm{n}^{\circ}$ 37) montre des perles de forme hexagonale qui s'avèrent être des béryls, des cristaux vert foncé identifiés en tant que pâtes de verre, et une perle fine ;

- l'intaille d'une bague en or (catal. $n^{\circ} 40$ ) est constituée d'une couche inférieure violette et d'une couche supérieure blanchâtre. Le minéral identifié est du quartz plus ou moins bien cristallisé.

Pierre Michel, Michelline Boudeulle et Gérard Panczer

Université Claude Bernard, Lyon I 


\section{Les monnaies du trésor de Vaise}

\section{Gérard Aubin}

112- Les monnaies du trésor de Vaise

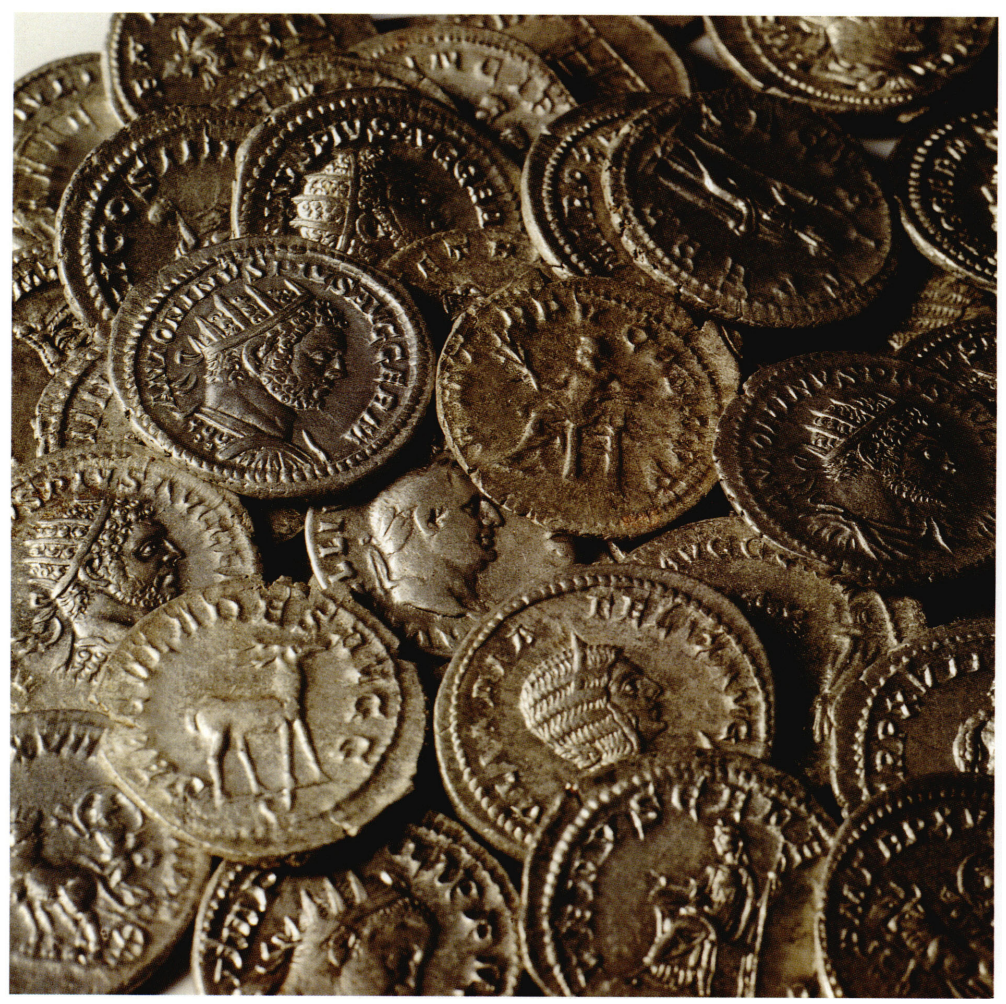

1 Au fond de la caisse en bois du second dépôt, à côté de vaisselle d'argent et de cuillers, et au-dessous de bijoux, une bourse, en matière organique, contenait 81 monnaies en argent - 29 deniers et 52 antoniniens - en bon état de conservation, dont l'arc chronologique s'étend de Vitellius (69) au règne conjoint de Valérien et Gallien (253-260), soit sur un laps de temps de presque deux siècles. 
2 Il faut y ajouter, au-dessus de la bourse, une monnaie d'or montée en pendentif déjà présenté dans la section précédente, mais qui avant d'être un bijou a eu une fonction monétaire.

3 Je donnerai d'abord le catalogue des monnaies, suivi de commentaires sur ce classement, avant de réfléchir à la composition de ce petit magot et de proposer une hypothèse sur les modalités de sa constitution.

\section{Catalogue des monnaies}

4 Les monnaies sont classées par règne, puis par atelier et lorsque cela est possible par émission. Chaque monnaie d'argent porte un numéro d'ordre de 1 à 81 . Le médaillon en or, décrit ici pour sa partie monétaire, n'est pas numéroté. La notice catalographique comporte : la légende du droit et le code indiquant le type du buste; la légende du revers accompagnée de la description du type ; le poids en grammes; la référence aux ouvrages usuels de classement. Les lettres illisibles sont restituées en minuscule entre crochets ; les césures dans les légendes sont indiquées par des tirets.

5 Toutes les monnaies sont reproduites (photographies de moulages). Elles sont conservées au Musée de la civilisation gallo-romaine à Lyon sous les numéros d'inventaire nº 9311051 à 81.

\section{Code descriptif des bustes}

\section{A : têtes}

$\mathrm{Al}$ : Tête à droite

A2 : Tête à droite avec pan de draperie sur l'épaule

\section{B : bustes cuirassés}

B1 : Buste cuirassé, vu de face, tête à droite

B2 : Buste cuirassé, vu de dos, tête à droite

\section{$8 \quad \mathrm{C}:$ bustes drapés}

$\mathrm{Cl}$ : Buste drapé, vu de face, tête à droite

$\mathrm{C} 2$ : Buste drapé, vu de dos, tête à droite

\section{$\mathrm{D}$ : bustes drapés et cuirassés (pteryges à l'épaule)}

D1 : Buste drapé et cuirassé, vu de face, tête à droite

D2 : Buste drapé et cuirassé, vu de dos, tête à droite

\section{E : impératrices}

El : Buste drapé, tête à droite

E2 : Buste drapé, tête diadémée à droite

E4 : Buste drapé, tête diadémée et voilée à droite

11 Ces descriptions concernent des antoniniens dont les effigies sont caractérisées par des têtes radiées (empereurs) ou des bustes sur croissant (impératrices). Pour les deniers (et pour Vaureus), la lettre code est précédée du signe * (tête laurée pour les empereurs, absence de croissant pour les impératrices) ou du signe + (tête nue des empereurs). 


\section{Les monnaies d'argent}

Références bibliographiques

BMC : Mattingly (H.), Carson (R.A.G.) et Hill (P.V.). - Coins of the Roman Empire in the British Museum. V-2 : Pertinax to Elagabalus. 2e ed., Londres, 1975.

Clay : Clay (C.L.) - The Roman Coinage of Macrinus and Diadumenian. Numismatische Zeitschrift, 93, 1979, p. 2140, pl. 4-5.

Cunetio = Besly (E.), Bland (R.) - The Cunetio Treasure. Roman Coinage of the Third Century AD. Londres, British Museum, 1983, 199p., 40pl.

Eauze $=$ Schaad (D.) ed. - Le trésor d'Eauze (Gers). Bijoux et monnaies du IIIe s. ap. J.-C. Toulouse, ed. APAMP, 1992, LXXIX + 435 p., 93 pl.

Elmer : Elmer (G.). - Die Münzprägung der gallischen Kaiser in Köln, Trier und Mailand. Darmstadt, 1941 (extrait des Borner Jahrbiicher, cahier 146, p. 1-106).

Hill : Hill (P.V.). - The Coinage of Septimius Severus and his Family of the Mint of Rome AD 193-217. Londres, 2e éd., 1977, 42 p., 2 pl.

RIC : The Roman Imperial Coinage.

I2. 31 BC - AD 69. Londres, 1984 (C.H.V. Sutherland)

II. Vespasian to Hadrian. Londres, 1926 (H. Mattingly et E.A. Sydenham)

III. Antoninus Pius to Commodus. Londres, 1930, (H. Mattingly et E.A. Sydenham)

IV-1. Pertinax to Geta. Londres, 1936 (H. Mattingly et E.A. Sydenham).

IV-2. Macrinus to Pupienus. Londres, 1938 (H. Mattingly, E.A. Sydenham et C.H.V. Sutherland).

IV-3. Gordian III to Uranius Antoninus. Londres, 1949 (H. Mattingly, E.A. Sydenham et C.H.V. Sutherland).

V-l. Valerian to Florian. Londres, 1927 (P.H. Webb).

\section{Vitellius (69)}

Atelier de Rome

Avril-juillet 69 
1.
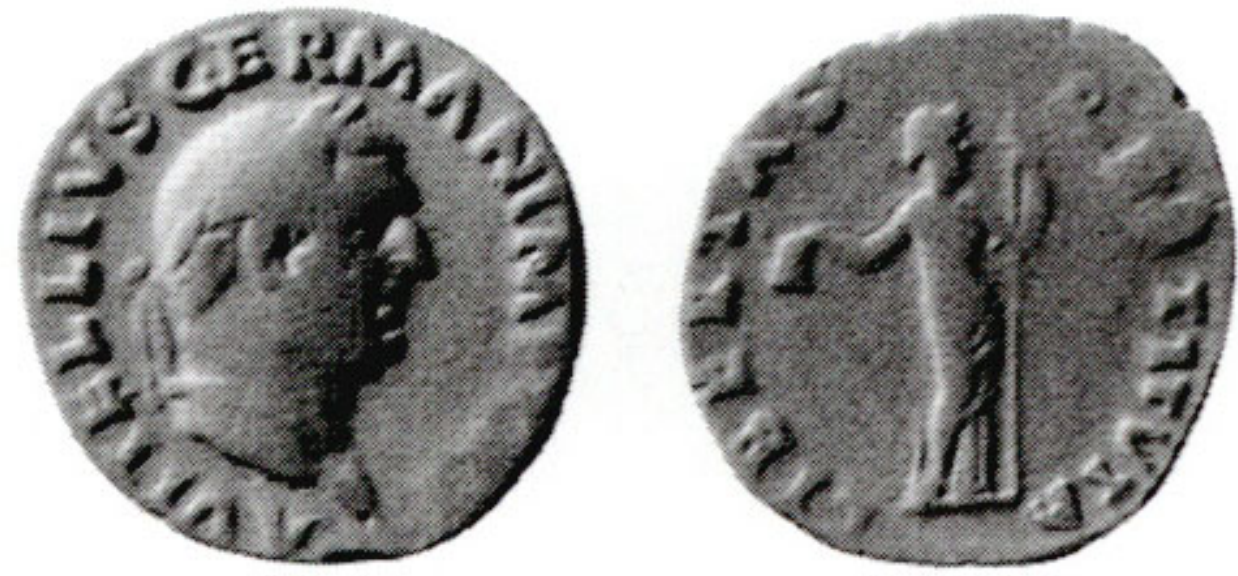

A VITELLIVS GERMAN IMP TR P, *A1

LIBERTAS RESTITVTA, Libertas, debout à droite, tenant de la main droite un bonnet phrygien et de la gauche, un sceptre vertical.

$3,09 \mathrm{~g}$

$R / C I^{2}$, p. $272 \mathrm{n}^{\circ} 81$

\section{Domitien (81-96)}

14 Atelier de Rome

$15 \quad 92$

2.
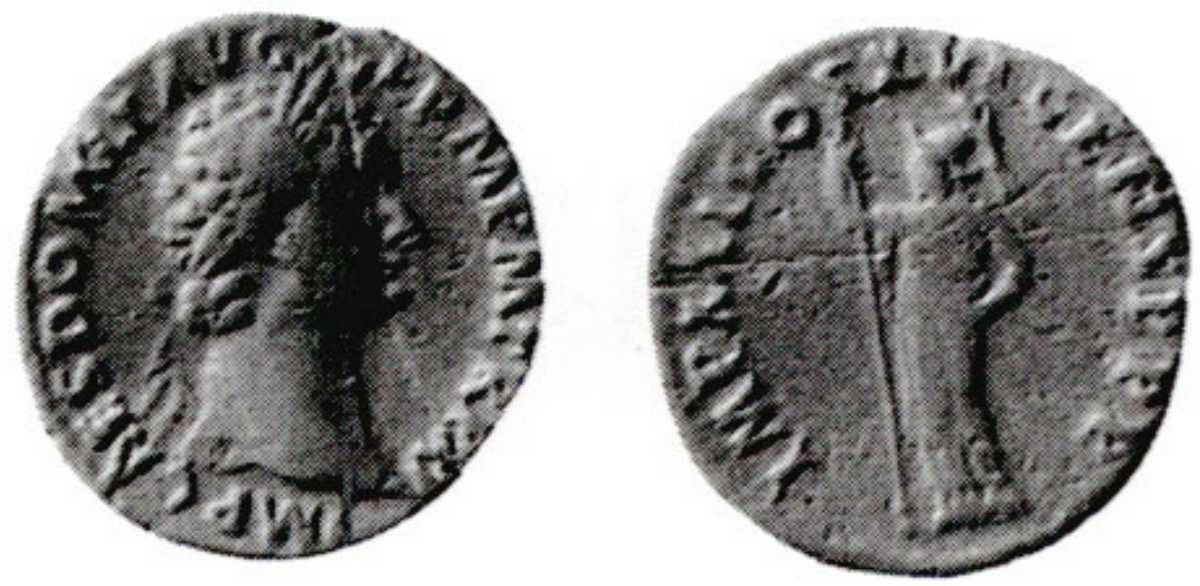

IMP CAES DOMIT AVG GERM P M TR P XI, *A1

IMP XXICOS XVI CENS P P P, Minerve à gauche tenant une lance de la main droite. 3,24 g RIC II, p. $173 \mathrm{n}^{\circ} 169$

Trajan (98-117)

16 Atelier de Rome

$17 \quad 100$ 
3.
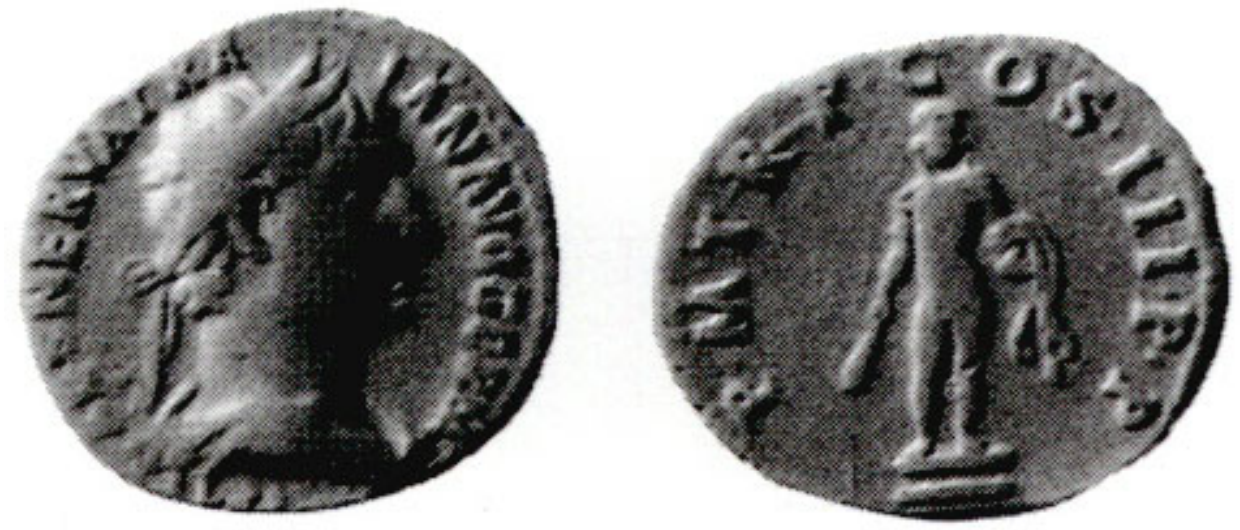

[imp c]AES NERVA TRA-IAN AVG GERM, *C1

$\mathrm{PM}$ TR $P \cdot \operatorname{COS} \cdot I I I \cdot P \cdot P$, Hercule debout de face, sur un autel tenant une massue et une peau de lion. $3,18 \mathrm{~g}$

RIC II, p. $247 n^{\circ} 37$

\section{$106-111$}

4.
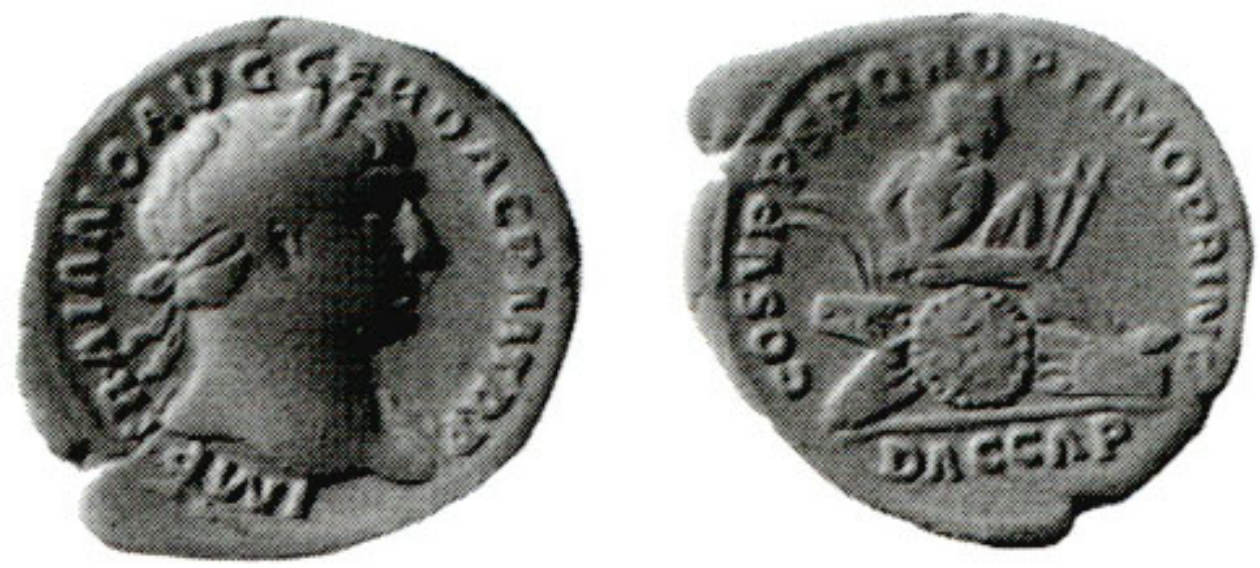

IMP TRAIANO AVG GER DAC P M TR P, *A2

COS V P P S P Q R OPTIMO PRINC, à l'exergue DAC CAP, Dace assis à droite, sur un trophée d'armes, bras attachés derrière le dos.

$3,12 \mathrm{~g}$

RICII, p. 250 n $^{\circ} 96$

106-111 
5.
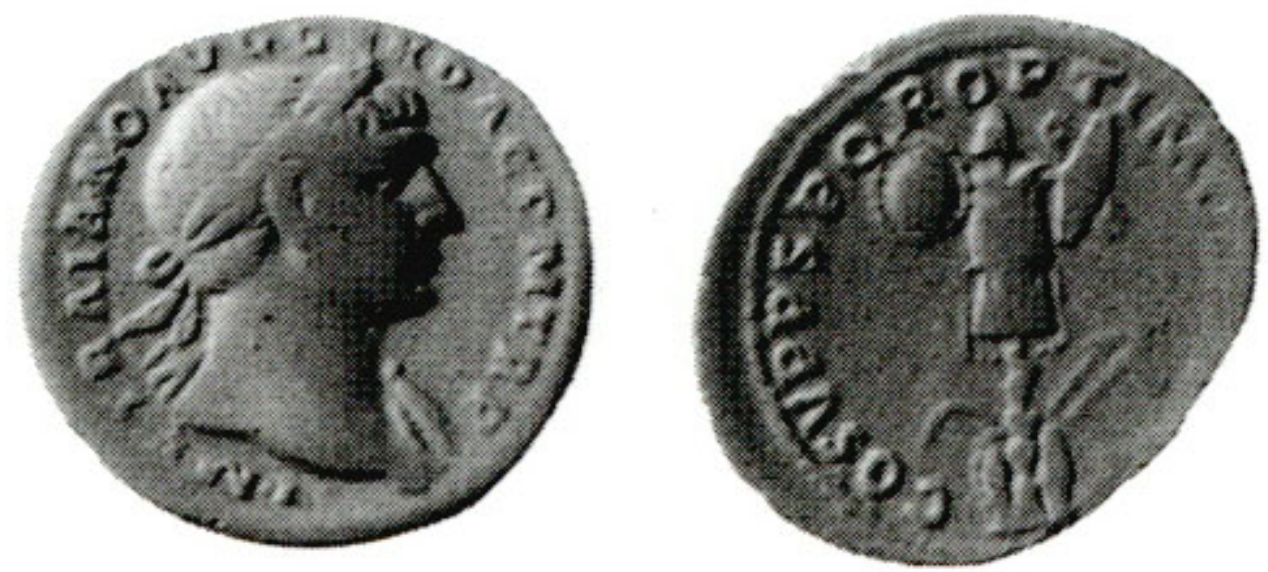

IMP TRAIANO AVG GER DAC P M TR P, *A2

COS V P P S P Q R OPTIMO [princ], trophée d'armes : un bouclier rond à gauche, deux ( ?) oblongs à droite ; au pied, deux petits boucliers, une épée recourbée à gauche et deux lances à droite.

$3,10 \mathrm{~g}$

RIC II, p. $254 n^{\circ} 147$ b

6.
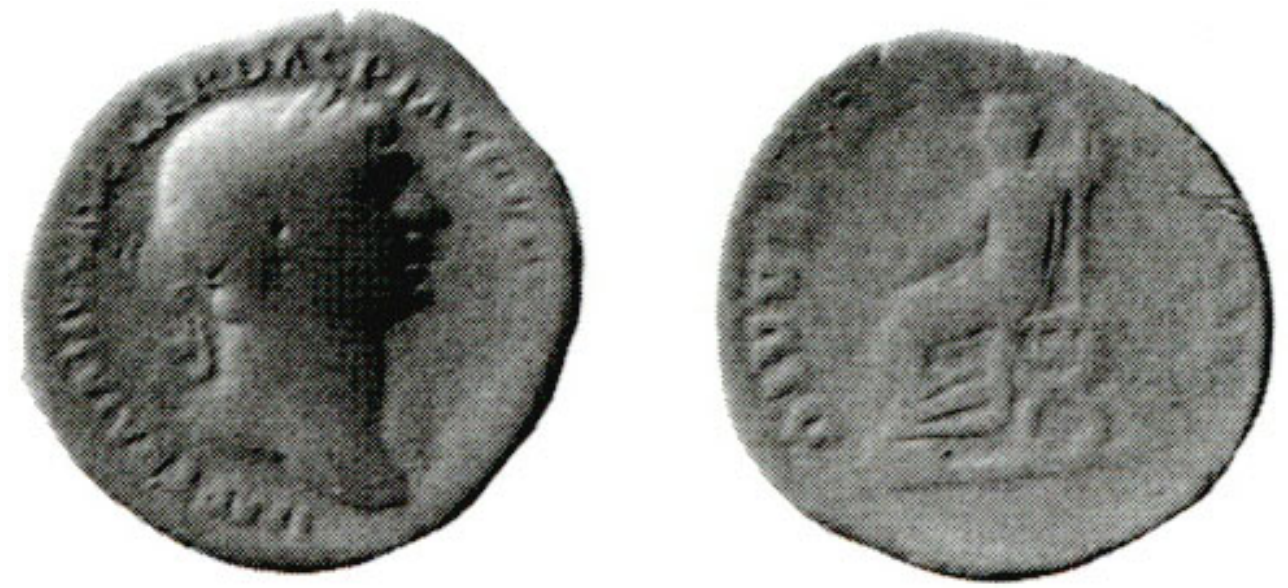

IMP TRAIANVS AVG GER DAC P M TR P COS VIP P, *A2

DIVVS PATER - TRAIAN, Trajan senior assis à gauche tenant une patère et un sceptre vertical.

$3,08 \mathrm{~g}$

RIC II, p. $261 \mathrm{n}^{\circ} 252$

\section{Antonin le Pieux (138-161)}

21 Atelier de Rome

$22 \quad 140$ 
7.
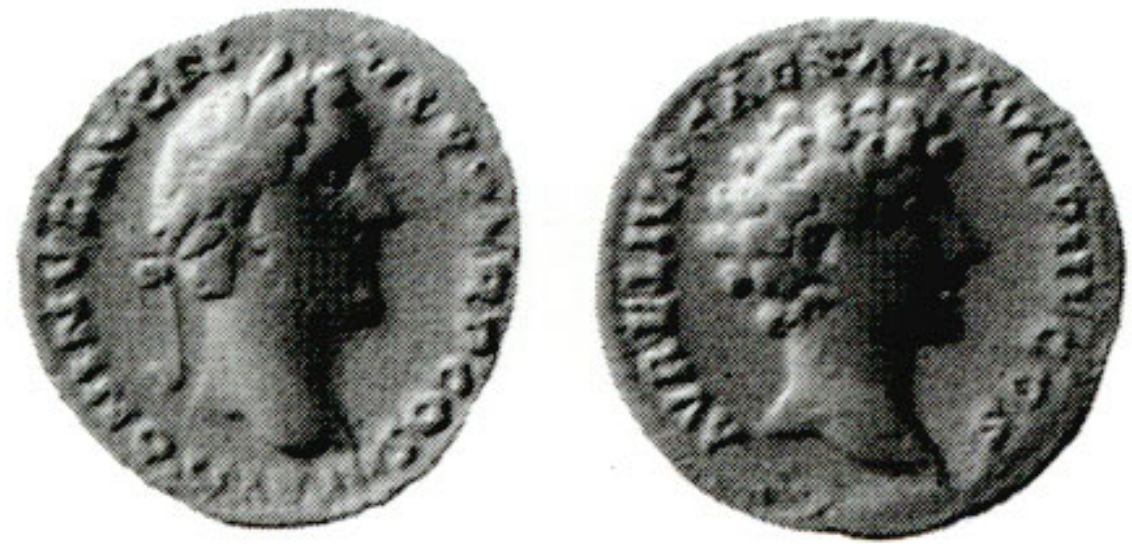

ANTONINVS AVG PI-VS P P TR P COS III, *A1

AVRELIVS CAESAR AVG PII F COS, tête nue de Marc-Aurèle à droite

$3,06 \mathrm{~g}$

RIC III, p. $78 n^{\circ} 417$ a

8.
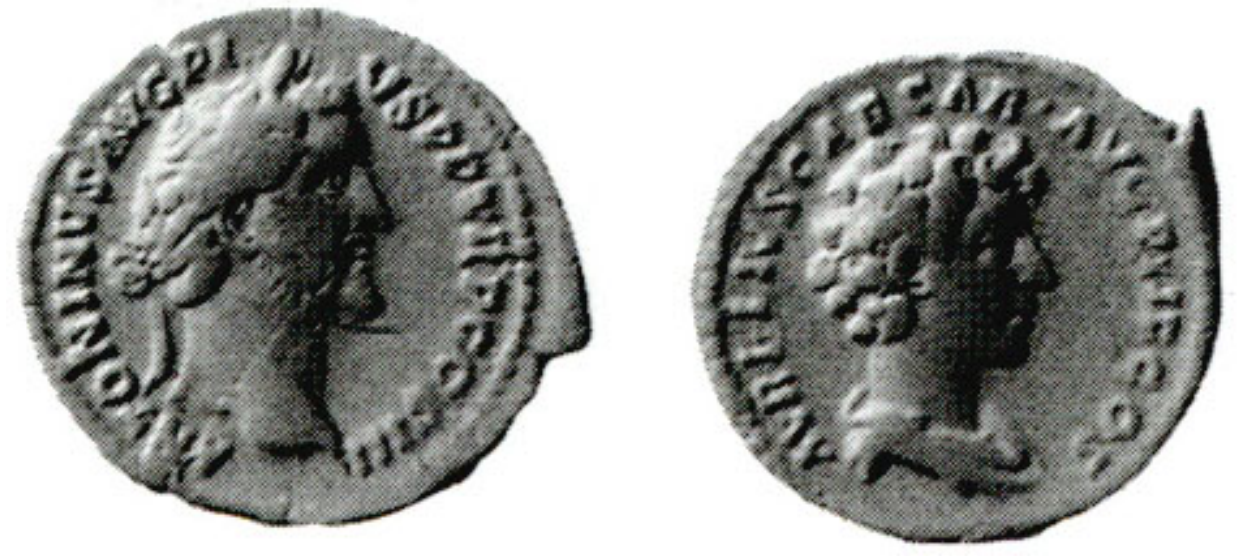

Même droit que $n^{\circ} 7$

AVRELIVS CAESAR AVG PII F COS, buste drapé, tête nue de Marc-Aurèle à droite. $2,98 \mathrm{~g}$

RIC III, p. $78 n^{\circ} 417$ b

151-152, pour Aelius César

9. 

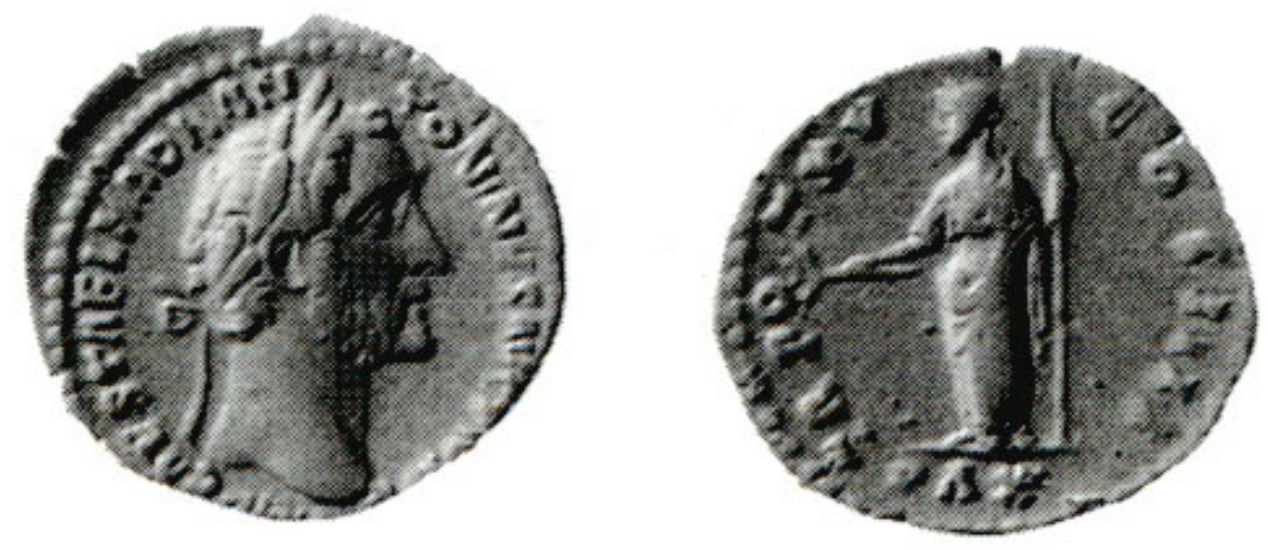

[imp] CAES T AEL HADR AN-TONINVS AVG PIVS P P, *AT

TR PO-T XV - COS IIII, à I'exergue PAX, Pax debout à gauche tenant un rameau et un long sceptre vertical.

$3,66 \mathrm{~g}$

RIC III, p. $52 n^{\circ} 216$ a (a)

\section{$159-160$}

10.
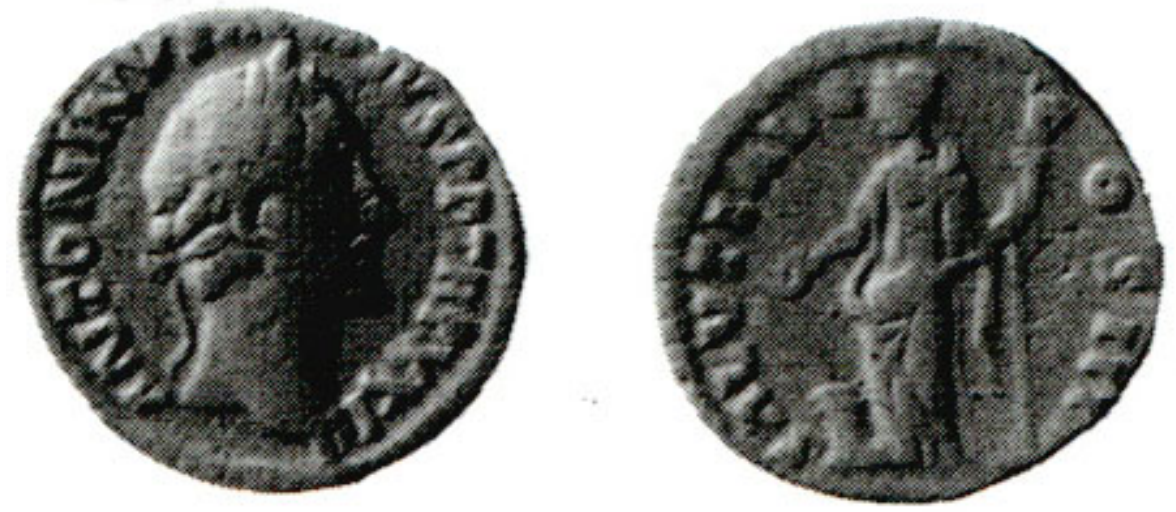

ANTONINVS AVG PIVS P P TR P XXIII, *A1

SALVTI AVG - COS IIII, Salus debout à gauche, nourrissant un serpent sortant d'un autel et tenant un sceptre.

$2,88 \mathrm{~g}$

RIC III, p. $63 n^{\circ} 305$

\section{Marc-Aurèle (161-180)}

25 Atelier de Rome

$26161(?)$ 
11.
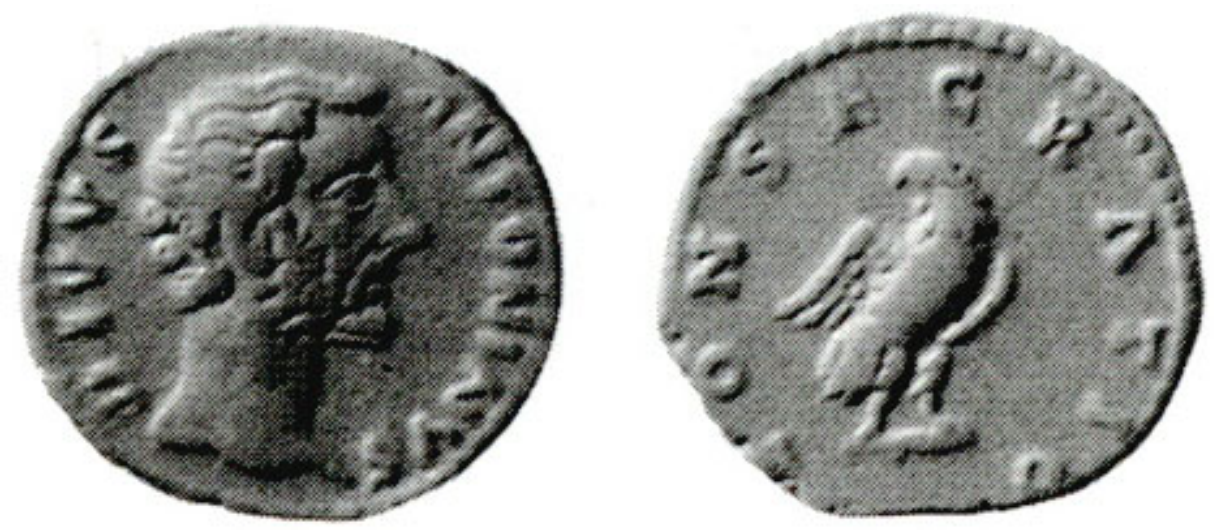

DIVVS ANTONINVS, +A1 CONSECRATIO, aigle à droite, tête à gauche $3,76 \mathrm{~g}$

RIC III, p. $247 n^{\circ} 429$

27 Décembre 161-décembre 162, pour Lucius Verus

12.
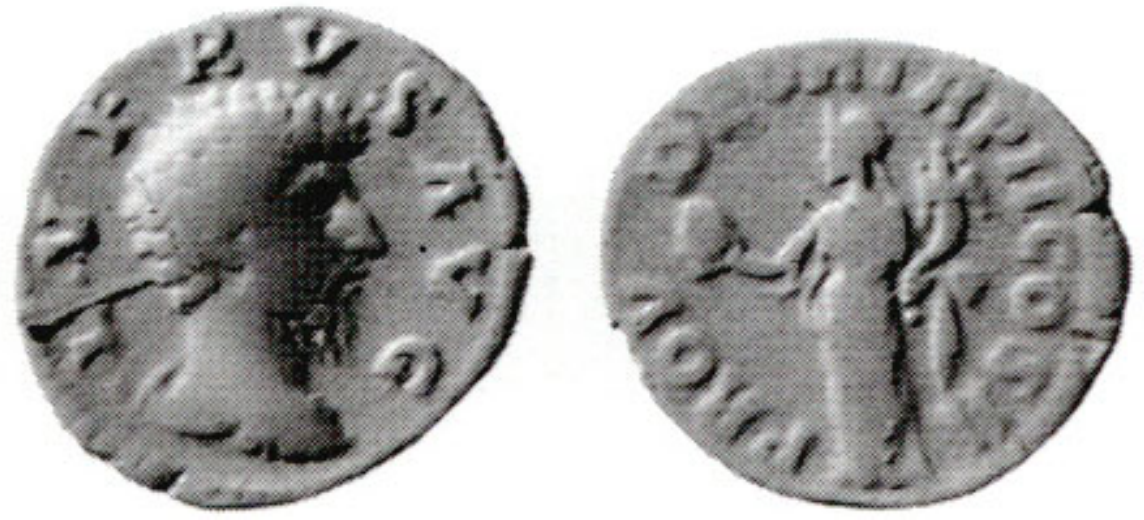

$L$ VERVS AVG,$+B 2$

PROV- DEOR TR P COS II, Providentia debout à gauche, tenant un globe et une corne d'abondance. $2,88 \mathrm{~g}$

RICIII, p. $253 n^{\circ} 485$

161-176, pour Faustine II 
13.
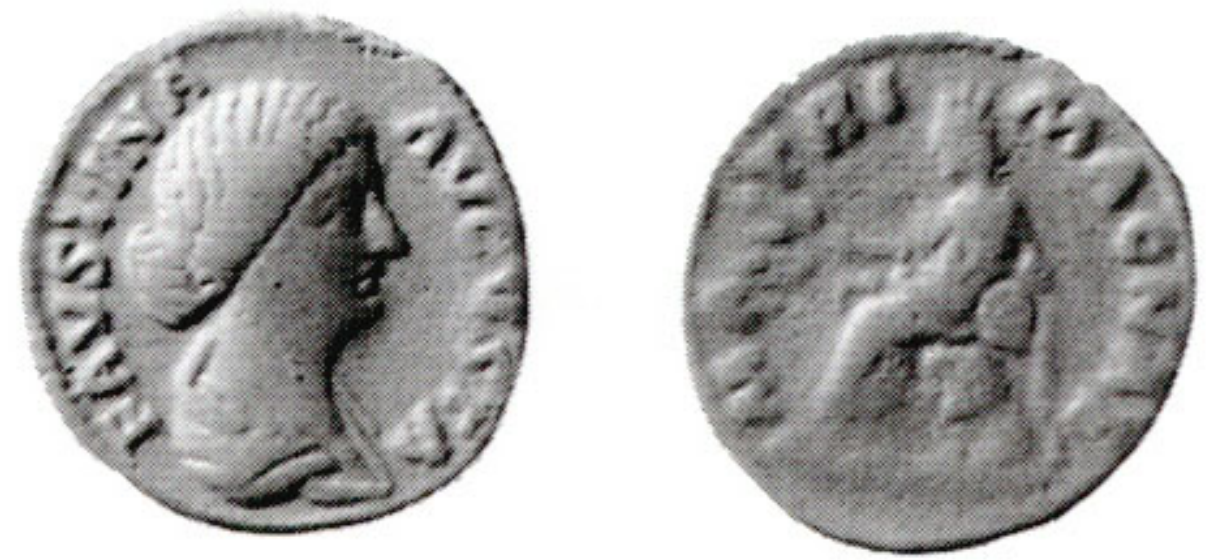

FAVSTINA - AVGVSTA, *E1 MA-TRI MAGNAE, Cybèle assise à gauche, tenant un rameau, le coude posé sur un tambour ; à côté, un lion.

$3,14 \mathrm{~g}$

RIC III, p. $270 n^{\circ} 706$

Décembre 168-décembre 169

14.
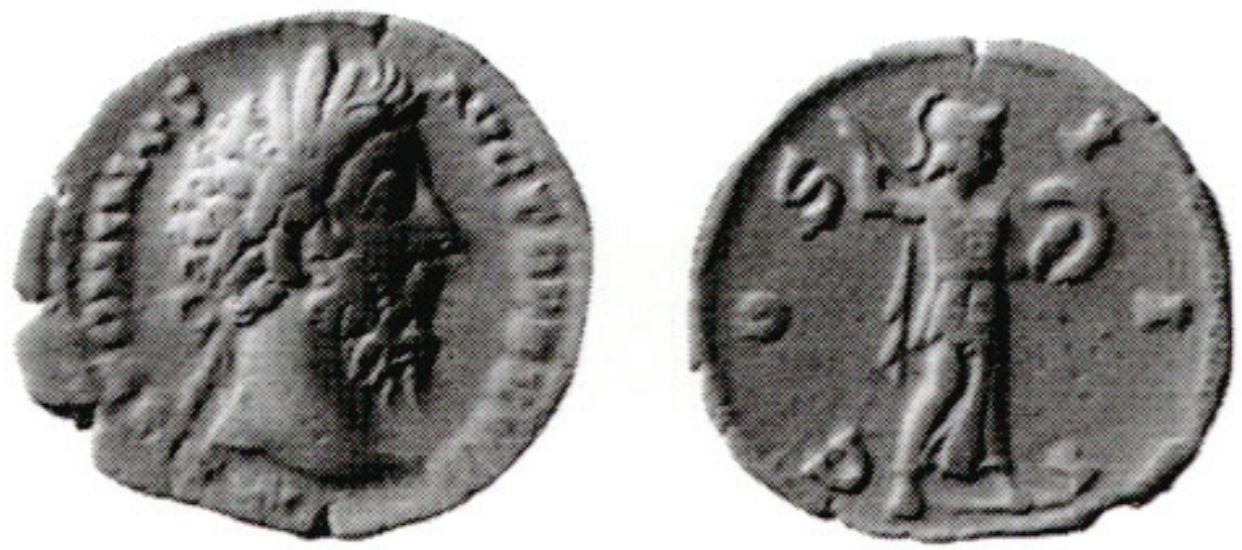

M ANTONINVS AVG TR P XXIII, *A 1

COS- III, Minerve debout à droite, brandissant une javeline dans la main droite et tenant un bouclier dans la main gauche.

3,54 g

RIC III, p. 229 n 198

\section{Commode (180-192)}

Atelier de Rome

$31 \quad 181$ 
15.
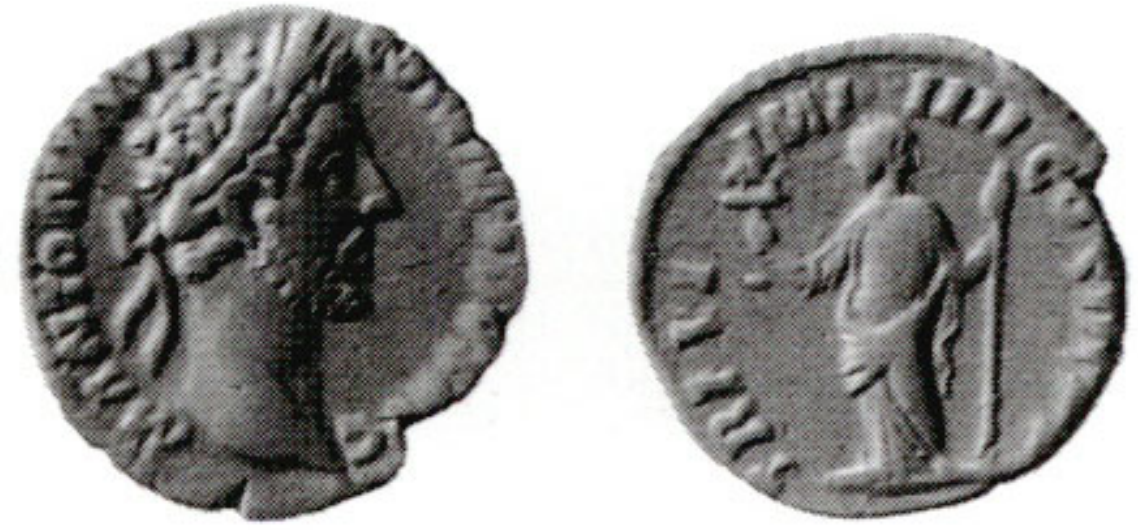

M ANTONINVS - COMMODVS AVG, *AT

TR P VI IMP IIII COS III P P, Félicitas debout à gauche tenant un caducée et un sceptre. $2,85 \mathrm{~g}$

RIC III, p. 368 n $^{\circ} 15$

32 Décembre 181-décembre 182, 2ème émission

16.
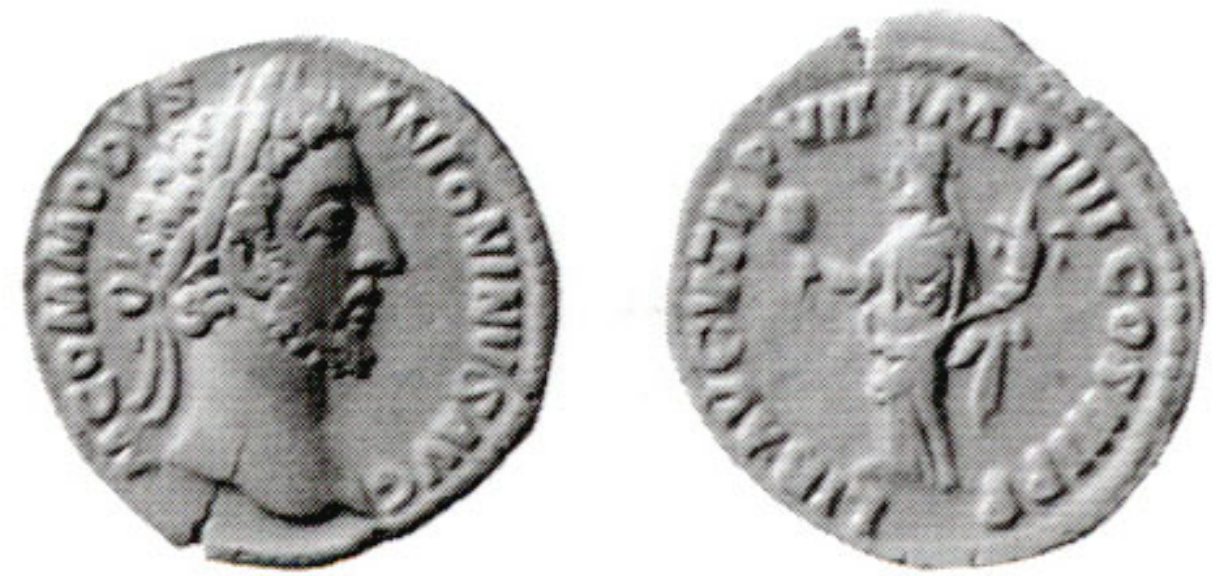

M COMMODVS - ANTONINVS AVG, *A1

LIB. AVG V V. TR P VII IMP IIII COS III P P, Liberalitas debout à gauche tenant un abaque et une corne d'abondance

2,98 g

RIC III, p. $370 n^{\circ} 36$

33183 , 1ère émission

17. 

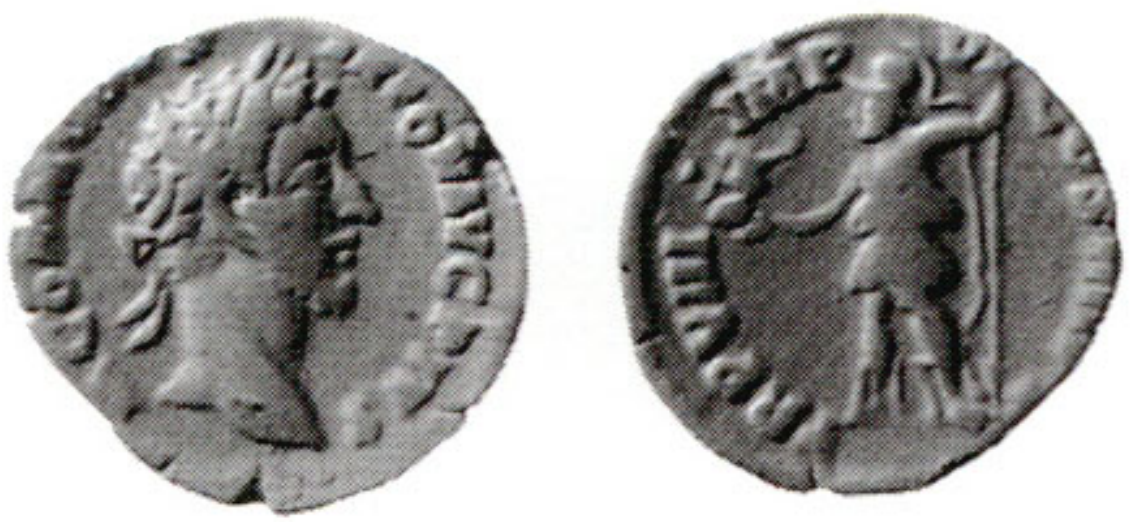

M COMMODVS - ANTON AVG PIVS, *A1

TR P VIII - IMP - V COS IIII P P, Rome debout à gauche tenant une victoire et une lance $2,66 \mathrm{~g}$

RIC III, p. $371 \mathrm{n}^{\circ} 49$

\section{Décembre 187-décembre 188}

18.
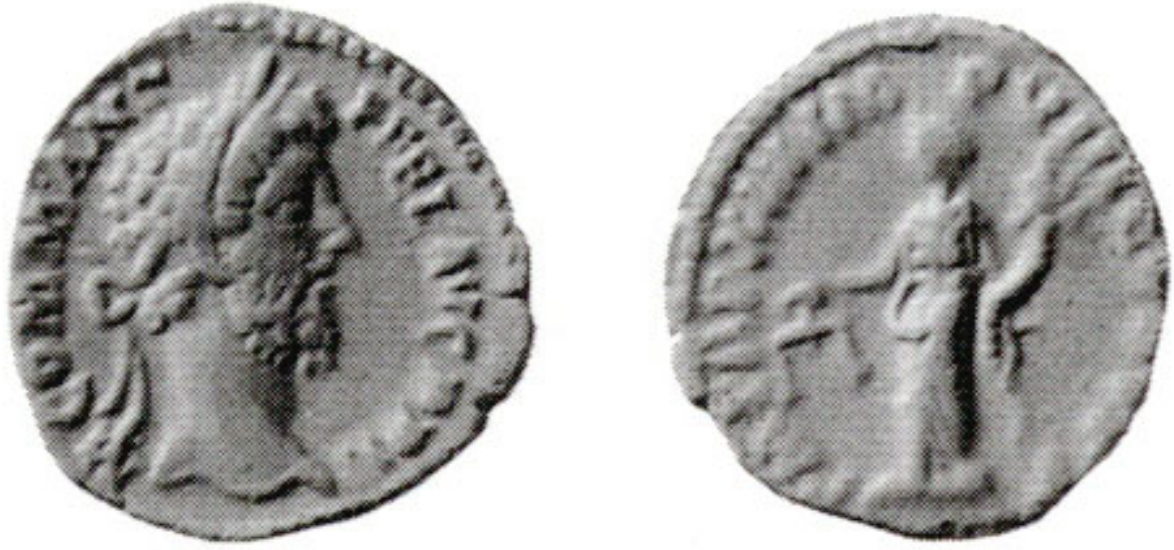

M COMM ANT - P FEL AVG BRIT, *AT

P M TR P XIIIIM-P VIII COS [V p p], Aequitas debout à gauche tenant une balance et une corne d'abondance.

$3,09 \mathrm{~g}$

RIC III, p. $384 n^{\circ} 164$

192, phase b 
19.
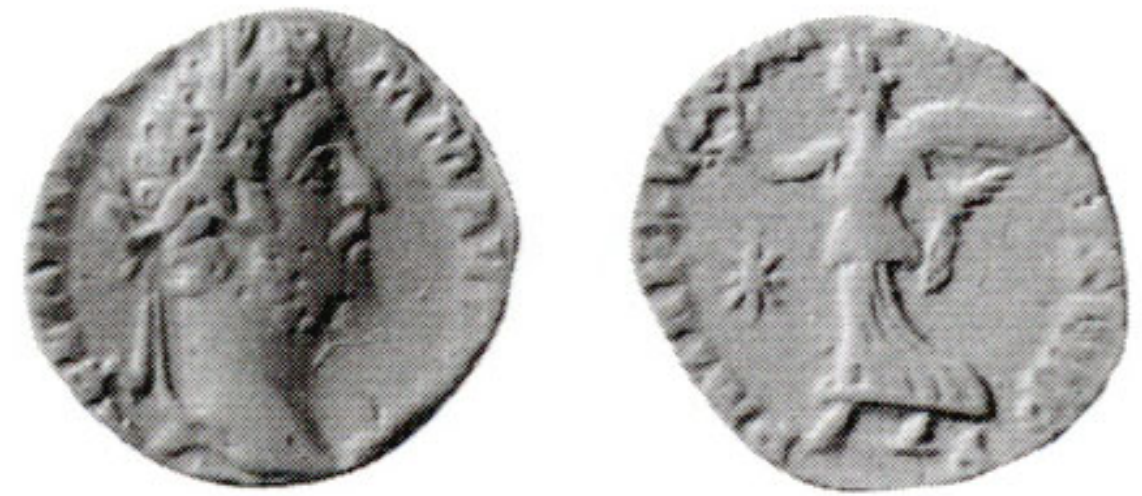

[1 a]EL AVREL CO-MM AVG [p fel], *A1 P M TR P XVIIIM-P [viii] - COS VII P - P, étoile dans le champ à gauche, Victoire à gauche tenant une couronne et une palme. $2,06 \mathrm{~g}$

RIC III, p. $393 n^{\circ} 237$

Septime Sévère (193-211). Règne conjoint de Septime Sévère et Caracalla (198-209).

20.
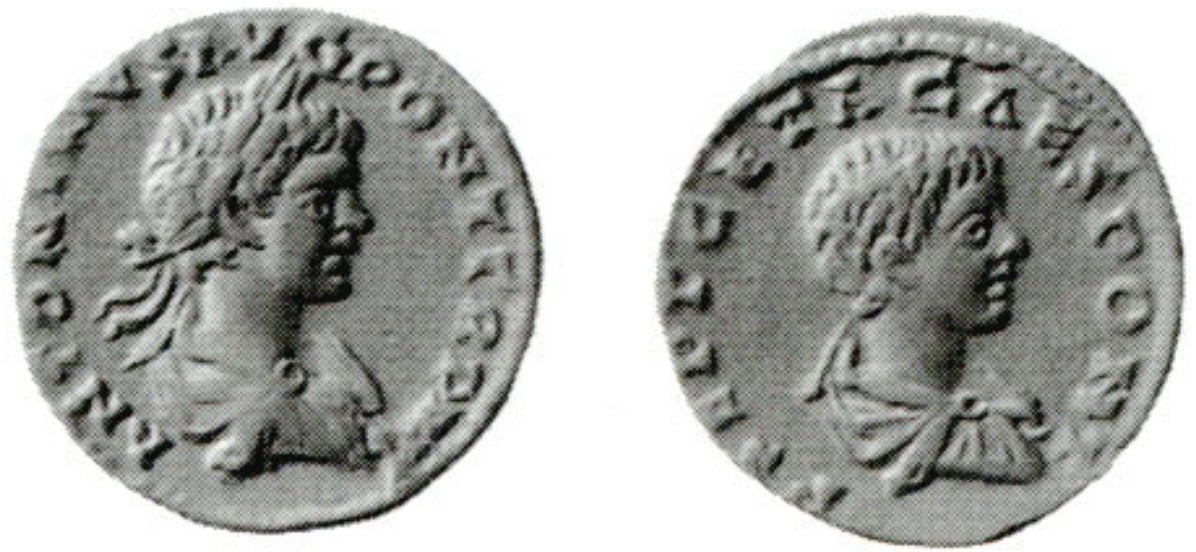

ANTONINVS AVG PONT TR PIIII, *D2 P SEPT GETA CAES PONT, buste de Géta drapé, cuirassé, vu de dos, tête nue, à droite.

$3.08 \mathrm{~g}$

$\mathrm{R} 1 \mathrm{CIV} / 1$, p. $266 \mathrm{n}^{\circ} 345$; BMC V, p. $299 \mathrm{n}^{\circ} 729$

Atelier de Rome

Première émission dynastique : 201 
21.
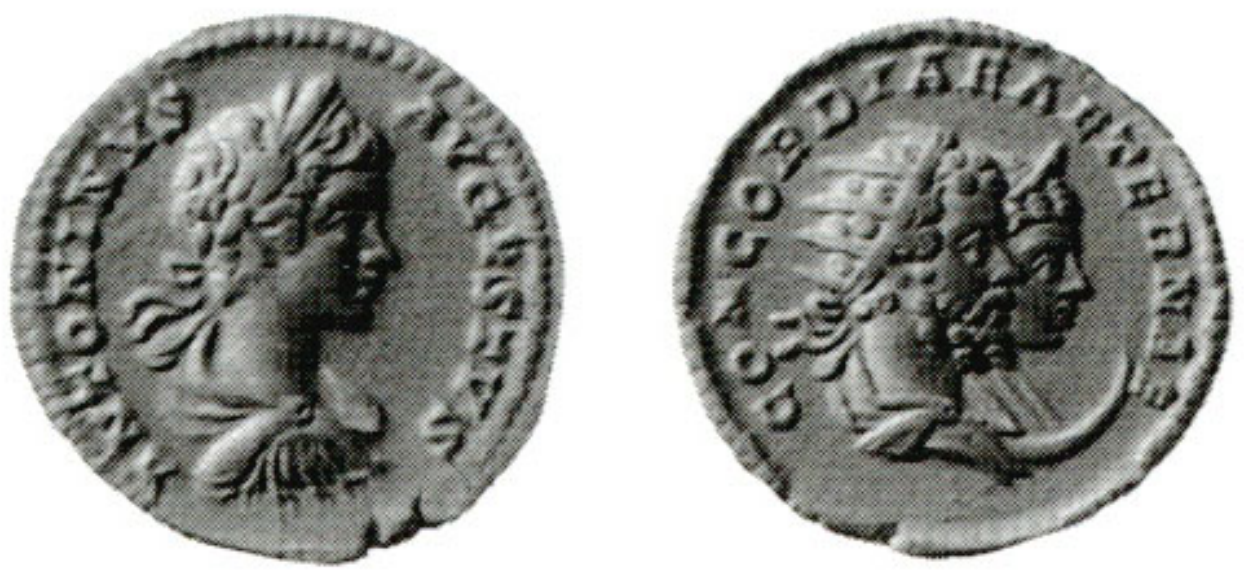

ANTONINVS AVGVSTVS, *D2

CONCORDIAE AETERNAE, bustes accolés à droite de Septime Sévère, radié, drapé, cuirassé vu de trois quarts en arrière et de Julia Domna, diadémée, drapée, sur un croissant.

$3,34 \mathrm{~g}$

Même coin de revers que $n^{\circ} 24 \mathrm{RICIV} / 1$ - ; BMC V - ; Hill -

Inédit

40 Deuxième émission dynastique : 201

22.
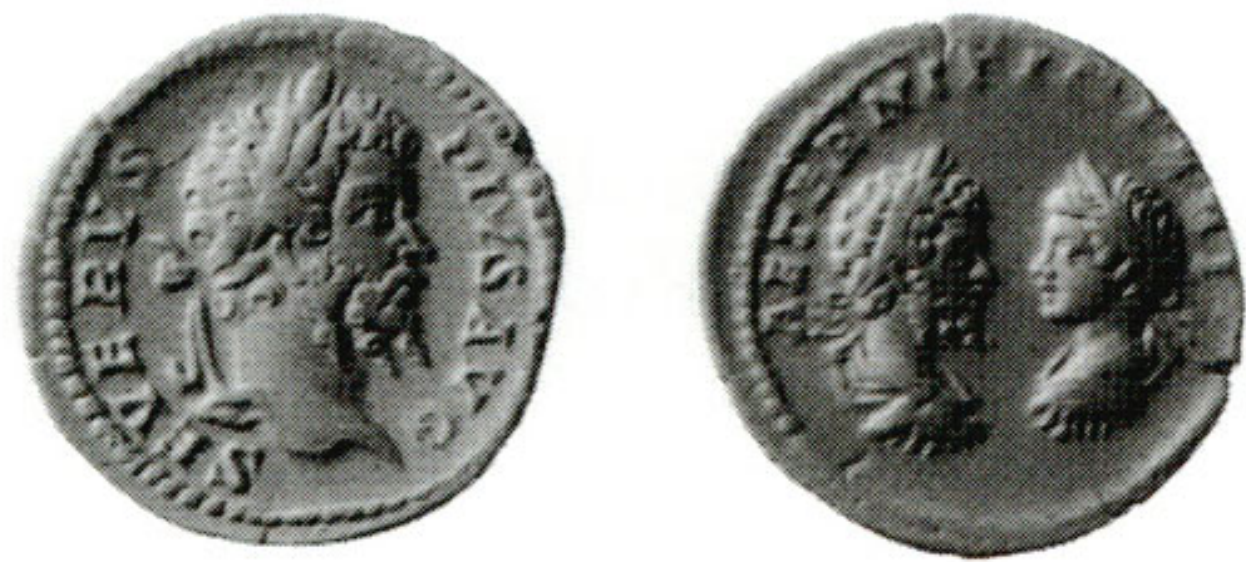

SEVERVS - PIVS AVG, *AT

AETERNIT IMPERI, bustes de Septime Sévère et de Caracaila se faisant face, laurés, drapés et cuirassés, vus de trois quarts en arrière.

$3.09 \mathrm{~g}$

RIC IV/1, p. 123 n² 250 ; Hill 516 
23.
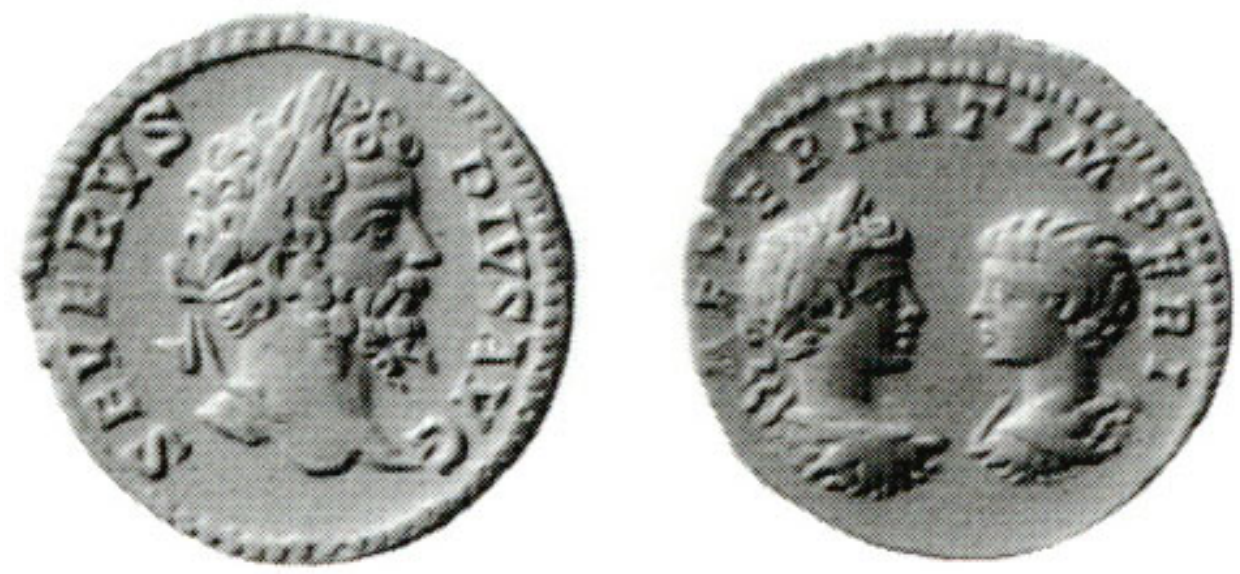

SEVERVS - PIVS AVG, *AT

AETERNIT IMPERI, bustes de Caracaila (lauré, drapé et cuirassé vu de trois quarts en arrière) et de Géta (buste drapé et cuirassé vu de trois quarts en arrière, tête nue) se faisant face.

$3,44 \mathrm{~g}$

RIC IV/1, p. $123 n^{\circ} 251$; Hill 517

24.
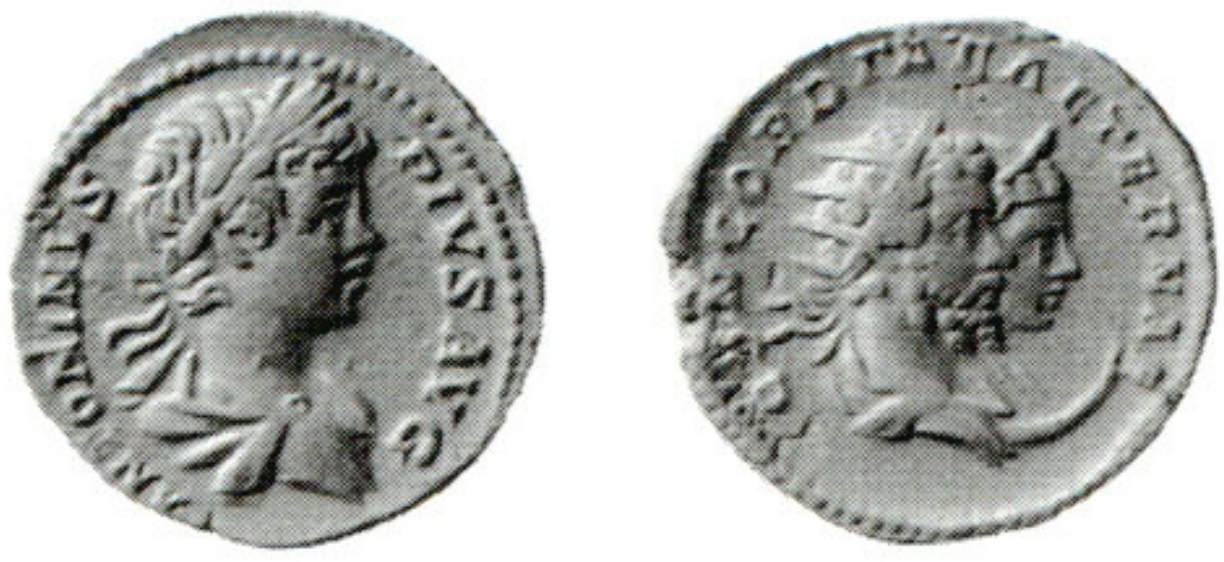

ANTONINVS - PIVS AVG ${ }^{*} \mathrm{C} 2$

CONCORDIAE AETERNAE, même description que $n^{\circ} 21$.

$3,18 \mathrm{~g}$

Même coin de revers que $n^{\circ} 21$ RIC IV/1, p. $231 n^{\circ} 125$ a : Hill 521 
25.
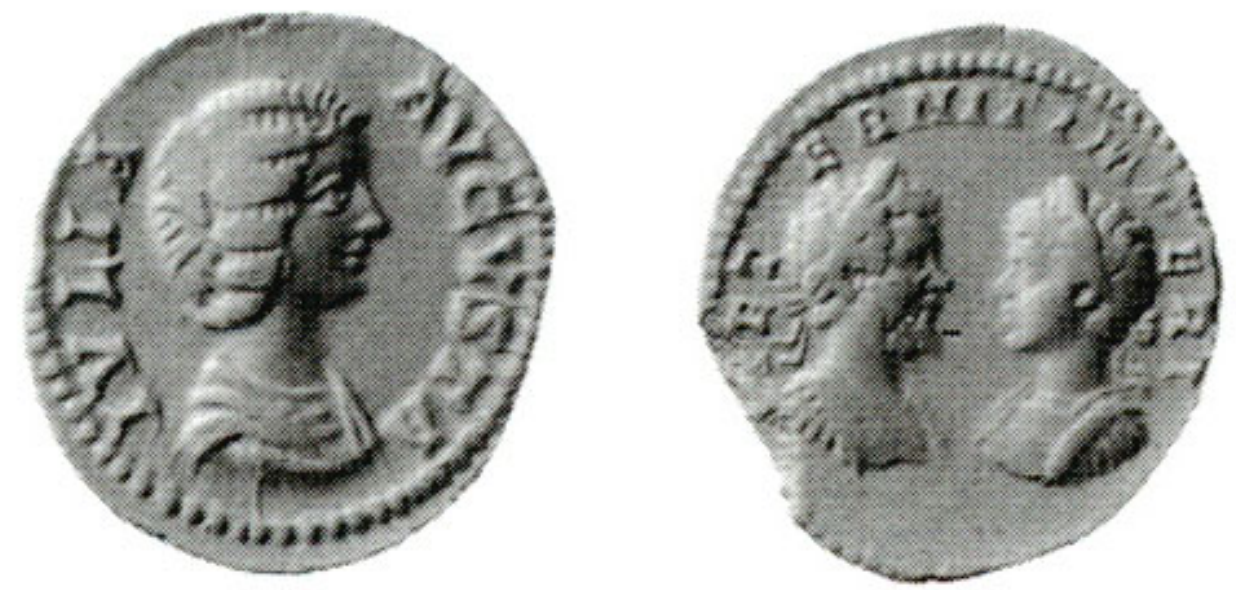

IVLIA - AVGVSTA, *E1

AET-ERNIT IMPERI. bustes de Septime Sévère et Caracaila se faisant face, laurés, cuirassés vus de trois quarts en avant.

$3,49 \mathrm{~g}$

Même coin de droit que $n^{\circ} 26$ et 27

RIC IV/1, p. 166 n $^{\circ} 539$ b; Hill 523

26.
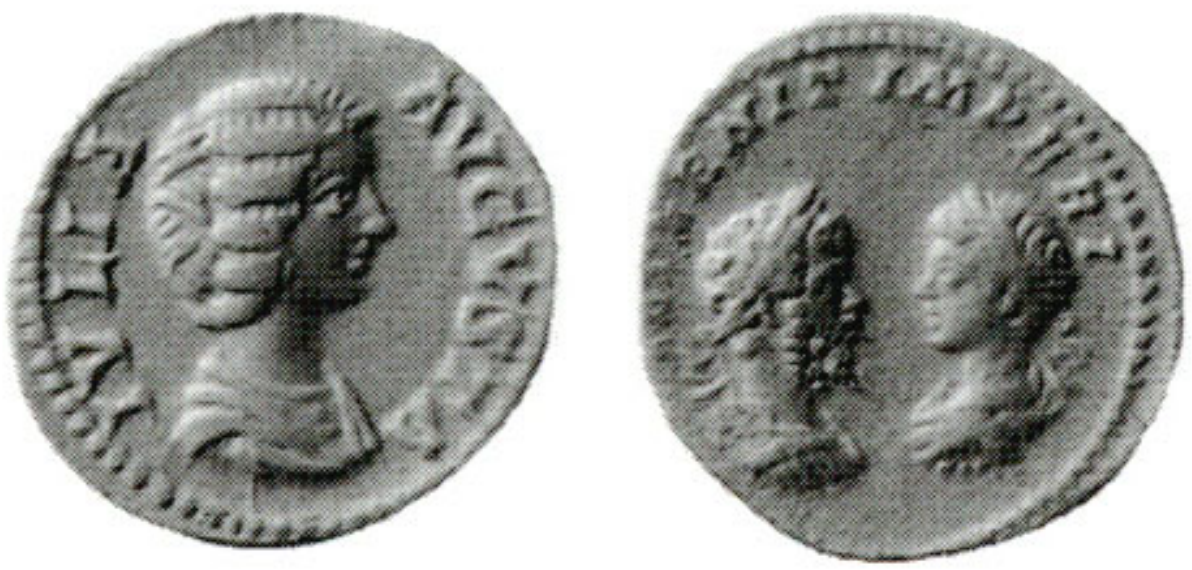

IVLIA - AVGVSTA, *E1

AETERNIT IMPERI, bustes de Septime Sévère et Caracaila se faisant face, laurés, drapés, cuirassés vus de trois quarts en arrière.

$2,94 \mathrm{~g}$

Même coin de droit que $\mathrm{n}^{\circ} 25$ et 27

RIC IV/1, p. 166 n $^{\circ} 539$ b ; Hill 523 
27.
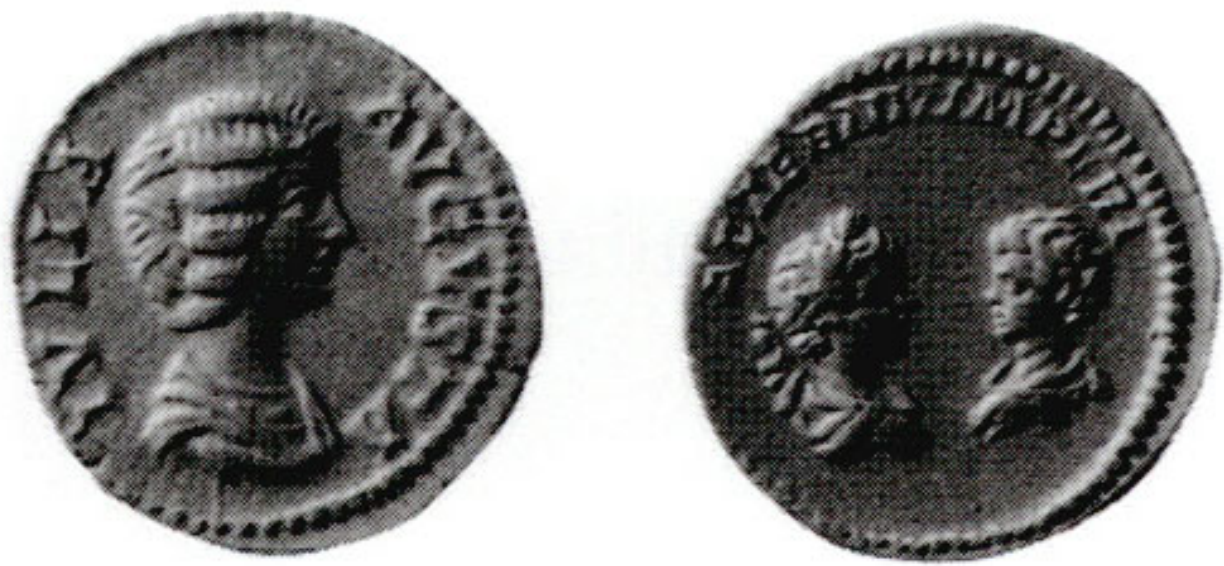

IVLIA - AVGVSTA, *E1

AETERNIT IMPERI, bustes de Caracalla (lauré, drapé, vu de trois quarts en arrière) et de Géta (tête nue drapé, vu de trois quarts en arrière) se faisant face.

$3,32 \mathrm{~g}$

Même coin de droit que $n^{\circ} 25$ et 26

RICIV/7, p. 166 n 540 ; Hill 524

Caracalla, règne seul (212-217).

41 Atelier de Rome

42 8e émission : 215 (antoniniens)

ANTONINVS PIVS AVG GERM 

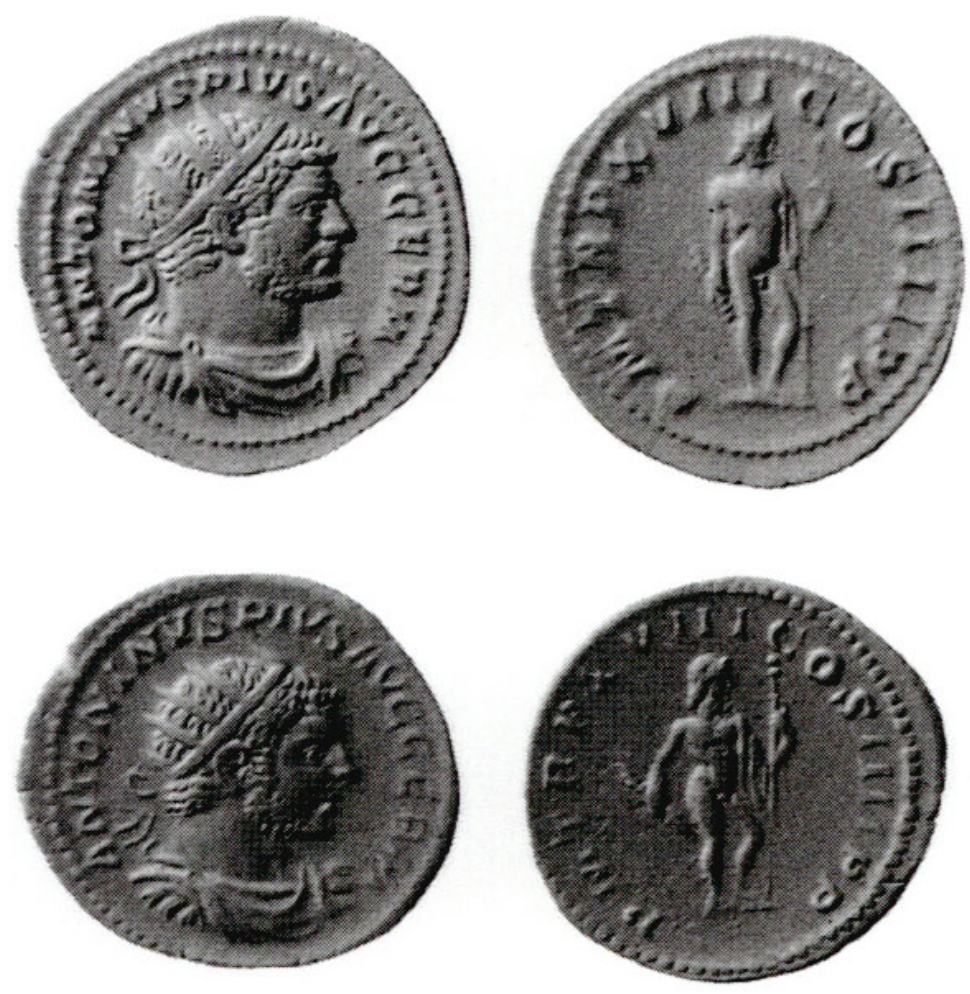

D1

P M TR P XVIII COS IIII P P, Jupiter debout de face, tête à droite, la main droite tenant un foudre et la main gauche un long sceptre vertical.

4,$63 ; 4,64 \mathrm{~g}$

RIC IV/1, p. 249 n² 258 a ; Cf. Hill 1465 (Jupiter debout à gauche)

30.
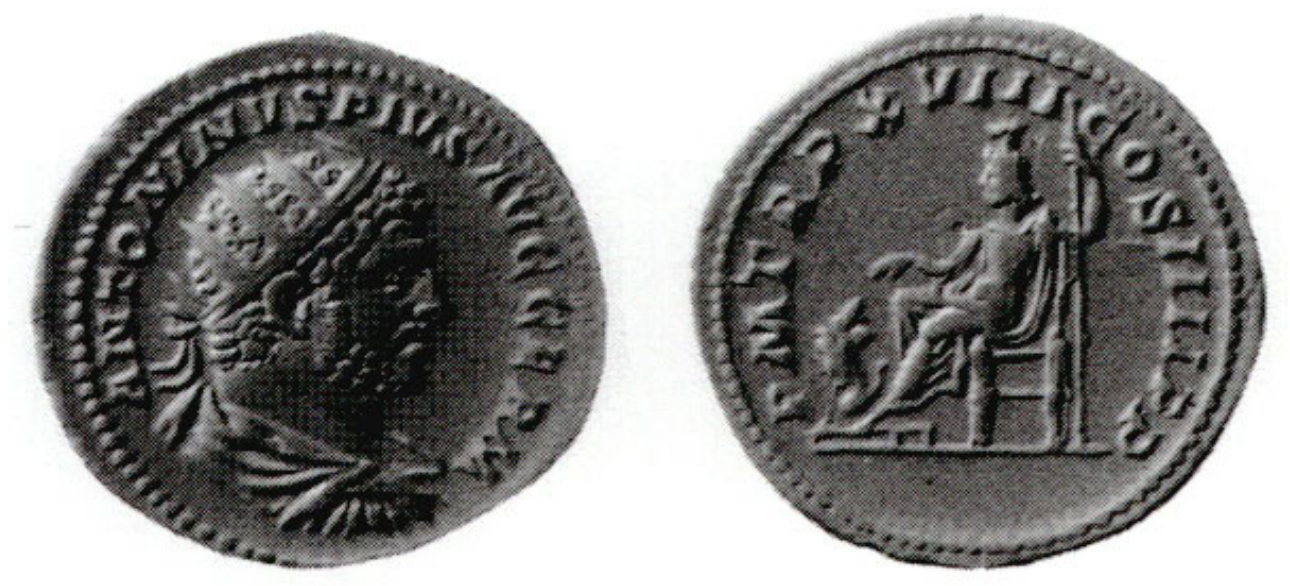

D2

P M TR P XVIII COS IIII P P. Pluton assis à gauche la main droite étendue, la main gauche tenant un long sceptre vertical ; à ses pieds à gauche, Cerbère.

$5,62 \mathrm{~g}$

R/CIV/1, p. 249 n 261 d; Hill 1489 
31.
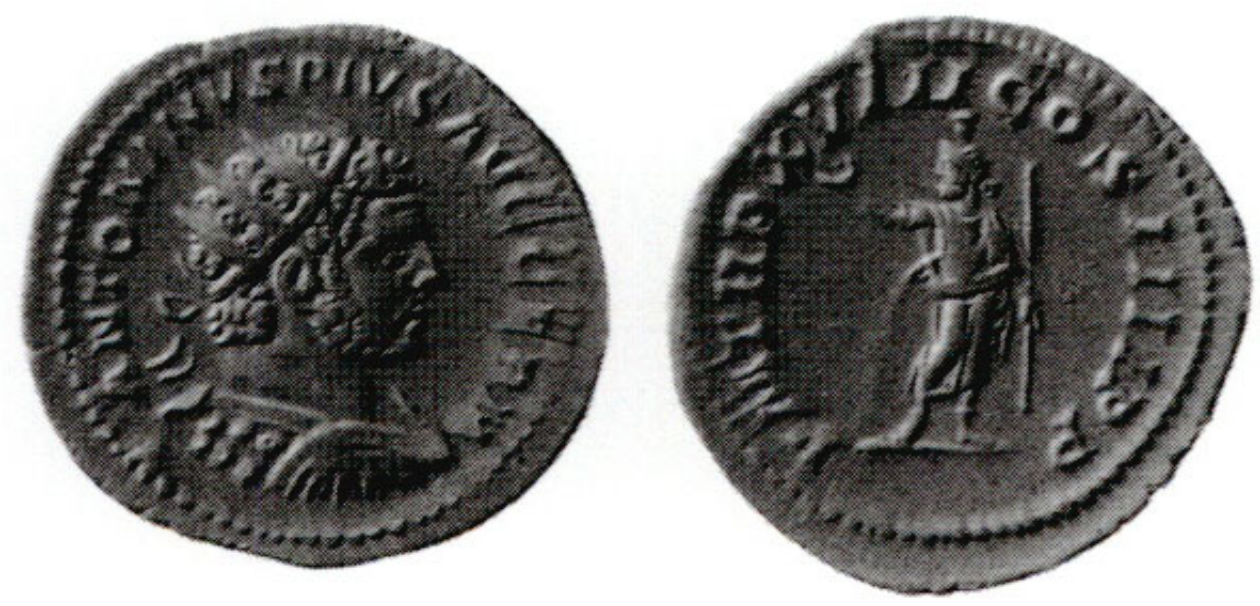

B2 P M TR P XVIII COS IIII P P, Sérapis debout de face, tête à gauche ; le bras droit est levé et la main gauche tient un long sceptre vertical.

$4,72 \mathrm{~g}$

RIC IV/1, p. 250 n 263 d; Hill 1467

32.
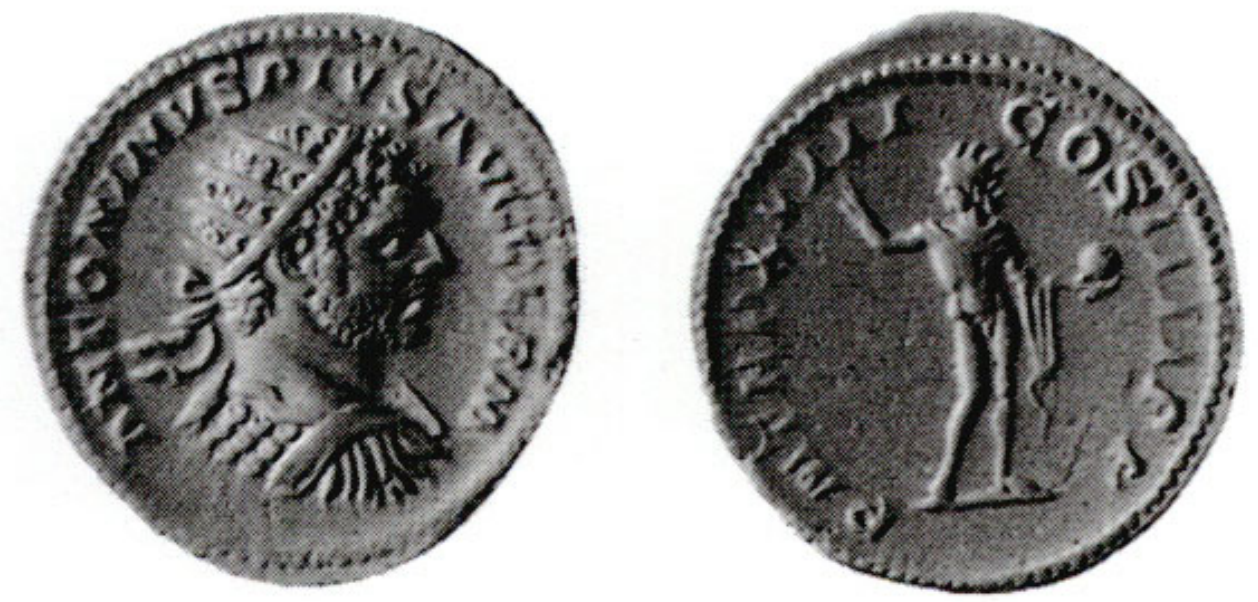

B2

P M TR P XVIII COS IIII P P, Sol radié debout de face, tête à gauche ; le main droite est levé, la main gauche tient un globe.

$5,01 \mathrm{~g}$

RIC IV/1, p. 250 n 264 b ; Hill 1468 
33.
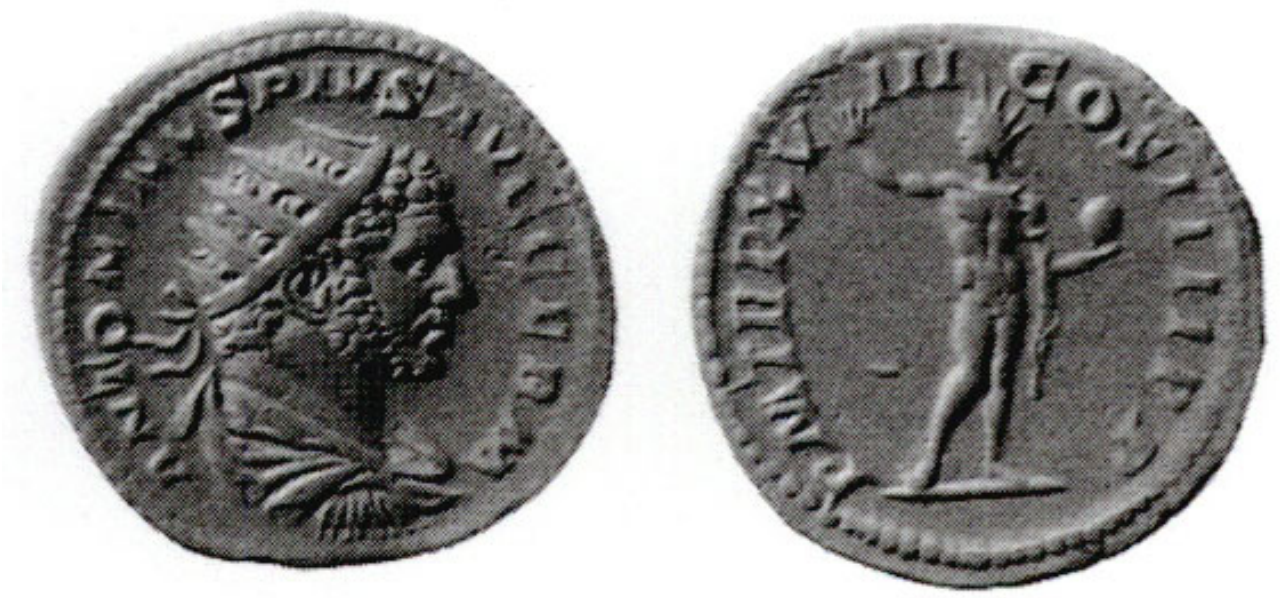

D2

P M TR P XV-III COS IIII P P, Sol comme au n 32. $5,29 \mathrm{~g}$

RIC IV/1, p. 250 n 264 c ; Hill 1468

34.
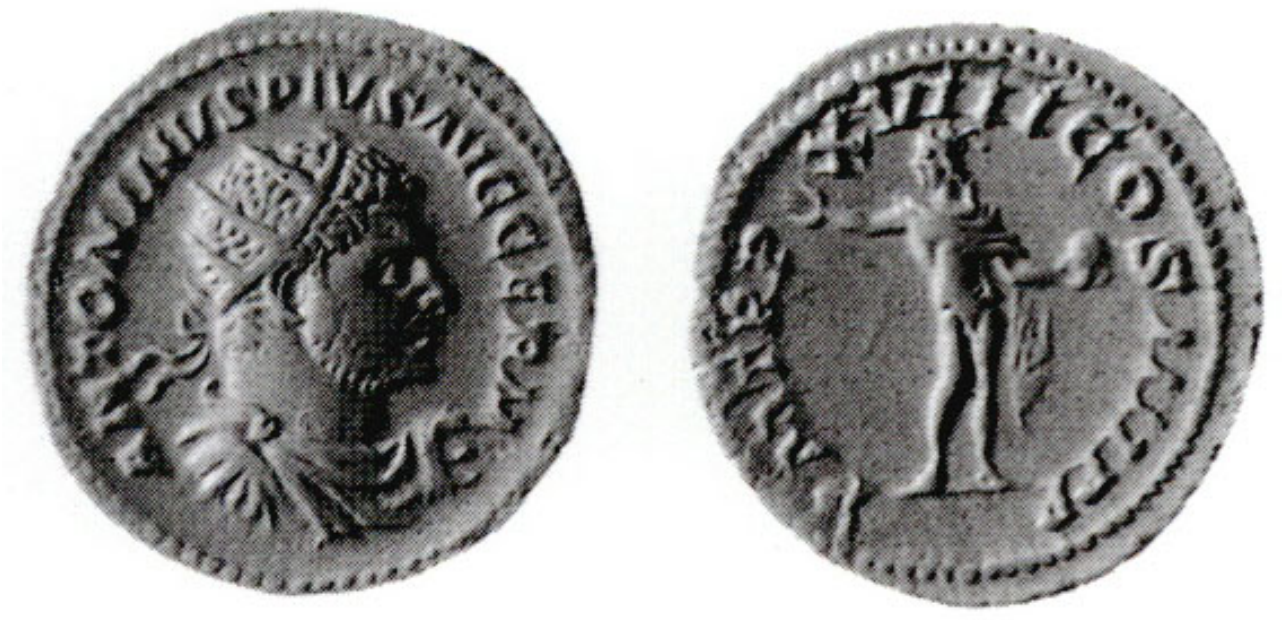

D1

P M TR P - XVIII COS IIII P P, Sol comme au n ${ }^{\circ} 32$

$4,94 \mathrm{~g}$

RIC IV/1, p. 250 n 264 c ; Hill 1468

35-36. 

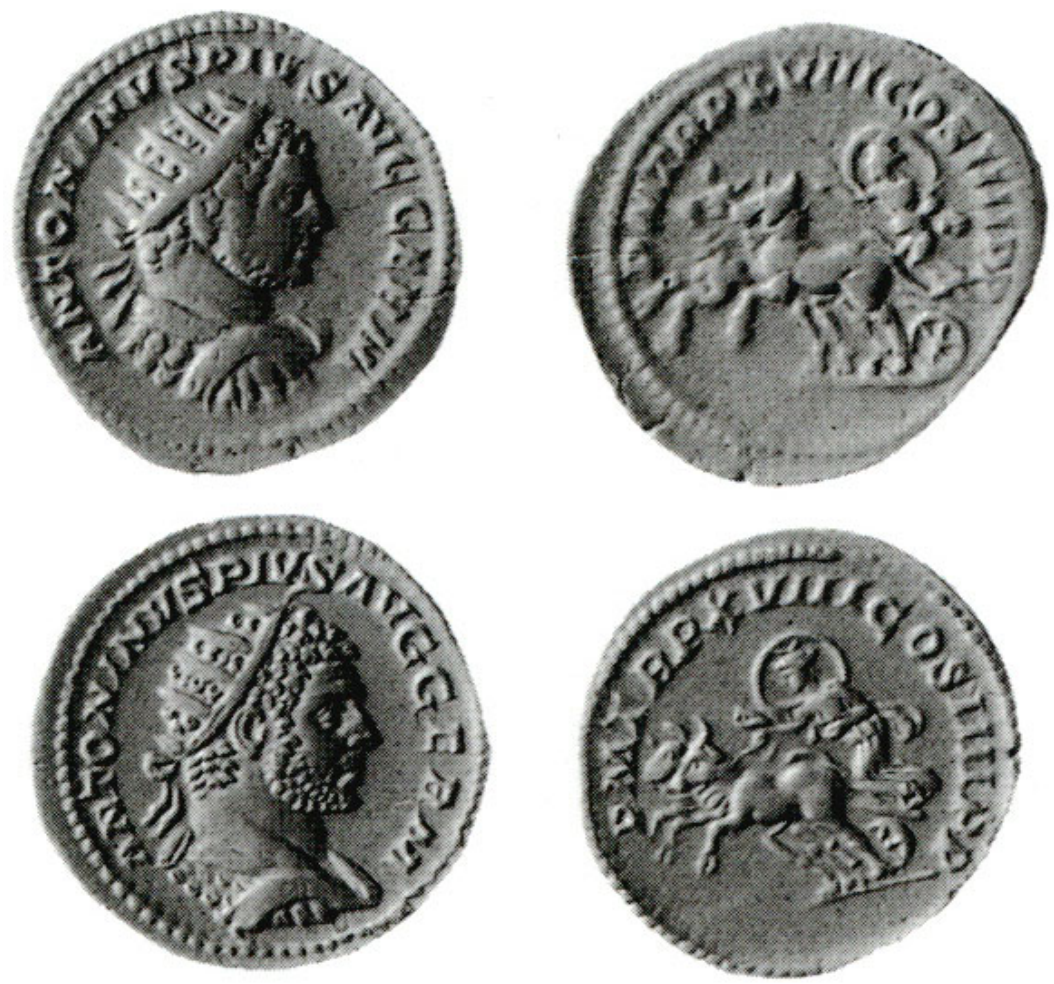

B2

P M TR P XVIII COS IIIIP P, Luna conduisant un bige de taureaux à gauche. 4,$04 ; 4,47 \mathrm{~g}$

RICIV/1, p. $248 n^{\circ} 256$ b ; Hill 1466 
37-38.
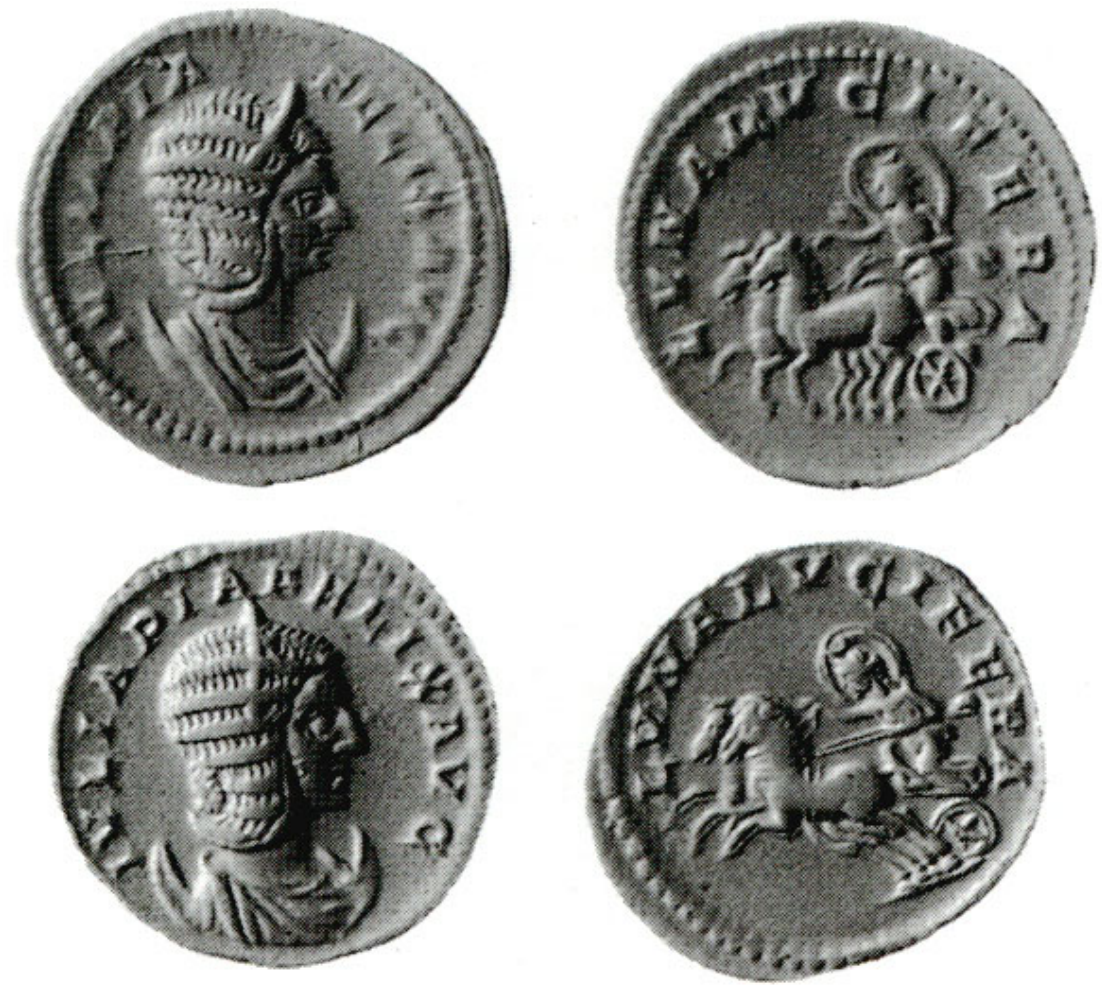

IVLIA PIA FELIX AVG, E2

LVNA LVCIFERA, Luna (croissant sur la tête) conduisant un bige de chevaux à gauche. $4,92 \mathrm{~g}$ (césure PIA - FELIX); 5,09 g

RIC IV/1, p. 273 n $^{\circ} 79$ a; Hill 1472

9e émission : 216 (antoniniens)

ANTONINVS PIVS AVG GERM

39.
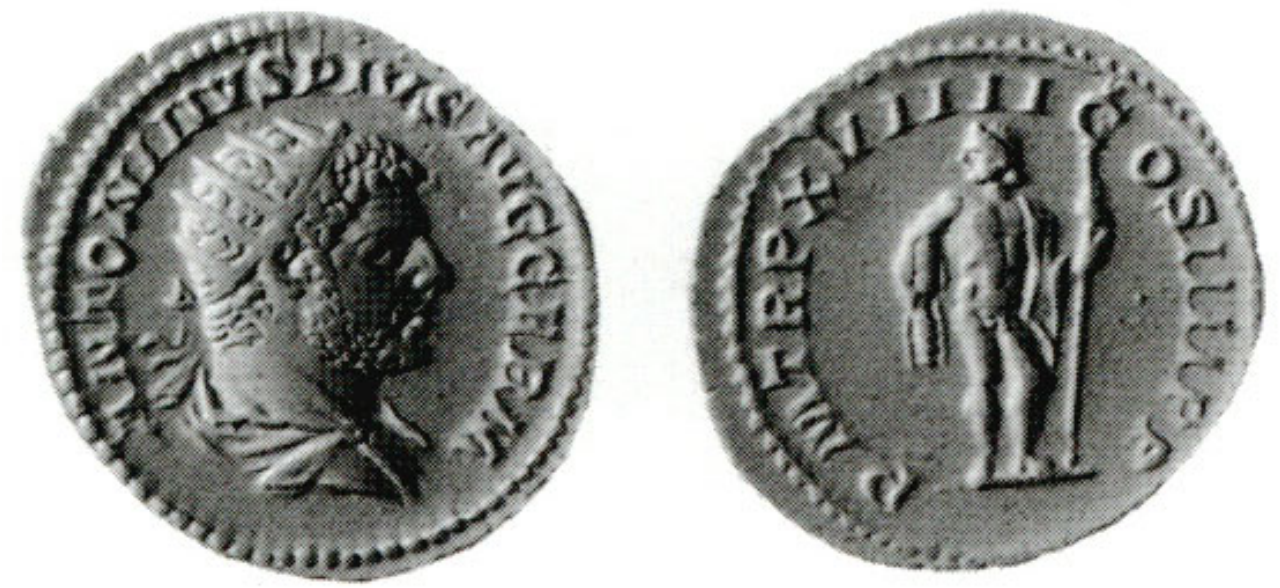

C2

P M TR P XVIIII COS IIII P P, Jupiter debout de face comme le $n^{\circ} 28$, mais tête à gauche.

$5,41 \mathrm{~g}$

RIC IV/1, p. 252 n 275 b; Hill 1520 
40.
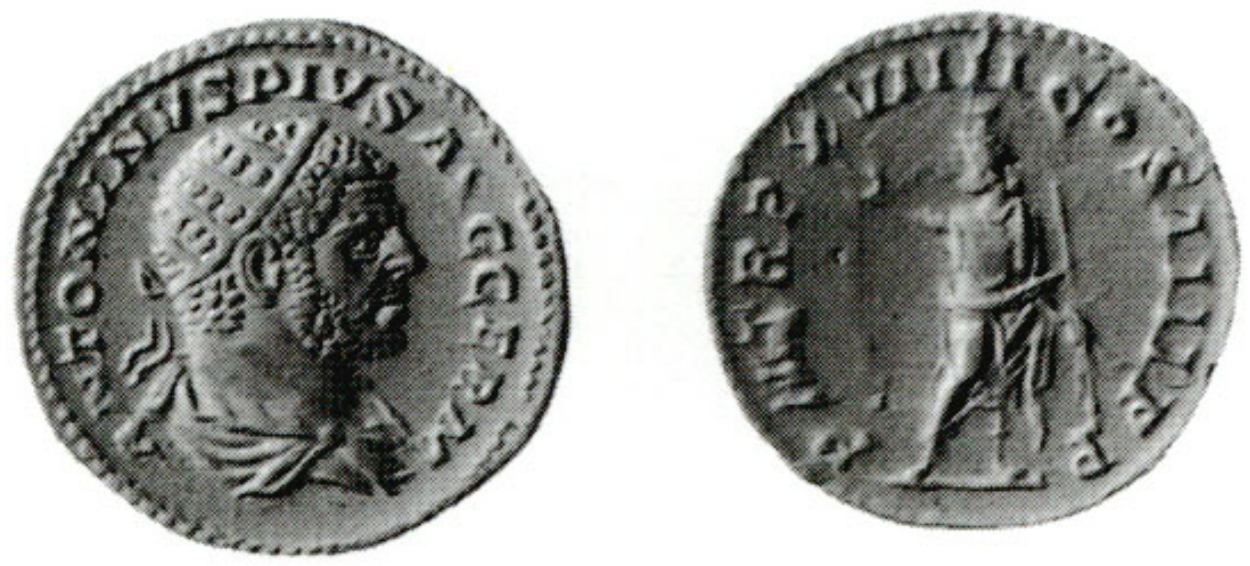

C2

P M TR P XVIIII COS IIII P P, Sérapis comme au n 31.

$5,07 \mathrm{~g}$

RIC IV/1, p. 253 n² 280 d; Hill 1523

41.
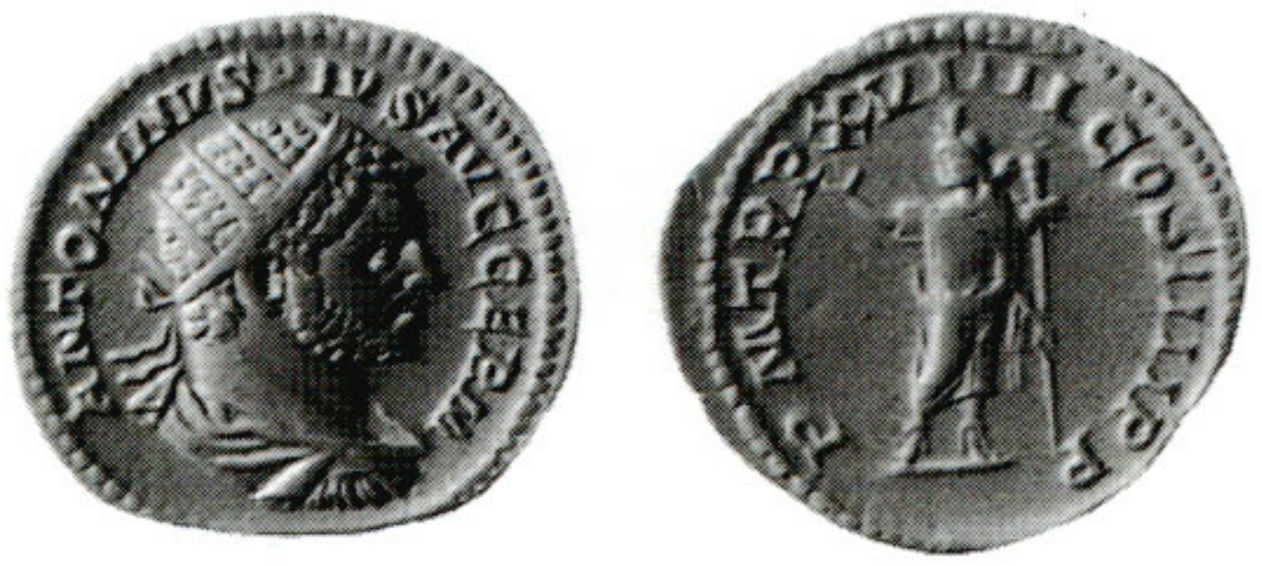

D2

P M TR P XVIIII COS IIII P P, Sérapis comme au n 31.

$4,74 \mathrm{~g}$

RIC IV/1, p. 253 n² 280 e ; Hill 1523 
42-43.
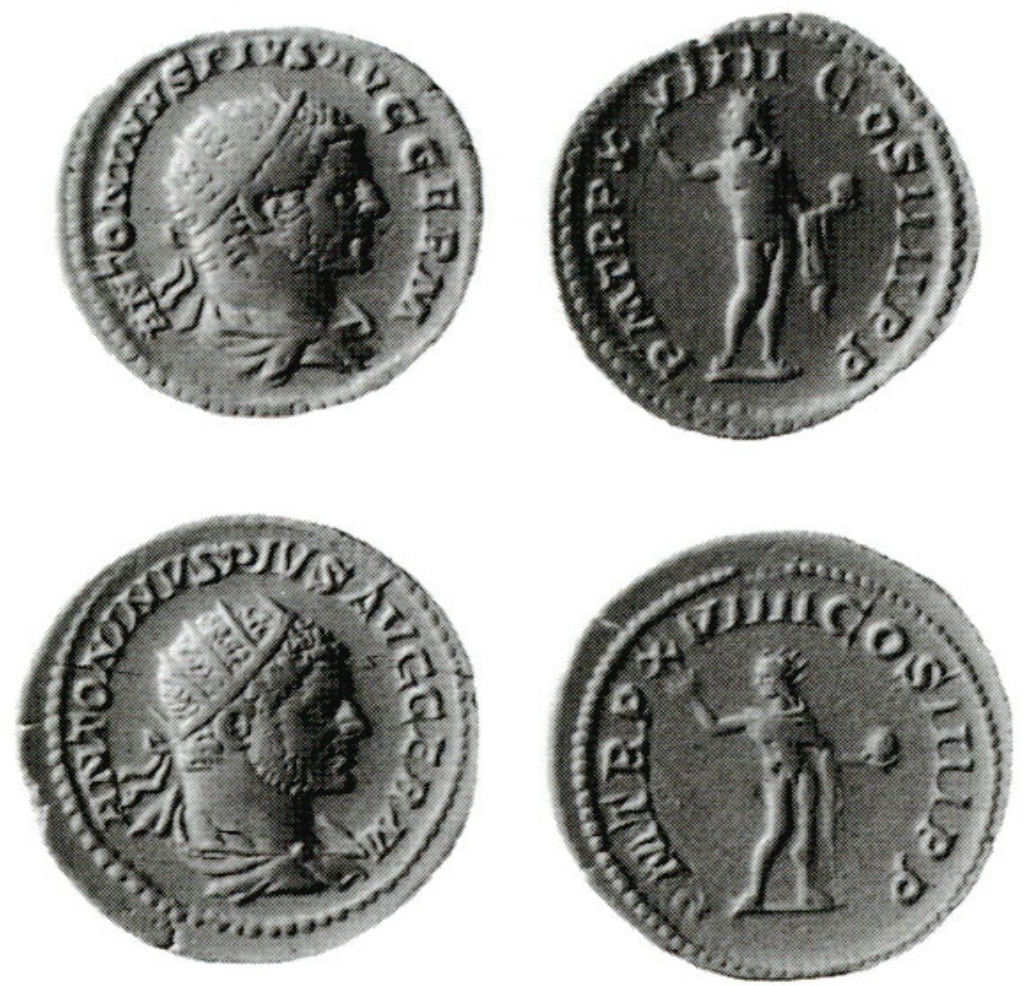

C2

P M TR P XVIIII COS IIII P P, Sol comme au $n^{\circ} 32$.

4,$27 ; 4,88 \mathrm{~g}$

RIC IV/1, p. 253 n 281 a ; Hill 1524

44.
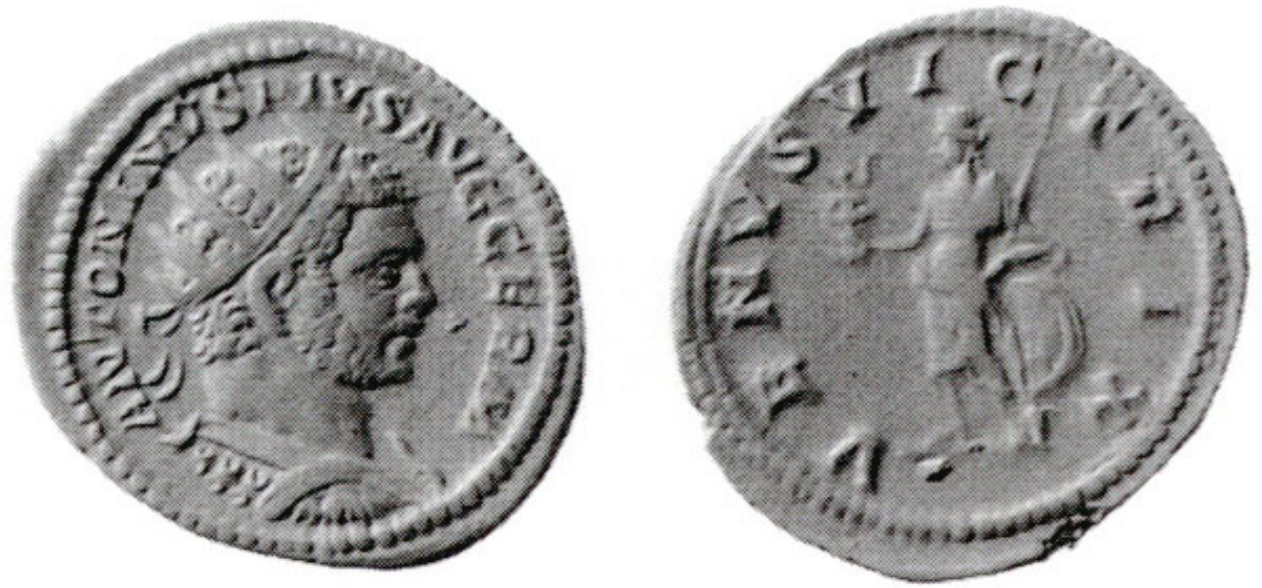

B2

VENVS VIC-TRIX, Venus debout à gauche, la main droite tenant une victoire et la main gauche un sceptre transversal ; le coude gauche s'appuie sur un bouclier.

4,64 g

RICIV/7, p. 529 n 311 c (variante de buste) ; Hill 1525 
45-46.
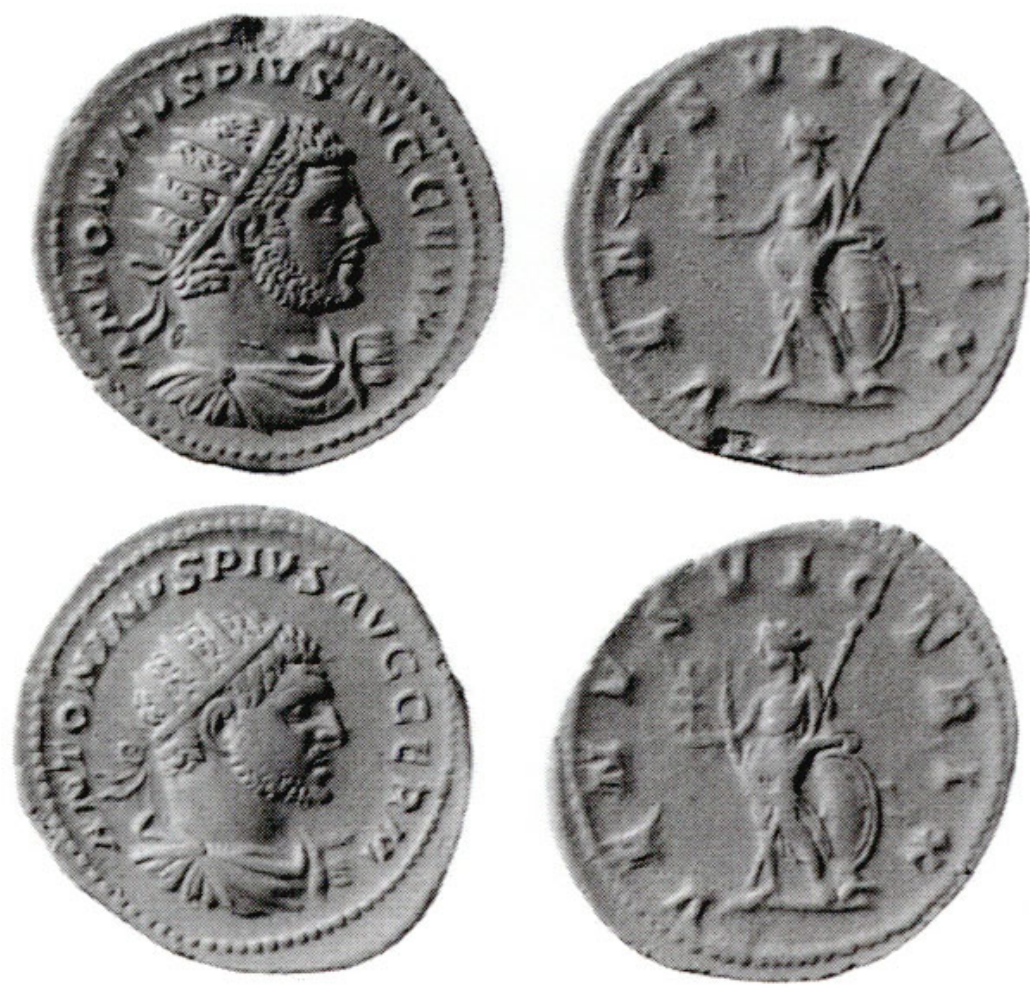

D1

VENVS VICTRIX, Venus comme au $n^{\circ} 44$ 4,$96 ; 5,44 \mathrm{~g}$

Même coin de revers

RIC IV/1, p. 259 n 311c ; Hill 1525

44 Julia Domna

IVLIA PIA FELIX AVG, E2 
47-56.
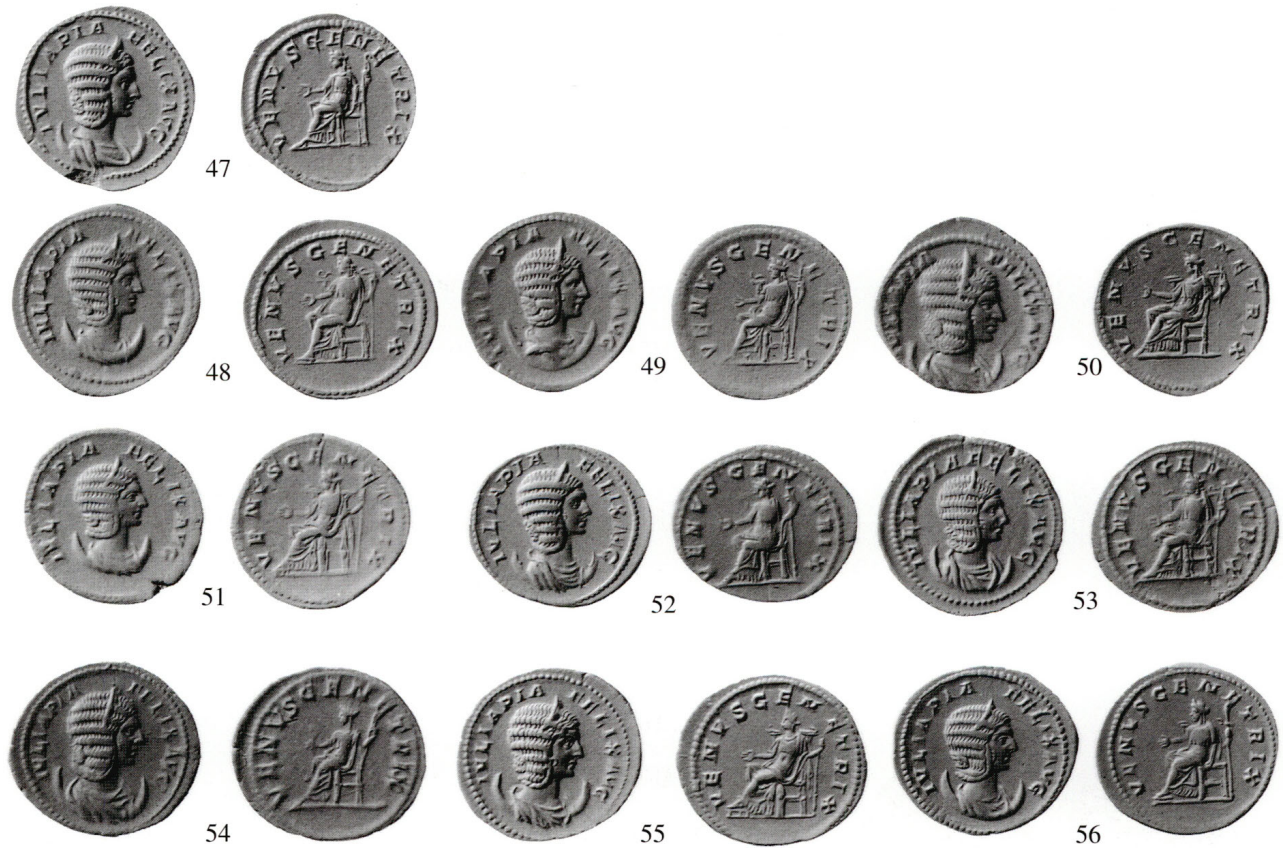

VENVS GENETRIX, Venus assise à gauche, la main droite tendue et la main gauche tenant un long sceptre vertical.

4,$74 ; 4,75 ; 4,83 ; 4,88 ; 5,02 ; 5,05 ; 5,08 ; 5,18 ; 5,28 ; 5,56 \mathrm{~g}$

RIC IV/1, p. 274 n $^{\circ} 388$ a; Hill 1528

\section{Campagne orientale : 216 (denier)}

57.
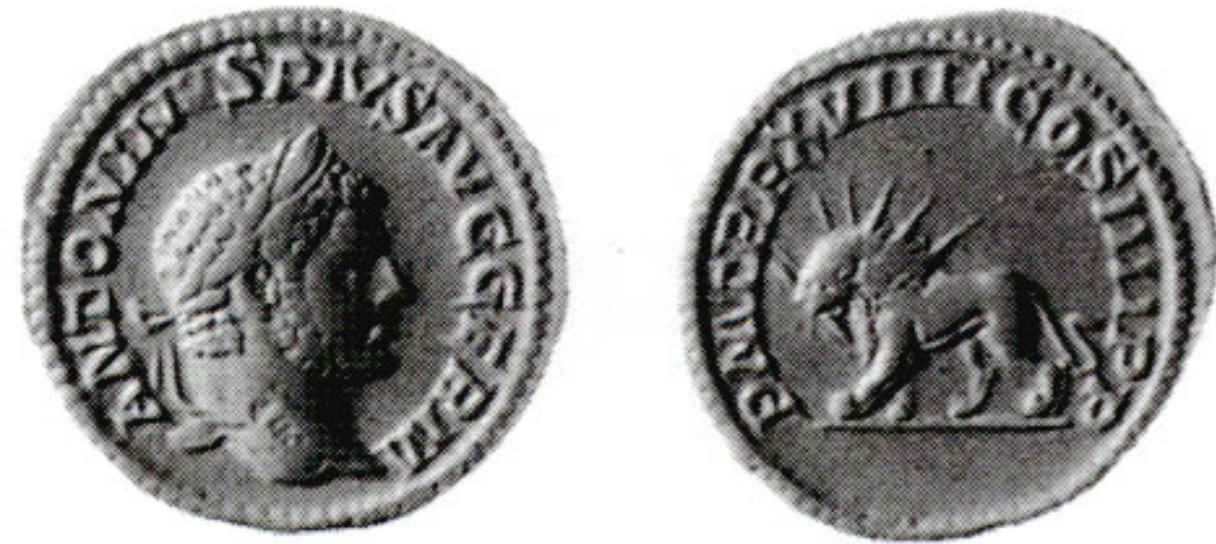

ANTONINVS PIVS AVG GERM, *A1

P M TR P XVIIII COS IIII P P, Lion radié marchant à gauche, tenant un foudre dans la gueule. $2,27 \mathrm{~g}$

RIC IV/1, p. 254 n 283 c ; Cf. Hill 1550 (buste drapé)

Campagne orientale : 217 (antoniniens)

ANTONINVS PIVS AVG GERM 
58.
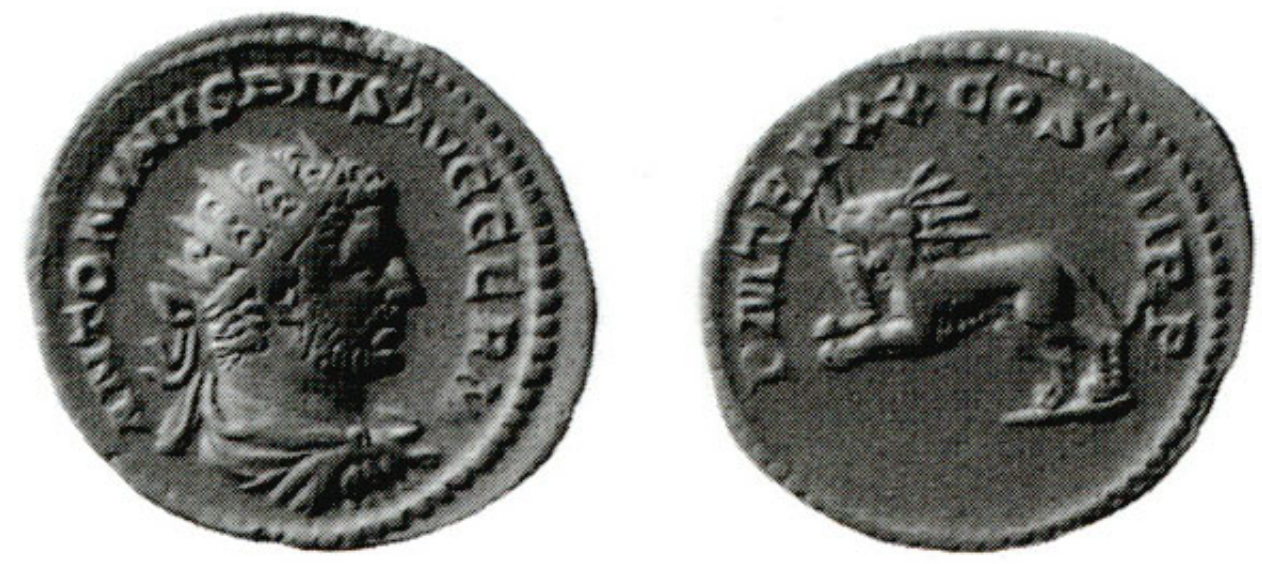

D2

P M TR P XX COS IIIIP - P, Lion radié bondissant à gauche, tenant un foudre dans la gueule.

$4,97 \mathrm{~g}$

RIC- ;BMC- ; Hill 1558

59.
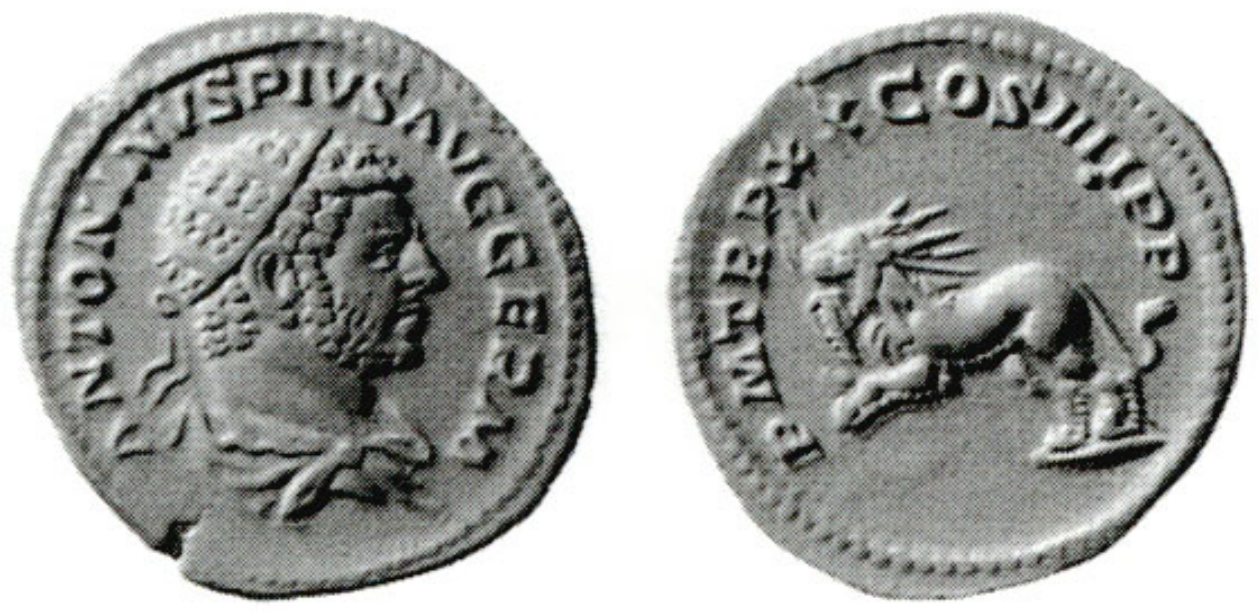

$\mathrm{C} 2$

P M TR P XX COS IIII P P, Lion comme au $n^{\circ} 58$

$5,71 \mathrm{~g}$

RIC- ; BMC - ; Hill 1558

10e émission : 217 (antoniniens) 
60.
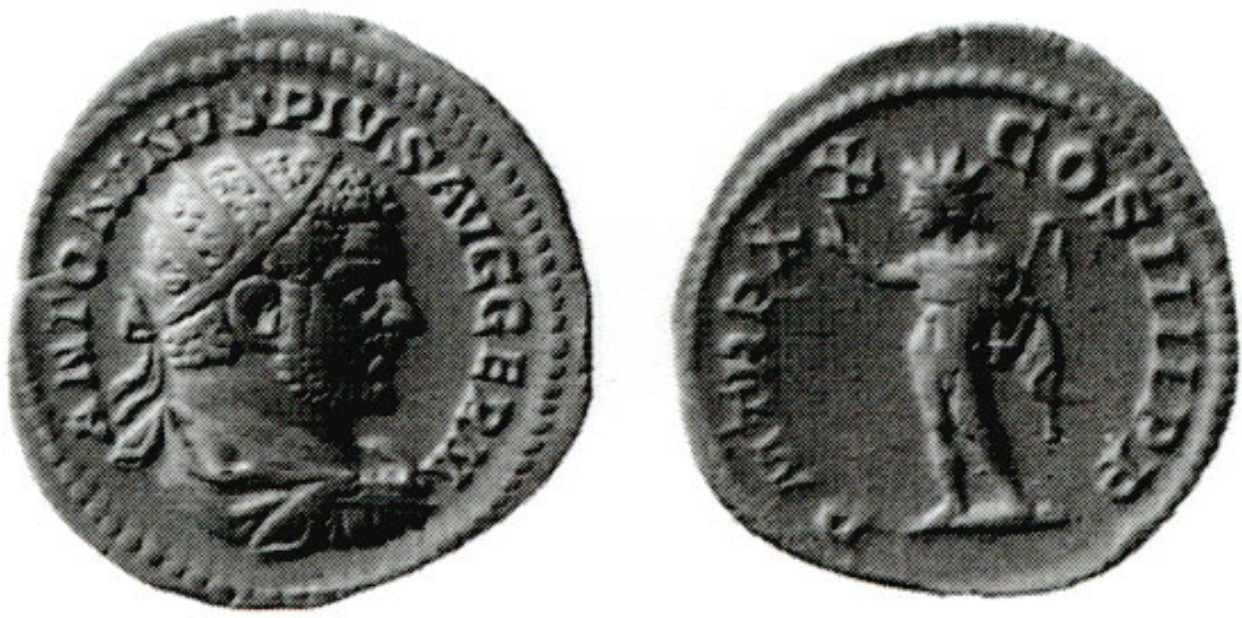

ANTONINVS PIVS AVG GERM, D2

P M TR P X-X COS IIII P P, Sol debout à gauche ; le bras droit est levé, la main gauche tient un fouet. $5,25 \mathrm{~g}$

RIC IV/1, p. 256 n $^{\circ} 293$ f; Hill 1581

61.
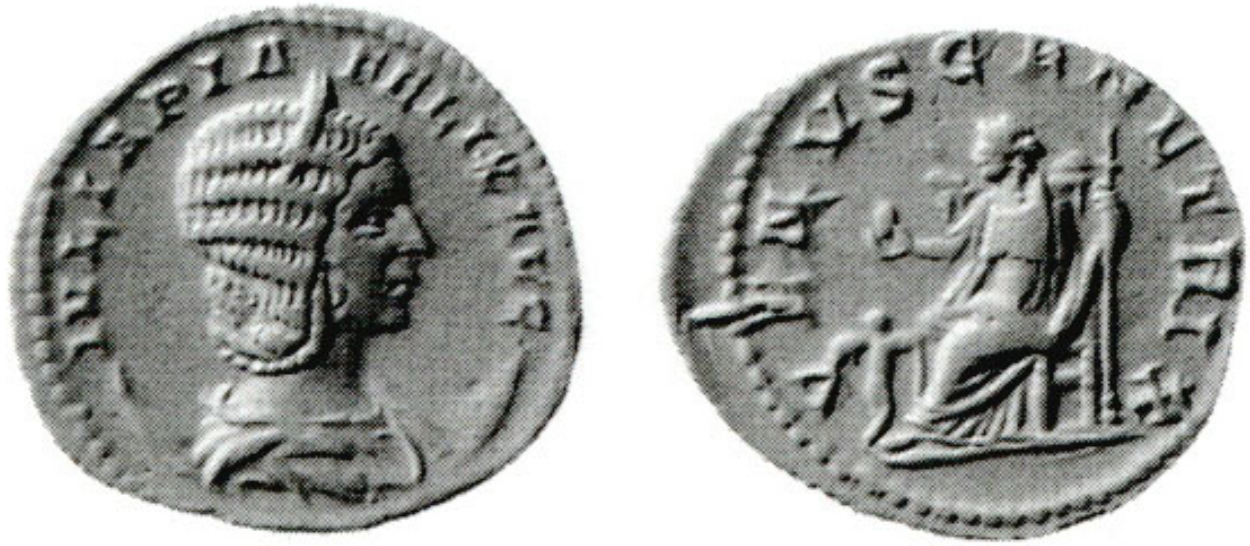

IVLIA PIA - FELIX AVG, E2

VENVS GENETRIX, Venus assise à gauche, la main droite tendue tenant une pomme et la main gauche tenant un long sceptre vertical ; à ses pieds, un enfant debout. $4,84 \mathrm{~g}$

RIC IV/1, p. 274 n 389 a; Hill 1582

Diaduménien (217-218)

Atelier de Rome

2e émission : juillet 217-mars 218 (denier) 
62.
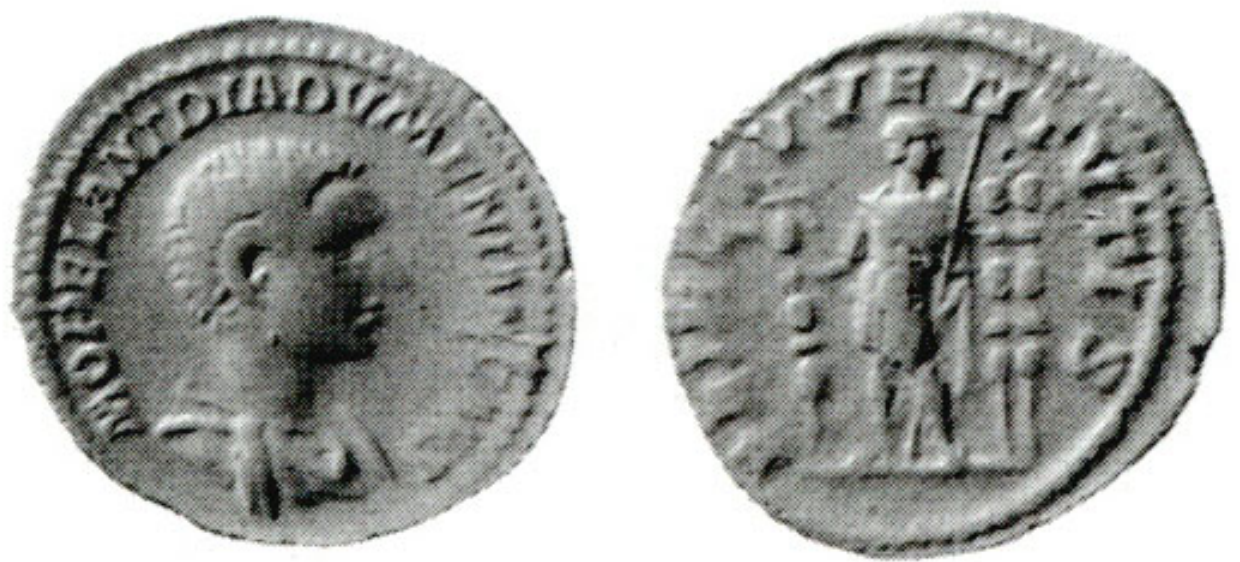

M OPEL ANT DIADVMENIAN CAES, +C1

PRINC IVVENTVTIS, Diaduménien debout de face, tête à droite, la main droite tenant une enseigne verticale et la main gauche un long sceptre transversal; deux enseignes verticales à droite. $3.09 \mathrm{~g}$

RIC IV/2, p. $13 n^{\circ} 102$; Clay, p. 22, 34

\section{Gordien III (238-244)}

\section{IMP GORDIANVS PIVS FEL AVG, D2}

63.
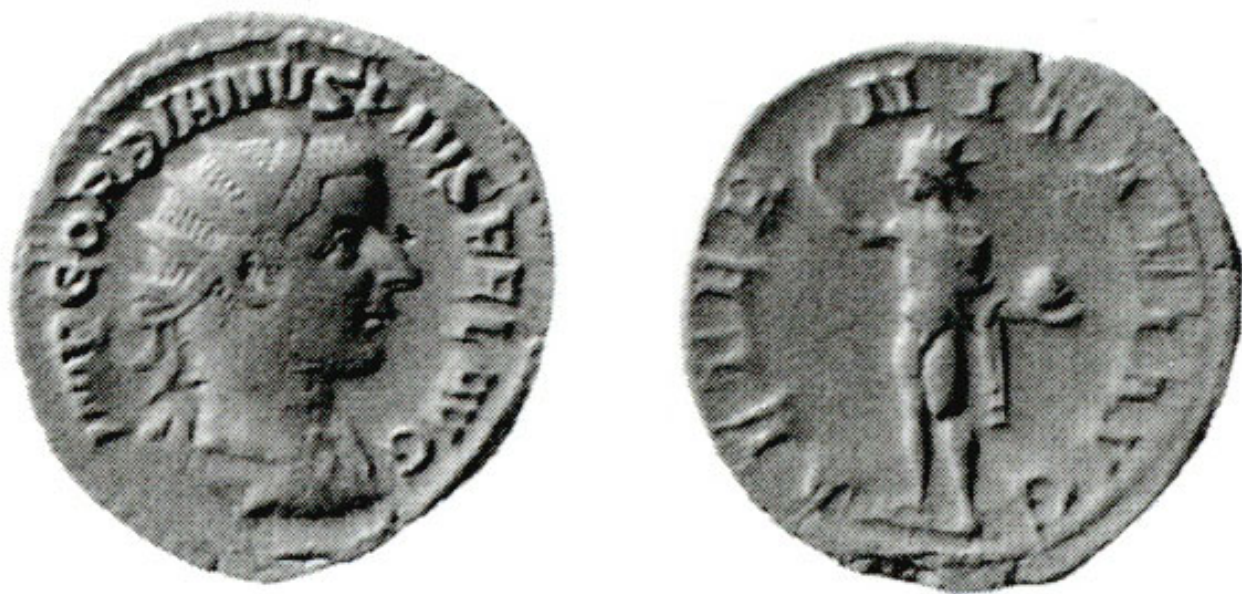

AETER - NITATI AVG, Sol debout de face, tête à gauche, la main droite levée, la main gauche tenant un globe.

3,44 g

RIC IV/3, p. 24 n 83 ; Eauze 698 
64.
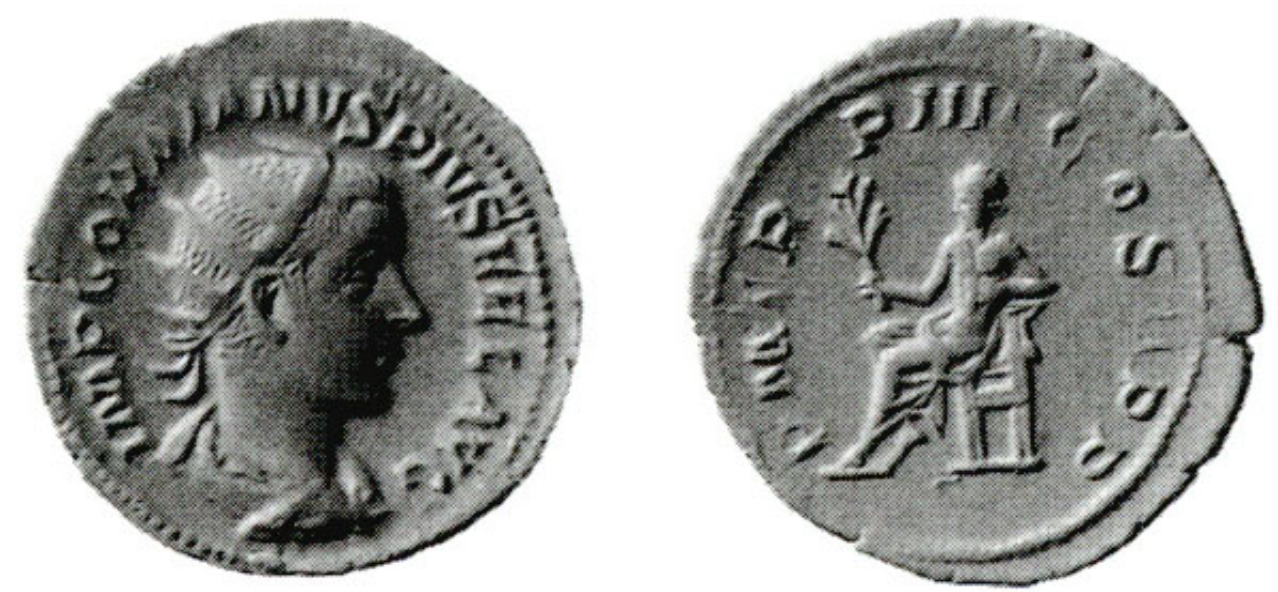

P M TR - P IIII COS II P P, Apollon assis à gauche, la main droite tenant un rameau, le coude gauche s'appuyant sur une lyre.

$3,84 \mathrm{~g}$

RIC IV/3, p. 25 n 88 ; Eauze 710

65.
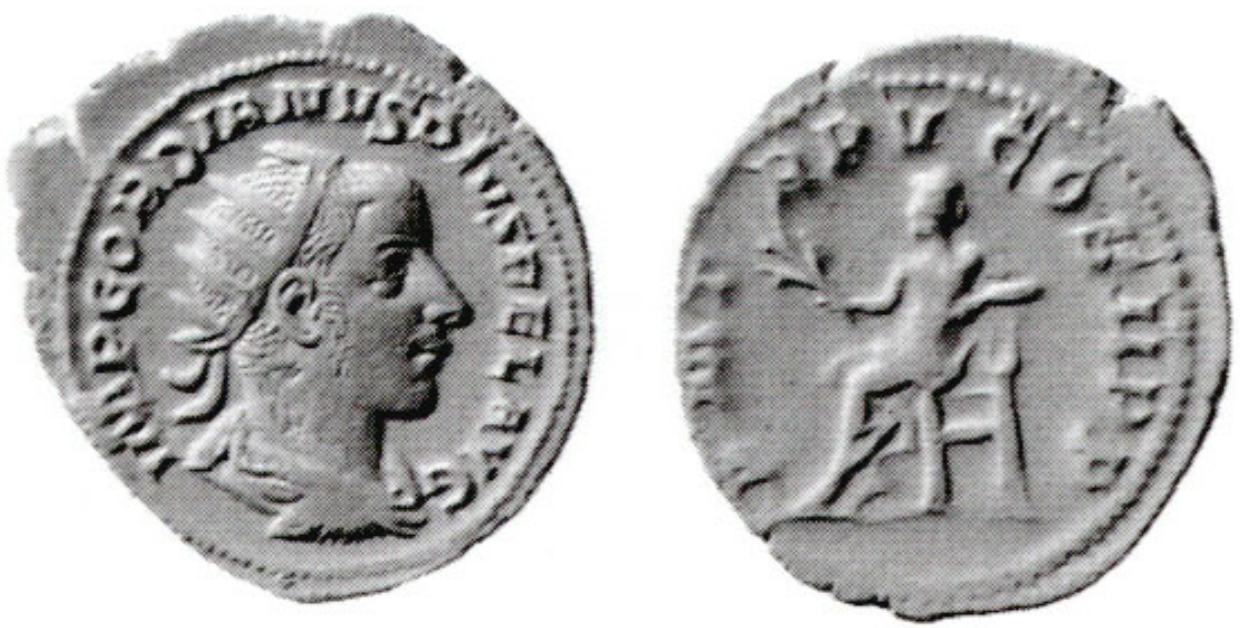

P M T-R P V COS IIP P, Apollon comme au n 64

$3.23 \mathrm{~g}$

RICIV/3, p. 25 n 89 ; Eauze 712

5e émission : 243-244

IMP GORDIANVS PIVS FEL AVG, D2 
66.
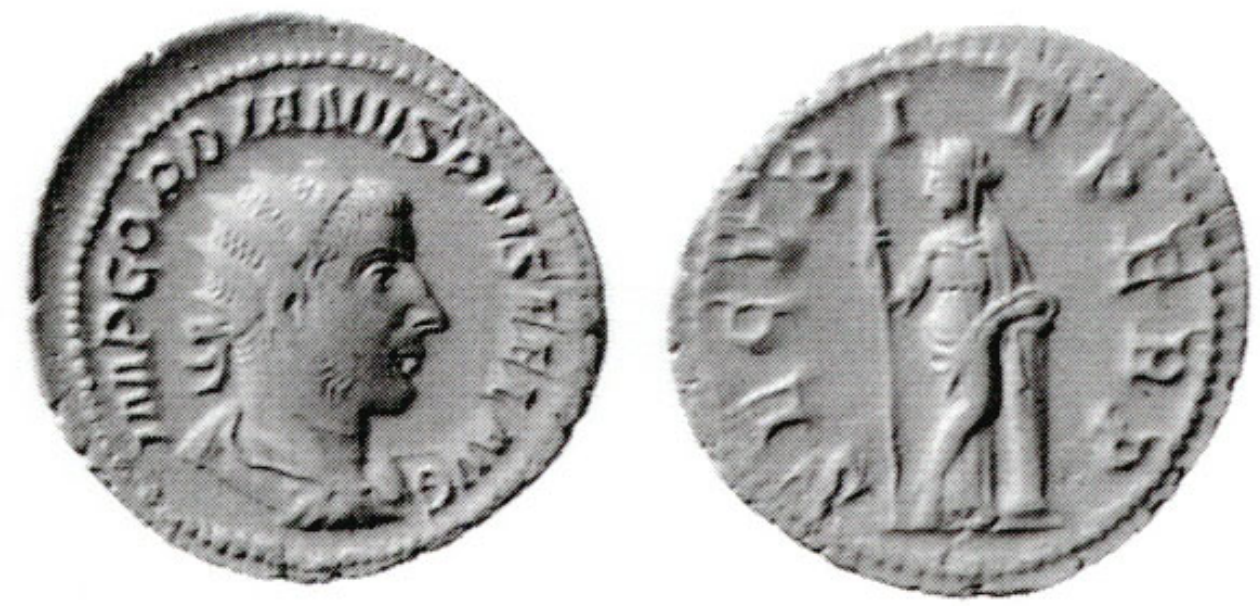

SECVR - IT PERP, Securitas debout de face, tête à gauche, la main droite tenant un long sceptre vertical, le coude gauche s'appuyant sur une colonne.

$4,14 \mathrm{~g}$

RIC IV/3, p. 31 n 151 ; Eauze 730

\section{Philippe (244-249)}

Atelier de Rome

\section{2e émission : 245}

67.
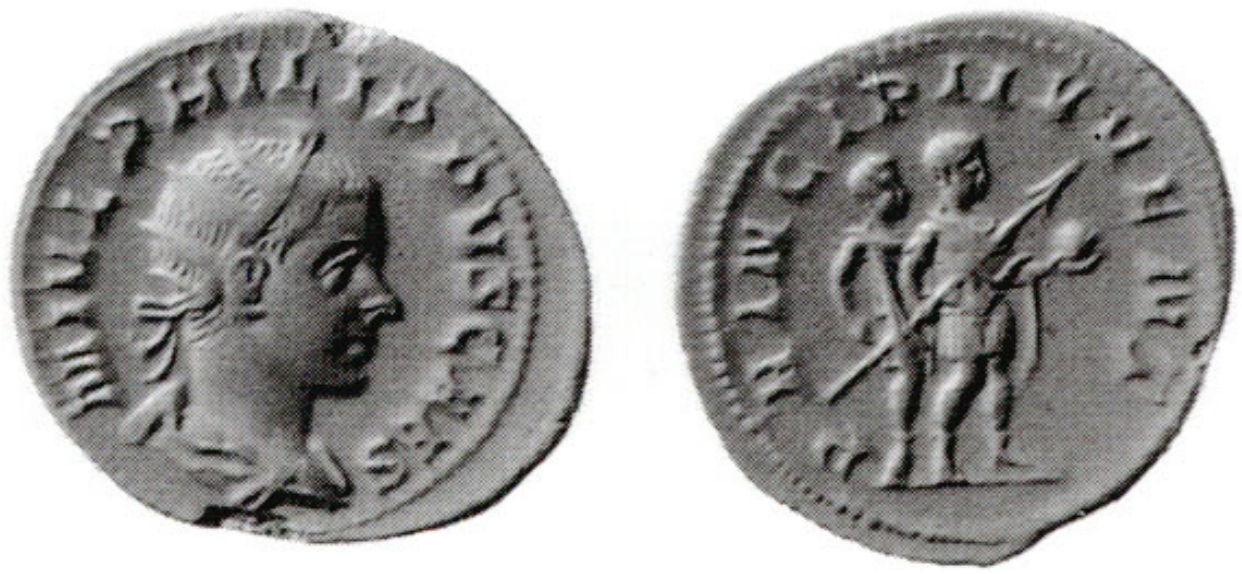

MIVL PHILIPPVS CAES, C2 (Philippe II)

PRINCIPIIVVENT, Philippe II debout à droite, la main droite tenant une lance transversale dirigée vers l'avant et la main gauche un globe ; derrière lui, un jeune homme. $4,35 \mathrm{~g}$

RIC IV/3, p. 95 n² 217 ; Eauze 845

3e émission : 245-247 
68.
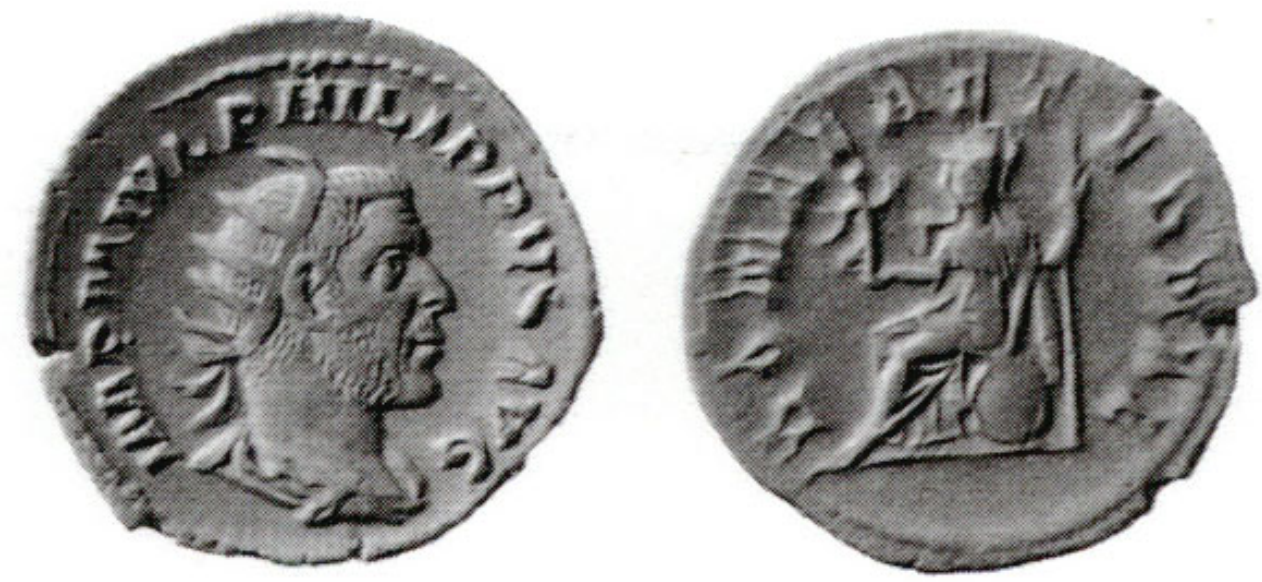

IMP M IVL PHILIPPVS AVG, D2 (Philippe I)

ROMAE AETERNAE, Rome assise à gauche sur un bouclier, la main droite tenant un globe nicéphore et la main gauche un long sceptre vertical.

$3,71 \mathrm{~g}$

RIC IV/3, p. 73 n 44 b; Eauze 795

69.
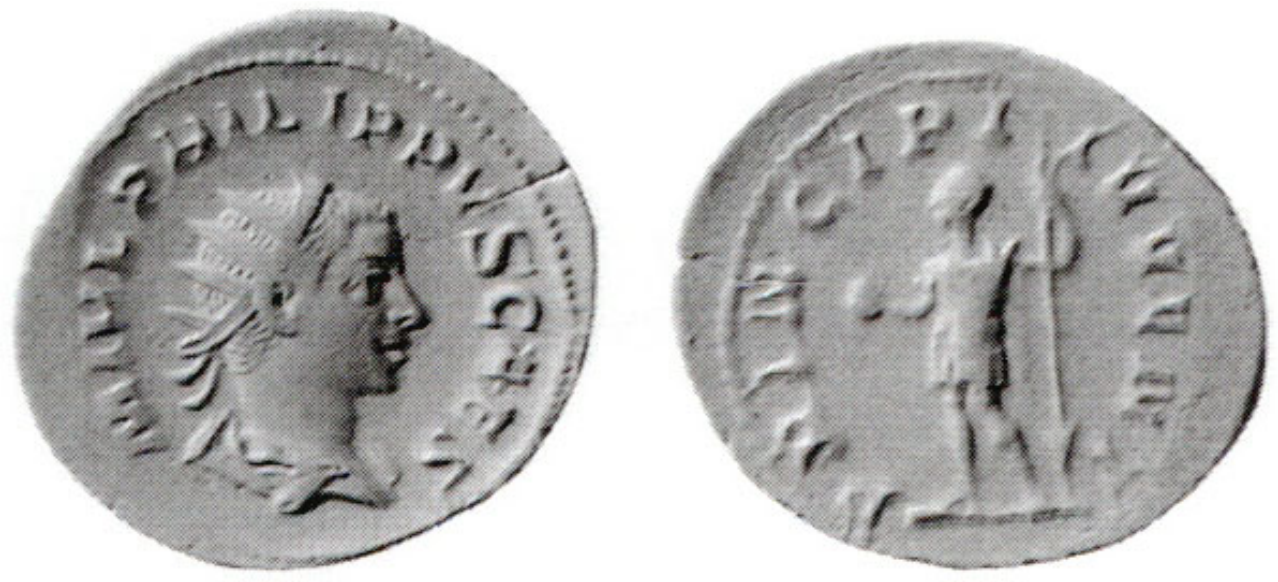

M IVL PHILIPPVS CAES, C2 (Philippe II)

PRINCIPI- IVVENT, Philippe II debout à gauche, la main droite tenant un globe et la main gauche une lance verticale inversée.

$2,91 \mathrm{~g}$

RIC IV/3, p. 96 n² 218 d; Eauze 839

5e émission : 248 
70-71.
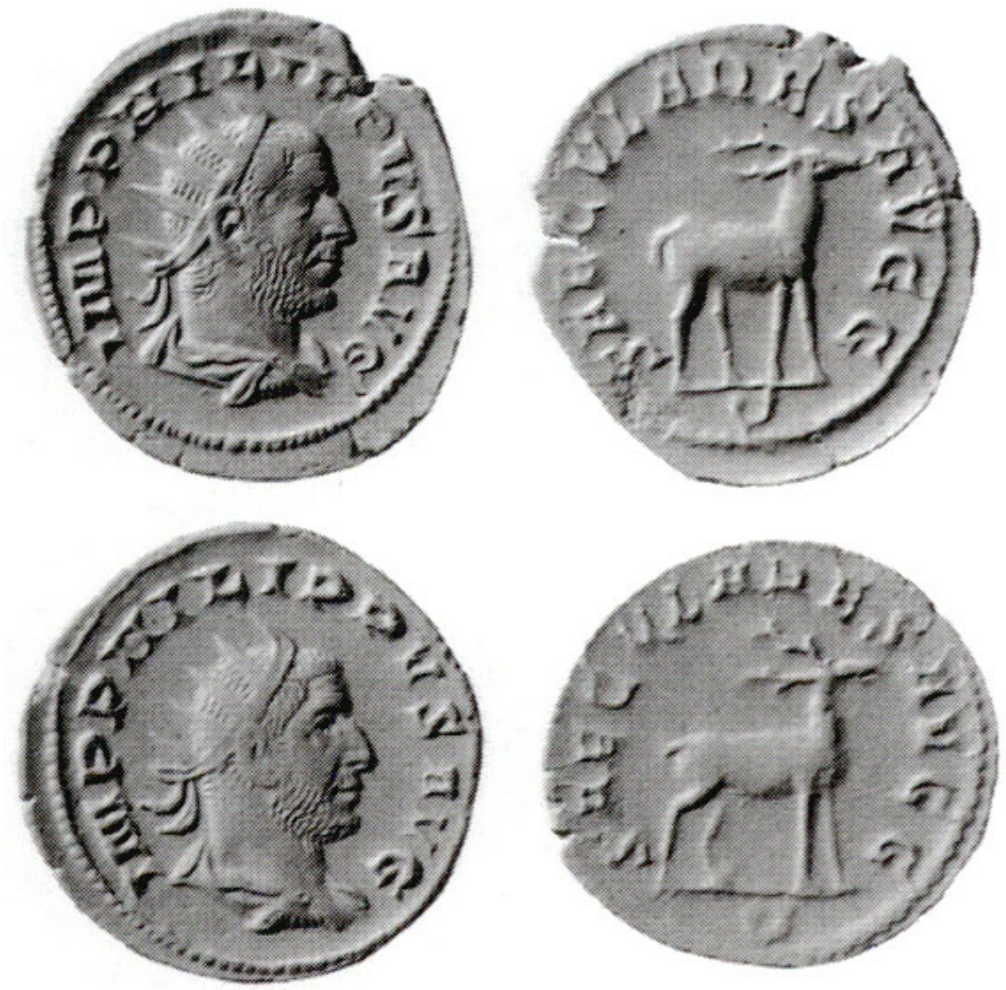

IMP PHILIPPVS AVG, D2 (Philippe I)

SAECVLARES AVGG, $V$ à l'exergue, cerf marchant à droite. 3,$06 ; 3,69 \mathrm{~g}$

RIC IV/3, p. 70 n 19 ; Eauze 807

6e émission : 248

72.
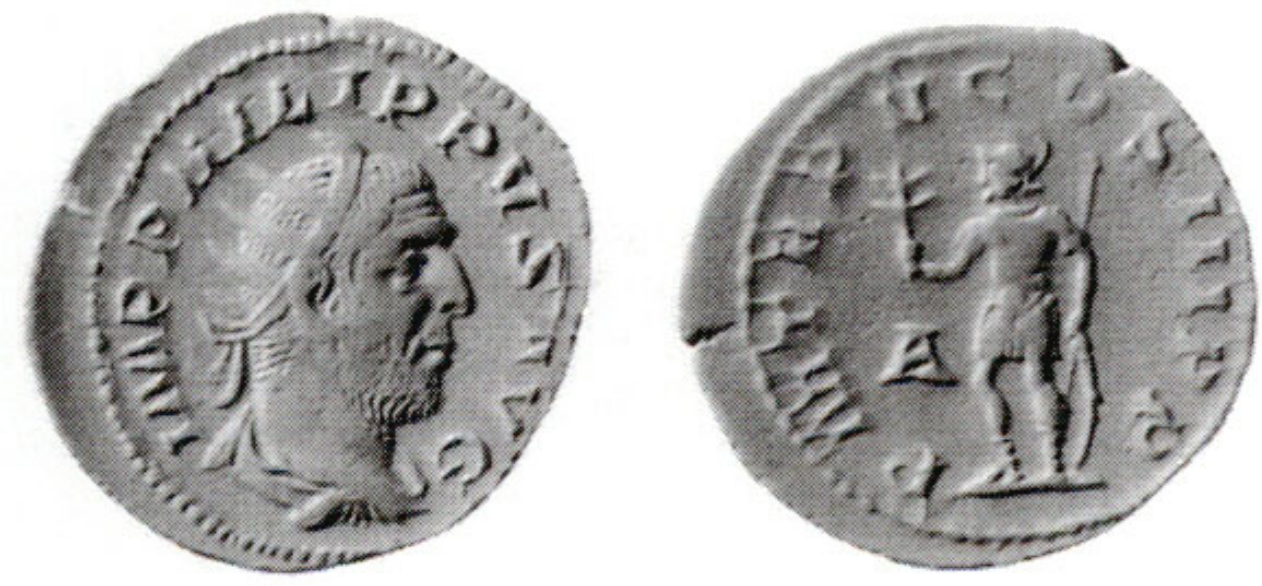

IMP PHILIPPVS AVG, D2 (Philippe I)

P M TR P V COS III P P, A dans le champ à gauche, Mars debout à gauche, la main droite tenant un rameau et la main gauche une lance et un bouclier.

$5,08 \mathrm{~g}$

RIC IV/3, p. $69 n^{\circ} 7$; Eauze 810 
73.
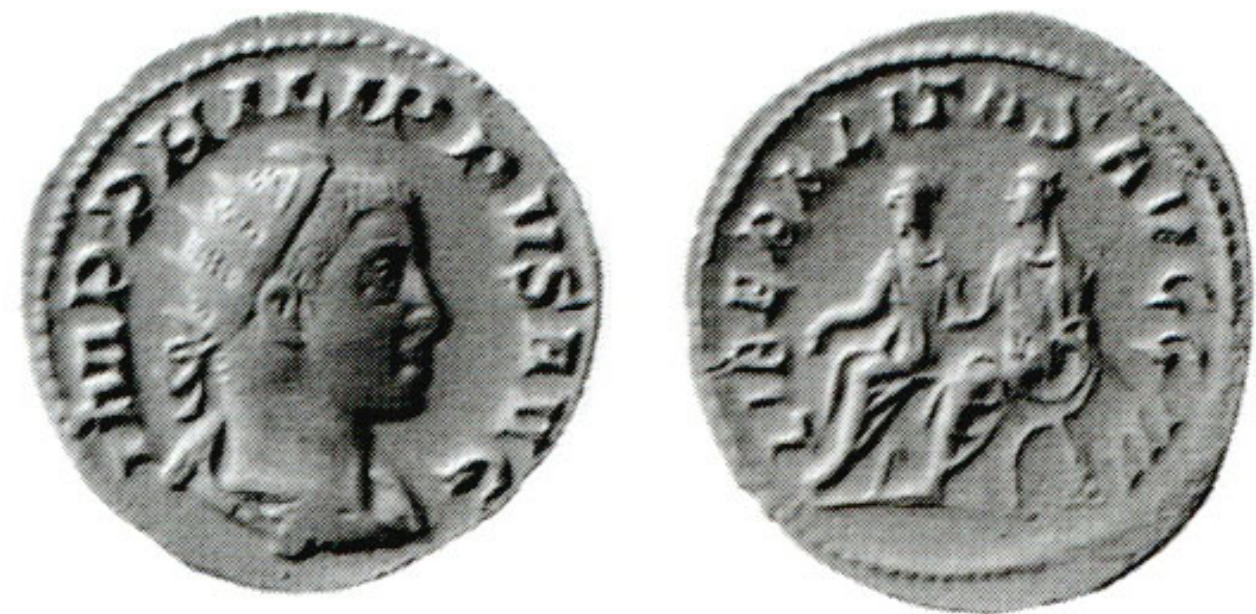

IMP PHILIPPVS AVG, D2 (Philippe II)

LIBERALITAS AVGG III, Philippe I et Philippe II assis à gauche sur des chaises curules.

$4,77 \mathrm{~g}$

RICIV/3, p. 97 n 230 ; Eauze 851

Trajan Dèce (249-251)

74.
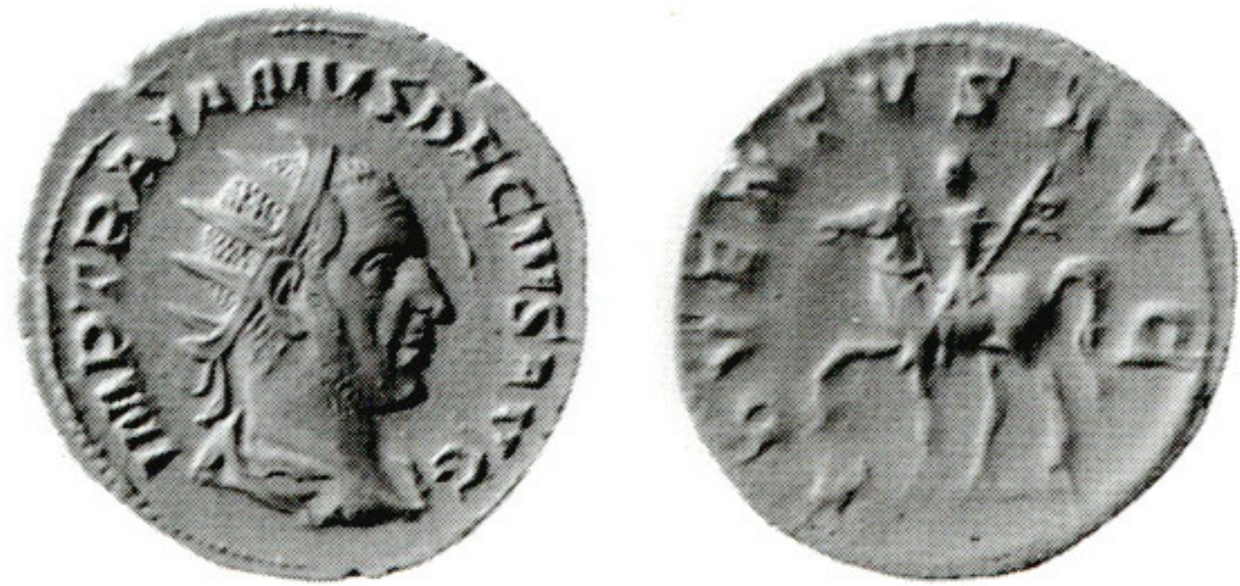

IMP TRAIANVS DECIVS AVG, D2

ADVENTVS AVG, Trajan Dèce à cheval à gauche, le bras droit levé, la main gauche tenant un sceptre transversal.

$4,05 \mathrm{~g}$

RIC IV/3, p. $120 \mathrm{n}^{\circ} 1$ b ; Eauze 877

61

\section{Etruscilla}


75.
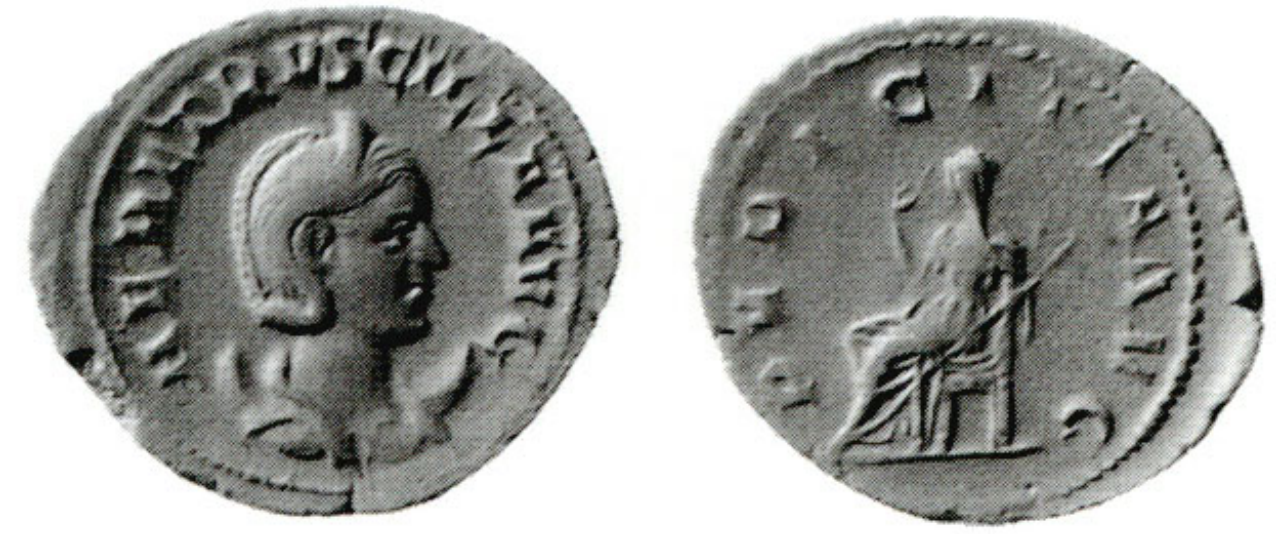

HER ETRVSCILLA AVG, E2 (cheveux plats)

PVDICITIA AVG, Pudicitia assise à gauche, la main droite touchant son voile et la main gauche tenant un long sceptre transversal.

$5,14 \mathrm{~g}$

RIC IV/3, p. 127 n 59 b ; Eauze 940

76.
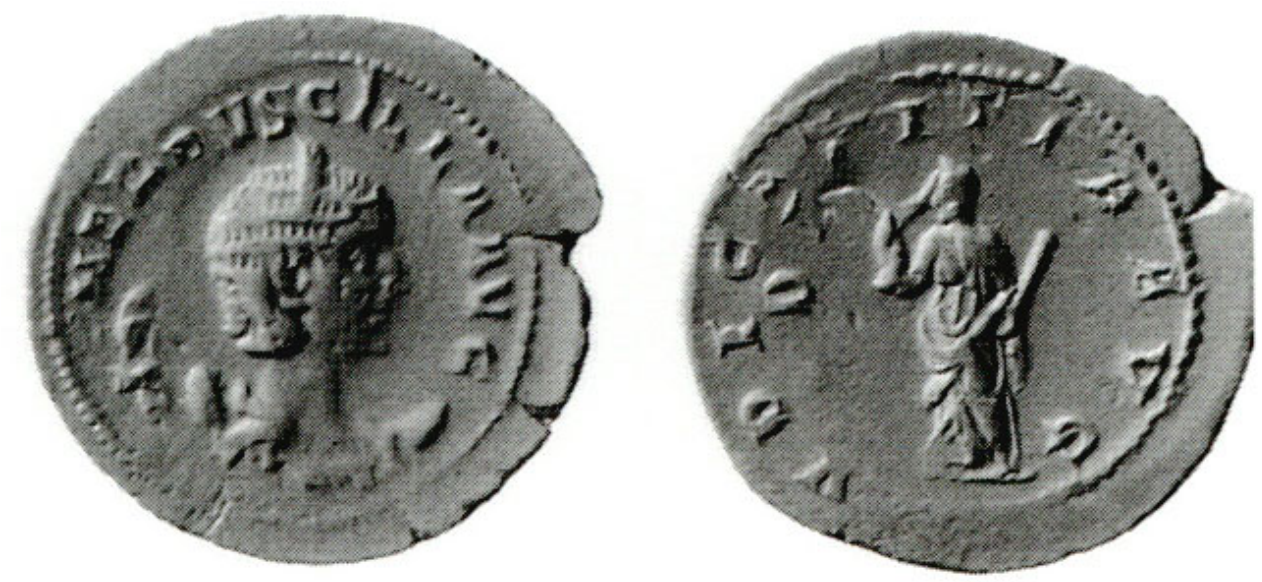

HER ETRVSCILLA AVG, E2 (cheveux ondulés)

[P]VDICITIA AVG, Pudicitia debout à gauche, la main droite touchant son voile et la main gauche tenant un sceptre transversal.

$4,70 \mathrm{~g}$ (monnaie tréflée au revers).

RIC IV/3, p. 127 n 58 b ; Eauze 939

\section{Valérien (253-260)}

Atelier de Rome

\section{Mariniane}


77.
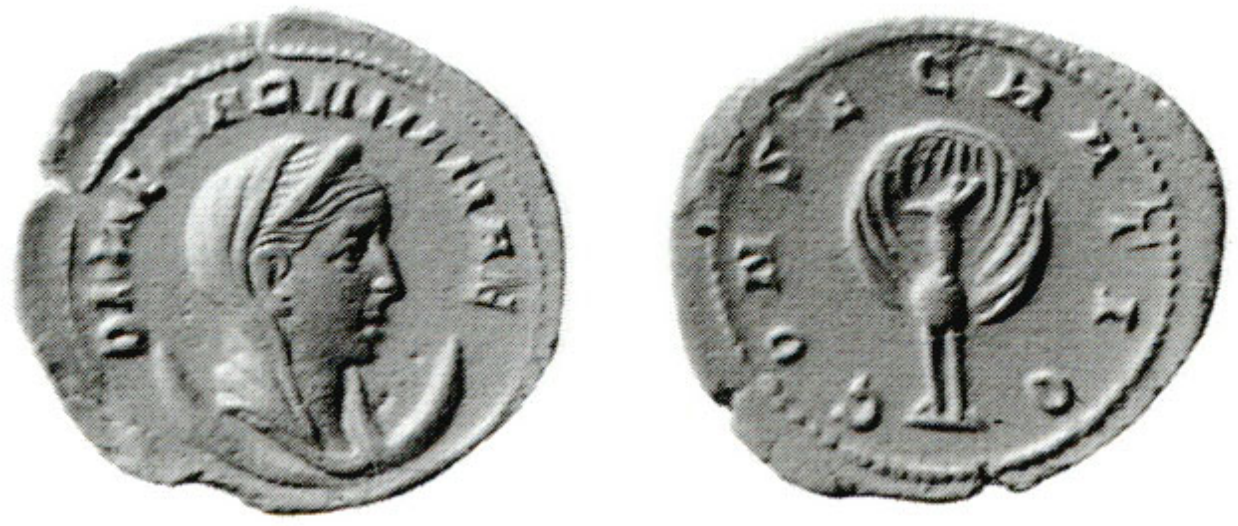

254-mi258

DIVAE MARINIANAE, E4

CONSECRATIO, Paon faisant la roue, debout de face avec la tête à gauche.

$86 \mathrm{~g}$

RIC, V/1, p. $64 n^{\circ} 3$; Cunetio p. $102 n^{\circ} 646$ (Rome) ; Eauze p. 188 n 1427 (Viminacium)

78.

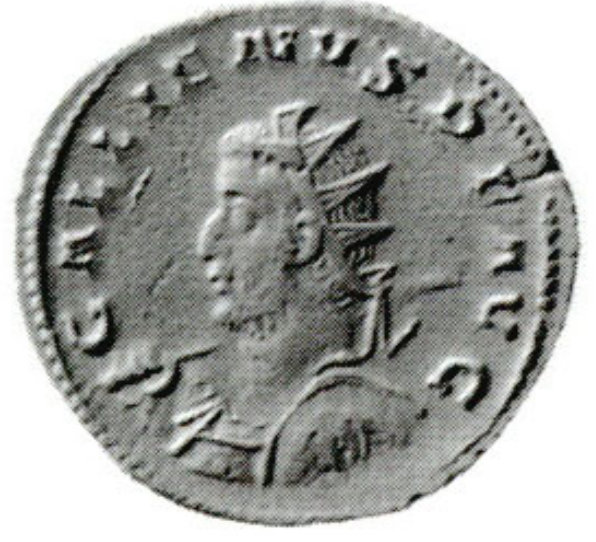

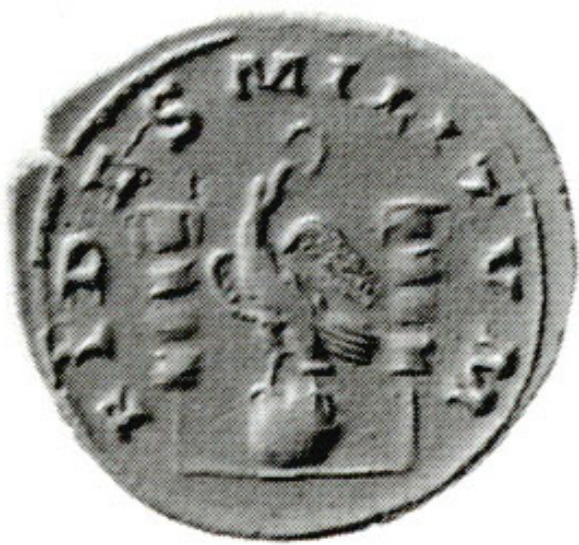

GALLIENVS P F AVG, buste radié cuirassé à gauche, vu de face, avec une lance sur l'épaule droite et un bouclier couvrant l'épaule gauche.

FIDES MILITVM, aigle debout à droite sur un globe, entre deux enseignes, la tête à droite et tenant une couronne dans le bec.

$3,03 \mathrm{~g}$

Elmer 39 ; Eauze 1478

67 4e émission, phase $b: 258$ 
79.
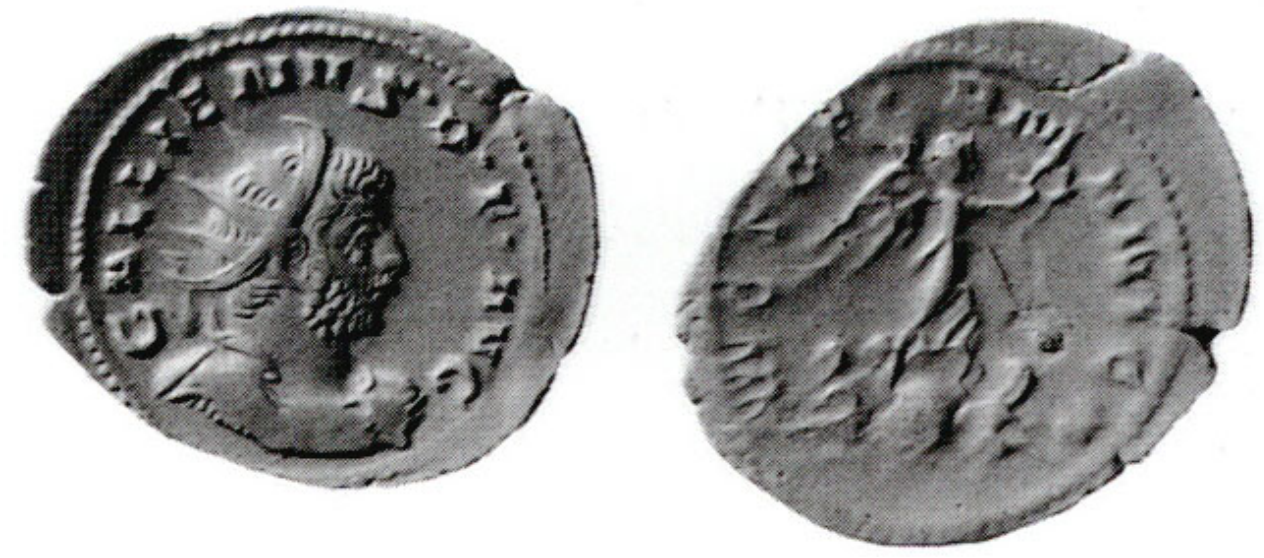

GALLIENVS $\cdot P \cdot F \cdot A V G, B 1$

VICT GE-RM-ANICA, Victoire debout à droite sur un globe entre deux captifs assis et enchaînés ; la main droite tient une couronne et la main gauche un trophée.

$3,60 \mathrm{~g}$

Elmer 57 ; Eauze 1492

\section{Salonine}

69

257-258

80.
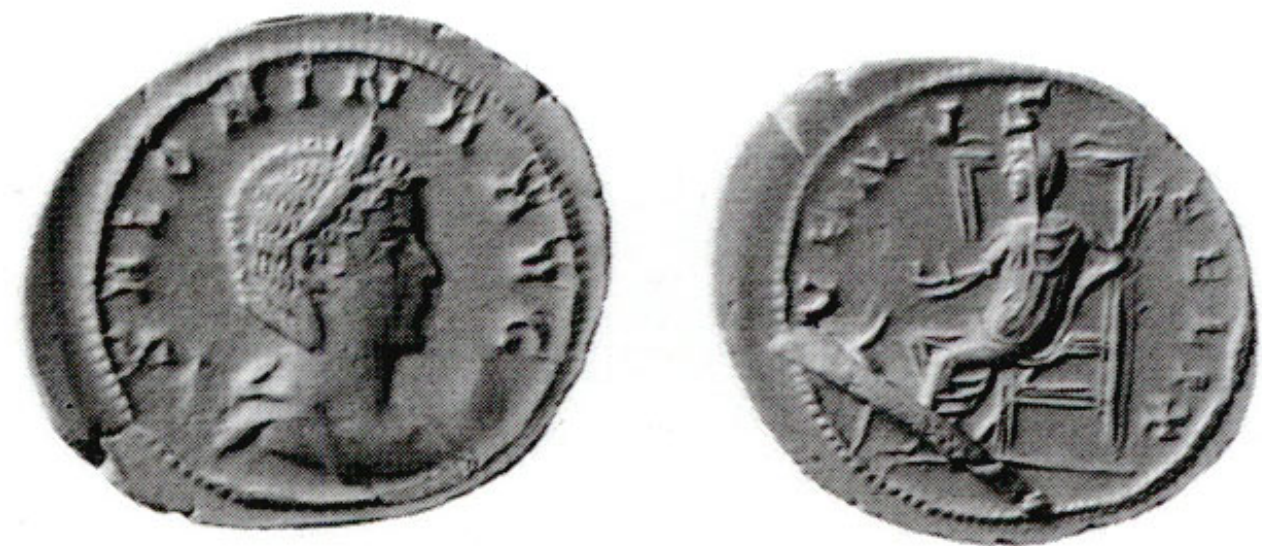

SALONINA AVG, E2

VENVS- FELIX, Venus voilée, assise à gauche, le bras droit tendu tenant une pomme, la main gauche tenant un long sceptre transversal; à gauche et à ses pieds, un enfant debout à droite, les deux bras levés.

$3,08 \mathrm{~g}$

Elmer 60 ; Eauze 1513

\section{Valérien II}

4e émission, phase $b$ : 258 

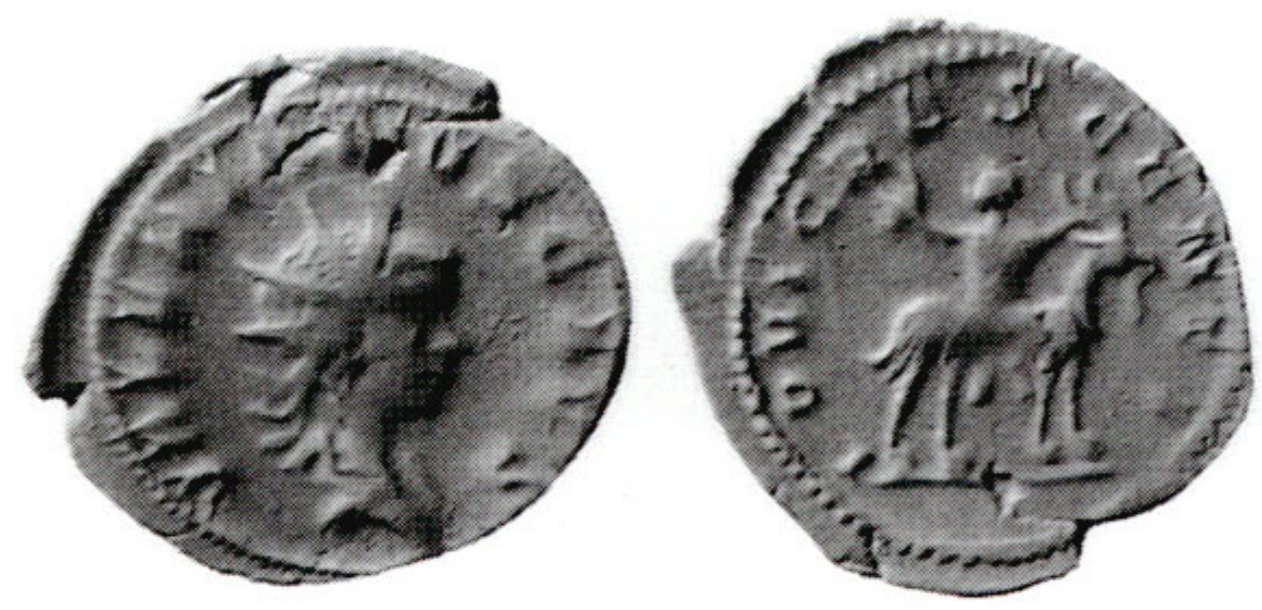

VALERIANVS CAES, C2 (buste étroit)

IOVI CRESCENTI, Jupiter enfant assis sur une chèvre debout à droite. $2,54 \mathrm{~g}$

Elmer 67 a ; Eauze 1526

\section{La monnaie d4or (montée en médaillon)}

Aureus de Gordien III. Rome

4 e émission (241-243) : 242
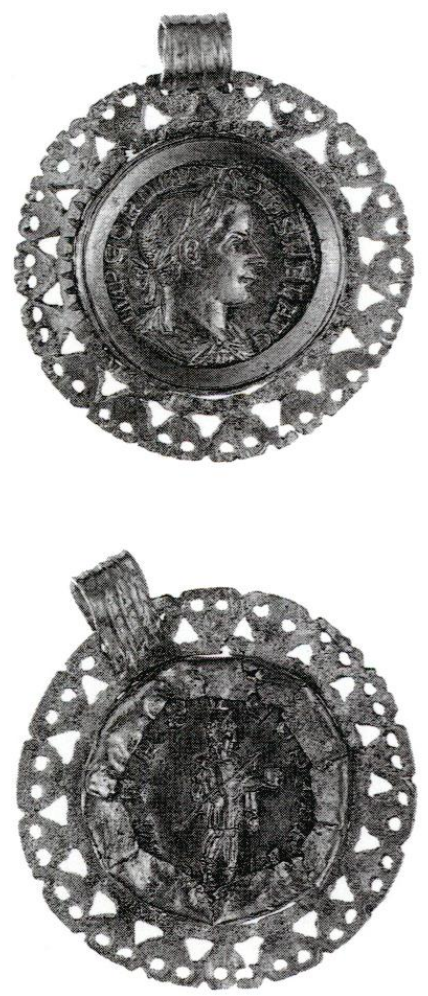

IMP GORDIANVS PIVS FEL AVG, *D2

P M TR P V COS II P P, Gordien, en habits militaires, debout à droite, la main droite tenant une lance transversale et la main gauche un globe. non pesable

RICIV/3, p. $26 n^{\circ} 106$

(fig. 107) 


\section{Le classement des monnaies}

72 Le classement des monnaies a été effectué à l'aide des catalogues britanniques (RIC, BMC) ou allemands (Elmer) traditionnels, corrigés et complétés par les éditions de trésors monétaires de plus en plus nombreuses et de plus en plus complètes depuis une trentaine d'années, notamment dans la collection Trésors Monétaires de la Bibliothèque nationale de France, et les "gros» trésors de Cunetio et d'Eauze, ce dernier ayant l'avantage d'offrir une abondante illustration.

\section{Les ateliers}

73 On notera quelques problèmes d'ordre général concernant les ateliers. Certes, la majeure partie des monnaies est issue de l'atelier romain, ce qui ne saurait étonner puisque jusqu'au début du IIIe s., l'hôtel des monnaies de la capitale fournit l'essentiel du numéraire impérial ; toutefois, le phénomène de mise en place d'un réseau d'ateliers impériaux périphériques, destinés notamment à satisfaire les besoins militaires - lever, nourrir et équiper les troupes - en rapprochant l'appareil de production des théâtres d'opérations (Christol 1977) se manifeste dans les dernières frappes du trésor, sous le règne de Valérien (253-260). La monnaie de consécration à la mémoire de Mariniane, épouse de Valérien (cat. $\mathrm{n}^{\circ}$ 77) est classée par les éditeurs du trésor d'Eauze (Schaad 1992, p. 295) à l'atelier mésien de Viminacium (aujourd'hui, Kostolac, Serbie), ouvert en 253 : cette attribution repose sur un détail iconographique (le buste de Mariniane serait représenté voilé à Rome, mais voilé et diadémé à Viminacium) et sur une possible différence de titre et de poids en faveur de Viminacium ; mais on relève que ce type de buste voilé et diadémé existe aussi avec un revers romain (RIC V/l, p. $64 \mathrm{n}^{\circ} 6$; Eauze 1320) même si Schaad note qu'il s'agit d'un cas exceptionnel; d'autre part la comparaison de teneurs en argent obtenues avec des méthodes différentes (analyse chimique dans un cas, micro analyse $\mathrm{X}$ dans l'autre) est délicate. Faute d'être convaincu par cette proposition d'attribution, j'ai donc préféré conserver le classement romain traditionnel à l'instar d'autres publications récentes de trésors de cette époque.

En revanche, les quatre dernières monnaies du trésor appartiennent à la production de l'atelier rhénan ouvert par Gallien, dans la seconde moitié de l'année 256. La localisation de cet atelier dont l'école anglaise avait d'abord attribué la production à Lyon (RIC V/l), n'est pas assurée. À la suite des travaux d'Elmer, en 1941, l'accord se fit sur Cologne, capitale de la Germanie Inférieure, cet atelier principal étant doublé par un atelier secondaire ouvert à Trêves en 269 (Lafaurie 1975a, p. 879-881). Ce schéma classique fut remis en cause dans la publication du trésor de Cunetio (Besly et Bland, 1983), puis dans celle de Normanby (Bland, Burnett 1988, p. 147) qui inversèrent les localisations : l'atelier gaulois ouvert par Gallien en 256 " was probably situated at Trier " et devint l'atelier principal de Postume en 260 ; en 268 , un deuxième atelier fut ouvert à Cologne et signa ses premières productions des lettres CCAA (Colonia Claudia Ara Agrippinensium). Cette thèse, adoptée par l'historien britannique Drinkwater (1987, p. 132-147), puis exposée ou défendue par des numismates français (Hollard 1990; Gricourt 1990), est considérée avec sympathie, mais sans emporter totalement l'adhésion (Callu 1989, p. 370 ; Schaad 1994) ni faire l'unanimité. Je me bornerai donc à employer l'expression d'atelier de Gaule. 

monnaies coulées à l'aide de moules dont on sait que la production frauduleuse a sévi en Gaule et notamment à Lyon où sept trouvailles de moules en argile ont été recensées (Turcan 1982; Aubin, Monin, 1996). Mais il est vrai que l'incertitude règne sur la période d'activité des faussaires: dès le règne d'Alexandre Sévère (222-235) ou postérieurement à 260 ?

\section{Monnaies remarquables}

Par ailleurs, deux cas particuliers méritent examen.

Un denier (cat. $\mathrm{n}^{\circ}$ 20) associe un revers de P SEPT GETA CAES PONT, buste de Géta, à un droit de Caracalla ANTONINVS AVG PONT TR P IIII. Cette monnaie au style particulier, peu romain, (traitement de l'œil et de la chevelure, disposition des légendes, forme de certaines lettres comme le E) semble issue de la même paire de coins que l'exemplaire du British Museum n ${ }^{\circ} 729$, attribué à l'atelier oriental de Laodicea ad Mare (Lattaquié, Syrie). Cet atelier, entre 196 et 202, a concentré la frappe des deniers en Orient et est devenu une véritable filiale de l'atelier romain, faisant appel au même stock thématique (Callu 1969, p. 163; BMC, V, p. clxi). Ainsi, ce "colle-giategiate type", associant le jeune auguste et son frère promu césar, utilisé dans cet atelier oriental, se trouve abondamment frappé à Rome tant en or (Hill 465, 513) qu'en argent (Hill 475, $522,538)$ mais avec des légendes de droit variées qui s'égrènent tout au long de l'année $201^{7}$.

Un denier de Caracalla (cat. $n^{\circ} 21$ ) semble inédit, au moins dans ce métal : il s'agit d'un droit à légende ANTONINVS AVGVSTVS associé à un revers CONCORDIAE AETERNAE, bustes accolés de Septime Sévère et de Julia (fig. 116). Ce couplage est connu pour l'or par un rare aureus de la collection Jameson (1913: $n^{\circ} 192$, pl. IX) qui est daté par Hill de la première émission dynastique de $201^{8}$, mais n'est pas répertorié pour l'argent. Le même revers est utilisé avec des droits aux légendes différentes, mentionnant l'épithète PIVS, lors de la deuxième émission dynastique de 201, pour l'or (Hill 512) et pour l'argent (Hill $521 ; c f$. supra cat. $n^{\circ} 24$ ). Le denier de Vaise, en attestant l'emploi de ce revers pour l'argent dès la première émission, permet donc d'obtenir un parallélisme entre les deux premières émissions dynastiques ${ }^{9}$. Il serait d'ailleurs préférable de parler de phases d'émission distinguées par de simples changements de titulature. Ces phases durent se succéder rapidement puisque deux deniers de Vaise ( $n^{\circ} 21$ et 24$)$ appartenant selon le classement de Hill à deux émissions différentes sont liés par l'utilisation du même coin de revers. (cf. infra: Les émissions dynastiques de 201-202)

\section{Les émissions dynastiques de 201-202}

À l'issue de la seconde guerre parthique, glorieuse campagne en Mésopotamie (197-199), et d'une tournée en Syrie et en Egypte, Sévère revint à Rome, au premier semestre 202, pour célébrer le dixième anniversaire de son règne, son triomphe et le mariage de Caracalla. Ce retour de Sévère et des siens fut préparé à Rome par des émissions monétaires spéciales célébrant la famille impériale, dans le dessein d'affirmer la naissance d'une dynastie pour le plus grand bien de l'Empire. Sévère souhaitait faire oublier l'origine militaire de son pouvoir. Son souci insistant de se rattacher, par adoption posthume, à la dynastie des Antonins et d'acquérir ainsi une apparence de légitimité n'eut d'égal que sa volonté d'inscrire son règne dans 
le cadre d'une dynastie nouvelle.

La famille impériale

- Severus Pius : Lucius Septimius Severus, né en 145, africain d'origine, sénateur, proclamé empereur par ses troupes danubiennes en avril 193, vainqueur de la guerre civile de 193-197, se proclame fils adoptif de Marc Aurèle, et frère de Commode.

- Iulia Augusta : Julia Domna, princesse syrienne de la famille des grands prêtres du culte de Baal d'Emèse, seconde épouse de Septime Sévère en 187.

- Antoninus Augustus : Lucius Septimius Bassianus, premier fils de Sévère, né à Lyon le 4 avril 188, alors que son père était gouverneur de Lyonnaise ; nommé César en avril ou juin 196 sous le nom de Marcus Aurelius Antoninus (les noms de Marc Aurèle), puis Auguste le 28 janvier 198. Connu sous le sobriquet de Caracalla (caracallus : nom d'un manteau long à capuchon militaire dont il se vêtait).

- P. Sept Geta : Publius Septimius Géta, deuxième fils de Sévère, né en 189, nommé César le 28 janvier 198, ne devint Auguste qu'à l'automne 209.

Structure des émissions

Un tableau (fig. 113) ordonnant les pièces connues et retenues dans l'ouvrage de Hill (1977), sur le monnayage de Septime Sévère et de sa famille dans l'atelier de Rome en dépit de ses imperfections, permet de mieux percevoir le programme. Contrairement aux émissions habituelles ou ordinaires, ces émissions spéciales n'étaient frappées qu'en or (AV) et en argent (AR), ce qui suggère qu'elles étaient d'abord destinées à des cadeaux. Hill distingue 4 émissions - numérotées de 1 à 4 sur le tableau -, les trois premières en 201, la quatrième en 202. Elles se différencient par des modifications des titulatures :

- entre la 1ère et la 2e émission, Sévère et Caracalla reçoivent le titre de PIVS ; - entre la 2e et la 3e émission, Sévère passe de la 9e à la 10e puissance tribunicienne et Caracalla de la 4e à la 5e puissance tribunicienne (10 décembre 201) ;

- entre la 3e et la 4e émission. Sévère revêt un 3e consulat et Caracalla revêt son 1er consulat (1er janvier 202).

Les monnayeurs doivent donc utiliser de nouveaux coins de droit qui tiennent compte de ces modifications. On serait donc enclin à n'envisager qu'une seule émission dynastique déclinée en différentes phases s'étalant au moins sur la deuxième moitié de 201 et le début de 202.

L'architecture de ces émissions est assez simple puisqu'elle s'appuie sur la combinaison de quatre droits (les quatre personnes de la domus divina) et de sept types de revers : trois revers présentent individuellement les membres de la famille associés à l'empereur avec leur nom en légende ; trois autres associent deux membres (Sévère/Julia ; Sévère/Caracalla ; Caracalla/Géta) et enfin un revers réunit trois membres.

En ce qui concerne les types présentant un portrait sur chaque face, la distinction entre droit et revers est aléatoire ! Les classements modernes s'appuient, semble-til, sur le principe que le personnage de rang le plus élevé figure au droit ; ce qui explique que Géta bien représenté en nombre de revers n'en commande aucun. Sur les autres frappes figurant au revers deux portraits accolés ou confrontés, ou trois portraits, la règle semble être de ne pas doubler le portrait du personnage du droit sur le revers. Un tableau de synthèse (fig. 114) montre bien ce principe qui ne subit que trois exceptions au profit de Sévère (Hill 516) et de Caracalla (Hill 474, 
520) pour des deniers à légende aeternit imperi. Faut-il y voir des anomalies et des erreurs de monnayeurs ? Dans ce dernier cas, on aurait utilisé pour Sévère ou Caracalla des coins de revers destinés normalement à des frappes pour Domna ou Géta. Par ailleurs, le tableau comporte des blancs ; la construction logique de cette émission implique l'existence de monnaies non encore connues : un aureus de Sévère avec un revers pour Géta (répondant au denier Hill 473) ; un denier de Géta au revers Concordiae aetemae, Sévère-Julia (répondant à l'aureus Hill 467). En revanche, le type Felicitas aux trois portraits n'a sans doute été frappé qu'en or.

113- Les émissions dynastiques de 201-202 à Rome.

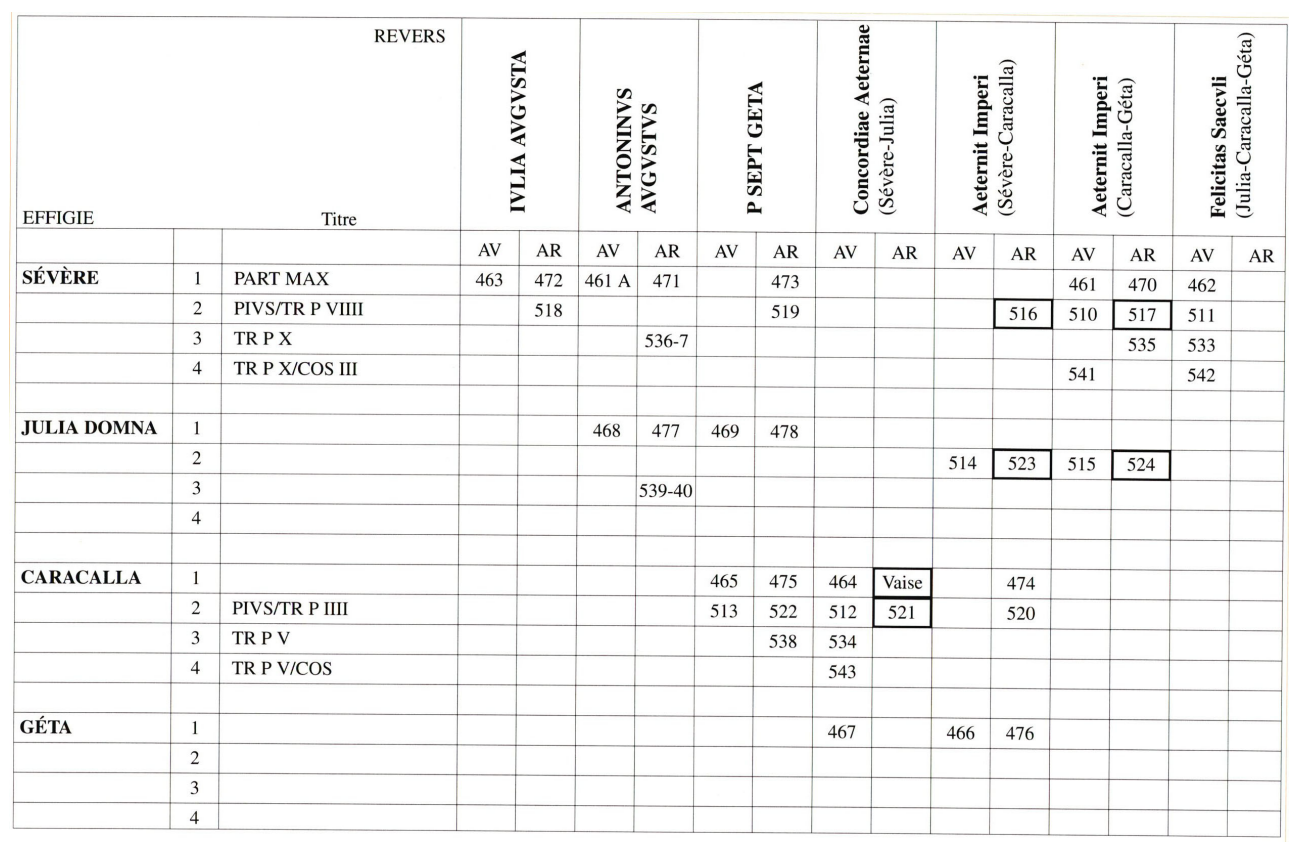

LES NUMÉROS RENVOIENT AU CATALOgUE DE HILL (1977). LES EXEMPLAIRES REPRÉSENTÉS À VAISE SONT ENCADRÉS

114- Synthèse des émissions dynastiques de 201-202 à Rome.

\begin{tabular}{|c|c|c|c|c|c|c|c|c|c|c|c|c|c|c|}
\hline \multirow[t]{2}{*}{ EFFIGIE } & \multicolumn{2}{|c|}{ 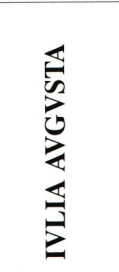 } & \multicolumn{2}{|c|}{ 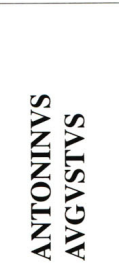 } & \multicolumn{2}{|c|}{ 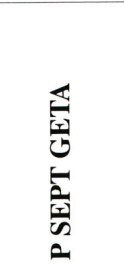 } & \multicolumn{2}{|c|}{ 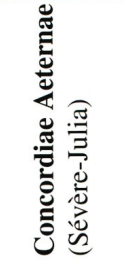 } & \multicolumn{2}{|c|}{ 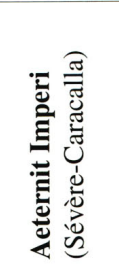 } & \multicolumn{2}{|c|}{ 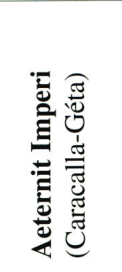 } & \multicolumn{2}{|c|}{ 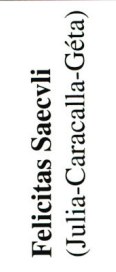 } \\
\hline & AV & AR & AV & AR & AV & AR & $\mathrm{AV}$ & AR & $\mathrm{AV}$ & AR & $\mathrm{AV}$ & AR & $\mathrm{AV}$ & AR \\
\hline SÉVÈRE & 1 & 2 & 1 & 2 & & 2 & & & & 1 & 3 & 3 & 4 & \\
\hline JULIA DOMNA & & & 1 & 2 & 1 & 1 & & & 1 & 1 & 1 & 1 & & \\
\hline CARACALLA & & & & & 2 & 3 & 4 & 2 & & 2 & & & & \\
\hline GÉTA & & & & & & & 1 & & 1 & 1 & & & & \\
\hline
\end{tabular}

Dans chaque cellule figure le nombre de types recensés par Hill (1977). Les cases grisées correspondent à des impossibilités théoriques 


\section{Les thèmes}

Trois légendes accompagnent les portraits multiples:

La légende AETERNIT(as) IMPERI(i), l'Eternité de l'Empire, diffuse l'idée que la continuité de l'Etat est liée à celle de la famille impériale (fig. 115). Au sortir de quatre années de guerres civiles (193-197) pendant lesquelles Sévère s'était imposé face à trois compétiteurs (Julianus, Niger, Albinus), les images de l'association entre les deux augustes Sévère et Caracalla, et entre les deux fils de l'empereur, montraient que la succession était assurée. On notera sur ces portraits affrontés la différence de traitement entre Caracalla et Géta : alors que le premier porte la couronne laurée impériale, Géta - simple césar - est représenté tête nue, conformément à la tradition.

La légende CONCORDIAE AETERNAE, à la Concorde éternelle, accompagne les bustes accolés de Sévère et de Domna (fig. 116). Sévère est représenté avec la couronne radiée de Sol et Julia avec le croissant de Luna, allusion possible aux cultes solaires, notamment syriens ; ces signes distinctifs seront employés treize ans plus tard par Caracalla sur sa nouvelle espèce d'argent, l'antoninien. Le culte impérial favorise l'identification du prince à Hélios et de son épouse à Séléné ou aux Mères orientales. Le thème de l'harmonie, traditionnel pour les mariages impériaux, sera aussi utilisé en 202 dans une émission spécifique pour le mariage de Caracalla (Hill p. 21 n 576, p. 37 n 575 A) : la représentation de l'union entre le jeune Auguste et Plautille est placée sous la même invocation. Les monnaies célèbrent alors sur tous les tons la concorde (CONCORDIA AVGG ou CONCORDIA FELIX), et la perpétuation de l'Empire (PROPAGO IMPERI).

La réunion de la famille impériale sur une même monnaie représente la tentative la plus aboutie de propagande sévérienne ${ }^{10}$. N'existant qu'en or, elle n'est pas représentée dans le trésor de Vaise ; l'exemplaire illustré ci-contre (fig. 117), sans provenance connue, est conservé à la Bibliothèque nationale de France (collection Beistegui 120) : au droit, Sévère ; au revers, Julia Domna, de face, entourée de ses deux fils. La légende FELICITAS SAECVLI, le bonheur du siècle, annonce un âge d'or dont la première décennie va être prochainement célébrée.

Pendant la même période, les émissions ordinaires déclinent les thèmes de la victoire parthique. Sévère, PART(icus) MAX(imus) (très grand parthique), est qualifié de FVNDATOR PACIS (fondateur de la paix), RESTITVTOR VRBIS (restaurateur de la Cité), tandis que Caracalla, RECTOR ORBIS (conducteur du monde) est assimilé à Sol. La fin des guerres civiles et étrangères garantissent ROMA AETERNA (Rome éternelle) et assurent, avec la prospérité, SAECVLI FELICITAS (la félicité du siècle).

Ces émissions sont donc une des expressions de la politique dynastique menée par Septime Sévère qui célébra la domus divina et manifesta, en assurant sa succession de son vivant, sa volonté de fonder une dynastie comme les Antonins auxquels il se réfère et se rattache. La glyptique en offre d'autres manifestations, qu'il s'agisse d'intailles ou du camée du cabinet des Médailles (Bastien 1992-1994 : III, pl. 79). Les thèmes dynastiques se poursuivent bien au delà de cette année 202. Citons, par exemple, la commémoration des victoires parthiques, en 204, avec au droit de l'or et de l'argent la légende IMPP INVICTI PII AVGG (les empereurs invaincus, pieux, Augustes) et les portraits accolés de Sévère et Caracalla ${ }^{11}$; ou encore, l'étonnant aureus émis en 209 pour l'accession de Géta à l'augustat, montrant au droit les 
bustes de Sévère et de Julia avec la légende FELICITAS PVBLICA, le bonheur public, et au revers, les bustes des deux jeunes empereurs avec la légende PERPETVA

CONCORDIA, la concorde perpétuelle ${ }^{12}$. Cette insistance sur le thème de l'harmonie entre les deux frères, comme garant de la pérennité et de la prospérité de l'Empire ne visait qu'à masquer leur hostilité. Le 27 février 212, un an après la mort de leur père, Caracalla fit assassiner Géta, réfugié dans les bras de leur mère.

115- Caracalla et Géta au revers d'un denier de Septime Sévère

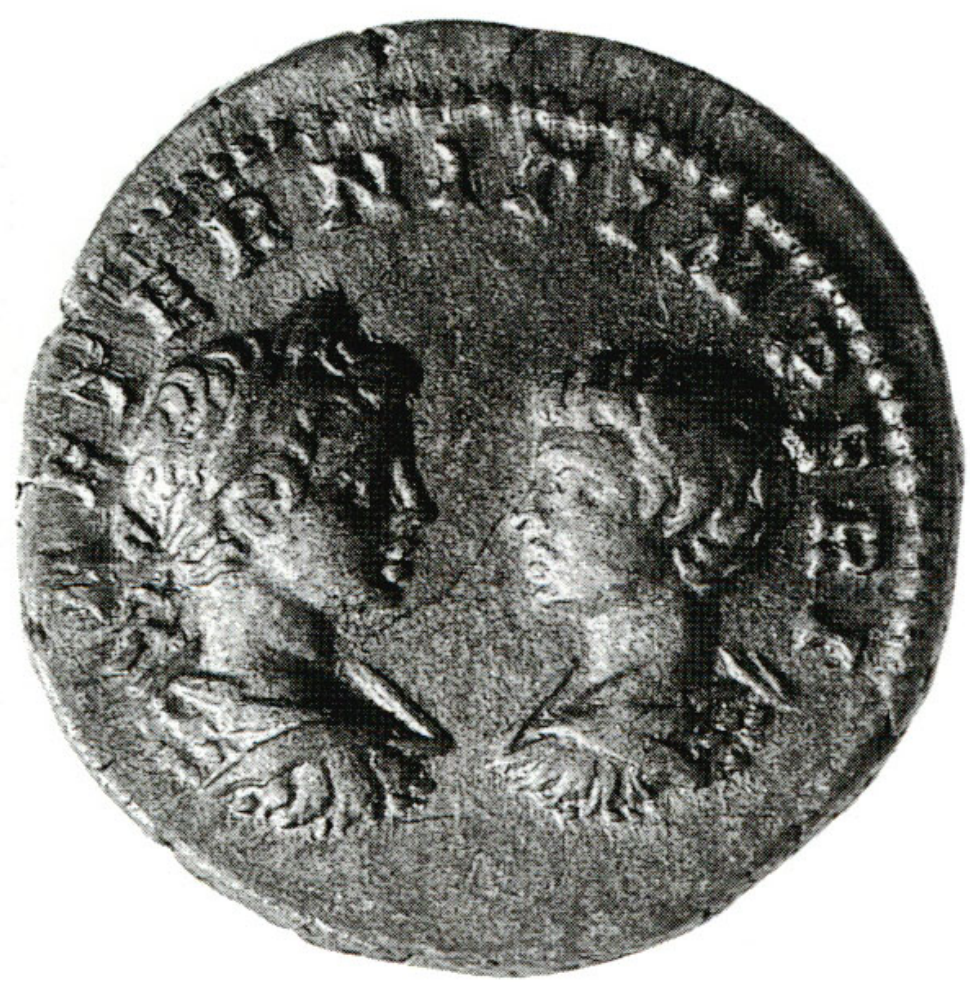

(catal. $n^{\circ} 23$ ) 


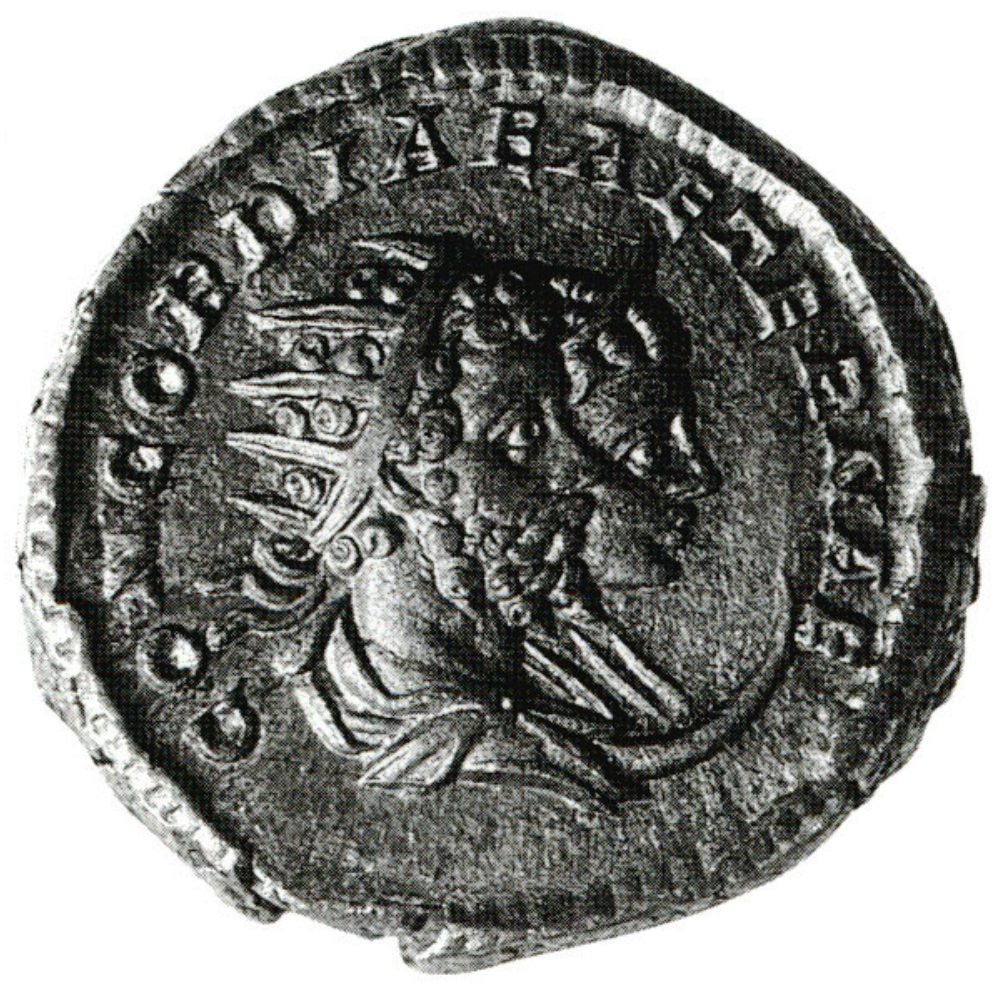

(catal. $\left.n^{\circ} 21\right)$
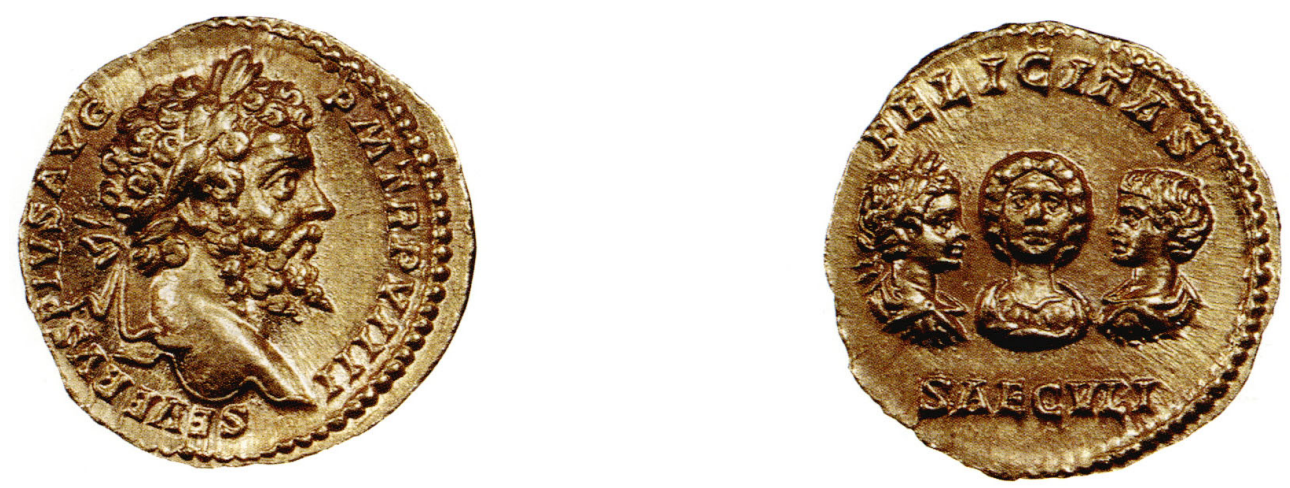

117- Aureus de Septime Sévère.

Au revers, Julia Domna de face, entourée de ses deux fils, Caracalla à gauche, Géta à droite $(x 2,5)$.

Cliché Bibliothèque nationale de France, Paris. Coll. Beistegui n 120

\section{Les liaisons de coins}

79 Trois séries de liaisons de coins ont été relevées: les deux revers CONCORDIAE AVGVSTAE signalés ci-dessus (cat. $\mathrm{n}^{\circ} 21$ et 24 ) ; trois droits de Julia Domna (cat. $\mathrm{n}^{\circ} 25$, $26,27)$; enfin deux revers VENVS VICTRIX (cat. $n^{\circ} 45$ et 46 ). Fait remarquable, ces monnaies issues de mêmes coins datent des règnes de Septime Sévère et de Caracalla : cinq d'une émission dynastique de 201, deux d'une émission de 216. La conservation de ces groupements au sein du trésor ne peut s'expliquer que par une thésaurisation 
proche de la date d'émission. En effet, une circulation monétaire active aurait eu tôt fait de provoquer leur dispersion.

\section{Les dernières monnaies}

La détermination de la monnaie la plus récente d'un trésor est toujours un exercice délicat; c'est elle qui fixe le terminus post quem de la fermeture du trésor, date trop souvent confondue avec celle de son abandon. À Vaise, il n'y a pas une, mais quatre dernières monnaies (cat. $\mathrm{n}^{\circ}$ 78-81), datant du règne de Valérien et aux effigies de membres de sa famille: son fils Gallien, associé à l'empire, la femme de ce dernier, Salonine, et leur fils Valérien le Jeune, nobilissimus Caesar. Elles sont toutes issues de l'atelier rhénan, localisé, on l'a vu, à Cologne ou à Trêves. A. Bourgeois et M. Christol (1973 ; Christol 1975, p. 814) fixent la date d'ouverture de cet atelier avant le 1er janvier 257, et sans doute dans la deuxième moitié de 256, peu après l'association de Valérien le Jeune au pouvoir avec le titre de césar qui se place en août-septembre 256 (Christol 1997, p. 246). Selon le classement d'Elmer, ces monnaies appartiennent à la 4e émission, scindée en deux phases, et dont la date peut être fixée dans le courant de l'année 258. Mais cette date ne constitue qu'un terminus post quem.

81 Les progrès des classements des émissions monétaires, depuis quelques décennies, et la plus grande abondance des documents comparatifs nous ont habitués à des datations précises, à quelques mois près, des «fermetures » de trésors du IIIe s. Dans un trésor plus abondant, on n'hésiterait guère à tirer argument de l'absence de monnaies de consécration du jeune Valérien II (mort, en opérations (?), en Illyricum au printemps de 258) ou de monnaies du second fils de Gallien, Salonin, élevé au césarat au même moment et installé sur le Rhin, à Cologne (Christol 1997). Dans un lot numériquement si faible leur absence pourrait être aléatoire. En revanche l'absence de tout monnayage de l'usurpateur Postume dont on connaît l'abondance et la bonne qualité est un argument recevable pour fixer avant la fin de l'année 260 la fermeture du trésor, c'est-à-dire l'arrêt de son alimentation. Mais encore une fois, cette date ne doit être avancée qu'avec une extrême prudence dans le cas d'une cachette qui n'est pas essentiellement monétaire.

\section{La composition}

\section{1 monnaies : un petit nombre}

Premier fait frappant, il s'agit d'un petit trésor: 81 monnaies pour un arc chronologique de 189 ans (69-258)! Même si l'on considère que l'épargne n'a débuté qu'en 201, sous Septime Sévère, pour s'achever en 258, la moyenne annuelle n'est que de 1,40 monnaies. La consultation de la liste des trésors enfouis en 259/260 en Gaule du Sud-Est (Py, Hiernard, Richard 1983) montre, en dépit d'incertitudes documentaires, que ce cas n'est pas isolé, même si y dominent les trésors regroupant plusieurs centaines de monnaies.

Selon l'équivalence habituellement admise $(1 \text { antoninien }=2 \text { deniers })^{13}$ ce trésor représente au mieux une somme de $29+(52 \times 2)=133$ deniers ou, si l'on préfère compter en bronze, 532 sesterces, ou encore 5,5 pièces d'or. Il s'agit d'une petite somme ; la solde annuelle du légionnaire, payée en trois fois, s'élève à 600 deniers en 197 sous Septime Sévère qui en a doublé le montant invarié depuis Domitien, et de 900 deniers en 212 
sous Caracalla (Speidel 1992). Il est vrai qu'à cette époque, le soldat est pratiquement nourri et vêtu, et que sa solde est complétée par des gratifications occasionnelles - le donativum sur lequel on reviendra - qui représentent au IIIe s. une part non négligeable des versements monétaires. Mais l'épargne de Vaise, à peine deux mois de solde, ne permettait guère que de vivre modestement pendant quelques mois ${ }^{14}$.

\section{9 deniers et 52 antoniniens} de lune des impératrices. Avec un aloi d'environ 50 \% (le même que celui du denier) et un poids moyen de $5,02 \mathrm{~g}$, sa valeur aurait dû être de 1 denier et demi ; or il semble qu'elle ait été fixée à 2 deniers, ce qui constituait une dévaluation de fait et explique que cette monnaie fut d'abord mal acceptée et que sa frappe fut abandonnée dès 219 pour ne reprendre qu'en 238. Monnaie militaire, a-t-on dit (Callu 1969, Christol 1977, Hollard 1995, Estiot 1996), destinée à permettre l'augmentation des soldes et à financer d'abord les guerres parthiques puis, tout au long du siècle, un budget durablement déficitaire. À partir de Gordien, l'antoninien est imposé et le denier cesse alors d'être frappé, sinon de manière épisodique. Mais le poids de la nouvelle espèce et son titre ne cessent de décroître puisque les manipulations monétaires sont le moyen choisi pour combler le passif. En 238, l'antoninien pesait $4,35 \mathrm{~g}$ pour un titre de $43 \%$ d'argent, soit un poids de fin moyen d'environ $1,87 \mathrm{~g}$; en $256 / 258$, il ne pèse plus qu'environ $3,50 \mathrm{~g}$ et ne titre que $36,2 \%$ d'argent, du moins à l'atelier de Gaule où la qualité était meilleure qu'à Rome, soit un poids de fin de 1,26 g. Cette perte d'un tiers de valeur en vingt ans n'est que le prélude à une dépréciation accélérée pendant la décennie suivante, jusqu'à la réforme d'Aurélien (Callu 1969, p. 237-248; Carcassonne, Christol 1974). Ces dévaluations monétaires incessantes conduisaient naturellement les particuliers à conserver précieusement les monnaies les mieux titrées, notamment les deniers anciens accumulés en raison de leur valeur intrinsèque, voire à les rechercher. 
118- Tableau de composition du trésor de Vaise

\begin{tabular}{|c|c|c|c|c|c|}
\hline & Dates & Denier & Antoninien & Total par règne & $\%$ \\
\hline Vitellius & 69 & 1 & & 1 & 1,2 \\
\hline Domitien & $81-96$ & 1 & & 1 & 1,2 \\
\hline Trajan & $98-117$ & 4 & & 4 & 4,9 \\
\hline Antonin le Pieux & $138-161$ & 4 & & 4 & 4,9 \\
\hline Marc Aurèle & $161-180$ & 1 & & 4 & 4,9 \\
\hline Antonin divus & & 1 & & & \\
\hline Lucius Verus & & 1 & & & \\
\hline Faustine II & & 1 & & & \\
\hline Commode & $180-192$ & 5 & & 5 & 6,1 \\
\hline Septime Sévère & $193-211$ & 2 & & 8 & 10 \\
\hline Julia Domna & & 3 & & & \\
\hline Caracalla & & 3 & & & \\
\hline Caracalla & $211-217$ & 1 & 20 & 34 & 42 \\
\hline Julia Domna & & & 13 & & \\
\hline Diaduménien & $217-218$ & 1 & & 1 & 1,2 \\
\hline Gordien III & $238-244$ & & 4 & 4 & 4,9 \\
\hline Philippe & $244-249$ & & 4 & 7 & 8,6 \\
\hline Philippe II & & & 3 & & \\
\hline Trajan Dèce & $249-251$ & & 1 & 3 & 3,7 \\
\hline Etruscille & & & 2 & & \\
\hline Gallien r. conjoint & $253-260$ & & 2 & 5 & 6,1 \\
\hline Mariniane & & & 1 & & \\
\hline Salonine & & & 1 & & \\
\hline Valérien II & & & 1 & & \\
\hline Total & & 29 & 52 & 81 & \\
\hline$\%$ & & 35,8 & 64,2 & & 99,7 \\
\hline
\end{tabular}

Le tableau de composition du trésor de Vaise (fig. 118) montre bien la succession des espèces dans la thésaurisation et le relais pris par l'antoninien à partir du règne de Gordien III (238-244). Mais le tableau de synthèse (fig. 119a) permet de saisir une image plus fragmentée que la lecture du catalogue permettait déjà de pressentir. Ce trésor présente un profil heurté qui ne traduit pas une composition normale (fig. 119b). 


\begin{tabular}{|c|c|c|c|c|}
\hline Période & D & Ant & Total & \% \\
\hline Ier siècle & 2 & & $\mathbf{2}$ & 2,4 \\
\hline IIe siècle & 17 & & $\mathbf{1 7}$ & 21 \\
\hline $193-218$ & 10 & 33 & $\mathbf{4 3}$ & 53 \\
\hline $238-251$ & & 14 & $\mathbf{1 4}$ & 17,2 \\
\hline $253-258$ & & 5 & $\mathbf{5}$ & 6,1 \\
\hline Total & $\mathbf{2 9}$ & $\mathbf{5 2}$ & $\mathbf{8 1}$ & \\
\hline$\%$ & 35,8 & 64,2 & & \\
\hline
\end{tabular}

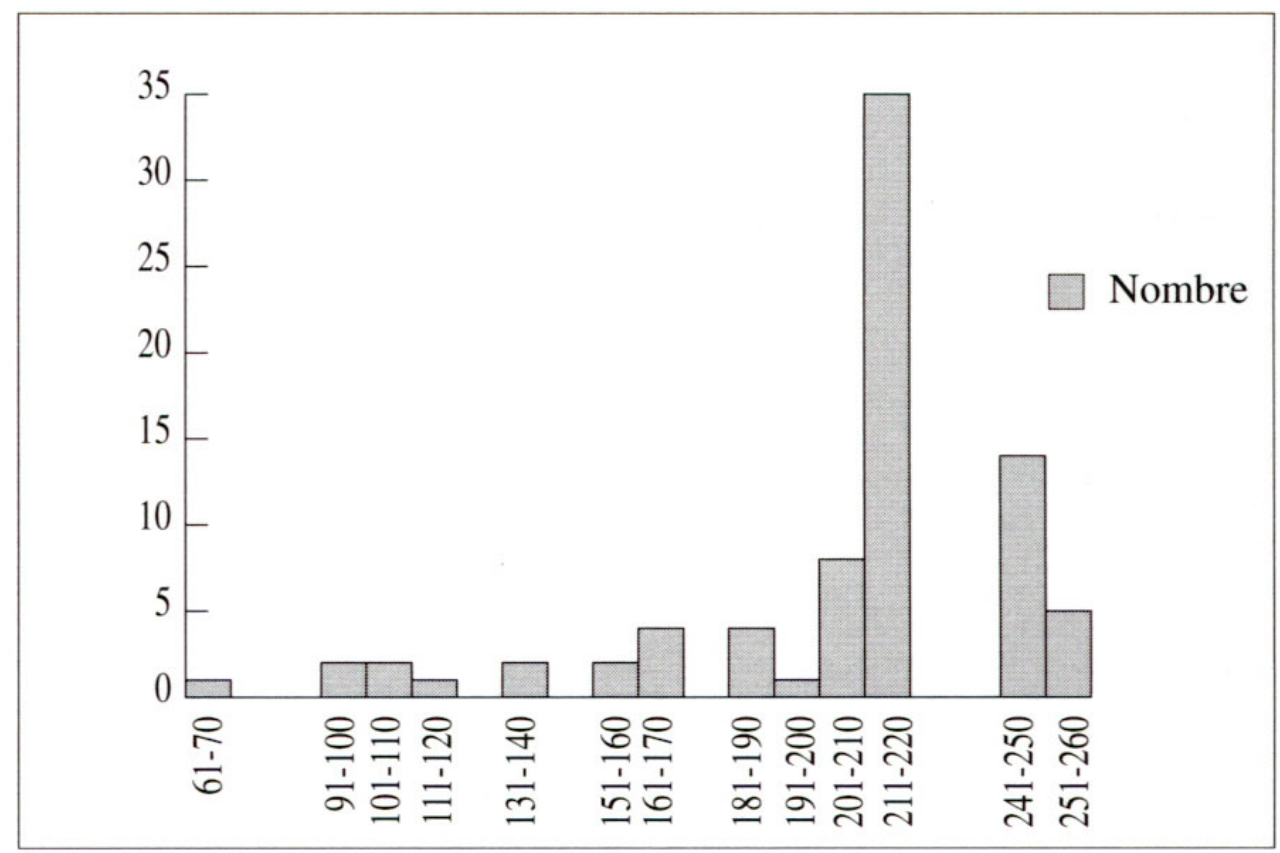

\section{Une ou deux thésaurisations?}

Les numismates ont l'habitude de distinguer trésors de circulation et trésors de thésaurisation. Les premiers seraient formés par prélèvement sur la masse monétaire disponible dont ils fourniraient un instantané. Ces trésors sont alors constitués de monnaies récentes, ce terme s'entendant au moment de leur prélèvement, et 
généralement d'espèces de même valeur métallique. Les cas les plus exemplaires sont les petits ensembles qualifiés de bourses, tel le trésor de Nages (Gard) qui comptait 19 antoniniens aux effigies de Valérien I et de sa famille émis entre 254 et 259(Py, Hiernard, Richard 1983). À l'inverse, les thésaurisations épargnent sur la longue durée en s'efforçant de sélectionner les meilleures monnaies, entendez les plus lourdes et les plus titrées, dans la mesure où cette distinction est accessible au thésauriseur.

Prenons l'exemple du vicus de Velzeke, en Flandre orientale, qui a livré deux trésors aux dates de fermeture très voisines (fig. 120). Le premier, fermé en 259/260, composé d'un denier de Sévère Alexandre et de 235 antoniniens de Caracalla à Valérien et Gallien, représente le type classique du trésor de circulation : nombre insignifiant de monnaies antérieures à 238 (1,6\%), fort contingent de monnaies récentes $(54,2 \%$ pour les dix dernières années) (Lallemand 1971 ; Lallemand, van Heesch 1994). En revanche, Velzeke II, fermé en 264/265, avec 539 deniers de Trajan à Gordien III et 538 antoniniens de Caracalla à Postume, illustre bien un souci de thésaurisation : majorité de frappes antérieures à 238 (54\%), faible représentation - au moins en pourcentage (6,7 \%) - des dix dernières années (Thirion 1974 ; Lallemand, van Heesch 1994).

La majorité des trésors se situe entre ces deux pôles. D'abord parce que à côté des désirs, le trésor reflète aussi le possible. Ensuite parce qu'un trésor n'est jamais figé que par l'événement qui empêche sa récupération; réserve d'espèces, il vit et évolue; il peut grossir ou maigrir au gré des besoins et des possibilités. Bref, reflet partiel d'états de la circulation monétaire et de comportements thésaurisants, il est aussi le reflet d'histoires individuelles.

De fait, le pécule de Vaise se compose d'au moins deux « lots» :

- un premier ensemble de 62 monnaies, soit $76 \%$ des espèces, s'achève en 218 . Il contient 19 «vieux » deniers de Vitellius à Commode, 8 deniers plus récents tous datés de 201, 33 antoniniens et 1 denier frappés de 215 à 217 pour Caracalla ; ce lot se termine par un denier de Diaduménien, en 217-218.

- un second lot se compose de 19 antoniniens émis entre 241 et 258 , soit sur une durée de 17 ans.

Entre les deux, de 218 à 241 , on constate une interruption de 23 ans couvrant les règnes d'Élagabale, de Sévère Alexandre, de Maximin, de Gordien I et II, de Balbin et Pupien et les premières années de Gordien III. Cette lacune correspond en termes monétaires à l'effacement temporaire de l'antoninien, abandonné par Elagabale en 219 et restauré par Balbin et Pupien en 238. Le propriétaire de Vaise aurait-il été - contrairement à toute attente des numismates - sensible à l'aspect flatteur des premiers antoniniens brillance de la surface et finesse de la gravure - encore perceptible aujourd'hui, et rebuté par les nouveaux deniers de poids allégé et dont l'aspect terne, par exemple sous Alexandre Sévère, trahissait le mauvais aloi ? Dans cette hypothèse, la deuxième naissance de l'antoninien s'accompagnerait alors d'une reprise de la thésaurisation. Mais s'agit-il du même individu et faut-il analyser ce trésor en termes strictement monétaires?

Revenons à chacun de nos deux lots.

Le premier ensemble se caractérise par une quasi équivalence entre deniers et antoniniens : 29 contre 33. homogène, datée de 201. Des recherches faites sur la circulation monétaire, il ressort 
que la première moitié du IIIe s. est marquée par une élimination progressive et inexorable du denier et surtout du bon denier - entendez avant son affaiblissement par Septime Sévère en 194. L'analyse des trésors permet de suivre pas à pas le processus ; les monnaies d'argent antérieures à 193, encore assez abondantes jusqu'en 217, tombent à $20 \%$ dans les trésors enfouis sous Elagabale (218-222), $15 \%$ sous Alexandre Sévère (222-235), et à 3 à $6 \%$ après 230 : «L'élimination de la monnaie d'argent de bon titre était pratiquement terminée»(Kunisz 1990). Ensuite, le retrait des deniers s'accélère et concerne aussi les espèces sévériennes notamment sous Trajan Dèce (249-251) et sous Trébonien Galle (251-253) ${ }^{15}$. À cet égard, à Vaise, la représentation des deniers $(35 \%)$ et surtout des deniers antérieurs à $193(23 \%)$ indique bien un dépôt de thésaurisation conservant des espèces qui, en 258 , ne circulent plus couramment. Quand en ont-elles été soustraites? La comparaison avec d'autres trésors nous oriente vers une date antérieure à 230 mais la présence de la petite série sévérienne de 201, avec ses liaisons de coins et son faible degré d'usure, plaide en faveur d'un retrait simultané de cette série et des deniers du IIe s., sans doute dès 201 ou peu après.

Pour autant il n'y a pas forcément eu choix délibéré de l'épargnant dont les capacités sont restreintes: le maniement des pourcentages ne doit pas faire oublier le petit nombre de monnaies concernées.

97 La présence de 33 antoniniens des années 215217 est remarquable; en effet, la faible durée de leur frappe (quatre années, de 215 à 219) rend leur représentation dans les trésors assez faible : le gros trésor d'Eauze (Schaad 1992, p. 12, 305) n'en contenait que 10 pour 28000 monnaies d'argent (dont 361 deniers de la période 193-218) ${ }^{16}$. Là encore, l'homogénéité de la série de Vaise jointe à son excellent état de conservation et à la présence d'une liaison de coins plaide en faveur d'un retrait précoce ${ }^{17}$.

Le second ensemble n'appelle que peu de commentaires. On relèvera que les règnes les plus anciens de Gordien et de Philippe sont les mieux représentés (11 ex.), ce qui est habituel en raison du meilleur aloi de leurs monnaies ; que le règne de Trébonien Galle et Volusien est totalement absent et enfin que le règne conjoint de ValérienGallien ne figure que par 5 monnaies. Mais, sur un total de 19 monnaies, est-ce bien significatif ? Retenons que ce petit lot d'antoniniens correspond bien à ce qui pouvait circuler en 258. Ainsi, à Lisieux (Calvados), dans une construction incendiée, un rouleau de 6 antoniniens se composait de 2 Gordien III, 1 Volusien, 1 Salonine, 1 Valérien I, 1 Valérien II (Elmer 63/65, donc daté de 258) (Pilet-Lemière 1994).

Cette composition explique la difficulté de trouver des parallèles au trésor de Vaise. Certes, d'autres épargnes peuvent être évoquées qui mettent l'accent sur la thésaurisation des deniers ou la faible représentation des derniers règnes (fig. 120). Comment ne pas songer à l'exemple régional de Faverges (Haute-Savoie) qui contenait 2306 monnaies de Néron à Trébonien Galle, dont 1778 deniers? Thésaurisation puissante "amassée sans doute par plusieurs générations" (Pflaum, Huvelin 1981). Autre comparaison suggestive, le trésor de Caisterby-Yarmouth (Norfolk), avec son fort contingent de deniers sévériens, mais dont la fermeture est plus tardive, sans doute pas avant 266 (Jenkins 1947). 
120- Trésors de circulation et trésors de thésaurisation : composition en pourcentage

\begin{tabular}{|l|c|c|c|c|c|c|c|c|c|}
\hline Trésor & Fermeture & Total & av. 193 & $193-218$ & $218-238$ & $238-249$ & $249-253$ & $253-260$ & $260-268$ \\
\hline Lyon-St Jean (Rhône) & $258-260$ & 173 & 0 & 0,5 & 0 & 23,1 & 13,8 & 62,4 & 0 \\
\hline Velzeke I (Flandre or.) & 259 & 236 & 0 & 0,8 & 0,8 & 44,1 & 13,1 & 41,1 & 0 \\
\hline Velzeke II (Flandre or.) & $264-265$ & 1077 & 2,04 & 32,2 & 19,7 & 32,8 & 6,3 & 3,2 & 3,5 \\
\hline Caister (Norfolk) & 266 & 847 & 11,9 & 59,6 & 6,6 & 18,1 & 2,1 & 1,1 & 0,6 \\
\hline Lyon-Vaise (Rhône) & 258 & 81 & 23,4 & 53 & 0 & 13,5 & 3,7 & 6,1 & 0 \\
\hline Faverges (Hautes-Savoie) & 253 & 2306 & 33,9 & 27,5 & 16,9 & 20 & 1,5 & 0 & 0 \\
\hline
\end{tabular}

100 Toutefois, tel qu'il est constitué, le trésor de Vaise conserve son originalité et ne correspond à aucun autre trésor connu dans la région RhôneAlpes. À Lyon, il est par exemple très différent du trésor de Saint-Jean (Phoungas 1990), presque contemporain puisque fermé en 258/60 (?), dont le profd, beaucoup plus normal, correspond à une épargne récente. Des 173 antoniniens du militaire de Saint-Jean, 108, soit 62,4\%, dataient du règne conjoint de Valérien et Gallien, c'est-à-dire des six années précédant la fermeture du dépôt.

\section{La monnaie d'or (montée en médaillon)}

La seule monnaie d'or présente dans le trésor a perdu sa fonction monétaire :l'aureus de Gordien III (cf. supra: les bijoux) est serti dans une monture à décor de peltes tout à fait caractéristique d'une époque, le troisième siècle, et d'une zone géographique, la Lyonnaise. Sa présence mérite quelques commentaires numismatiques.

Les monnaies d'or de Gordien III découvertes en Gaule ne sont pas très nombreuses. Trois découvertes de monnaies isolées sont connues (Callu, Loriot 1990): une en Narbonnaise à Montgirod (Savoie) et deux en Lyonnaise à Mably (Loire) et à Mailly (Saône-et-Loire). Deux trésors en recélaient un plus grand nombre :

- À Bordeaux (Gironde), 13 Gordien III dans un trésor de 43 aurei allant de Vespasien à 257/259 (TAF, VI, p. $\left.19 \mathrm{n}^{\circ} 6\right)$.

- À Labretonie (Lot-et-Garonne), 3 Gordien III dans un trésor de composition incertaine, mais fermé peut-être sous Claude II (TAF, VI, p. 61 n 7).

Il faut y ajouter un aureus de Gordien III, découvert à proximité du tunnel ferroviaire de l'Epine (Savoie), peut-être dans un trésor d'antoniniens enfoui sous Claude II ou postérieurement (TAF, V/2, p. $\left.75 \mathrm{n}^{\circ} 18\right)$.

104 Le fait que le propriétaire avait réussi à conserver des deniers anciens, mais aucun aureus, hormis ce bijou monétaire, ne doit pas surprendre. Cette relative rareté de l'or dans les thésaurisations des années 235-269 se déduit de l'observation des trésors : en Occident (sauf l'Italie), deux trésors seulement sont composés uniquement de monnaies d'or (Huvelin, Loriot 1992, p. 226), la règle générale étant les trésors dits mixtes, c'estàdire composés de monnaies de différents métaux, mais aussi de bijoux et d'argenterie. Mais la part des aurei y est toujours faible. Aux exemples réunis par Callu (1969, p. 425-426), on ajoutera le trésor de Mâcon des années 253/260, d'environ 30000 monnaies, de très nombreux bijoux et statuettes, mais seulement 11 aurei (CAG 71/4 : 305-306), ou plus récemment, celui d'Eauze avec 28000 monnaies d'argent, mais seulement 6 aurei dont 3 sertis (Schaad et al. 1992). À l'extrême, certains trésors, surtout dans la période 253-275, ne conservent même qu'une ou deux monnaies d'or : le trésor de Coulanges-lès-Nevers (Nièvre) composé de 352 monnaies de Marc Aurèle à Postume et de quelques bijoux ne renfermait qu'une monnaie d'or de Septime Sévère ${ }^{18}$; à Givry 
(Hainaut), un dépôt monétaire de 56 deniers de Sévère à Gordien III et de 5 antoniniens de Philippe à Valérien était accompagné par un aureus de Macrin (Doyen 1984).

La disparition des grandes épargnes homogènes d'or au profit de la thésaurisation de quelques monnaies d'or mêlées à d'autres espèces ou à des objets précieux a suscité quelques tentatives d'explication (Callu 1969, p. 428 ; Huvelin, Loriot 1992, p. 238-239) : moindre production de monnaies d'or pendant la période 222-253, défiance du public face à une instabilité pondérale. Le poids moyen de l'aureus depuis Hadrien s'établit à $7,19 \mathrm{~g}$ soit une taille au 1/45e de livre (Callu 1969, p. 430 n. 2) ; le décrochage initié sous Caracalla $(6,77 \mathrm{~g})$ s'accentue sous ses successeurs Élagabale et Sévère Alexandre $(6,32$ g); la rupture est encore plus nette sous Gordien III où le poids moyen de 91 exemplaires s'établit à $4,83 \mathrm{~g}$ soit à un pied plus proche du 1/67e de livre que du $1 / 64 \mathrm{e}$ généralement retenu pour cet empereur ${ }^{19}$. Le nouvel aureus ne pèse plus que les deux tiers de l'aureus des Antonins. Comment, alors que son taux de change avec le denier ne pouvait plus demeurer constant, n'aurait-il pas suscité la défiance des usagers? Mais, dans ce contexte, on doit aussi envisager une difficulté d'accès à l'or ou plutôt de conservation de l'or. Dans les milieux " populaires ", l'or coûte cher, entendez que sa thésaurisation immobilise du pouvoir d'achat.

À quelle date cet aureus de Gordien III a-t-il pu être retiré de la circulation ? L'examen des trésors monétaires indique la possibilité théorique de réunir des aurei de Gordien III jusque sous Claude II, en 270. Les bijoux fournissent la même indication ; le trésor mixte de Petrijanec (Croatie), fermé vers 300, contient deux bracelets réunissant chacun 4 aurei : Antonin, Vérus, Domna, Gordien III pour le premier, Marc Aurèle, Caracalla, Gordien III, Claude II pour le second (Mowat 1888, p. 232-233 ; Brenot, Metzger 1992, p. $336 \mathrm{n}^{\circ}$ 64). Dans le cas de Vaise, le bon état de l'aureus monté en pendentif suggère une faible circulation; en outre, la chute pondérale de la monnaie d'or se poursuivant $(3,53$ g sous Galle en $251 ; 3,12$ à $2,28 \mathrm{~g}$ sous Valérien à Rome de 253 à 259 ), il eut sans doute été assez difficile de le trouver dans la circulation après 250 .

La relative rareté des aurei de ce premier troisième siècle s'accompagne d'une fréquente utilisation de l'or monétaire sous forme de bijoux: bagues à chaton, bracelets, colliers ou pendentifs utilisent des aurei sertis, enchassés, montés. Cette pratique se développe à partir du règne de Septime Sévère pendant tout le troisième siècle. Ce détournement de la monnaie d'or de sa fonction monétaire correspond davantage à un phénomène de mode qu'à une immobilisation métallique ou à une recherche de plus value.

108 J.-P. Callu s'était interrogé sur le caractère militaire des pendentifs monétaires à l'instar des fibules ornées de monnaies d'or données aux officiers au Bas-Empire. Pour $\mathrm{X}$. Loriot, les trouvailles de Rennes, Vieil-Evreux, Heuqueville, Rouen, ne présentent pas de caractère militaire (Loriot 1983). J.-P. Bost et J.-M. Gurt après avoir envisagé cette hypothèse de cadeau militaire pour les trois pendentifs d'Eauze y renoncent également, faute de preuves (Schaad, 1992, p. 303-304 et $\mathrm{n}^{\circ}$ 12). Pourtant, les colliers monétaires de Beaurains ou du trésor dit de la dyarchie, le premier de 8 à 10 médaillons, le second de 7 pendentifs, accompagnent des monnaies d'or interprétées comme les produits de donativa (Bastien, Metzger 1977 ; Catalogue Greek and Roman Coins 1991). Ne serait-ce pas, à un échelon plus modeste, le cas du trésor de Vaise?

Un exemple de largesse impériale 
À titre d'exemple, on évoquera les fêtes décennales de Septime Sévère qui eurent lieu le 9 avril 202 et les jours suivants. Ce jubilé - en fait le $9 \mathrm{e}$ anniversaire de sa proclamation comme empereur par les soldats à Camuntum, le 9 avril 193, et donc le début de la dixième année de règne - fut jumelé avec le triomphe parthique de l'empereur (victoires sur les Parthes de 197 à 199) et le mariage du prince héritier, Caracalla, avec Plautilla, la fille du Préfet du Prétoire, âgée de 14 ans. Sévère et la famille impériale venaient d'arriver d'Antioche.

À cette occasion, rapporte Dion Cassius, sénateur, historien bithynien et témoin oculaire, "Sévère gratifia l'ensemble de ceux qui bénéficiaient des distributions de blé et les soldats de la garde d'un nombre de pièces d'or égal pour chacun à celui des années du règne, ce dont il se glorifia hautement. Et à vrai dire, il est exact que jamais personne ne leur avait distribué autant puisque, pour cette libéralité, furent dépensés cinquante millions de drachmes [= 2 millions d'aurei]". Puis Dion décrit le banquet de noces "de style mi-impérial mibarbare" et "les spectacles variés en l'honneur du retour de Sévère, de ses décennales et de ses victoires", notamment des jeux au Colisée ou au Cirque Maxime où furent exhibés et massacrés sept cents animaux. La fête dura sept jours. (Dion, 76, 1, 1-5 ; trad. A. Chastagnol)

Hérodien de Syrie (actif vers 230) lui fait écho ; "II fut accueilli en triomphateur par le peuple de Rome, avec de grandes acclamations et marques de dévotion. Il accorda au peuple des sacrifices et cérémonies religieuses ainsi que des réjouissances au cours desquelles il procéda à de généreuses distributions d'argent et offrit des jeux sacrés pour fêter ses victoires". (Hérodien, III, 10, 1-2 ; trad. A. Chastagnol)

Ainsi Sévère distribua-t-il 2000000 d'aurei à raison de 10 aurei par bénéficiaire, ce qui implique un nombre de 200000 personnes : 15000 soldats du prétoire et environ 185000 membres de la plèbe urbaine dite frumentaire car ayant droit aux distributions de blé gratuit. Donativum aux soldats et congiaire à la plèbe romaine vont toujours de pair, nous dit Paul Veyne pour qui « les empereurs considéraient que soldats et citoyens de Rome étaient sous leur protection particulière » (Veyne 1976, p. 614). Il s'agit de sommes considérables, d'autant qu'on conçoit mal que l'ensemble de l'armée ait pu être exclue des largesses de ce jubilé.

Quelles étaient les monnaies réellement distribuées ? Parmi les monnaies émises à Rome en 202, celles relatives aux vœux décennaux existent sous forme de bronze et d'argent ; en revanche, celles célébrant la famille impériale, le retour des empereurs ou leurs victoires existent en argent et en or. Il est vraisemblable que les distributions aient été faites non seulement en or, mais aussi en deniers (soit 250), voire partiellement en sesterces (soit 1000) pour la plèbe. Enfin, toujours en 202, Hill propose une émission spéciale de quinaires d'or (soit la moitié d'un aureus) pour la troisième libéralité du règne.

Selon A. Chastagnol, « Septime Sévère a voulu conférer à son jubilé un retentissement plus grand que celui de toutes les fêtes données par ses prédécesseurs et faire de lui l'apogée de son règne, la date symbolique qui marquait sa gloire personnelle et le succès de ses armes comme de sa politique et, en voyant plus loin, l'affirmation solennelle du principe dynastique en faveur de sa famille ». (Chastagnol 1984 a, p. $119 ; 1984$ b, p. 104).

Cette largesse était la 3e du règne après celle d'accession (en 193), et celle de l'élévation de Caracalla au césarat (en 196). D'autres libéralités du règne nous sont connues : la 4e pour les jeux séculaires (en 204), la 5e pour le deuxième consulat de 
Caracalla et le premier de Géta (en 205), la 6e pour l'élévation de Géta à l'augustat (en 209) ; quatre autres suivront sous le règne de Caracalla.

\section{Origine du lot monétaire}

La composition particulièrement atypique du trésor de Vaise, avec son contingent de deniers anciens et sa composition fragmentée m'incite à proposer une hypothèse sur la constitution et la conservation de ce lot.

Partons d'une certitude. L'apport principal est sévérien puisque 43 monnaies sur 81, soit $53 \%$, sont comprises entre 201 et 218. Il est clair que ces monnaies n'ont pu être réunies qu'au moment de leur émission. En effet, il aurait été extrêmement difficile de rassembler un ensemble aussi cohérent, en outre avec des liaisons de coins, quelques années et $a$ fortiori quelques décennies après leur émission. Mais à l'intérieur même des espèces sévériennes, on relève une solution de continuité que ne suffit pas à expliquer la faiblesse statistique du lot: 8 deniers de 201 dont 5 concernés par des liaisons de coins, puis 33 antoniniens des années 215-217 auxquels on peut ajouter 1 denier de 216 et 1 denier de 217-218. Pourquoi ce trou entre 201 et 215? Aucun argument numismatique ne peut être avancé qui le justifie. L'hypothèse est que le propriétaire de Vaise a reçu deux paiements, l'un en 201 ou peu après, l'autre en 217 ou 218, dont nous n'avons peut-être que des reliquats.

111 Le caractère particulier du premier lot constitué de revers dynastiques, rarement réunis en aussi grand nombre ${ }^{20}$, m'incite à envisager le fruit d'une libéralité impériale. Il pourrait en effet s'agir des reliquats soit d'un donativum, distribution exceptionnelle de monnaies en faveur des soldats, soit d'un congiarum, cadeau destiné à la plèbe. Ces largesses sont nombreuses au IIIe s., à l'occasion des avènements, des anniversaires, des succès militaires, etc. (Bastien 1988). Ainsi, entre 196 et 214, Caracalla compte neuf largesses, commémorées et numérotées sur des monnaies à légende LIBERALITAS.

112 À titre d'exemple, on évoquera les fêtes décennales de Septime Sévère, en 202, objet de largesses en monnaies d'or pour les 15000 prétoriens (10 aurei), et vraisemblablement en argent ( 250 deniers) voire en bronze (1000 sesterces) pour les 185000 bénéficiaires de la plèbe. (cf. : Un exemple de largesse impériale)

Le second sous-ensemble des Sévères, avec ses 33 monnaies regroupées sur un laps de temps de trois ans, est composé d'émissions « ordinaires » sauf le $n^{\circ} 30$ correspondant à des vœux pour la santé de Caracalla en 215. Mais Bastien (1988, p. 37) précise bien qu’à côté de monnaies émises spécialement en vue de largesses impériales, on utilisait aussi des monnaies courantes pour des distributions plus modestes. On pourrait ainsi songer aux vicennalia de Caracalla, le 28 janvier 217 (Chastagnol 1984a, p. 121), ou encore au donativum d'avènement de Macrin dont témoignerait le denier du césar Diaduménien. Selon l'Histoire Auguste (SHA, Diad., II, 1; Bastien 1988:12, 15), Macrin distribua à chaque soldat 3 aurei pour sa proclamation à l'Empire et 5 aurei pour l'élévation de son fils Diaduménien au césarat (fin mai 217). Rien ne s'opposerait à un paiement en argent (200 deniers ou 100 antoniniens), au cours du deuxième semestre 217, les paiements étant souvent différés et en utilisant des monnaies récentes de Caracalla. En effet, Macrin, bien qu'étant à l'origine du complot contre Caracalla, se comporta au moins face à l'armée comme son continuateur. 
114 À côté de ces événements exceptionnels relatés par les textes ou les légendes monétaires, d'autres circonstances pouvaient provoquer des distributions de routine en espèces, par exemple les anniversaires annuels de la prise du pouvoir. Les trésors identifiés comme le produit de distributions impériales sont peu nombreux et concernent surtout des personnages de rang supérieur. Voyez la fortune mobilière du propriétaire de Beaurains (Pas-de-Calais) qui s'élevait à au moins 370 monnaies d'or dont 22 multiples d'aurei, des bijoux, des pièces d'argenterie. La qualité des monnaies qu'il a reçues montre qu'il a «participé à la plupart des distributions impériales depuis la prise du pouvoir de Dioclétien en Italie et en Gaule après la bataille de Margus [mi 285] jusqu'en 310, année où il bénéficie du donativum offert pour les quinquennalia de Constantin le 25 juillet» (Bastien, Metzger 1977). Autre exemple, le trésor dit de la Dyarchie, de provenance inconnue, qui outre un collier monétaire de 7 aurei d'Hadrien, Marc Aurèle, Septime Sévère, Macrin, Gordien III (2 ex.), Valérien I (binio), comptait au moins 46 aurei presque tous frappés pour le donativum d'avril 286, deux fibules en or, et d'autres bijoux (Catalogue Greek and Roman Coins 1991, p. 194203) (fig. 108). À chaque fois, les commentateurs disent avoir affaire à des officiers de haut rang. À Vaise, au contraire, rien de tel. Si donativum il y a, c'est celui donné à un homme de troupe, légionnaire ou prétorien, et dont il ne reste qu'une modeste partie. Mais encore une fois, cette évocation relève de l'hypothèse, puisque bien d'autres occasions s'offraient à notre lyonnais de recevoir des paiements.

\section{Conclusion}

$115 \mathrm{Au}$ total, la partie monétaire du trésor de Vaise, atypique dans sa composition, se définit comme une petite collection de monnaies anciennes, fragmentée en prélèvements effectués en $201 / 202$ et en $217 / 218$, à laquelle on a ajouté une poignée de monnaies récentes. Faut-il parler de thésaurisation? Plus que la constitution du lot, c'est sans doute sa conservation qui est remarquable. Admettre notre hypothèse de retraits successifs, à l'occasion de cadeaux ou de paiements, conduit à envisager soit une épargne de jeunesse conservée jusqu'à la fin de sa vie par le même homme (avoir 20 ans vers 200 et 78 ans vers 258 ) soit un héritage transmis et faiblement augmenté. En tout état de cause, on songera à un patrimoine familial conservé par attachement sentimental, peut-être à une génération de distance, plutôt qu'à une réserve de valeur dont on a dit la modestie.

\section{NOTES}

7. Le RIC (IV/1, p. $216 \mathrm{n}^{\circ} 29$ B) répertorie à Rome, d'après Cohen (IV, p. $244 \mathrm{n}^{\circ}$ 5), un denier offrant le même couplage Caracalla/Géta mais avec la mention TR P III, ce qui nous situe en 200 ; le $B M C(V, p$. cxli) fait aussi allusion à ce couplage à Rome en 200 , mais ne le répertorie pas à la page attendue (p. 190) bien que l'index des légendes invite aussi à se reporter à la p. 190 ; Enfin Hill (1977) ne le mentionne pas, ce qui semble logique puisque ces types dynastiques relèvent d'un programme iconographique qui ne se développe à Rome qu'en 201 et 202. On peut donc 
s'interroger sur l'existence de ce denier qui n'est peut-être qu'une création due à une mauvaise lecture. Voyez le denier de Vaise sur lequel la numération de la puissance tribunicienne, peu distincte, se lit d'abord III.

8. Hill $464=$ RIC IV $/ 1$, p. $218 \mathrm{n}^{\circ} 36=$ BMC V, p. 185 .

9. Ce revers est essentiellement réservé à Caracalla pour lequel il est encore frappé dans une troisième émission dynastique en 201 (aureus, Hill 534), et enfin dans une émission dynastique de 202 (aureus ; Hill 543 dont l'indication du droit est manifestement à corriger par une légende $13=$ $B M C, V$, p. $233 n^{\circ}$ 389). En 202, les émissions du mariage de Caracalla reprennent cette légende de CONCORDIAE AETERNAE, mais avec la représentation de Caracalla et de Plautille (BMC, V, p. 233-235). Toutefois, ce revers dynastique intervient aussi une fois dans le monnayage de Géta (Hill 467) et curieusement une fois pour Septime Sévère, dans un atelier oriental (RIC $522=$ BMC p. 298).

10. Cette monnaie se prête admirablement à une utilisation comme élément de décor de bijoux. Le collectionneur Jules Charvet possédait un bracelet orné d'un aureus à ce type (Charvet $1863 \mathrm{p}$. 15). Un exemplaire (RIC 181 c, Hill 542) est monté dans un pendentif en or contemporain dont le sertissage privilégie le revers (Triton I, 2-3 décembre 1997, New York, $\mathrm{n}^{\circ} 1534$ ).

11. Hill 674 et 677 (AVG errore) ; BMC, V, t. 1 p. cxlv et t. 2 p. 205 n $^{\circ} 265$ date cette monnaie de 201 ou de 202.

12. $B M C, V$, t. 2 p. 360 = Hill 1032 ; l'existence de cette monnaie n'était attestée que par la description d'une monnaie du Cabinet des médailles, volée en 1831 ; aujourd'hui, elle ne semble connue qu'à un exemplaire : Sotheby's. Two hundred highly important greek and roman coins. London, 5th july 1995, p. 118-119 n 142, fig. (poids : 7,22 g).

13. L'autre valeur parfois proposée pour l'antoninien : 1,50 deniers. Il est vraisemblable que sa valeur a varié entre sa création en 215 et sa disparition en 274 ; on a par exemple avancé 1,25 deniers pour l'antoninien de Gordien et de Philippe. Les équivalences utilisées ici sont les suivantes : 1 aureus $=25$ deniers $=100$ sesterces

14. On notera qu'un centurion de légion recevait 15 fois la solde de base d'un légionnaire qu'il commandait, soit 13500 deniers, et un primipile 60 fois, soit 54000 deniers (Speidel 1992, p. 105-106). Sur les soldes, on consultera utilement la riche documentation réunie par Callu (1969, p. 295-300, 309-313). Sur les prix, Mrozek (1975), Corbier (1985). Sur les effets de l'inflation sur la société et l'économie, Corbier (1989). Il est difficile de fournir des prix significatifs, surtout pour la Gaule. Au fil de la lecture de l'ouvrage de Mrozek, j'ai relevé que le salaire d'un travailleur manuel en Occident, au Haut-Empire, ne dépassait pas un denier par jour, nourriture en sus (p. 75); avec 150 deniers, on pouvait acheter en Afrique en 202 (table de Zraïa) : deux tuniques ( $\mathrm{p}$. 39) ou un âne ou quinze amphores de vin de 26,26 litres, mais la somme était insuffisante pour un cheval (p. 49: 400 deniers) ou un esclave (500 deniers). Enfin, sur le donativum, outre Bastien (1988), je recommande la lecture des réflexions de Paul Veyne (1976, p. 609-621) sur les rapports entre l'empereur et l'armée.

15. Callu 1969, p. 257. Christol a bien montré comment cette récupération systématique par l'Etat avait pour objet une refonte pour dévaluer et accroître les moyens de paiement de l'Etat ; l'atelier rhénan en fut l'instrument qui avec la quantité de métal fin d'un denier fabriqua d'abord une pièce valant 50 à $100 \%$ de plus, puis ensuite une pièce et demie, puis deux pièces (Christol 1977, p. 262). La présence de deniers est naturellement possible dans des trésors fermés un plus tardivement ; par exemple en 267/268 à Souzy-la-Briche, Essonne : 260 deniers de Septime Sévère à Gordien III sur 5446 monnaies il est vrai (Foucray 1995, p. 37 : tableau de répartition des deniers dans six trésors enfouis entre 253 et 262).

16. Autres exemples d'antoniniens de Caracalla dans des trésors : à Nanterre, fermé en 256, 19 sur un total de 1968 monnaies (Le Gentilhomme 1946); à Dorchester, fermé en 257, 26 sur un total de 20748 monnaies (Mattingly 1939) ; à Viuz-Faverges (Haute-Savoie), fermé en 253, 36 sur un total de 2306 monnaies (Pflaum, Huvelin 1981). Enfin à Velzeke II (Flandre Orientale), fermé 
en 264-265, 19 sur 1077 monnaies (Lallemand, Van Heesch, 1994 rectifiant Thirion 1974). Les nombres absolus sont comparables, mais les pourcentages sont inférieurs à $2 \%$ (contre $40 \%$ à Vaise).

17. Les poids des 33 antoniniens de Caracalla du trésor de Vaise s'échelonnent de 4,04 g à 5,62 g ; le poids moyen est de 4,96 g, soit un chiffre très proche de celui de 5,02 g constaté au British Museum sur 58 exemplaires (BMC, V, p. xx).

18. Les notices sur ce trésor sont fautives depuis Blanchet $\left(1900, n^{\circ} 269\right)$ qui omet de la liste Gallien, Salonine, Valérien le jeune et surtout Postume. Il faut se reporter à la source princeps : Gillet (P.), Annuaire du département de la Nièvre pour l'an XI [1803], p. 63. Pour ne l'avoir pas fait, la récente CAG de la Nièvre (1996, p. 125 commune 088) transmet la même erreur de terminus et ajoute à la liste Trajan errore.

19. Valeur retenue par X. Loriot 1975, p. 733 (d'après S. Bolin) ; Lafaurie 1975b, p. 133.

20. Je n'en ai pas trouvé d'exemples. L'impressionnant dépôt de Réka-Devnia (Bulgarie), de plus de 80000 monnaies, n'en contenait que 3 sur 19073 monnaies de la seule famille de Septime Sévère (Mouchmov 1934). Un exemple régional de leur rareté est donné par le trésor de ViuzFaverges (Haute-Savoie) qui n'en contient qu'un exemplaire $\left(n^{\circ} 1003=\right.$ Hill 538) pour 584 deniers de la période 193-217 (Pflaum, Huvelin 1981). 


\section{Nature et datation du trésor de Vaise à Lyon}

1 Composé d'éléments variés - statuaire, vaisselle, bijoux, monnaies - le trésor de Vaise est bien représentatif des trésors mixtes qui accumulent des métaux précieux sous différentes formes. Après le catalogue raisonné qui considère chacun des éléments, il faut s'intéresser à l'ensemble qui pose de multiples questions.

\section{Un ou deux dépôts?}

2 Pour J.-P. Lascoux qui a dirigé la fouille, l'existence de deux fosses, leur localisation et les modalités d'enfouissement plaident en faveur de deux dépôts distincts. Dans un cas (dépôt $\mathrm{n}^{\circ} 2$ ), une fosse est creusée dans l'angle d'une pièce, aux dimensions d'un coffre dans lequel les objets ont été ensuite soigneusement déposés; dans l'autre (dépôt $\mathrm{n}^{\circ} 1$ ), une "excavation de fortune" accueille un sac, probablement en toile, dans lequel des statuettes complètes sont disposées tête-bêche et d'autres objets (bracelets en argent, aile de statuette, fragments...) imbriqués dans les espaces libres pour réduire l'encombrement. Mais, du fait de l'arasement des niveaux archéologiques des IIIe et IVe s., aucun argument stratigraphique ne vient étayer l'hypothèse de deux enfouissements distants dans le temps, a fortiori effectués par deux personnes différentes. Comme à Vienne (Baratte et al. 1990), nous postulons qu'il s'agit d'un enfouissement unique, entendez accompli par une même personne, ainsi divisé pour des raisons pratiques.

Les données archéologiques ne permettent pas non plus de dater ces enfouissements sinon dans une fourchette très large. Le dépôt $n^{\circ} 1$ recoupe une fosse qui contenait du mobilier céramique dont la production s'étale du 1er s. ap. J.-C. à 230 au plus tôt; le dépôt $n^{\circ} 2$, dans la mesure où il serait lié à l'habitat, aurait été enfoui avant l'installation d'un atelier de potier dont le four principal ne peut être antérieur à 310/311. Mais, on ne peut être assuré de l'état du quartier au moment de l'enfouissement: en activité ou en déshérence? En d'autres termes, cachette de l'habitant des lieux ou d'un individu de passage ? Le riche trésor d'Eauze était dissimulé en bordure de la zone urbaine, dans un quartier artisanal occupé par des potiers, et 
donc - selon nos collègues - sans lien avec l'activité du propriétaire (Schaad 1992, p. 336). Il faut donc en revenir au mobilier et d'abord au trésor $n^{\circ} 2$.

\section{Le dépôt $n^{\circ} 2$ : vaisselle d'argent, bijoux d'or, deniers et doubles deniers}

Rappelons en rapidement les composantes :

- Le lot d'argenterie comporte une coupe décorée d'une effigie de Mercure, deux petits plateaux ornés sur leur marli, le premier de poursuites d'animaux, le second de scènes mipastorales, mi-cultuelles (l'un d'entre eux est seulement en bronze plaqué d'argent), de quatorze cuillers, dont certaines avaient des fonctions multiples, regroupées en petites séries et les fragments de deux petits ustensiles mal définis (le premier se terminait peutêtre par une passoire).

- Les bijoux, au nombre de 10, offrent l'image d'une cassette féminine : un collier polychrome de

- d'or et de perles d'émeraude, une paire de bracelets torsadés en or, une paire de pendants d'oreilles en or, émeraude, perle et pâte de verre, une autre paire elle aussi composite (or, grenat, émeraude et perle), une bague en or, une bague en or avec intaille, et enfin un médaillon monétaire (aureus de Gordien III).

- Une bourse compte 81 monnaies en argent - 29 deniers et 52 antoniniens (ou doubles deniers) - dont la majorité sont des frappes anciennes. La partie sévérienne du lot est interprétée comme un reliquat de libéralité impériale.

5 C'est donc un ensemble modeste à tous points de vue: en poids, l'argenterie ne représente que 709, $35 \mathrm{~g}$ (853,65 g en comptant l'assiette plaquée dont le noyau est en bronze), c'est-à-dire à peine plus de deux livres d'argent, ce qui est fort peu de choses en valeur. La qualité des objets est en outre tout à fait ordinaire : le décor de la coupe, gravé, et des deux plateaux, en relief, trouve des équivalents directs sur le plan de la facture et de la technique dans l'argenterie contemporaine, en Gaule ou ailleurs - nous en avons cités dans le catalogue-, mais dans la production la plus banale. La bijouterie est de bonne qualité, mais sans rien d'exceptionnel ni en quantité ni en rareté; on notera même sur l'une des paires de pendants d'oreilles, l'utilisation de pâte de verre pour remplacer l'émeraude. Enfin, les monnaies qui représentent l'équivalent d'une livre de métal $(324,73 \mathrm{~g})$ ou d'une petite somme de 133 deniers, relèvent davantage de la collection que de la réserve de valeur.

C'est aussi, il est vrai, un ensemble plutôt homogène : du point de vue de la date tout d'abord. Dans les formes, celle des cuillers en particulier, comme dans le décor l'emploi du nielle dans un des cuillerons, celui des reliefs ou de la gravure - tout s'accorde avec la chronologie suggérée par les monnaies : le IIIe s. Le svastika niellé, la présence sur la même cuiller d'une tête de griffon, la présence de deux couverts individuels en plus des cuillers orientent probablement vers la fin de la période, c'est-àdire les alentours de 260 ap. J.-C., pour certaines des pièces d'argenterie tout au moins. Il n'est pas exclu toutefois que d'autres puissent être un peu plus anciennes, sans remonter, nous semble-t-il, au-delà de la fin du IIe s. Les bijoux s'inscrivent aisément dans la production d'orfèvrerie courante en Gaule au IIIe s., plutôt dans la première moitié, même si certains d'entre eux, comme le collier - n'oublions pas qu'il a subi une réparation - peuvent avoir une date plus ancienne. Mais il est vrai que les monnaies influent sur la datation de l'ensemble. Les dernières monnaies sont frappées dans le 
cours de l'année 258. Cette date tend à fournir un terminus ante quem pour la fabrication de l'argenterie et des bijoux, et un terminus post quem pour la fermeture du trésor. Mais il ne s'agit que d'une probabilité car il faut bien distinguer l'arrêt de l'accumulation, l'enfouissement du trésor et l'événement qui empêche sa récupération. Quel laps de temps sépare ces épisodes : quelques jours, quelques mois ou quelques années ? À titre d'hypothèse, nous avons envisagé la fourchette 258/260 ; certains seront peut-être tentés d'aller jusqu'en 270 . Il est vrai que c'est durant cette décennie que se concentrent les grands trésors mixtes.

7 Mais l'homogénéité se remarque aussi dans les fonctions : même si le nombre des pièces d'argenterie est limité, ce sont les formes présentes dans les autres trésors contemporains que l'on rencontre à Vaise, c'est-à-dire des formes ouvertes, plates, destinées au service de la nourriture ou à la présentation d'autres objets, et des cuillers à l'exclusion de la vaisselle à boire. On pourra il est vrai s'interroger sur le rôle de la coupe au Mercure. Sa présence dans le trésor n'implique en rien le caractère religieux de celui-ci: nous nous trouvons dans le même cas qu'à Chaourse où, au sein d'un ensemble de vaisselle important, apparaît une coupe de forme équivalente à celle de Vaise, décorée elle aussi d'une figure de Mercure. En revanche, il n'est pas certain qu'elle ait eu une fonction pratique et qu'elle ait servi à autre chose qu'à mettre en évidence la silhouette du dieu, à des fins de dévotion ou largement décoratives.

En ce qui concerne les cuillers, on observera qu'il s'agit de toute évidence d'un ensemble constitué progressivement d'exemplaires de formes diverses et de fonctions également différentes. Les cochlearia l'emportent très nettement en nombre sur les ligulae, et on note la présence d'une cuiller dont le manche est terminé par un sabot fourchu (ou une pince) qui devait avoir un rôle particulier. S'y ajoutent deux autres cuillers que complétaient sans doute à l'extrémité du manche opposée au cuilleron une lame de couteau.

9 On ne saurait évidemment parler de service. L'un d'entre nous a d'ailleurs eu l'occasion à plusieurs reprises de dire sa réserve devant une analyse trop systématique des trésors d'argenterie dans la perspective de l'identification de tels services, qui auraient compris par principe un nombre déterminé d'objets de différents types (Baratte 1993, p. 259-260). La notion de "service" de vaisselle (ministerium, dans le vocabulaire latin) existait à coup sûr, les textes suffisent à l'attester (cf. par exemple ceux rassemblés par W. Hilgers, Hilgers 1969, p. 222-223 ; Thésaurus Linguae Latinae, VIII, s.v. ministerium, col. 1014) : ils désignent toutefois des ensembles de vases en argent sans préjuger de leur contenu. Mais le caractère particulier de l'argenterie, un produit de luxe, suffit aussi à expliquer que les amateurs pouvaient se contenter de pièces ou de groupes de pièces isolés qu'ils se procuraient en fonction de leurs moyens ou au hasard de la rencontre avec des objets qui les séduisaient, sans idée d'organisation systématique. L'argenterie ne constituait en outre, dans bien des cas, qu'une partie de la vaisselle de table de son possesseur: une pièce, ou quelques-unes, qui pouvaient être mêlées à d'autres moins précieuses.

10 Le petit nombre de bijoux évoque une parure féminine courante. Bracelets, collier et pendants d'oreilles sont des bijoux relativement étroits $(40 \mathrm{~cm}$ de long seulement pour le collier ; 6,9 à 7,5 cm de diamètre pour les bracelets) et légers. Par opposition les deux bagues apparaissent comme d'assez grandes dimensions (diam. int. : 1,85 et $2 \mathrm{~cm}$ ) et pesantes (27 et 13,4 g), sans que l'on puisse pour autant y voir des bijoux masculins, l'une d'elles apparaissant comme anneau de mariage ou de fiançailles. Le médaillon 
monétaire peut aussi avoir été porté par une femme comme sembleraient l'attester des découvertes de médaillons de l'empereur Postume dans des tombes féminines (Lafaurie 1975a, p. 981). Mais on peut songer aussi à un cadeau masculin, au même titre que les monnaies d'argent sévériennes.

11 Les objets de Vaise peuvent sans difficulté être considérés comme un tout appartenant à un moment donné à une unique personne, ou mieux à un couple. Mais on ne peut exclure non plus tout à fait qu'il s'agisse d'un ensemble hétéroclite, constitué au moment de la cachette en un même lot de pièces de provenances diverses.

\section{Le dépôt $n^{\circ} 1$ : les statuettes}

12 Quant aux statuettes, elles représentent dans l'état actuel 1864,30 g d'argent, soit près de 6 livres (y compris les tenons en un autre métal des parties rajoutées). Mais le lot est sans doute incomplet, puisque plusieurs fragments, une aile, la couronne tourelée, un bras, attestent l'existence d'autres objets, en nombre indéterminé. Trois statuettes sont conservées entièrement: Hélios, la déesse aux oiseaux et la Fortune. Deux têtes elles aussi peuvent être considérées comme constituant à elles seules des entités plus ou moins complètes, celles de Jupiter et du personnage aux deux excroissances sur le crâne. Une seule des trois statuettes dotées d'un socle possède une inscription : une dédicace au numen d'un empereur. Il n'est pas indifférent de noter que c'est précisément Hélios qui la porte, et peut-être doit-on penser que l'empereur en question avait un attachement particulier pour un culte solaire.

13 Cette dédicace intervient aussi dans la réflexion sur la nature du trésor. Les trois divinités - les deux Fortune tout particulièrement, mais aussi Hélios - seraient parfaitement à leur place dans un laraire privé par leurs dimensions comme par leur nature. Le reste, vaisselle et bijoux, assez modeste à vrai dire, à peine plus de 10 livres d'argent et très peu d'or (une partie des bijoux est en argent, ce qui est plutôt rare) constituerait une autre partie des biens de son dernier possesseur.

14 Le trésor de Vaise serait ainsi dans sa composition, à une échelle certes bien différente, l'équivalent du grand trésor découvert en 1768 à Mâcon (Trésors d'orfêvrerie, p. 185 ; CAG $71 / 4$, p. 305306) qui, outre un lot très important de monnaies, renfermait des bijoux, de la vaisselle d'argent, pas très abondante, et des statuettes de petites dimensions.

15 S'ajoute à Vaise une pièce particulière, le buste probablement impérial. L'identité du personnage représenté reste imprécise: à titre d'hypothèse nous avons proposé Gallien, mais d'autres identifications restent tout à fait possibles, en particulier pour des empereurs de la seconde moitié du IIIe s. Mais à la question de la fidélité éventuelle du portrait s'en ajoute une autre, celle de la justification de sa présence dans le trésor d'une part, à Lyon d'autre part. La réponse donnée à la première de ces deux questions, nous y revenons plus loin, pèse évidemment sur l'idée que l'on se forme de la nature de l'ensemble de Vaise.

16 Pour apprécier l'intérêt de la trouvaille de Vaise, on soulignera qu'elle renforce sérieusement la liste des statuettes en argent de moyennes dimensions, en fournissant trois exemplaires complets et deux têtes, trouvés en outre dans un contexte connu, ce qui est plutôt rare : au trésor de Chaourse (une statuette de Fortuna, à laquelle on ajoutera le poivrier en forme d'esclave noir endormi, Trésors d'orfêvrerie, $\mathrm{n}^{\circ} 81$ et 83), caché vers 260, à celui, contemporain, de Nicolaevo en Bulgarie (une boîte à épices en forme d'enfant tenant un chien) (Filow 1914, p. 1-48), à celui de Daphné, enfin, près 
d'Antioche, sensiblement plus tardif (la cachette doit avoir eu lieu dans le courant du IVe s., et contenait une Aphrodite diadémée) (Ross 1953, p. 40-41), on ne peut guère ajouter que la récente trouvaille de Clermont-Ferrand: mais la technique de la statuette de bronze et d'argent qu'elle a livrée est différente, comme celle de la Vénus de Kaiseraugst, entièrement fondue.

Des exemples nombreux existent de statuettes en argent ayant appartenu à un laraire privé, à Pompéi notamment. Le dépôt découvert naguère à Clermont-Ferrand, déjà mentionné en raison de l'existence d'une Fortuna en bronze et en argent, appartient aussi, sans doute, à cette catégorie. Si l'on étend la définition du laraire à tout ce qui concerne la dévotion privée, on pourra considérer également comme des objets de cette nature la Fortune de Chaourse (Trésors d'orfèvrerie, $\mathrm{n}^{\circ}$ 83), dans la seconde moitié du IIIe s. (bien qu'elle se trouve au milieu d'un important ensemble de vaisselle), celle du petit trésor d'Antioche-Daphné au début du IVe s. (quelques pièces de vaisselle et une statuette) ou la Vénus de Kaiseraugst au milieu du IVe s.

\section{Objets votifs?}

18 Un passage peu connu d'Ammien Marcellin (XXII, 13, 3), qui se passe sous le règne de l'empereur Julien, donne pour la fin de l'Antiquité une curieuse illustration de ces dévotions privées qui pouvaient en même temps prendre, d'une certaine manière, un caractère public, ou tout au moins, semi-public: le philosophe Asclépiadès avait l'habitude d'emporter avec lui en voyage une petite image en argent de la déesse Caelestis devant laquelle il priait chaque soir en allumant des cierges; or il advint un jour qu'après avoir déposé pour la nuit cette icône aux pieds de la statue d'Apollon dans le célèbre sanctuaire du dieu à Daphné, près d'Antioche, il mit le feu au temple pour avoir oublié d'éteindre les chandelles. De cette anecdote on retiendra que des dévotions avaient lieu dans un sanctuaire public, mais avec des objets privés: les statuettes pouvaient sans doute parfois jouer le même rôle que la petite icône du philosophe, que l'on imaginera à l'image des feuilles en argent que l'on connaît bien par ailleurs.

19 Mais la dédicace au numen impérial donne à la statuette d'Hélios le caractère d'un objet votif, qu'avaient par exemple la statuette de Rosmerta, en argent sans doute elle aussi, qui n'est plus connue que par son socle en métal précieux inscrit au nom de la déesse, découvert il y a quelques années à Dompierre-sur-Authie (Somme), sur la côte de la Manche (inédit), ou bien encore la grande statuette découverte à Tunshill (Lancashire) en Grande-Bretagne, dédiée par un certain Valerius Rufus à la Victoire de la VIe légion Victrix, dont il ne subsiste plus qu'un bras (on retiendra que pour cet objet la dédicace figure sur une petite plaque sommaire gravée en lettres pointillées et attachée au bras ; on s'interrogera sur la raison d'être de cette curieuse façon de faire : n'y avait-il plus déjà au moment de l'offrande que le bras, et dans ce cas de quelle manière était-il entré en possession de Valerius Rufus?). Un parallèle intéressant au trésor de Vaise est fourni enfin par la trouvaille de Champoulet, dans le Loiret, qui rassemblait vaisselle et statuettes de bronze (Joffroy 1978; CAG 45, p. 46). L'importance de l'ensemble est équivalente, qui réunissait des statuettes, identifiées comme des pièces votives offertes à Apollon, à Mercure et à Rosmerta par les inscriptions qui figurent sur leur socle, et plusieurs coupes qui présentent elles aussi des dédicaces : il s'agit sans nul doute d'un dépôt sacré. La trouvaille de Weissenburg (Kellner-Zahlhaas 1993), en Bavière, constitue elle aussi une partie du trésor d'un sanctuaire : ce sont les feuilles votives et 
les dédicaces sur certaines pièces de vaisselle qui permettent d'écarter pour les statuettes en bronze l'idée d'un laraire, même si elles ne comportent elles-mêmes aucune inscription; l'anecdote d'Asclépiadès, citée plus haut, montre néanmoins que des feuilles de ce type pouvaient éventuellement avoir un caractère privé.

La question des dépôts de vaisselle et de statuettes, et de leur appartenance éventuelle à un laraire privé ou à un temple, est reprise de manière approfondie par A. KaufmannHeinimann à propos des trouvailles d'Augst (Kaufmann-Heinimann 1998, p. 253, fig. 205). Cet ouvrage est malheureusement paru trop tard pour que nous puissions l'utiliser dans cette publication.

21 Ainsi le trésor de Vaise proviendrait-il d'un sanctuaire plutôt que d'un laraire privé. Mais la dédicace sur l'Hélios est le seul indice en faveur de cette solution. Ni les bijoux, ni la vaisselle, ni les monnaies ne l'appuient, mais ils ne l'écartent pas davantage. À Acci (auj. Guadix, province de Grenade), en Hispanie Citérieure, mais à la limite de la Bétique, une inscription datée du IIe s. relate l'offrande à Isis d'une statue en argent d'un poids de 36,66 kg et de sa parure, effectuée par Fabia Fabiana en l'honneur de sa petite fille. Le texte détaille les bijoux offerts (diadème, pendants d'oreilles, collier, bracelets, bagues) et leur composition ; au total, une grosse perle, 53 autres perles, au moins 36 émeraudes, 26 cylindri - type de pierre ou élément en or ? - et 6 autres pierres précieuses (CIL, II, 3386 ; Schaad 1992, p. 67, 336). Mais, peu fréquentes, ces dédicaces associant une statue, souvent en argent, et sa parure (cum suis ornamentis) semblent une particularité de la Bétique où une demi-douzaine de cas sont répertoriés (Beltrân Fortes, Ventura Villanueva 1992-93) : particularité régionale du culte ou de l'ostentation artistocratique? Enfin, les monnaies représentent l'offrande la plus commune dans un sanctuaire. Tout objet peut devenir votif par l'usage qu'on en fait, d'où la difficulté de décider de la fonction d'un dépôt en l'absence de données complémentaires, qu'il s'agisse du lieu de découverte ou de dédicaces portées sur des offrandes. En outre, la localisation dans un sanctuaire ne serait peut-être pas un critère suffisant selon H. Guiraud (Schaad 1992, p. 65) qui fait une série de remarques suggestives sur les trésors de bijoux réunis comme offrande. Ainsi le trésor de Cracouville (Evreux, Eure) retrouvé près d'un portique de fanum est-il une offrande à une divinité ou une cachette placée sous la protection du dieu? On notera qu'il a été trouvé dans des couches de démolition (CAG 27, p. 155 ; sur les trésors de temples, cf. Baratte 1992).

La mention vraisemblable de ratiarii, des bateliers, et le rapprochement avec l'inscription d'Ostie mentionnant des statuettes de la Victoire, de la Fortune et des effigies impériales en argent au siège d'un collège conduisent même à se demander si une partie du trésor de Vaise n'aurait pas pu appartenir, à un moment donné à une association.

Une dernière possibilité doit être examinée concernant la constitution de l'ensemble de Vaise, qui dans une certaine mesure exclut à la fois l'une et l'autre des hypothèses précédentes : si l'on considère qu'il ne s'agit pas de biens appartenant à un unique propriétaire, on se demandera si les différents éléments du dépôt n'ont pas été rassemblés progressivement, de manière aléatoire, autrement dit s'ils ne représentent pas soit le fruit de rapines successives, soit des objets préparés pour le refonte; la présence de fragments pourrait s'expliquer de cette manière. Mais cette interprétation ne s'impose pas. 
trésor familial, trésor de sanctuaire ou butin de malandrins, les dépôts de Vaise n'ont pas été récupérés et il reste à s'interroger sur cet abandon. Hormis le rôle d'événements d'ordre privé, on songera aux effets de troubles politiques, sociaux ou militaires qui affectent la Gaule pendant plus d'un quart de siècle. La problématique sur les invasions n'a pas - on le sait - bonne presse. Les causes possibles de cachettes sont trop nombreuses pour pouvoir toujours en retenir une de façon assurée. Nous avons pourtant tenu à inviter notre collègue Xavier Loriot à présenter la carte du "Schatzfundhorizont" de 259/260, qui renouvelle la documentation disponible, jusquelà limitée au quart sud-est de la Gaule (Py, Hiernard, Richard 1983), et dont l'intérêt vient d'être relancé par la découverte d'une inscription à Augsbourg (Bakker 1993; Lavagne 1994). Le trésor de Vaise, à l'instar de celui de Lyon Saint-Jean, est un nouveau document à verser à ce dossier.

\section{L'invasion alamannique de 259-260 en Gaule \\ D'après les sources littéraires, épigraphiques et numismatiques}

L'invasion des Alamans en Gaule et en Italie, dont une inscription récemment découverte à Augsbourg $(\mathrm{AE}, 1993,1231)$ a révélé qu'elle fut accompagnée d'une incursion simultanée des Juthunges en Rhétie et en Italie du Nord, aboutissant à couper les communications entre le limes germanorhétique et le pouvoir impérial, fut, plus encore que la capture de Valérien vers la mi-260, la cause déterminante de l'usurpation de Postume, qu'il est désormais possible de dater entre le 25 avril et le 11 septembre $260^{21}$.

Ces événements sont probablement les moins mal documentés de tous ceux qui accablèrent la Gaule durant les années noires de la crise du IIIe s. L'on dispose en effet de trois sources littéraires indépendantes, quoique toutes très tardives. La plus explicite est Grégoire de Tours : il relate dans ses Histoires (I, 32-34), comment, sous le règne de Valérien et Gallien, le roi des Alamans, nommé Chrocus, envahit la Gaule. Il détruit à Augustonemetum (Clermont-Ferrand) le temple de Vassogalate, martyrise à Mende l'évêque de Javols saint Privat avant d'être capturé et exécuté en Arles $^{22}$. Au début du VIIe s., le médiocre chroniqueur burgonde connu sous le nom de pseudoFrédégaire mentionne la destruction par les barbares d'Aventicum (Avenches), capitale des Helvètes ${ }^{23}$. Enfin le byzantin Zonaras (XII, 24) nous apprend que Gallien vainquit devant Milan à la tête de dix mille hommes une bande d'Alamans trois fois supérieure en nombre. Or ces témoignages, à commencer par celui de Grégoire, apparaissent fiables, à quelques détails près. Ainsi, le nom de «Vassogalate » n'est pas celui d'un temple, mais du dieu qu'on y honorait, lequel, d'après une inscription de Beda (Bitburg), en territoire trévire, était une divinité celtique assimilée à Mercure (CIL, XIII, 4130 ${ }^{24}$. La description du sanctuaire, remarquablement précise, paraît empruntée à quelque chronique arverne, dont Grégoire, né à Clermont, aurait pu disposer ${ }^{25}$. La destruction d'Avenches semble confirmée par la découverte dans le théâtre, en 1875, d'un petit ensemble de monnaies des Tétricus, indice peut-être de la transformation de l'édifice en zone d'habitat ${ }^{26}$. Par ailleurs, l'inscription d'Augsbourg corrobore indirectement le témoignage de Zonaras, puisqu'il en ressort que les Juthunges firent irruption en Italie, à ce qu'il semble dans le cours de 259 .

La numismatique est-elle à même de compléter le témoignage de ces sources, en précisant notamment l'itinéraire suivi par les barbares, grâce au pointage 
cartographique des trouvailles ou " trésors » ? C'est un vieux débat. L'ouvrage pionnier d'Adrien Blanchet, Les trésors de monnaies romaines et les invasions germaniques en Gaule, Paris, 1900, développait, en la systématisant, l'idée selon laquelle les enfouissements monétaires étaient essentiellement liés aux événements politiques ou militaires contemporains. Cette problématique, qui néglige les faits proprement monétaires (dévaluations, décris), comme les considérations pratiques (les Anciens ne possédaient ni coffres-forts ni banques où déposer leurs économies) est aujourd'hui largement remise en cause ${ }^{27}$. Toutefois, le pointage des trouvailles ayant un même terminus n'est pas sans signification, si d'autres indices peuvent être allégués, comme c'est ici le cas. Divers savants s'y sont essayés, en particulier F. Staehelin et É. Demougeot, dont les études sont malheureusement entachées de nombreuses erreurs, liées à l'utilisation trop confiante de sources non vérifiées. Plus récemment, M. Py, J. Hiernard et J.-C. Richard ont dressé, pour un grand quart sud-est de la Gaule, région où les dépôts abondent, un inventaire détaillé (Py, Hiernard, Richard 1983) que Michel Amandry et moi-même avons tenté de mettre à jour en 1986 (Amandry, Loriot 1986, note 6). Il apparait toutefois nécessaire de replacer ces trouvailles dans l'ensemble de l'espace gaulois. La carte ci-jointe (fig. 121), élaborée à l'occasion de la Table ronde tenue à Paris sur l'inscription d'Augsbourg, a été enrichie de quelques trouvailles supplémentaires - dont deux proviennent de Lyon ${ }^{28}$. Ce document est certes impressionnant, mais de lecture et d'interpétation difficiles. Nombre des documents pointés (ou " spots ») correspondent à des trésors très mal connus ; que dire par exemple de celui de Digne (Alpes-de-Hte-Provence) : monnaies « de l'époque de Gallien » ou encore de celui d'Andancette (Drôme), qui consiste en imitations coulées. Une première constatation fait apparaitre l'absence ou l'extrême rareté des dépôts dans l'Ouest ou le Sud-Ouest. On observe en revanche une forte concentration dans le sillon rhodanien et dans les Alpes du Nord, en particulier dans les départements de la Côte-d'Or (15), de la Saône-et-Loire (13), mais aussi de l'Ain, du Rhône, de la Loire, de la Haute-Savoie et de l'Isère, avec des prolongements vers le Massif Central (Allier, Puy-deDôme) et la basse vallée du Rhône (Drôme, Gard). D'autres trouvailles, provenant d'Alsace ou de Suisse (trésor de la Rennweg à Zurich, de Baden, de Cœuve ${ }^{29}$ ), semblent jalonner un autre itinéraire, empruntant le versant oriental du Jura. Opérant depuis le secteur nord des Champs Décumates (trésor de Niederbieber), les Alamans se seraient ainsi divisés en deux bandes principales, l'une empruntant l'axe Moselle-Saône-Rhône, l'autre le coude de Bâle et le plateau suisse, avant d'obliquer vers l'Italie, les uns par le Petit-Saint-Bernard et le Mont-Cenis, les autres par le Grand-Saint-Bernard, ces deux routes convergeant sur Aoste. Quant aux Juthunges, ils auraient suivi, par Bregenz et Coire, la via Claudia pour franchir les Alpes par Foetis (Fiissen), le Fernpass et la vallée de l'Adige, pour déboucher en Italie à Pons Drusi (Bolzano), et de là à Trente et Vérone. Peut-être est-ce à eux que se rapporte la notice d'Eutrope (VIII, 7) relative à un raid de "Germains" en direction de Ravenne. Il convient toutefois de remarquer que de fort nombreuses trouvailles ont été signalées dans le Nord de la Gaule, en particulier en Belgique occidentale (Hainaut, Picardie, Champagne, avec une concentration autour de Reims). Les Alamans seraient-ils parvenus jusque-là en 259/260 ? ou faut-il incriminer d'autres barbares, les Francs par exemple, dont cependant l'on ne rencontre aucune mention certaine avant le règne de Probus ${ }^{30}$ ? Force est également de constater que ces régions sont celles 
qui, à toutes époques, fournissent de nombreux trésors (ainsi le volume VIII/1 du $T A F$ répertorie-t-il 150 dépôts pour le seul département de la Somme). L'on en revient ainsi à la question initialement posée quant à la validité méthodologique de l'utilisation des trésors. Il convient d'envisager le problème avec pragmatisme, pour ainsi dire au cas par cas. À cet égard, il n'est pas indifférent de rapprocher le trésor de Lyon-Vaise de celui de Nivolas-Vermelle, lieu-dit Ruffieu (TAF, V/2, Isère 19), où fut exhumé en 1837 un trésor comprenant 6 aurei, des bijoux et de l'argenterie, daté par les monnaies de 254/255. Or il appartenait à un soldat de la légion IIe Augusta de Bretagne ${ }^{31}$.On conçoit mal que sa présence en ce lieu ne soit pas liée à quelque événement à caractère militaire. Il se pourrait que ce légionnaire ait appartenu aux troupes dépêchées par Postume pour garder les cols des Alpes ${ }^{32}$.

\section{Xavier Loriot}

112- Trésors monétaires enfouis en 25-260

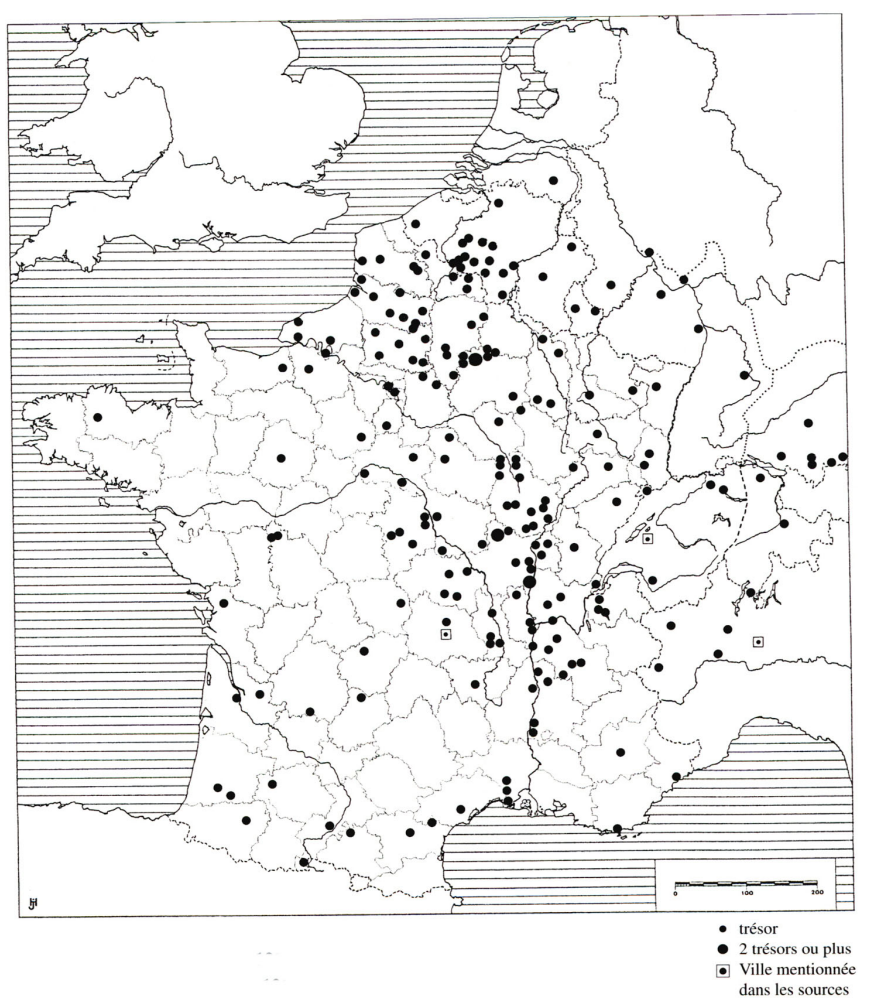

\section{NOTES}

21. La bibliographie de ce document est déjà longue. Outre la publication originale de L. Bakker, "Raetien unter Postumus - Das Siegesdenkmal einer Juthungenschlacht im Jahre $260 \mathrm{n}$. Chr. aus Augsburg", Germania, 71, 1993, p. 369-386, on citera : H. Lavagne, "Une nouvelle inscription 
d'Augsbourg et les causes de l'usurpation de Postume", CRAI, 1994, p. 431-446 ; P. Le Roux, "Armées, rhétorique et politique dans l'Empire gallo-romain. À propos de l'inscription d'Augsbourg", ZPE, 115, 1997, p. 281-290. Une table-ronde de l'URA 1979 lui a été consacrée le 19 novembre 1994 : on en trouvera les actes dans les Cahiers du Centre Gustave-Glotz, 8, 1997, p. 224-260 (communications de S. Demougin, M. Christol et J. Hiernard, avec présentation d'ensemble de M. Christol et X. Loriot).

22. Étude détaillée de ce témoignage par É. Demougeot, "Les martyrs imputés à Chrocus et les invasions alamanniques en Gaule méridionale", Annales du Midi, 74, 1962, p. 5-28 ; sur un plan plus général : La formation de l'Europe et les invasions barbares, I, Des origines germaniques à l'avènement de Dioctétien, Paris, 1969, p. 493-499. Voir aussi L. Schmidt, Geschichte der deutschen Stämme, Die Westgermanen, Munich, 1938-1940, rééd. 1970, p. 233-235.

23. MGH, II, 40, Scriptores rerum Meroving., II, p. 64.

24. In h(onorem) d(omus) d(iuinae) deo Mercurio Vassocaleti Mandalonius Gratus d(ono) d(edit).

25. Sur la localisation du temple et ses vestiges, $c f .:$ CAG, 63/1, Paris, 1994, p. 143-151.

26. E. Secrétan, Bull, de l'Association "Pro Aventico", 2, 1888, p. 27-28; cf. id., Aventicum, son passé et ses ruines, Lausanne, 1919, p. 29. Discussion des témoignages archéologiques chez F. Staehelin, Die Schweiz in romischer Zeit, 3e éd., Zurich, 1948, p. 260-262 et D. Van Berchem, "Aspects de la domination romaine en Suisse", Revue suisse d'histoire, 5, 1955, p. 162-164 (destruction d'un temple, cachette d'un buste d'or de Marc Aurèle).

27. Voir notamment R. Delmaire, "Les enfouissements monétaires, témoignages d'insécurité ?", Revue du Nord, 77, 1995, p. 21-26 (cf. ibid.. 74. 1992, p. 152) ; S. Estiot, "Le troisième siècle et la monnaie : crise et mutations", dans J.-L. Fiches (éd.), Le IIIe siècle en Gaule Narbonnaise, SophiaAntipolis, 1996, p. 56-61.

28. Trésors de Lyon-Vaise et celui de l'avenue AdolpheMax, au pied de Fourvière (Villedieu 1990, p. 162). Ajouter, dans la même région, un nouveau trésor mis au jour à Sillingy (Haute-Savoie) : D. Rizzo, Revue savoisienne, 1992, p. 39-40 (trouvaille peut-être incomplète).

29. Sur ce trésor, découvert en 1840 et partiellement conservé à Porrentruy, voir l'étude de Y. Muhlemann, Association des Amis du Cabinet des Médailles [de Lausanne]. Bulletin, 9, 1996, p. 21-26.

30. Cf. T.D. Barnes, "The Franci before Diocletian », Historiae Augustae Colloquium Genevense. Atti dei convegni sulla Historia Augusta. II, Bari, 1994, p. 11-18.

31. CIL, XII, 2355 : C(ai) Didi Secundi mil(itis) leg(ionis) II Aug(ustae) c(enturiae) Mari.

32. Cf. Anonymus post Dionem, fragment 6 (FGH, IV, p. 194-195 Miller); X. Loriot, D. Nony, La crise de l'Empire romain, 235-285, Paris, 1997, p. 67-68 n² 29 B. 


\title{
Annexe
}

\author{
François Planet
}

\section{Trésors anciens du Rhône et de la région Rhône-Alpes}

1 Quelle place les trouvailles monétaires occupent-elles réellement dans le patrimoine d'une ville, d'un département ou d'une région? Sans être extrêmement fréquentes, elles ne sont pas rares puisque la liste établie en 1987 et 1988 pour les seuls volumes du corpus des Trésors monétaires Antiques de la France (TAF, tome V) consacré à la région Rhône-Alpes est riche de deux cent quarante-trois trouvailles locales recensées depuis le XVIe s. ${ }^{33}$ (fig. 122). Malheureusement, les circonstances de leurs découvertes souvent fortuites - et la dispersion qui s'ensuit nous privent trop souvent des informations qu'on aurait été en droit d'en tirer. Nous devons alors composer à partir des données disponibles: articles de presse de l'époque, correspondances, comptes rendus de séances de sociétés historiques locales ou nationales et surtout inventaires de collections publiques ou privées.

2 Tous ces trésors ne sont pas de la même importance, ni de la même nature. La plupart, composés de monnaies courantes, sont dissimulés en vue de paiements futurs et abandonnés ensuite (trésor de Chaponnay - TAF V/l, Rhône, 3). D’autres, constitués de monnaies en or, sont de véritables réserves de valeur dépassant les seuls besoins d'une personne (trésors de Perrache à Lyon ou de Lentilly - TAF V/l, Rhône, 7 et 9). Certains enfin appartiennent à des particuliers désirant, à la faveur d'une crise locale, dissimuler leur patrimoine, comme par exemple à Lyon, le trésor du Clos des Lazaristes (TAF V/l, Rhône, 12) ou, dans une moindre mesure, celui de Vaise. Des bijoux précieux, de la vaisselle et même des statuettes d'argent côtoient des monnaies d'or en nombre limité, parfois conservées sous la forme de bijoux monétaires. 


\begin{tabular}{|c|c|}
\hline $\begin{array}{c}\text { Départements de la } \\
\text { région Rhône-Alpes }\end{array}$ & $\begin{array}{c}\text { Nombre de trésors } \\
\text { recensés dans les TAF }\end{array}$ \\
\hline Ain & 46 \\
\hline Rhône & 27 \\
\hline Loire & 15 \\
\hline Ardèche & 9 \\
\hline Drôme & 44 \\
\hline Isère & 48 \\
\hline Savoie & 18 \\
\hline Haute-Savoie & 36 \\
\hline
\end{tabular}

3 L'ensemble peut être associé à de larges séries de numéraire récent en argent. Ces monnaies correspondent alors à des liquidités initialement soustraites à la circulation monétaire pour un laps de temps plus court et abandonnées dans les mêmes circonstances que le reste du dépôt.

\section{Réserves d'espèces courantes}

4 Les trésors lyonnais ou régionaux sont le plus souvent constitués de monnaies en argent ou en bronze. Bon nombre d'entre eux sont abandonnés au cours de la seconde moitié du IIIe s. après J.-C. Les vagues successives d'invasion qui ravagent les Gaules de 253 à 276 sont habituellement évoquées pour expliquer cette sur-représentation ( $c f$. Loriot supra). De manière générale, les guerres et l'insécurité qui les suit ont évidemment leur importance dans l'abandon des trésors monétaires. Le cas le plus fréquent est celui du dépôt que l'on ne vient jamais reprendre, comme par exemple les trésors d'Ouroux (TAF V/l, Rhône, 19) et de Sainte-Colombe (TAF V/l, Rhône, 20). Plus rare est celui d'une caisse militaire dissimulée avant la bataille comme, peut-être, à Genas (TAF V/l, Rhône, 4). En 197 après J.-C., les armées de Clodius Albinus, usurpateur soutenu par les Lyonnais, affrontent les troupes de l'empereur Septime Sévère dans la plaine de la Saône. Les troupes d'Albin sont défaites et Lyon est mise à sac. Personne ne vint rechercher cette partie de la solde après la défaite et il faut attendre 1826 pour voir plusieurs milliers ${ }^{34}$ de beaux deniers d'argent (frappés à Lyon en vue du paiement des troupes), apparaître sur le marché des monnaies anciennes (fig. 123). Outre le trésor du Clos des Lazaristes déjà cité, d'autres découvertes monétaires associées à des sépultures semblent liées à cet épisode de guerre civile :

- À Saint-Didier-au-Mont-d'Or, un squelette enveloppé d'une sorte de cotte de mailles avait à ses côtés un cœur en bronze doré, dix-sept deniers d'argent et quatre pièces de bronze allant de Vespasien à Septime Sévère (TAF V/l, Rhône, 34) ;

- À Lyon, au sud-est de la Croix Rousse, rue des Fantasques, un autre squelette de soldat romain portait une ceinture contenant un pécule d'un sesterce et douze deniers dont le plus récent était de 194 (TAF V/l, Rhône, 33 ; Audra, Mathey 1992, p. 54-55). 

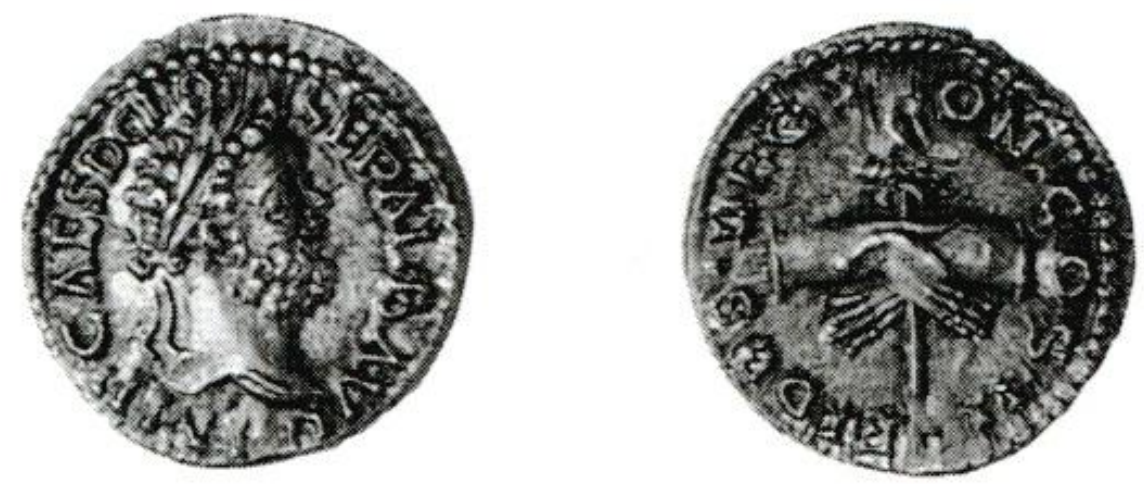

MBA Lyon, ML. 139

5 Ces deux derniers ensembles sont interprétés comme des bourses de soldats romains tués lors de la bataille de 197. Plus récemment, à Vaise, quartier Saint-Pierre, un charnier renfermant les restes osseux de quatre humains et cinq chevaux a été rapproché, avec les réserves d'usage, des mêmes événements (Chastel 1995, p. 63).

L'enfouissement d'un trésor monétaire peut s'expliquer également par des considérations économiques, voire purement monétaires (Estiot 1996). Le IIIe s. de notre ère est une période d'inflation pendant laquelle la teneur de la monnaie en métal précieux ne cesse de chuter. En moins de cinquante ans, le double denier ou antoninien, créé par Caracalla en 215, voit sa teneur en argent fin passer du simple au tiers avant d'être réduite à une fine pellicule déposée à la surface d'une pièce de cuivre à partir de 266 (Carcassonne, Christol 1974). Dans un tel contexte, mieux vaut conserver la bonne et vieille monnaie qui, officiellement, n'a pas plus de valeur que la nouvelle, alors que sa valeur intrinsèque est largement supérieure. Les importantes réformes monétaires mises en place dans l'Empire sous Aurélien en 274 (Callu 1969) puis sous Dioclétien en 294-295 (Callu 1960) peuvent alors expliquer l'existence de trésors postérieurs aux invasions. L'obligation de rendre la monnaie ancienne en échange des nouvelles dénominations a pu provoquer l'enfouissement des anciennes espèces dans les années suivant les réformes. Ainsi, l'apparition en 294-295 de la monnaie taillée au 1/32 de la livre romaine et appelée communément follis ou nummus aurait été suivie par l'enfouissement d'importants dépôts monétaires dans la région lyonnaise; les trésors de Lancié-Morancé ${ }^{35}$ (TAF V/l, Rhône, 5 et 18) et de Villette-d'Anthon (Isère) (Amandry et al. 1996, p. 117123) trouveraient ainsi leur origine ${ }^{36}$.

\section{Trésors de monnaies en or}

$7 \quad$ Les trésors composés d'aurei sont naturellement beaucoup plus rares que les autres. Il est donc tout à fait exceptionnel de pouvoir évoquer trois trésors importants pour le seul département du Rhône.

Le trésor de Lentilly (TAF V/l, Rhône, 7) est le plus spectaculaire. Avec plus de deux cents monnaies, il constitue la plus belle trouvaille de monnaies d'or antiques faite à ce 
jour dans la région Rhône-Alpes. À l'époque de sa découverte, en 1866, son propriétaire eut la bonne idée de le proposer au Musée des Beaux-Arts de Lyon. Le conservateur du musée, Edme-Camille Martin-Daussigny, étudia le trésor dans son ensemble et acquit pour le musée un lot représentatif de dix-huit exemplaires. Grâce à ces exemplaires et en s'appuyant sur différents témoignages de l'époque, il a été possible de reconstituer le trésor dans sa globalité, puis de l'étudier précisément (Planet 1992).

On peut également signaler le trésor de Perrache (TAF V/l, Rhône, 9), dégagé en 1832 à l'occasion de la construction de la célèbre gare lyonnaise. Malheureusement, aucun inventaire détaillé des cinquante-quatre monnaies n’a été rédigé lors de l'entrée des pièces au musée de Lyon et seul un aureus unique de Faustine peut aujourd'hui être identifié dans le fonds ancien du Médaillier de Lyon (fig. 124). Nous savons cependant que sa constitution est antérieure au règne de Commode puisque les monnaies les plus récentes remontent à Lucius Vérus (trois aurei de Lucius Vérus et deux pour sa sœur Lucille). Toutes les autres monnaies sont postérieures à la réforme monétaire de Néron de 64 et les plus anciennes datent de l'époque flavienne (huit aurei de Vespasien et cinq de Domitien). La distribution chronologique du trésor est ensuite très classique : trois aurei de Trajan, trois d'Hadrien, dix-huit pour Antonin le Pieux, huit pour Faustine et trois pour Marc-Aurèle. On notera la forte concentration de monnaies de l'époque antonine ainsi que l'absence de monnaies de Commode, ces deux éléments limitant la possibilité d'un enfouissement en relation avec les événements de 197. À cet ensemble très cohérent viennent s'ajouter trois mystérieuses monnaies d'or « douteuses ${ }^{37}$, sans qu'aucune autre précision ne soit apportée dans l'Etat descriptif du trésor rédigé à l'occasion de son dépôt au Musée par le Maire de Lyon, le docteur Prunelle, le 12 juin $1832^{38}$

124- Aureus unique de Faustine provenant du trésor de Perrache (Lyon-1832).
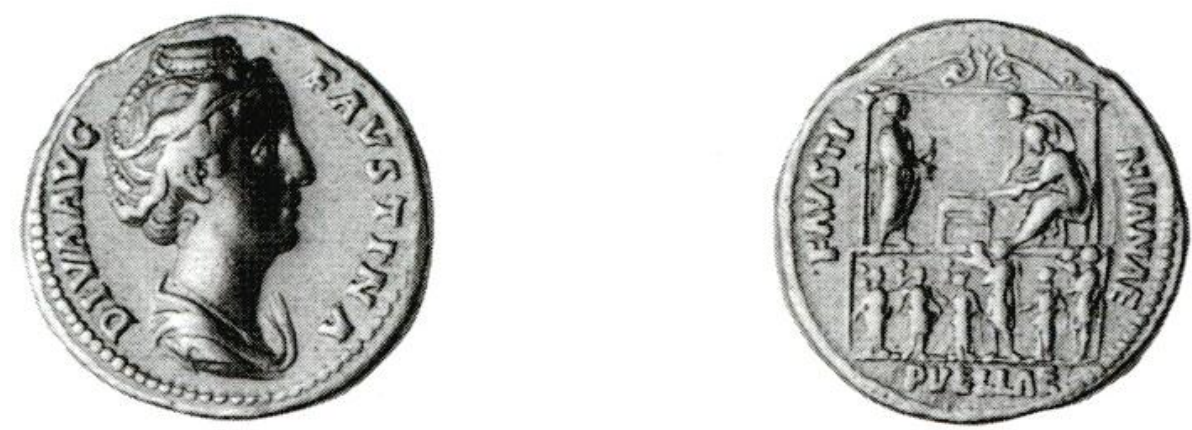

MBA Lyon, MAV. 240

Quant au trésor de Villeurbanne (TAF V/l, Rhône, 27), il est le plus récent (1936), mais aussi le plus mystérieux puisque les circonstances de sa découverte, sa composition exacte et sa localisation actuelle sont encore mal connues (Rémy 1984, p. 564-566).

\section{Trésors mixtes}

11 Alors que les réserves d'espèces courantes ou de monnaies en or correspondent généralement à des retraits massifs et ponctuels de numéraire en circulation, les trésors mixtes tel celui de Vaise semblent avoir été constitués sur le long terme ${ }^{39}$. Il s'agit le plus souvent de dépôts regroupant l'ensemble des biens précieux d'une même 
famille, ce patrimoine ayant été amassé sur plusieurs générations. Le trésor du Clos des Lazaristes à Lyon (TAF V/l, Rhône 12) contient des monnaies d'argent que l'on ne peut malheureusement pas identifier aujourd'hui (de Vespasien à Septime Sévère), mais également quelques monnaies en or et de superbes bijoux connus à l'époque de leur découverte, en 1841, sous le nom d'écrin d'une dame romaine (Comarmond 1844 ; supra $\mathrm{p}$. 98) (fig. 125). Le trésor de Ruffieu (TAF V/2, Isère, 19) associe lui aussi des monnaies d'or allant d'Hadrien (117-138) à Gallien (260-268), des bijoux de provenance locale des IIe et IIIe s. et de la vaisselle d'argent ${ }^{40}$. Trois autres trésors mixtes de la région RhôneAlpes, découverts au XIXe s., méritent d'être mentionnés :

- À Saint-Georges-de-Reneins (TAF V/l, Rhône, 25), une cachette double a été exhumée en 1880 et 1901. Le premier lot renfermait dans une sorte de sac (?) cent cinquante monnaies d'argent associées à trois bagues en or et sept cuillers en argent. Le second était formé d'un bloc d'environ cinq cents deniers et antoniniens de Marc Aurèle au règne de Gallien (260-268). Seule une monnaie plus tardive puisque frappée par Carin pour sa femme Magnia Urbica reporte le terminus en 283-285 (à condition bien sûr qu'elle n'ait pas été " associée » à la trouvaille après sa découverte). Un petit vase d'argent contenant un collier (chaîne avec trois émeraudes et deux perles), dix bagues en or et quatre en argent (ornées pour la plupart d'intailles) se trouvait à proximité du second dépôt monétaire ;

- À Saint-Genis-Pouilly (TAF V/l, Ain, 36), on découvrit en 1821, un trésor réunissant trois monnaies d'or et environ deux cents deniers et antoniniens de Vespasien à Valérien et Gallien (vers 260) à de la vaisselle d'argent (une petite assiette, une casserole à manche et deux cuillers), des bijoux et d'autres objets précieux. On trouva également une douzaine de monnaies de bronze, dont une à l'effigie de Iulia Domna sertie dans une monture d'argent (Loriot 1991, p. 81-83);

- Au Sault-Brénaz (TAF V/l, Ain, 41) enfin, près du Sault du Rhône (qui donne au trésor son nom usuel), à environ trois cents mètres du fleuve, un vase de bronze fut déterré par un cultivateur en 1862. Il contenait six monnaies d'or de Vitellius à Gallien, deux multiples d'or dont l'un de Gallien serti dans une monture circulaire, au moins quatre cent quatre-vingtcinq deniers et antoniniens de Néron à Postume (260-269) et un sesterce d'Hadrien. On dégagea également un collier d'or, un gros anneau d'or orné d'une intaille en nicolo et plusieurs bagues en argent. À cet ensemble assez classique dans sa composition viennent s'associer à des fragments d'un torque d'or, de bagues et de bracelets d'argent ainsi qu'un lingot d'argent. Ces derniers éléments amenèrent le premier éditeur de la trouvaille, J. Charvet (Charvet 1863), à penser que le propriétaire du trésor était un bijoutier colporteur (fig. 126). 
125- Monnaies en or provenant du trésor du Clos des Lazaristes à Lyon. Quinaire en or unique et aurei de Commode (177-192)
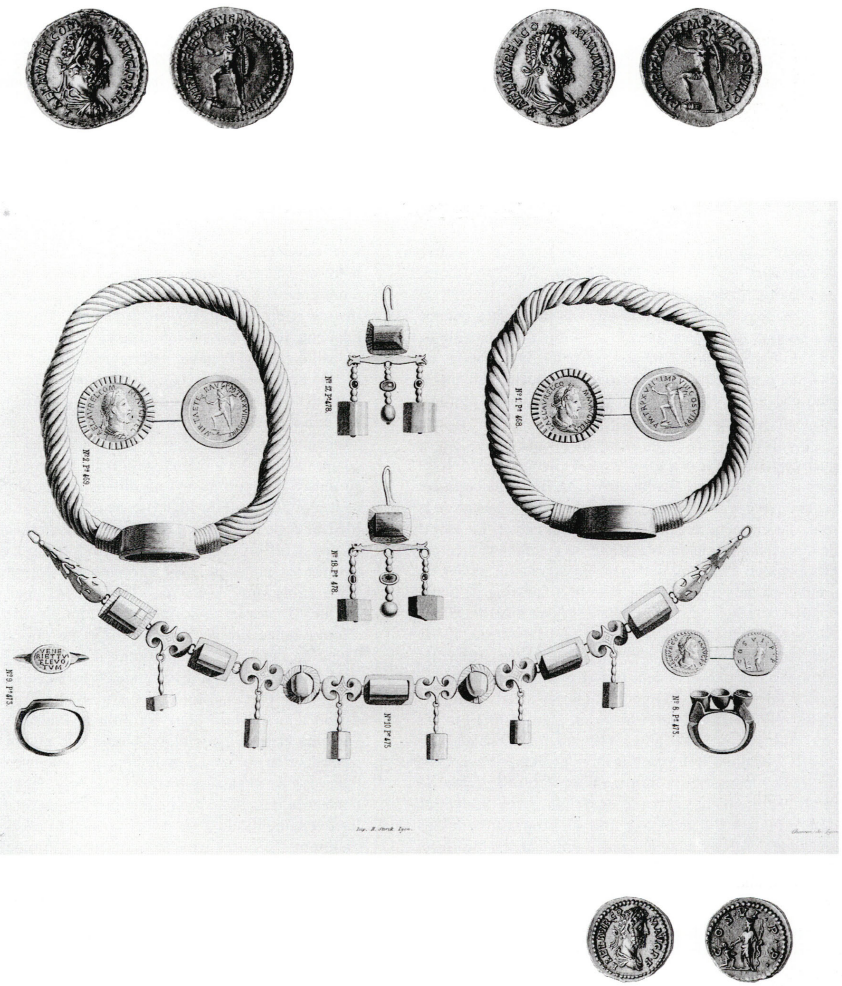

À l'origine, les deux dernières monnaies d'or étaient enchâssées dans de lourds bracelets du même métal (voir gravure ci-dessus). Dans la nuit du 14 février 1901, des monnaies et des bijoux en or furent dérobés au Musée de Lyon. Alors que la plupart des monnaies furent retrouvées à Paris, Bordeaux et Marseille, certains bijoux furent fondus. Les deux bracelets monétaires du trésor du Clos des Lazaristes ont été détruits dans ces circonstances et seuls les aurei subsistent. La planche gravée à l'occasion de la publication du trésor (Comarmond 1844) est le seul document iconographique qui rappelle les bracelets disparus en 1901.

(MBA Lyon, MAV. 280, MAV. 281 et MAV. 282) 
126- Le trésor du Sault du Rhône ou de Sault-Brénaz (Ain-1862).

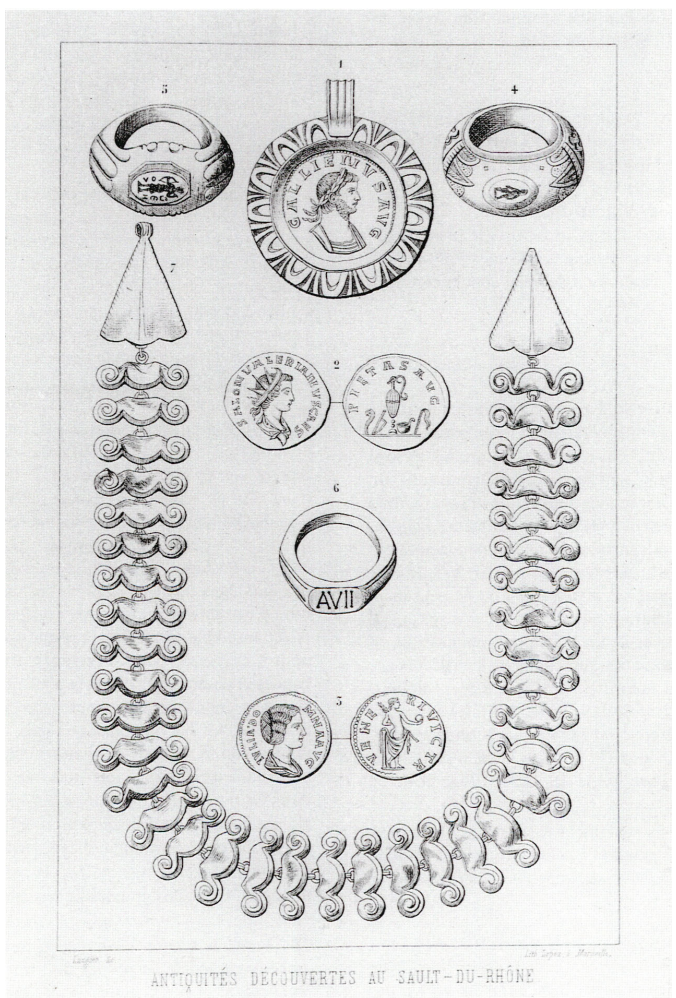

Planche extraite de J. Charvet, Notice sur des monnaies et bijoux antiques découverts en 1862 au Sault-du-Rhône, Paris, 1863

12 Aux deux cent quarante-trois trésors antiques recensés dans la région Rhône-Alpes en 1988 (fig. 127, 128) s'ajoutent déjà de nouvelles trouvailles ${ }^{41}$, dont celle de Vaise. Derrière ce nombre élevé de découvertes se dissimule une variété de cas qu'il n'est pas toujours simple de discerner. Certaines grandes lignes dans la composition du matériel thésaurisé peuvent cependant être relevées et nous apporter des indications partielles sur sa nature et son origine. Mais il est toujours délicat de se tenir aux seuls faits historiques, quand ils sont connus, pour expliquer les causes d'un enfouissement. Et il est plus difficile encore de comprendre les raisons qui amènent un propriétaire à ne pas récupérer son bien. Ces multiples dépôts correspondent à autant des cas individuels ou collectifs que l'historien n'a pas toujours lieu de connaître. C'est bien là que la part de mystère qui accompagne la découverte d'un trésor reste complète. 


\section{7- Principaux trésors anciens du Rhône}

\begin{tabular}{|c|c|c|c|c|c|c|c|}
\hline TAF & $\begin{array}{c}\text { Localisation } \\
\text { trouvailles }\end{array}$ & Date & Localisation actuelle & Nature & Type & Composition & Datation \\
\hline 3 & Chaponnay & av. 1673 & inconnue & monnaies AR & $\begin{array}{l}\text { réserve de } \\
\text { liquidités }\end{array}$ & env. 700 antoniniens (?) & IIle s. ? \\
\hline 1 & Belleville & 1799 & inconnue & monnaies AR & $\begin{array}{l}\text { réserve de } \\
\text { liquidités }\end{array}$ & 34 antoniniens & $\begin{array}{l}\text { Trajan Dèce (?)- } \\
\text { Dyarchie }\end{array}$ \\
\hline 21 & Sainte-Consorce & vers 1808 & $\begin{array}{l}\text { MCGR Lyon**, MBA } \\
\text { Lyon* et dispersion }\end{array}$ & $\begin{array}{l}\text { monnaies AR, } \\
\text { bijoux AR et AV }\end{array}$ & $\begin{array}{l}\text { trésor } \\
\text { mixte }\end{array}$ & $\begin{array}{c}\text { env. } 700 \text { deniers*, des bagues AV, } \\
1 \text { bague AR avec nicolo* dans } 1 \text { casserole } \mathrm{AE} \\
\text { inscrite** }\end{array}$ & $\begin{array}{l}\text { Auguste- } \\
\text { Septime Sévère } \\
\text { (Maximin?) }\end{array}$ \\
\hline 4 & Genas & 1826 & dispersion & monnaies AR & $\begin{array}{l}\text { réserve de } \\
\text { liquidités }\end{array}$ & au moins 2000 deniers & $\begin{array}{l}\text { Clodius Albinus } \\
\text { (197 ap. J.-C.) }\end{array}$ \\
\hline 9 & Lyon-Perrache & 1832 & MBA Lyon & monnaies AV & $\begin{array}{c}\text { trésor de } \\
\text { monnaies d'or }\end{array}$ & 54 aurei & $\begin{array}{l}\text { Vespasien-Lucius } \\
\text { Vérus }\end{array}$ \\
\hline 26 & $\begin{array}{l}\text { Villeurbanne } \\
\text { Charpennes }\end{array}$ & av. 1835 & MCGR Lyon $(?)^{* *}$ & $\begin{array}{l}\text { monnaies AR et } \\
\text { bijoux AV }\end{array}$ & $\begin{array}{l}\text { trésor } \\
\text { mixte }\end{array}$ & $\begin{array}{l}\text { antoniniens "du temps de Probus" et } 2 \text { boucles } \\
\text { d'oreilles en or et pierres dures (?)** }\end{array}$ & 2nde moitié du IIIle s \\
\hline 11 & Lyon-Brotteaux & $\begin{array}{c}\text { peu av. } \\
1836\end{array}$ & inconnue & $\begin{array}{l}\text { monnaies AR, } \\
\text { bijoux AR et AV }\end{array}$ & $\begin{array}{l}\text { trésor } \\
\text { mixte }\end{array}$ & $\begin{array}{l}\text { antoniniens, } 1 \text { collier } \mathrm{AV} \text { et } 3 \text { bagues } \mathrm{AR} \text { avec } \\
\text { intailles dans vase } \mathrm{AE}\end{array}$ & fin IIIle s. \\
\hline 12 & $\begin{array}{l}\text { Lyon-Clos des } \\
\text { Lazaristes }\end{array}$ & 1841 & $\begin{array}{l}\text { MCGR Lyon**, MBA } \\
\text { Lyon* et dispersion }\end{array}$ & $\begin{array}{c}\text { monnaies AR et AV } \\
\text { bijoux AV }\end{array}$ & $\begin{array}{l}\text { trésor } \\
\text { mixte }\end{array}$ & quinaire $\mathrm{AV}^{*}$, deniers et antoniniens, bijoux $\mathrm{AV}^{* *}$ & $\begin{array}{l}\text { Néron-Septime } \\
\text { Sévère }\end{array}$ \\
\hline 7 & Lentilly & 1866 & $\begin{array}{l}\text { MBA Lyon* et } \\
\text { dispersion }\end{array}$ & monnaies AV & $\begin{array}{c}\text { trésor de } \\
\text { monnaies d'or }\end{array}$ & $\begin{array}{c}\text { plus de } 200 \text { aurei } \\
\text { (17 ex. conservés au MBA Lyon*) }\end{array}$ & $\begin{array}{l}\text { Tibère-Néron } \\
\text { (av. } 60 \text { ap. J.-C.) }\end{array}$ \\
\hline 20 & Sainte-Colombe & 1870 & inconnue & monnaies AR & $\begin{array}{l}\text { réserve de } \\
\text { liquidités }\end{array}$ & 113 ex. : qqs. deniers et des antoniniens & Gordiens III-Gallien \\
\hline $5 / 18$ & Lancié-Morancé & 1880 & $\begin{array}{l}\text { dispersion (Lepaulle), } \\
\text { MBA Lyon* et MCGR** }\end{array}$ & monnaies AR & $\begin{array}{l}\text { réserve de } \\
\text { liquidités }\end{array}$ & plus de 1806 antoniniens* dans un vase $\mathrm{AE}^{* *}$ & $\begin{array}{l}\text { Gallien-Tétrarchie } \\
\text { (réforme de 294-295) }\end{array}$ \\
\hline 25 & $\begin{array}{l}\text { Saint-Georges-de- } \\
\quad \text { Reneins }\end{array}$ & $\begin{array}{c}1880 \text { et } \\
1901\end{array}$ & dispersion, MCGR** & $\begin{array}{c}\text { monnaies } A R, \\
\text { bijoux } A R \text { et } A V \text { et } \\
\text { vaisselle } A R\end{array}$ & $\begin{array}{l}\text { trésor } \\
\text { mixte }\end{array}$ & $\begin{array}{l}\text { env. } 650 \text { deniers et antoniniens, } 17 \text { bagues AV et AR } \\
\text { (dont } 14 \text { au MCGR**), lcollier AV, } 1 \text { vase AR et } \\
7 \text { cuillers AR }\end{array}$ & $\begin{array}{l}\text { Vespasien-Gallien } \\
\text { (260-268) ou Carin? } \\
\text { (283-285) }\end{array}$ \\
\hline 27 & Villeurbanne & 1936 & $\begin{array}{l}\text { dispersion (18 ex dans } \\
\text { coll. part. et } 9 \text { ex. à la } \\
\text { mairie de Villeurbanne } \\
\text { en } 1937\end{array}$ & monnaies AV & $\begin{array}{c}\text { trésor de } \\
\text { monnaies d'or }\end{array}$ & 45 aurei & $\begin{array}{l}\text { Néron-Trajan } \\
\text { (103/111 ap. } \\
\text { J.-C.) }\end{array}$ \\
\hline 19 & Ouroux & $1945 / 6$ & inconnue & monnaies AR & $\begin{array}{l}\text { réserve de } \\
\text { liquidités }\end{array}$ & 353 deniers et antoniniens & $\begin{array}{c}\text { Marc Aurèle- } \\
\text { Gallien } \\
\text { (253/260 ap. J.-C.) }\end{array}$ \\
\hline
\end{tabular}

128- Principaux trésors mixtes en Rhône-Alpes (hors département du Rhône).

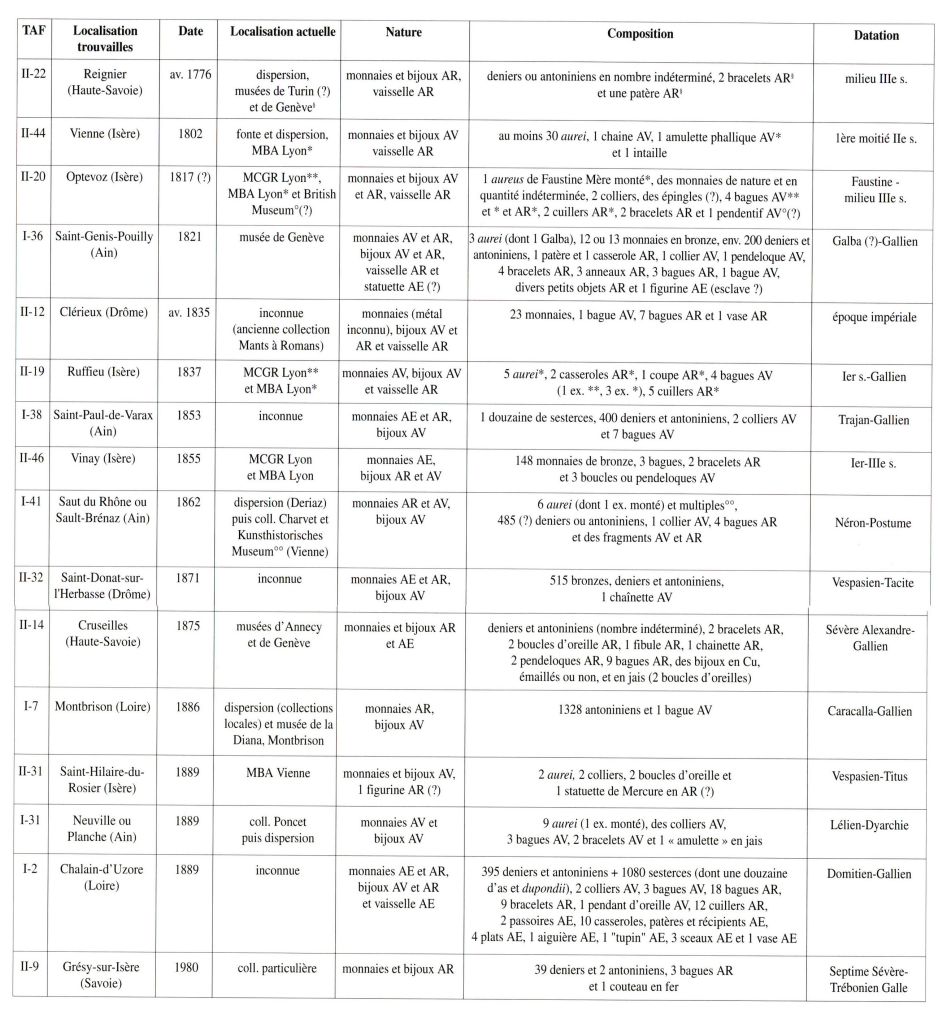

Les tableaux ont été rédigés à partir des TAF, augmentés et corrigés par les inventaires du MBA de Lyon. Les informations apportées ici sont provisoires et les localisations actuelles (* pour MBA Lyon, ** pour MCGR Lyon etc.) concernent seulement une partie du matériel 


\section{NOTES}

33. Seuls les trésors monétaires attestés ont été comptabilisés pour la présente étude (à l'exception des trouvailles récentes, inédites ou publiées depuis la parution des TAF). Les auteurs des TAF distinguent plusieurs catégories de trouvailles: les trésors certains ou probables, les dépôts significatifs (funéraires, votifs, dépôts de fondeur, bijoux monétaires, etc...) et les trésors douteux. Un trésor conservé au musée des Beaux-Arts de Lyon (Inventaire X, numéro 190, le 20 février 1855) a échappé aux rédacteurs des TAF : « 28 deniers en argent découverts sur le terrain des Hospices, place Grollier (Lyon) en 1855. Dans le nombre se trouve Othon, des Domitien, Vespasien, Maximin et Gordien père (Gordien Ier ?) ».

34. Le nombre estimé d'exemplaires varie entre 2000 et 5000 suivant les sources.

35. Ce trésor est recensé par les rédacteurs des TAF sous la forme de deux ensembles distincts. La lecture des inventaires du MBA de Lyon montre qu'il s'agit certainement d'une seule et même trouvaille.

36. Selon les édits d'Aphrodisias et du Maximum de 301, le nummus vaut 10 ou 12,5 deniers et pèse $10 \mathrm{~g}(1 / 32$ de la livre romaine). $\mathrm{Si}$, à l'époque de l'enfouissement des trésors de LanciéMorancé et de Villette-d'Anthon (vers 294-295), le nouvel antoninien ou aurelianus de 274 vaut toujours 2 deniers pour un poids théorique de 4,03g (1/80 de la livre romaine), sa valeur réelle est nettement supérieure à celle du nummus. En effet, ces deux dénominations sont similaires dans leur composition métallique (cuivre recouvert d'argent). Or le nummus vaut officiellement 5 ou 6 antoniniens, alors qu'il ne pèse pas 3 fois plus. Sa valeur effective est donc deux fois plus grande. Les propriétaires d'antoniniens n'ont donc pas intérêt à changer leurs doubles deniers contre des nummi, faute de quoi ils perdraient près de la moitié de leur capital.

37. Nous connaissons seulement leurs poids ( 5 deniers 18 grains, 5 deniers 18 grains et 5 deniers 16 grains) qui sont comparables à ceux des aurei contemporains contenus dans le trésor.

38. Archives départementales du Rhône, série R-78WP8.

39. Voir en dernier lieu François Baratte, La vaisselle d'argent en Gaule dans l'Antiquité tardive, Paris, 1993, p. 23-40 (rappel des vingt-quatre dépôts connus mêlant monnaies et argenterie). À propos du trésor de Vaise, voir Gérard Aubin dans ce même volume.

40. François Planet, « Autour du trésor de Ruffieu : une archéologie locale au service de l'histoire nationale », La monnaie, la cité, l'histoire : le Médaillier de Lyon, LyonParis, 1992, p. 56-67

41. Voir en dernier lieu Trésors Monétaires XVI, région Rhône-Alpes, Paris, 1997. 


\section{Bibliographie}

\section{Concernant le trésor de Vaise}

Durand-Godiveau 1994 : DURAND-GODIVEAU (H.). - Le trésor de Lyon-Vaise. Archéologia, ${ }^{\circ}$ 301, mai 1994, p. 18-23.

Patrimoine(s) 1992 : PATRIMOINE(S). - Les trésors de Charavay. DRAC Rhône-Alpes, 2, sept. 1992, p. 3

Le trésor de Vaise 1994 : LASCOUX (J.-P.), BARATTE (F.), METZGER (C.), AUBIN (G.), M.-CL. DEPASSIOT (M.-CL.). - Le trésor de Vaise. Lyon - Rhône 1994. Lyon : Musée de la civilisation galloromaine, $1994,36 \mathrm{p}$.

Pliska 1998 : PLISKA (P.). - La restauration du trésor de Lyon-Vaise. In : Nicolini (G.) dir. et Dieudonné-Glad (N.) dir. - Les métaux antiques: travail et restauration. Actes du colloque de Poitiers, 28-30 septembre 1995. Montagnac : éd. M. Mergoil, p. 181-185.

L'usure du temps 1997 : L'usure du temps. La restauration des objets du Patrimoine. Catalogue d'exposition, SaintRomain-en-Gal, 7 novembre 1997 - 31 mars 1998. nº 14-15, p. 32-35.

Mistero di una fanciulla 1995 : Mistero di una fanciulla. Catalogue d'exposition, Accademia Valentino, 17 déc. 199518fév. 1996. Milan : Skira éd., 1995, p. 101-117.

Lascoux 1996 : LASCOUX (J.-P). - Rue du Chapeau Rouge. In: Aubin (G.). - Rhône-Alpes (1988-1991), Gallia-Informations 1996, p. 190-191, fig. 121-124.

\section{Bibliographie générale}

Allmer 1888: ALLMER (A) et DISSARD (P.). - Inscriptions antiques du Musée de Lyon. Lyon, 1883-1893, 5 vol.

Amandry, Loriot 1986 : AMANDRY (M.), LORIOT (X.). - Fragment d'un trésor d'antoniniens probablement découvert à Annecy. BSFN, 41, 1986, p. 108-111.

Amandry et al. 1987 : AMANDRY (M.), DELESTREE (L.-P.), HOLLARD (D.) et METZGER (C.). - Le trésor de Mons-Boubert (Somme). Deniers et antoniniens de Lucille à Postume. Trésors Monétaires, IX, 1987, p. 31-45, pl. A (en frontispice) et IX-XI. 
Amandry et al. 1996 : AMANDRY (M.), ESTIOT (S.), GAUTIER (G.), PLANET (F.), et VICHERD (G.). Le trésor d'antoniniens des "Communaux d'Arésieux" à Villetted'Anthon (Isère), Actes des Journées Numismatiques de Lyon, 31 mai-2 juin 1996, BSFN, 51, juin 1996, p. 117-123.

Antique Silver 1994 : POPOVIC (I.), (éd.), Antique silver from Serbia. Belgrade, 1994.

Arias 1946-1948 : ARIAS (P. E). - Il piatto argenteo di Cesena. Annali della Scuola Archeologica di Atene, 24-26, 1946-1948, p. 309-344.

Arlaud et al. 1994 : ARLAUD (C.), BURNOUF (J.), BRAVARD (J.-P. ), LUROL (J.-M.), VEROTBOURRELY (A.). - Lyon Saint-Jean. Les fouilles de l'îlot Tramassac. Lyon: Service régional de l'Archéologie, DARA n 10, 1994, 151p. (série lyonnaise $n^{\circ} 4$ ).

Artaud 1846 : ARTAUD (J.-F.). - Lyon souterrain ou observations archéologiques et géologiques faites dans cette ville depuis 1794 jusqu'en 1836. Lyon. 1846, 259 p.

Aubin, Monin 1996 : AUBIN (G.), MONIN (M.). - Les moules monétaires du IIIe s. de la rue Soeur Bouvier à Lyon (Rhône), BFSN, 51, juin 1996, p. 112-116.

Audin 1956: AUDIN (A.). - Essai sur la topographie de Lugdunum. Institut des études rhodaniennes, Lyon, 1956,176 p.

Audra, Mathey 1992 : AUDRA (A.), MATHEY (P.). - Monnaies romaines. Archéologie et numismatique à Lyon (Ier s. av. - IIIe s. ap. J.-C.). Lyon : ALPARA, 1992, 81p.

Babelon 1916 : BABELON (E.). - Le trésor de Berthouville, Paris, 1916.

Bakker 1993: BAKKER (L.). - Raetien unter Postumus: Das Siegesdenkmal einer Juthungenschlacht im Jahre 260 n. Chr. aus Augsburg, Germania, 71, 1993, p. 369-386.

Baratte 1981 : BARATTE (F.). - Le trésor d'argenterie gallo-romaine de Notre-Dame d'Allençon (Maineet-Loire), 40e suppl. à Gallia, Paris, 1981.

Baratte 1986 : BARATTE (F.). - Le trésor d'orfêvrerie romaine de Boscoreale, Paris, 1986.

Baratte 1988 : BARATTE (F.).(éd.) - Argenterie romaine et byzantine, Actes de la table ronde tenue à Paris le 11-13 Oct. 1983, Paris, 1988, p. 66, nº 3

Baratte et al. 1990 : BARATTE (F.), LE BOT-HELLY (A.) et al., Le trésor de la place Camille-Jouffray à Vienne (Isère). Paris : CNRS, 1990, n 5-6, p. 53-64 (50e suppl. à Gallia).

Baratte 1992 : BARATTE (F.). - La vaisselle d'argent d'époque romaine en Normandie, In: L'art en Normandie. Actes du XXVIe Congrès des Sociétés Historiques et archéologiques de Normandie, Caen, 1992.

Baratte 1992 : BARATTE (F.). - Les trésors de temples dans le monde romain : une expression particulière de la piété. In : Boyd (S.), Mundell Mango (M.), Ecclesiastical Silver Plate in sixth-century Byzantium. Baltimor-Washington 1986, Washington, 1992, p. 111-121.

Baratte 1993 : BARATTE (F.). - La vaisselle d'argent en Gaule dans l'Antiquité tardive. Paris, 1993, p. 259-260.

Baratte 1998 : BARATTE (F.). - Il tesoro di Marengo, In : Archeologia in Piemonte, Mercando (L.) dir. Turin, 1998, p. 369-379.

Bastien 1988: BASTIEN (Р.). - Monnaie et Donativa au Bas-Empire. Wetteren: Editions Numismatique Romaine. 1988, 139 p., 7 pl. (Numismatique Romaine. Essais, Recherches et Documents, XVII).

Bastien 1992-1994 : BASTIEN (P.). - Le buste monétaire des empereurs romains. Wetteren : Editions Numismatique Romaine. 1992-1994, 3 vol.,768 p. + 48 p. et 266 pl. (Numismatique Romaine. Essais, Recherches et Documents, XIX). 
Bastien, Metzger 1977 : BASTIEN (P.) et METZGER (C.). - Le trésor de Beaurains (dit d'Arras). Wetteren : Editions Numismatique Romaine, 1977, 258 p., 18 pl. (Numismatique romaine. Essais, recherches et documents, $\mathrm{X}$ ).

Becker 1989 : BECKER (C.), JACQUIN (L.). - La sigillée du Centre de la Gaule. In : Trois ensembles de la fin du IIIe au milieu du IVe ap. J.-C. sur le site de l'îlot Vieille Monnaie à Lyon. SFECAG. Actes du Congrès de Lezoux, 1989, p. 93-100.

Bendinelli 1937 : BENDINELLI (G.). - Il tesoro di argenteria di Marengo. Turin, 1937.

Bellon 1995 : BELLON (C.). - L'occupation gallo-romaine de Gorge de Loup. In : Delavai (E.) et al. Vaise, un quartier de Lyon antique. Lyon: Service régional de l'Archéologie, DARA n 11, p. 130-178 (série lyonnaise $\mathrm{n}^{\circ}$ 5).

Beltrân Fortes, Ventura Villanueva 1992-93 : BELTRÂN FORTES (J.), VENTURA VILLANUEVA (A.). - Basis marmorea cum signo argenteo, Tabona, VIII-II, 1992-93, p. 373-389.

Bernhard et al. 1990 : BERNHARD (H.), et al. - Der romische Schatzfund von Hagenbach, Mayence, 1990.

Besly, Bland 1983 : BESLY (E.), BLAND (R). - The

Cunetio Treasure. Roman Coinage of the Third Century AD. Londres : British Museum, 1983, 199 p., 40 pl.

Blanchet 1900 : BLANCHET (A.). - Les trésors de monnaies romaines et les invasions germaniques en Gaule. Paris, 1900, $332 \mathrm{p}$.

Bland, Burnett 1988 : BLAND (R.), BURNETT (A.). - The Normanby hoard and other Roman coin hoards. Londres : British Muséum. 1988, 238 p., 40 pl. (Coins Hoards from Roman Britain, vol. VIII).

Boucher 1976 : BOUCHER (S.). - Recherches sur les bronzes figurés de la Gaule pré-romaine et romaine. Paris, 1976, p. 114.

Bourgeois, Christol 1973 : BOURGEOIS (A.), CHRISTOL (M.). - A propos d'un antoninianus inédit de Valérien le Jeune provenant du trésor de Chézelles (Indre) : la date d'ouverture de l'atelier de Cologne. BSFN, 28, juillet 1973, p. 415-418.

Braemer 1968 : BRAEMER (F.). - Sculptures en métal battu et repoussé de la Gaule romaine et des régions limitrophes. Revue archéologique, 1968, p. 327-354.

Brenot, Metzger 1992 : BRENOT (C.) et METZGER (C.). - Trouvailles de bijoux monétaires dans l'Occident romain. In : Brenot (C.) dir. et Loriot (X.) dir. - L'or monnayé III. Trouvailles de monnaies d'or dans l'Occident romain. Actes de la table ronde tenue à Paris les 4 et 5 décembre 1987, Paris : CNRS, 1992, p. 313-371, Pl. 1-4 (Cahiers Ernest-Babelon, 4).

CAG : CARTE ARCHÉOLOGIQUE DE LA GAULE (sous la dir. de M. Provost)

27. Eure, par Cliquet (D.), Académie des Inscriptions et Belles-Lettres 1993, 285 p.

45. Loiret, par Provost (M.), Paris : Académie des Inscriptions et Belles-Lettres, 1988, 249 p.

58. La Nièvre, par Bigeard (H.), Paris : Académie des Inscriptions et Belles-Lettres, 1996, 300 p.

59. Le Nord, par Delmaire (R.) Paris : Académie des Inscriptions et Belles-Lettres, 1996, 497 p.

63. Auvergne, par Provost (M.) et Mennessier-Jouannet (C.), Clermont-Ferrand,/l. Paris : Académie des Inscriptions et Belles-Lettres, 1994.

71/4. Saône-et-Loire, par Rebourg (A.), Paris : Académie des Inscriptions et Belles-Lettres, 1994, 552 p.

Cahn, Kaufmann 1984 : CAHN (H.), KAUFMANN (A.) éd., - Der spàtrömische Silberschatz von Kaiseraugst. Derendingen, 1984.

Callu 1960 : CALLU (J.-P). - Genio Popvli Romani (295316). Contribution à une histoire numismatique de la Tétrarchie, Paris, 1960, $134 \mathrm{p}$. 
Callu 1969: CALLU (J.-P.). - La politique monétaire des empereurs romains de 238 à 311. Paris : De Boccard, 1969. 562 p., 3 pl. (Bibl. des Ec. fr. d'Athènes et de Rome, 214).

Callu 1989 : CALLU (J.-P.). - L'empire gaulois selon J.-F. Drinkwater. Journal of Roman Archaeology, 2,1989, p. 362-373.

Callu, Loriot 1990 : CALLU (J.-P.), LORIOT (X). - L'or monnayé II. La dispersion des aurei en Gaule romaine sous l'Empire. Juan-les-Pins : Edition A.P.D.C.A., 1990, 591 p. (Cahiers Emest Babelon, 3).

Carcassonne, Christol 1974 : CARCASSONNE (C.) et CHRISTOL (M.). - L'aloi de l'antoninianus dans l'atelier de Cologne sous le règne de Valérien et de Gallien (256-260) : étude statistique. BSFN, 29, juillet 1974, p. 598-604.

Catalogue 1905 : Catalogue de la collection des objets antiques et du Moyen-Age provenant de la collection de M. Guilhou. Paris, 1905.

Catalogue Greek and Roman Coins 1991 : Catalogue Greek and Roman Coins from a distinguished american collection. Zurich : Bank Leu TDD, 15 mai 1991, p. 194-203.

Chastel 1995 : CHASTEL (J.), PLASSOT (E.), THIÉRIOT (F.). - Le quartier Saint-Pierre, la voie et les sépultures gallo-romaines. In : Delaval (E.) et al. - Vaise un quartier de Lyon antique. Lyon: Service régional de l'Archéologie, DARA n 11, p. 36-70 (série lyonnaise $n^{\circ}$ 5).

Charvet 1863 : CHARVET (J.). - Notice sur des monnaies et des bijoux antiques découverts en 1862 au Sault du Rhône. Paris, 1863, 17 p., 1 pl.

Chastagnol 1984a : CHASTAGNOL (A.). - Les jubilés décennaux et vicennaux des empereurs sous les Antonins et les Sévères. Revue numismatique. 1984, p. 104-124, pl. X-XX.

Chastagnol 1984b : CHASTAGNOL (A.). - Les fêtes décennales de Septime-Sévère. Bulletin de la société nationale des Antiquaires de France, 1984, p. 91-107, pl. VII, 3-4.

Christol 1975: CHRISTOL (M.). - Les règnes de Valérien et de Gallien (253-268) : travaux d'ensemble, questions chronologiques. Aufstieg und Niedergang der romischen Welt. II2, Berlin - New York : Walter de Gruyter, 1975, p. 803-827.

Christol 1977 : CHRISTOL (M.). - Effort de guerre et ateliers monétaires de la périphérie au IIIe s. ap. J.-C. L'atelier de Cologne sous Valérien et Gallien. In: Armées et fiscalité dans le monde antique : Paris, 14-16 octobre 1976. Paris, CNRS, 1977, p. 235-275 (Colloques nationaux du CNRS, n 936).

Christol 1990 : CHRISTOL (M.). - [Compte rendu de Drinkwater 1987]. Revue numismatique, 1990, p. 308-313.

Christol 1997 : CHRISTOL (M.). - Les déplacements du collège impérial de 256 à 258 : Cologne, capitale impériale. Cahiers Glotz, VIII, 1997, p. 243-253.

CIL : Corpus inscriptionum latinarum : MOMMSEN (T.) éd., HÜBNER (E.) éd., HIRSCHFELD (O.) éd., et al., 16 t. Berlin 1863 ss.

Collection Jameson 1913 : - Collection R. Jameson, t. II : Monnaies impériales romaines. Paris : Feuardent Frères, 1913, 96 p., 23 pl.

Comarmond 1844 : COMARMOND (A.). - Description de l'écrin d'une dame romaine trouvé à Lyon en 1841. ParisLyon, 1844,48 p., 5 pl.

Corbier 1985: CORBIER (M.). - Dévaluations et évolution des prix (Ier-IIIe s.). Revue numismatique, 6e série, XXVII, 1985, p. 69-106.

Corbier 1989 : CORBIER (M.). - Dévaluations, inflations et circulation monétaire au IIIe s. In : Morrisson (C.) éd., Lefort (J.) éd. - Hommes et richesses dans l'Empire byzantin, I. Paris, 1989, p. 195-211.

Curie 1923 : CURLE (A. O.). - The Treasure of Traprain. Edimbourg, 1923, n' 30 p. 36-39, $\mathrm{n}^{\circ} 44$ p. 49 et $\mathrm{n}^{\circ} 208$, p. 72. 
Das Haus lacht vor Silber 1987 : Das Haus lacht vor Silber. - Prittwitz von (H.-H)., H. Mielsch (H.), (éd.). Catalogue d'exposition, Bonn, 1997.

Delavai 1995 : DELAVAL (E.). - L'habitat privé de deux insulae de la ville haute de Lugdunum (Lyon) sous le HautEmpire romain. Thèse de doctorat nouveau régime. Université de Provence (AixMarseille), Aix-en-Provence, 1995.

Delaval et al. 1995 : DELAVAL (E.), BELLON (C.), CHASTEL (J.), PLASSOT (E.), TRANOY (L.). - Vaise un quartier de Lyon antique. Lyon: Service régional de l'Archéologie, DARA n 11, 291 p. (série lyonnaise $n^{\circ} 5$ ).

Demougeot 1962: DEMOUGEOT (E.). - Les martyrs imputés à Chrocus et les invasions alamanniques en Gaule méridionale. Annales du Midi, 74, 1962, p. 5-28.

Demougeot 1969 : DEMOUGEOT (E.). - La formation de l'Europe et les invasions barbares, t. 1 : Des origines germaniques à l'avènement de Dioctétien. Paris : Aubier, 1969, 615 p., 18 cartes h.-t.

Demougeot 1985 : DEMOUGEOT (E.). - Autun et les invasions germaniques de la seconde moitié du IIIe s.. In: Sept siècles de civilisation gallo-romaine vus d'Autun. Autun: Société Eduenne des Lettres, Sciences et Arts, 1985, p. 111-143.

Desbat 1984 : DESBAT (A.). - Les fouilles de la rue des Farges à Lyon 1974-1980. Lyon: Groupe Lyonnais de Recherche en Archéologie gallo-romaine, 1984, 107 p.

Doyen 1984 : DOYEN (J.-M.). - Le trésor de Givry (Hainaut) : aureus, deniers et antoniniens de Septime Sévère à Valérien. Helinium, XXIV, 1984, p. 247-263.

Drexel 1909 : DREXEL (Fr.). - Alexandrinische Silbergefasse der Kaiserzeit. Bonner Jahrb. 1909, p. 177-235,

Drinkwater 1987 : DRINKWATER (J.-F.). - The Gallic Empire. Separatism and Continuity in the NorthWestern Provinces of the Roman Empire A.D. 260-274. Stuttgart: Franz Steiner Verlag Wiesbaden $\mathrm{GmbH}, 1987,276$ p. (Historia Einzelschriften, 52).

Espérandieu 1910 : ESPERANDIEU (E.).-Recueil général des bas-reliefs, statues et bustes de la Gaule romaine III, Lyonnaise Iere partie. Paris, 1910.

Estiot 1996 : ESTIOT (S.). - Le troisième siècle et la monnaie : crise et mutations. In : Fiches (J.-L.) éd. - Le IIIe siècle en Gaule Narbonnaise. Données régionales sur la crise de l'Empire. Actes de la table ronde du GDR 954, tenue à Aix-en-Provence les 15-16 septembre 1995, Sophia Antipolis : Editions APDCA, 1996, p. 33-70.

Filow 1914 : Filow (B.). - Le trésor romain de Nicolaevo. Bull, de la Société arch. bulgare, 1914, p. 1-48.

Foucray 1995 : FOUCRAY (B.). - Le trésor de deniers et d'antoniniens de La Grosse Haie à Souzyla-Briche (Essonne). Trésors monétaires, XV, 1995, p. 35-70, pl. V-VII.

Gallia 1975 : Informations, Gallia, XXXII, 2, 1975, p. 547.

Garbsch. Overbeck 1989 : GARBSCH (J.), OVERBECK (B.). - Spätantike zwischen Heidentum und Christentum. Catalogue d'exposition, Prohistoriche Staatsammlung, Munich, 1989.

Geominy, Franchi,1995 : GEOMINY (W.), FRANCHI (Cl.). - Die Silberbiiste des Kaisers Galba. Il busto argenteo del imperatore Galba. Bonn, 1995.

Greifenhagen 1970-1975 : GREIFENHAGEN (A.). - Schmuckarbeiten in Edelmetall, I et II. Berlin, 1970-1975.

Gricourt 1990 : GRICOURT (D.). - Les premières émissions de Postume à Trèves. In: Trésors Monétaires, XII, 1990. Paris : Bibliothèque Nationale, 1991, p. 31-54, pl. IXXV.

Guillemain 1961 : GUILLEMAIN (Ch.). - Histoire de la commune de Vaise. Faubourg de Lyon. Album du Crocodile, 29e année, Lyon, 1961, p. 14-15. 
Guey 1962 : GUEY (J.). - L'aloi du denier romain de 177 à 211 après J.-C. (Etude descriptive). Revue numismatique, 1962, p. 73-140, pl. VI, 2 dépliants.

Guey 1965 : GUEY (J.). - Peut-on se fier aux essais chimiques ? Encore l'aloi du denier romain de 177 à 211 ap. J.-C. Revue numismatique, 1965, p. 110-122, 1 dépliant.

Guiraud 1988 : GUIRAUD (H.). - Intailles et camées de l'époque romaine en Gaule (territoire français). Paris : CNRS, 1988, 236 p., 68 pl. (48e supplément à Gallia).

Hauser 1992: HAUSER (S.). - Spàtantike undfrühbyzantinische Silberloffel. Bemerkungen zur Produktion von Luxusgiitern im 5. bis 7. Jahrhundert, JbAC, Ergänzungsband 19, Munster, 1992, $\mathrm{n}^{\circ}$ 50-52, p. 104, pl. 9a.

Hill 1977 : HILL (P. V.). - The coinage of Septimius Severus and his Family of the Mint of Rome AD. 193-217. 2eéd. Londres : Spink and Son Ltd, 42 p., 2 pl.

Hilgers 1969 : HILGERS (W.). - Lateinische Gefàssnamen, Diisseldorf, 1969.

Hollard 1990 : HOLLARD (D.). - [Compte-rendu de Bland, Burnett, 1988]. Revue numismatique, 1990, p. 314317.

Hollard 1995 : HOLLARD (D.). - La crise de la monnaie dans l'empire romain au IIIe s. après J.-C. Synthèse des recherches et résultats nouveaux. Annales Histoire, Sciences Sociales, 50, septembreoctobre 1995, p. 1045-1078.

Huvelin, Loriot 1992 : HUVELIN (H.). LORIOT (X.). - Les trouvailles de monnaies d'or dans l'Occident romain au IIIe s. de notre ère. In : Brenot (C.) dir. et Loriot (X.) dir. - L'or monnayé III. Trouvailles de monnaies d'or dans l'Occident romain. Actes de la table ronde tenue à Paris les 4 et 5 déc. 1987, Paris : CNRS, 1992, p. 215-272 (Cahiers Ernest-Babelon, 4).

Jenkins 1947 : JENKINS (G.K.). - The Caister-byYarmouth hoard. The Numismatic Chronicle, 6e s., VII, 1947, p. 175-179.

Joffroy 1978: JOFFROY (R. ). - Découverte d'une cachette de bronzes gallo-romains à Champoulet (Loiret). C.R.A.I., 1978, p. 795-814.

Johns 1996 : JOHNS (C.M.). - The Jewellery of Roman Britain. Celtic and classical traditions. Londres, 1996.

Johns, Potter 1983 : JOHNS (C.M.), POTTER (T.). - The Thetford Treasure. Roman Jewellery and Silver. Londres, 1983.

Kaufmann-Heinimann 1998 : KAUFMANN-HEINIMANN (A.). - Gotter und Lararien aus Augusta Raurica. Herstellung, Fundzusammenhänge und sakrale Funktion figürlicher Bronzen in einer römischen Stadt, Forschungen in Augst 26, GF 32, 1998.

Kellner, Zahlhaas 1993 : KELLNER (H. J.), ZAHLHAAS (G.). - Der Romische Tempelschatz von Weissenburg i. Bay. Mayence, 1993.

Koethe 1942 : KOETHE (H.). - Zur Geschichte Galliens im dritten Viertel des 3. Jahrhunderts. 32 Bericht der rômischgermanischen Kommission. Berlin, 1942 (paru en 1950), p. 199-224.

Kunisz 1990: KUNISZ (A.). - Remarques sur la circulation monétaire sur les territoires européens de l'Empire romain au cours de la première moitié du IIIe s. BSFN, 45, décembre 1990, p. $942-948$.

Künzl 1983 : KÜNZL (E.). - Zwei silberne Tetrarchenportrats im RGZM und die römischen Kaiserbildnisse aus Gold und Silber. Jahrbuch des rômischgermanischen Zentralmuseum Mainz, 30, 1983, p. 381-402.

Kiinzl 1993 : KÜNZL (E.). - Der Alamannenbeute aus dem Rhein bei Neupotz. Mayence, 1993.

Lafaurie 1975a: LAFAURIE (J.). - L'empire gaulois. Apport de la numismatique. Aufstieg und Niedergang der römischen Welt. II-2, Berlin - New York : Walter de Gruyter, 1975, p. 853-1012. 
Lafaurie 1975b: LAFAURIE (J.). - Réformes monétaires d'Aurélien et de Dioclétien. Revue numismatique, 17, 1975, p. 74-138, pl. VII-VIII.

Lallemand 1971 : LALLEMAND (J.). - Trésor de monnaies romaines à Oombergen : antoniniens de Caracalla à Valérien-Gallien (Salonin). Helinium, XI, 1971, p. 48-60.

Lallemand, Van Heesch 1994 : LALLEMAND (J), VAN HEESCH (J.). - La période romaine. In : Rogge (M.), Beeckmans (L.) éd. - Geld uit de grond : Tweeduizend jaar muntgeschiedenis in Zuid-OostVlaanderen. Zottegem, 1994, p. 210-211.

Laubenheimer 1985 : LAUBENHEIMER (F.). - La production des amphores en Gaule Narbonnaise. Paris : Les Belles-Lettres, 1985. (Annales Littéraires de l'Université de Besançon, 327).

Lavagne 1989: LAVAGNE (H.). - Us dieux de la gaule romaine. Luxembourg: galerie d'art municipale, 14 avril - 5 juin, 1989.

Lavagne 1994: LAVAGNE (H.). - Une nouvelle inscription d'Augsbourg et les causes de l'usurpation de Postume. CRAI, avril-juin 1994, p. 431-445.

Le Gentilhomme 1946 : LE GENTILHOMME (P.). - La trouvaille de Nanterre. Revue numismatique, IX, 1946, p. 15114, pl. II-IV.

Liénard 1881 : LIÉNARD (F.). -Archéologie de la Meuse, I, partie sud du département. Verdun, 1881.

LIMC : Lexicon Iconographicum Mythologiae Glassicae, Zurich. 1981-1997.

Loriot 1975 : LORIOT (X.). - Les premières années de la grande crise du IIIe s. : de l'avènement de Maximin le Thrace (235) à la mort de Gordien III (244). Aufstieg und Niedergang der rômischen Welt. II-2, Berlin - New York : Walter de Gruyter, 1975, p. 657-787.

Loriot 1983 : LORIOT (X.). - Bijoux monétaires du IIIe s. I. Le collier du Vieil-Evreux (Eure). BSFN, 38 , janvier 1983, p. 265-269.

Loriot 1991 : LORIOT (X.). - Un ancien témoignage sur le trésor de Saint-Genis-Pouilly (Ain), BSFN, 46, mai 1991, p. 81-83.

Mandy 1983 : MANDY (B.). - Le quartier antique du Verbe Incarné. Histoire et Archéologie, 78, 1983, p. 23-26.

Marshall 1911 : MARSHALL (F.H.). - Catalogue of the Jewellery, Greek, Etruscan and Roman, British Museum. Londres, 1911.

Martin-Kilcher 1986 : MARTIN-KILCHER (S.). - Ein silbernes Schwertortband mit Niellodekor. Jahresberichte aus Augst und Kaiseraugst, 5, p. 147-203.

Matchabély 1970 : MATCHABÉLY (K.). - Les coupes d'argent d'Armaziskhévi, Géorgie. Tbilissi, 1970.

Matchabély 1976 : MATCHABÉLY (K.). - La toreutique de la Géorgie dans l'antiquité tardive. Tbilissi, 1976.

Mattingly 1939 : MATTINGLY (H.). - The great Dorchester hoard of 1936. Numismatic Chronicle, 5e s., XIX, 1939, p. 21-61, pl. II-IV.

Mouchmov 1934: MOUCHMOV (N.A.). - Le trésor numismatique de Réka-Devnia (Marcianopolis). Sofia : Musée national bulgare, 1934, 139 p., 5 pl. (Extrait de : - Annuaire du musée national bulgare, $\mathrm{t}$. $\mathrm{V}$, supplément).

Mowat 1888 : MOWAT (M.). - De quelques objets antiques incrustés de monnaies. Mémoires de la Société des Antiquaires de France, 1888. p. 220-238.

Mrozek 1975 : MROZEK (Stanislaw). - Prix et rémunération dans l'Occident romain (31 av. n. è. - 250 de n. ̀̀.). Gdansk, 1975, $142 \mathrm{p}$.

Mutz 1990 : MUTZ (Fr.). - Das Kunstdrehen des Metalls bei den Römern. Munich, 1972, p. 86-87, fig. 202 ; Vienne 1990. 180 p.

Oliver 1977 : OLIVER (A.). - Silverfor the Gods. Toledo, 1977. 
Pelatan 1971 : PELATAN (J.P.). — La fouille de sauvetage du 21 quai Arloing (1967-1968). Bulletin mensuel de la Société Linéenne de Lyon, 1971, 10, p. 309-312.

Picard 1962: PICARD (Ch.). - Le Dieu thraco-phrygien Sabazios-Sabazius à Vichy. Revue archéologique du Centre de la France, I, 1, 1962, p. 10-30.

Planet 1992 : PLANET (F.). - Le trésor de Lentilly (1866), Bulletin des Musées et Monuments Lyonnais 1992/2, p. 18-31.

Plassot 1995 : PLASSOT (E.) et al. - Le quartier SaintPierre ; la maison aux Xenia. In: Delaval (E.) et al. - Vaise un quartier de Lyon antique. Lyon: Service régional de l'Archéologie, DARA ${ }^{\circ} 11 . \mathrm{p}$. 71-129, (série lyonnaise $n^{\circ}$ 5).

Pflaum, Huvelin 1981 : PFLAUM (H. G.), HUVELIN (H.). - Le trésor de Viuz-Faverges. In : Trésors monétaires, III, 1981, p. 33-76, pl. IX-XIV.

Phoungas 1990 : PHOUNGAS (A.). - Composition du lot de monnaies associé à l'équipement d'un militaire. In: Villedieu (F.). - Lyon St-Jean. Les fouilles de l'avenue Adolphe Max. Lyon: Circonscription des antiquités historiques, DARA n³,1990, p. 162, (série lyonnaise ${ }^{\circ} 2$ ).

Pilet-Lemière 1994 : PILET-LEMIERE (J.). - Les monnaies du castrum. In : - Lisieux avant l'an Mil. essai de reconstitution. Lisieux, 25 juin-29 août 1994. Lisieux, 1994, p. 58-59.

Pirzio 1992 : PIRZIO BIROLI STEFANELLI (L.). - L'Oro dei Romani, Gioielli di Età impériale. Rome, 1992.

Popovic 1996 : POPOVIC (I.). - Les bijoux romains du Musée national de Belgrade. Il, les bijoux d'or. Belgrade, 1996.

Py, Hiernard, Richard 1983 : PY (M.), HIERNARD (J.), RICHARD (J.-C.). - Le trésor de Nages-etSolorgues (Gard). In : Trésors Monétaires, V, 1983. Paris, Bibliothèque Nationale, 1983, p. 117-123, pl. XXXVII.

Remy 1984: RÉMY (R.) - Un trésor d'aurei à Villeurbanne (Rhône), BSFN, 10, déc. 1984, p. 564-566.

Riha, Stem 1982 : RIHA (E.), STERN (W. B.). - Die römischen Löffel aus Augst und Kaiseraugst, Forsch. in Augst 5, Augst, 1982.

Ross 1953 : ROSS (M.C.). - A silver treasure from DaphnéHarbié. Archaeology, 6-1, 1953, p. 40-41.

Sadurska, Bounni 1994 : SADURSKA (A.), BOUNNI (A.). - Les sculptures funéraires de Palmyre. Rome, 1994 (Rivista di archeologia, suppl. 13).

Schaad 1992: SCHAAD (D.) éd. - Le trésor d'Eauze (Gers). Bijoux et monnaies du IIle s. ap. J.-C. Toulouse : Ed. APAMP, 1992, LXXIX + 435 p., 93 pl. h.-t.

Schaad 1994 : SCHAAD (D.). - Les déplacements du Comitatus Gallieni entre 256 et 260, d'après une source épigraphique et des données numismatiques. BSFN, 49-6, juin 1994, p. 858-864.

Sena Chiesa 1998 : SENA CHIESA (G.). - In : L. Mercando [dir.] - Archeologia in Piemonte, II, L'età romana. Turin, 1998, p. 359-368.

Simonett 1946 : SIMONETT (Chr. ). - Der römische Silberschatz aus Wettingen. Zeitschrift fur Schweizerische Archaologie und Kunstgeschichte, 1946, p. 1-15.

Speidel 1992 : SPEIDEL (M. A.). - Roman Army Pay Scales. Journal of Roman Studies, 82, 1992, p. 87-106.

Steyert 1895 : STEYERT (A.). - Nouvelle histoire de Lyon et des provinces du Lyonnais, Forez, Beaujolais, Franc-Lyonnais et Dombes. Lyon : éd. Bernoux et Cumin, t. 1, 1895, 614 p.

Strong 1966 : STRONG (D. E.). - Greek and Roman Gold and Silver Plate. Londres, 1966. 
TAF : Corpus des trésors monétaires antiques de la France (publ. sous la dir. de X. Loriot et D. Nony), V/1 : RhôneAlpes, par X. LORIOT, B. REMY et A. BUISSON. Paris, 1987, 112 p. - V/2 : Rhône-Alpes, par X. LORIOT et B. REMY. Paris, 1988, 126 p.-VI : Aquitaine, par D. NONY, Paris, 1990, 106 p.

Thirion 1974 : THIRION (M.). - Le trésor de Zottegem - Velzeke II : argent jusqu'à Postume. Cercle d'Etudes Numismatiques. Bulletin, 11, 1, janvier-mars 1974, p. 12-15.

Tranoy 1995: TRANOY (L.) et GISCLON (J.-L.). - Le quai Arloing, artisanat et nécropole. In : Delaval (E.) et al. --Vaise un quartier de Lyon antique. Lyon : Service régional de l'Archéologie, DARA $\mathrm{n}^{\circ}$ 11, p. 179-254, (série lyonnaise $\mathrm{n}^{\circ}$ 5).

Trésors d'orfêvrerie 1989 : Trésors d'orfêvrerie GalloRomains, Baratte (éd.). Exposition Paris-Lyon, Musée du Luxemboug, Musée de la civilisation gallo-romaine, 1989. Paris : R.M.N. 302 p.

Turcan 1982: TURCAN (R.). - Les moules monétaires du Verbe Incarné (Lyon). In: Trésors Monétaires, IV, 1982, p. 929, pl. I-V.

Vermeule 1975 : VERMEULE (C.C.). - Numismatics in Antiquity. The Préservation and Display of Coins in Ancient Greece and Rome. Revue Suisse de Numismatique, 54, 1975, p. 5-32.

Veyne 1976 : VEYNE (P.) - Le pain et le cirque. Sociologie historique d'un pluralisme politique. Paris : Seuil, 1976, 800 p. (coll. l'univers historique).

Villedieu 1990: VILLEDIEU (F.). - Lyon Saint-Jean. Les fouilles de l'avenue Adolph Max. Lyon: Circonscription des antiquités historiques, DARA n 3,239 p. (série lyonnaise $n^{\circ} 2$ ).

Walker, Bierbrier 1997 : WALKER (S.), BIERBRIER (M.). - Ancient Faces. Mummy portraits from Roman Egypt. Londres : British Museum Press.

\section{Documents}

Battiga 1997 : BATTIGA (K.). - Meules et meuneries dans les villa du Rhône (époque gallo-romaine). Mémoire de maîtrise présenté sous la direction de J.-C. Beal, Université Lyon II, 1997, 103 p.

Bellon et al. 1994 : BELLON (C.), FRANC (O.), GENIN (M.), PERRIN (F.), DE SURY (B.), THÉVENIN (E.), et FELLMANN (D.). - 9, rue du docteur Horand, 69009, Lyon, Horand II 93, lots 5 bâtiments A et B. Document final de synthèse, AFAN. Conservé à la DRAC Rhône-Alpes, Service Régional de l'Archéologie, Lyon, 1994, 91 p. et annexes.

Bellon et al. 1996 : BELLON (C.), FRANC (O.), THÉVENIN (E.), et VICARD (T.). - 9, rue du docteur Horand, 69009, Lyon, Horand III 1995, lot 3. Document final de synthèse AFAN. Conservé à la DRAC Rhône-Alpes, Service Régional de l'Archéologie, Lyon, 1996,92 p. et annexes.

Celestin et al. 1993 : LE NÉZET-CELESTIN (M.), MONNOYEUR-ROUSSEL (V.), PLASSOT (E.). - Place Valmy, fouille de la future station de métro. Document final de synthèse, AFAN. Conservé à la DRAC Rhône-Alpes, Service Régional de l'Archéologie, Lyon, 1993, 303 p.

Chastel 1987 : CHASTEL (J.) et HÉNON (PH.). - Sondages archéologiques dans l'emprise de la ZAC Michel Berthet (Quartier Saint-Pierre de Vaise) à Lyon (Rhône). Rapport, DRAC Rhône-Alpes, Service Régional de l'Archéologie, Lyon, 1987.

Gisclon 1995 : GISCLON (J.-L.), BEL (V.). - Nécropole romaine et habitat médiéval, 54 bis, rue P. Audry, Lyon. Document final de sythèse, AFAN. Conservé à la DRAC Rhône-Alpes, Service Régional de l'Archéologie, Lyon, 1995.

Jacolin 1963 : JACOLIN (P.), PEILLON (P.). -

Découvertes archéologiques de la Solitude à Lyon (années 1961-1963). Rapport, DRAC Rhône-Alpes, Service Régional de l'Archéologie, Lyon, 1963, 71 p.

Jacquin et al. 1984 : JACQUIN (L.), et al. - îlot 24, 7-9-11 rue de la Monnaie. Rapport, S.A.M. DRAC Rhône-Alpes, Service Régional de l'Archéologie, Lyon, 1984, 87 p. 
Lascoux et al. 1987 : LASCOUX (J.-P), MARTIN (J.), WIDLAK (W.). - ZAC Saint-Vincent, secteurs I et Ib. Fouilles de sauvetage programmé, S.A.M. DRAC RhôneAlpes, Service Régional de l'Archéologie, Lyon, 1987, $93 \mathrm{p}$.

Lascoux et al. 1994a : LASCOUX (J.-P.), JACQUET (P.), NOURISSAT (S.), WIDLAK (W.). - ZAC SaintVincent, secteurs II, III et IV. Document final de synthèse, S.A.M. DRAC Rhône-Alpes, Service Régional de l'Archéologie, Lyon, 1994, volume I, 34 p. et annexes.

Lascoux 1994b : LASCOUX (J.-P). - Recherches archéologiques sur le site de la ZAC Charavay à Lyon (époque gallo-romaine). Mémoire de Maîtrise sous la direction de M. Yann Le Bohec. Université Lyon III. 1994, $184 \mathrm{p}$.

Lasfargues 1973 : LASFARGUES (J.). - Rapport de correspondant des Antiquités Historiques. Chantier de fouille de sauvetage rue P. Audry à Lyon 69005. Rapport Préliminaire. DRAC Rhône-Alpes, Service Régional de l'Archéologie, Lyon, 1973, 4 p.

Monin et al. 1995 : MONIN (M.), AYALA (G.), HORRY(A.). - 10, rue Marietton, 69009 Lyon, Document final de synthèse. S.A.M. et AFAN. DRAC Rhône-Alpes, Service Régional de l'Archéologie, Lyon, 1995, 86 p.

Rahatsöz et al. 1995 : RAHATSÔZ (M.), et al. - Rue du Bourbonnais "les Blanchisseries", Document finale de synthèse, AFAN. Conservé à la DRAC Rhône-Alpes, Service Régional de l'Archéologie, Lyon, 1995, 109 p. et annexes.

Tarras 1992: Tarras (M.). - Tranchée ouverte de l'extension de la ligne D, rue M. Berthet. Rapport, DRAC RhôneAlpes, Service Régional de l'Archéologie, Lyon, 1992, 2 p., 10 Pl.

\section{Abréviations}

APDCA : Association pour la Promotion et la Diffusion des Connaissances Archéologiques.

BSFN : Bulletin de la Société française de numismatique.

CIL : Corpus Inscriptionum Latinarum.

CRAI : Comptes rendus de l'Académie des Inscriptions et Belles-Lettres.

M.B.A. : Musée des Beaux-Arts.

MCGR : Musée de la civilisation gallo-romaine.

MGH : Monumenta germaniae historica.

S.A.M. : Service archéologique municipal de Lyon. 


\section{Crédits des illustrations (type annexe)}

\section{CRÉDITS DES ILLUSTRATIONS}

Nous remercions le Musée de la civilisation gallo-romaine à Lyon pour le prêt de nombreux documents.

\section{Contexte archéologique}

M.-N. Baudrand (dessins) : fig. 4 et 12

P. Gayte mise au point M.-N. Baudrand : fig. 1

S. Nourissat (AFAN) mise au point M.-N. Baudrand : fig. 2,

3,5 à 11.13 .14

Cl. S.A.M. : fig. 15

\section{Restauration}

Cl. Atelier Gabriel Chapotat, CREAM. Vienne : fig. 18, 19, 20

\section{Inventaire}

Cl. J.-M. Degueule, MCGR : fig. 21

\section{Vaisselle et statuaire}

Les clichés ont été effectués par $\mathrm{Ch}$. Thioc sauf :

M.-N. Baudrand (dessins) : fig. 23, 28, 29, 30, 31, 60, 61, 72, 77, 83

Cl. Bibliothèque Nationale de France : fig. 25, 75

Cl. British Museum : fig. 26, 33, 65, 76, 79, 80

Cl. Bulloz : fig. 34

Cl. P. Plattier : fig. 35

Cl. Chuzeville : fig. $32,69,70,74,82,86$

Cl. Kunsthistorisches Museum, Vienne : fig. 57

Le Caire, musée national, d'après Strzygowski : fig. 58

Cl. E. Schulz, Rumermuseum Augs : fig. 59

Cl. Rheinisches Landesmuseum, Bonn : fig. 62, 68

Cl. Römisch-Germanisches Museum, Cologne : fig. 63 
Cl. Mittelrheinisches Landesmuseum Mainz : fig. 64

Cl. Kunstwerke der Antike : fig. 73

Cl. Musée royal de Mariemont : fig. 81

Cl. Museo Archeologico, Aoste : fig. 88

F. Baratte : fig 36

\section{Les bijoux}

Les clichés ont été effectués par $\mathrm{Ch}$. Thioc sauf :

Cl. Bibliothèque Nationale de France, cabinet des médailles : fig. 98

Cl. J.-P. Godais, Musée d'Evreux : fig. 99

Cl. Jutta Tietz, Staatliche Museen, Antikensammlung Berlin : fig. 103

M.-N. Baudrand : fig. 102

Cl. British Muséum, Romano-British Antiquities : fig. 105

D'après Brenot Metzger, 1992, mise au point F. Vaireaux (AFAN) : fig. 109

\section{Les monnaies}

Les clichés des monnaies du trésor (catalogue) ont été effectués par le Cabinet des médailles de la Bibliothèque Nationale de France sur des moulages réalisés par $\mathrm{D}$. Clot.

Cl. Ch. Thioc : fig. 112, 115, 116

Cl. Bibliothèque Nationale de France : fig. 117

J. Hiernard et X. Loriot : fig 121

\section{Annexe}

Cl. Musée des Beaux-Arts, Lyon : fig. 123, 124, 125, 126 


\section{Résumé}

mots-clefs : Lyon/Lugdunum ; Vaise; Antiquité ; Gaule romaine; Bas-Empire ; urbanisme ; voie ; habitat ; entrepôt ; potier ; trésor ; dépôt ; vaisselle ; coupe ; plateau ; couvert ; cuillers ; statuette ; Fortuna : Helios ; Jupiter ; déesse ; busteportrait ; empereur ; bijou; bracelet; collier; pendant d'oreille; bague; intaille; médaillon monétaire; monnaie ; aureus ; denarius ; antoninianus ; or ; argent ; émeraude ; perle ; grenat ; nicolo ; pâte de verre.

2 La réalisation de fouilles archéologiques préventives lors d'un projet immobilier dans le quartier de Lyon-Vaise (ZAC Charavay) a permis la découverte d'un trésor du BasEmpire.

3 Dans l'Antiquité, cette partie de la plaine de Vaise se caractérise par le développement, hors les murs de Lugdunum, d'un secteur urbanisé le long d'un des axes majeurs du réseau mis en place par Agrippa (voie de l'Océan).

4 L'étude archéologique retrace l'évolution du site du Ier s. av. J.-C. au IVe s. ap. J.-C. Après les premières occupations pré-augustéennes, celui-ci est aménagé, au début du Ier s., en îlots comportant des habitations, des ateliers et des entrepôts (insulae) dont l'organisation est plus particulièrement perceptible du Ier au IIIe s. Le IVe s. semble marqué par un relatif déclin du quartier qui se resserre autour d'un noyau dont la périphérie est progressivement affectée à l'usage funéraire.

5 Le contexte de l'enfouissement du trésor, s'il est en partie connu, reste toutefois problématique: il s'agit en fait de deux dépôt, très proches mais biens distincts, d'objets précieux dans des caches ménagées sous le sol. Faut-il les attribuer aux occupants de l'habitat de la deuxième moitié du IIIe s. ou à ceux de l'atelier de potier qui leur succèdent au début du IVe s. ?

6 Le premier dépôt rassemble deux bracelets en argent et un groupe de statuettes du même métal, plus au moins fragmentaires. Trois sont complètes : un Hélios dédicacé au numen d'un empereur, une Fortuna à double corne d'abondance et une déesse plus énigmatique, qui tient une coupe dans laquelle sont posés deux oiseaux. Une tête de Jupiter et celle d'un étonnant personnage dont la chevelure s'orne de deux excroissances s'ajoutent à cet ensemble que complète un buste-portrait, probablement celui d'un empereur (Gallien?). 
7 Le second, renfermé dans une caisse de bois, consiste en vaisselle, bijoux et monnaies. L'argenterie comprend une coupe décorée d'un Mercure, deux petits plateaux ornés de reliefs (dont l'un n'est qu'en bronze plaqué d'argent), quatorze cuillers et deux couverts fragmentaires. Ces objets rentrent tous dans des séries bien connues en Gaule ; leur formes et leur décors permettent d'en situer l'exécution dans le courant du IIIe s. Le lot de bijoux réunit deux bracelets en or, deux paires de pendants d'oreilles en or, émeraude, perle et pâte de verre, deux bagues à chaton (dont un nicolo) et un médaillon monétaire (aureus de Gordien III, 238-244). Au fond de la caisse, une bourse contenait 81 monnaies en argent - 29 deniers et 52 antoniniens - en bon état dont l'arc chronologique s'étend de Vitellius (69) au règne conjoint de Valérien et Gallien (253-260). En fait, deux lots sont à distinguer: un premier de 62 monnaies, soit 19 "vieux" deniers de Vitellius à Commode, 8 plus récents tous datés de 201, 33 antoniniens et 1 denier frappés de 215 à 217 pour Caracalla et 1 denier de Diaduménien en 218 ; le second lot compte 19 antoniniens émis entre 241 et 258 .

8 On peut s'interroger sur la nature exacte de ces dépôts. Il y a là des sculptures dont la facture n'est guère homogène, peut-être une sorte de laraire privé ou encore des objets appartenant à un sanctuaire, puisque la plupart représentent des divinités dont l'une au moins, la déesse aux oiseaux, paraît avoir une forte connotation locale. L'argenterie représente à peine plus de deux livres de métal, et l'on parlera difficilement de "service", d'autant moins que les cuillers apparaissent plutôt dépareillées. Le lot de bijoux, davantage cohérent et de bonne qualité mais sans pièce exceptionnelle, illustre bien la parure en faveur dans les classes aisées de la Gaule vers le milieu du Me s. Quant aux espèces, divers arguments plaident en faveur d'une épargne anciennement constituée (peut-être à la suite de deux paiements principaux (donativa) en 202 et $217 / 218$, et maigrement complétée par un apport d'antoniniens, vers 258 - 260), un modeste patrimoine familial conservé par attachement sentimental, peutêtre à une génération de distance, plutôt qu'une réserve de valeurs.

9 Somme toute, il peut s'agir d'un trésor familial, y compris son laraire, caché dans l'attente de jours meilleurs, mais aussi d'un lot réuni au hasard par un artisan qui s'apprêtait à le refondre, ou même par un voleur. L'Hélios dédicacé oriente toutefois vers l'hypothèse d'un dépôt votif, qui n'est pas contradictoire avec la présence de biens domestiques, tout objet pouvant devenir votif par l'usage qu'on en fait.

\section{SUMMARY}

10 Keywords: Lyon/Lugdumim ; Vaise; Antiquity; Roman Gaul; Late Empire; town planning; way ; housing; warehouse ; potter ; treasure ; deposit ; crookery ; dish ; plate ; cutlery ; statuette ; Fortuna ; Helios ; Jupiter ; goddess ; bust-portrait ; emperor ; jewel ; bracelet; necklace ; pendant earring ; ring ; intaglio, coin medallion ; coins ; aureus; denarius; Antoninians ; gold ; silver ; emerald ; pearl ; garnet ; nicolo ; molten glass.

11 A treasure from the Late Empire was discovered in the area of Lyon-Vaise (Charavay urban development zone) thanks to preventive archaeological excavations which were conducted during a property plan. 
12 In the Antiquity, the characteristic feature of Vaise plain lays in the development, outside Lugdunum, of a urbanized zone along one of the major axis of the road System which was set up by Agrippa (Ocean way).

The archaeological study recounts the évolution of the site from the Ist cent. B.-C. to the IVth cent. A.-D. After the early pre-Augustinian occupations, it was organized into several blocks including housing, workshops and warehouses (insulae) especially between the Ist and the IIIrd cent. The IVth cent, seems to be marked by a relative decline of the area which draws in around a core, the periphery being progressively alloted to funeral purposes.

14 The context of the treasure burying, although partly known, remains problematic : in fact, there are two deposits of precious objects, very close to each other but quite distinct, inside caches which were laid under the ground. Should they be attributed to the occupants of the houses from the second half of the IIIr cent., or to the occupants of the potter's workshop following in the beginning of the IVth cent.?

The first deposit gathers together two silver bracelets and a group of statuettes in the same metal, more or less fragmentary. Three of them are fully preserved: one Helios dedicated to an emperor's numen, one Fortuna with a double horn of plenty and one goddess, more enigmatic, carrying a dish with two birds inside. The head of a Jupiter and of an amazing personage with two outgrowths as an ornament in the hair add to the whole which is completed by a bust-portrait, probably representing an emperor (Gallian?).

16 The second deposit, locked inside a wooden box, consists of crookery, jewels and coins. The silverware is made up of one dish decorated with a Mercure, two small trays ornated with reliefs (one of them just in bronze and silver plated), fourteen spoons and two fragmentary cutleries. All these objects belong to well known series in Gaul : their shapes and decoration show that their were made in the Illrd cent. The set of jewels includes two golden bracelets, two pairs of golden, emerald, pearl and molten glass pendant earrings, two bezel rings (one of them with a nicolo), and one coin medallion (Gordien III aureus, 238-244). In the bottom of the box, there was a purse with 81 well preserved silver coins - 29 denarius and 52 Antoninian coins - with a chronological curve stretching from Vitellius (69) to the joint reign of Valerian and Gallian (253-260). In fact, there are two different sets : one with 62 coins, that is to say 19 "old"denarius from Vitellius to Commode, eight more recent all dated 201, 33 Antoninians and one denarius struck from 215 to 217 for Caracalla as well as one denarius from Diadumenian in 218 ; the second set has 19 Antoninian coins issued between 241 and 258.

17 One can wonder about the precise nature of these deposits. The sculptures found there do not have quite an homogeneous construction ; maybe a kind of Lares altar or objects belonging to a sanctuary, as most of them represent divinities, and one at least, the birds goddess, seems to have a strong local connotation. The silverware hardly represents more than two pounds of metal; it is therefore difficult to speak of a "table set" all the less as the spoons are rather incomplete. The set of jewels, which is more coherent and of good quality but with no exceptional piece, illustrates well the favourite jewels in the well-off classes of Gaul around the middle of the Illrd cent. Regarding the coins, there are several arguments speaking for a saving, rather than for a reserve currency, which was formerly made (maybe following two main payments (donativa) in 202 and 217/218, and poorly completed by an Antoninian contribution 
around 258-260), a small family inheritance safeguarded for sentimental reasons, maybe one generation away,

18 All in all, it might be a family treasure, including its Lares, waiting hidden for better days, but also a set gathered by chance by a craftsman who was about to melt it or even by a thief. Nevertheless, the dedicated Helios leads to the hypothesis of a votive deposit, which is not in contradiction with the presence of domestic goods, as any object can become votive depending upon the use which is made out of it.

\section{RESUMEN}

19 Palabras claves : Lyon/Lugdunum ; Vaise ; Antigiiedad; Galia romana ; bajo Imperio ; urbanismo ; via,; habitacion ; almacén ; alfarero ; tesoro ; depôsito ; vajilla ; taza ; bandeja ; cubierto ; cuchara ; figurina ; Fortuna ; Helios ; Jupiter ; diosa ; busto retrato ; emperador ; joya ; correa ; collar ; pendiente ; anillo ; intaglio ; medallónnn monetario ; moneda ; aureus ; denarius ; antoninianus ; oro : plata ; esmeralda ; perla ; granate ; nicolo ; pasta de vidrio.

Unas excavaciones arqueológicasggicas preventivas, que fueron realizadas durante un proyecto inmobilario en el barrio de Lyon-Vaise (zona de ordenaciónn territorial concertada de Charavay) permitieron el descubrimiento de un tesoro del bajo Imperio.

21 En la Antigüedad, esta parte de la llanura de Vaise esta caracterizada por el desarollo de un sector urbanizado fuera de Lugdunum, a lo largo de uno de los mayores ejes de la red instaurada por Agrippa (via del Oceano).

El estudio arqueológicogigico describe la evoluciónn del sitio desde el siglo I antes de J.-

C hasta el siglo IV despues de J.-C. Despues de las primeras ocupaciones preagustinianas, el sitio esta organizado en cuadras al principio del siglo I, con habitaciones, talleres y almacenes (insulae), sobre todo desde el siglo I hasta el siglo III. El siglo IV parece marcado por una relativa decadencia del barrio que se estrecha alrededor de un nucleo cuya periferia esta progresivamente destinada al uso funerario.

El contexto del enterramiento del tesoro, aunque sea en parte conocido, queda siendo problematico: se trata en realidad de dos depositos, muy próximos pero bastante distintos, de objetos preciosos dentro de unos escondrijos bajo el suelo. La cuestión es de saber si hay que atribuirles a los ocupantes de la habitaciónn de la segunda parte del siglo III o a los del taller de alfarero que les suceden al principio del siglo IV.

El primero depósitosito reune dos correas de plata y un grupo de figurinas del mismo métal, mas o menos fragmentarias. Très son complétas : un Helios dedicado al numen de un emperador, una Fortuna con una doble cornucopia, y una diosa mâs enigmâtica que tiene una taza en la cual dos aves estánn posados. Una cabeza de Jupiter y la de un personaje asombroso, cuya cabellera esta ornada con dos excrecencias, anaden a este conjunto, completado por un busto retrato, probablemente lo de un emperador (Galeno ?).

El segundo, dentro de una caja de madera, contiene bajilla, joya y moneda. Los objetos de plata incluyen una taza decorada con un Mercurio, dos pequenas bandejas ornadas de relieves (uno siendo solamente de bronce chapado de plata), catorce cucharas y dos cubiertos fragmentarios. Todos estos objetos pertenecen a una serie bien conocida en Galia. Sus formas y decoraciones permiten situar su fabricaciôn en el transcurso del siglo III. El lote de joyas contiene dos correas de oro, dos pares de pendientes de oro, de 
esmeralda, de perla y pasta de vidrio, dos anillos con chaton (incluyendo un nicolo) y un medallónnn monetario (aureus de Gordiano III, 238244). En el fondo de la caja, una boisa contenta 81 monedas de plata - 29 denarios y 52 antoninarios - en buen estado, cuya cronologia se extiende desde Vitellius (69) hasta el regno conjunto de Valeriano y Galiano (253-260). En realidad, hay que distinguir dos lotes : el primero con 62 monedas, o sea 19 denarios "antiguos"desde Vitellius hasta Comodo, ocho mâs recientes todos datados de 201, 33 antoninarios y un denario de Diadumenio de 218; el segundo lote contiene 19 antoninarios emitidos entre 241 y 258.

Se puede interrogarse acerca de la natura exacta de estos depósitossitos. Hay esculturas cuya ejecucciónn no es muy homogénea, quizâs un especie de larario privado o unos objetos perteneciendo a un santuario, ya que la mayor parte representa unas divinidades y que una de ellas, por lo menos, la diosa de los aves, parece tener una conotaciónn local fuerte. Los objetos de oro no representan mâs que dos libras, y se hablara dificilmente de "vajilla", tanto menos que las cucharas estân desparejadas. El lote de joyas, mâs coherente y de buena cualidad pero sin ninguna obra excepcional, ilustra bien el aderezo preferido en las clases acomodadas de Galia alrededor del medio del siglo III. En cuanto a los metâlicos, ciertos argumentas hablan en favor de un ahorro antigüamente constituido (quizâs después de dos pagos principales (donativa), un patrimonio familiar modesto conservado por apego sentimental, quizâs a una generacin de distancia, mâs bien que de una réserva de valores.

En resumen, se puede tratar de un tesorio familiar, incluyendo su larario, escondido en espera de dias mejores, pero también de un lote reunido al azar por un artesano que estaba a punto de refundirlo, o incluso por un ladrón.nn. Sin embargo, el Helios dedicado orienta hacia la hipótesistesis de un depósitosito votivo que no estâ en contradicciónn con la presencia de objetos domésticos ya que cualquier objeto puede volver a ser votivo por el uso que se hace.

\section{RIASSUNTO}

parole chiave: Lione/Lugdunum ; Vaise ; Antichità ; Gallia romana ; basso-Impero ; urbanesimo ; strada ; abitato ; deposito ; ceramista ; tesoro ; ripostiglio ; vasellame ; coppa ; vassoio ; posata ; cucchiai ; statuetta ; Fortuna ; Helios ; Giove ; dea ; bustoritratto ; imperatore ; gioiello ; braccialetto ; collana ; orecchino ; anello ; intaglio ; medaglia monetaria; moneta; aureo; denaro; antoniano ; oro; argento; smeraldo; perla ; granato ; nicolo; pasta di vetro.

La realizzazione di scavi archeologici preventivi ad un progetto immobiliare nel quartiere di Lione-Vaise (ZAC Charavay) ha permesso la scoperta di un tesoro del bassoImpero.

Nell'Antichità, questa parte della pianura di Vaise si caratterizza per lo sviluppo, al di fuori delle mura di Lugdunum, di un settore urbanizzato lungo uno degli assi maggiori delia rete stradale attuata da Agrippa (strada dell'Oceano).

Lo studio archeologico ritraccia l'evoluzione del sito dal le secolo a.C. al IVe secolo d.C. Dopo le prime occupazioni pre-augusteiane la zona è organizzata, aU'inizio del Ie s., in isolati d'abitazione, officine e depositi (insulae) la cui organizzazione è meglio percettibile dal Ie al IIIe secolo. Il IVe s. sembra segnato da un relative declino del 
quartiere che si concentra in un nucleo la cui periferia è progressivamente destinata ad uso funerario.

Il contesto dell'interramento del tesoro, se è conosciuto in parte, resta comunque problematico : si tratta in effetti di due ripostigli di oggetti preziosi praticati sotto il livello del terreno, molto vicini ma ben distinti. Bisogna attribuirli agli occupanti dell'abitato delia seconda metà del IIIe secolo o a quelli dell'officina di ceramica che sono succeduti loro aU'inizio del IVe secolo ?.

Il primo ripostiglio comprende due braccialetti in argento e un gruppo di statuette nello stesso metallo, più o meno frammentarie. Tre sono complete : un Helios dedicato al numen di un imperatore, una Fortuna con un doppio corno d'abbondanza e una dea più enigmatica, che porta una coppa nella quale sono posati due uccelli. Una testa di Giove e quella di un personaggio sorprendente, con la capigliatura ornata da due escrescenze, completano questo insieme al quale si aggiunge un busto-ritratto, probabilmente di un imperatore (Gallieno ?).

Il secondo, rinchiuso in una cassa di legno, consiste in vasellame, gioielli e monete. L'argenteria comprende una coppa decorata di un Mercurio, due piccoli vassoi ornati in rilievo (uno dei due è in bronzo placcato d'argento), quattordici cucchiai e due posate frammentarie. Questi oggetti corrispondono tutti a delle serie ben conosciute in Gallia ; le loro forme e decorazioni permettono di situarne la fabbricazione nel corso del IIIe secolo. Il gruppo di gioielli comprende due braccialetti d'oro, due paia di orecchini d'oro, smeraldo, perla e pasta di vetro, due anelli a castone (fra i quali un nicolo), una medaglia monetaria (aureo di Gordiano III, 238-244). In fondo alla cassa, una borsa conteneva 81 monete d'argento - 29 denari e 52 antoniani - in buono stato e il cui arco cronologico va da Vitellio (69) al regno comune di Valeriano e Gallieno (253-260). In realtà si devono distinguere due gruppi : un primo di 62 monete, ossia 19 "vecchi" denari da Vitellio a Commodo, 8 più recenti tutti datati del 201, 33 antoniani e un denaro battuti dal 215 al 217 per Caracalla e un denaro di Diadumenio nel 218; il secondo gruppo comprende 19 antoniani emessi tra il 241 e il 258.

Ci si puo interrogare sulla natura esatta di questi ripostigli. Ci sono delle sculture di fattura non omogenea, forse una specie di larario privato o forse degli oggetti appartenenti a un santuario, dato che la maggior parte rappresenta delle divinità tra le quali una almeno, la dea con gli uccelli, sembra avere una forte connotazione locale. L'argenteria rappresenta poco più di un chilo di metallo e si parlerà difficilmente di "servizio" tanto più che i cucchiai sembrano piuttosto scompagnati. Il gruppo di gioielli, più coerente e di buona qualità ma senza un pezzo eccezionale, corrisponde bene alle gioie della classe agiata della Gallia verso la metà del IIIe secolo. Quanto alle monete, diversi argomenti sono in favore di un risparmio costituitosi anticamente (forse in seguito a due pagamenti principali (donativa) nel 202, nel 217/18, e completato poveramente da un'apporto di antoniani verso il 258260), un modesto patrimonio familiare conservato per attaccamento sentimentale, forse a una generazione di distanza, più che una riserva di valori.

In conclusione, puó trattarsi di un tesoro familiare, compreso il suo larario, nascosto in attesa di giorni migliori, ma anche di un'insieme riunito a caso da un'artigiano che si accingeva a rifonderlo, o anche da un ladro. L'Helios dedicato ci orienta piuttosto verso l'ipotesi di un deposito votivo, che non è in contraddizione con la presenza di beni domestici, ogni oggetto potendo diventare votivo secondo l'uso che se ne fa. 